Ko: $: 6020$

NP: 9097

MIXAАН I. ПAZAPZH

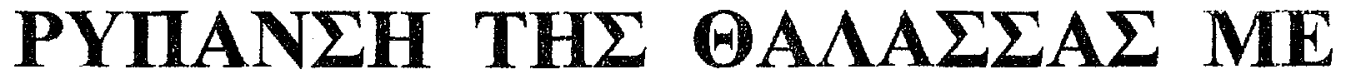 ПЕTPEАAIOEIАH АПО ПАОIA
}

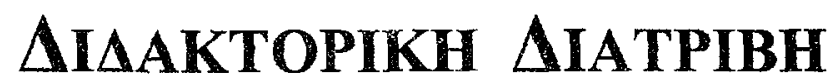

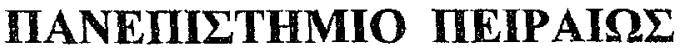

MEIPAIA 1992 
MIXAAH I. ПIAZAPZH

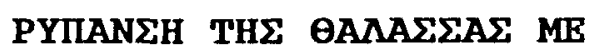

IIETPENAIOEIAH AПO ПINOIA

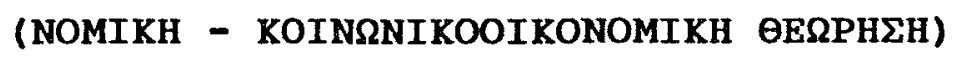

\author{
$\triangle I \triangle A K T O P I K H \quad \triangle I A T P I B H$

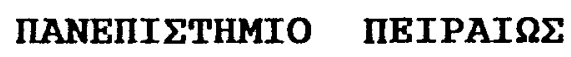 \\ MEIPAIAS 1992
}


$\Sigma E \Lambda I \Delta A$

MEPIEXOMENA

MEPOE A : OERPHTIKO TIAIEIO

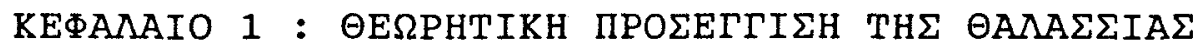
PYחAN $\Sigma$ H $\Sigma$

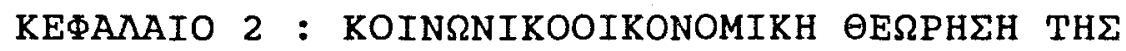

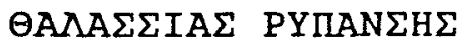

MEPOE B : NOMIKO IIAAIEIO

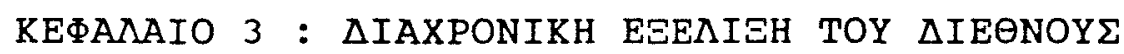

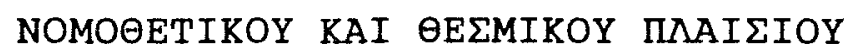

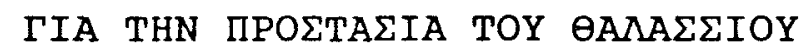
MEPIBA $\triangle O N T O \Sigma$

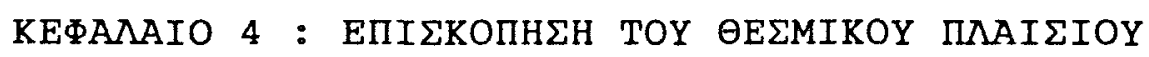

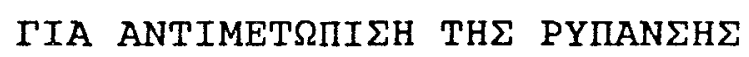

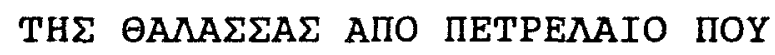
MPOEPXETAI AIO IINOIA 


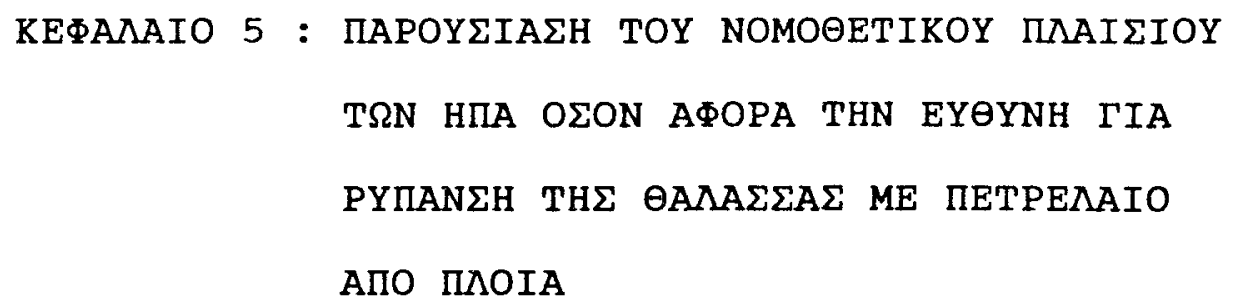

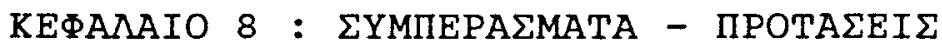

\section{BIB $\triangle I O \Gamma P A \Phi I A$}

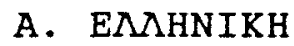
229
B. $\Xi E N O \Gamma \Lambda \Omega \Sigma \Sigma \mathrm{H}$
241

\section{ПAPAPTHMATA}




\section{EILAГRГH}

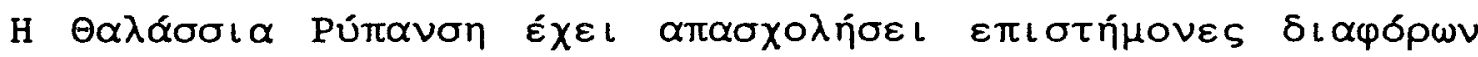

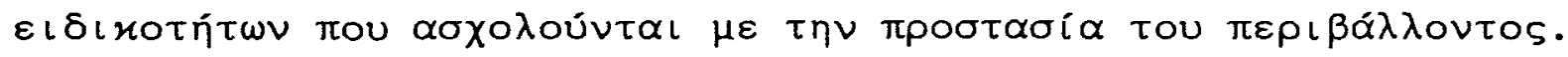

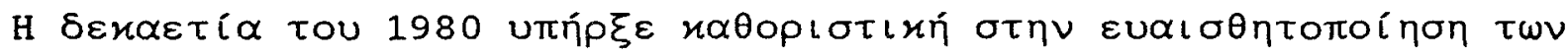

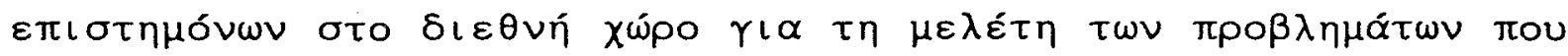

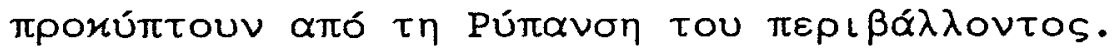

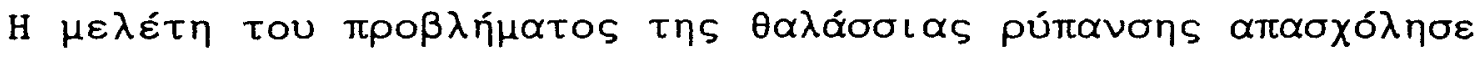

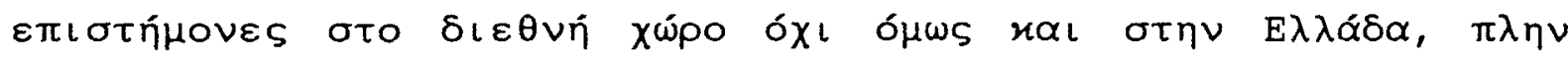

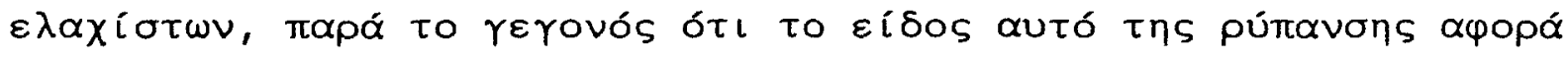

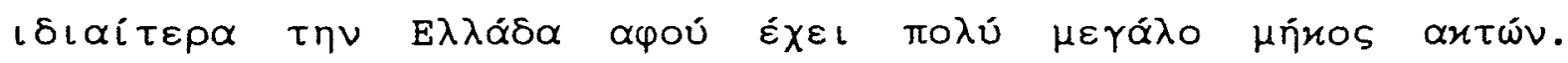

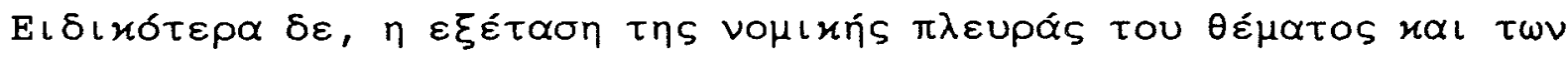

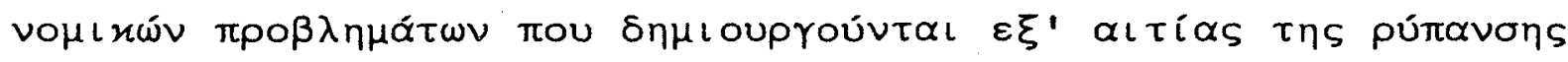

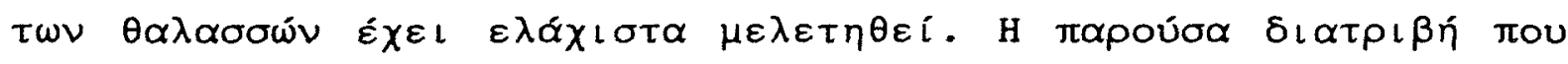

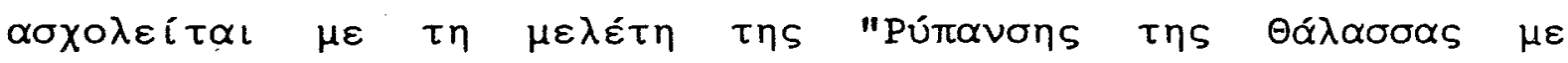

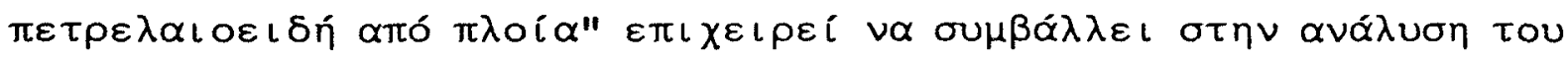

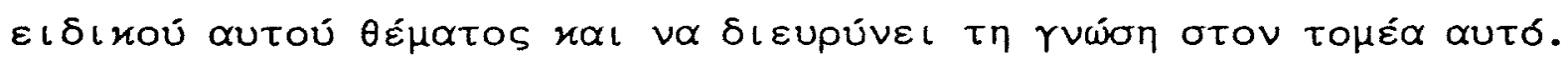

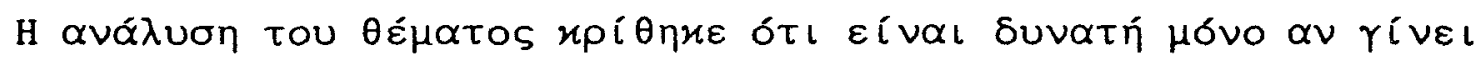

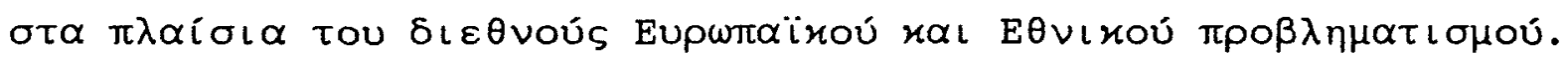

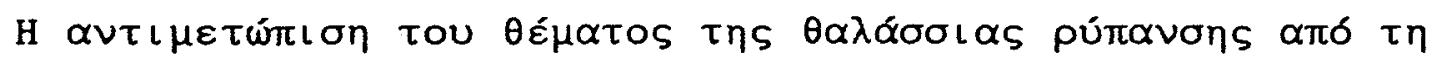




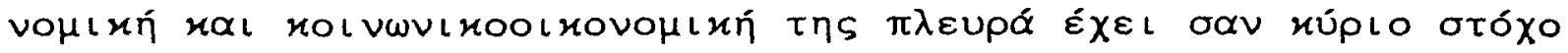

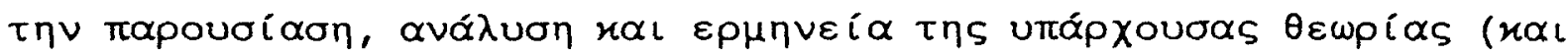

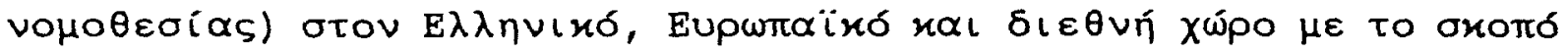

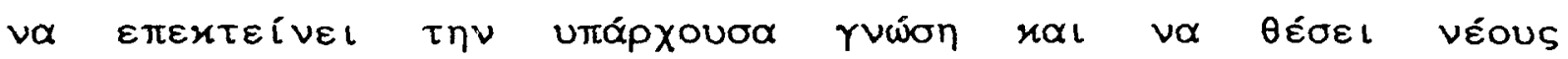

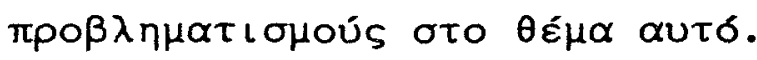

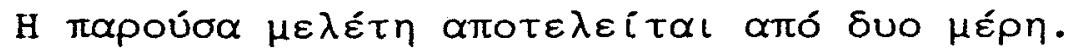

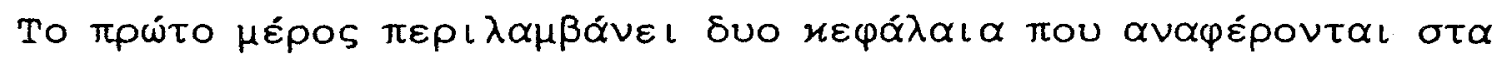

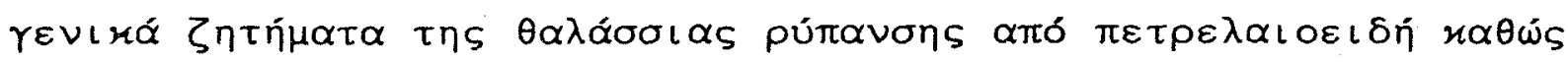

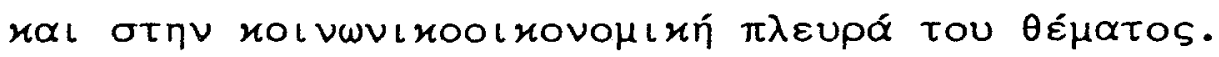

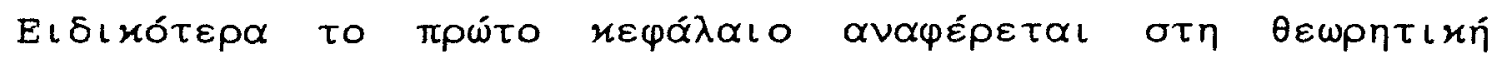

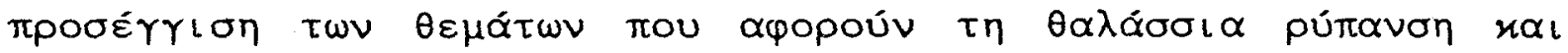

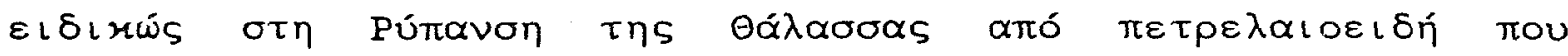

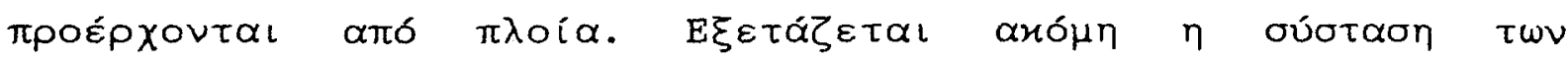

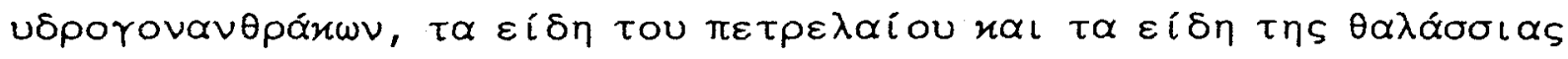

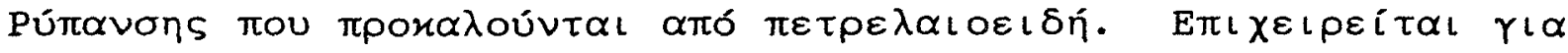

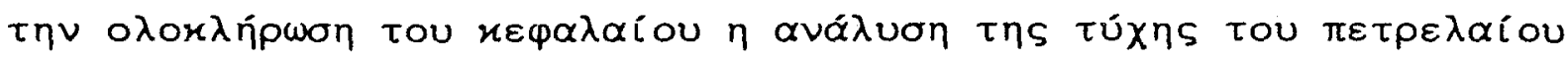

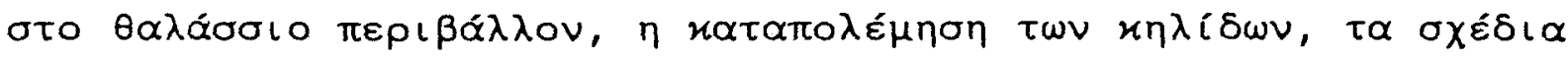

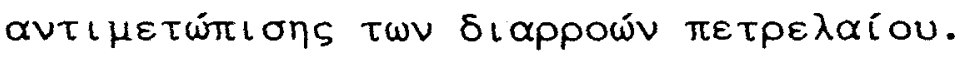

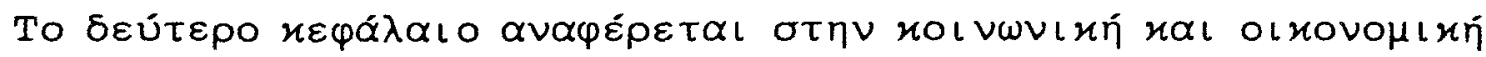

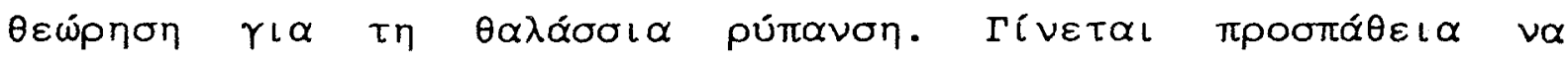

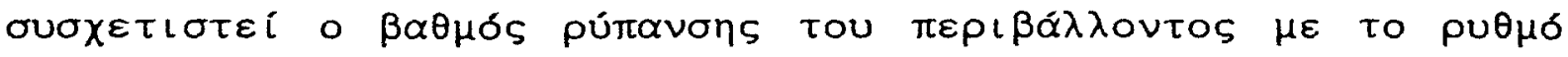

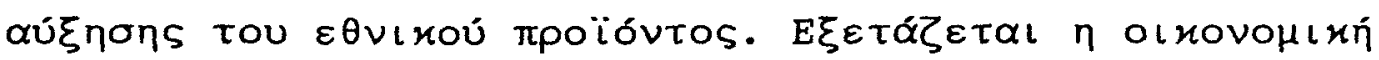




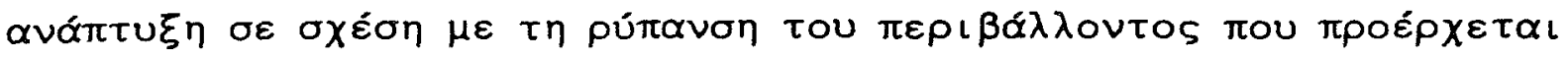

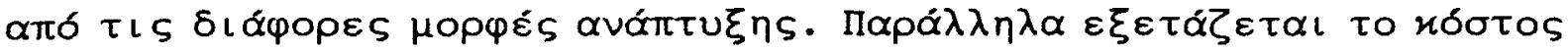

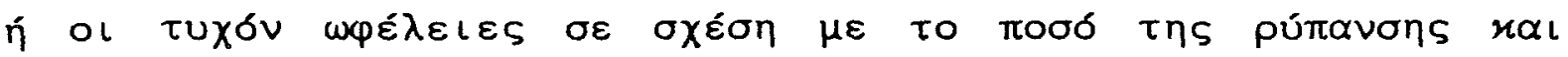

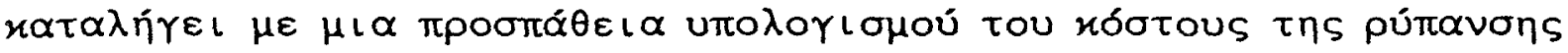

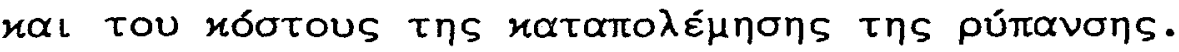

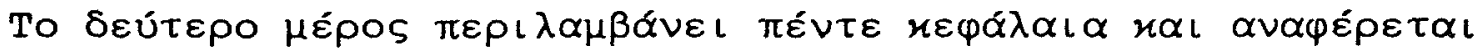

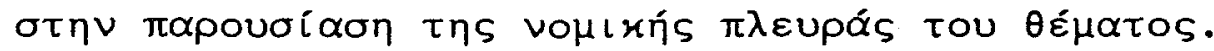

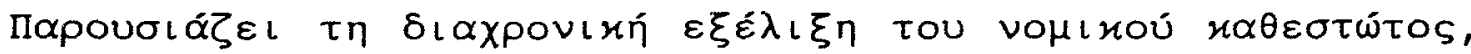

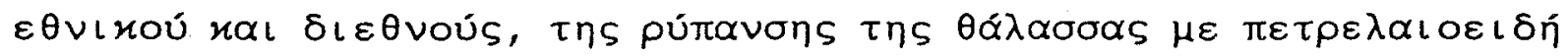

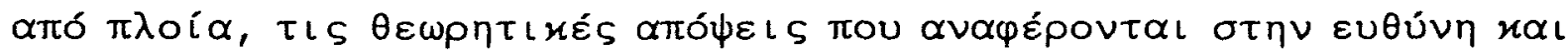

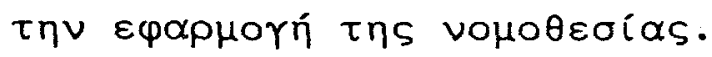

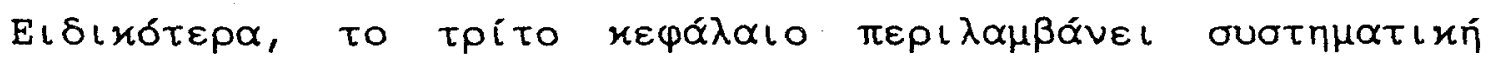

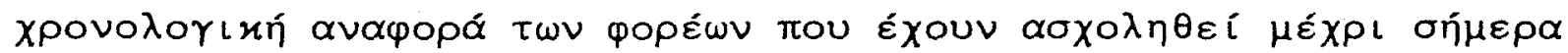

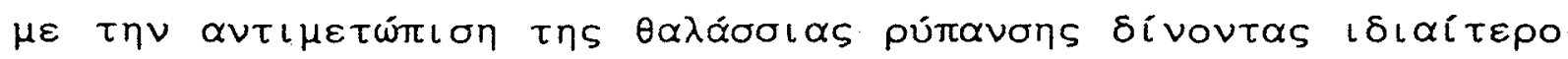

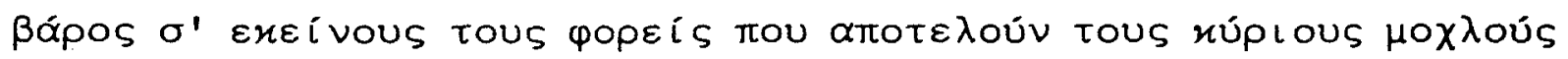

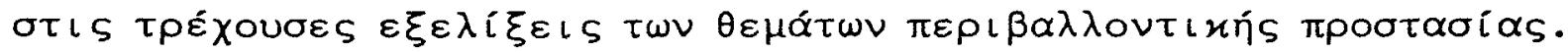

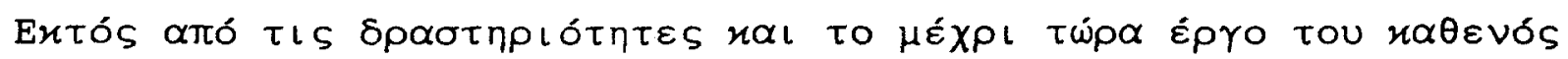

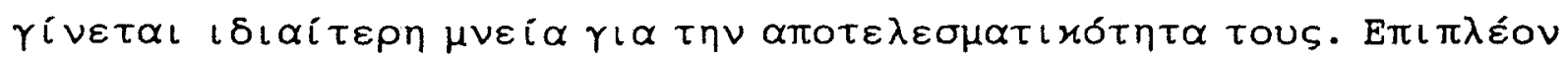

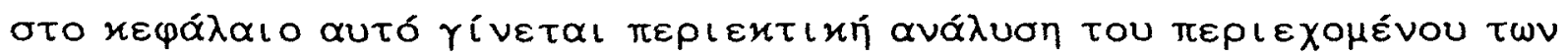

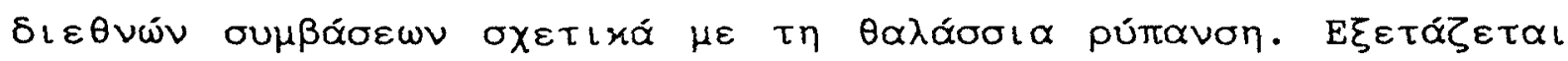

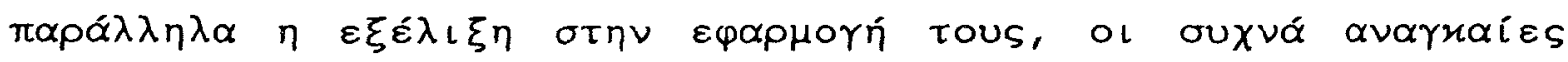

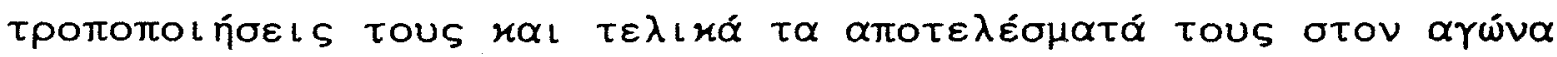




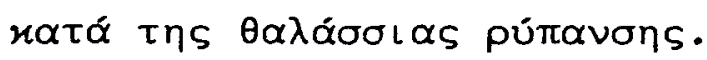

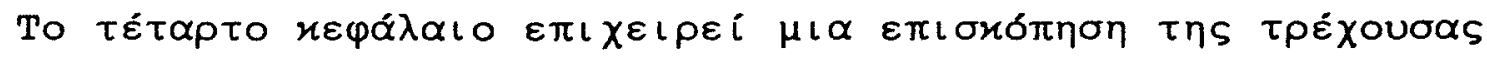

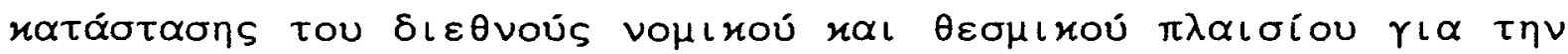

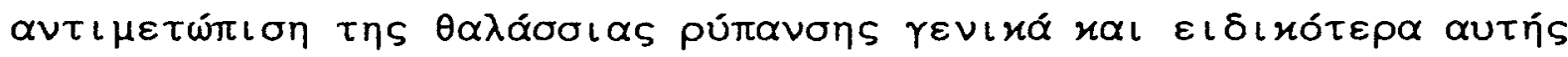

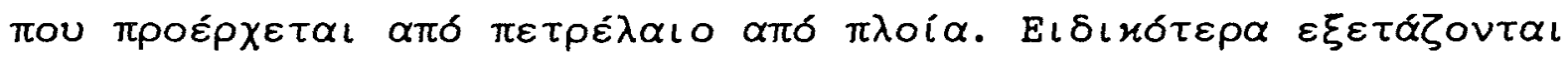

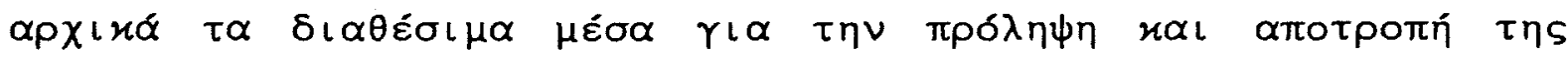

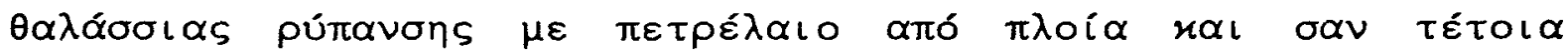

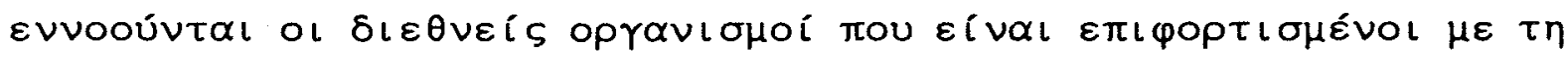

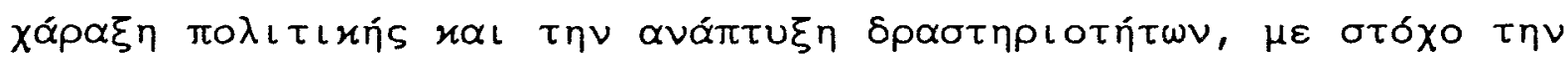

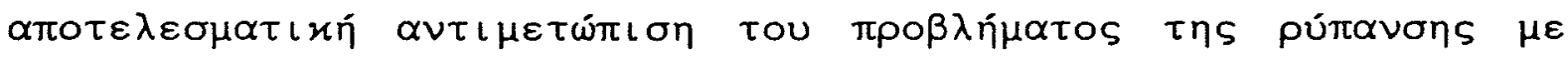

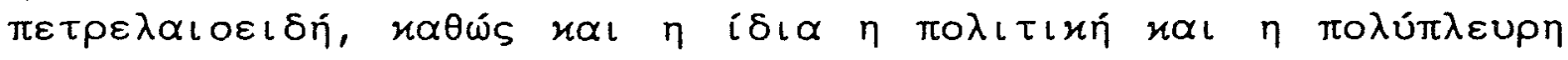

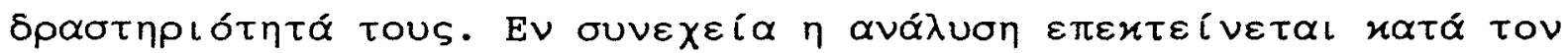

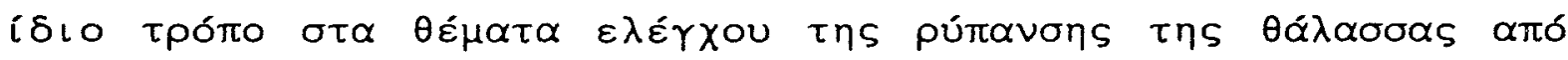

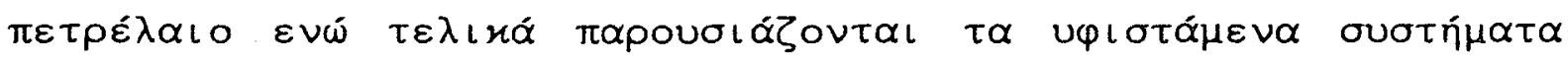

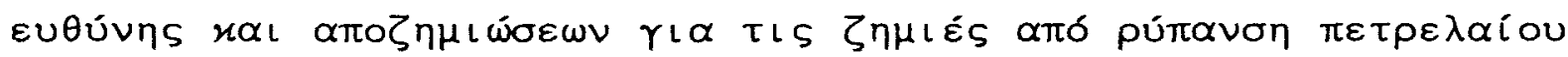

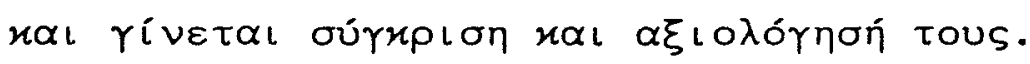

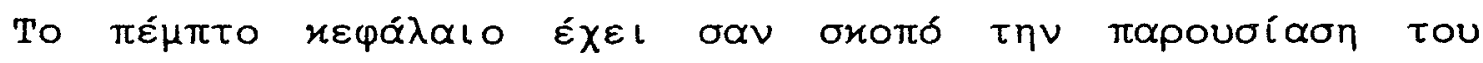

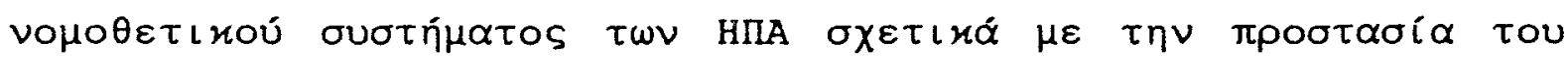

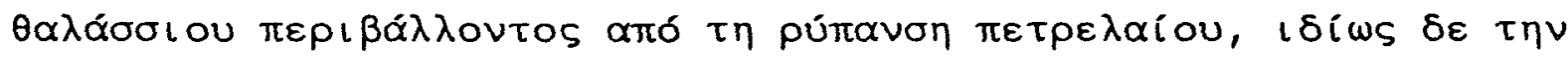

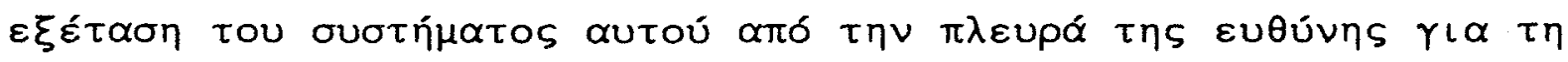

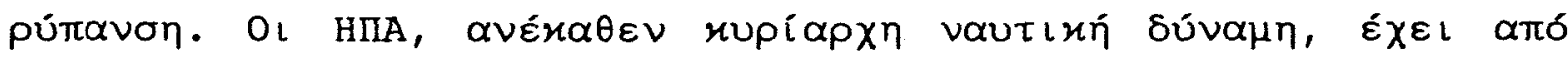
$\delta \varepsilon \varkappa \alpha \varepsilon \tau i \varepsilon S$ a 


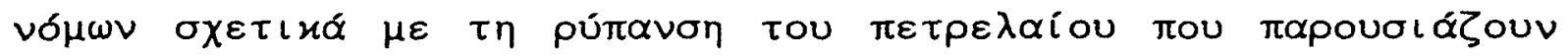

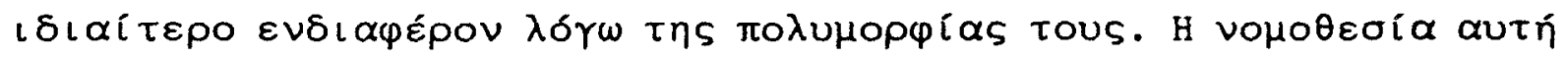

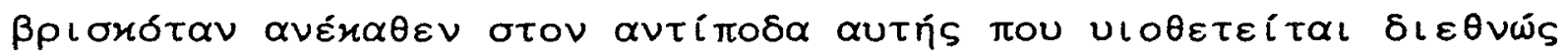

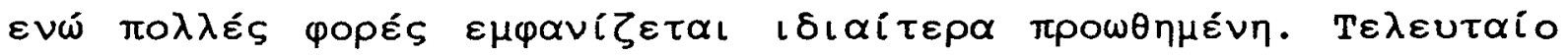

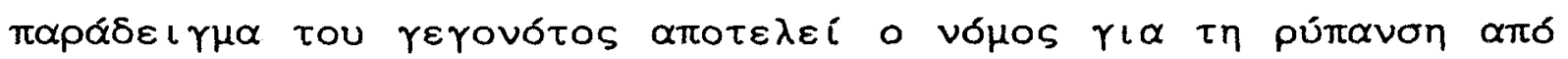

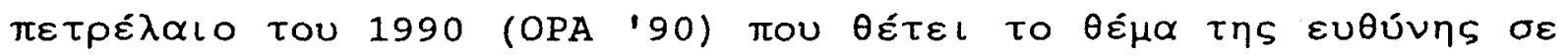

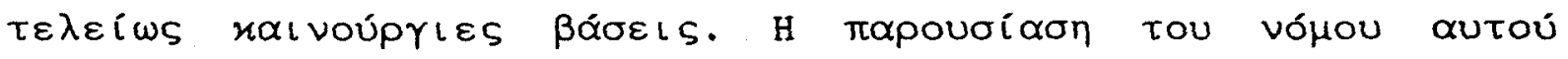

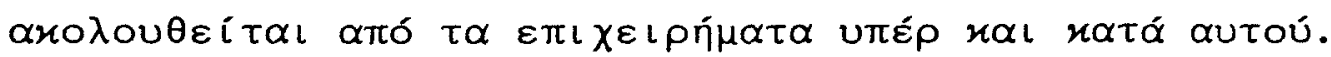

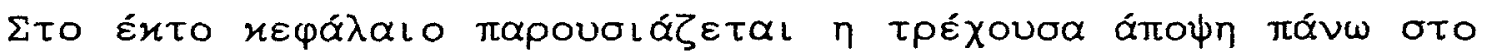

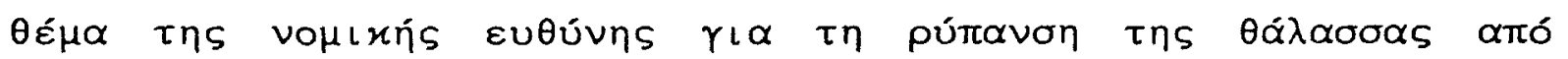

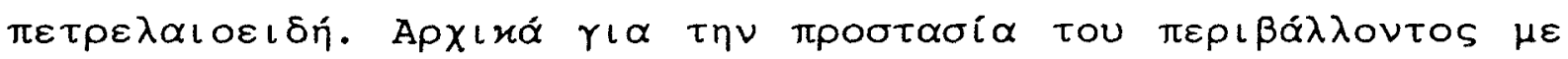

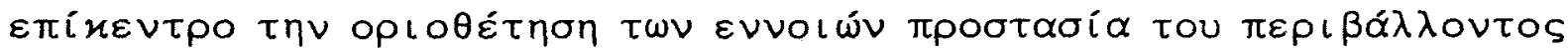

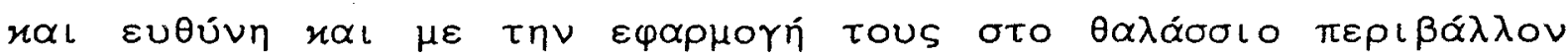

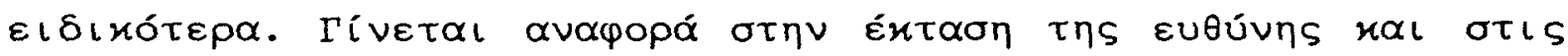

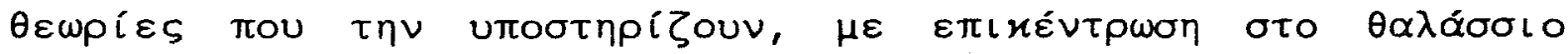

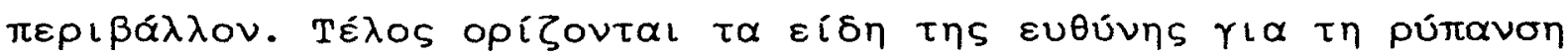

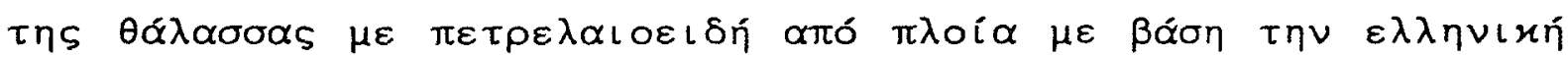

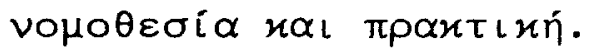

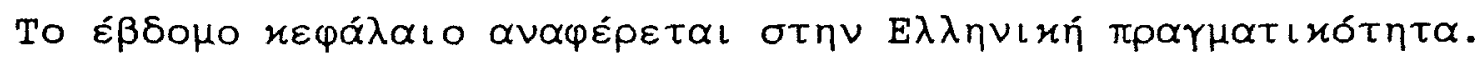

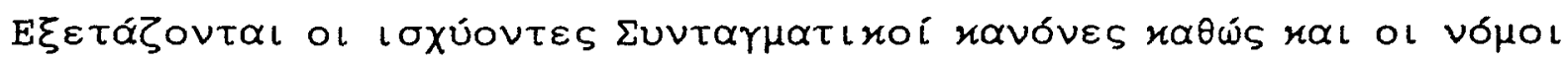

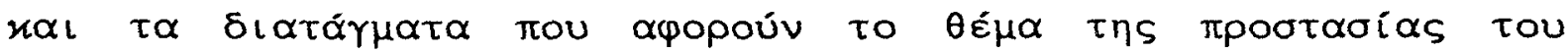

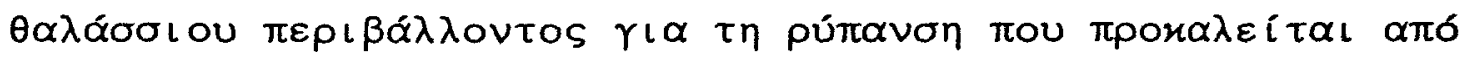




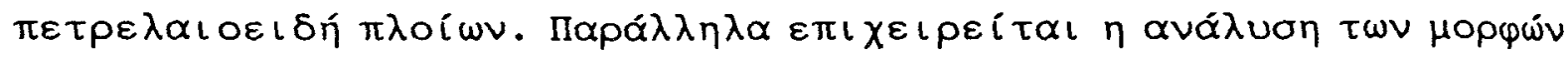

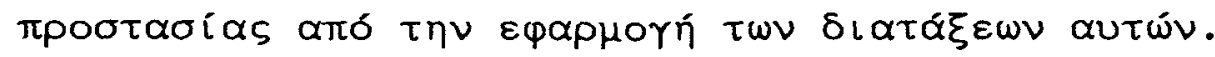

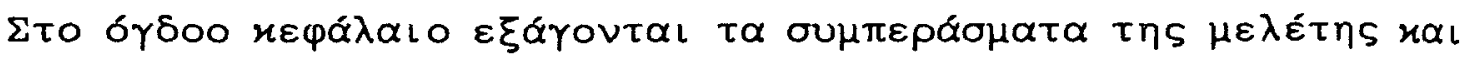

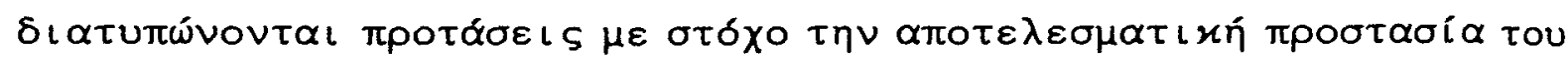

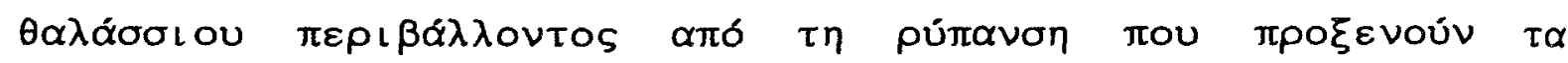
$\pi \varepsilon \tau \rho \varepsilon \lambda \alpha\llcorner O \varepsilon\llcorner\delta \eta ́ ~ \pi \lambda \circ i \omega \nu$.

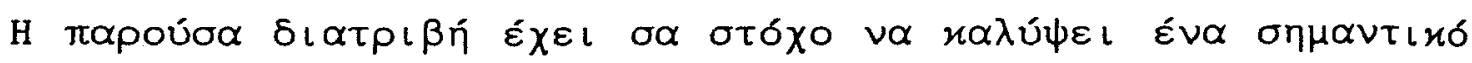

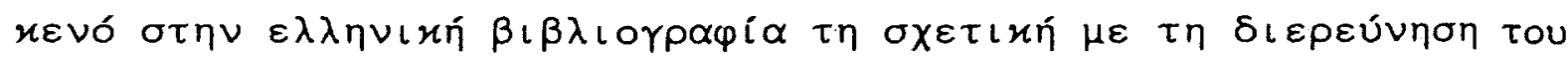

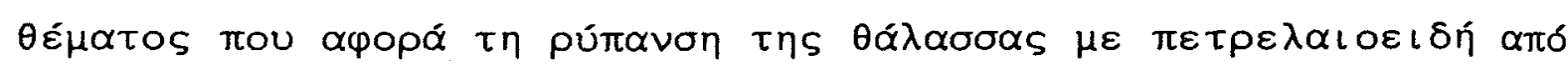
$\pi \lambda \circ i \alpha$. 


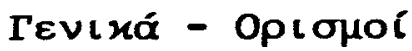

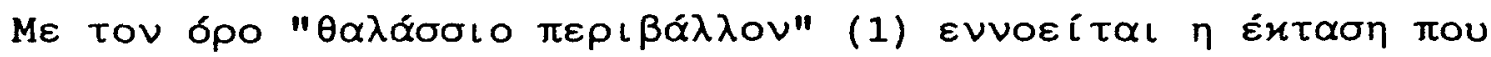

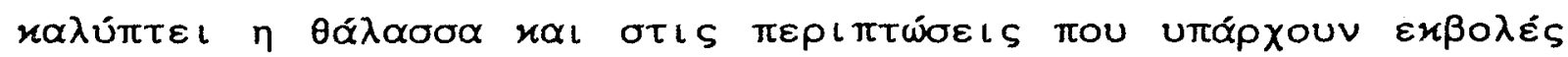

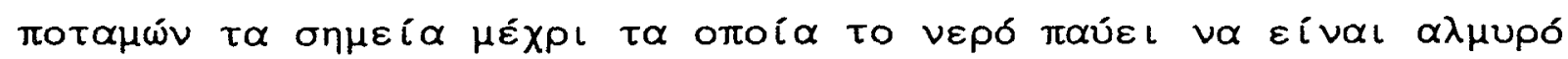

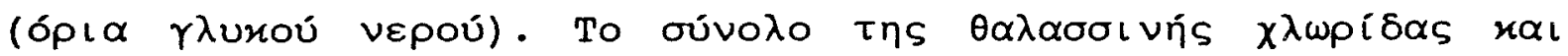

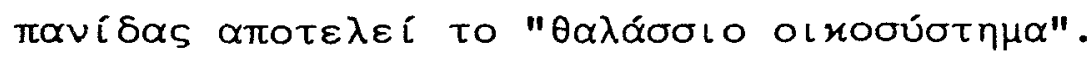

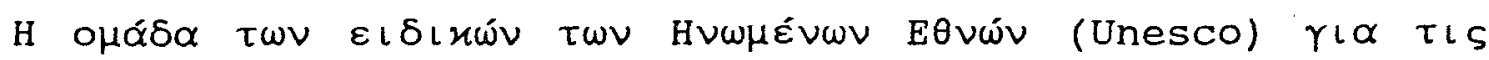

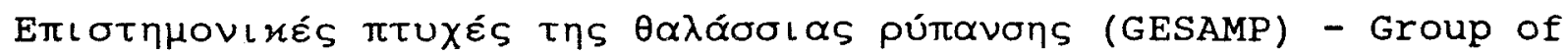
Experts on the Scientific Aspects of Marine Pollution $\delta\llcorner\alpha \mu o ́ p \varphi \omega \sigma \varepsilon$

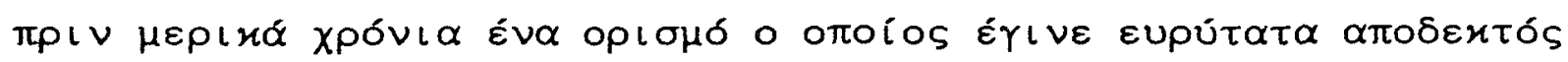

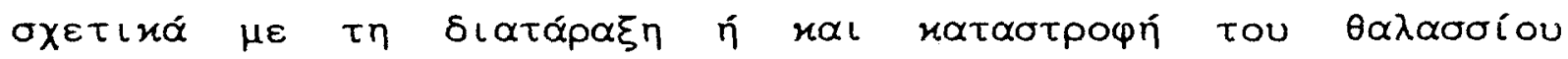

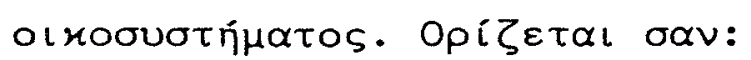

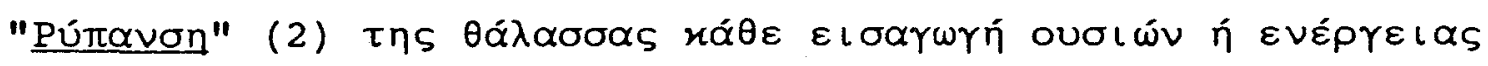

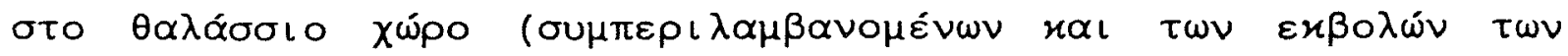

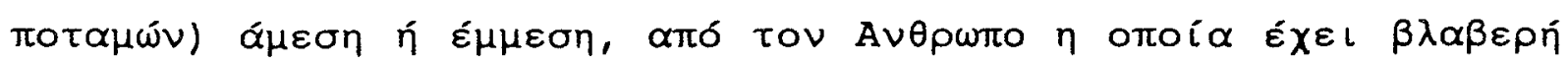

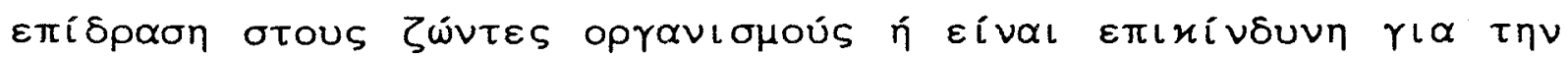

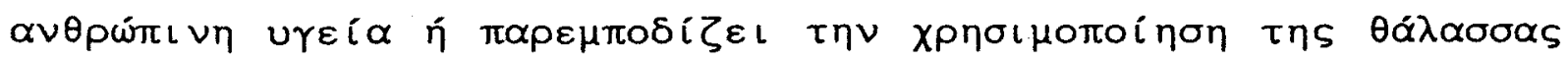

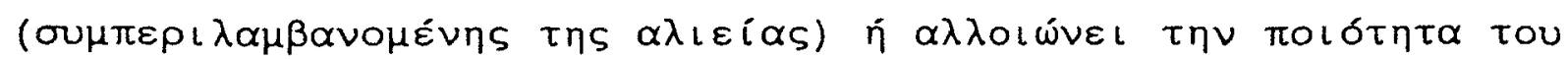

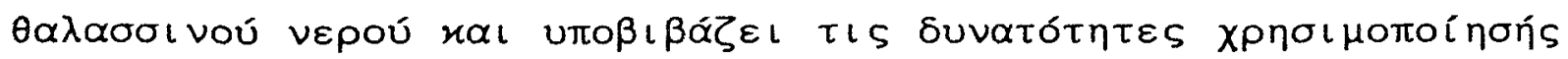

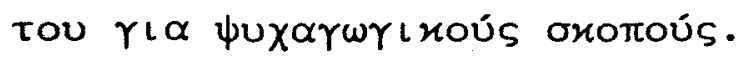




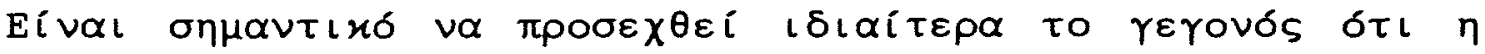

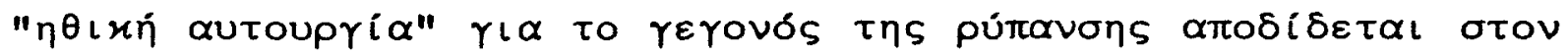

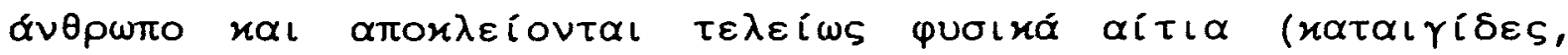

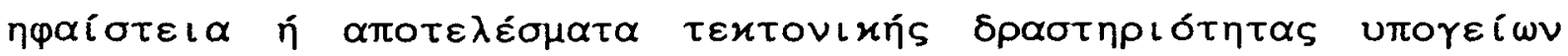

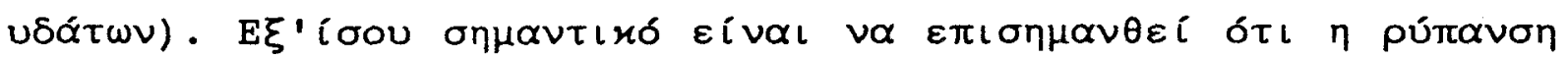

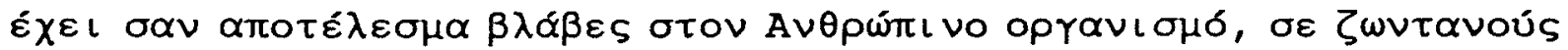

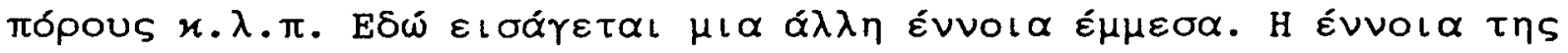

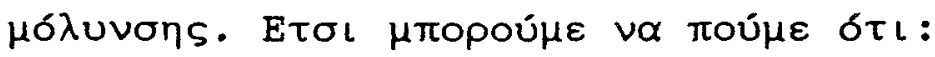

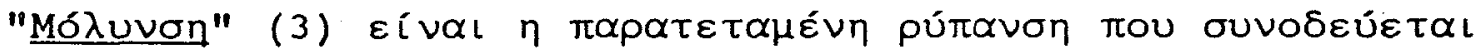

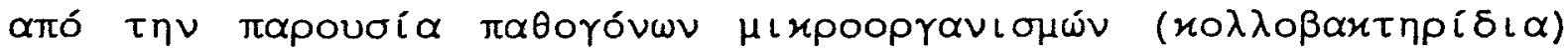

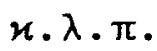

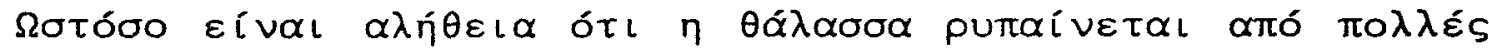

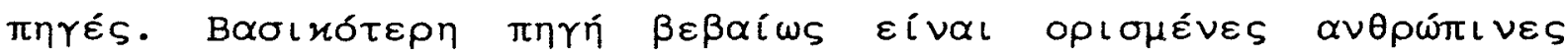

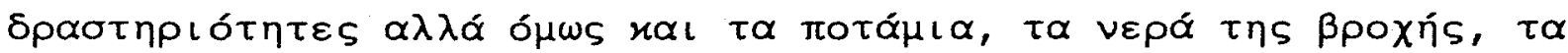

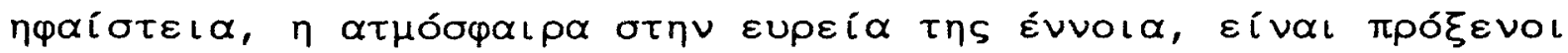

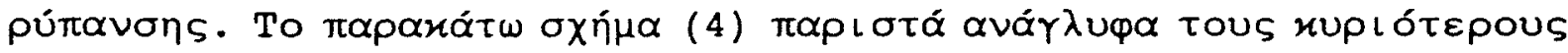

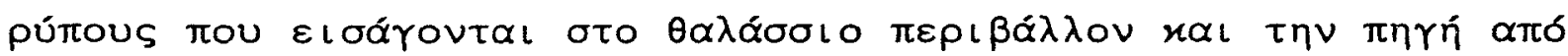

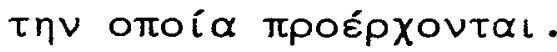

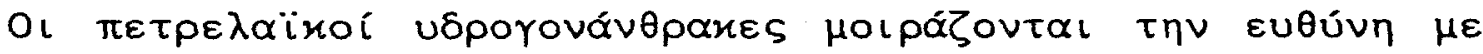

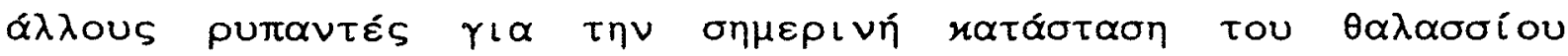

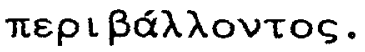




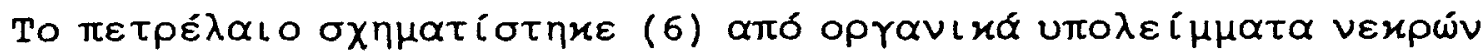

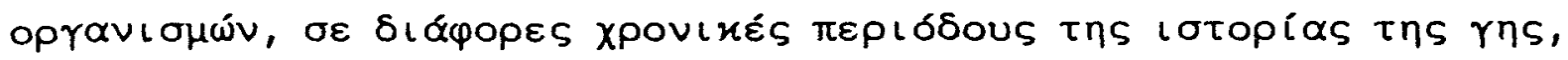

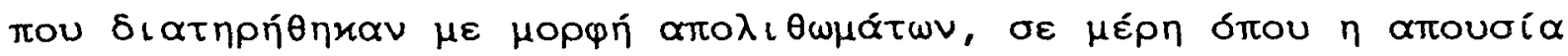

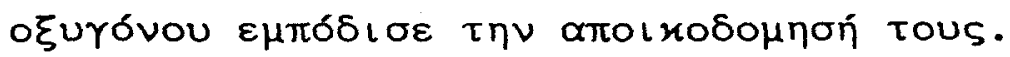

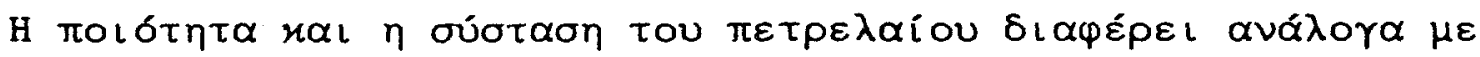

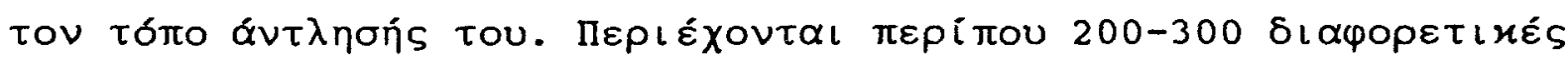

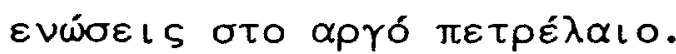

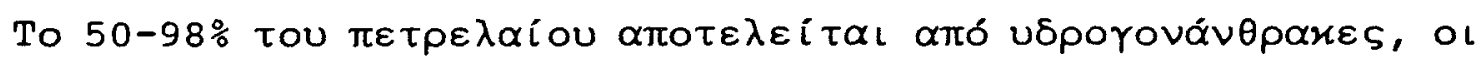

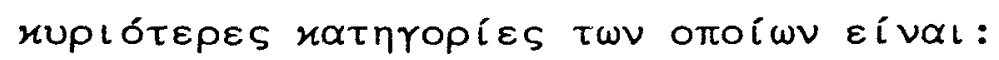

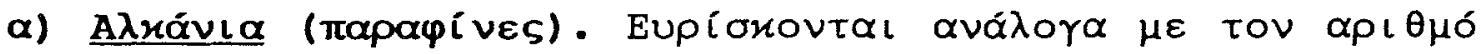

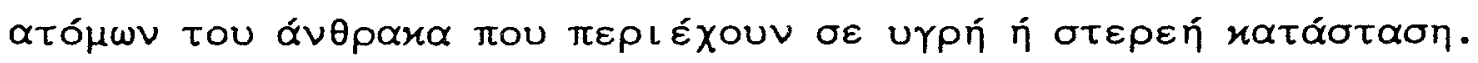

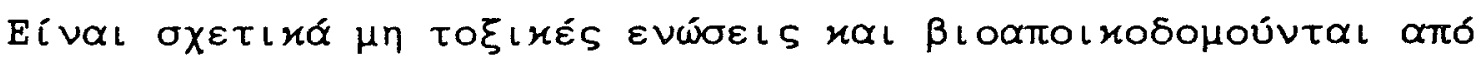

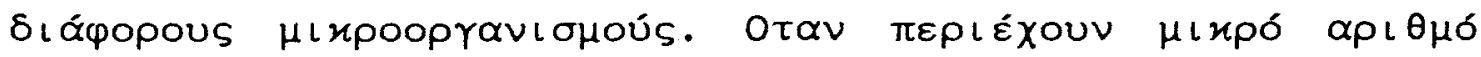

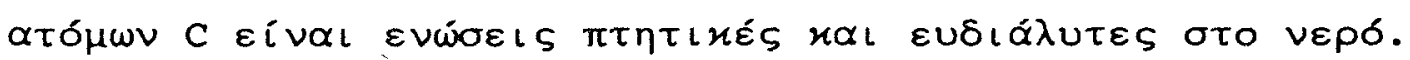

B) $\mathrm{Kux \lambda o \alpha \lambda x \alpha ́vL \alpha .} \mathrm{A \pi o \tau \varepsilon \lambda oúv} \mathrm{to} \mathrm{30-60 \%} \mathrm{\tau ou} \mathrm{\pi \varepsilon \tau \rho \varepsilon \lambda \alpha íou.} \mathrm{Eív \alpha L}$

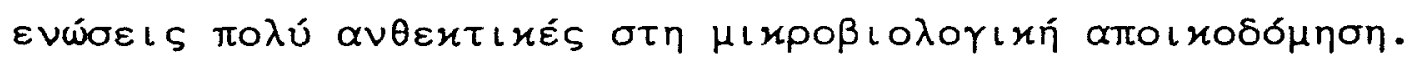

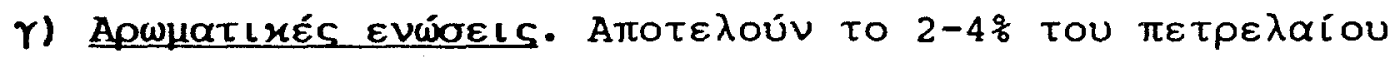

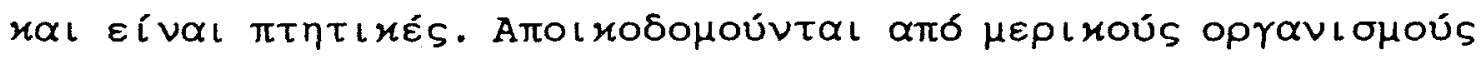
$\varepsilon \varkappa \lambda \varepsilon \varkappa \tau \iota \varkappa \alpha ́$. 


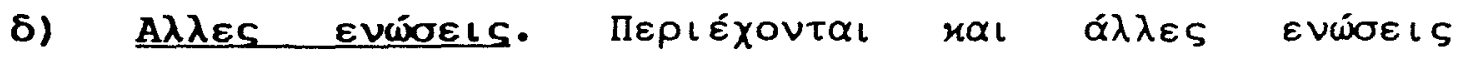

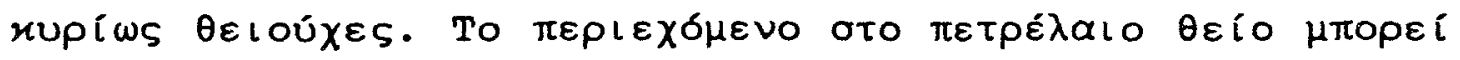

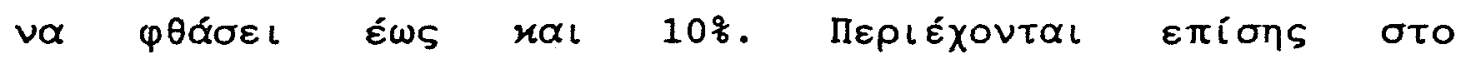
$\pi \varepsilon \tau \rho \varepsilon ́ \lambda \alpha \iota$ в

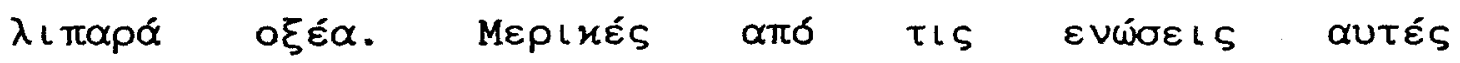

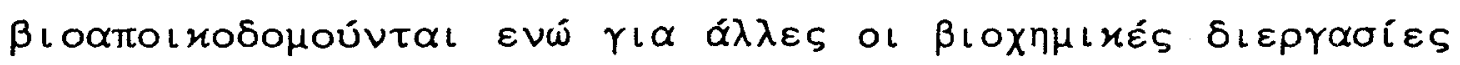

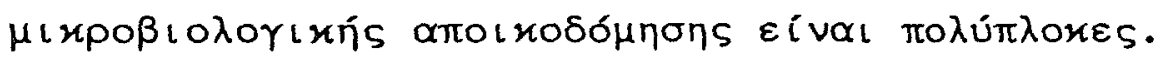

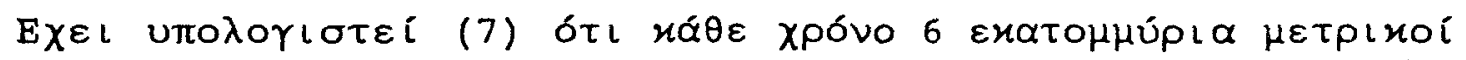

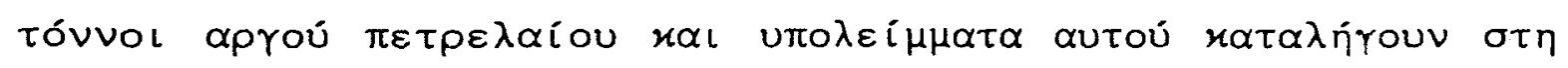

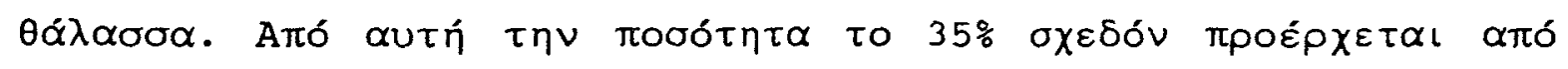

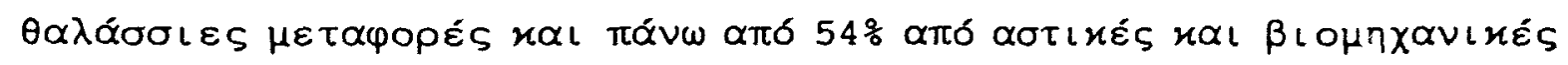

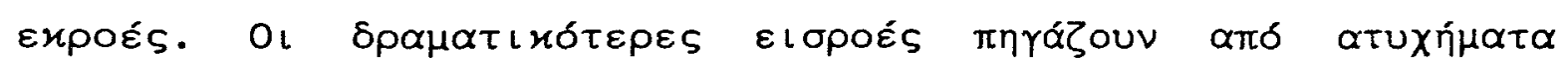
$\delta \varepsilon \xi \alpha \mu \varepsilon \nu \delta ́ \pi \lambda \circ \iota \omega \nu$. 


\section{BIAH APTOY METPENATOY}

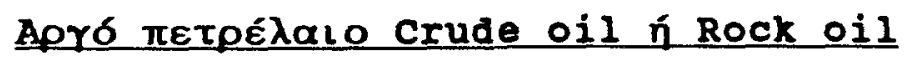

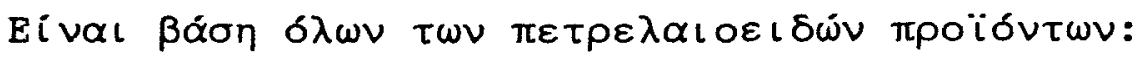

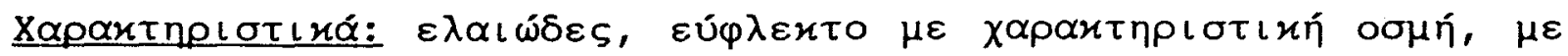

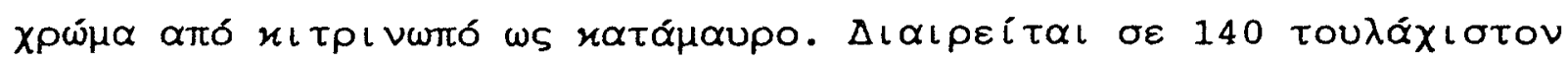

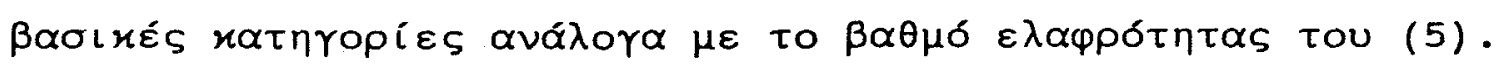

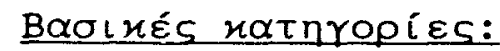

1. AVGAS (Aviation Gas).

2. TURBO FUEL (Kerozene based).

3. TURBO FUEL (Naptha Gas).

4. GASOLINE.

5. POWER FORMED FEED VIRGIN NAPTHA.

6. WHITE SPIRITS.

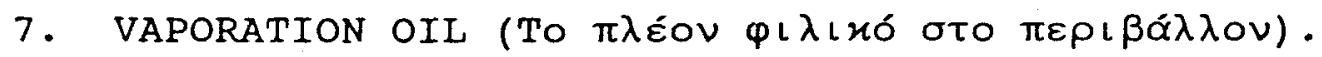

8. REGULAR KEROZENE.

9. PREMIUM KEROZENE.

10. DISTILLATE FUEL OIL (D.F.O)

11. GAS OIL. 
12. CRACKED GAS OIL.

13. MARINE DIESEL OIL.

14. FUEL OIL.

15. CUT FEED (AVCO-DIRTY).

16. CRUDE OIL.

17. CRUDE OIL COMPONENT/DISTILLATE SPIKE.

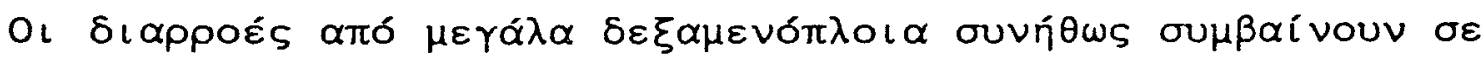

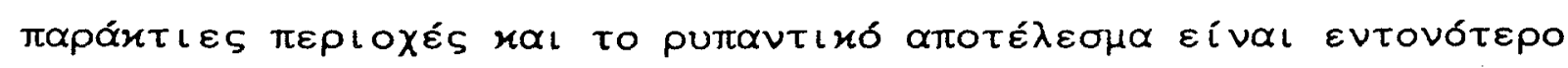

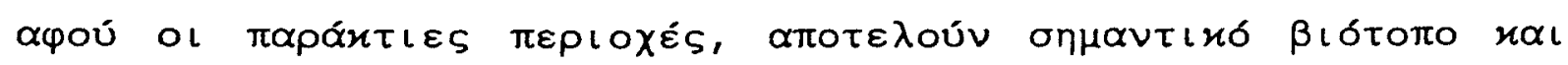

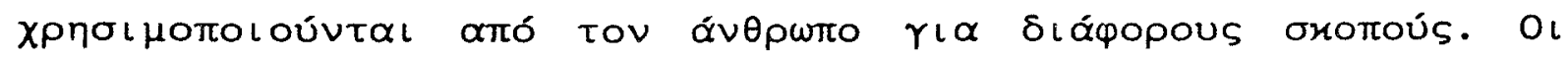

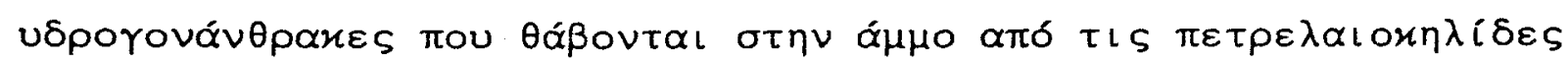

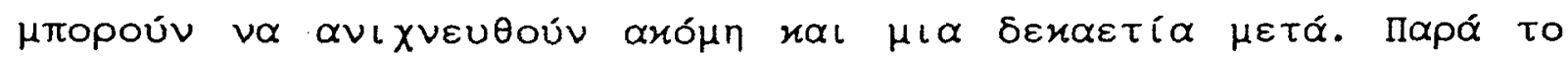

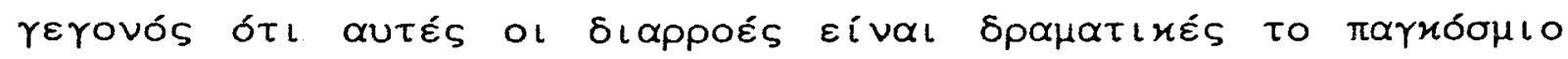

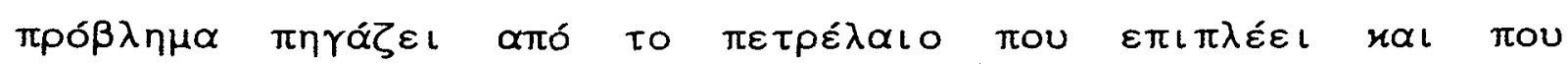

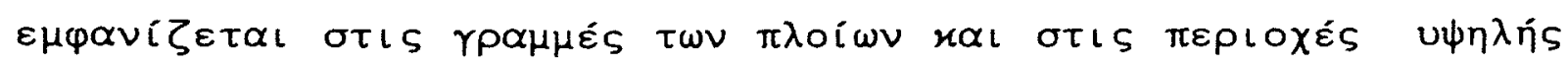

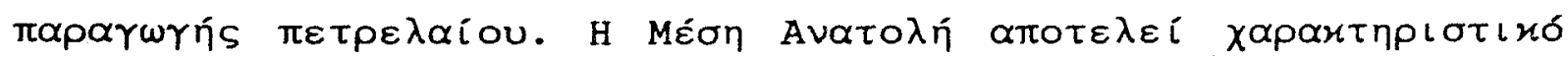

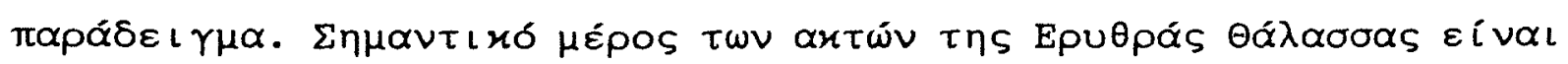

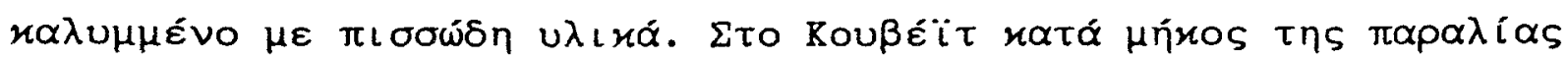

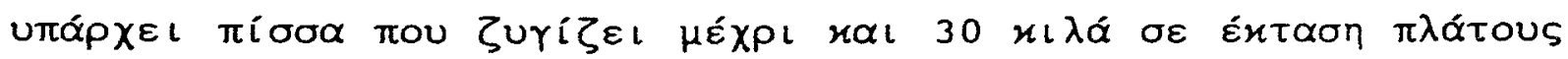

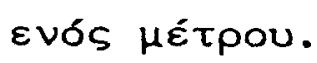

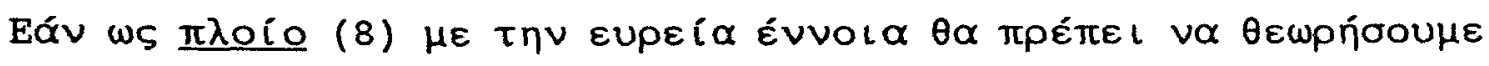


To ớ์

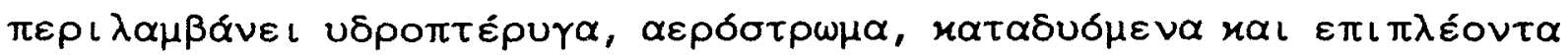

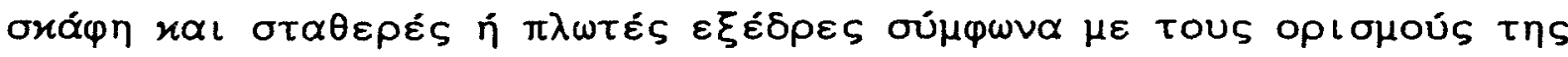

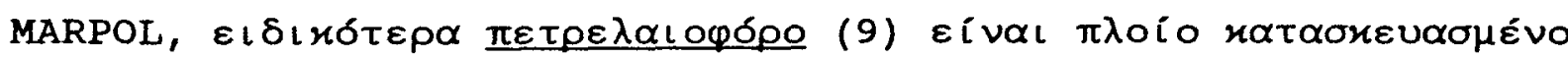

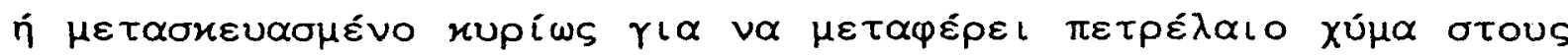

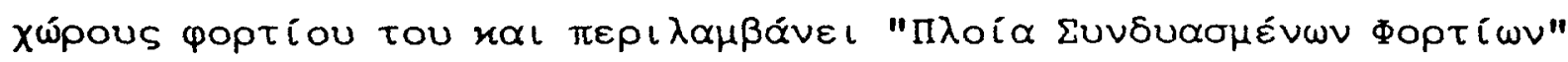

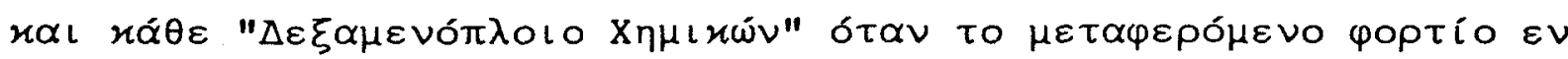

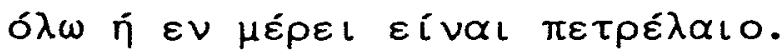

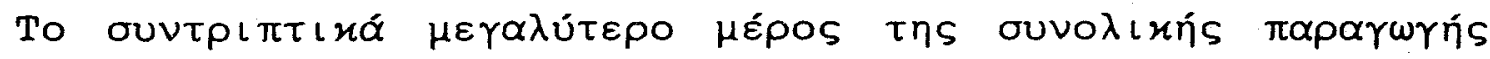

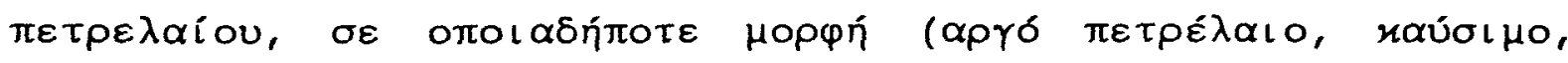

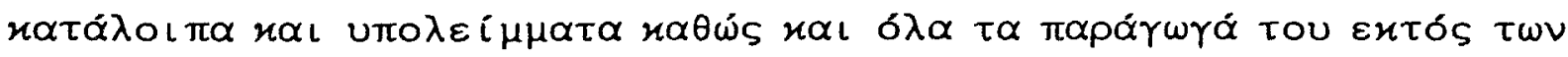

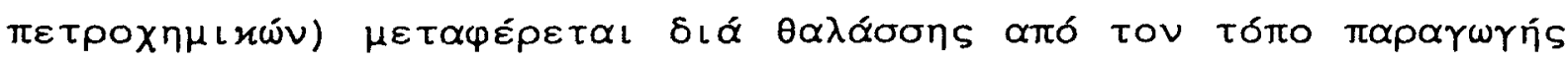

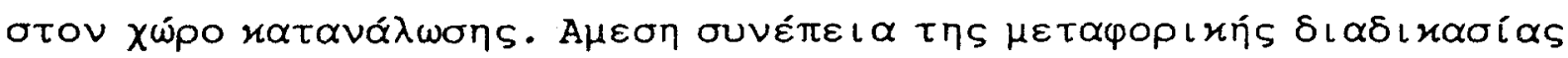

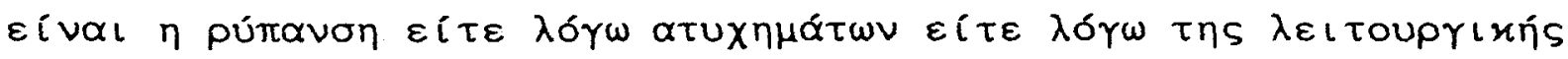

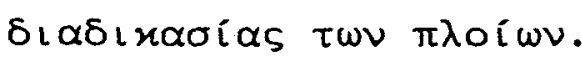

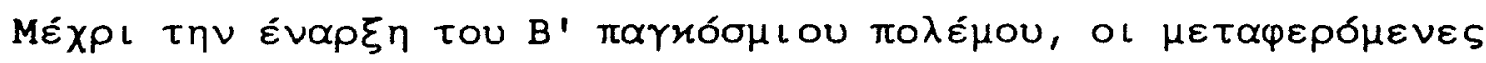

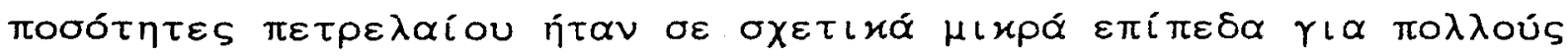

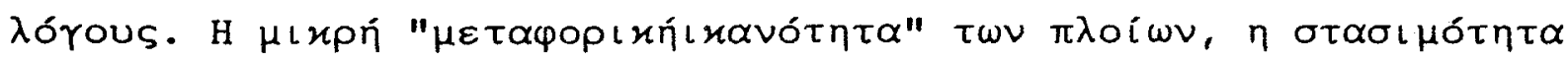

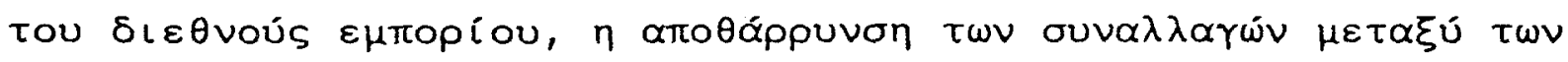

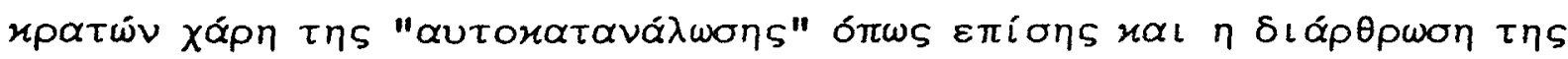

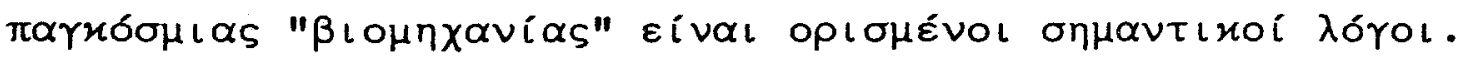




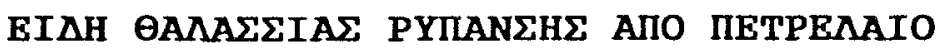

\section{Atuxnuatixń pứravan}

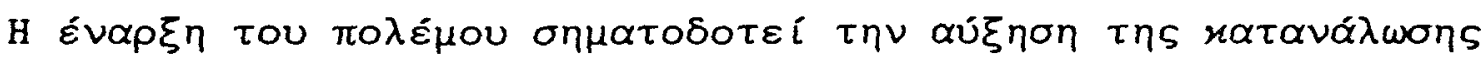

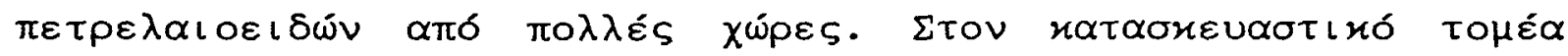

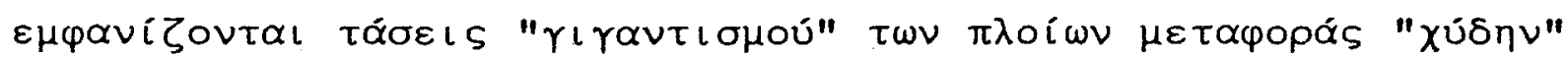

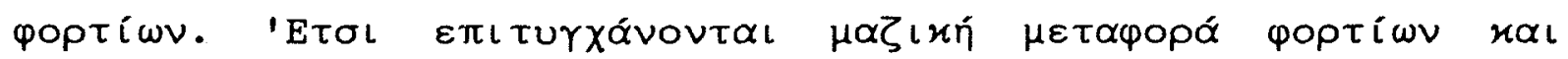

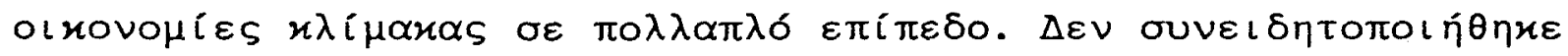

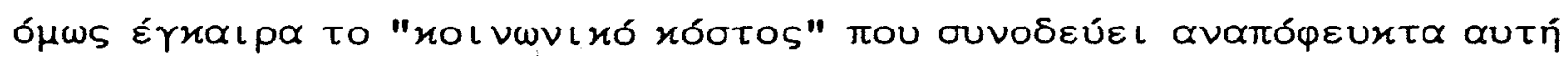

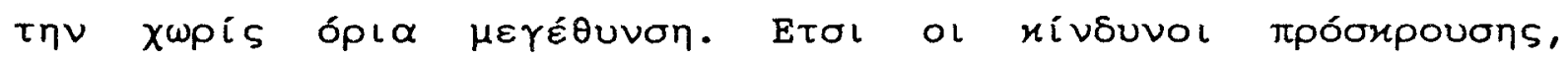

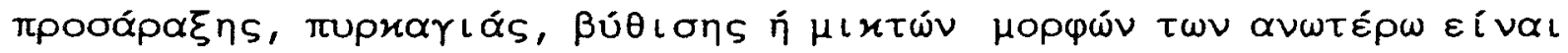

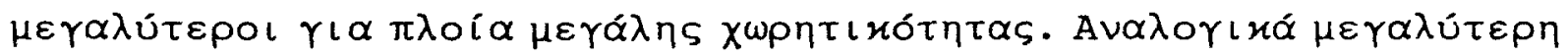

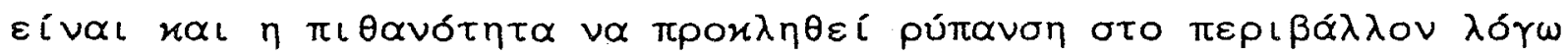

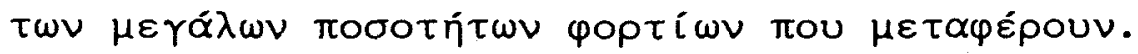

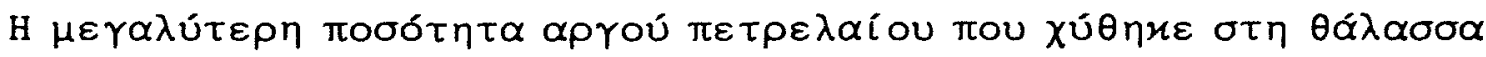

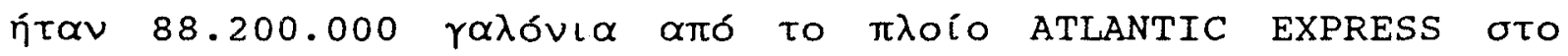

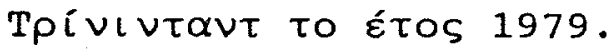

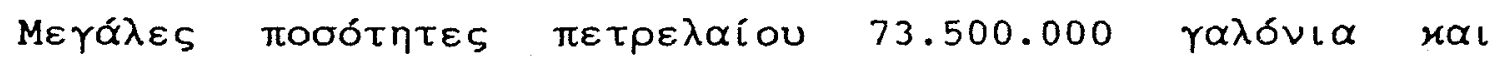

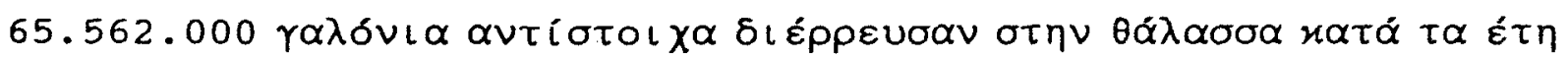

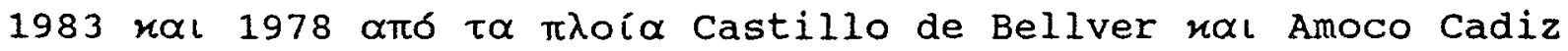

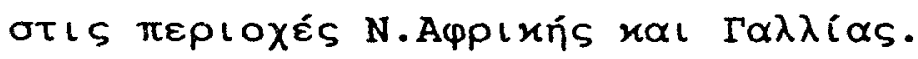




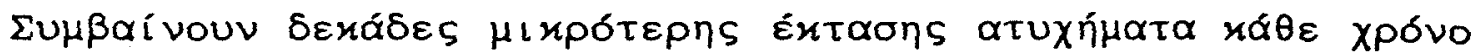

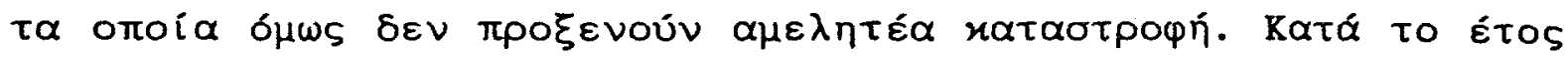

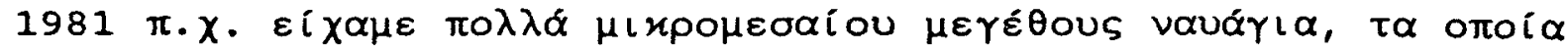

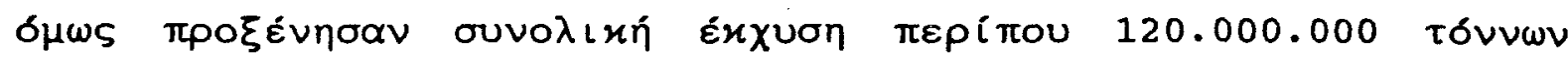

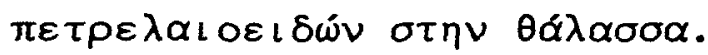

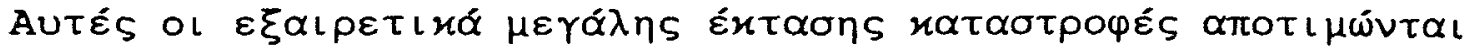

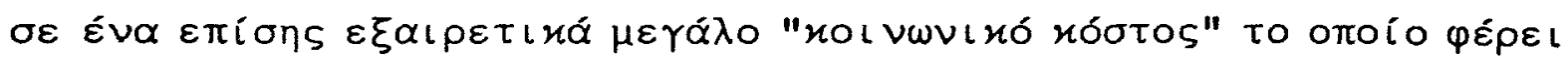

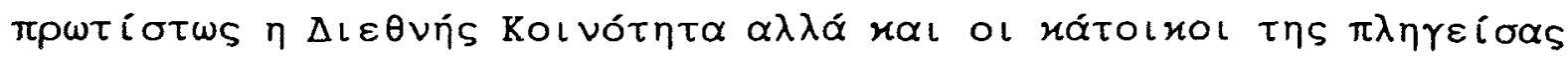

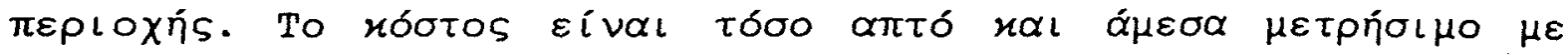

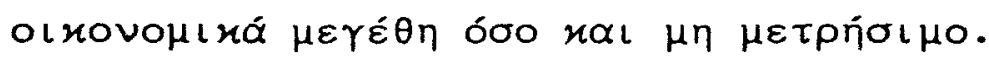

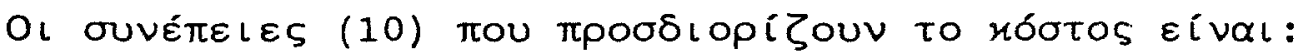

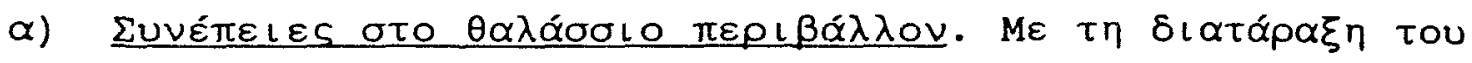

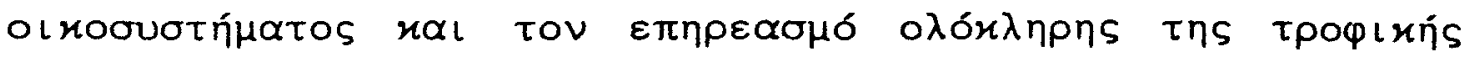

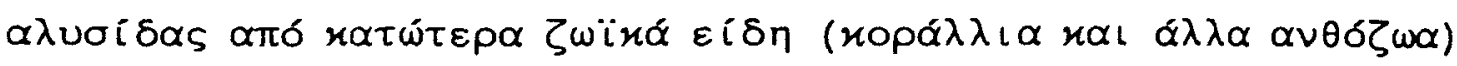

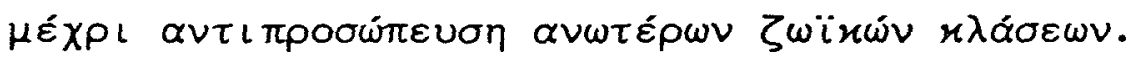

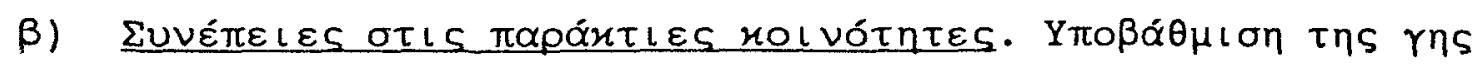

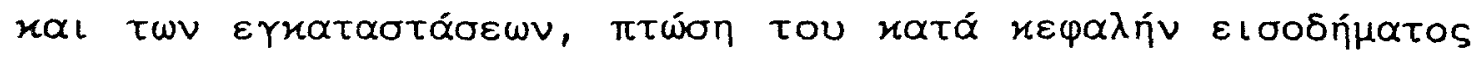

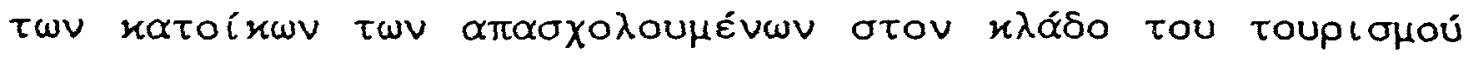

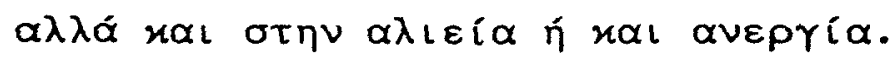




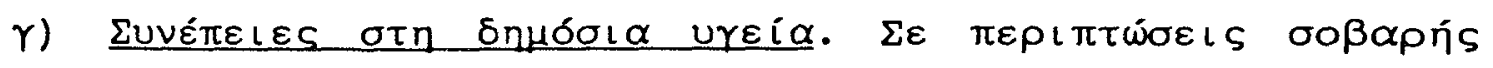

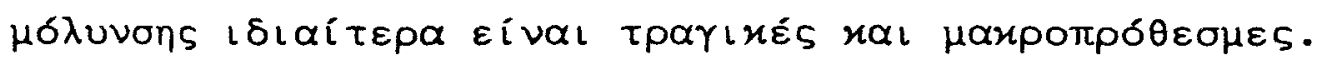

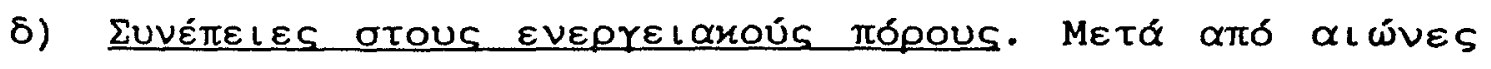

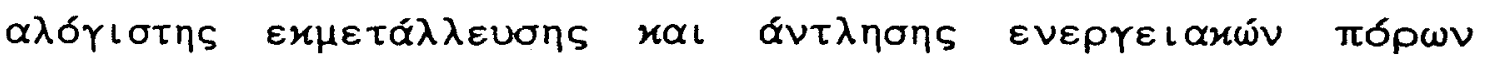

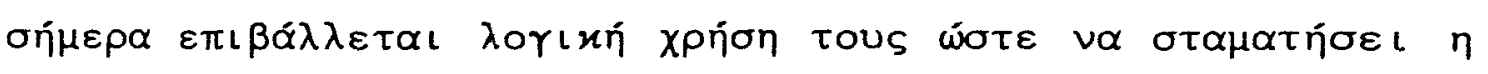
$\lambda \varepsilon \eta \lambda \alpha \sigma i \alpha$ tous.

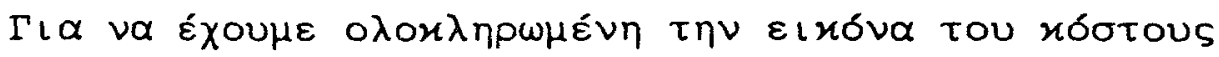

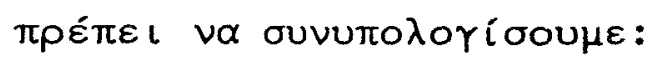

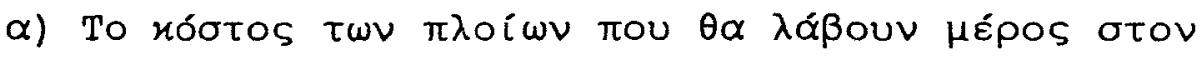

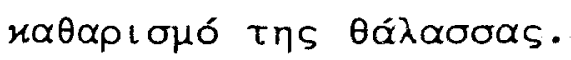

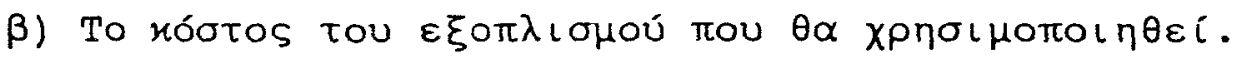

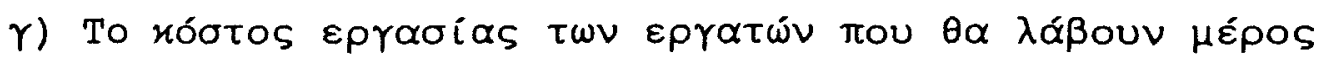

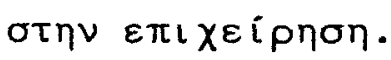

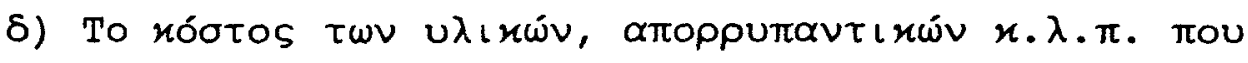

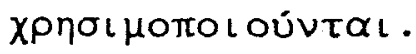

ع) To kóotos tou poptíou.

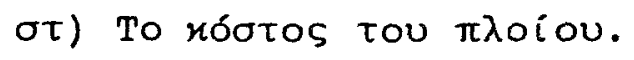

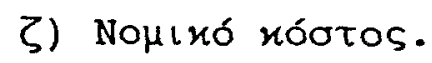

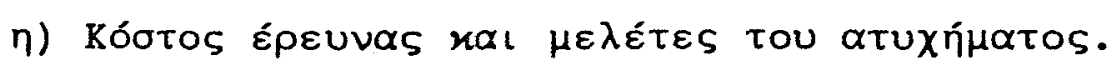




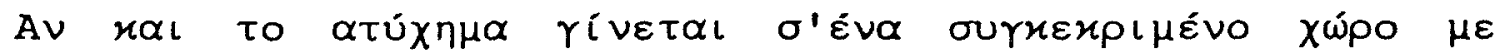

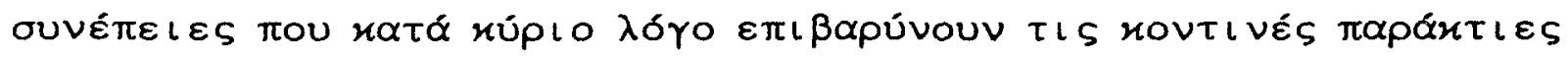

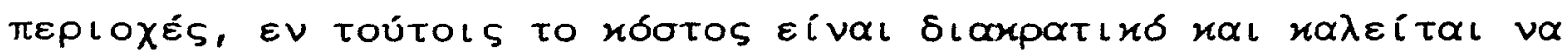

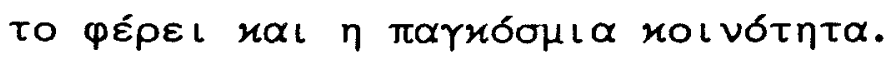

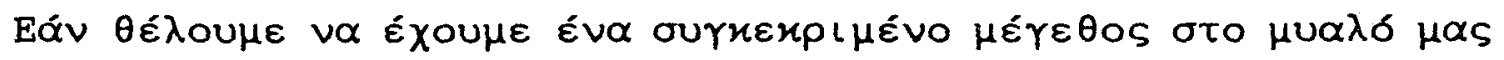

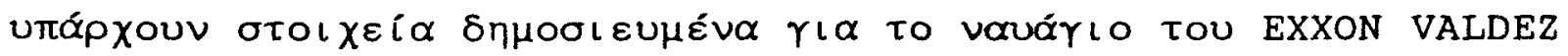

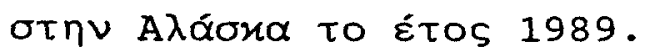

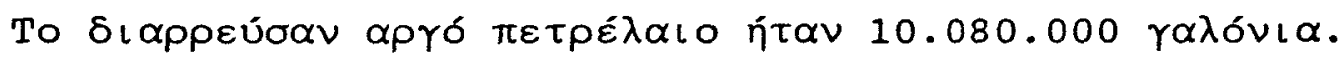

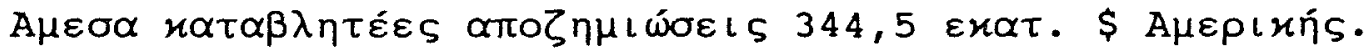

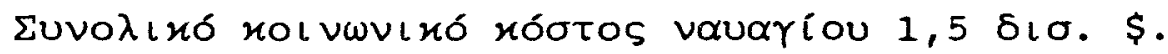

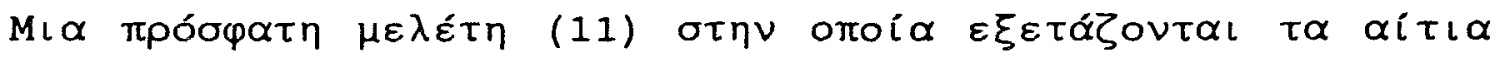

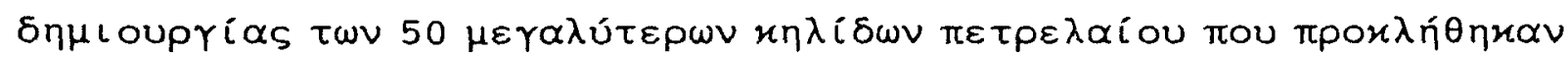

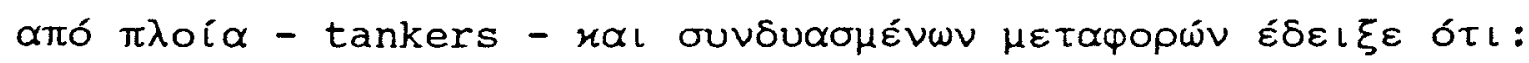

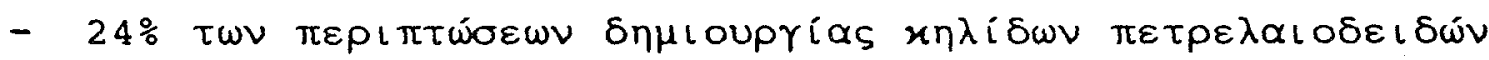

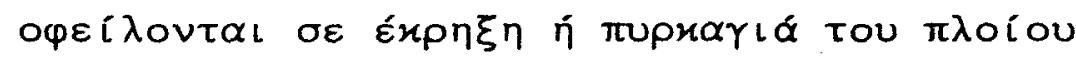

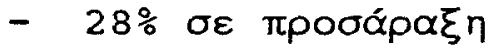

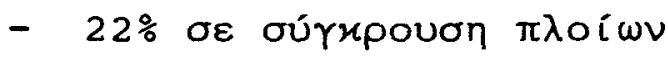

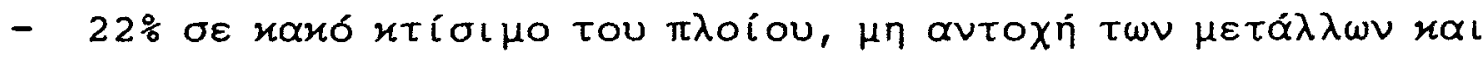
$\sigma \varepsilon$

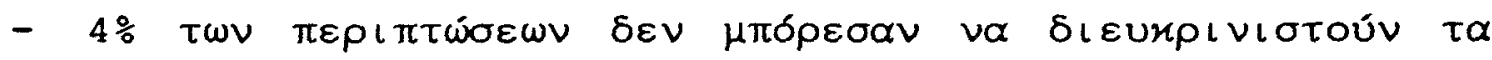
$\alpha i \tau \iota \alpha$. 


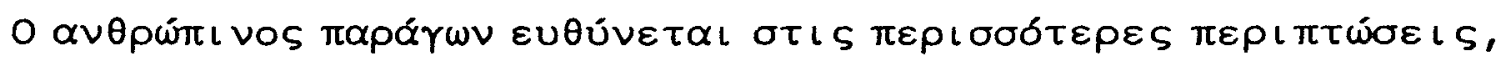

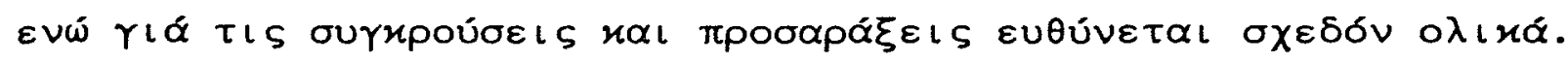

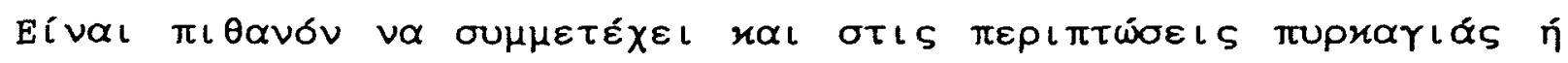

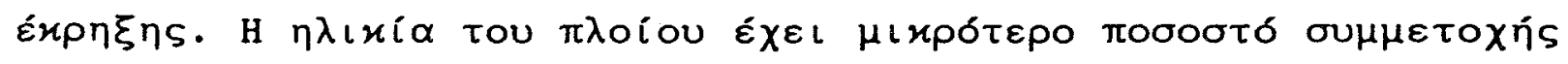

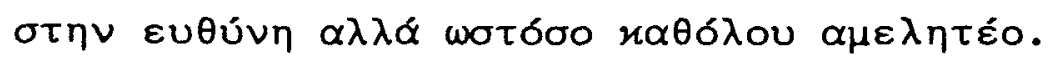




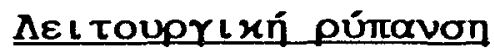

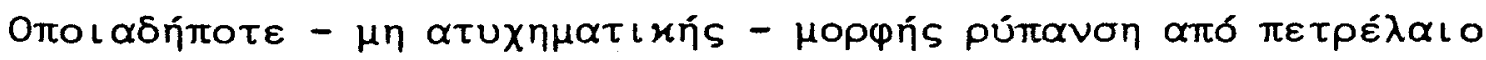

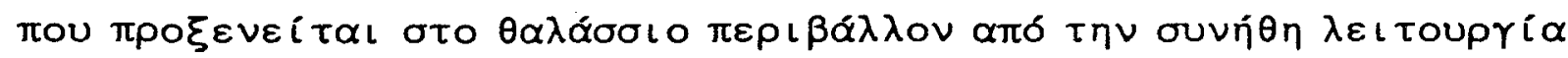

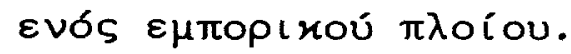

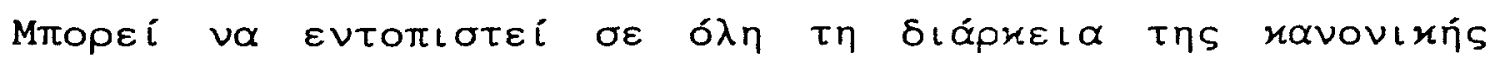

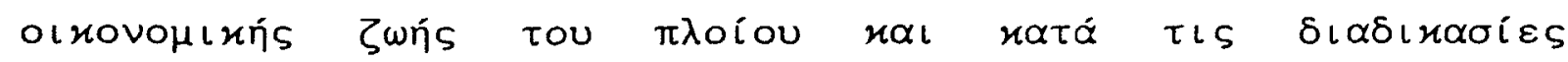

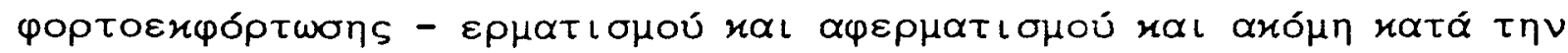
$\alpha \pi \circ \beta \circ \lambda \dot{~ \sigma \varepsilon v \tau \iota \nu \omega ́ v . ~}$

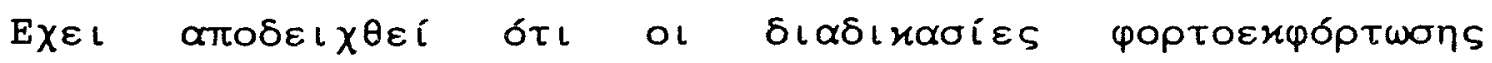

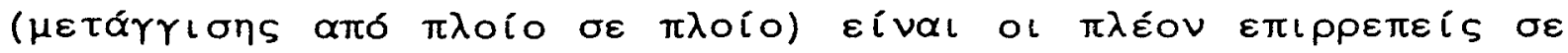

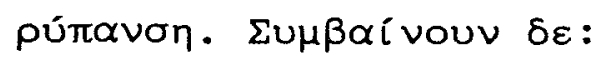

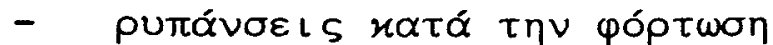

$-\quad n$

- $\quad 11$

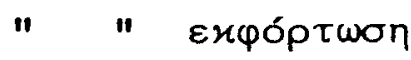

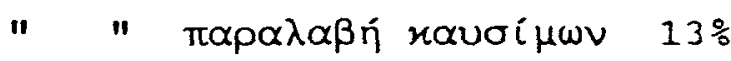

$22 \% \quad \tau \omega \nu \pi \varepsilon \rho\llcorner\pi \tau \omega ́ \sigma \varepsilon \omega \nu$

$$
34,4 \% \quad 11
$$

11

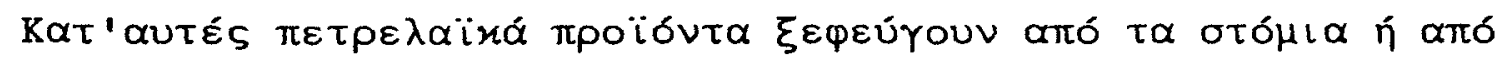

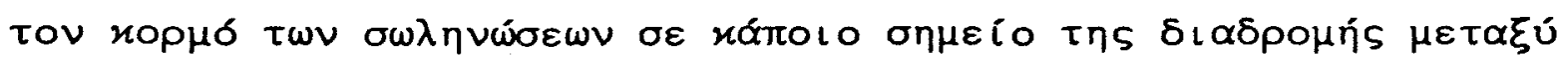




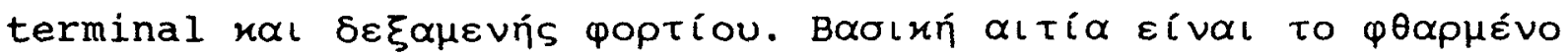

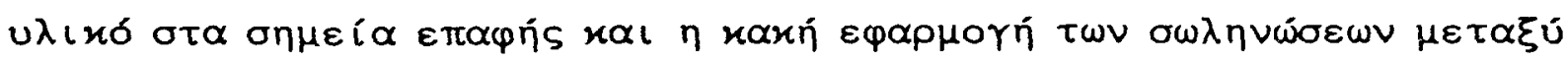
rous.

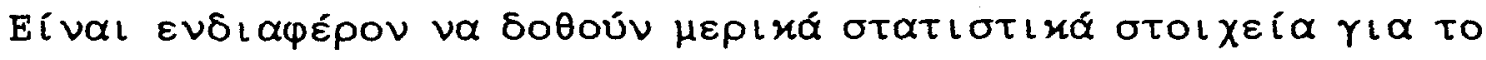

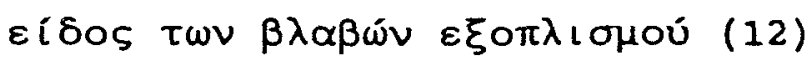

- $\delta ı \alpha p p o n ́ \beta$

- $\beta \lambda \alpha ́ \beta \eta \sigma \omega \lambda \eta \dot{\sigma} \sigma \varepsilon \omega \nu \quad 17 \%$

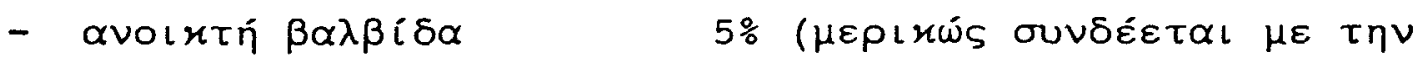

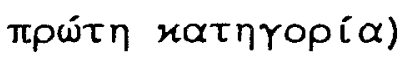

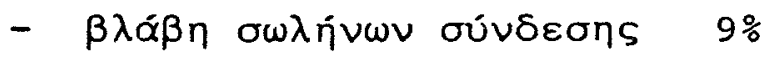

- $\alpha \lambda \lambda \varepsilon S \alpha \iota \tau i \varepsilon S$

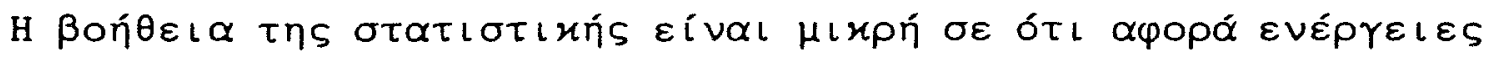

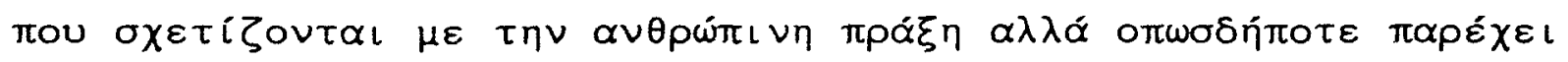

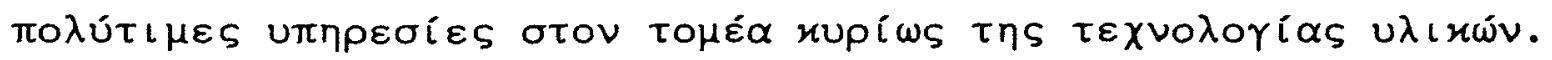

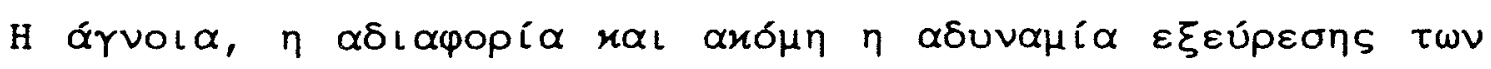

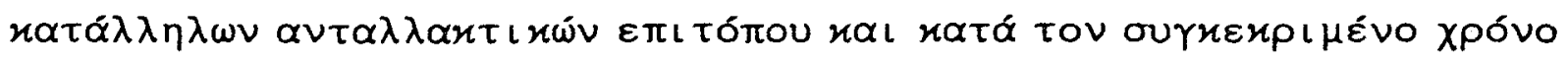

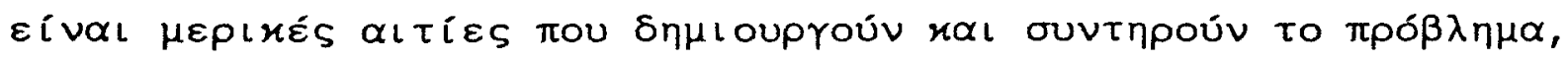

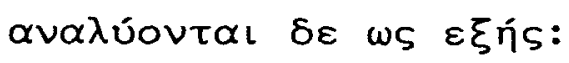




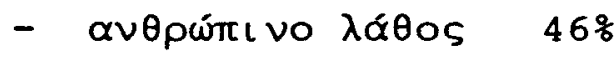

- $\beta \lambda \alpha \dot{\beta} \eta \varepsilon \xi \circ \pi \iota \sigma \mu \circ 0^{2} \quad 34,4 \%$

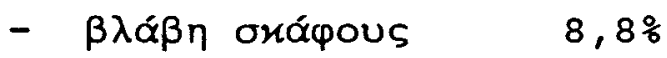

- $\alpha \lambda \lambda \varepsilon \varsigma \alpha i \tau i \varepsilon s \quad 10,8 \%$

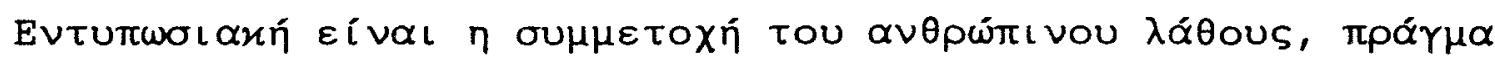

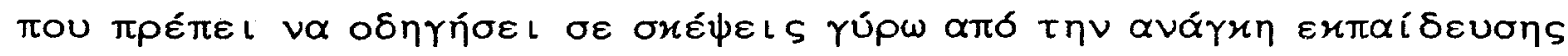

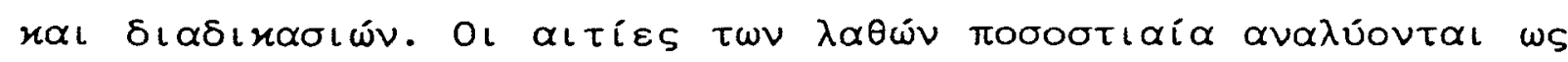
$\varepsilon \xi \tilde{s}$ :

$-\alpha \pi \rho \circ \sigma \varepsilon \xi\lceil\alpha$

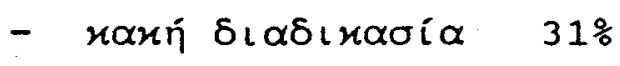

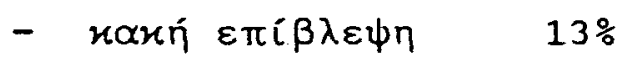

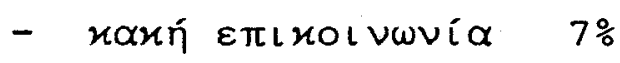

- $\alpha \lambda \lambda \varepsilon s \alpha i \tau i \varepsilon s \quad 9 \%$

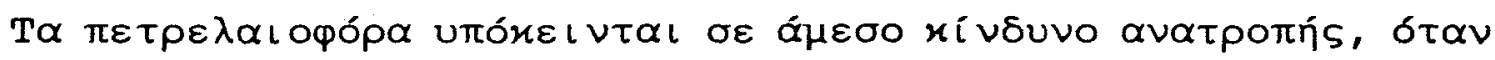

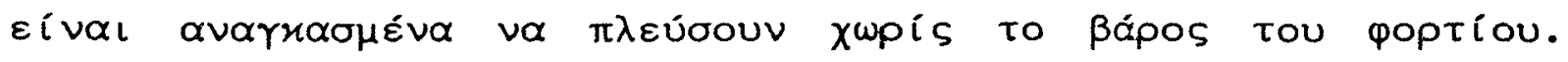

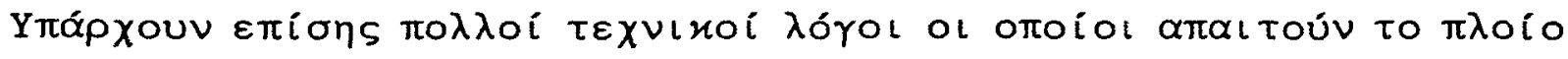

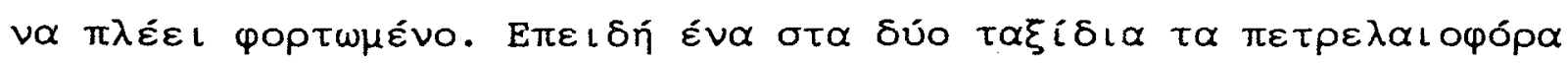

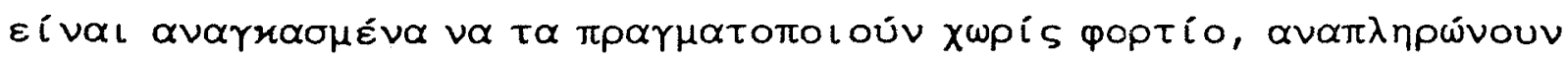

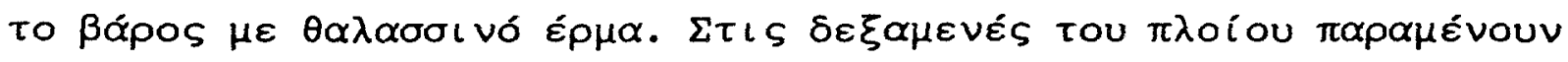

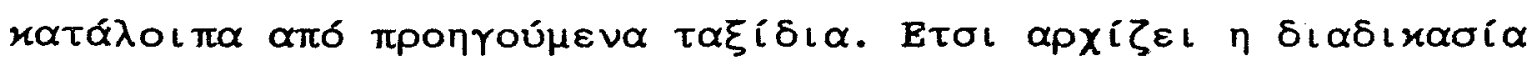




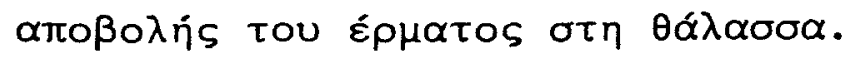

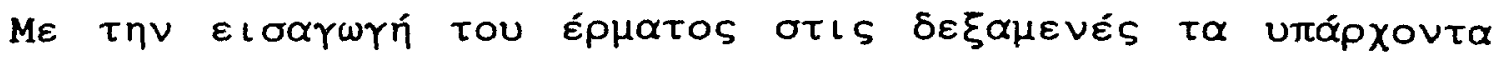

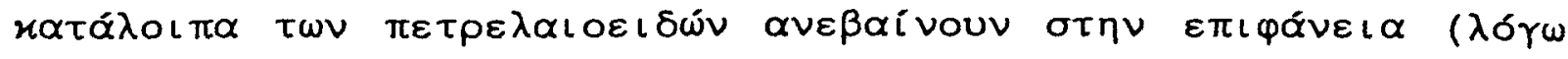

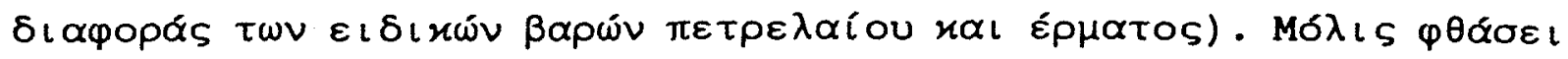

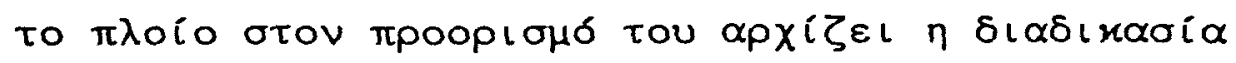

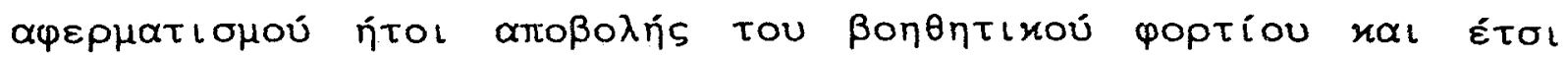

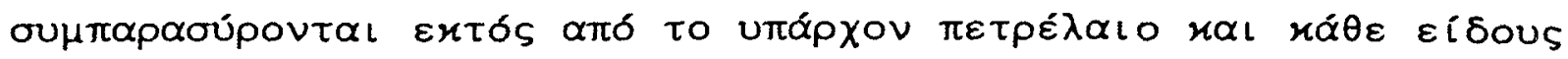

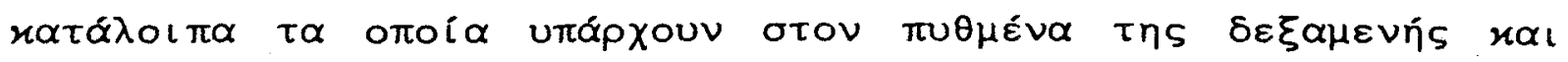

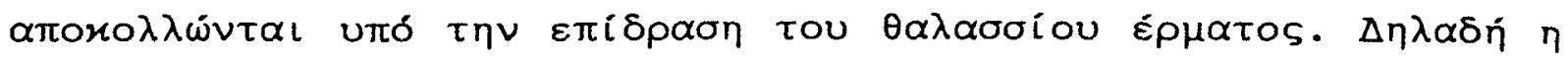

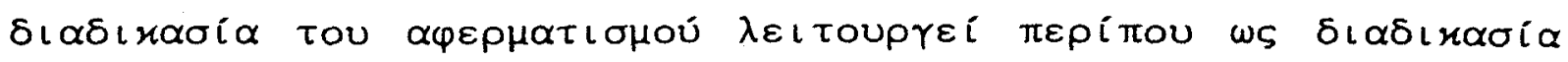

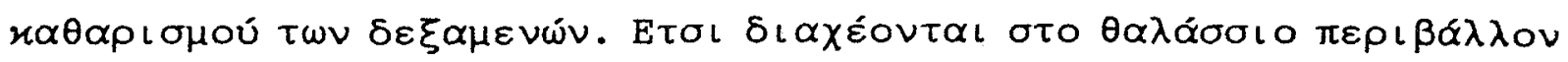

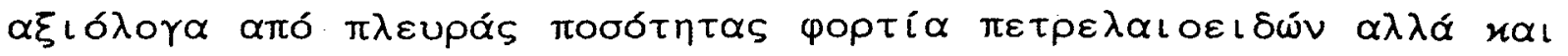

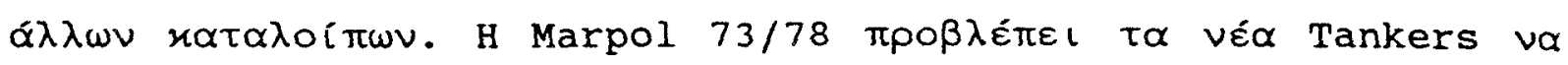

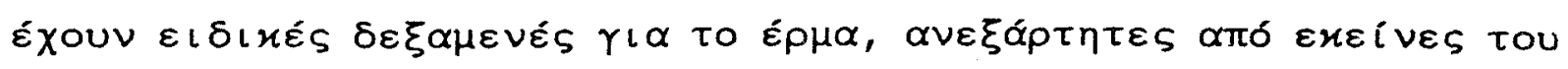

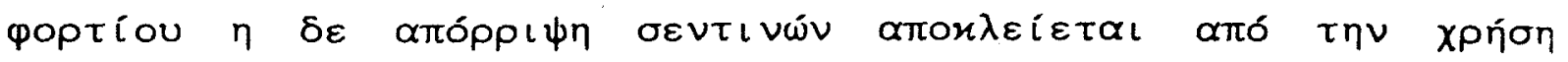

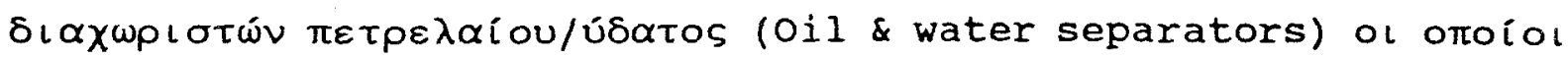

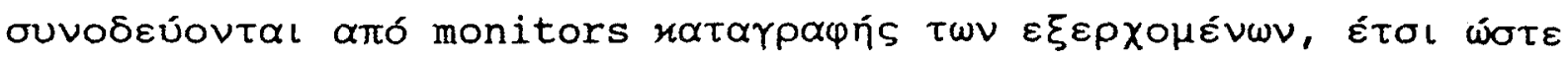

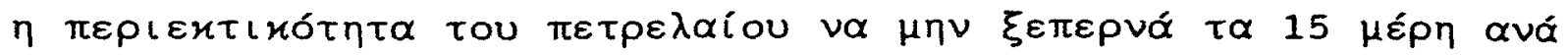

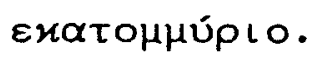

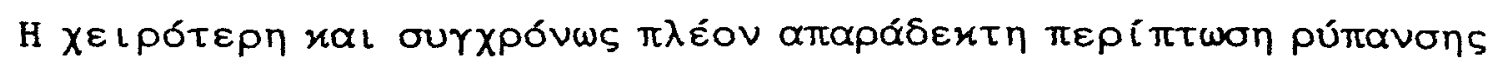

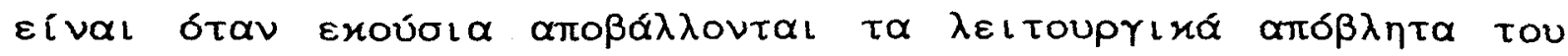

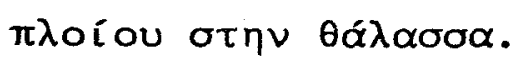


Eívaı రీ đutơ:

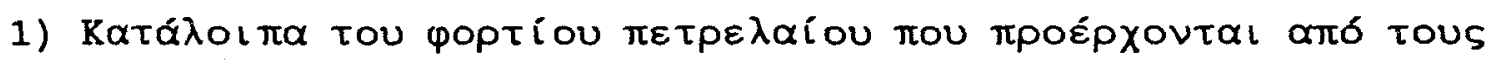

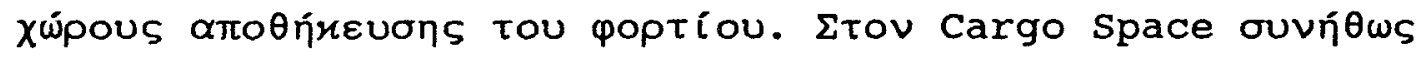

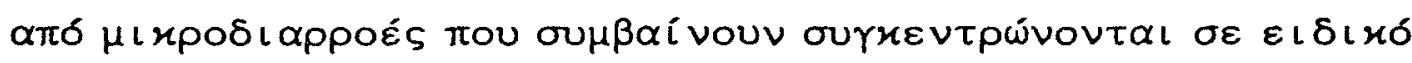

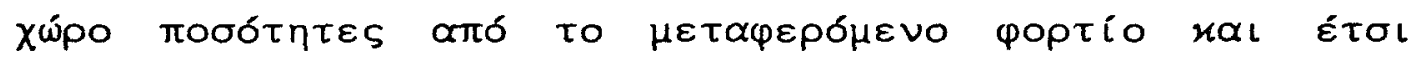

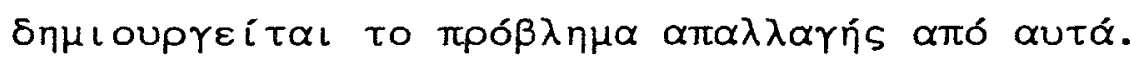

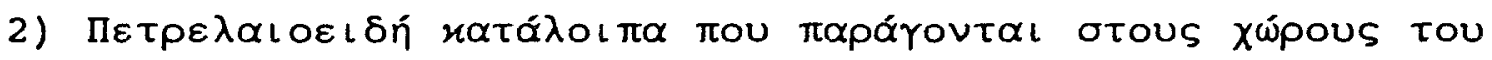

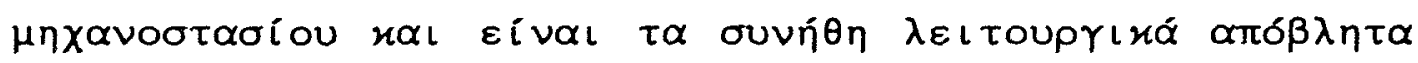

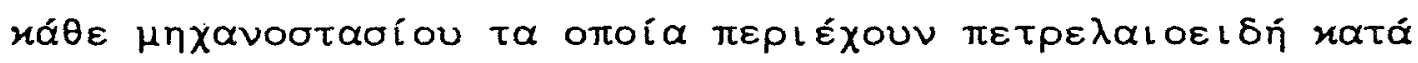

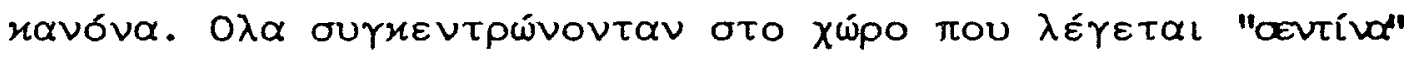

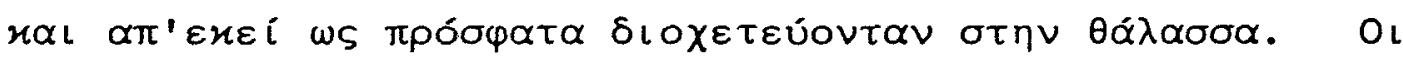
$\delta \eta \mu\llcorner$ о

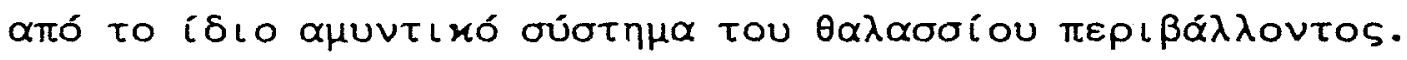

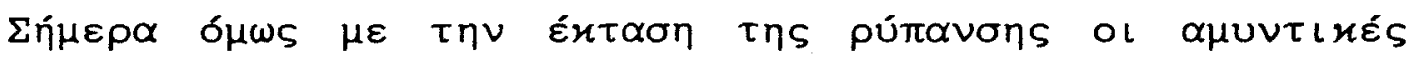

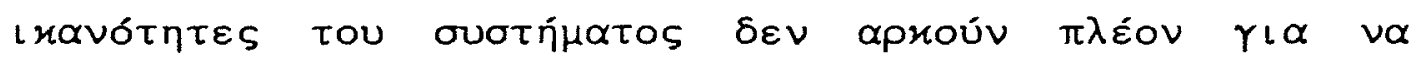

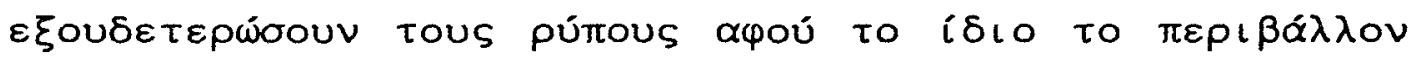

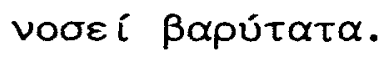




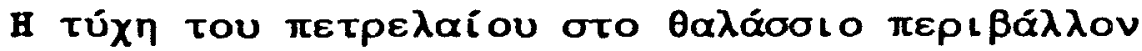

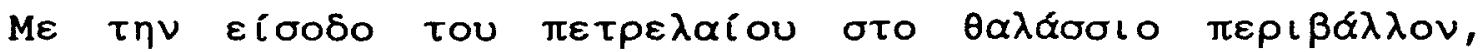

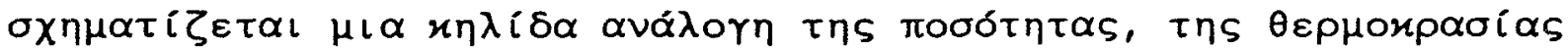

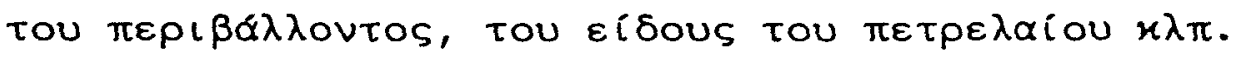

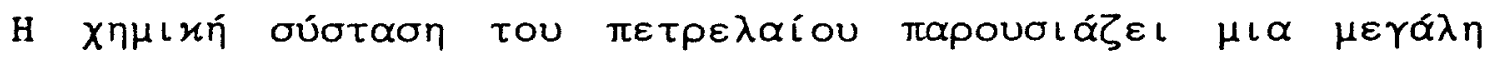

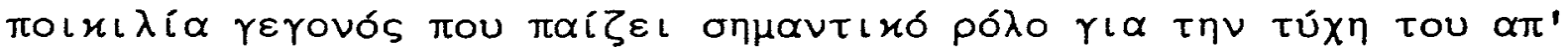

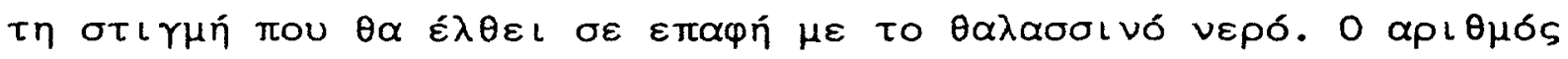

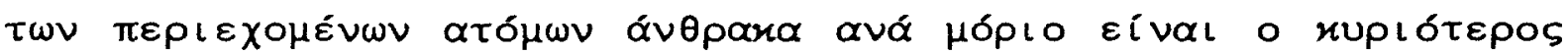

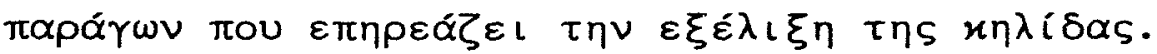

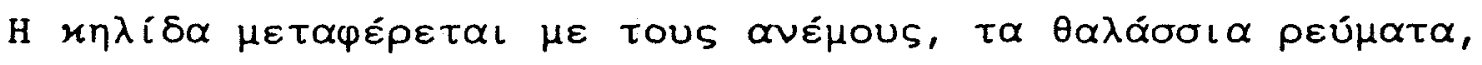

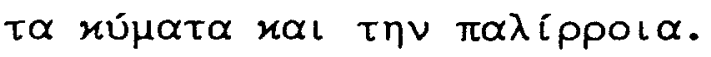

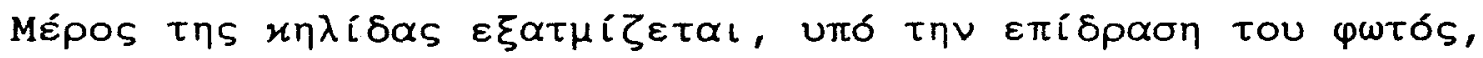

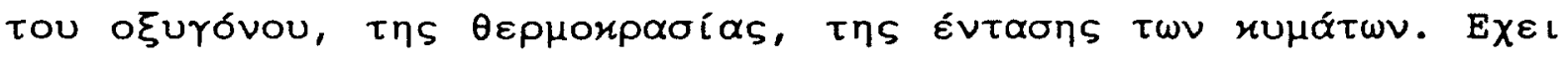

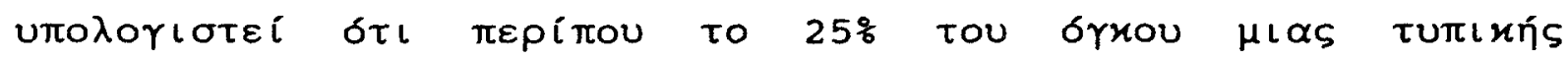

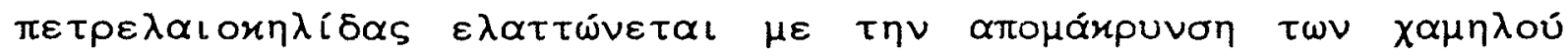

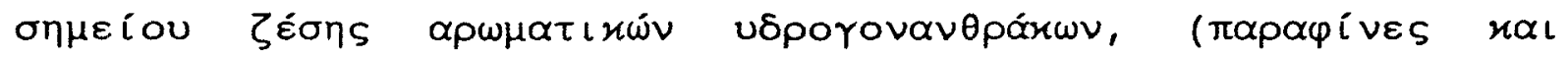

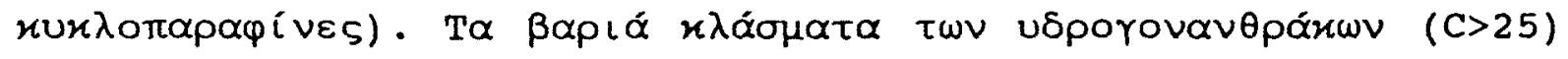

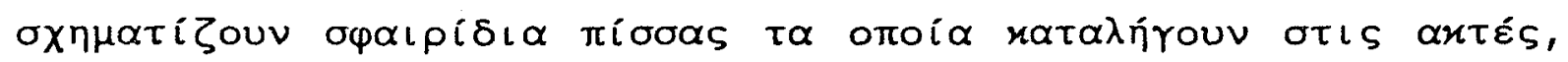

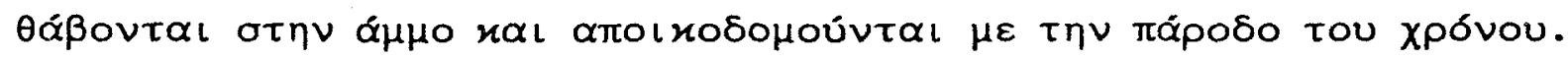

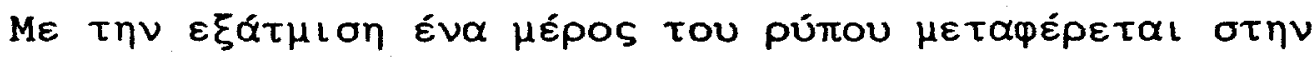




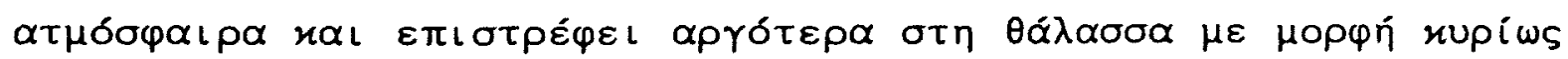

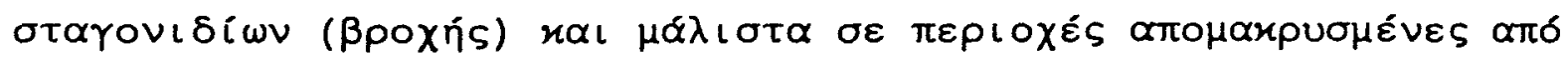

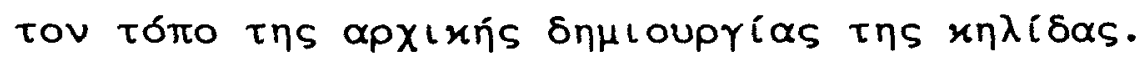

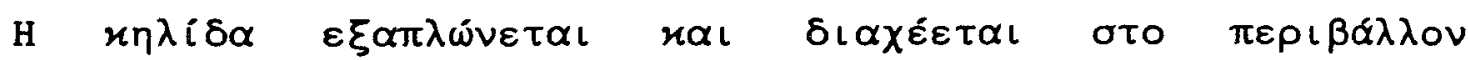

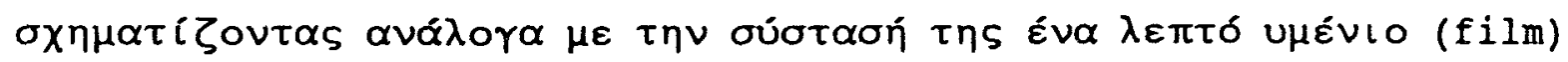

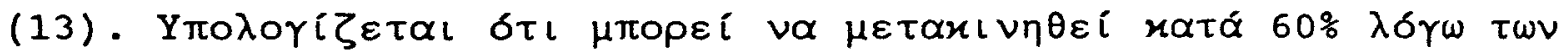

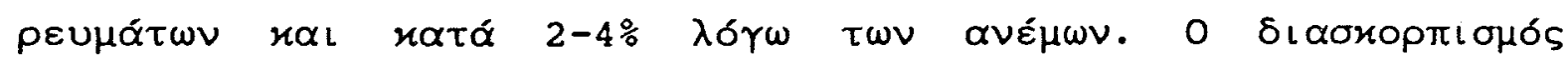

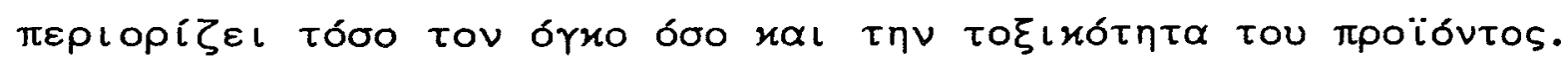

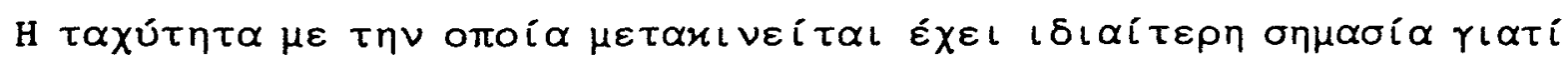

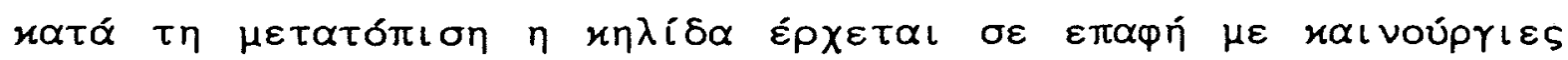

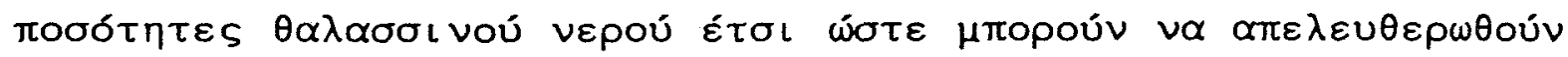

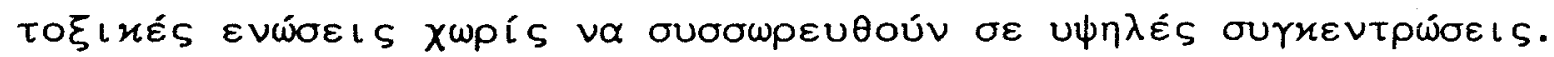

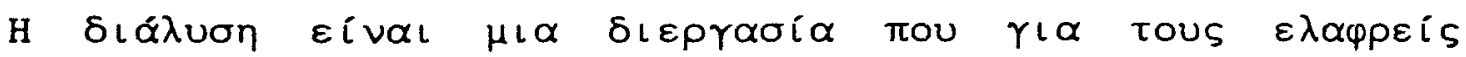

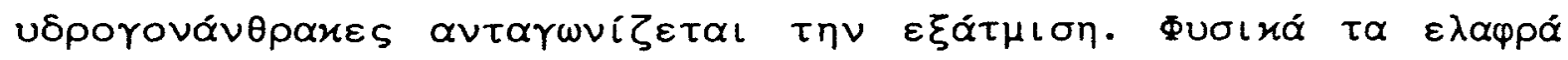

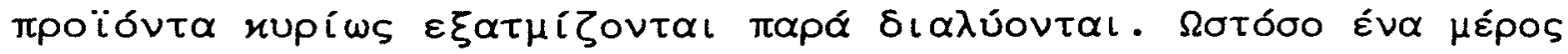

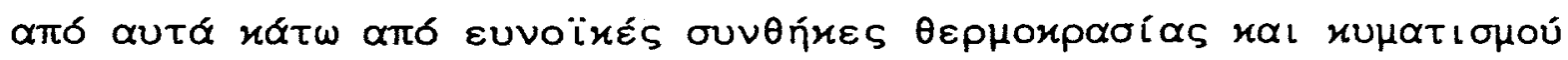

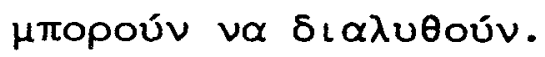

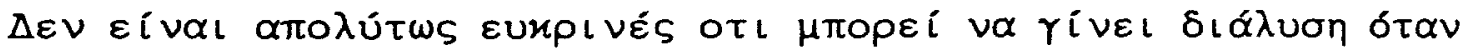

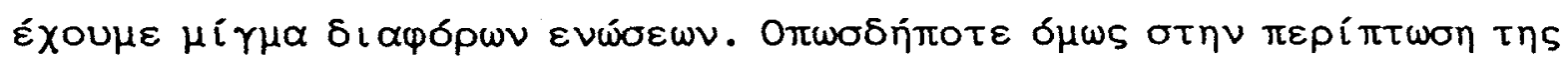

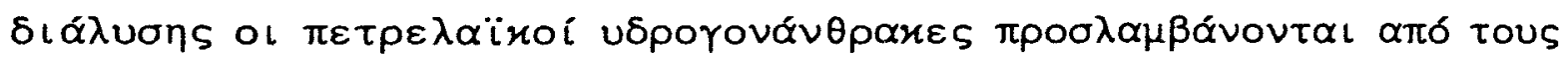

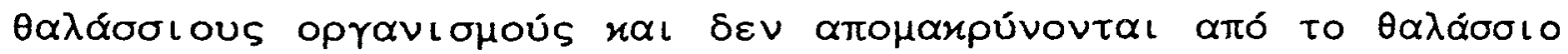
$\pi \varepsilon \rho\llcorner\beta \not ̛ ́ \lambda \lambda \circ \vee$. 


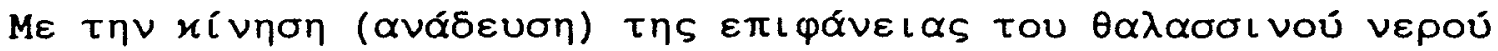

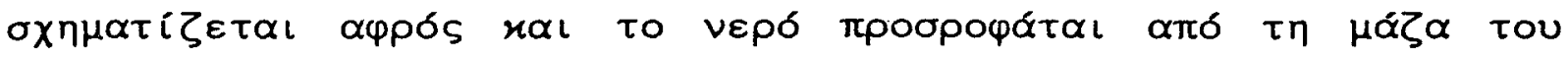
$\pi \varepsilon \tau \rho \varepsilon \lambda \alpha i ́$ U. Eín $\pi \varepsilon \tau \rho \varepsilon \lambda \alpha \iota$

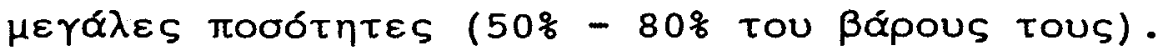

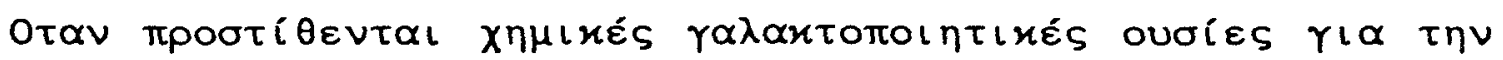

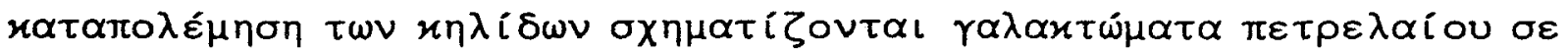

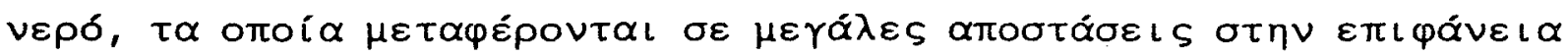

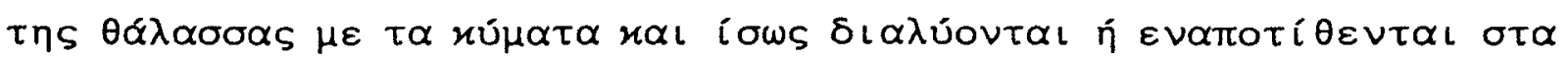

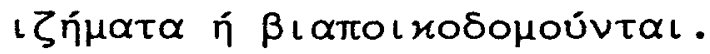

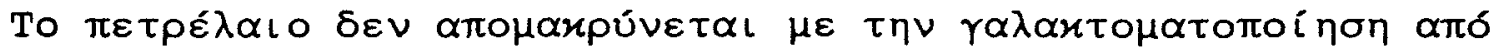

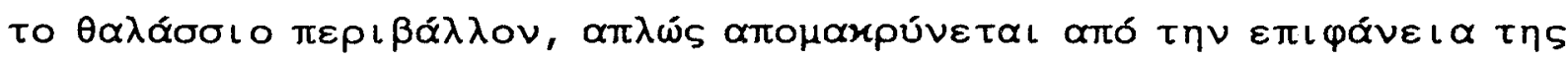

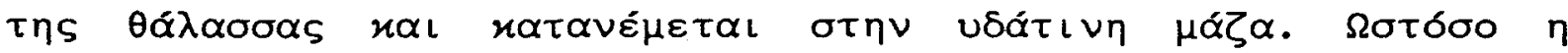

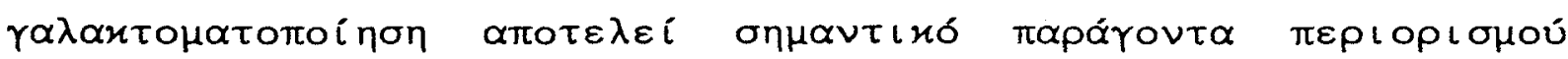

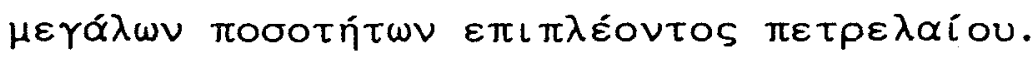

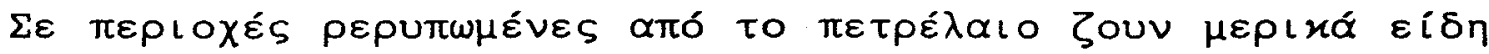

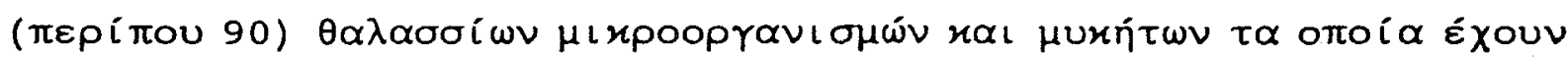

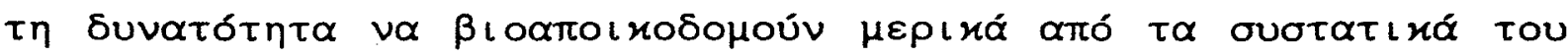

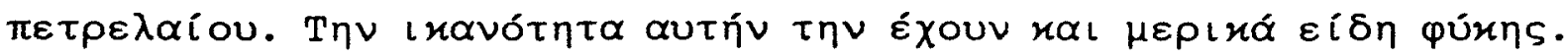

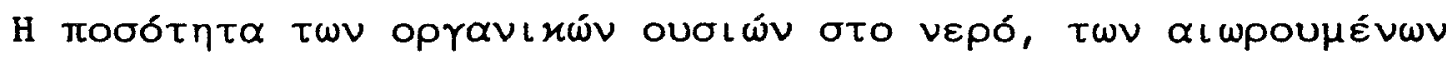

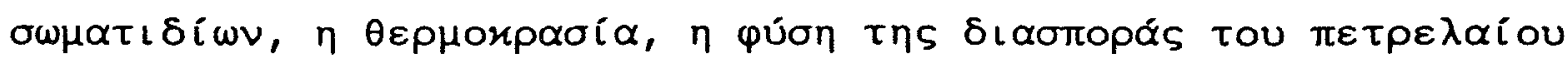

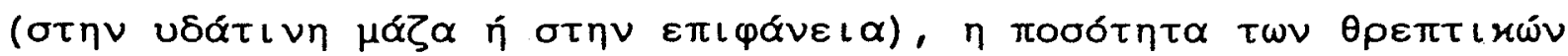

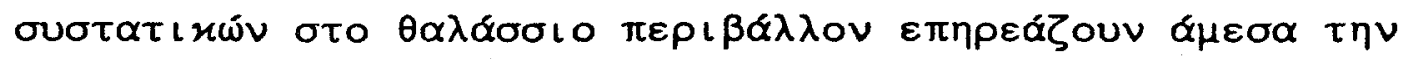




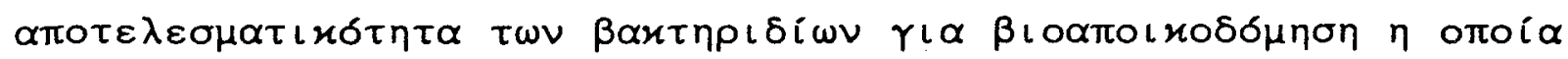

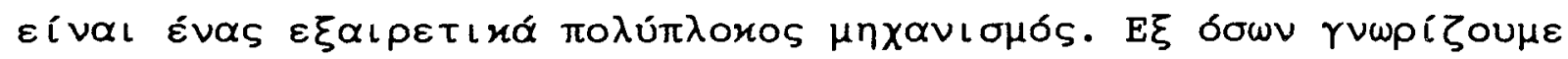

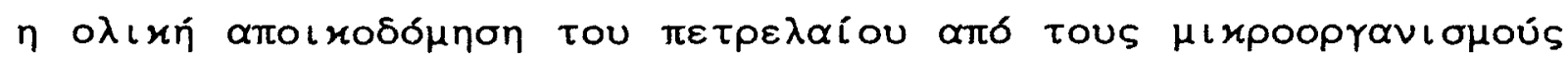

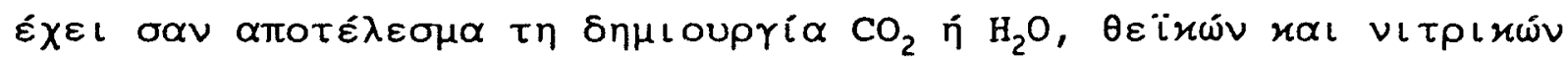

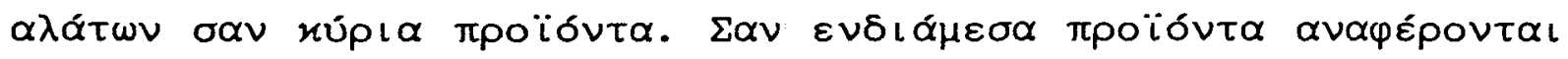

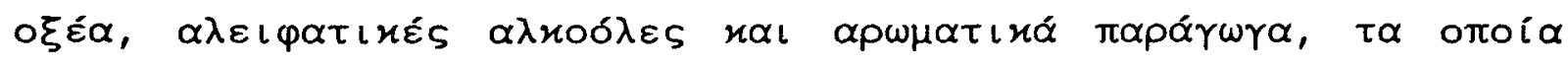

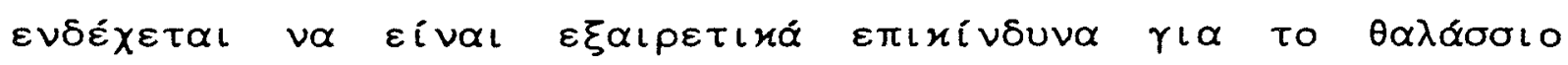

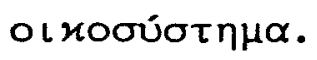

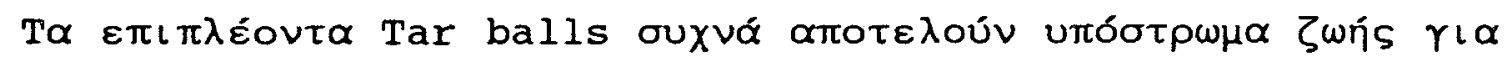

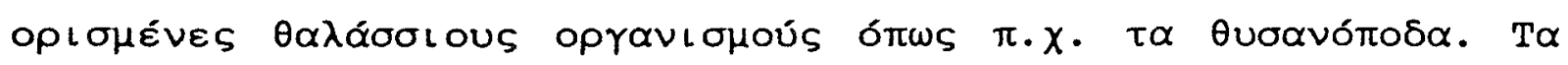

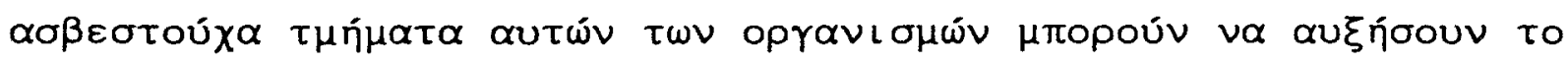

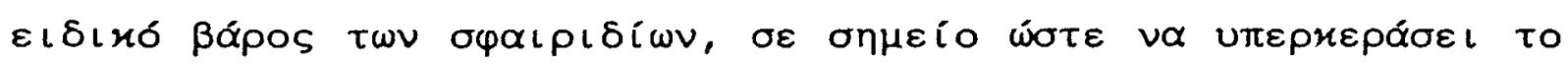

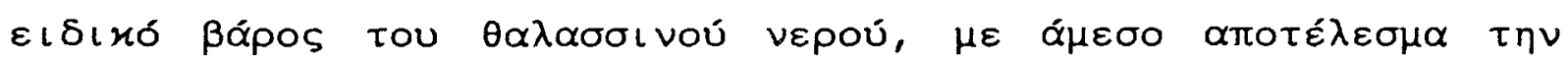

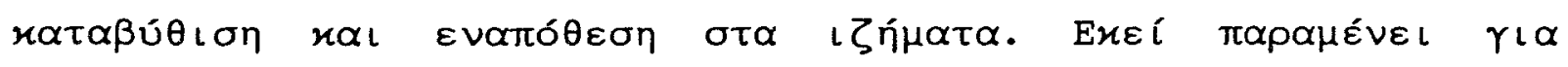

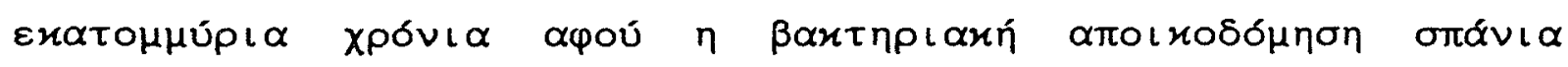

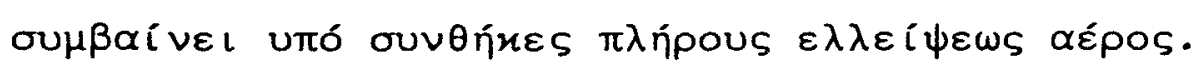

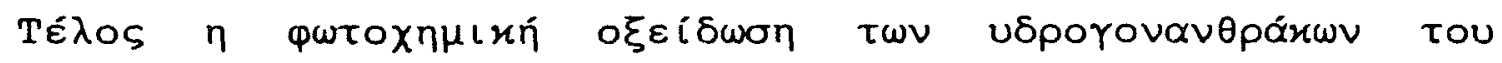

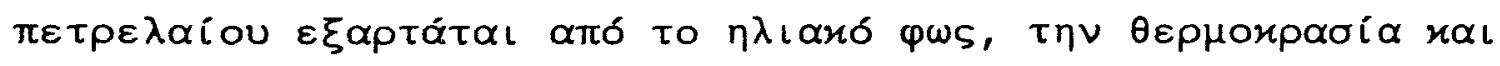

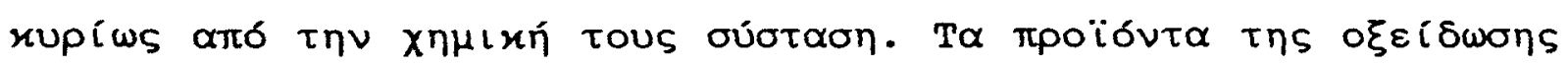

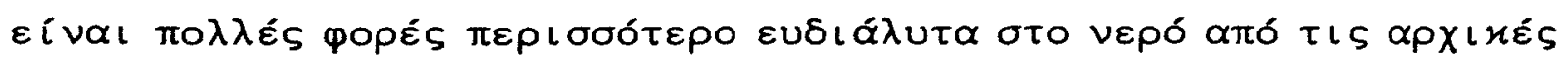

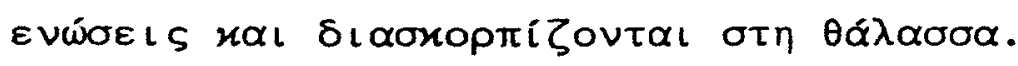




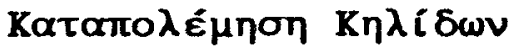

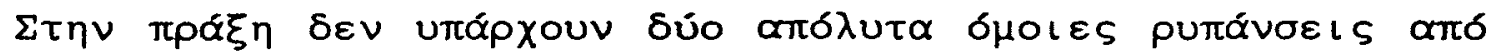

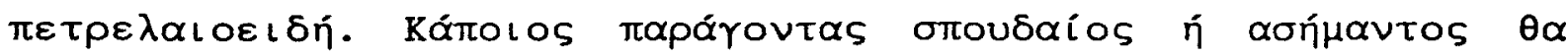

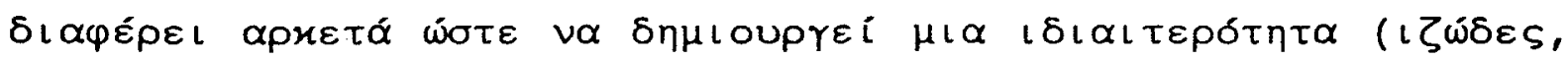

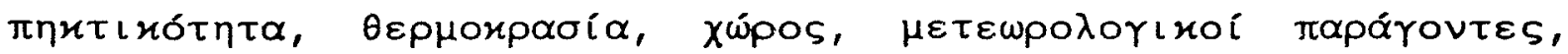

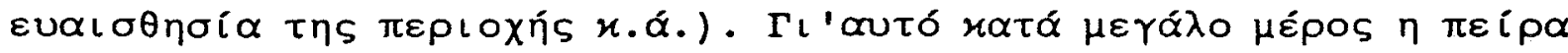

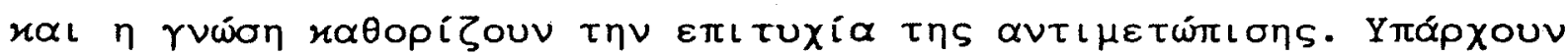

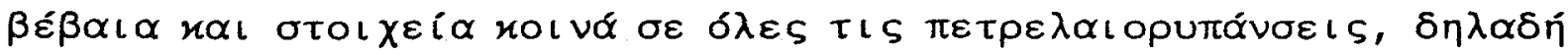

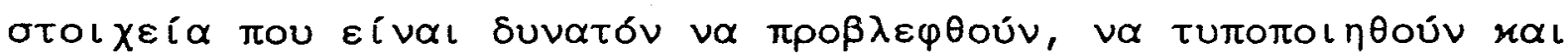

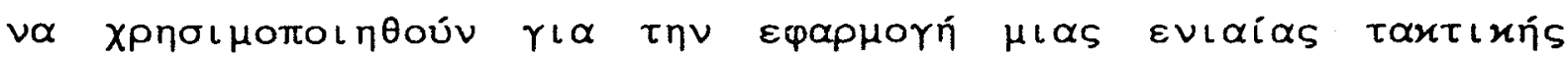

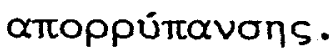

H $\varepsilon$ Uหว $\alpha$ Q

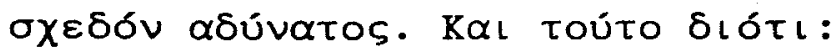

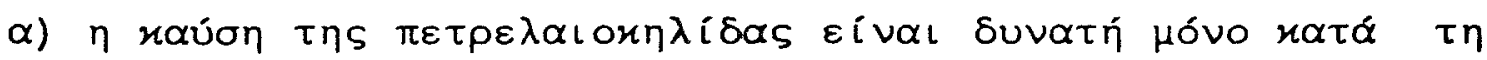

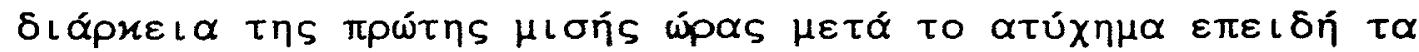

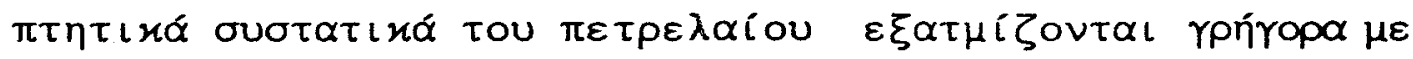

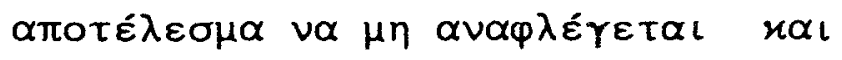




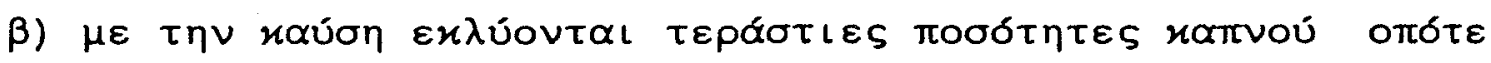

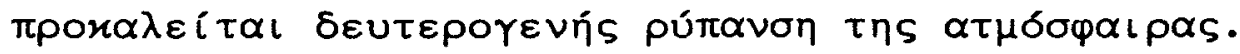

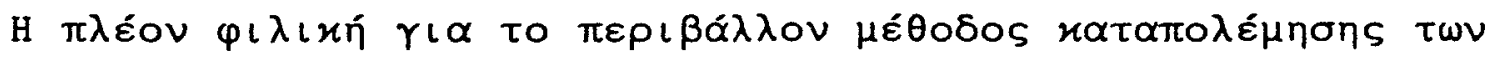

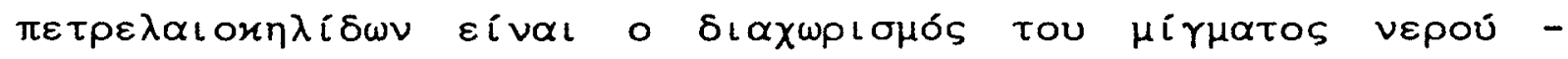

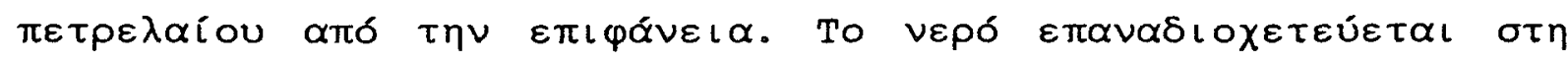

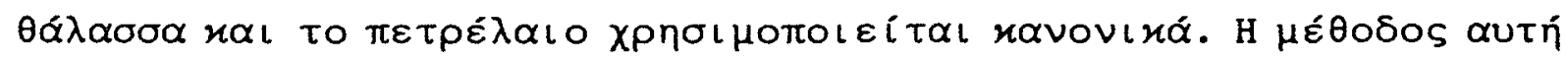

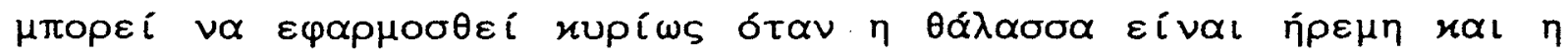

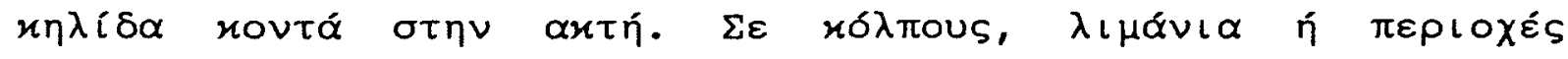

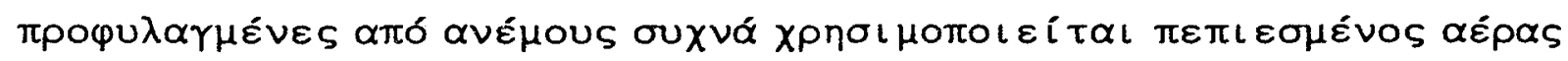

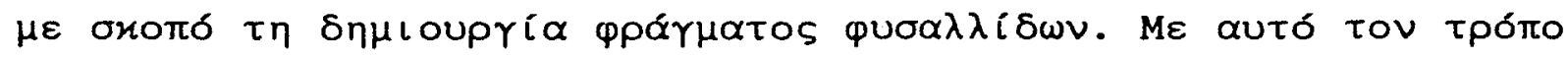

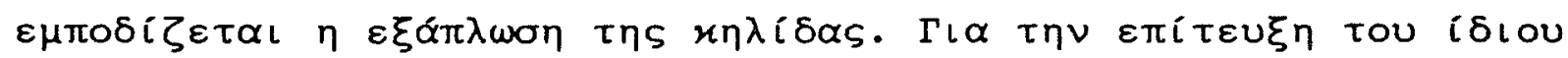

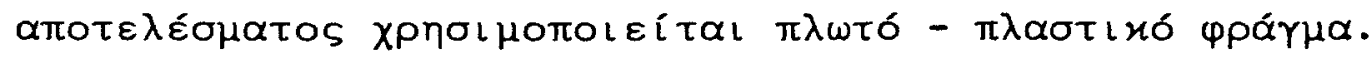

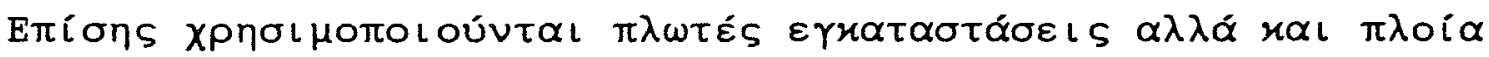

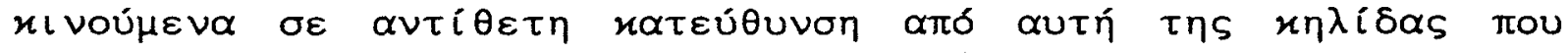

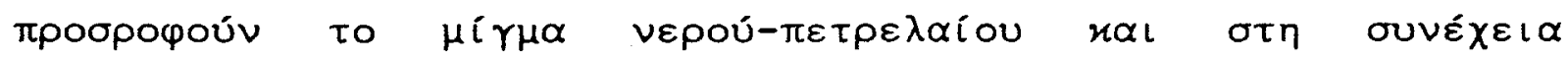

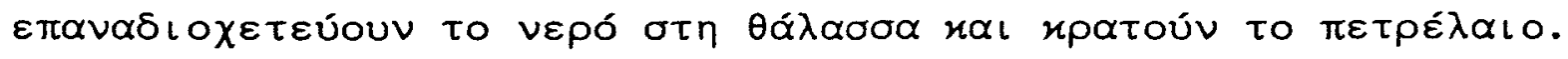

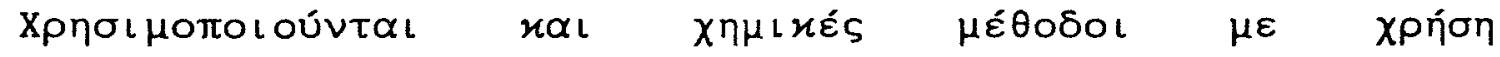

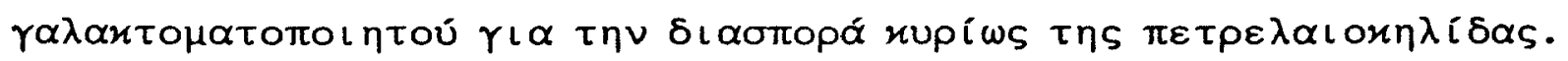

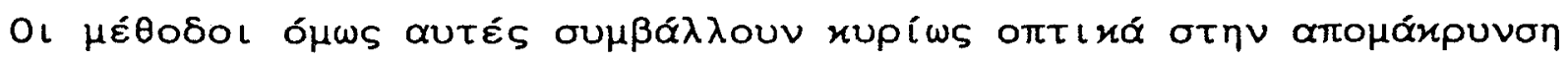

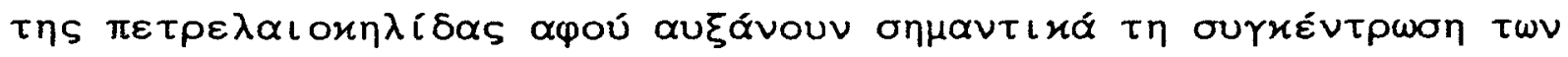

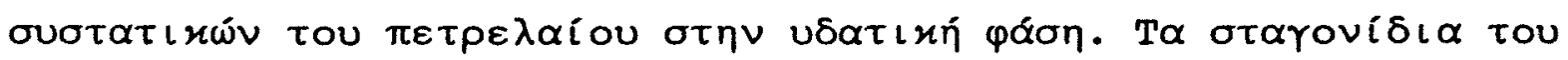




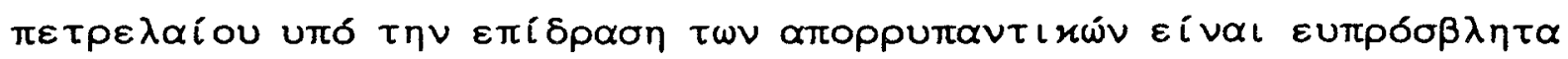

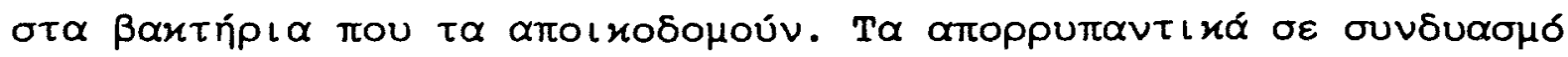

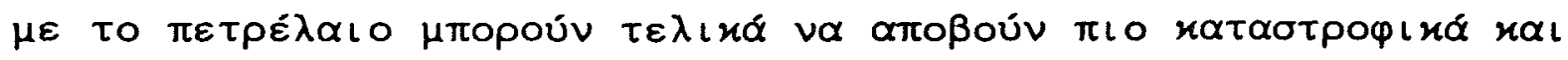

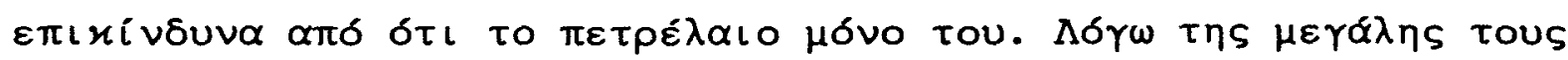

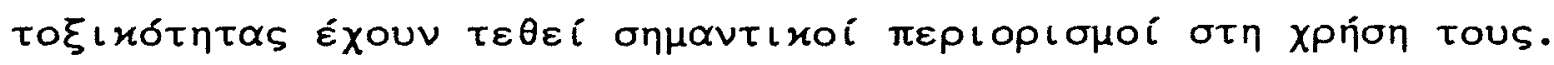

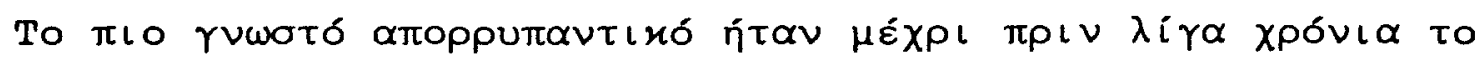

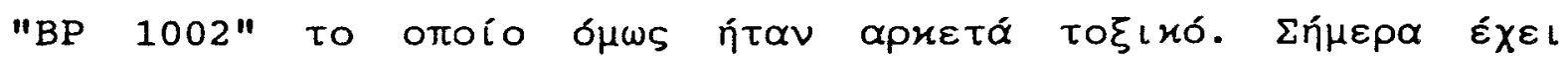

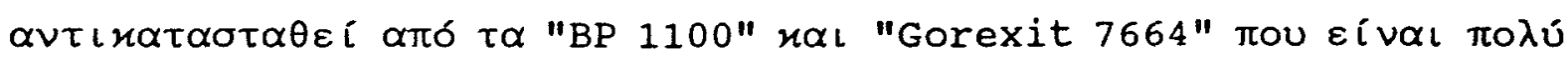

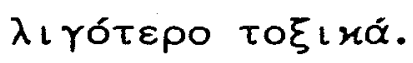

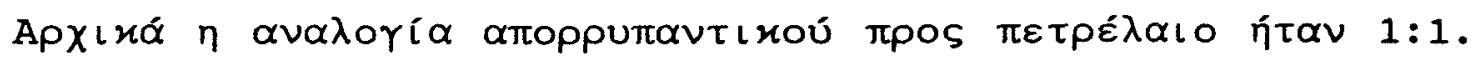

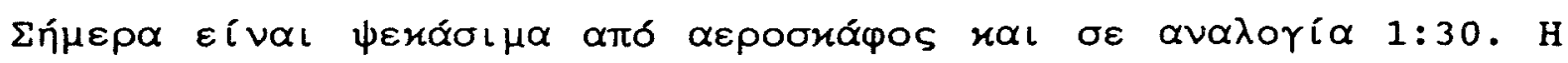

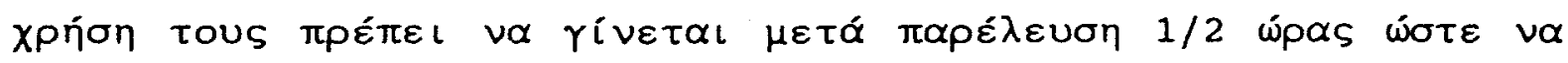

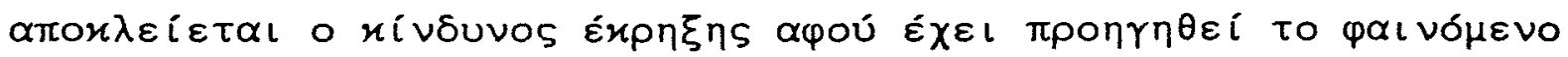

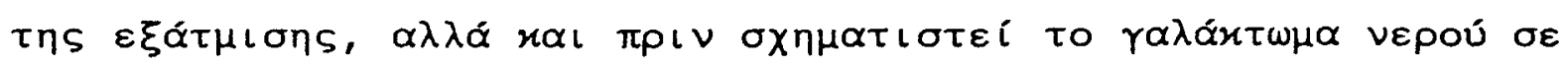

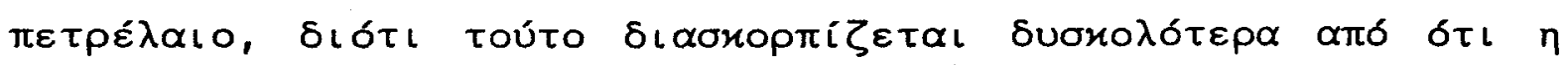
$\pi \varepsilon \tau \rho \varepsilon \lambda \alpha \iota$ oห $\eta \lambda i \delta \alpha$. 


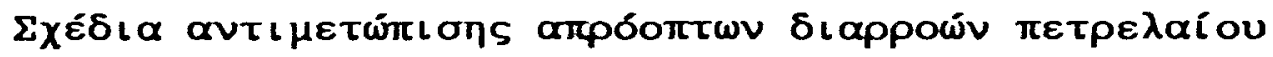

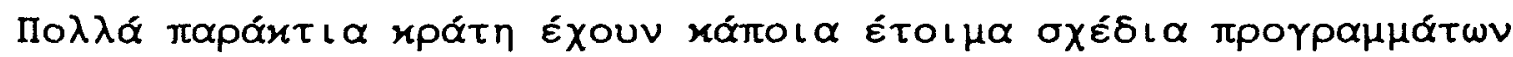

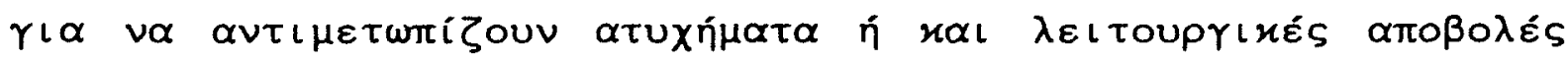

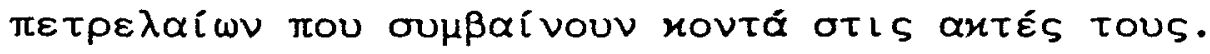

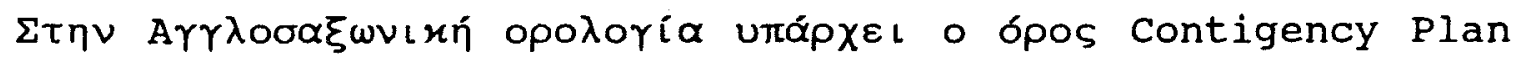

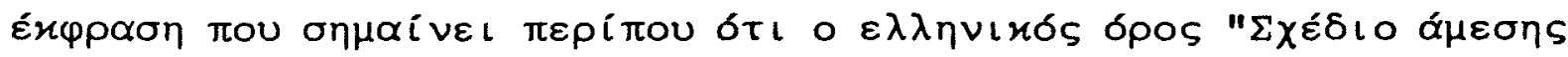

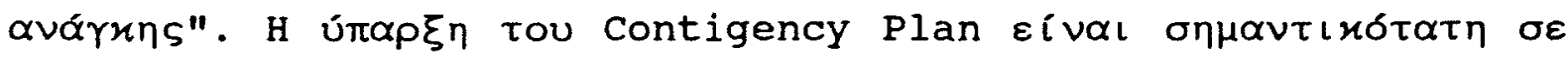
$\varkappa \alpha \dot{\theta} \theta \varepsilon \pi \varepsilon i \pi \tau \omega \sigma \eta \eta$

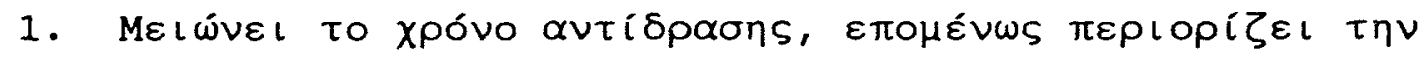

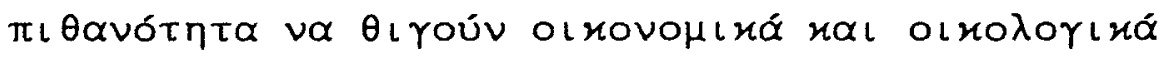

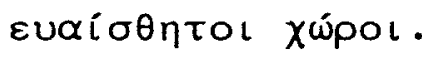

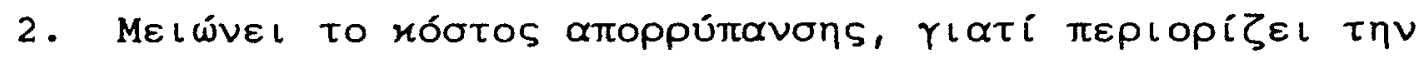

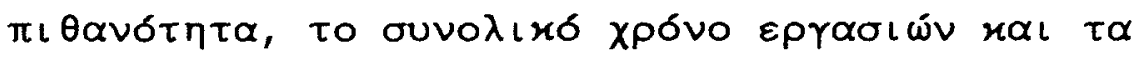
$\alpha \pi \alpha \iota \tau о \cup ́ \mu \varepsilon \vee \alpha \mu \varepsilon ́ \sigma \alpha$.

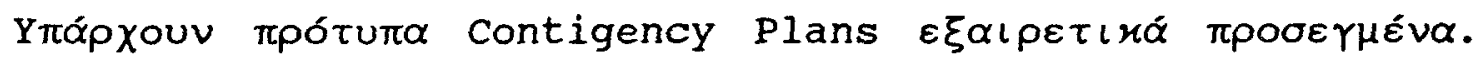

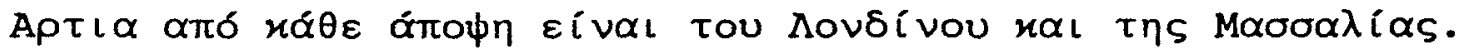

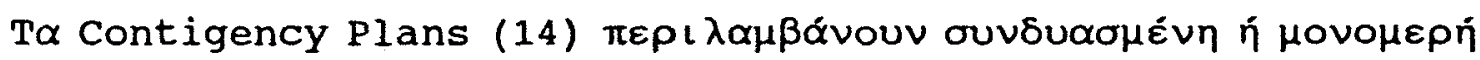




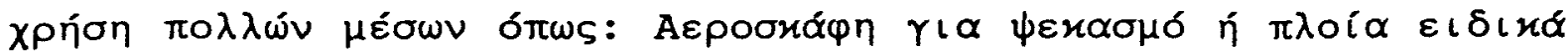

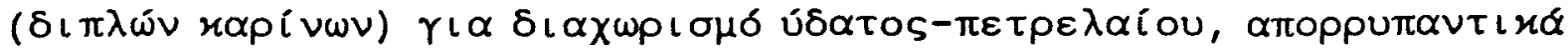

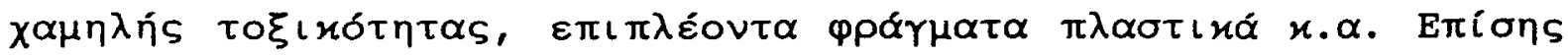

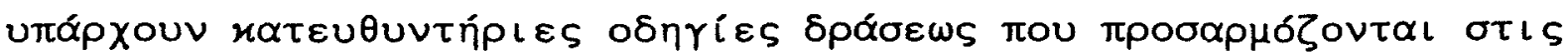

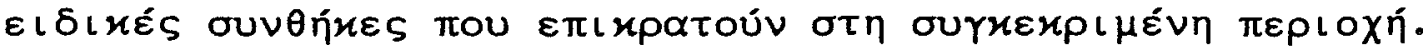

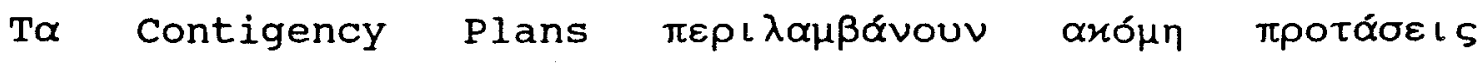

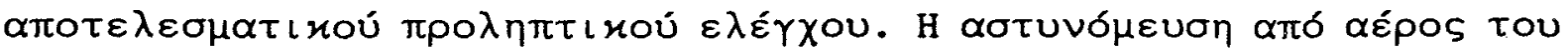

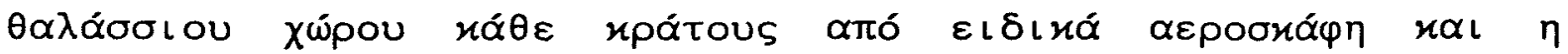

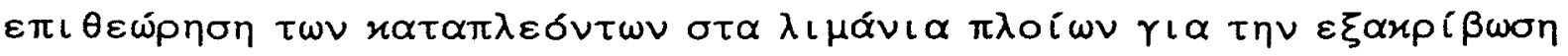

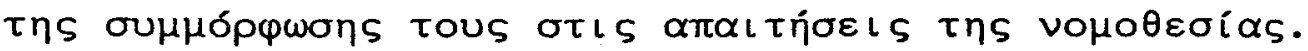




\section{$\underline{\boldsymbol{\Sigma}} \mathrm{HMBI \Omega \Sigma EI \Sigma}$}

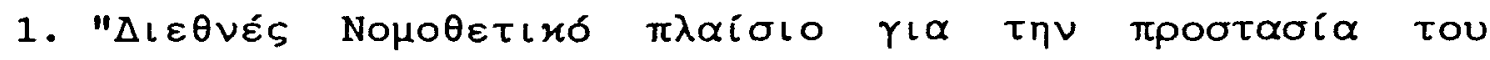

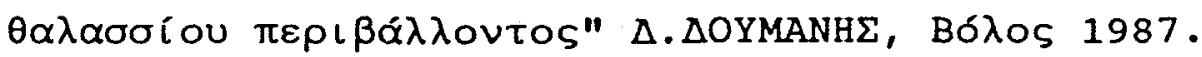

2. International Convention of 1973 for the prevention of Pollution from Ships (MARPOL 73/78) Annex I: Oil Pollution.

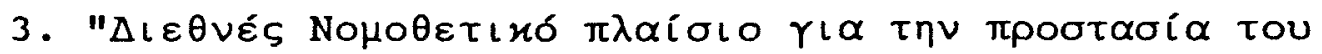

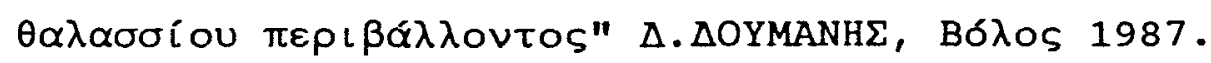

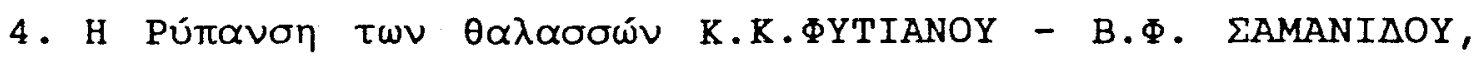
$\Theta \varepsilon \sigma \sigma \alpha \lambda o v i$ K 1988 .

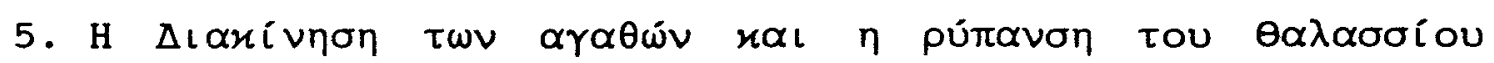

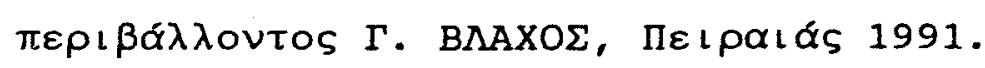

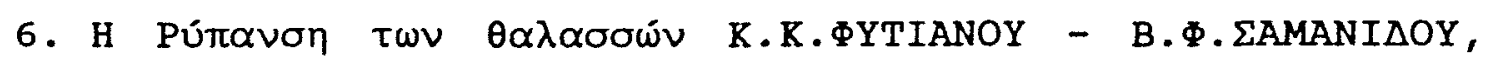

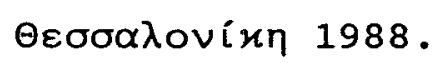




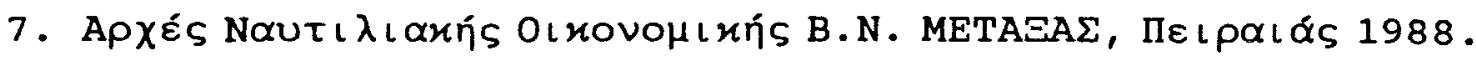

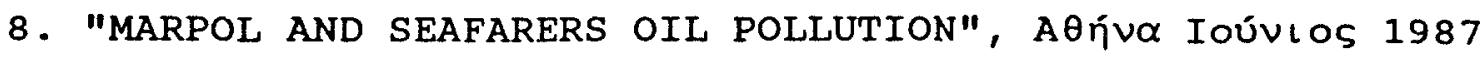

9. "MARPOL AND SEAFARERS OIL POLLUTION", A $\theta$ ñva Ioúvlos 1987

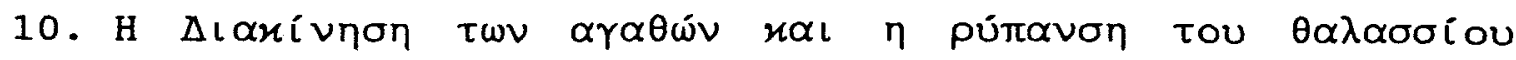

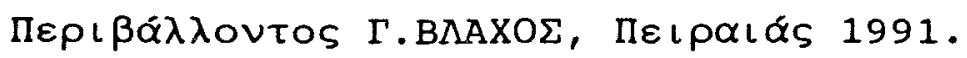

11. "Oil tanker design and pollution prevention - A report by the shipping industry", 1990.

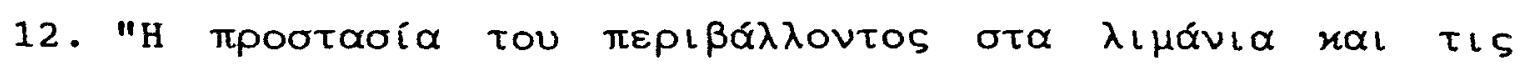

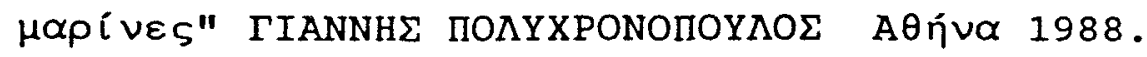

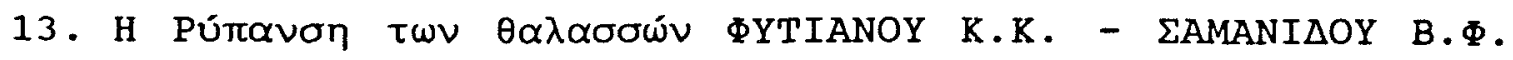

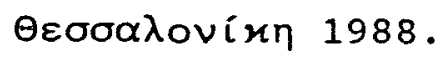

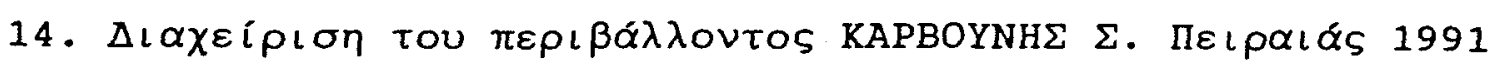




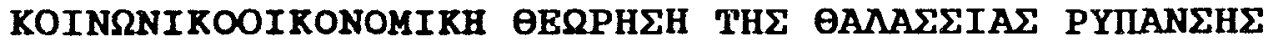

\section{IEPIBANAONTIKH PYIANEH}

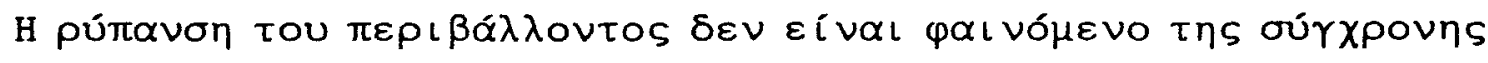

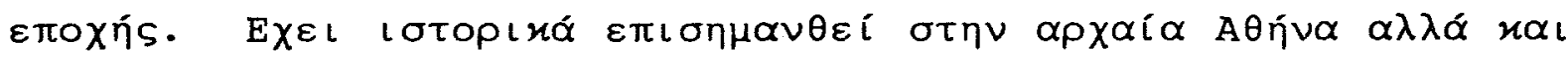

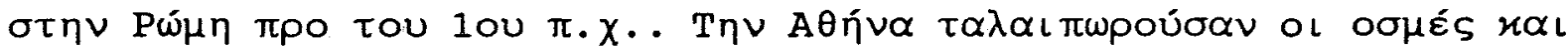

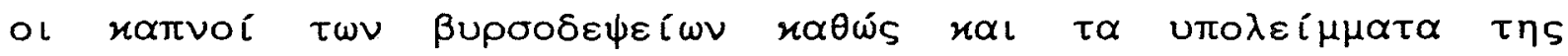

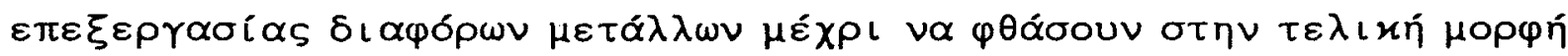

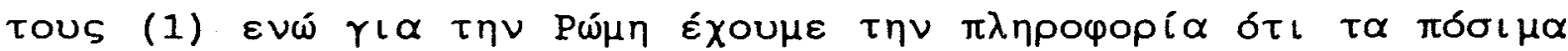

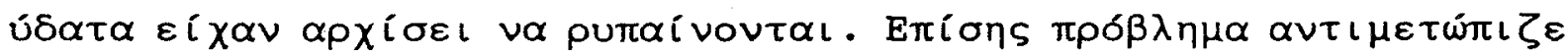

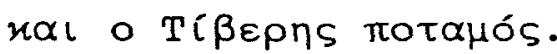

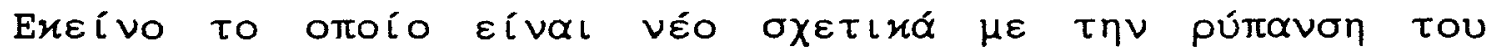

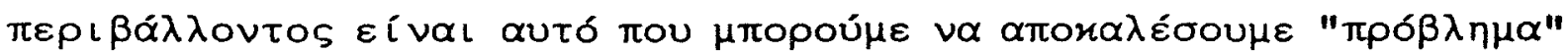

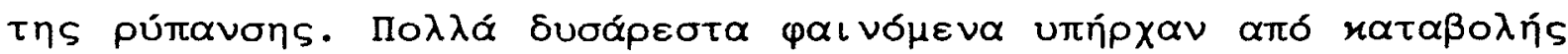

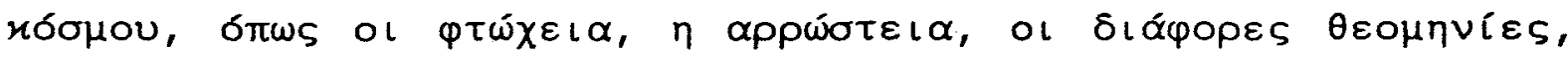

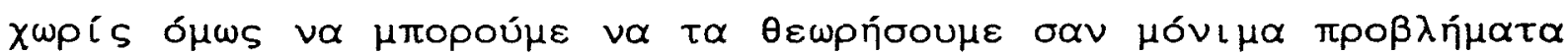

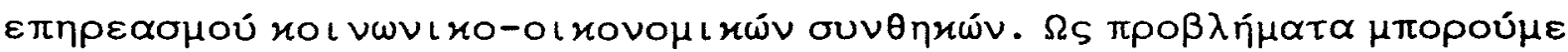

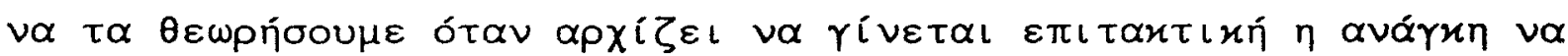

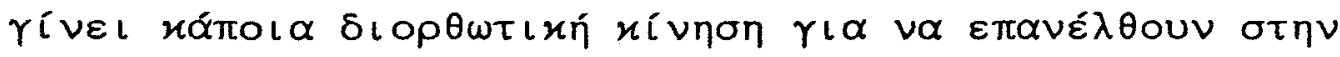

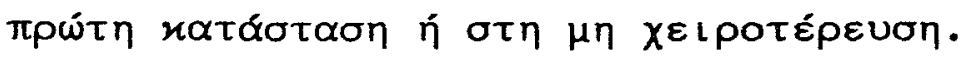




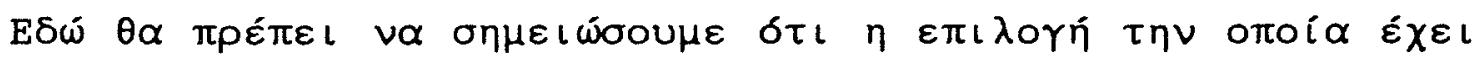

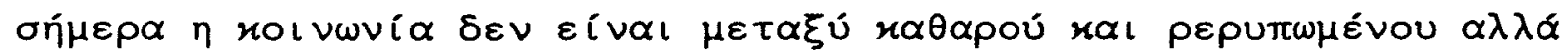

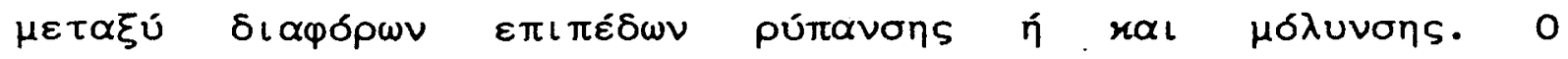

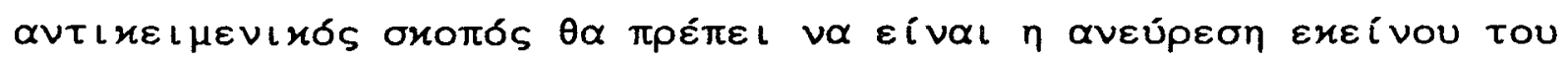

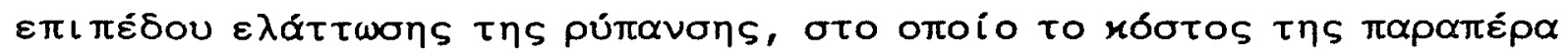

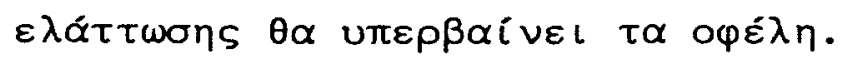

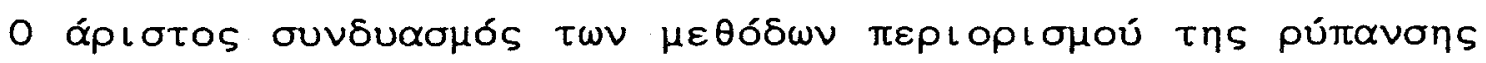

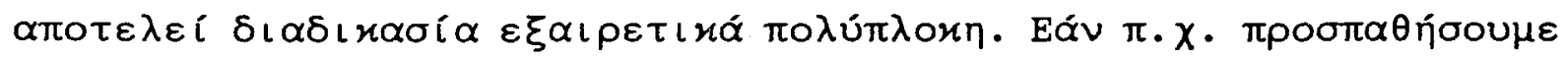

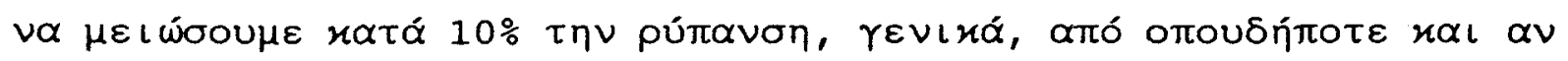

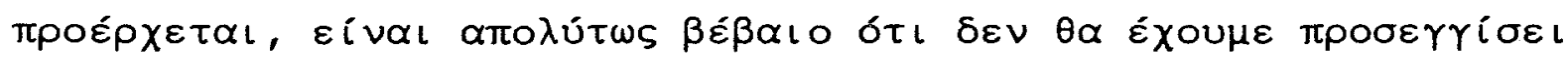

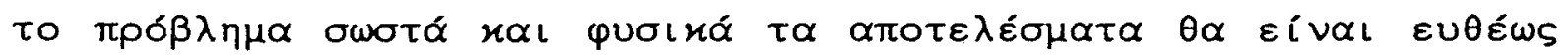

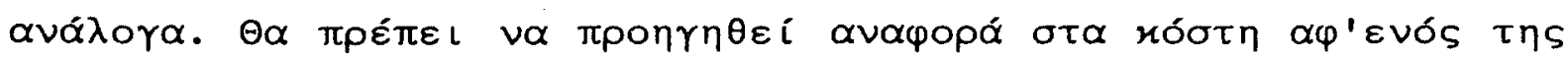

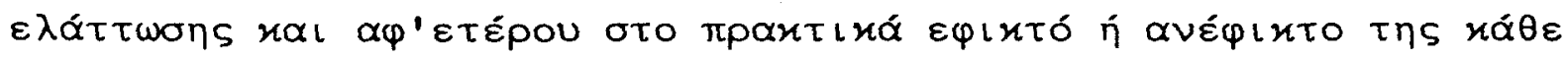

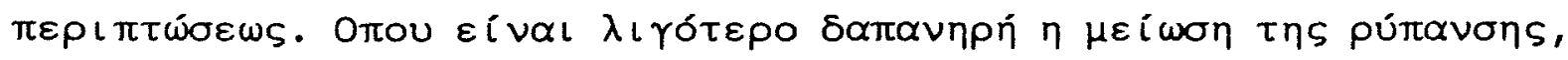

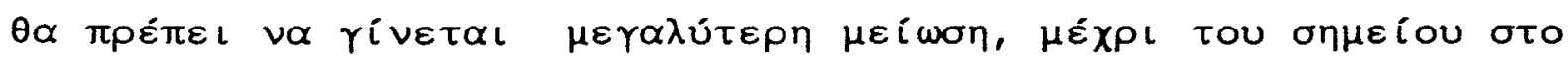

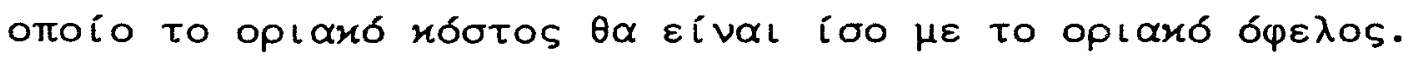

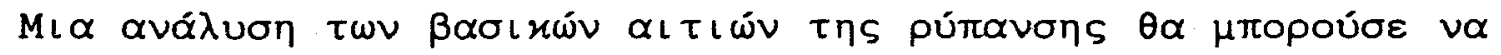

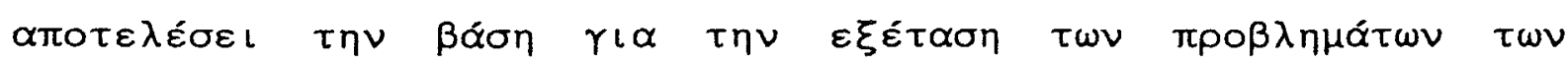

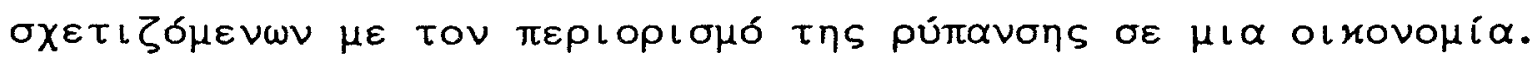

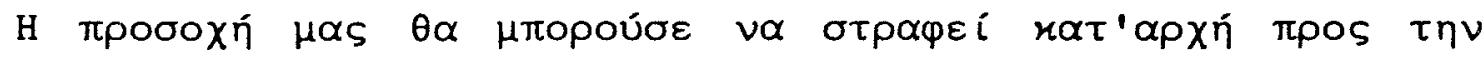

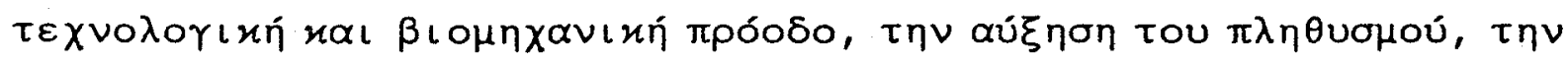


$\alpha \sigma \tau \iota$ หотої

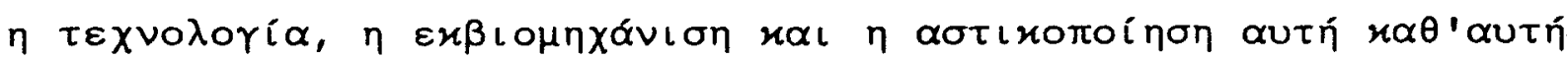

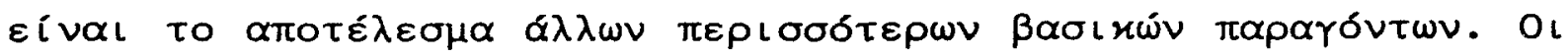

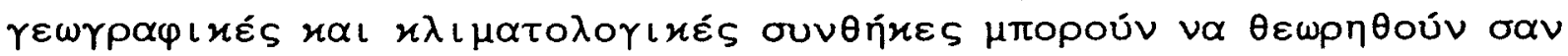

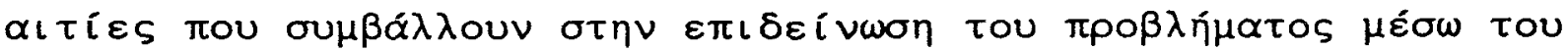

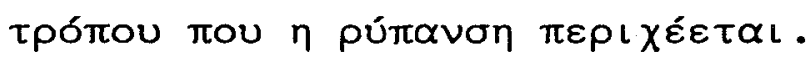

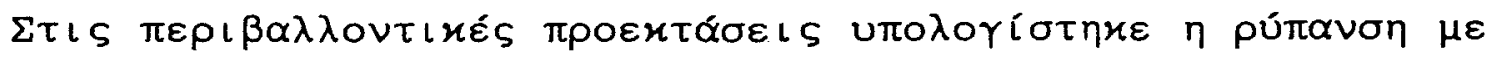

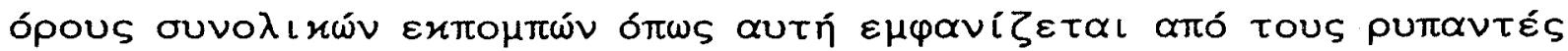

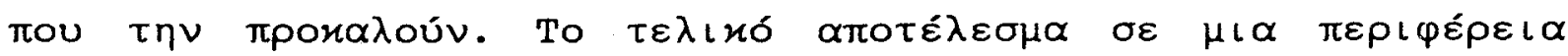

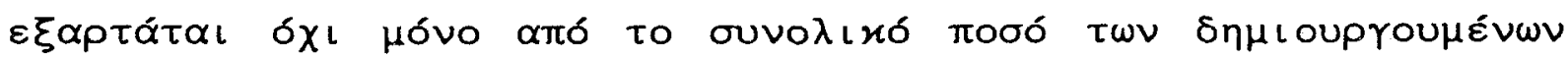

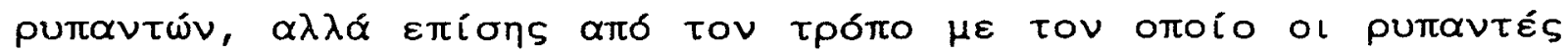

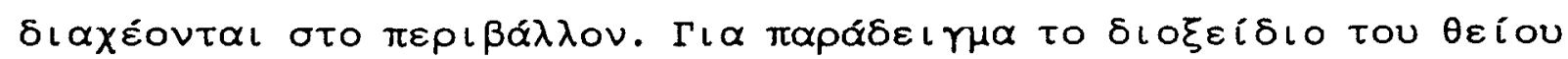

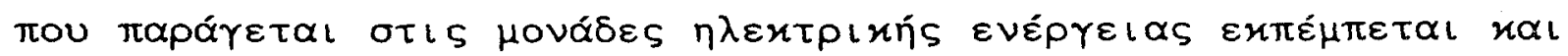

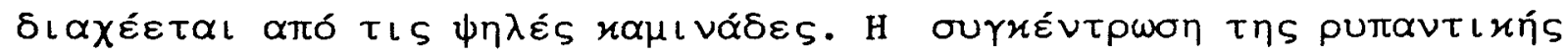

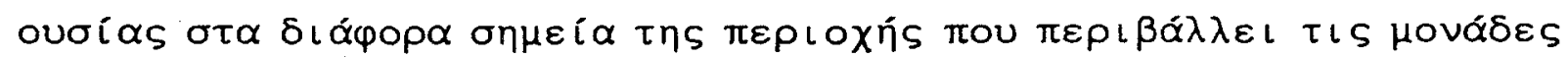

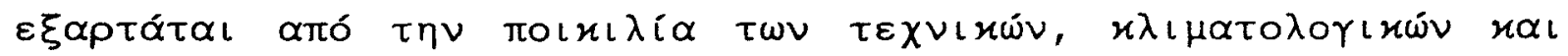

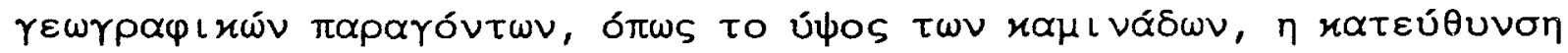

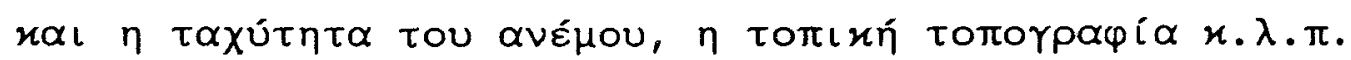

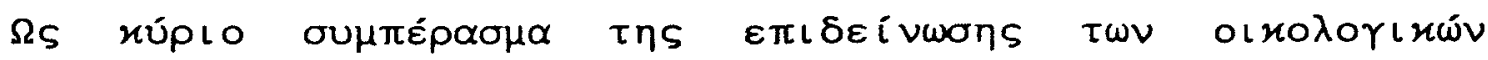

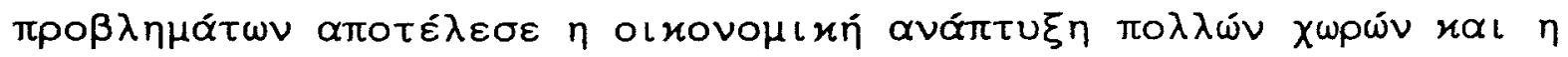

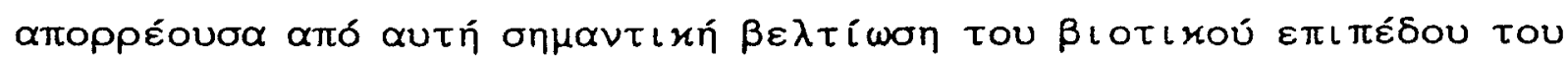
$\pi \lambda \eta \theta$ voroú tous (2). 


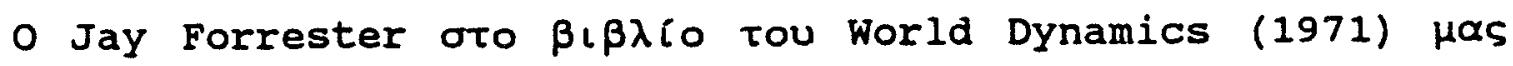

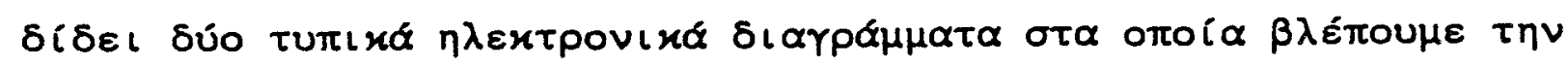

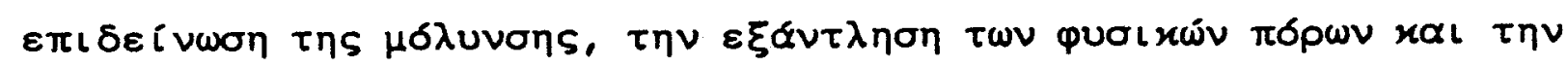

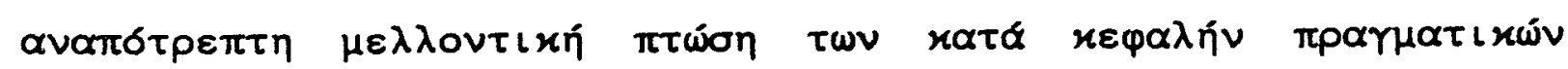

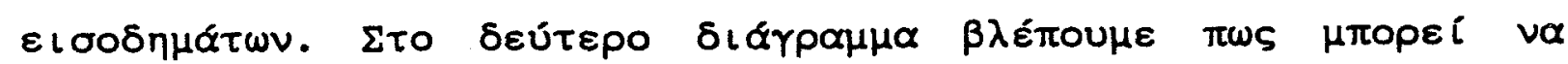

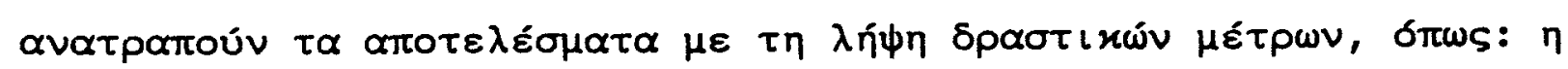

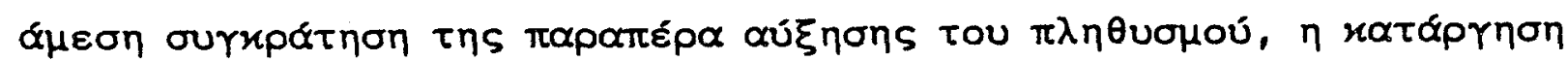

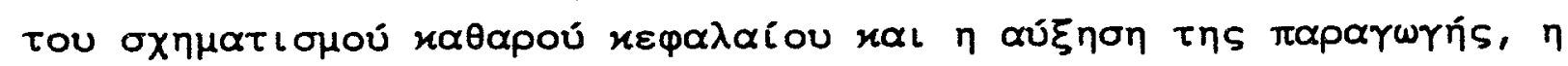

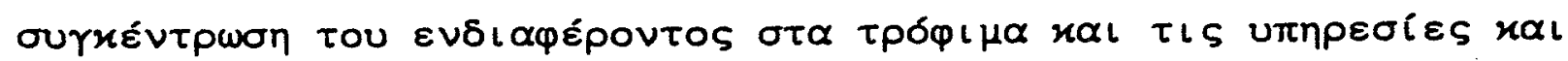

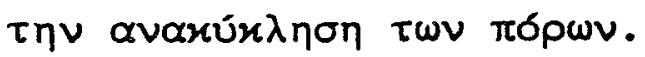

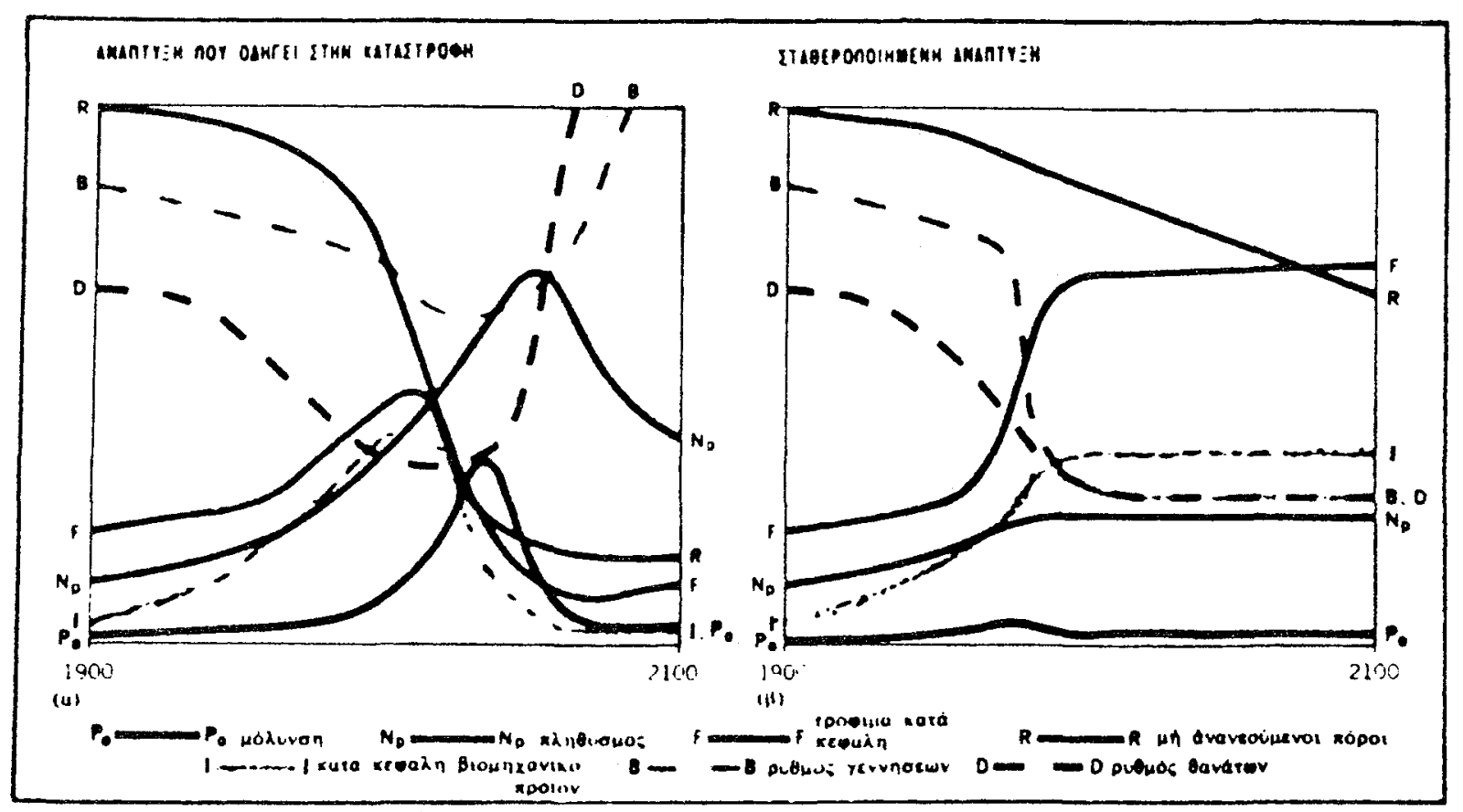

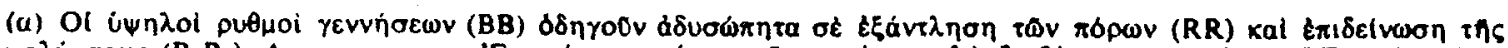

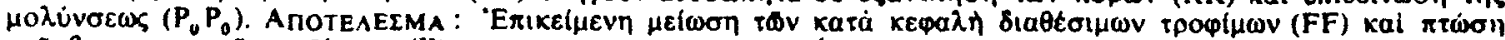

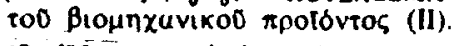

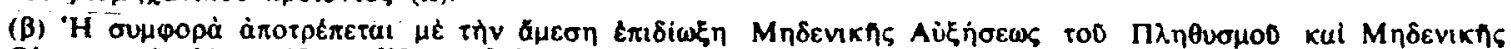

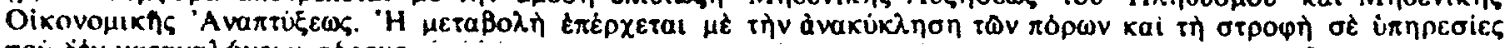
nou dév kutavahavouv rópous. 


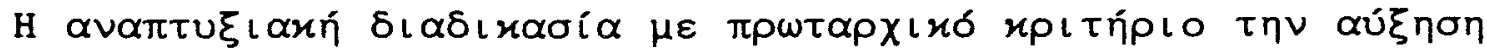

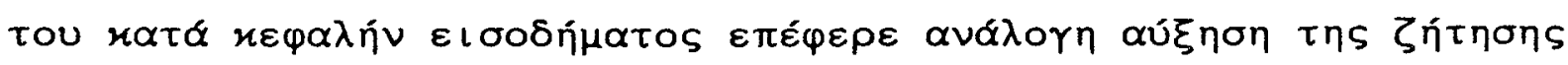

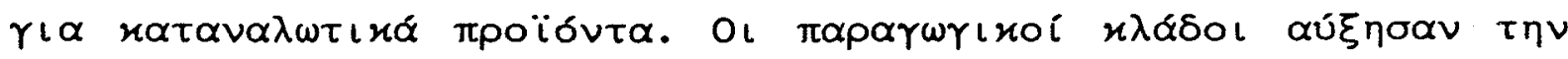

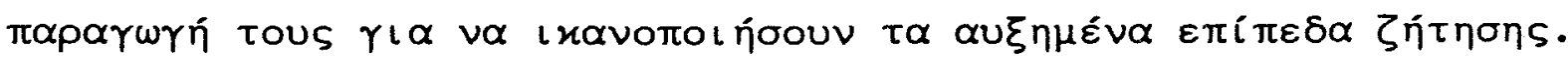

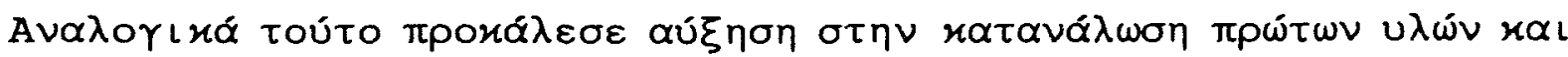

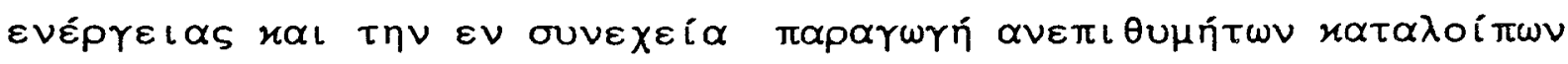

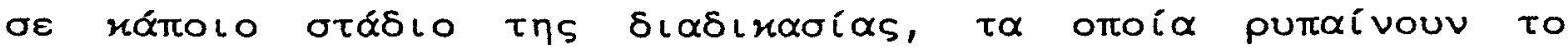
$\pi \varepsilon \rho\llcorner\beta \alpha ́ \alpha \lambda$ ov. (3)

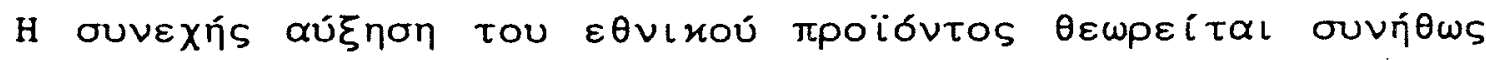

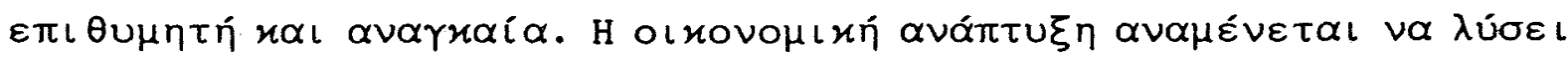

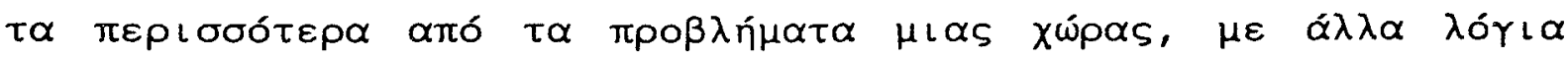

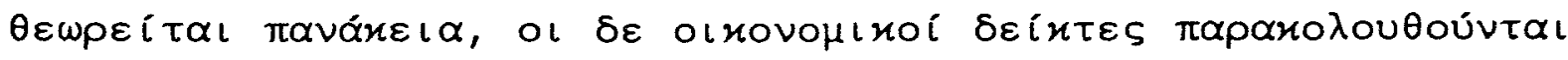

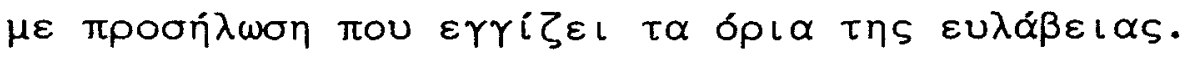

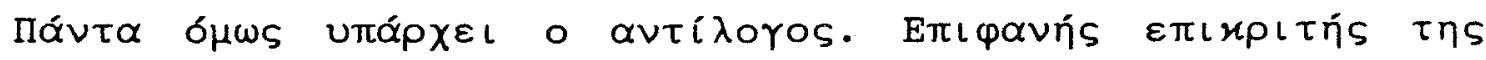

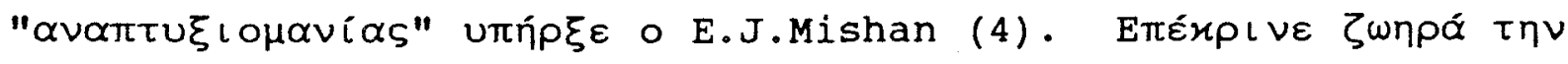

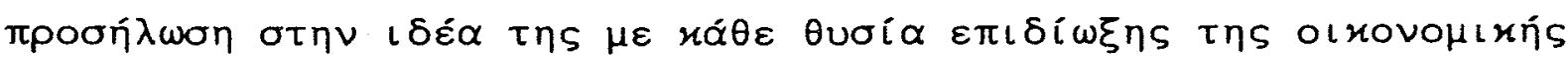

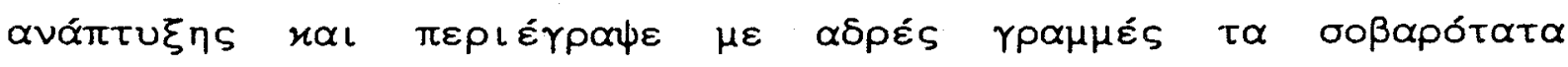

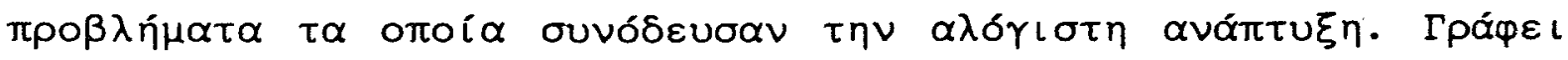

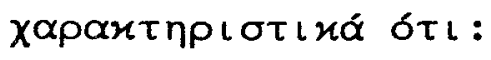

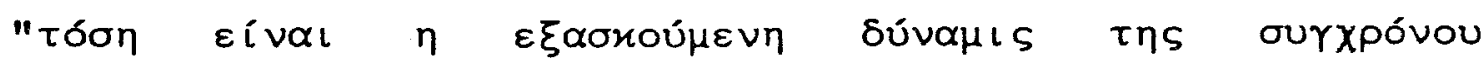

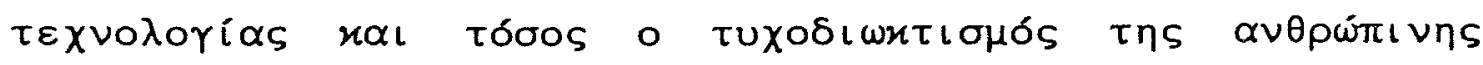

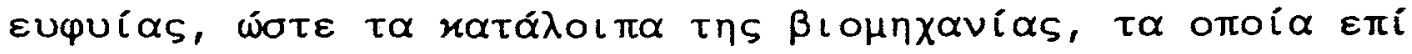




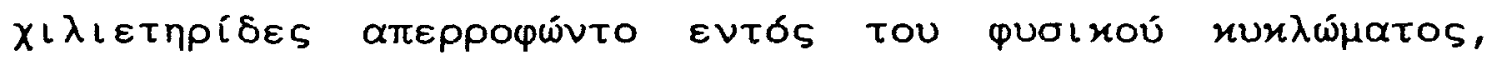

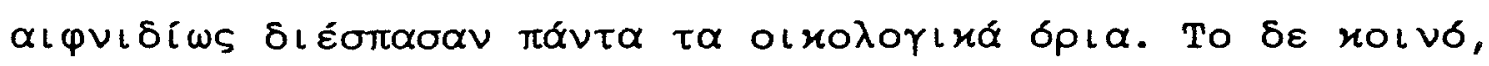

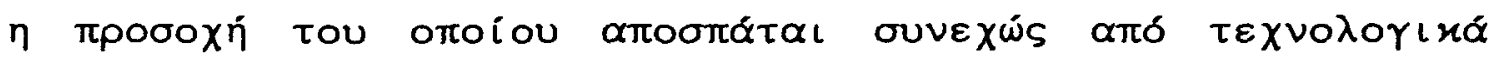

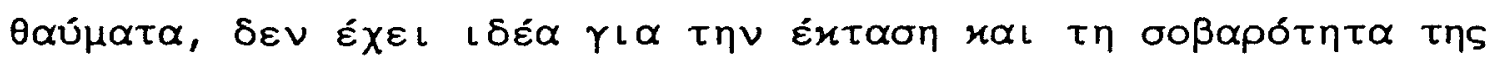

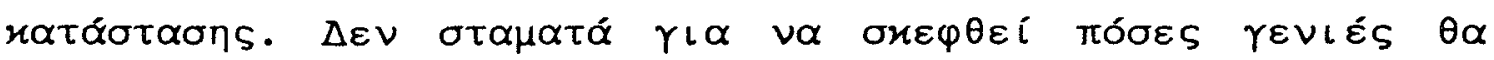

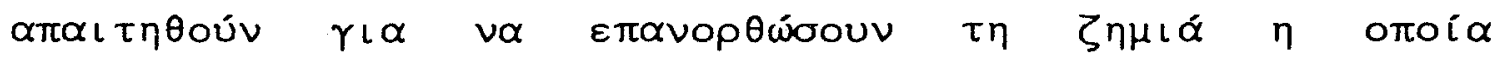

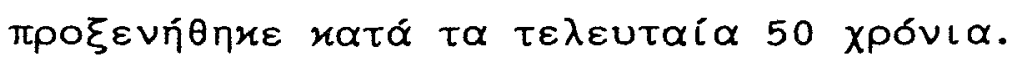

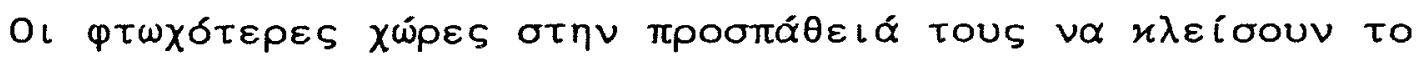

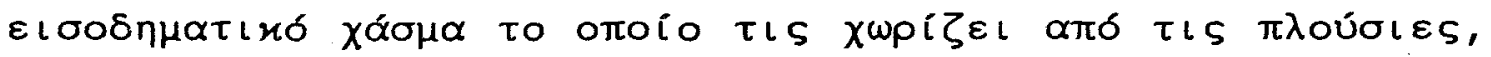

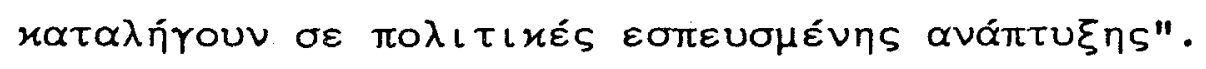

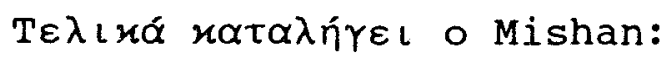

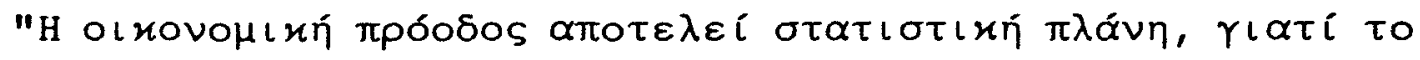

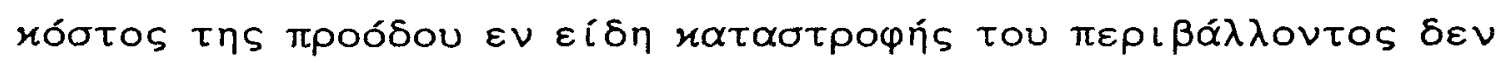

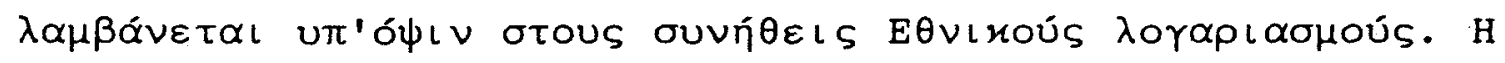

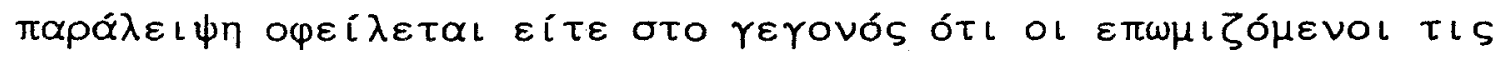

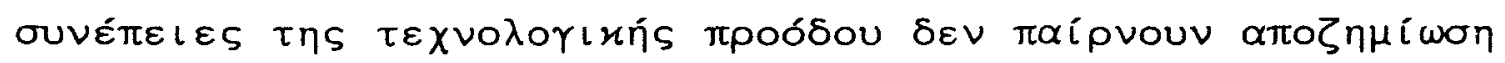

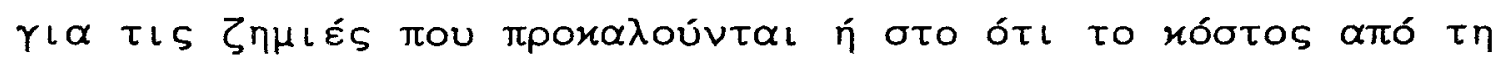

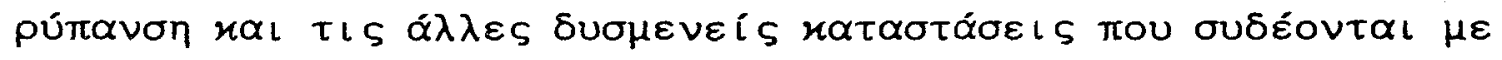

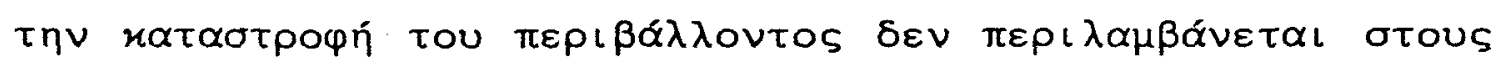

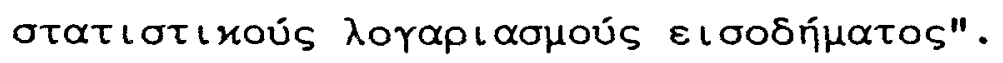




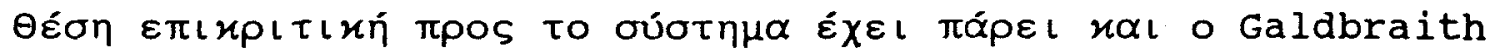

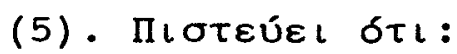

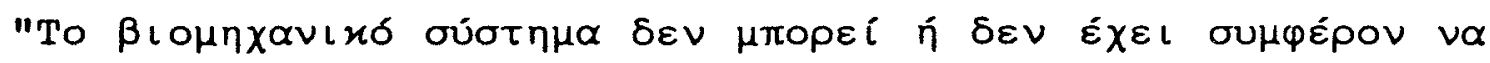

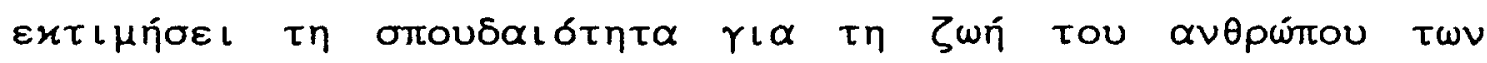

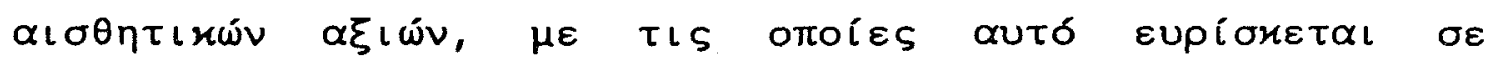

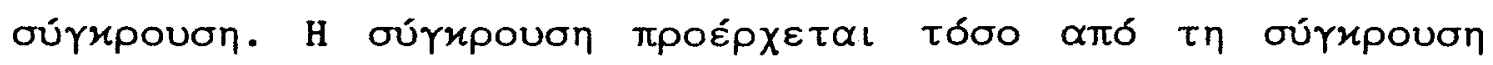

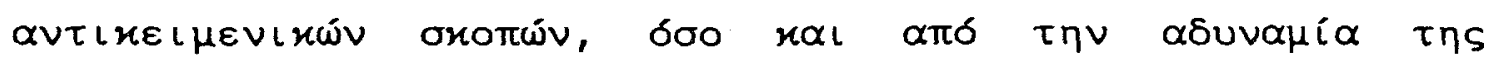

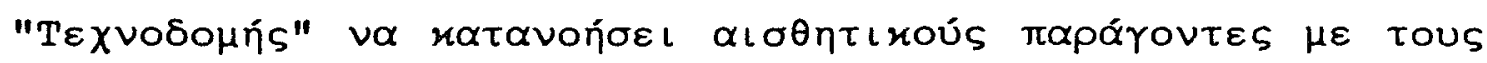

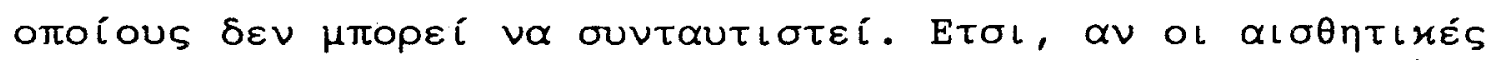

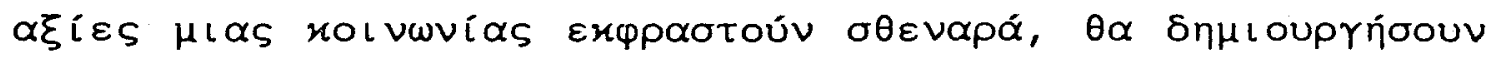

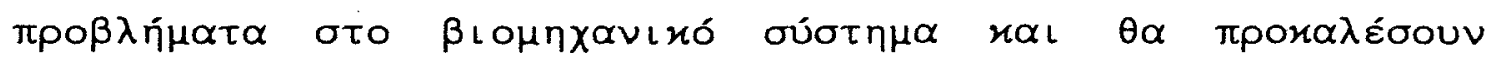

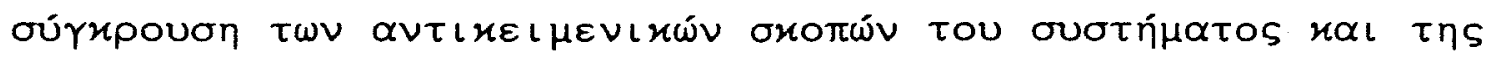

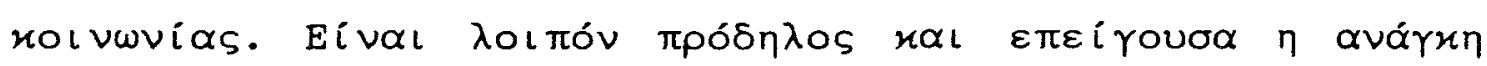

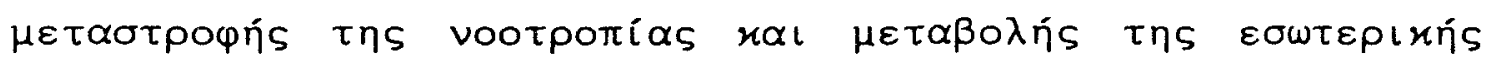

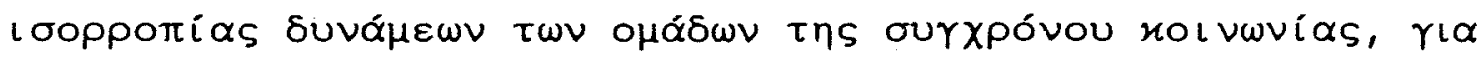

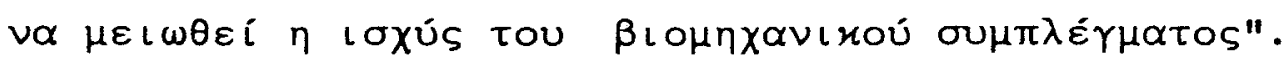

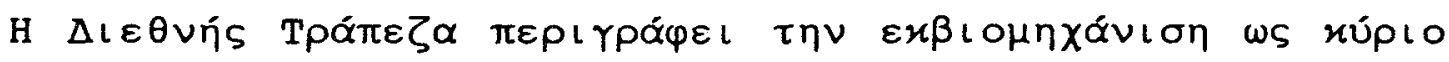

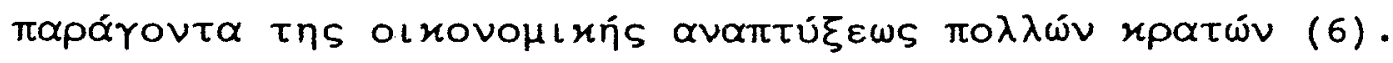

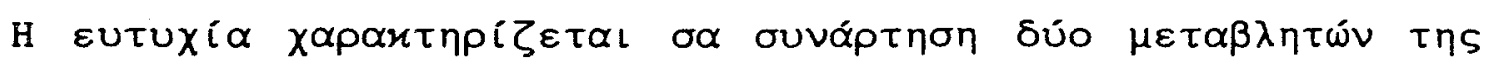

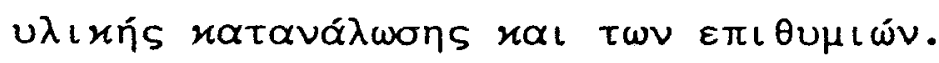

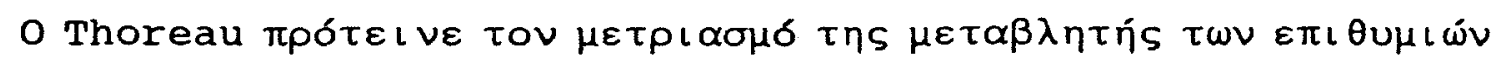




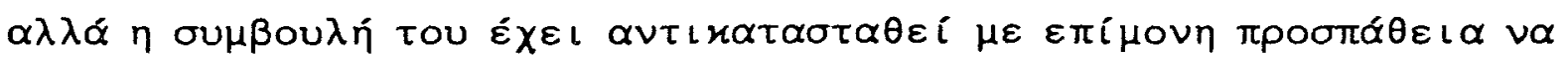

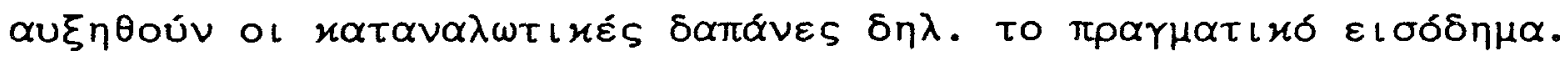

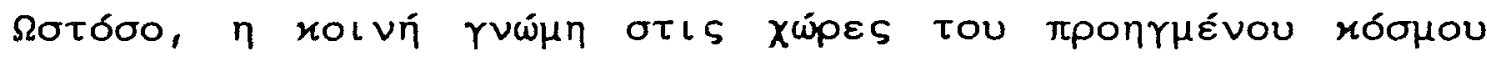

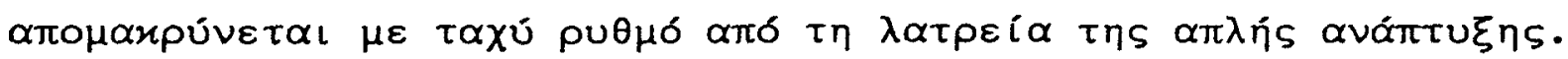

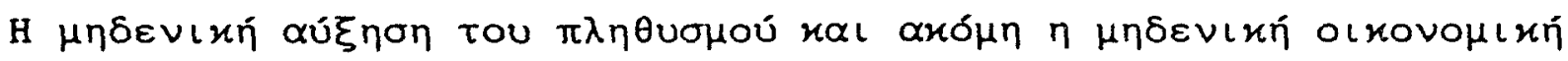

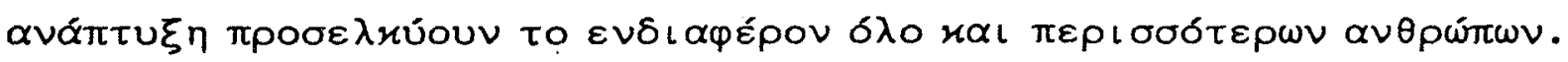

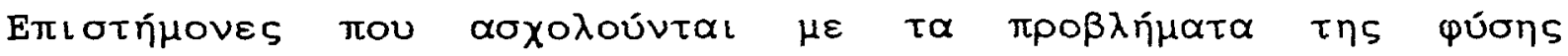

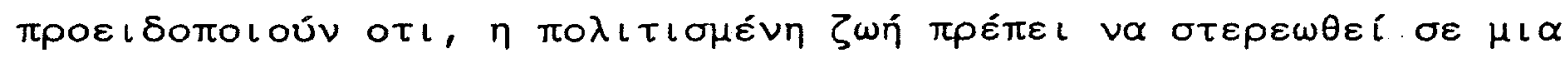

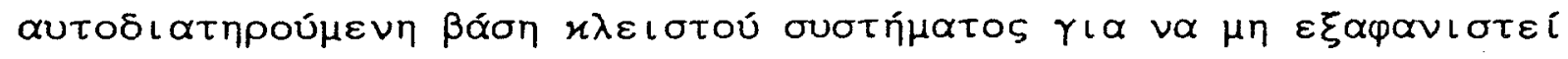

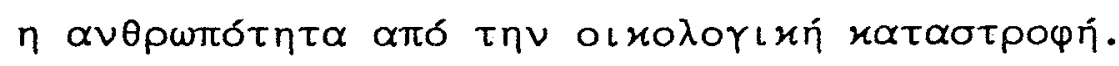

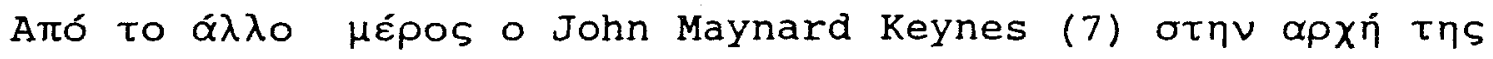

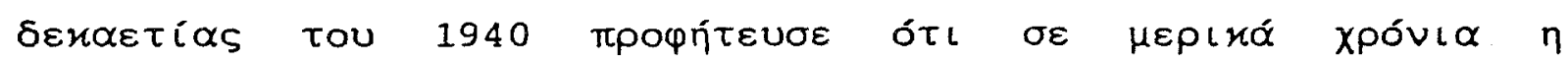

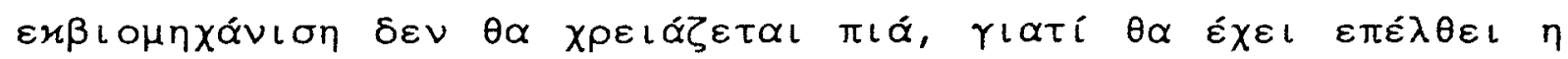

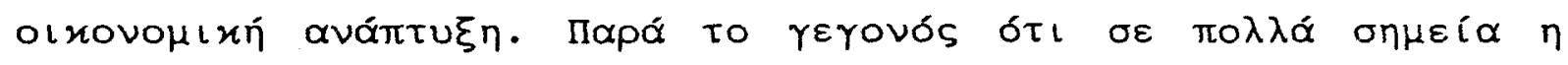

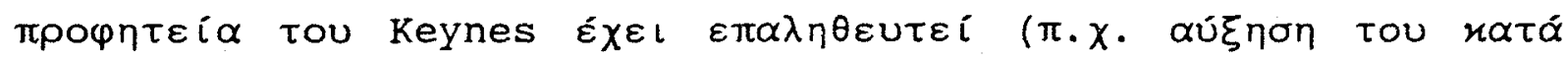

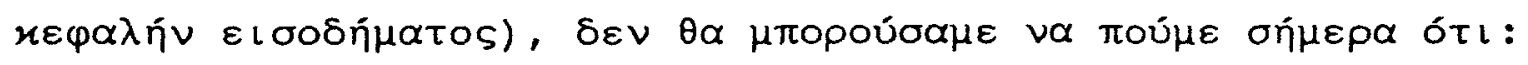

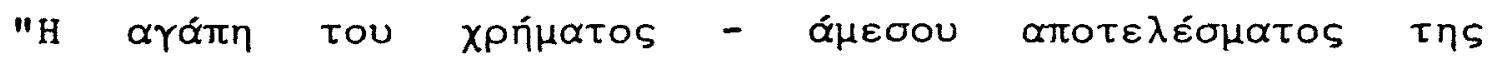

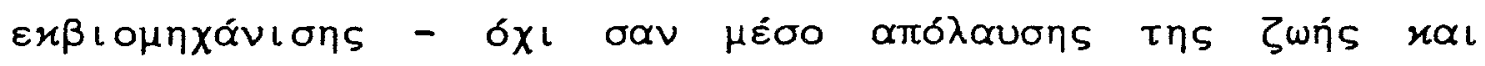

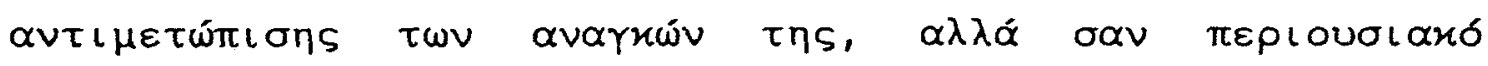

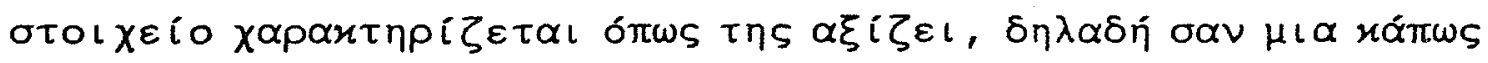

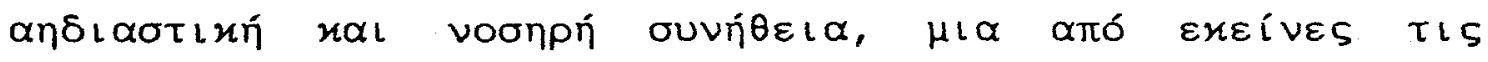
$\mu\llcorner\sigma o-\varepsilon \gamma \varkappa \lambda \eta \mu \alpha \tau \iota$ 


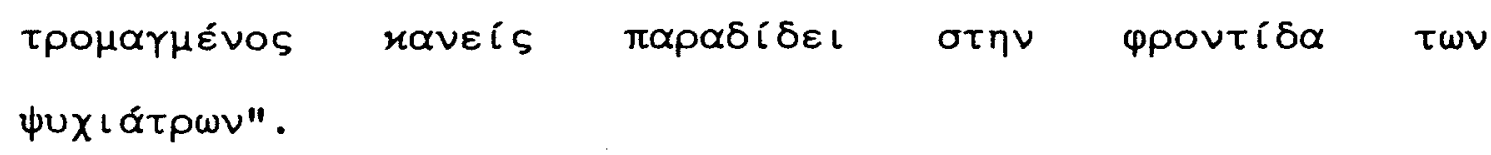

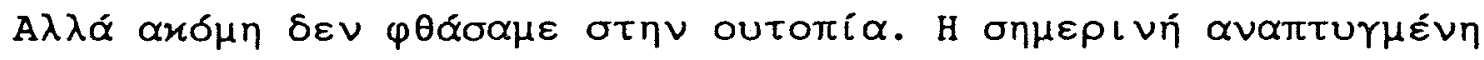

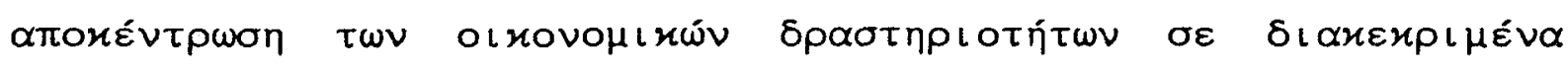

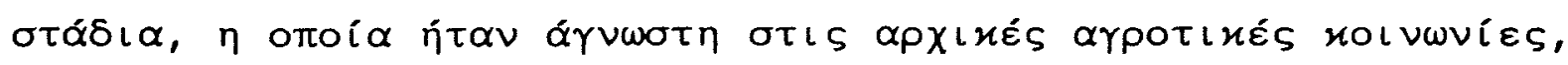

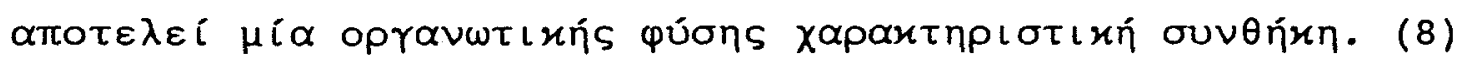

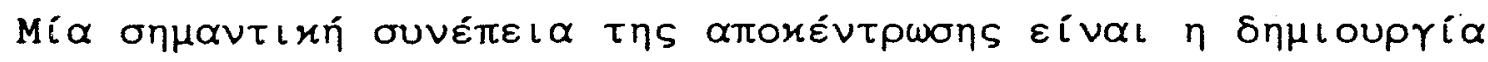

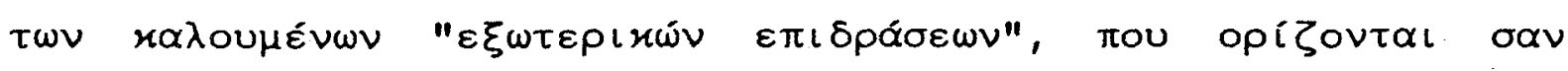

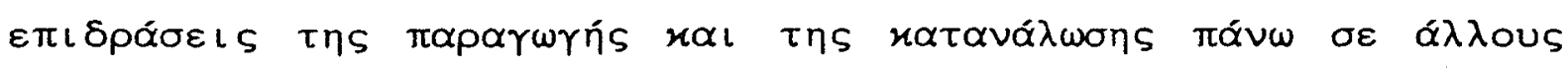

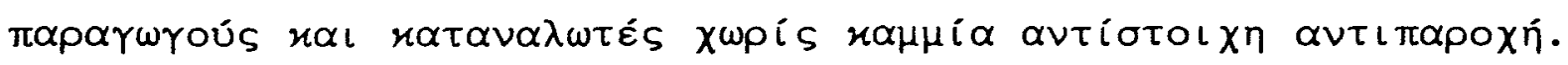

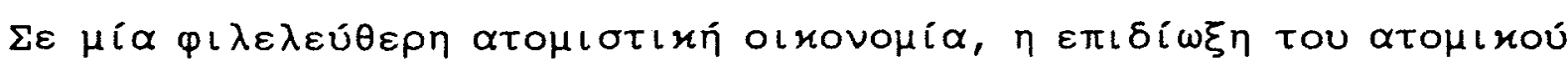

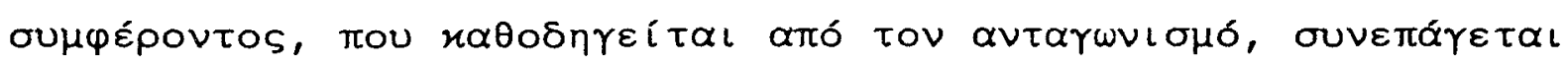

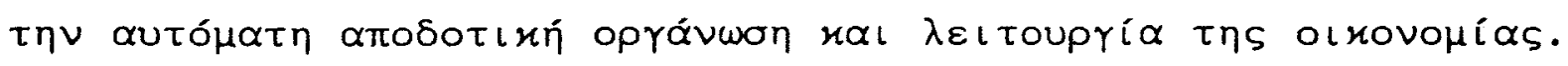

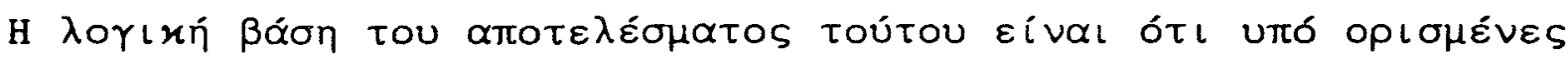

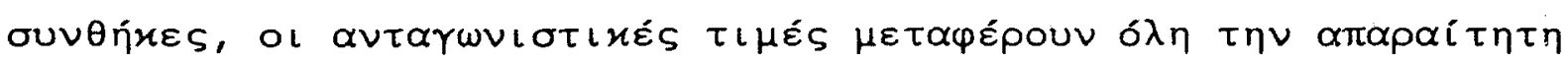

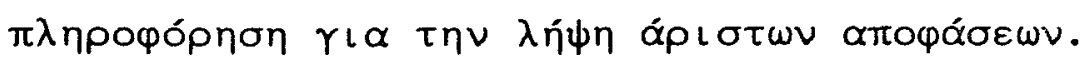

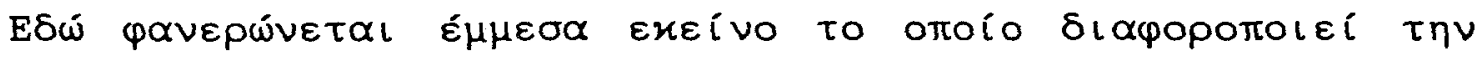

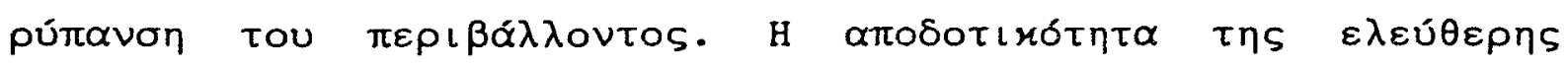

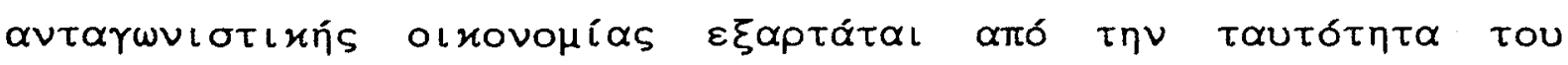

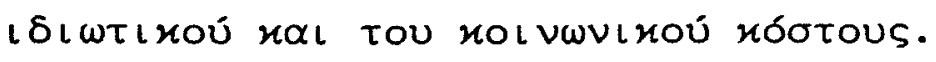




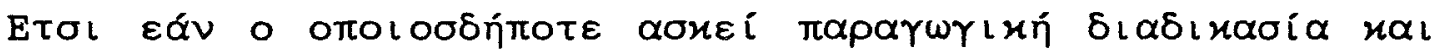

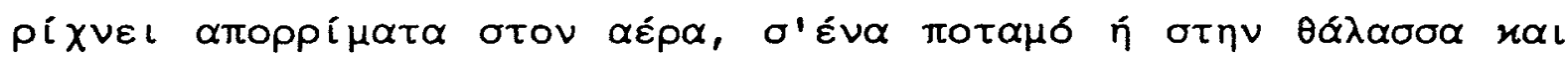
$\varkappa \alpha \mu \mu i \alpha \alpha \pi \circ \zeta \eta \mu i \omega \sigma \eta \delta \varepsilon \nu$ $\alpha \alpha \tau \alpha \beta \alpha \lambda \lambda \varepsilon\llcorner\quad \gamma \iota \alpha \tau \eta \nu \delta\llcorner\alpha \theta \varepsilon \sigma \eta \tau \omega \nu \alpha \pi \circ p \rho\llcorner\mu \alpha \tau \omega \nu$

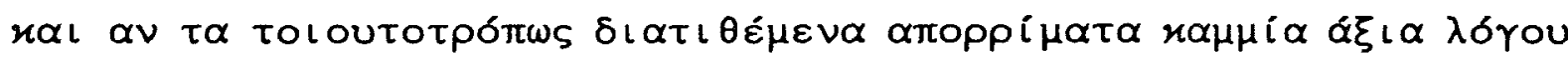

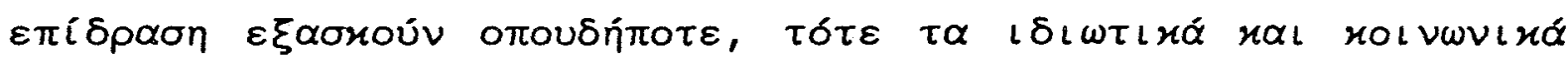

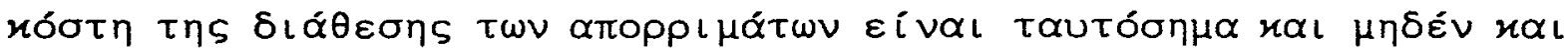

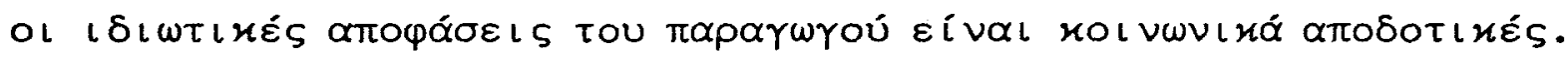

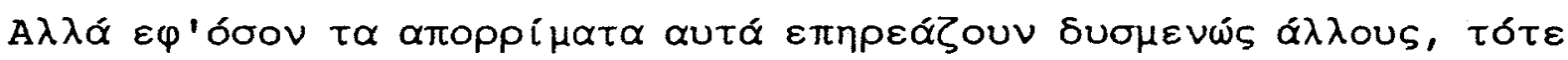

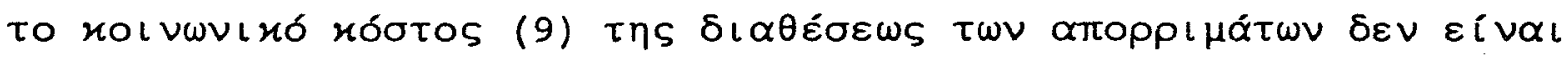

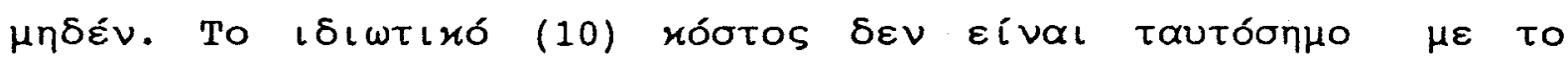

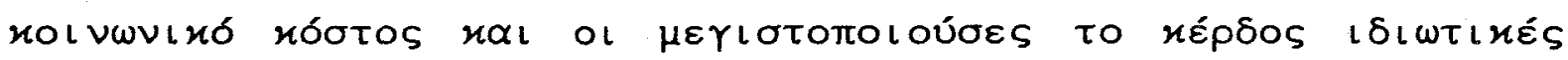

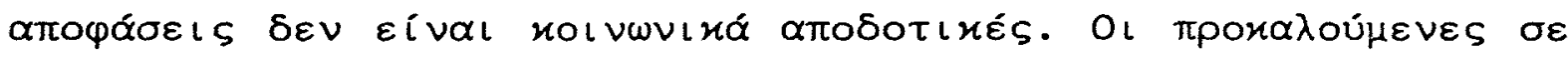

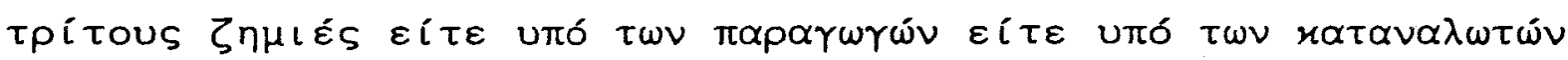

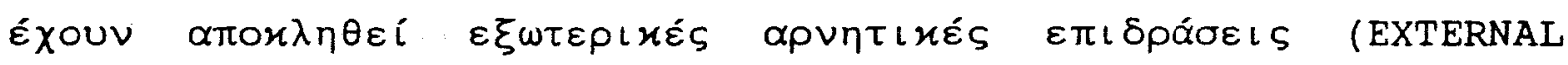
DISECONOMIES) (11).

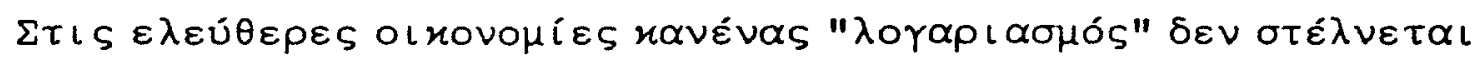

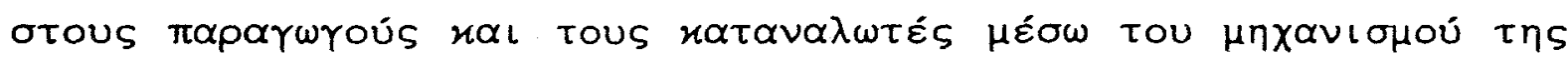

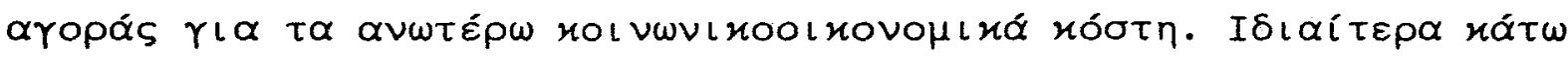

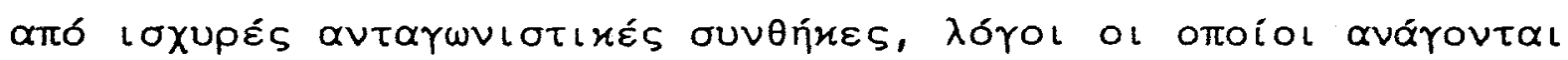

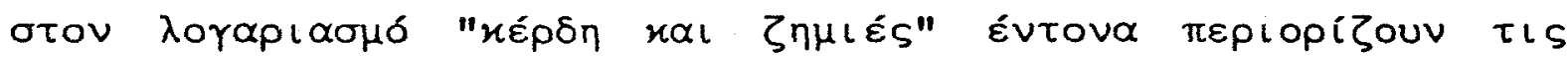

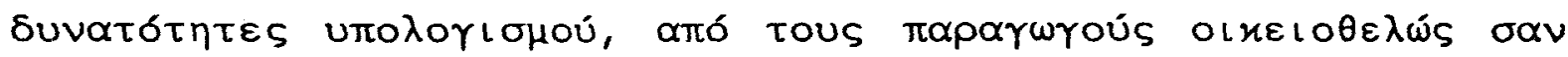

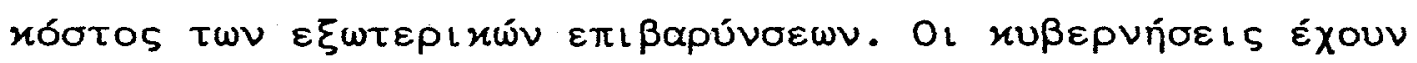




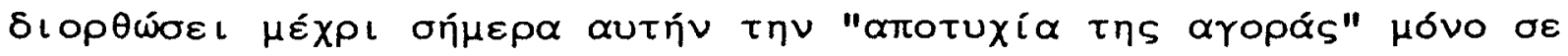

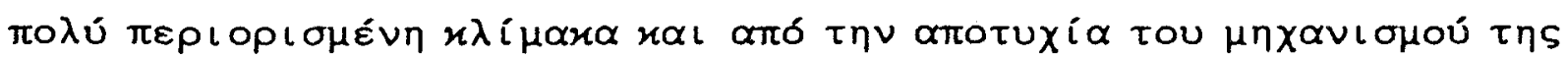

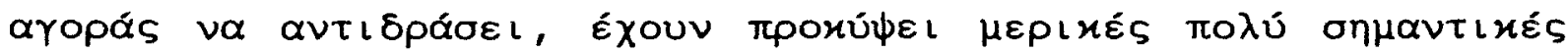

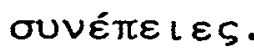

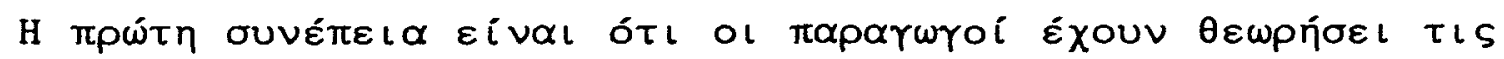

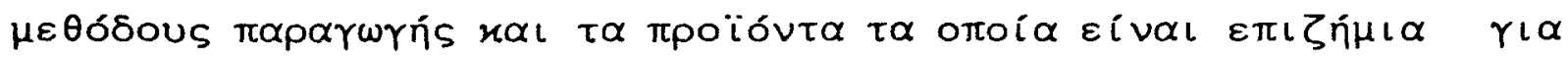

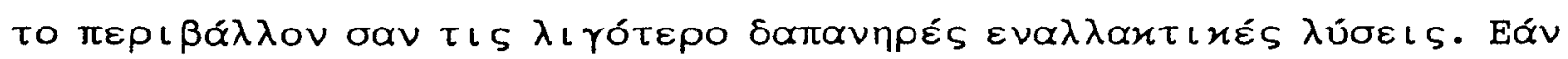

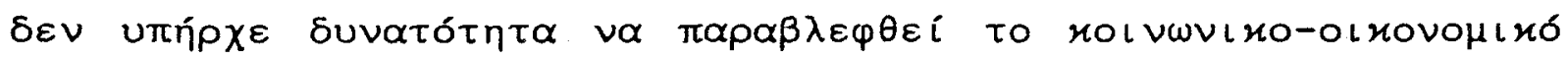

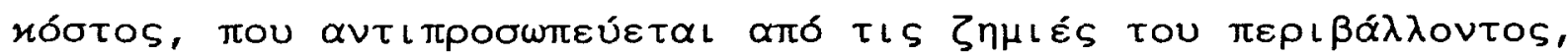

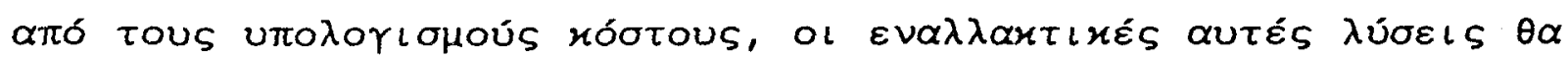

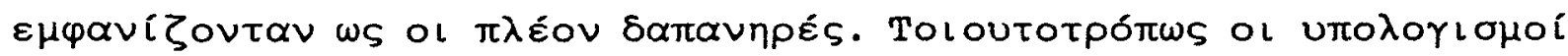

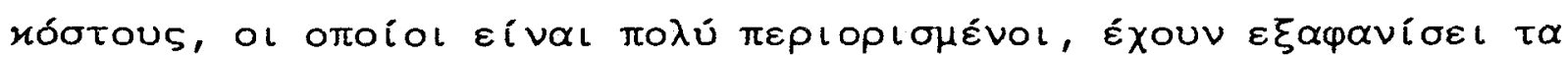

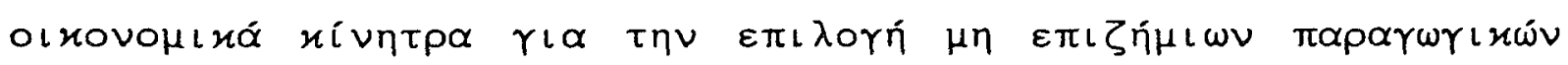

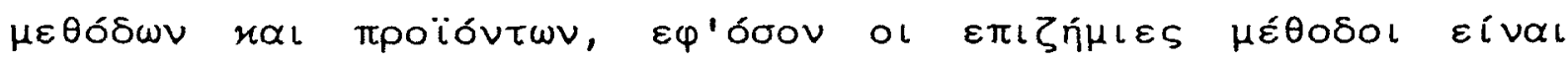
$\varphi \theta \eta \cup o ́ \tau \varepsilon \rho \varepsilon s$.

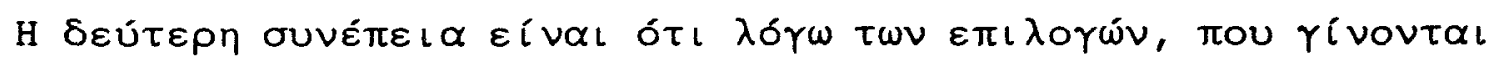

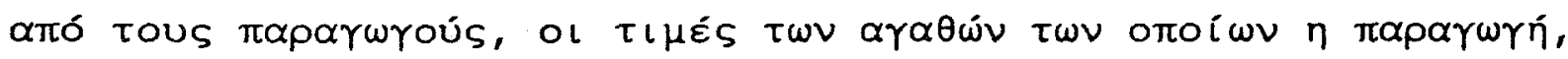

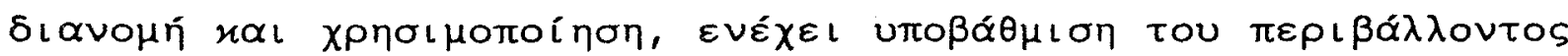

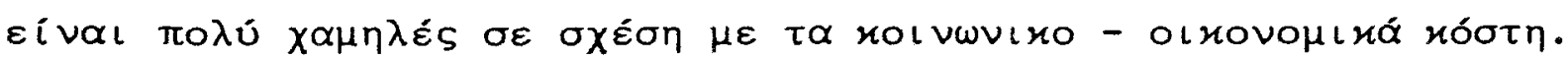

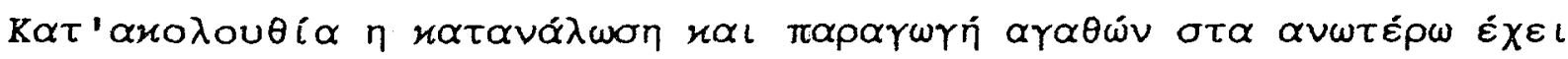

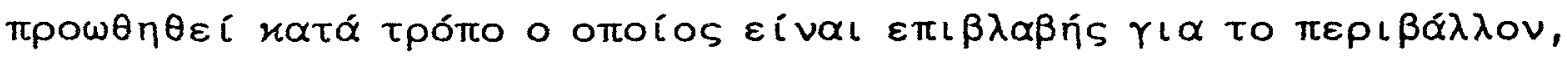

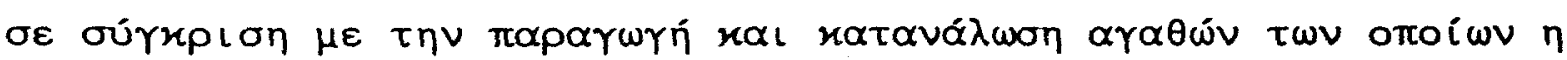









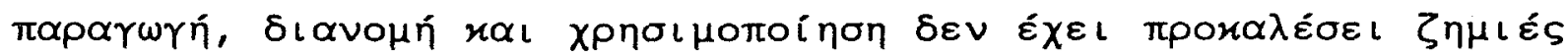

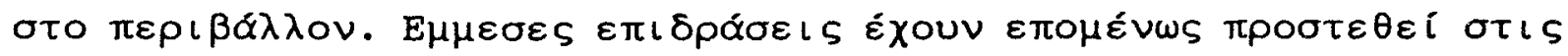

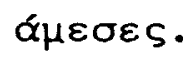

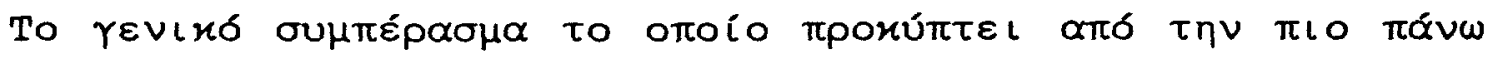

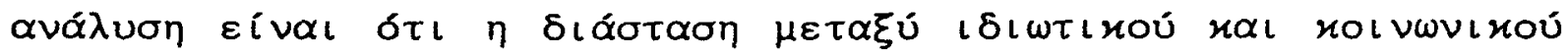

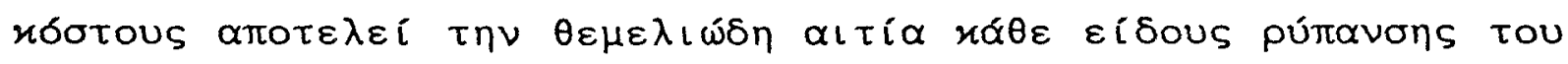

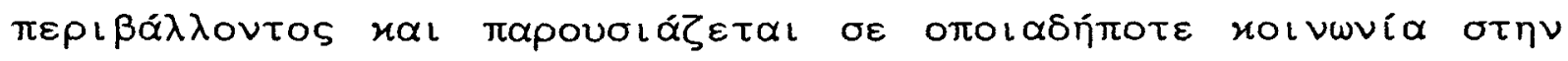

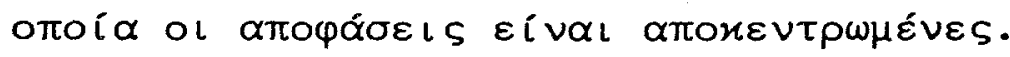

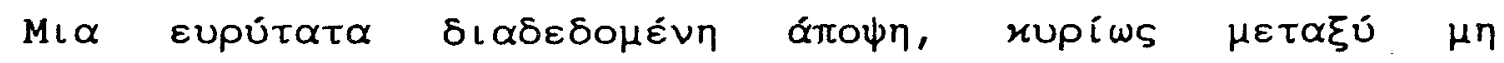

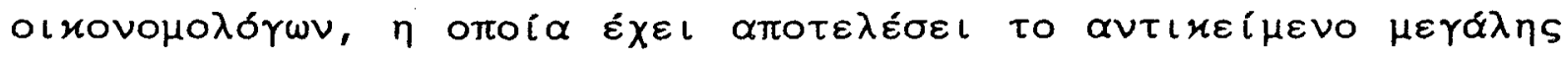

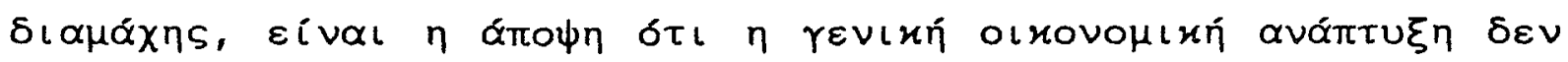

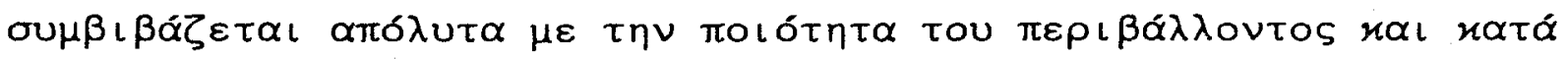

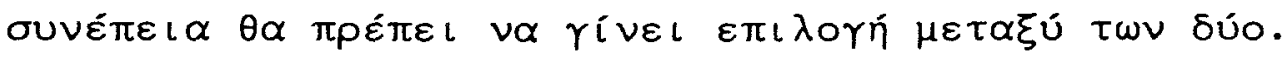

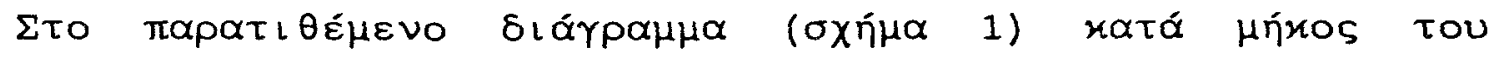

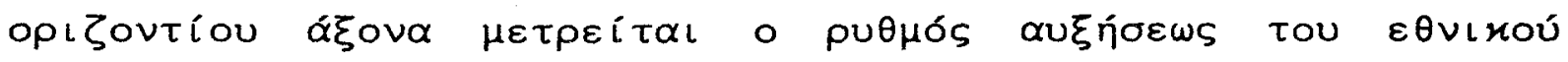

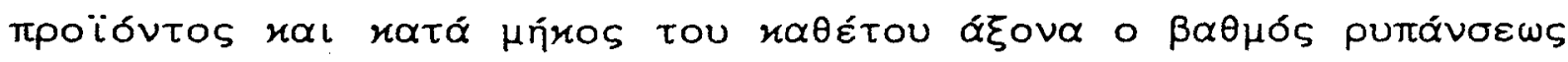

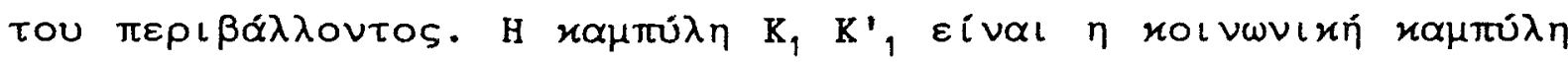

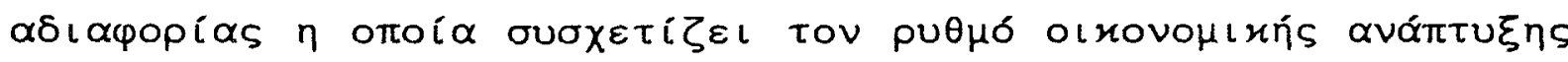

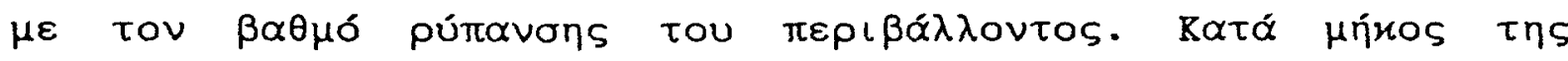

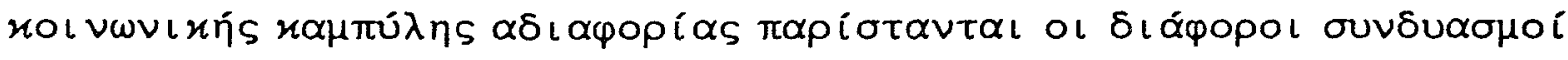

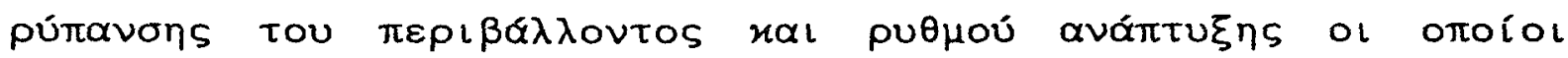

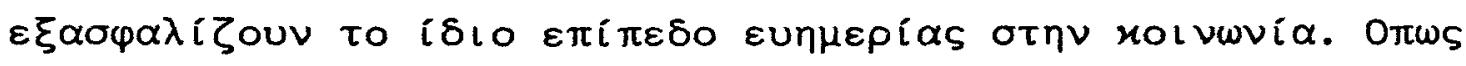


sXñua 1.

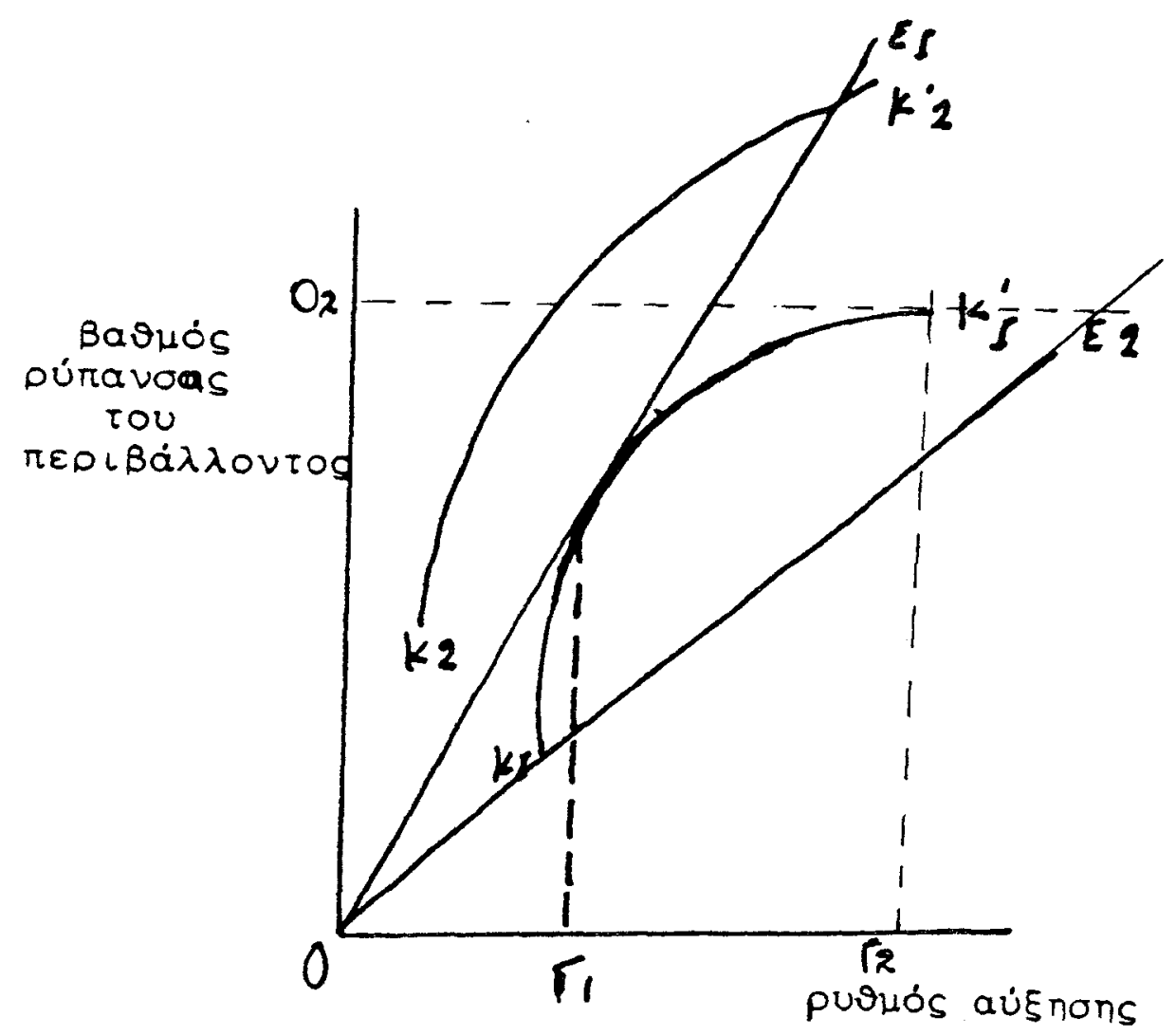

EQv. npoiört.s 
sxńla 1. 


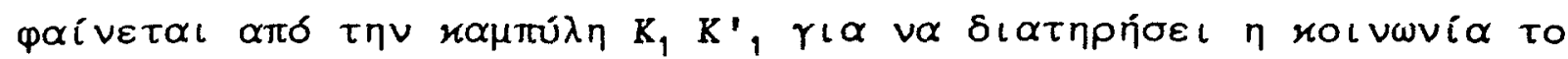

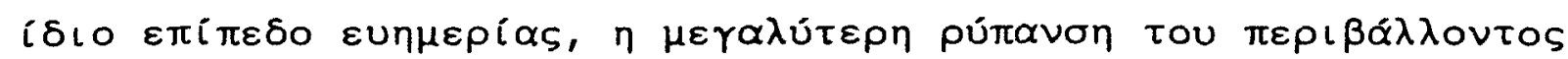

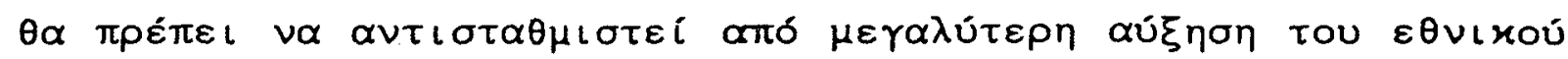

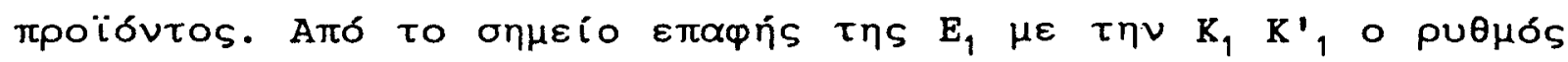

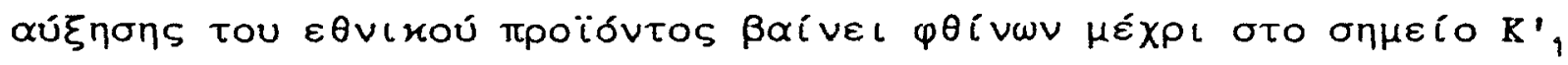

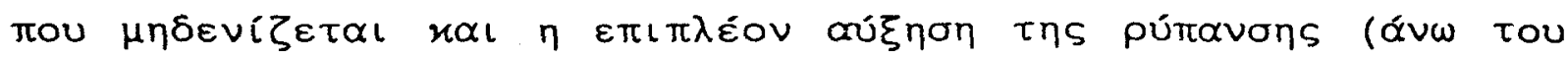

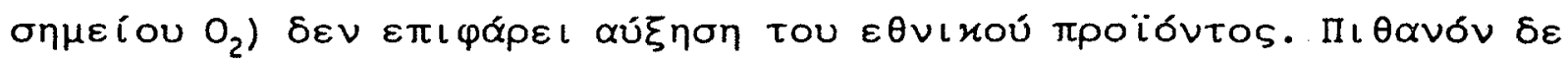

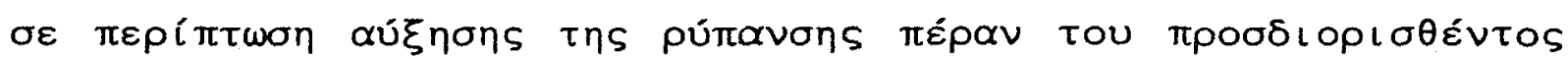

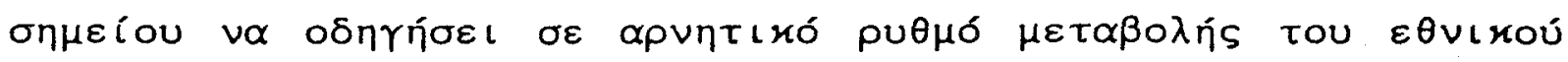
трo ióvtos.

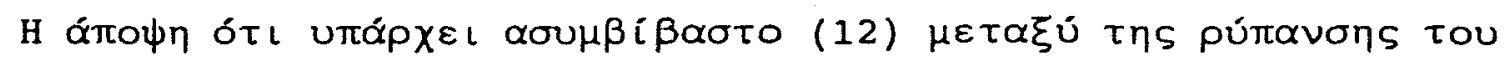

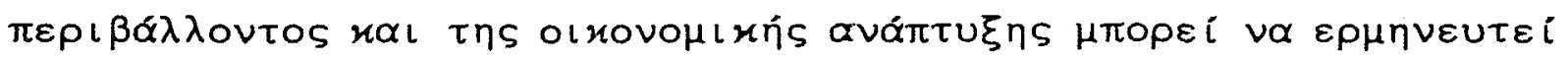

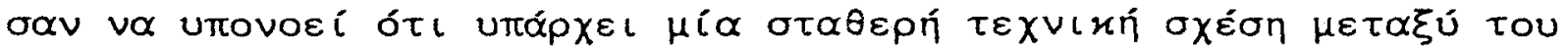

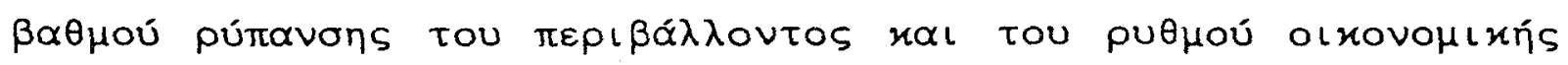

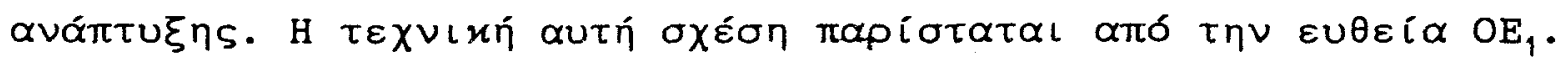

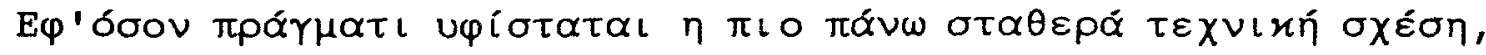

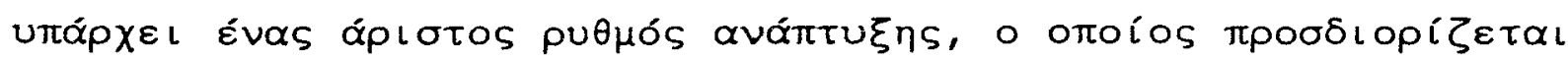

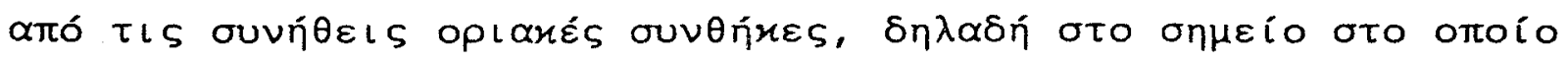

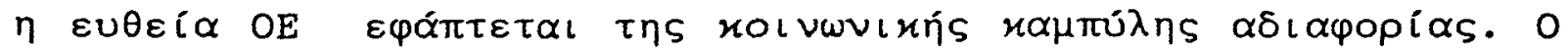

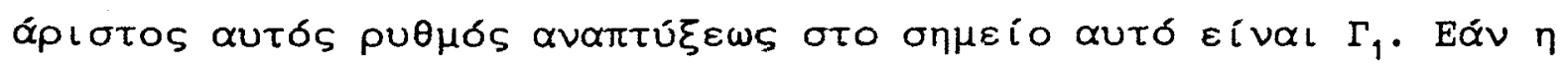

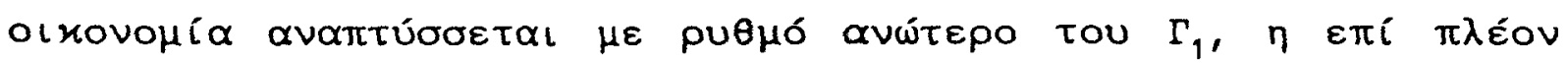

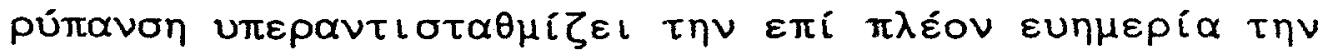




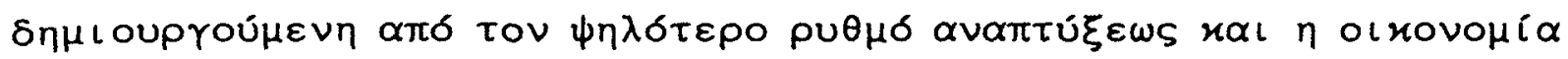

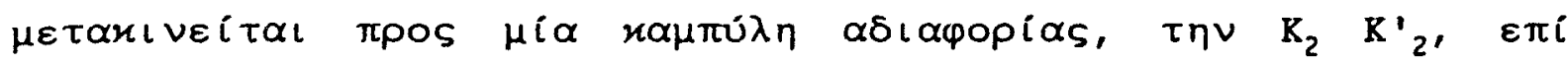

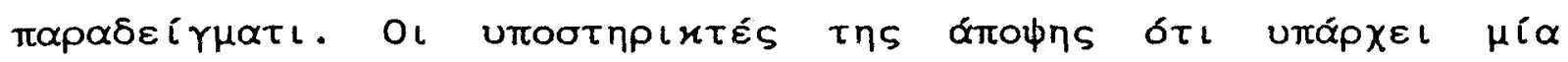

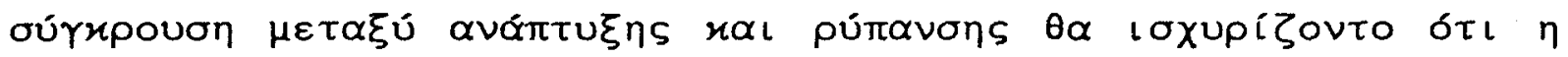

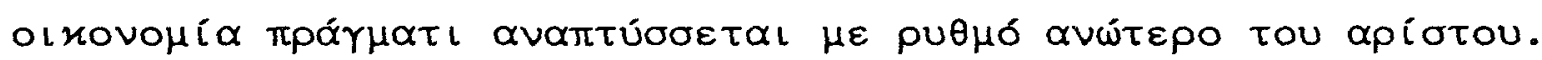

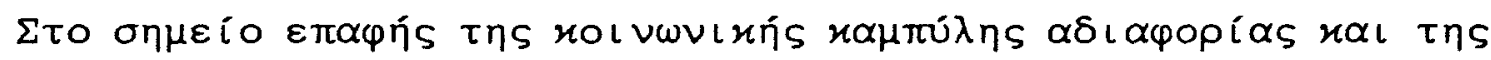

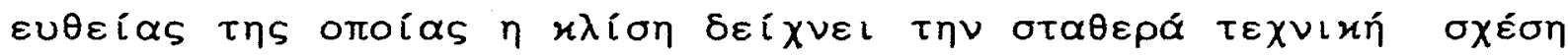

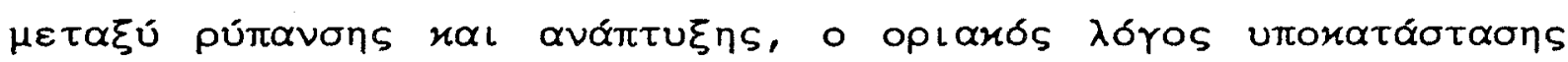

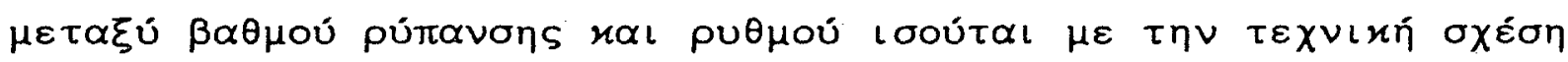

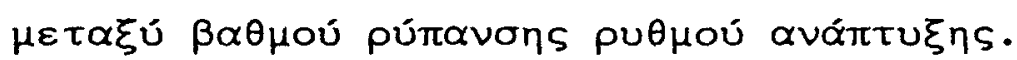

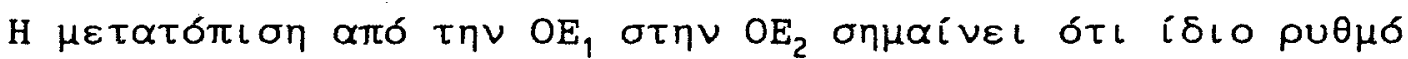

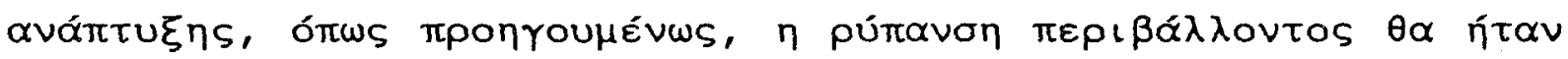

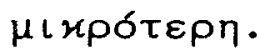




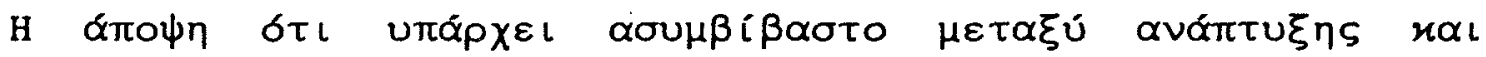

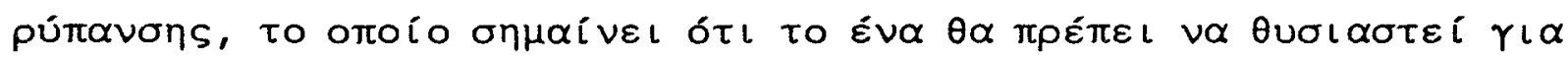

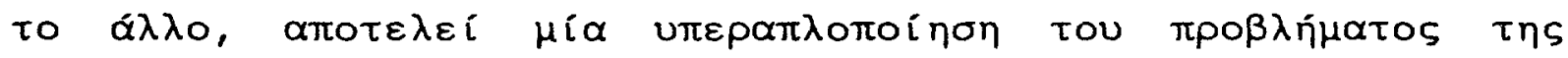

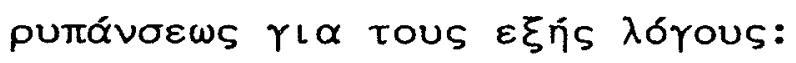

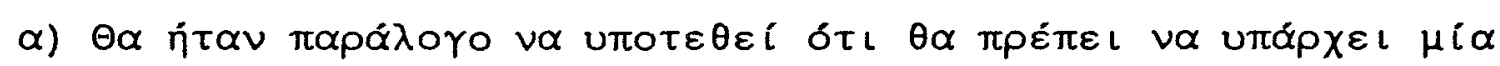

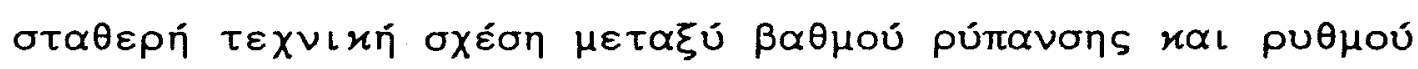

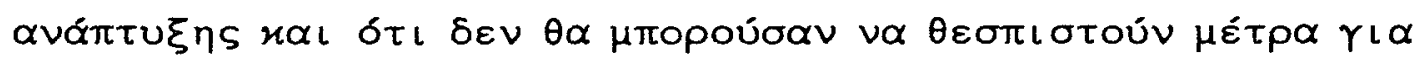

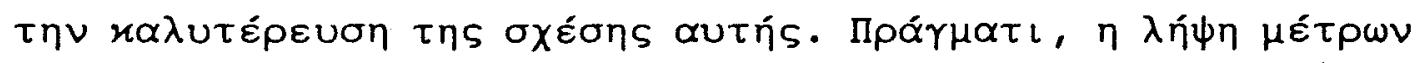

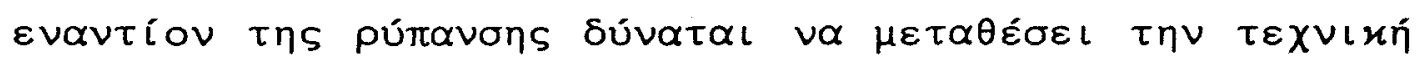

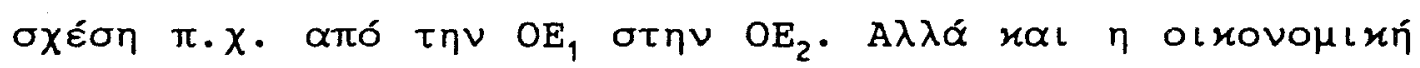

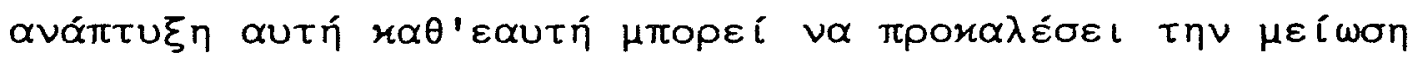

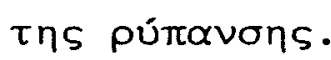

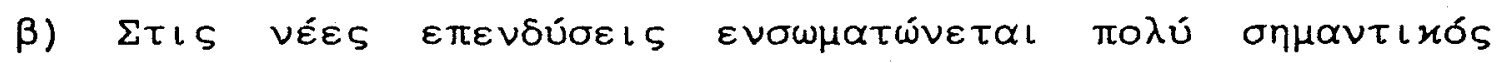

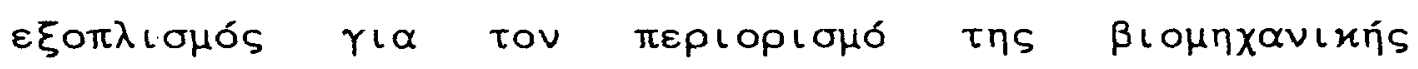
púravons.

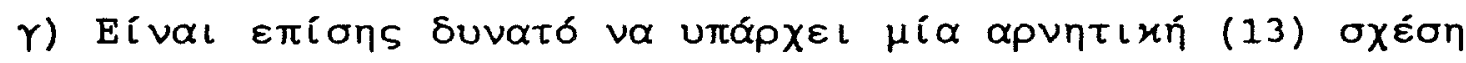

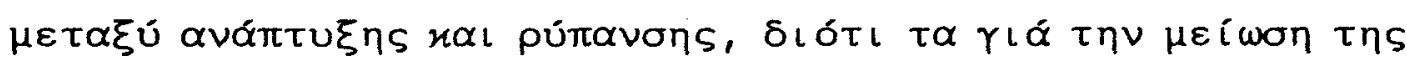

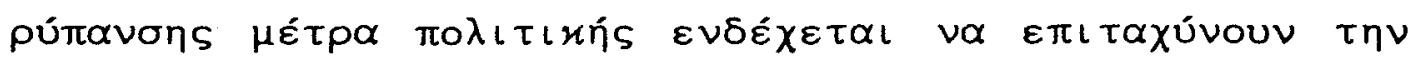

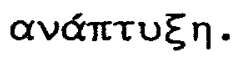




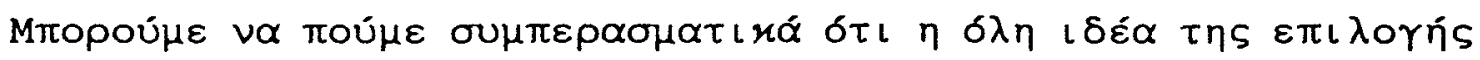

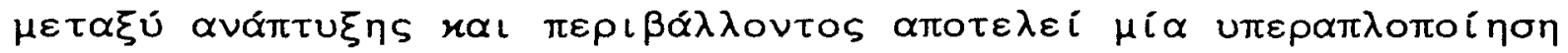

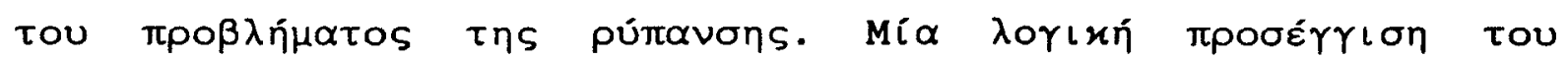

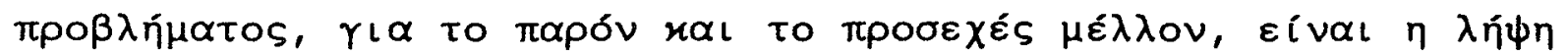

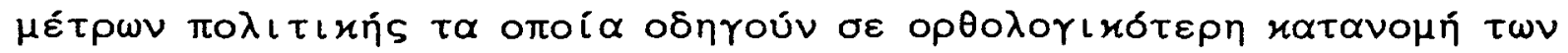

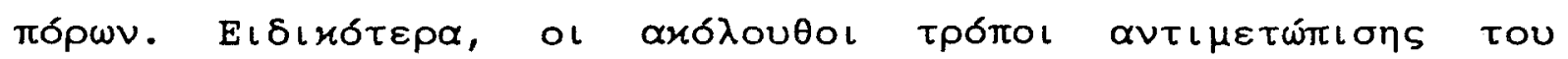

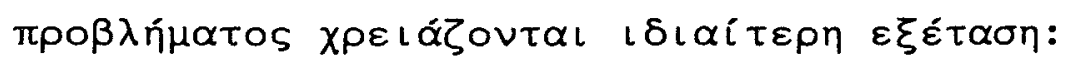

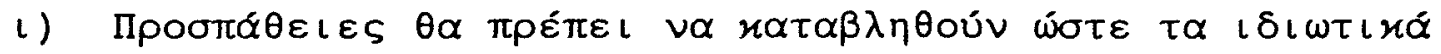

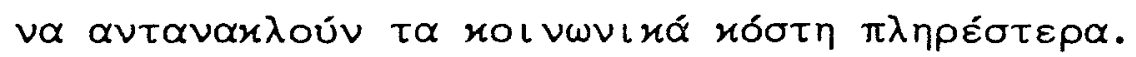

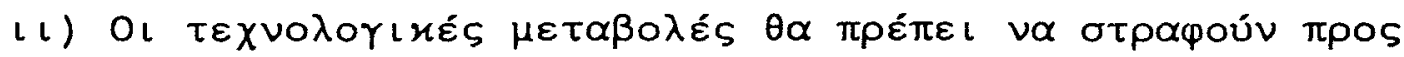

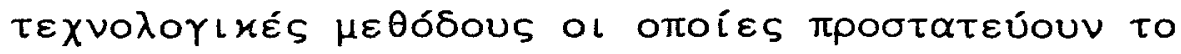
$\pi \varepsilon \rho\left\llcorner\beta \alpha \dot{\alpha} \lambda \lambda\right.$ v $^{\circ}$

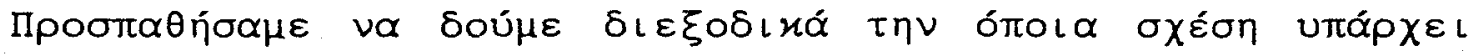

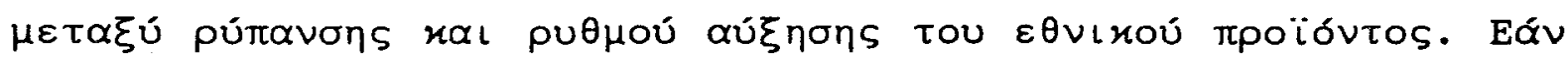

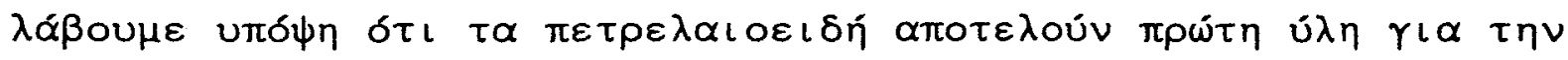

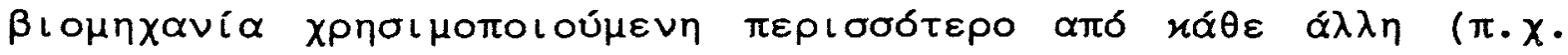

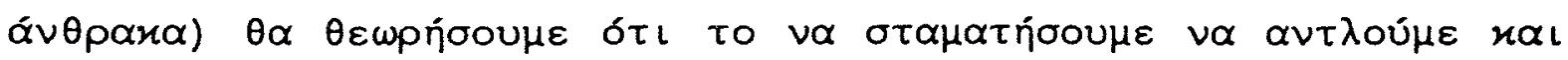

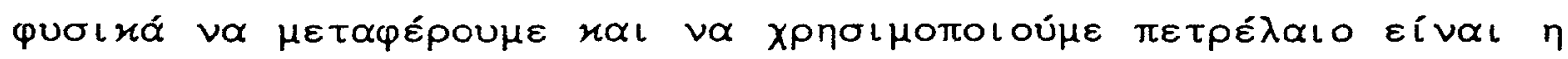

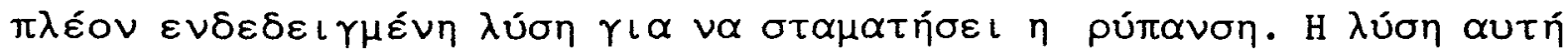

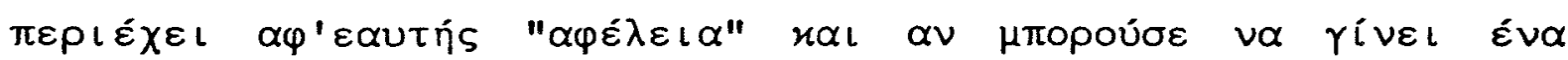

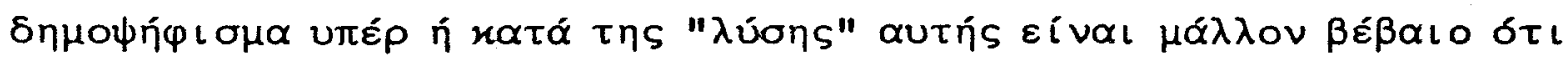




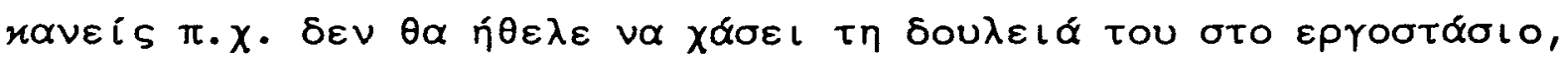

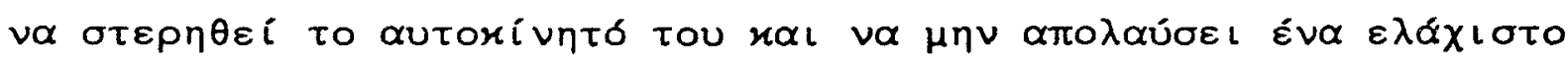

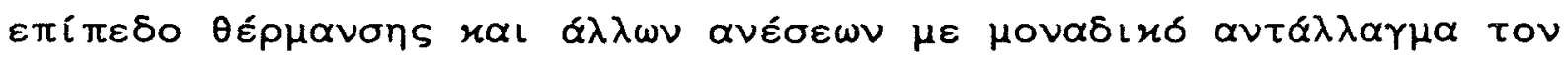

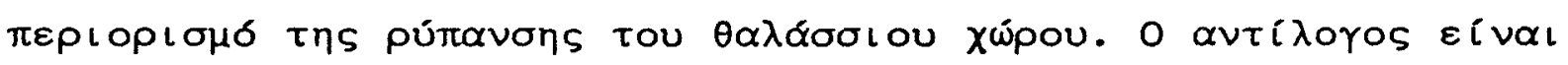

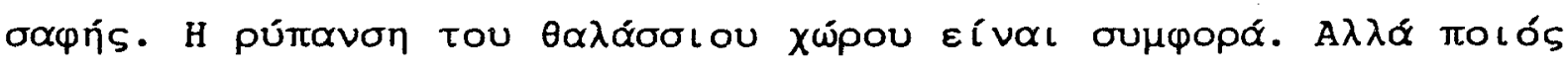

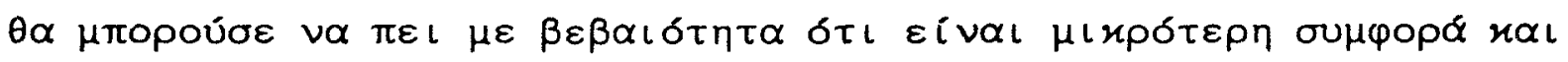

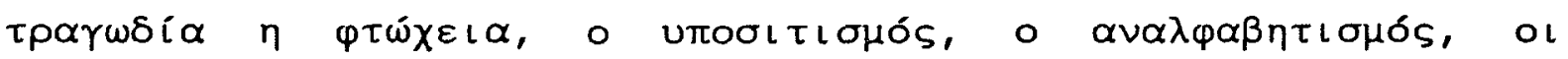
$\alpha \rho \rho \omega ́ \sigma \tau \iota \varepsilon s ;$

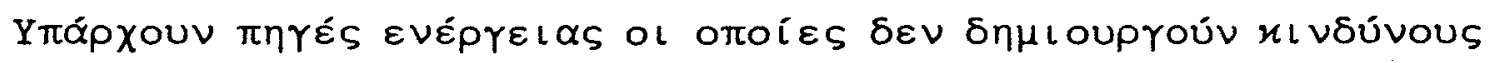

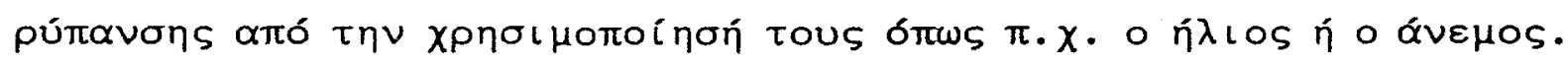

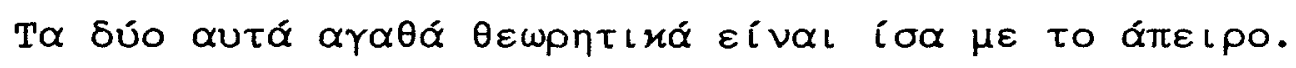

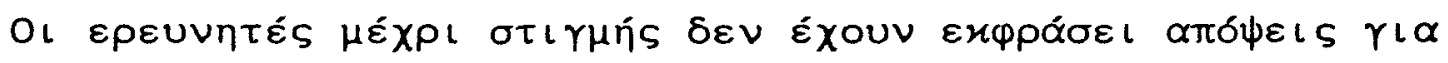

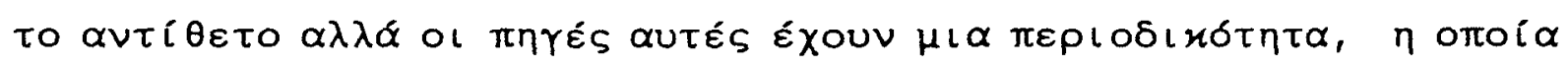

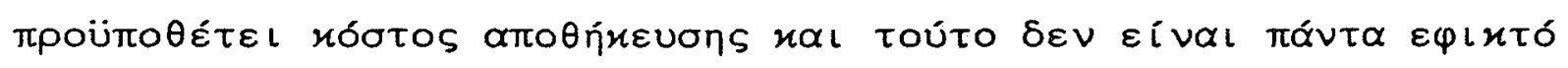

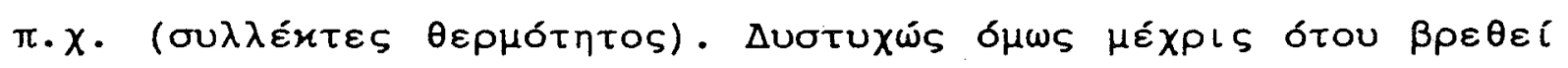

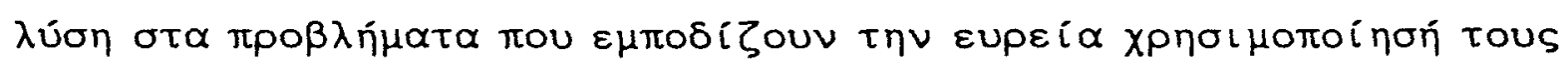

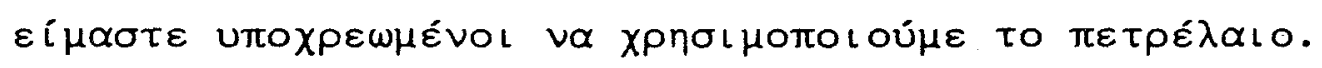

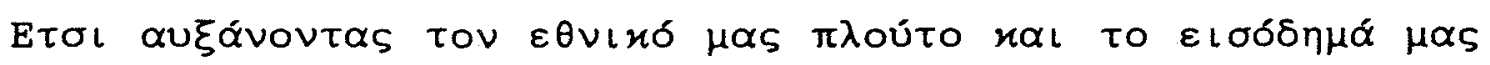

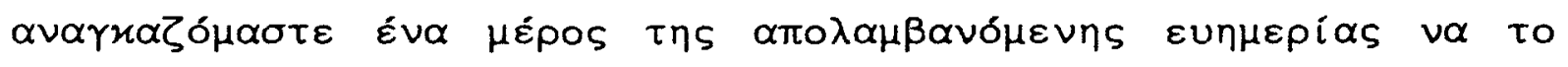

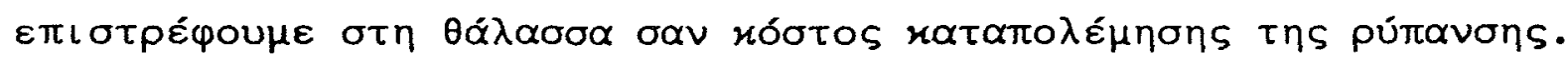




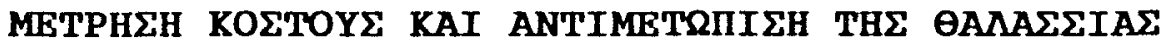

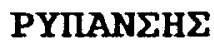

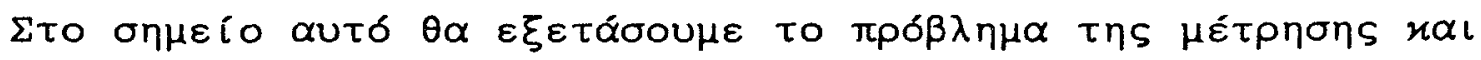

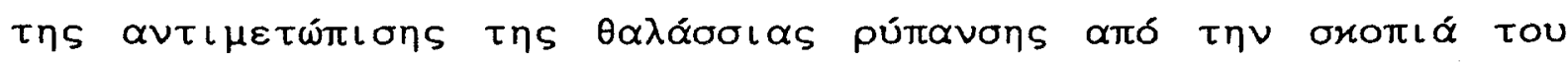
olxovouodórou (13).

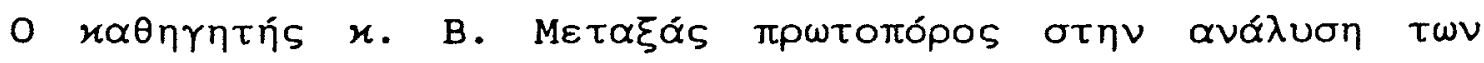

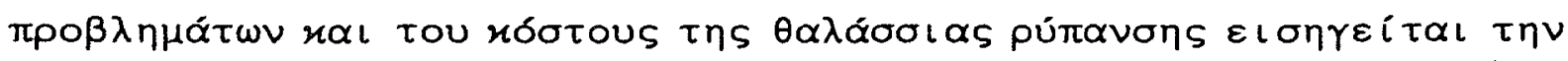

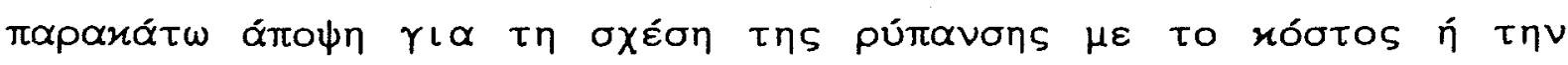
$\omega \varphi \varepsilon \dot{\lambda} \varepsilon\llcorner\alpha$.

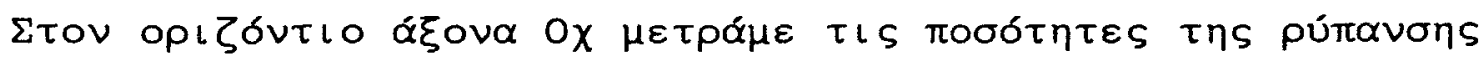

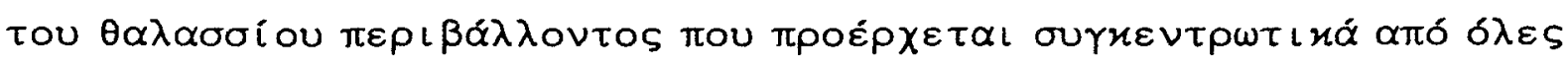

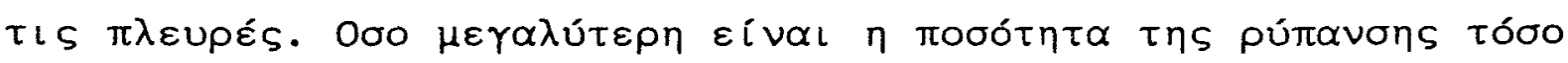

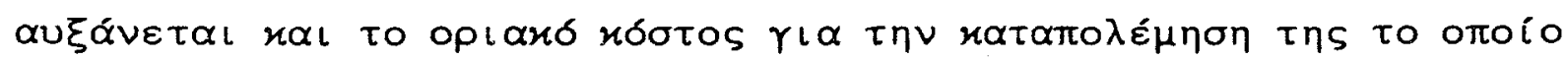

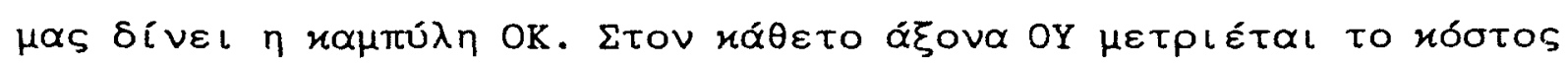

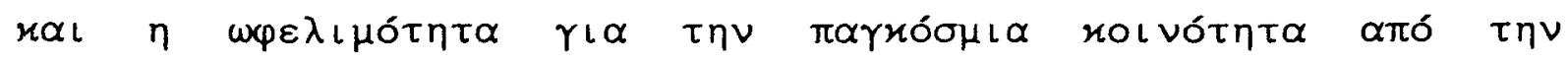

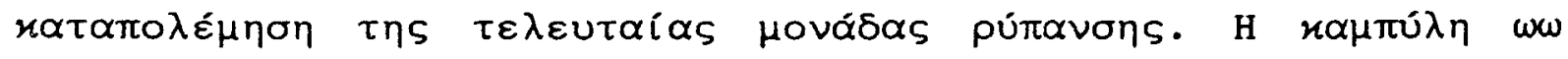

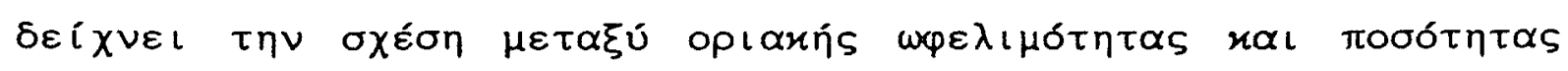

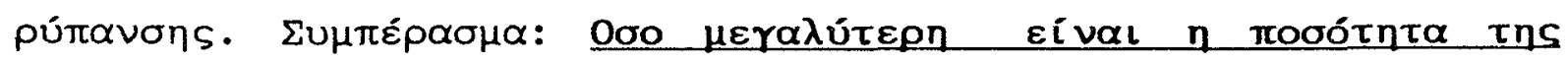

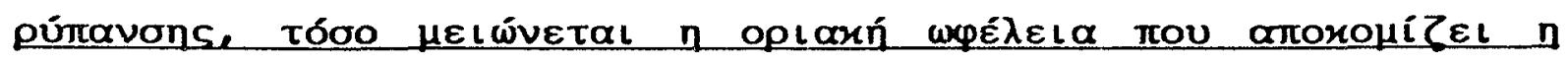

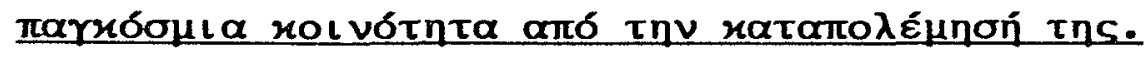


$\Sigma \chi n ́ \mu \alpha 2$. 


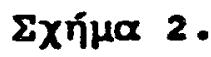

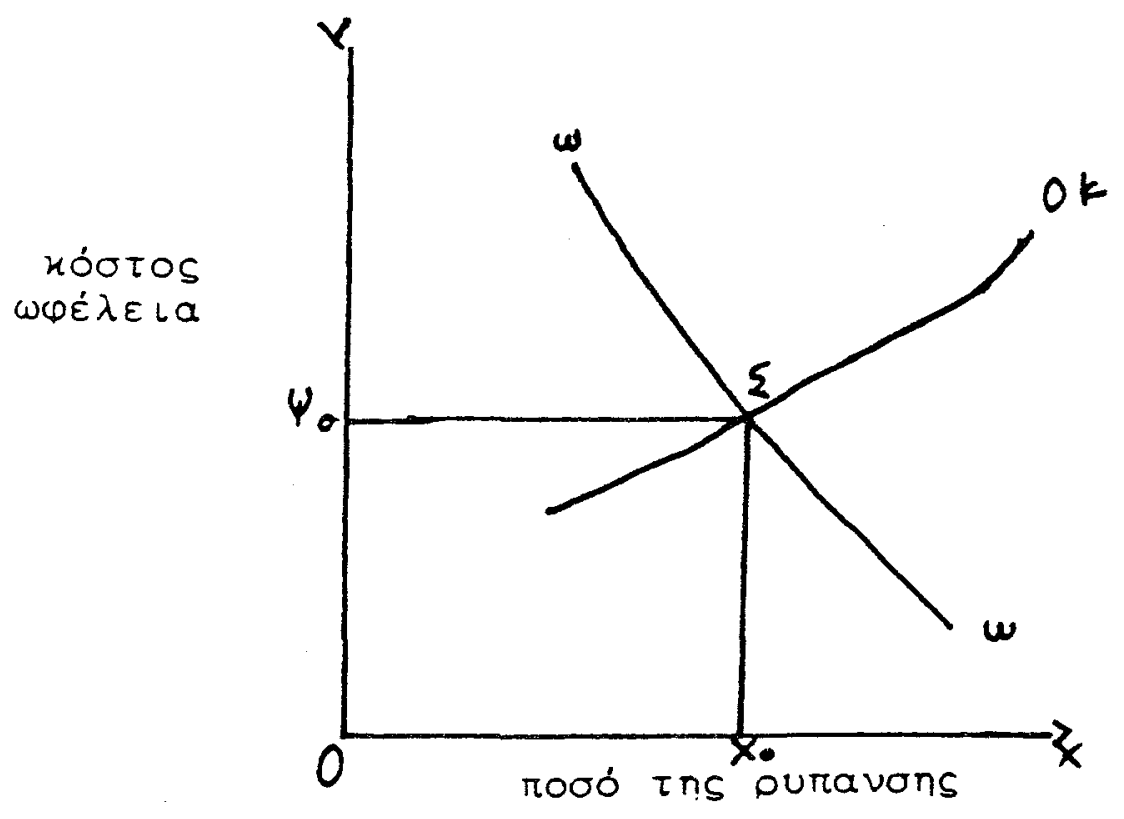




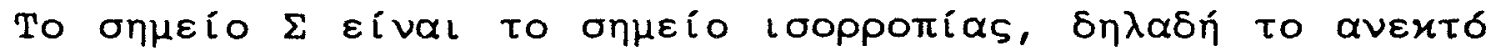

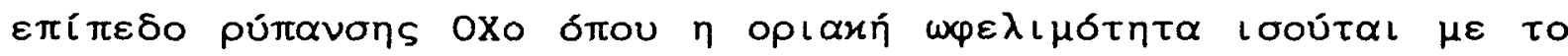

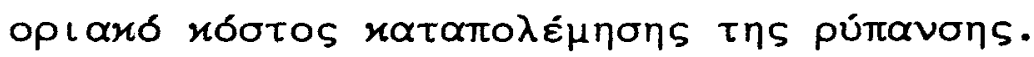

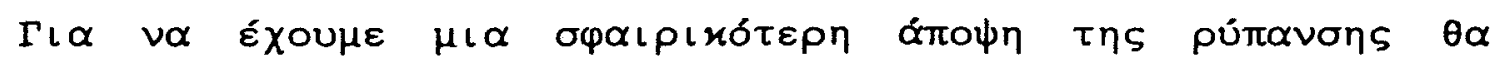

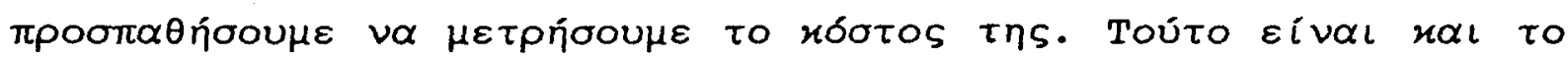

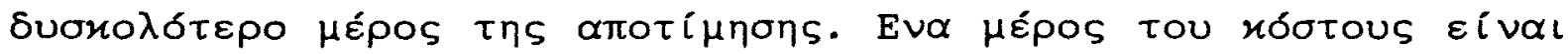

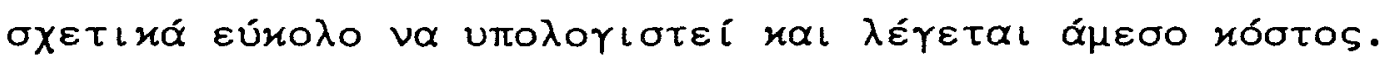

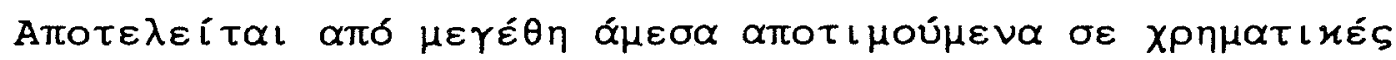

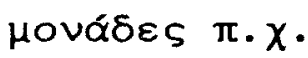

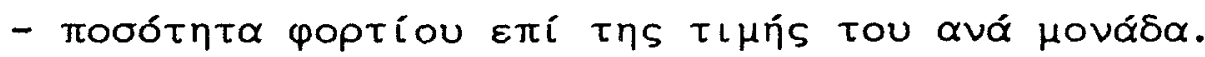

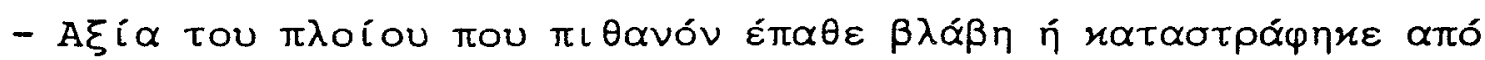

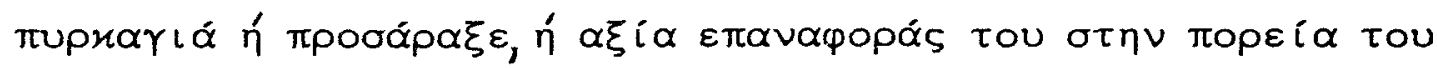

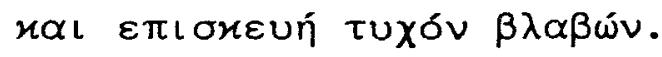

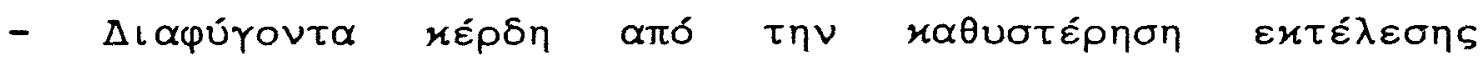

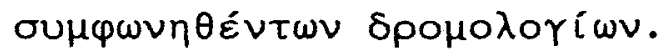

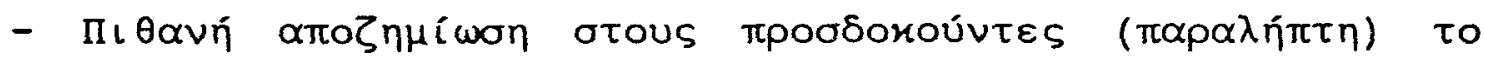
poptío. 


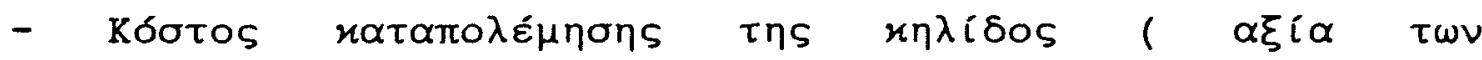

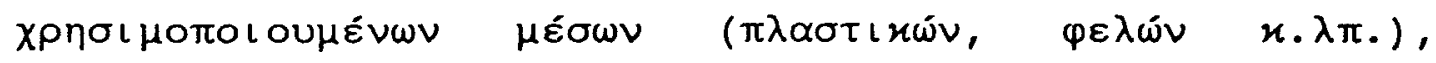

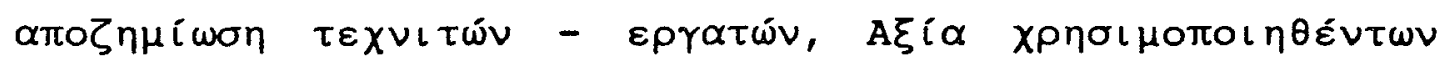
$\alpha \pi \circ \rho \rho \pi \alpha \nu \tau \tilde{v} v)$.

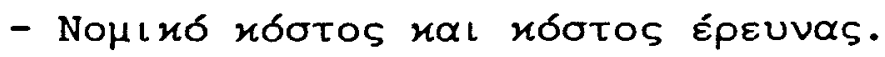

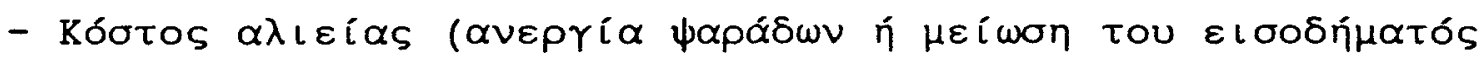

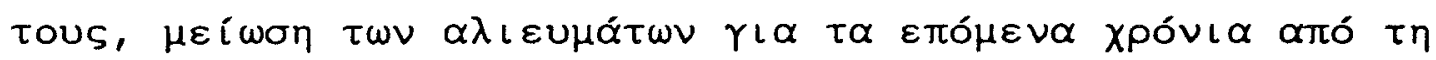

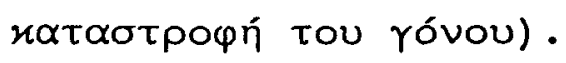

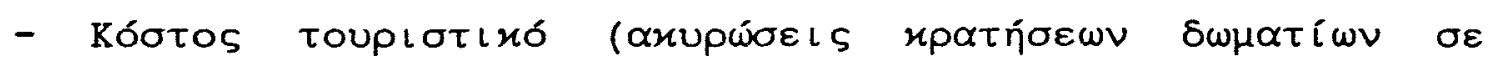

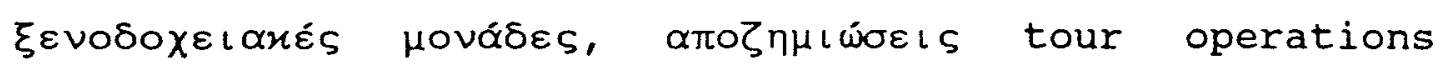
ห. $\lambda \pi$.

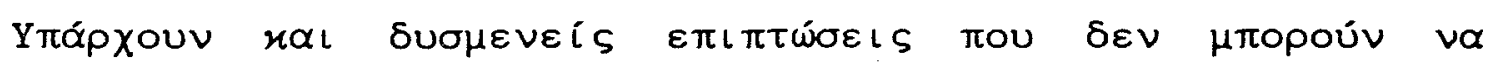

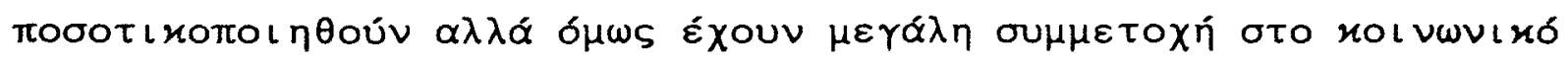

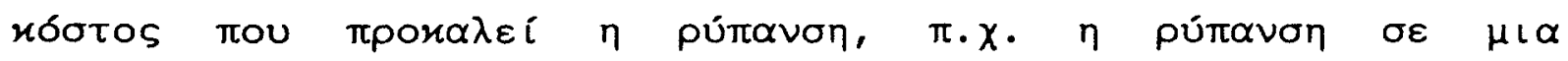

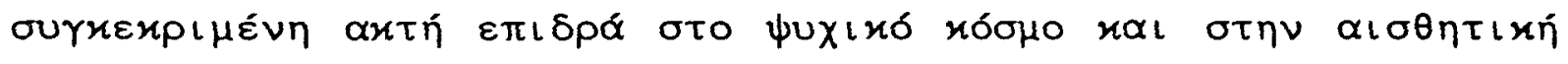

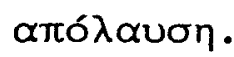

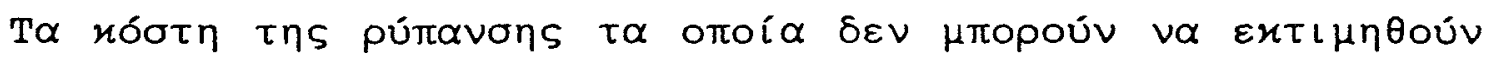

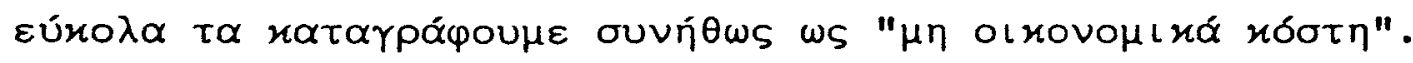

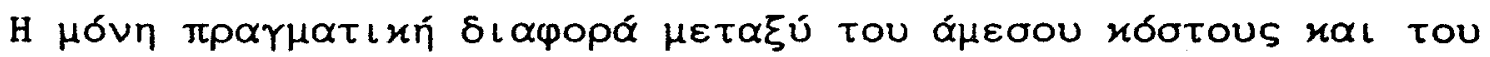

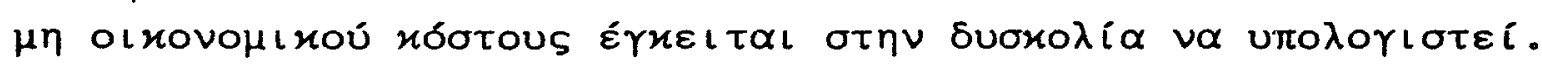




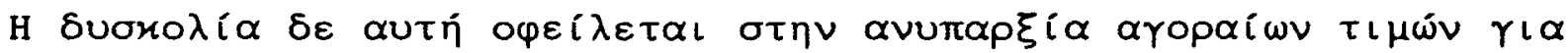

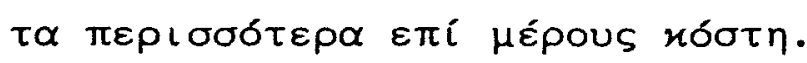

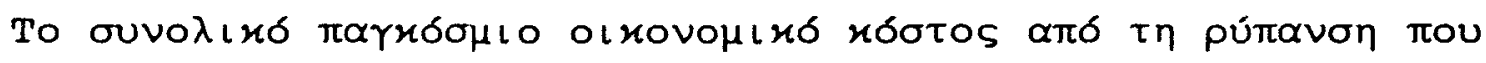

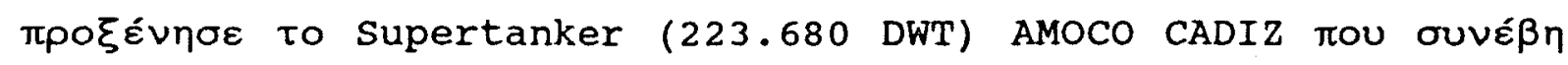

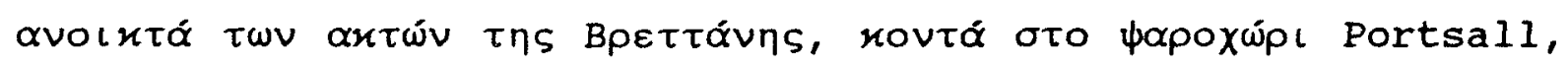

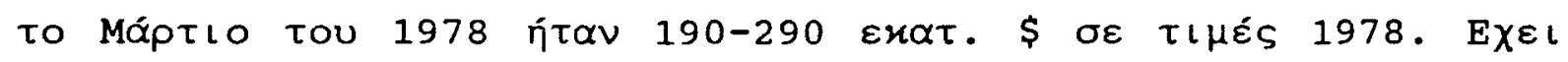
$\varepsilon \varkappa \tau \iota \mu \eta \theta \varepsilon i$ ót

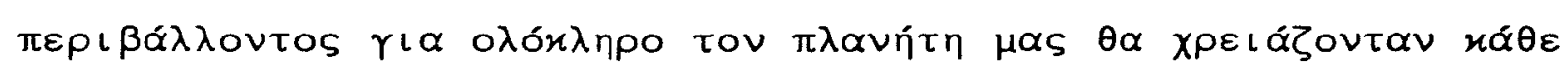
xpóvo 180 бıs $\$$. 


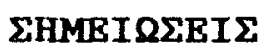

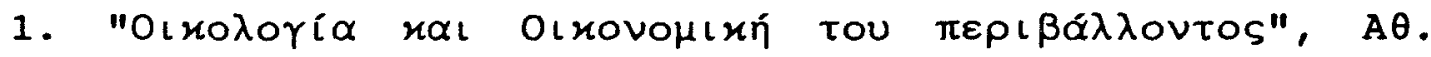

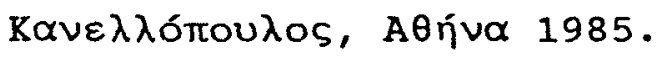

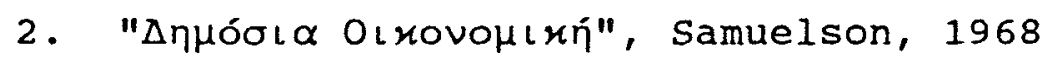

3. "Pollution: The Mess Around Us", Coldman.

4. "Technology and Growth, The Price We Pay", E.J.Mishan, Praeger Publishers, New York 1969.

5. "The New Industrial State", John Kenneth Galbraith, Houghton Miffin Company, Boston 1967.

6. "Industry, Sector Working Paper", World Bank operations, Sectoral Programs and Policies (Samuelson)

7. "Economic Possibillities for our Grandchildren", J.M.Keynes, 1930 


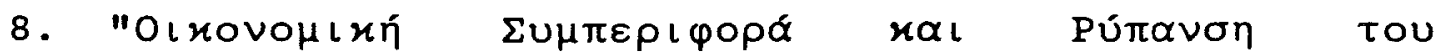

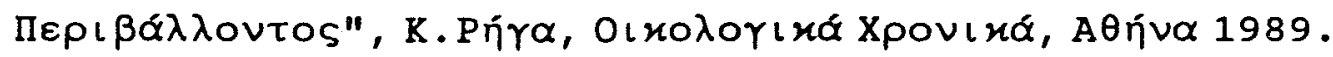

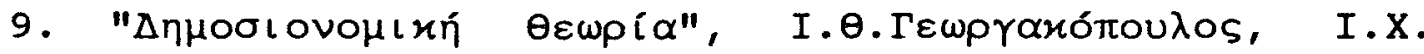

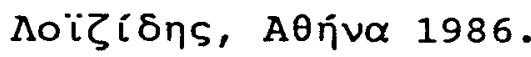

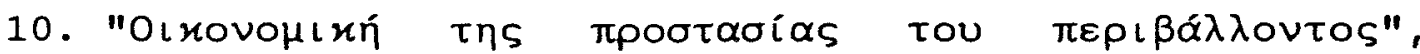

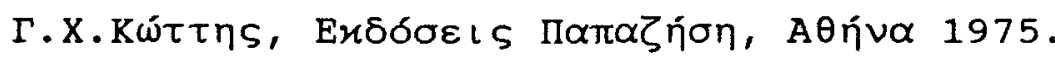

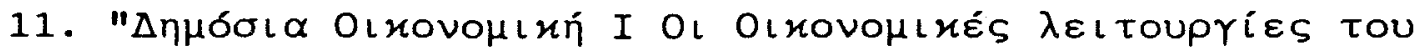

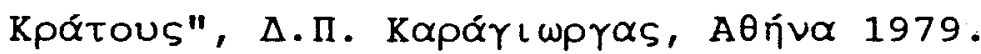

12. "The use of Economic Analysis in Valuing Natural Resourse Damages" National Oceanic and Athmocpheric Administration, Ioúvıos 1984.

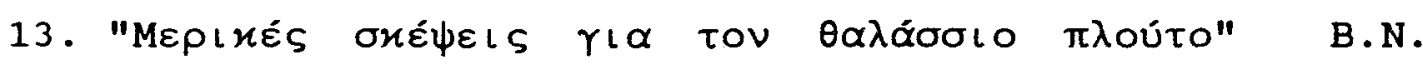

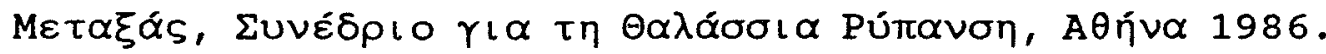




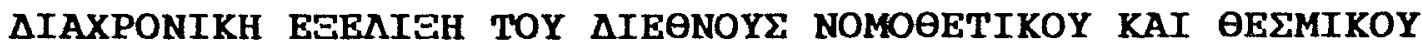 MAAIEIOY TIA THN MPOETAEIA TOY OANASEIOY MEPIBANNONTOE.
}

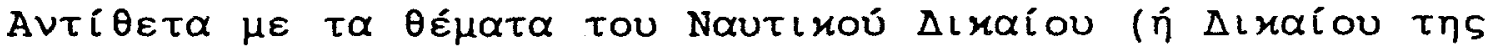

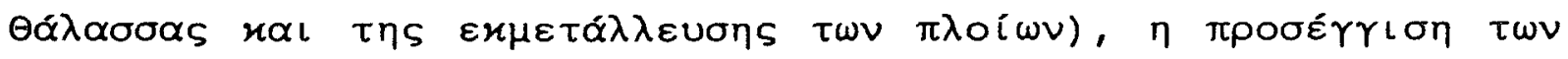

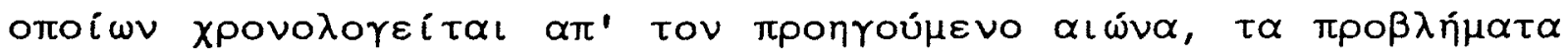

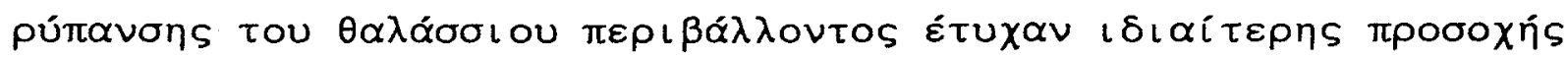

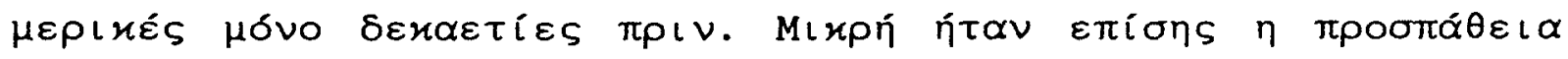

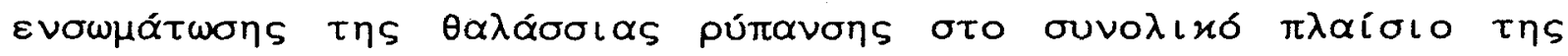

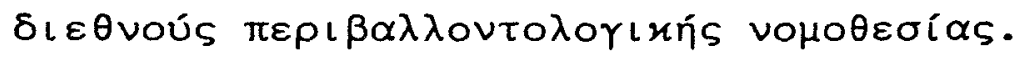

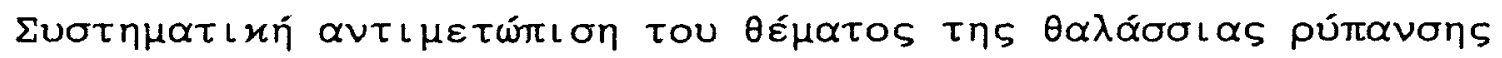

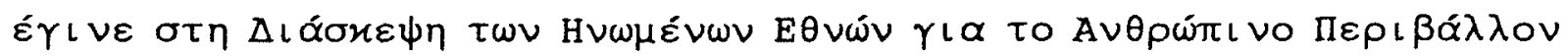

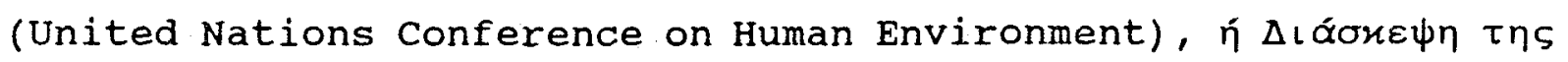

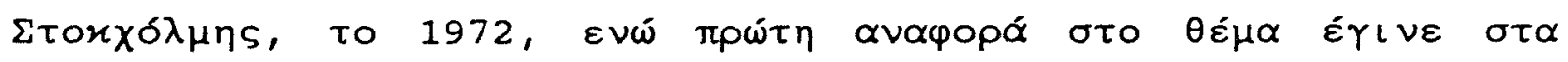

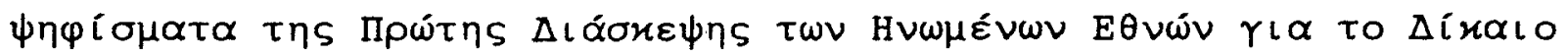
Tns $\theta \alpha \lambda \alpha \sigma \sigma \alpha s$ (First United Nations Conference on the Law of the

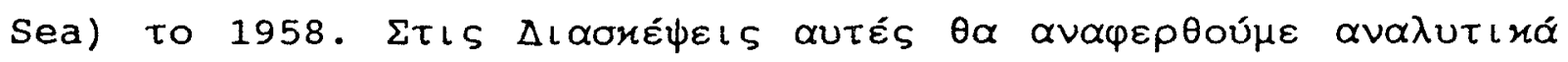

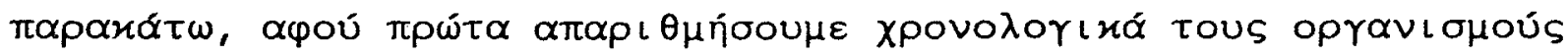

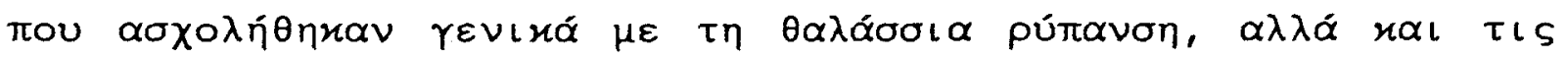

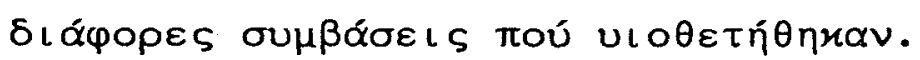




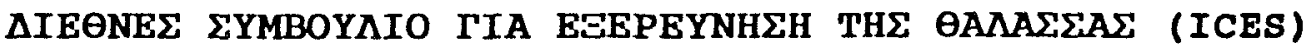 International Council for Exploration of the sea.}

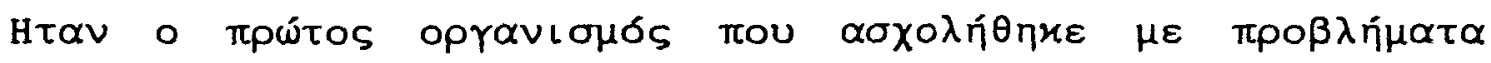

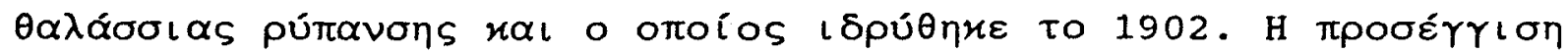

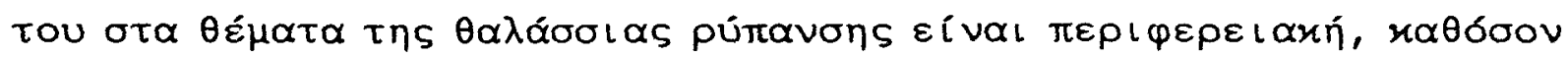

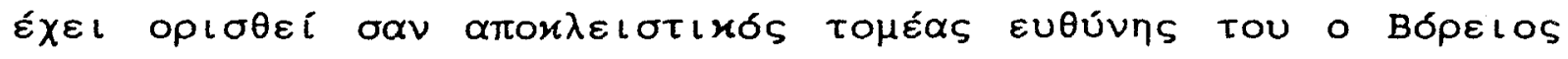

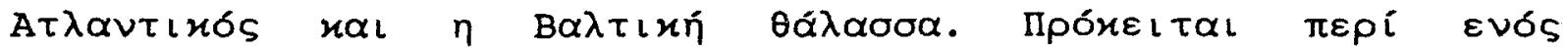

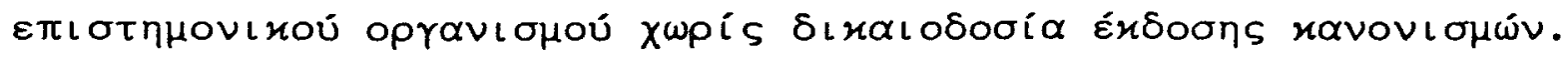

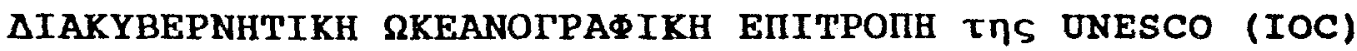 Intergovermental oceanographic commission.}

To $1950 \eta$ UNESCO

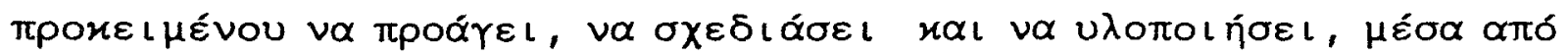

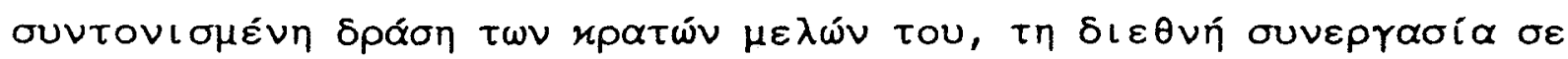

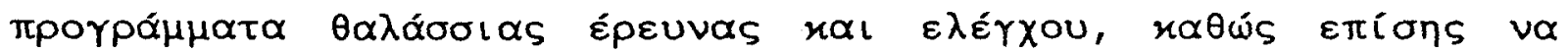

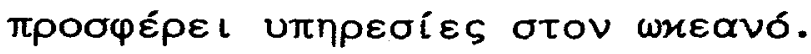




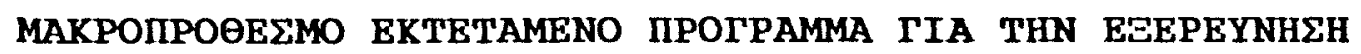

KAI EPEYNA TRN IKEANSN (LEPOR)

Long-Term Expanded Programme for Oceanic Exploration and Research.

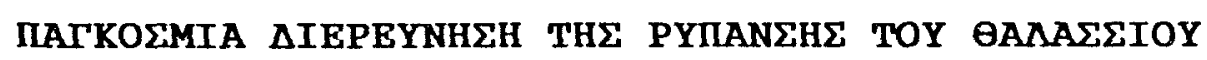
IIEPIBAANONTOE (GIPME)

Global Investigation of Pollution of the Marine Environment.

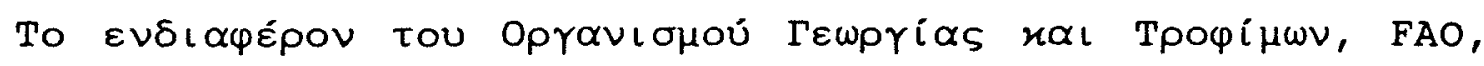

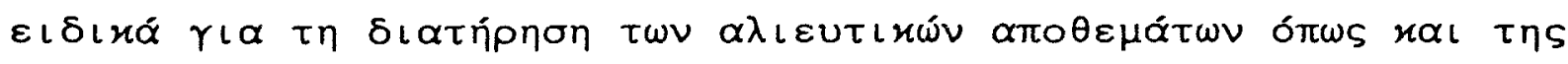

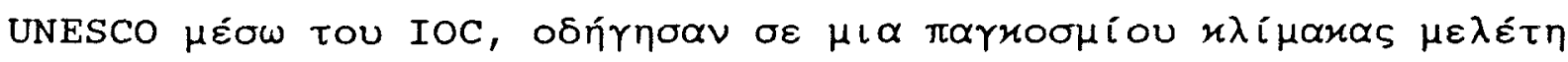

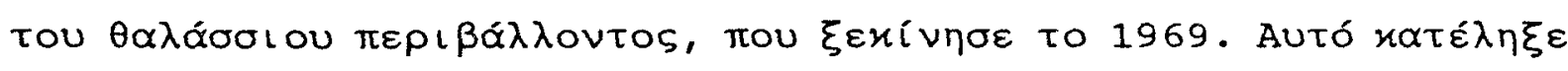

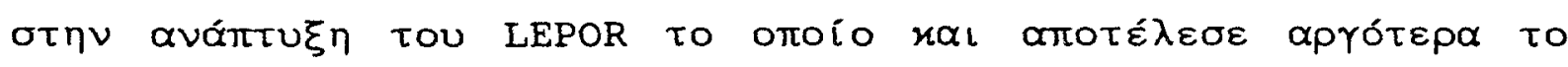

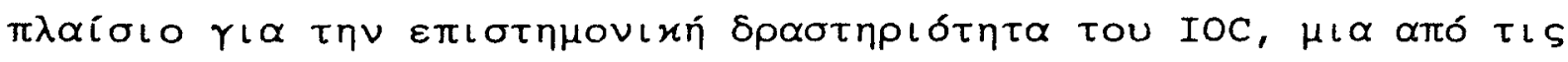

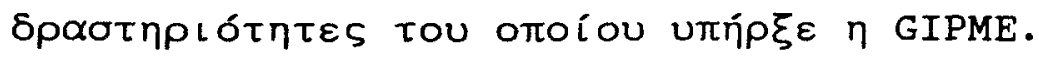

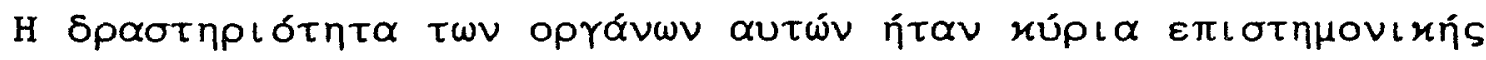

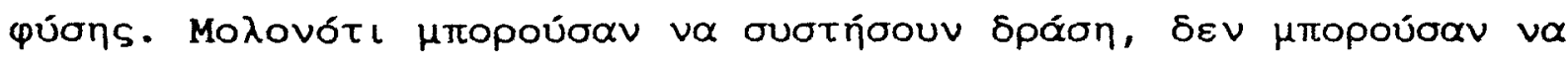

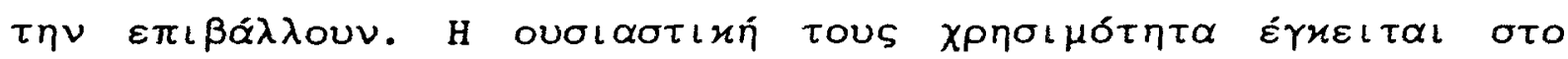

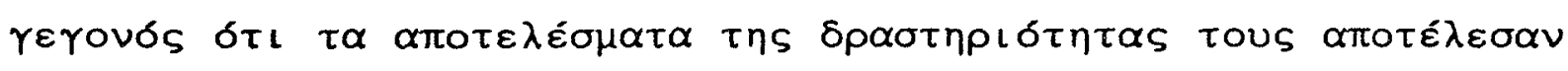

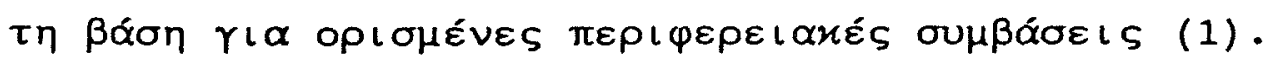




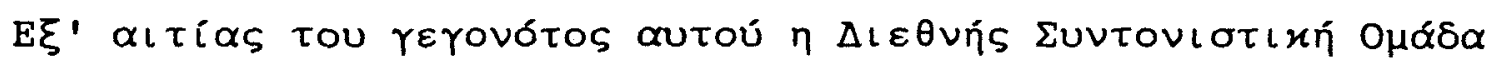

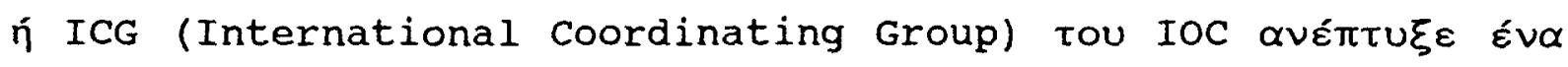

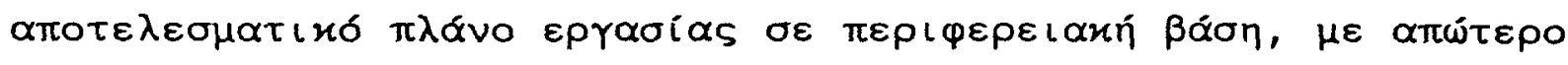

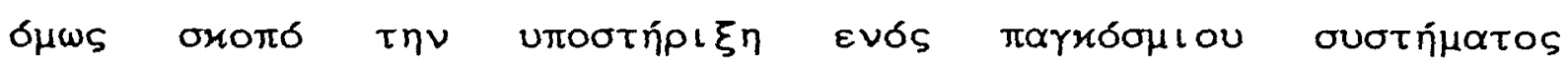

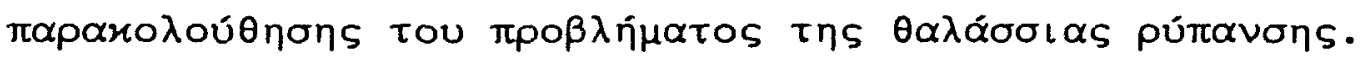

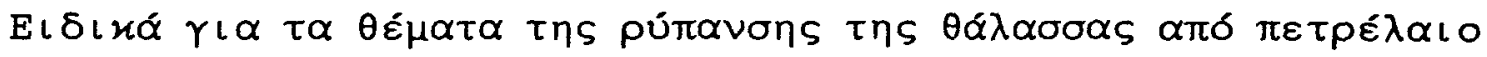

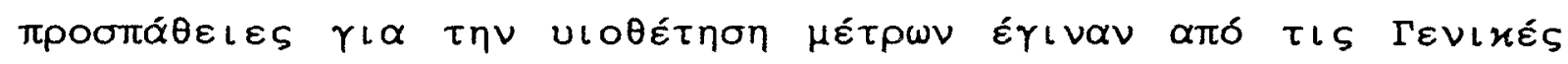

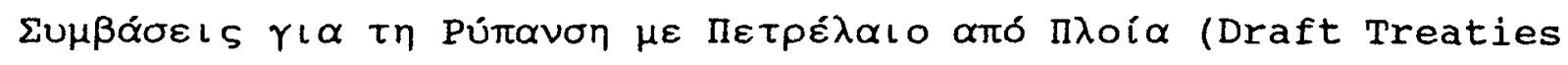
dealing with oil pollution from ships) to 1926 ral 1934. Ol

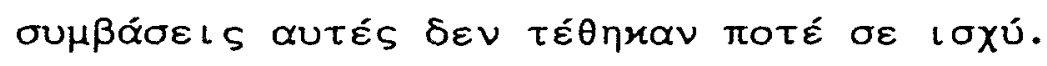


$\triangle I A-K Y B E P N H T I K O \Sigma$

NAYTIAIAKO

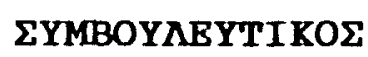

OPFANIEMOE

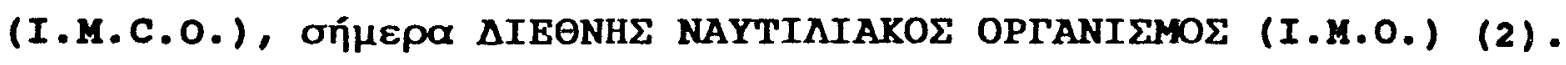
Inter-Governmental Maritime consultative organization, now International Maritime organization.

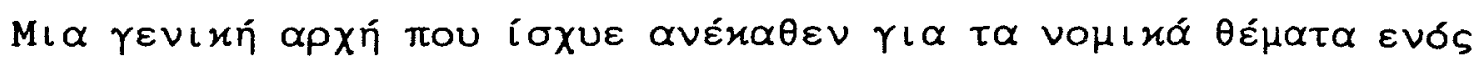

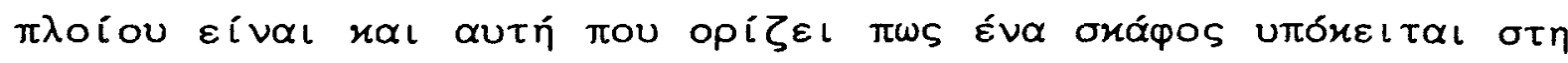

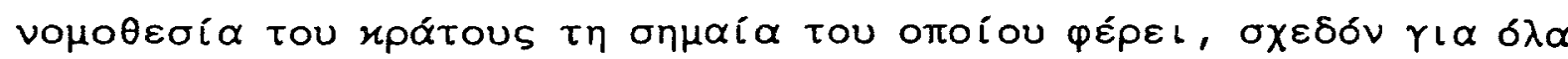

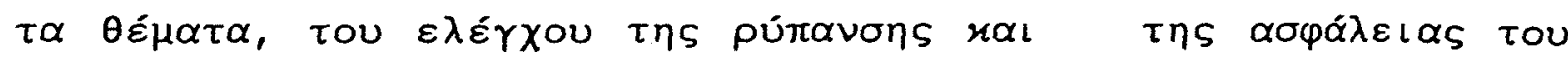

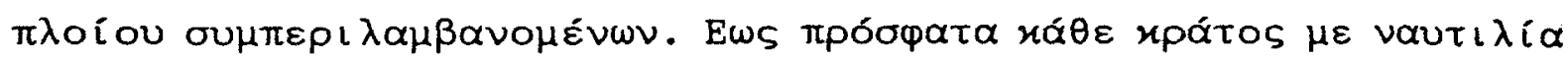

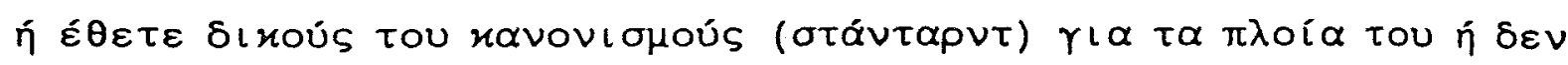
$\varepsilon \dot{\theta \varepsilon \tau \varepsilon ~ \varkappa \alpha \theta o ́ \lambda o u . ~}$

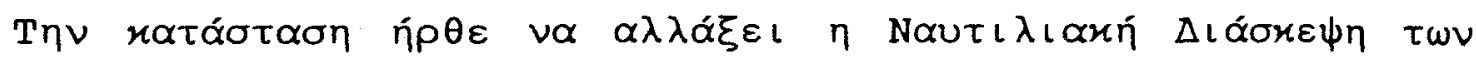

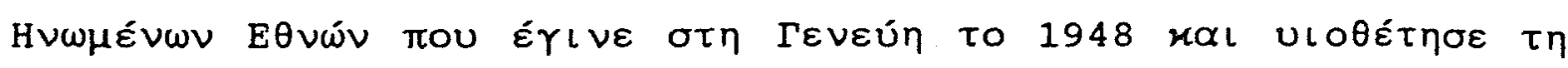

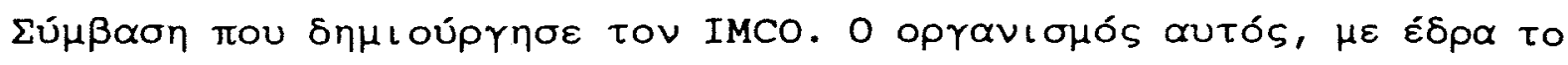

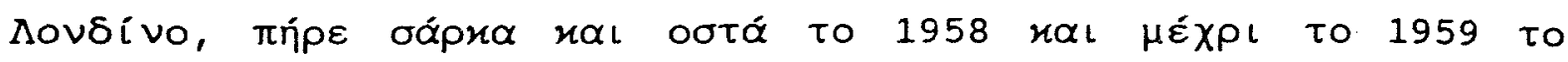

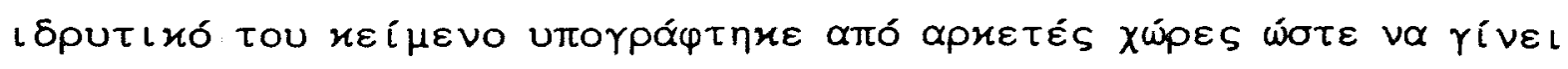

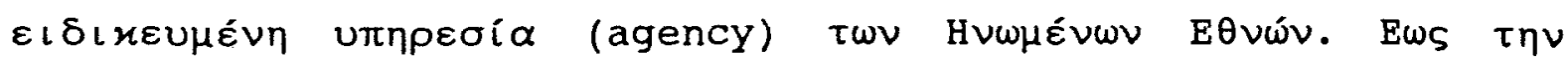

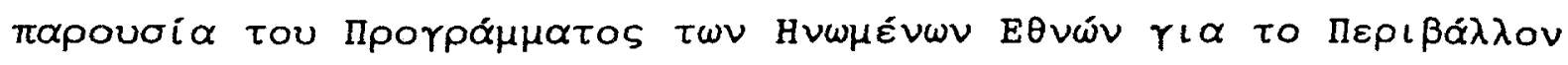

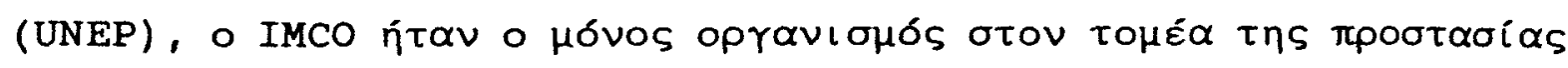

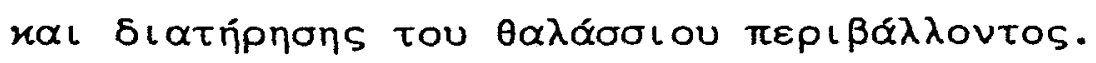




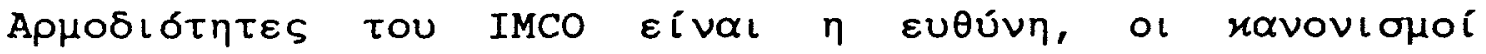

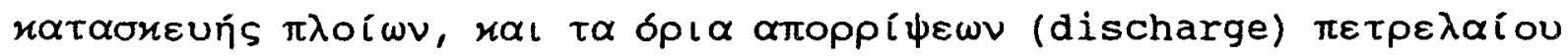

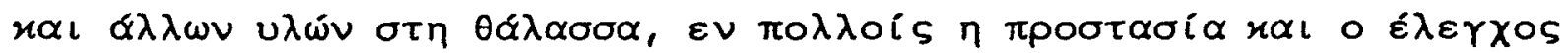

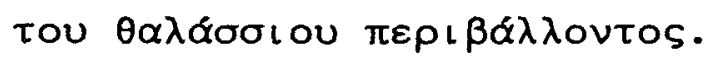

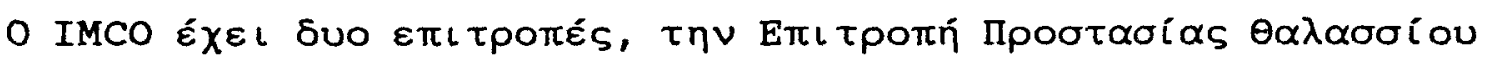

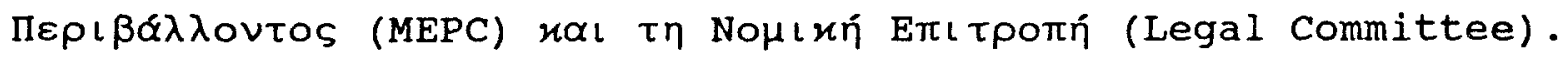

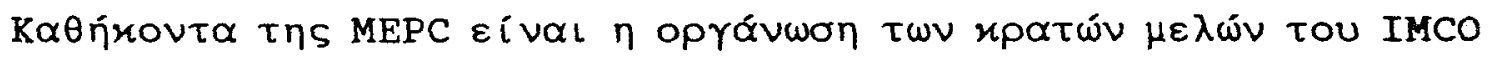

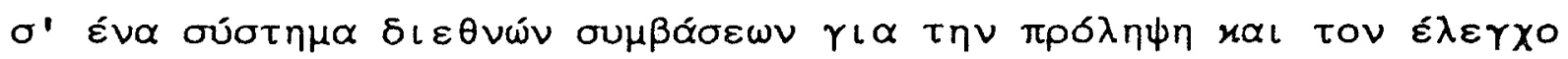

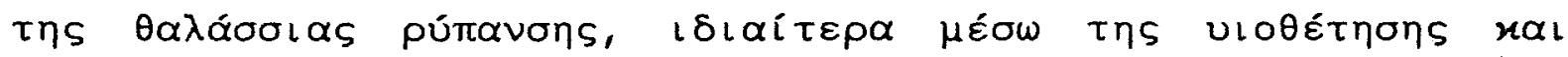

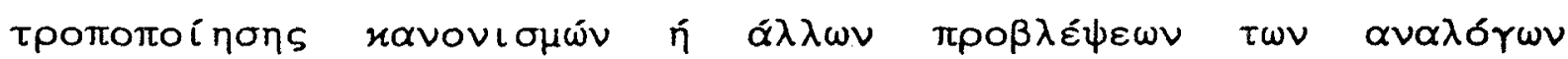

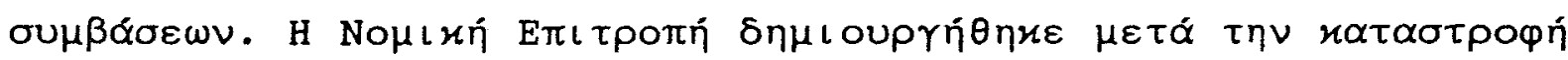

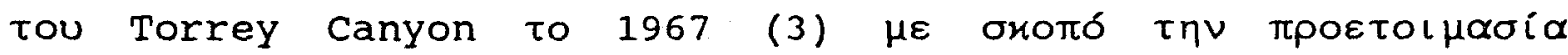

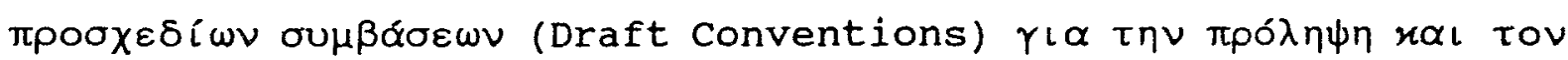

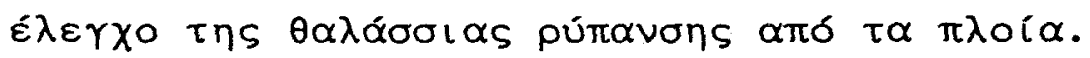

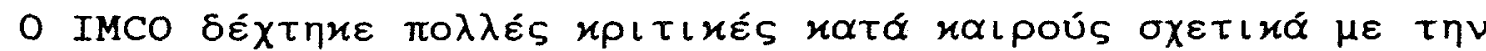

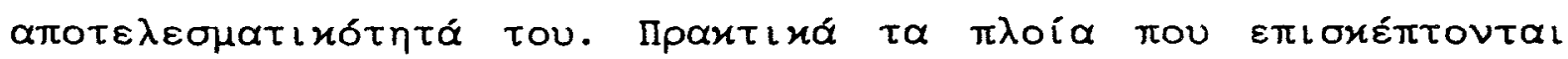

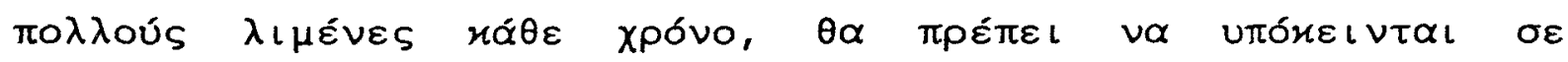

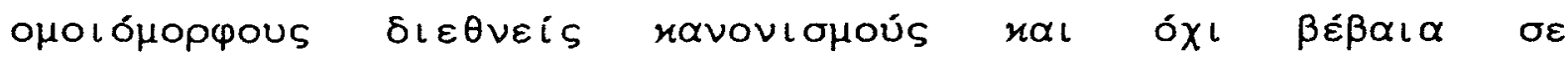

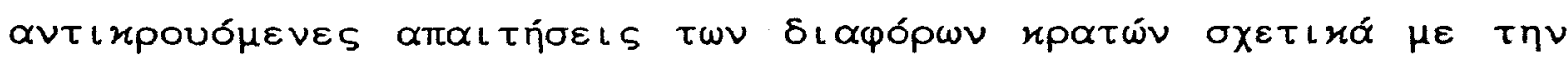

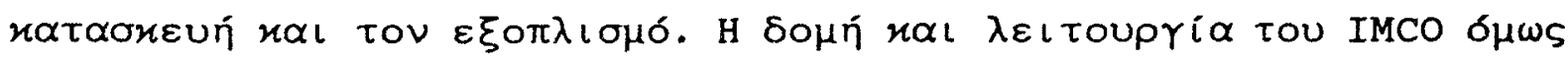

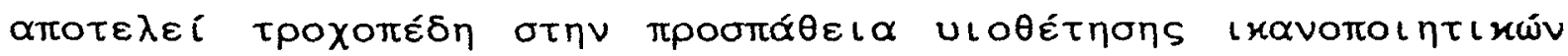

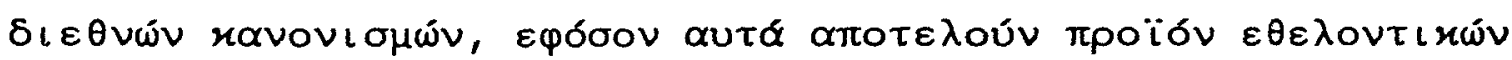




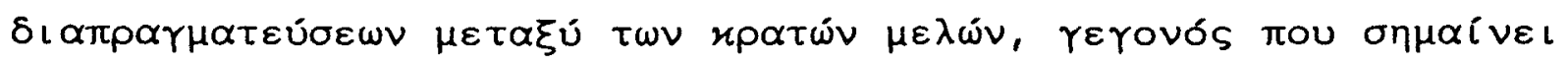

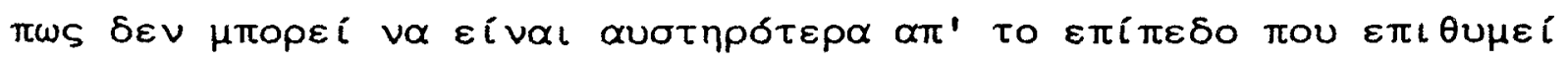

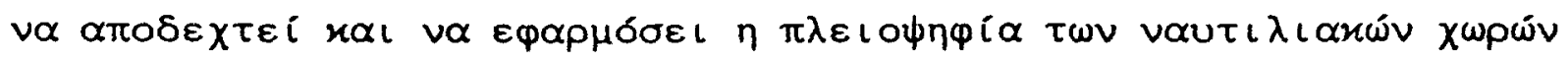
(4).

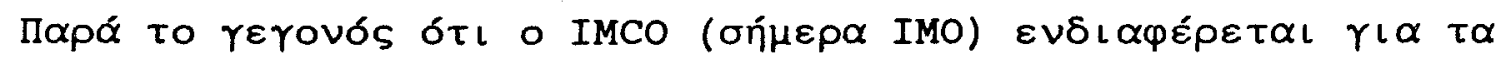

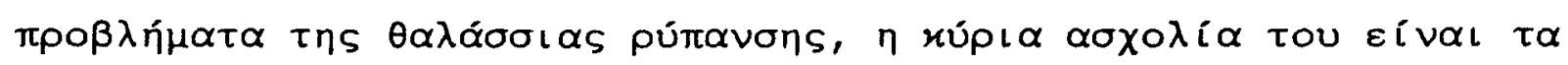

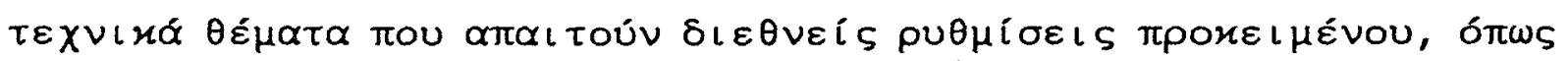

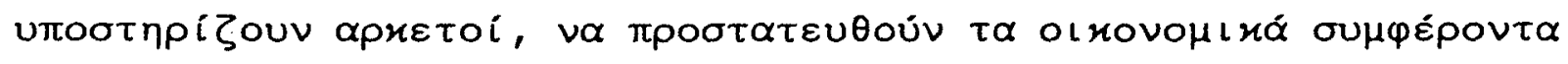

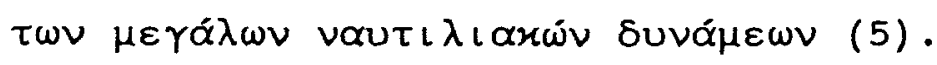

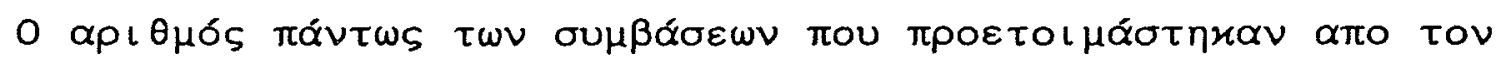

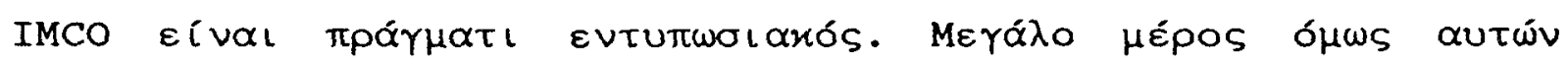
vเ

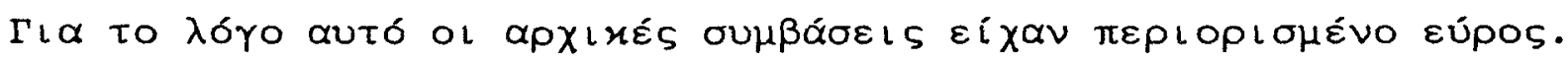

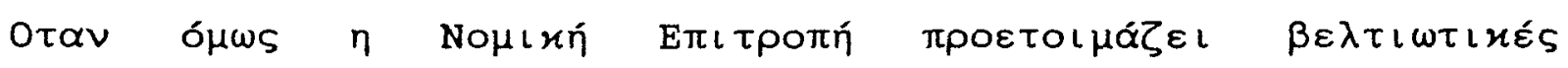

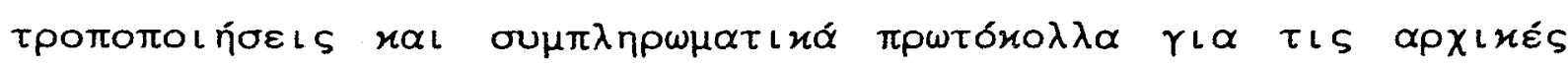

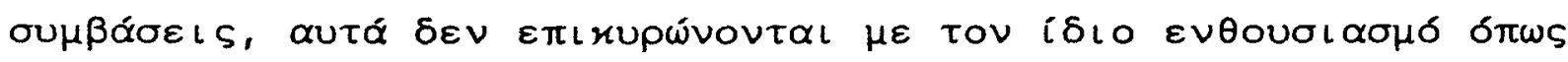

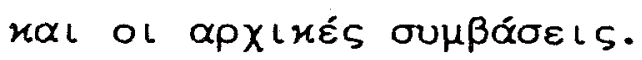

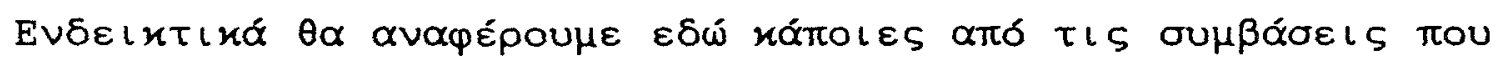

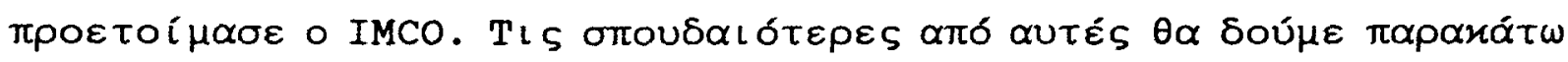
$\alpha \cup \alpha \lambda \cup \tau\llcorner$ ห́́. 


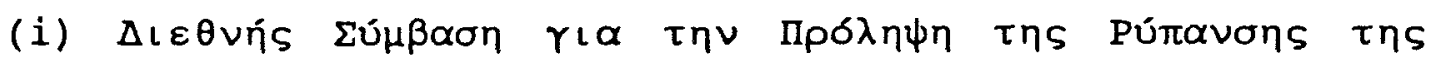

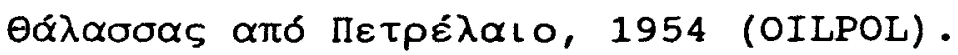

International Convention for the Prevention of Pollution of the Sea by Oil, 1954 (OILPOL).

- Tporotoínon tou 1962 (1962 Amendment)

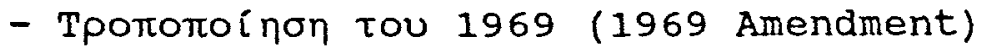

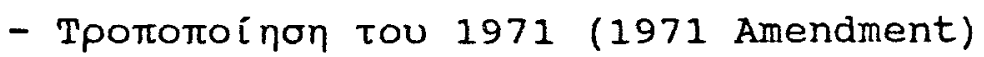

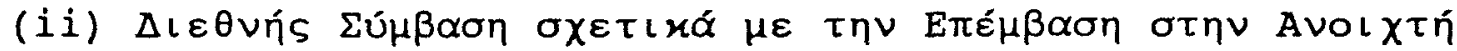

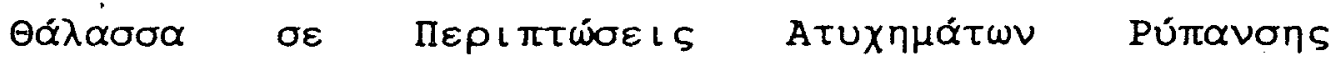

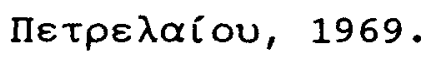

International convention Relating to Intervention on the High Seas in cases of oil pollution Casualties, 1969.

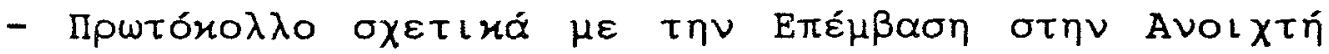

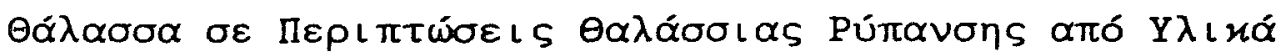
A $\lambda \lambda \alpha \varepsilon x \tau o ́ s ~ \Pi \varepsilon \tau p \varepsilon \lambda \alpha i o u, 1973$.

(1973 Protocol Relating to Intervention on the High Seas in Cases of Marine Pollution by Substances other than oil).

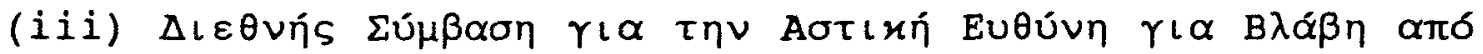

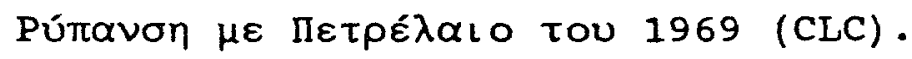

International Convention on civil Liability for oil Pollution Damage, 1969 (CLC). 


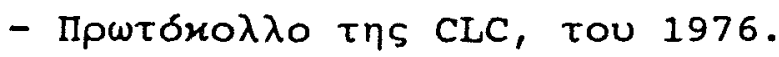

(1976 Protocol to the International Convention on Civil Liability for oil Pollution Damage).

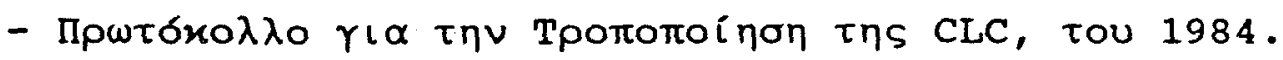
(Protocol of 1984 to Amend the International Convention on civil Liability for oil pollution Damage, 1969) .

(iv) $\Delta\llcorner\varepsilon \theta \cup \eta ́ s ~ \Sigma u ́ \mu \beta \alpha \sigma \eta \eta$

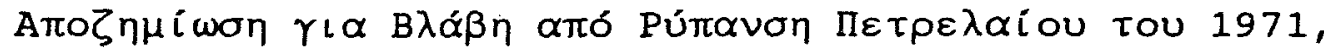
(FUND) •

(International Convention on the Establishment of an International Fund for Compensation for oil pollution Damage, 1971 (FUND).

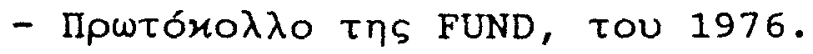
(1976 Protocol to the International convention on the Establishment of an International Fund for Compensation for oil Pollution Damage).

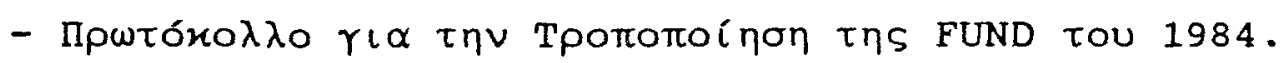
(Protocol of 1984 to Amend the International Convention on the Establishment of an International Fund for Compensation for Oil Pollution Damage). 


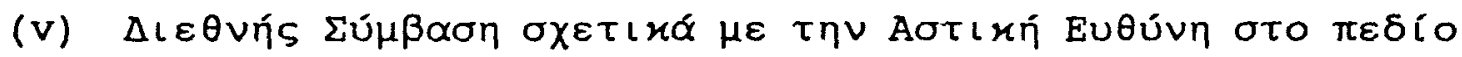

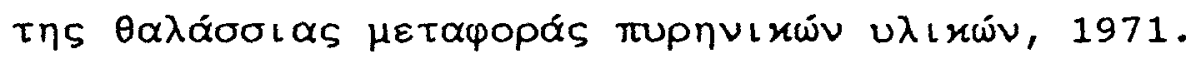
(International Convention Relating to Civil Liability in the Field of Maritime Carriage of Nuclear Material, 1971).

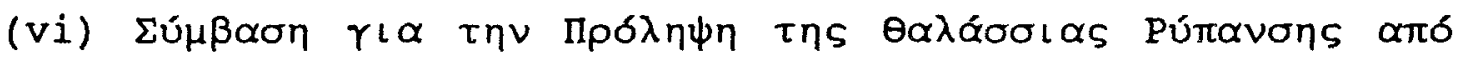

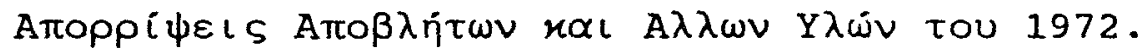

(Convention on the Prevention of Marine Pollution by Dumping of Wastes and other Matter, 1972).

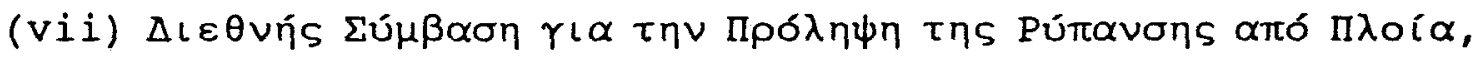
rou 1973 .

(International Convention for the Prevention of Pollution from Ships, 1973).

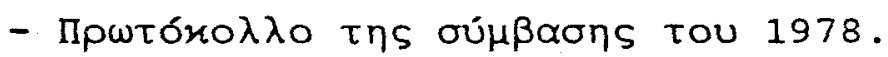

(Protocol of 1978 Relating to International Convention for the Prevention of Pollution from Ships, 1973). 
AIE METPENAIO, 1954 (OILPOL) KOL TPOIOIOIHEEIE TOY 1962, 1969 KAI 1971.

International convention for the prevention of pollution of the sea by Oil and Amendments of 1962, 1969 and 1971.

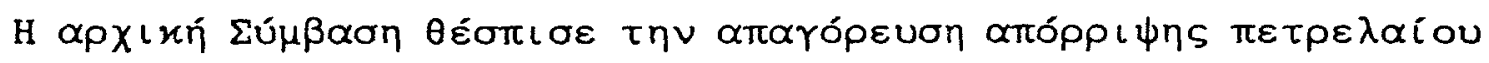

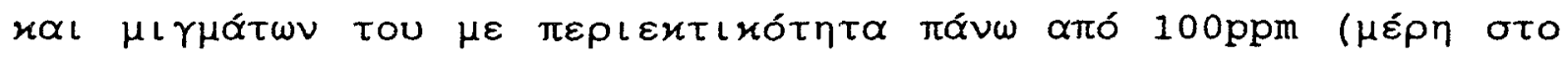

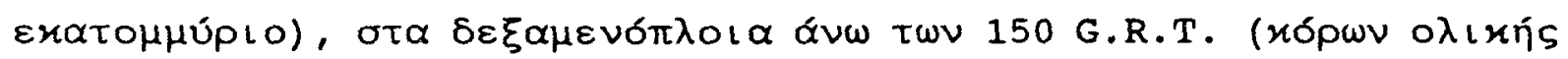

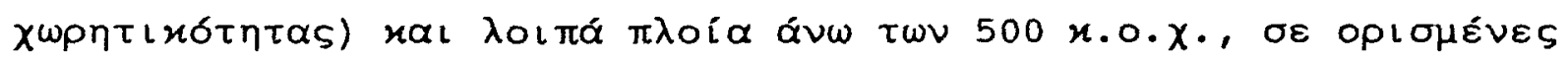

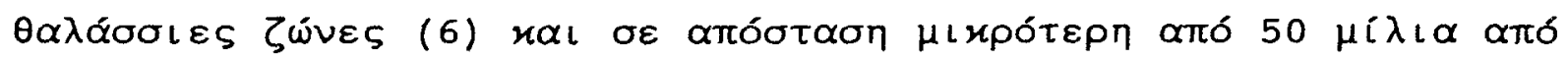

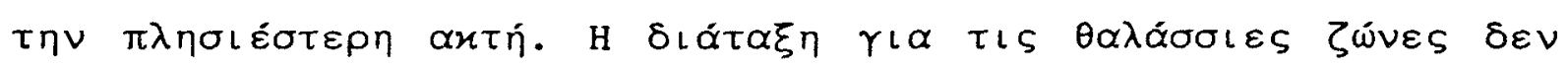

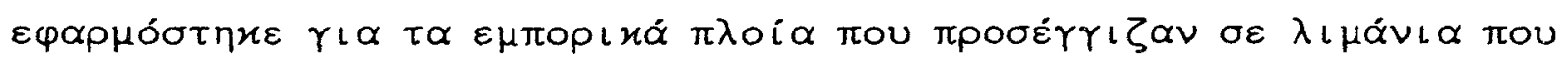

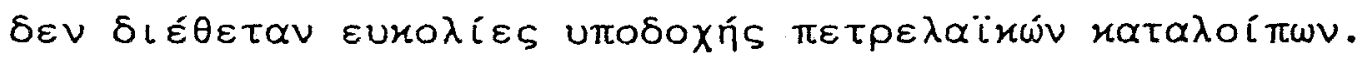

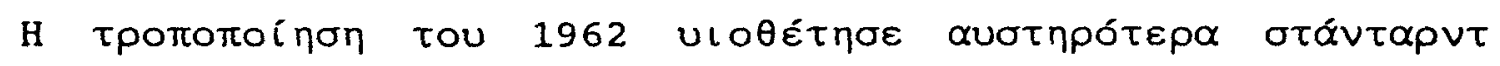

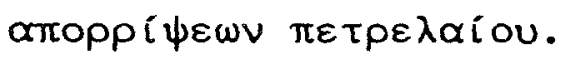

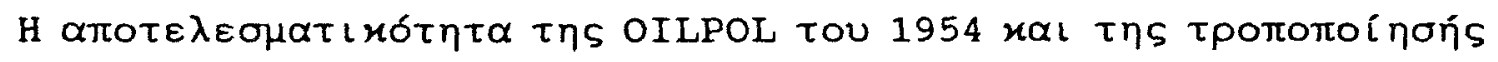

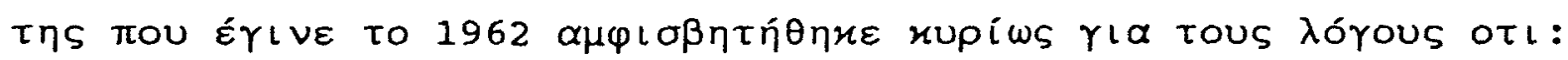

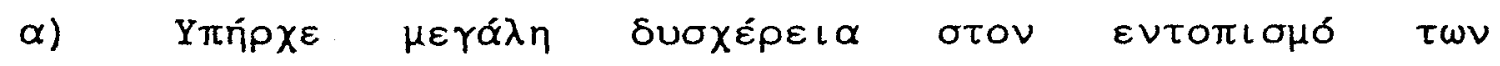

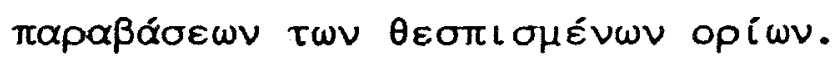




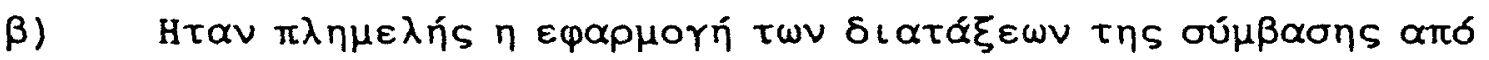

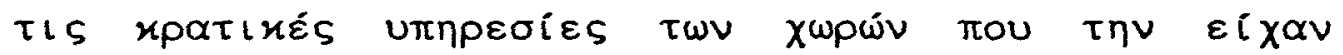

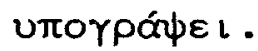

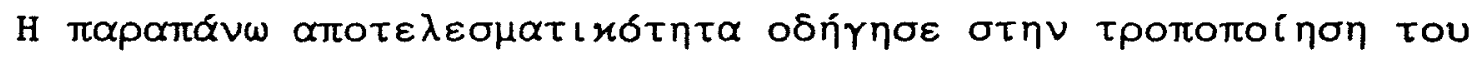

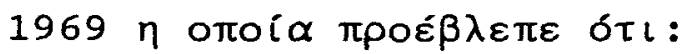

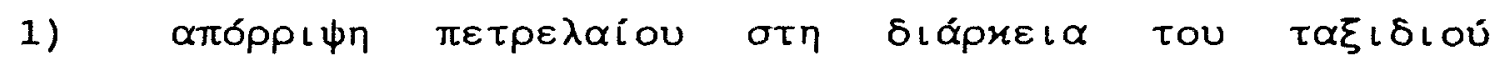

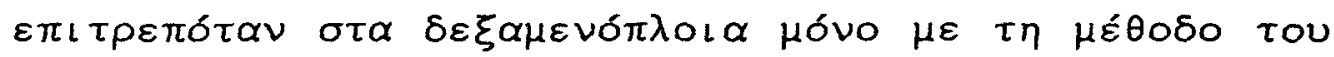

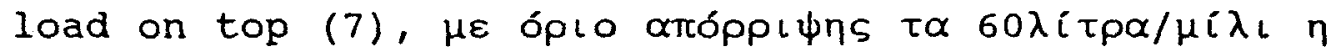

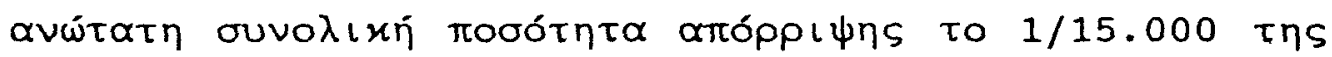
ouvoגıหńs $x$ wp

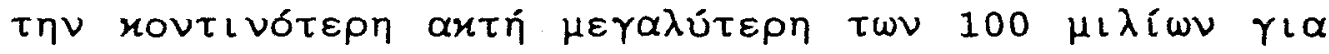

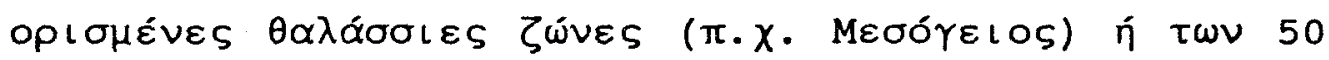

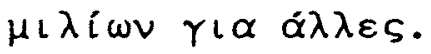

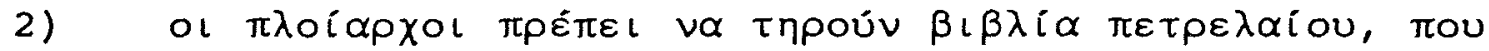

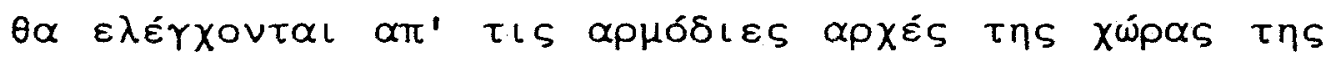
onjaías tou $\pi \lambda$ oĺou, $\gamma\llcorner\alpha$ :

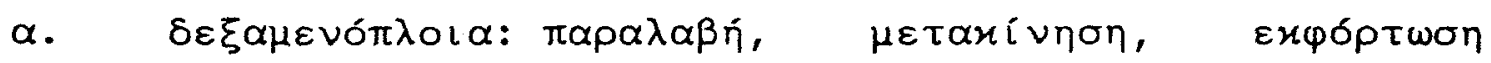

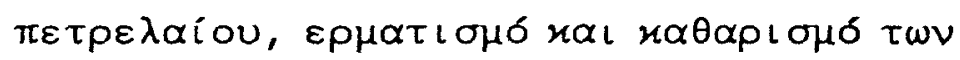
$\delta \varepsilon \xi \alpha \mu \varepsilon \vee \omega ́ v, \quad \alpha \pi o ́ p \rho\llcorner\psi \eta \quad \alpha \varkappa \dot{\alpha} \theta \alpha \rho \tau o u$

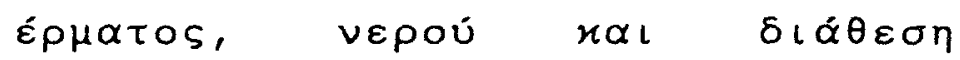

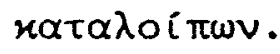




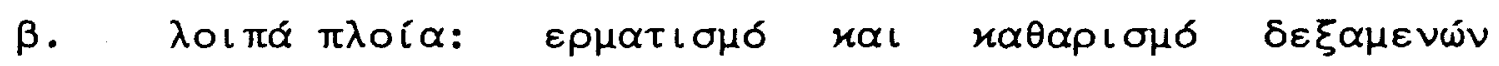

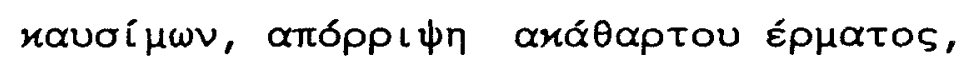

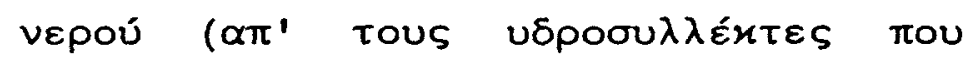

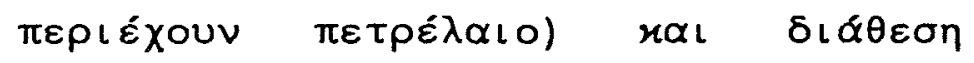
$\varkappa \alpha \tau \alpha \lambda \circ i \pi \omega \nu$.

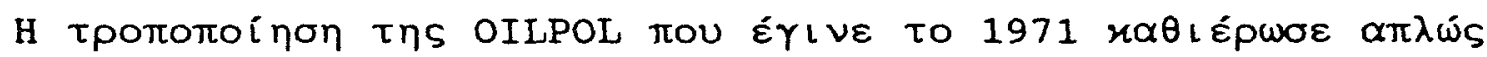

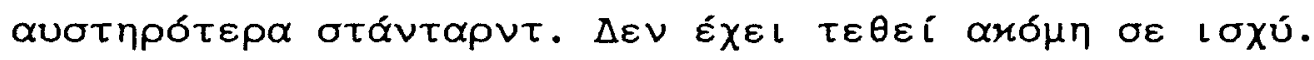

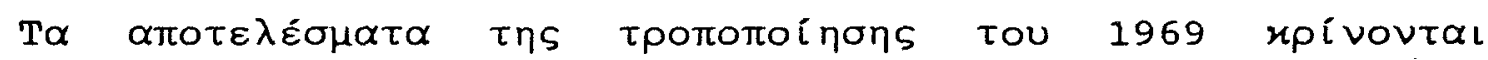

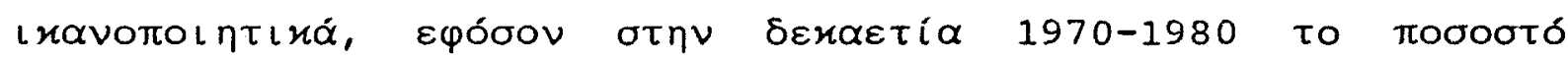

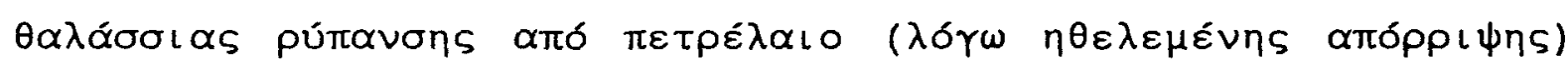

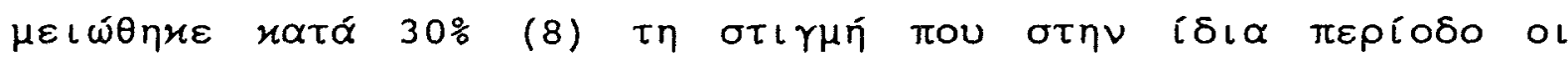

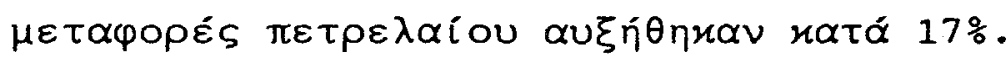

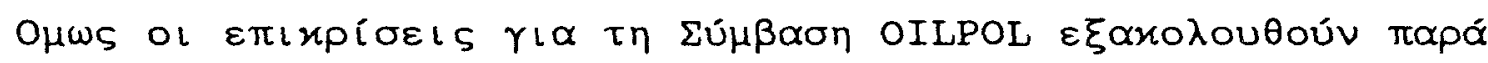

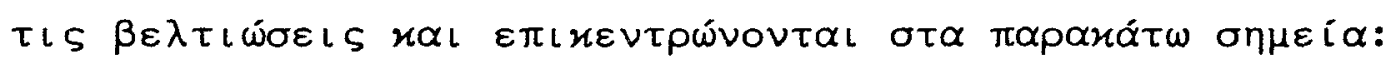

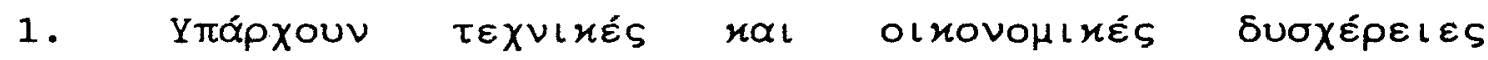

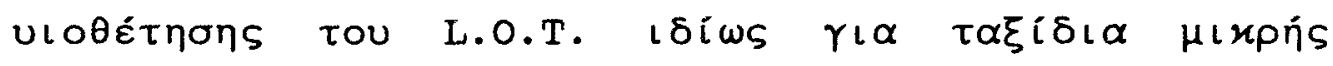

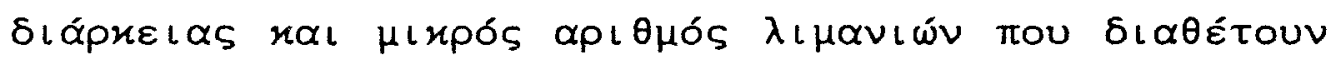

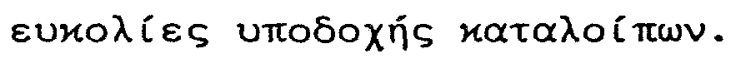

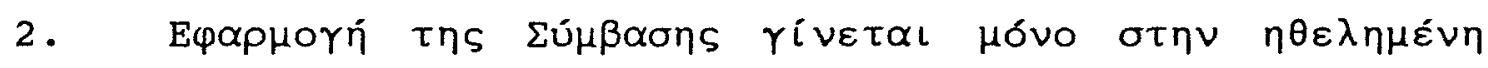

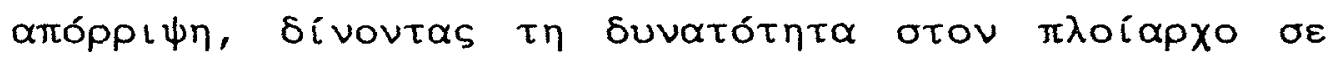

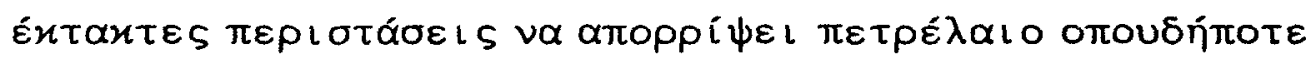




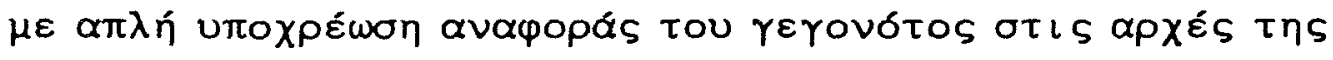

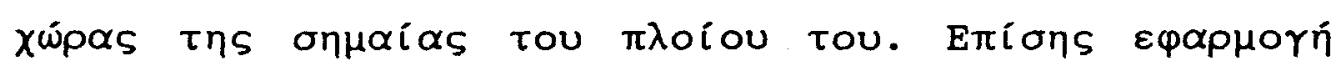

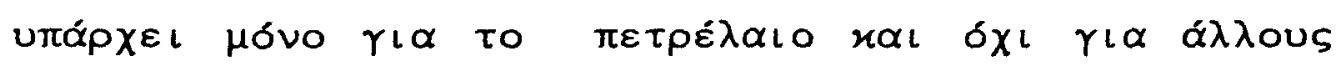

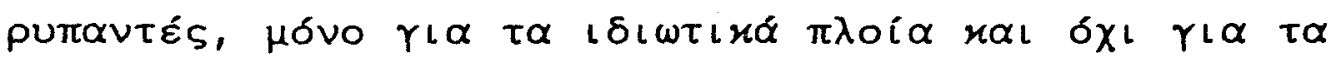
$\delta \eta \mu \delta \sigma \alpha$.

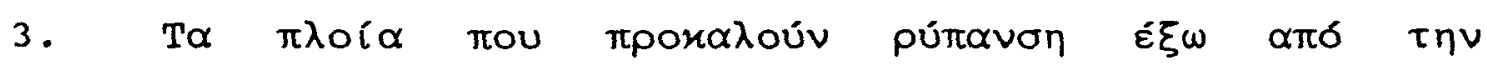

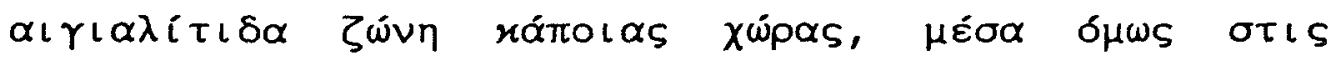

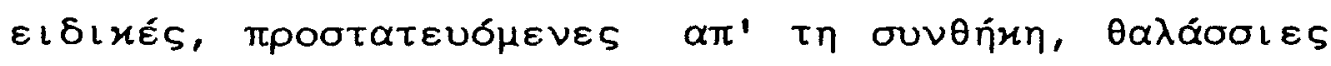

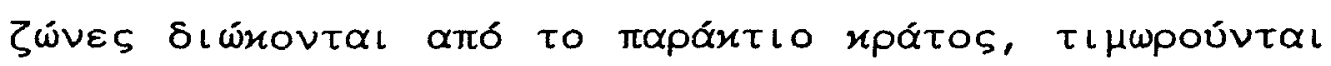

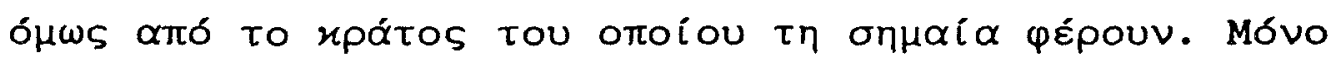

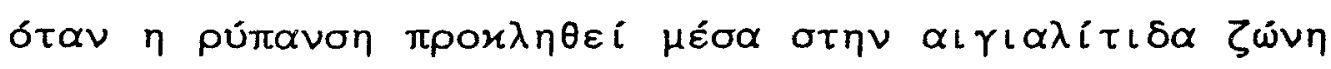

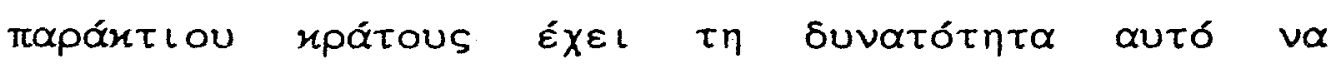

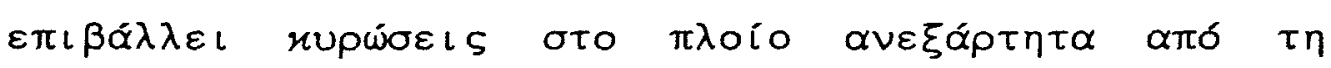
onjaía tou.

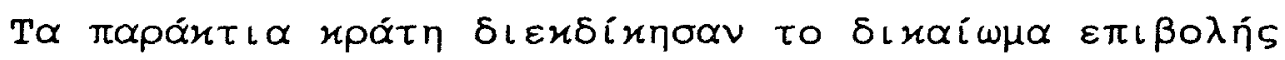

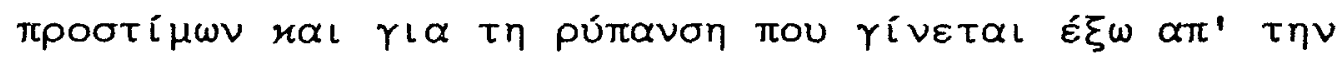

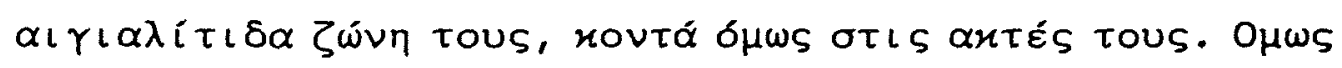

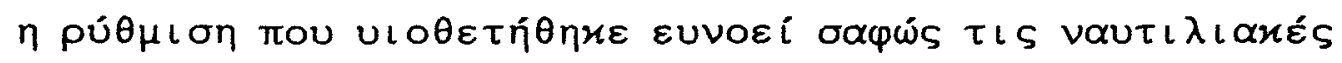

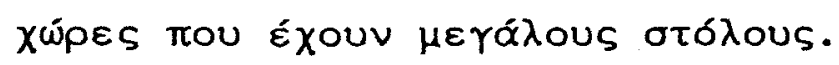

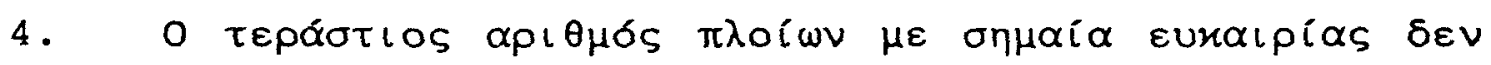

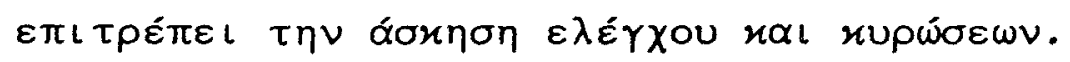




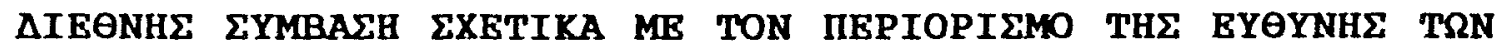

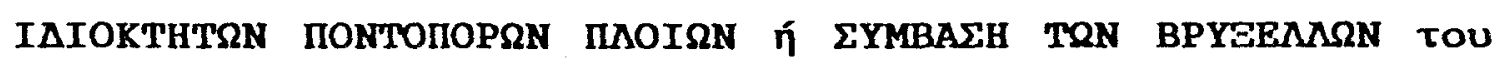
1957.

International convention Relating to Iimitation of the Liability of Owners of seagoing ships, 1957 or "the 1957 Brussels Convention".

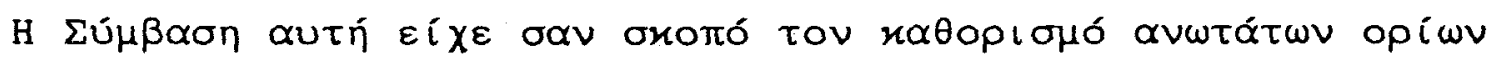

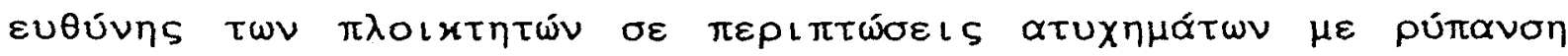
$\pi \varepsilon \tau \rho \varepsilon \lambda \alpha i$ ov.

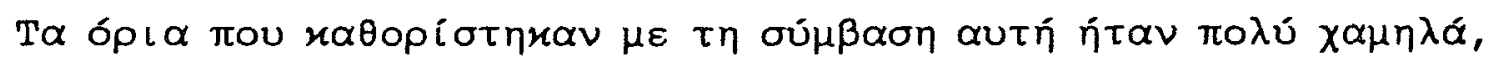

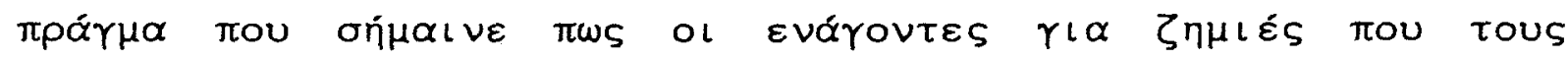

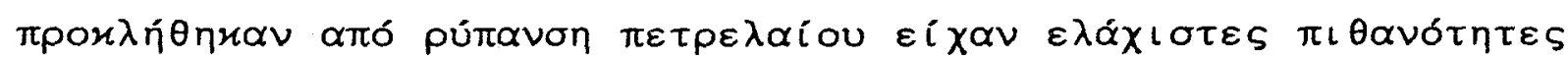
$v \alpha \alpha \pi \circ \zeta \eta \mu \iota \omega \theta$ oúv (9) .

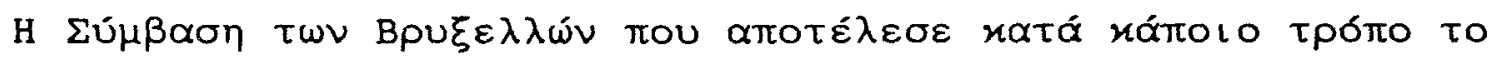

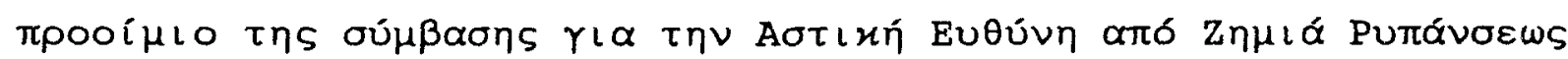

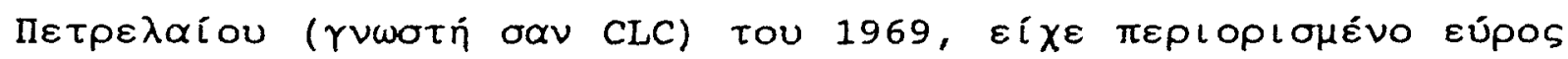

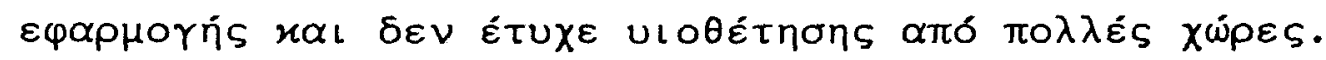




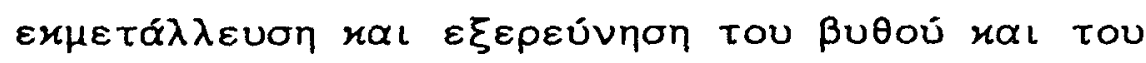

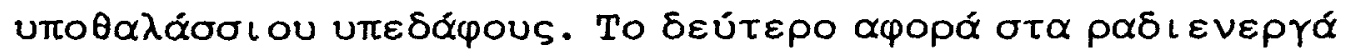
$\alpha \pi \delta \beta \lambda \eta \tau \alpha$.

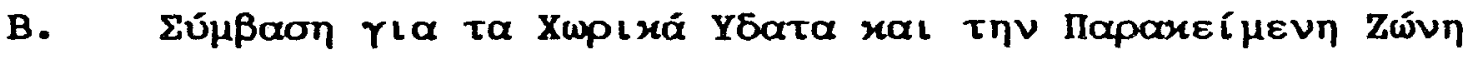
convention on the Territorial sea and the Contiguous zone).

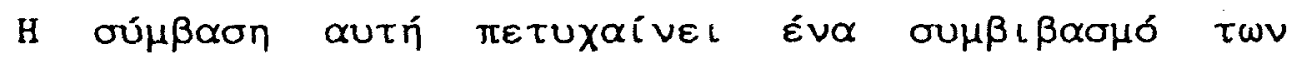
$\alpha \vee \tau \iota$ น

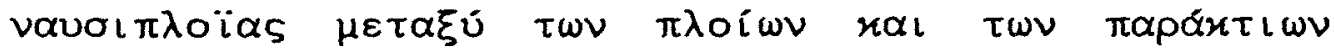

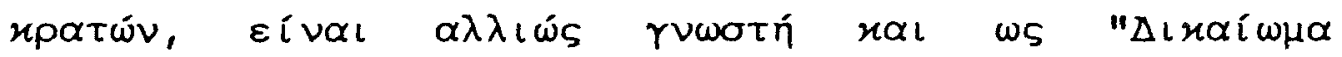

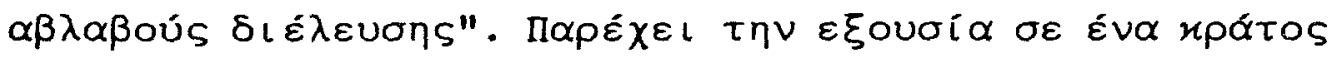

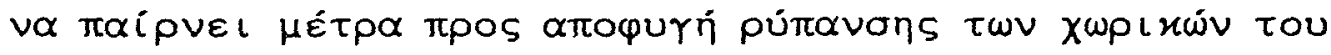

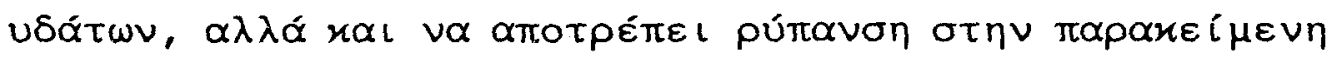

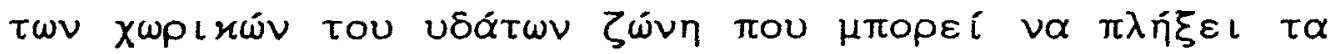

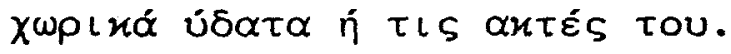

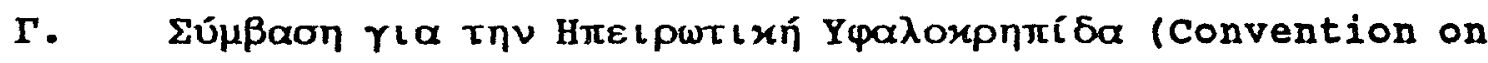
the continental shelf).

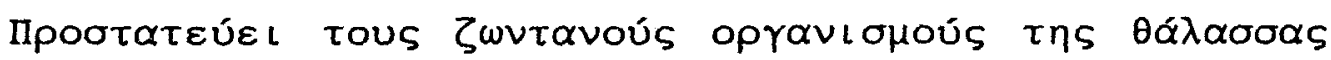

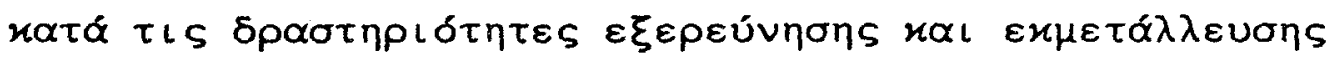

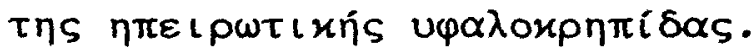




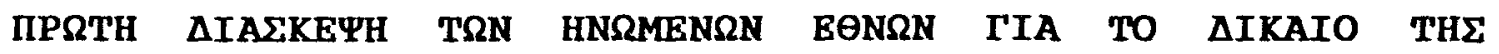

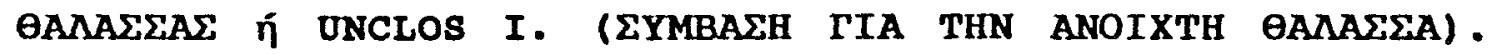
reveún 1958.

First United Nations conference on the Law of the sea. (High Seas Convention). Geneva, 1958.

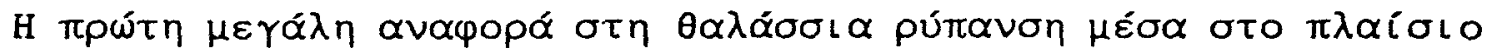

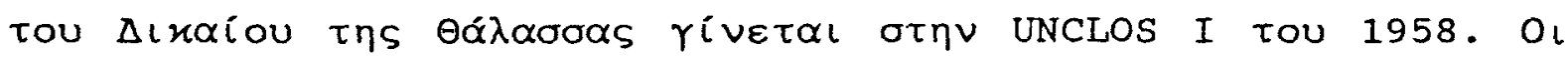

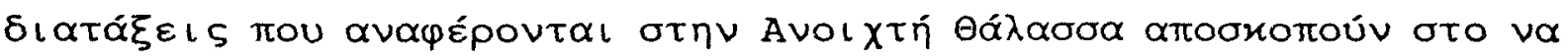

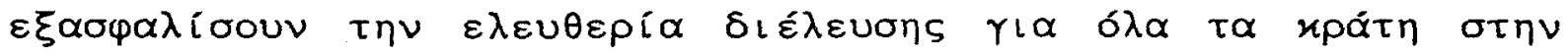

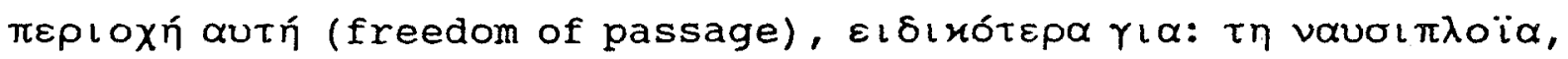

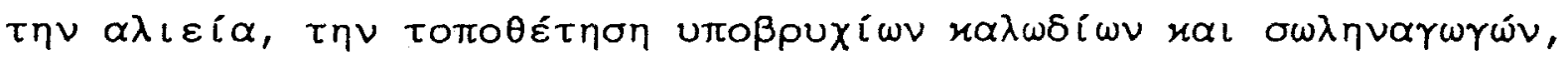

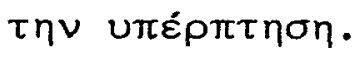

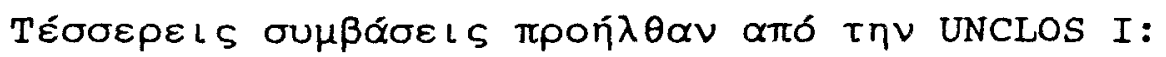

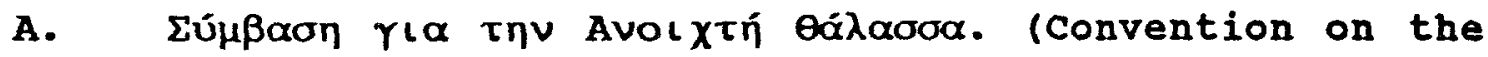
High seas).

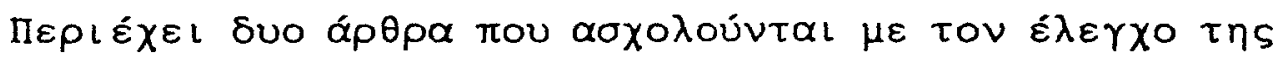
pútavons.

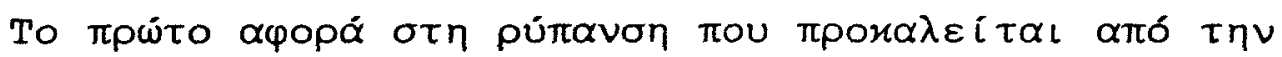

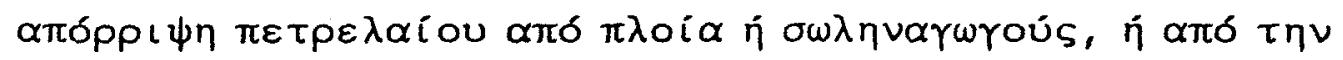




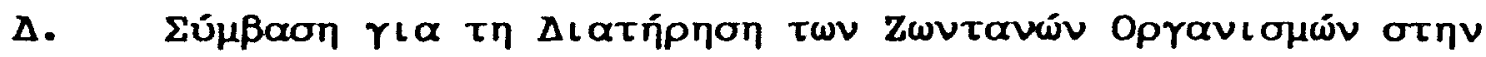

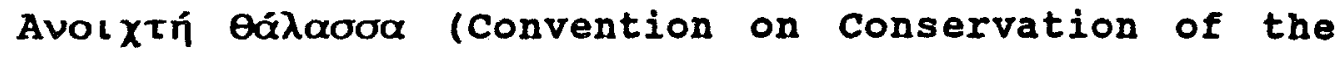
Living Resources of the High seas).

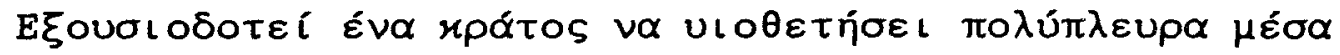

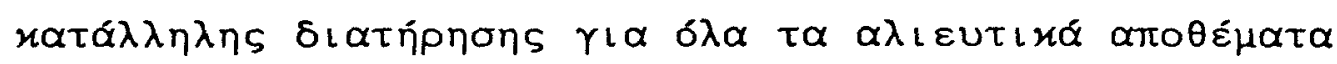

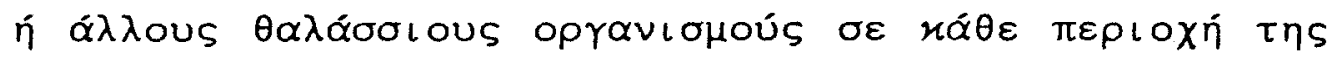

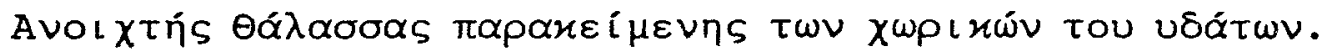

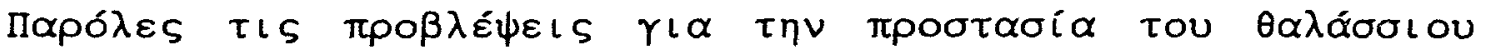

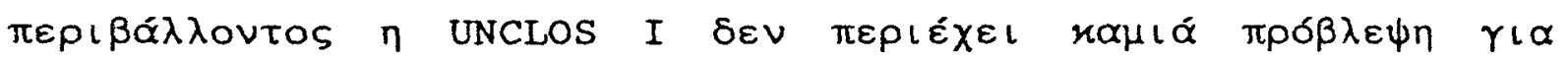

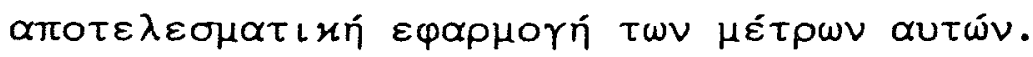

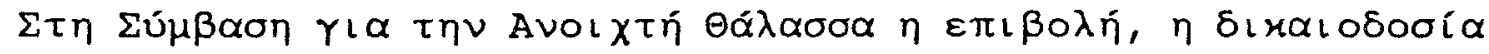

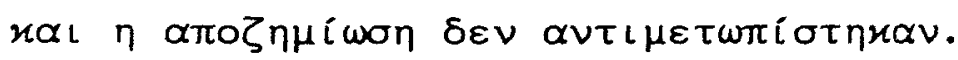

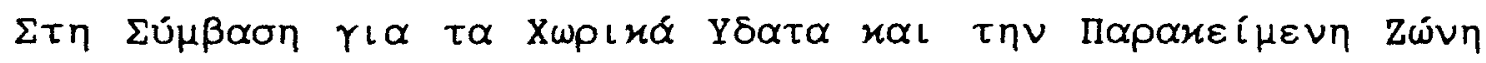

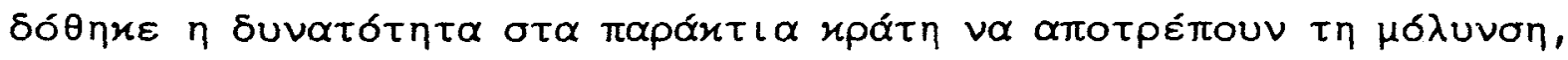

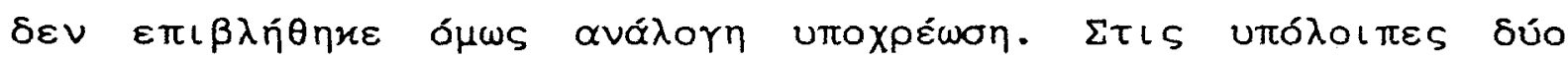

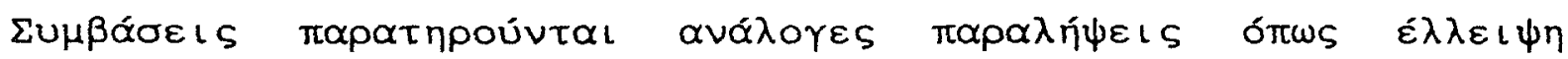

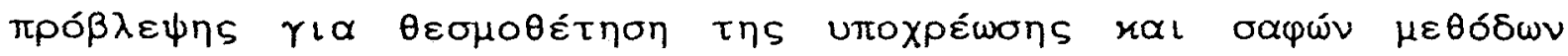

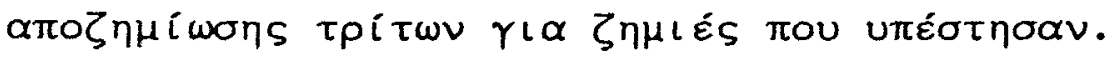




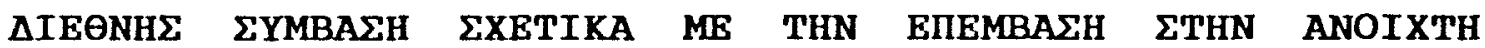

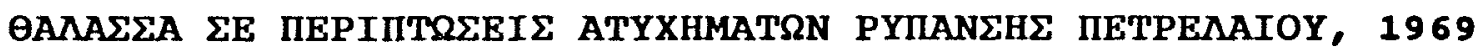

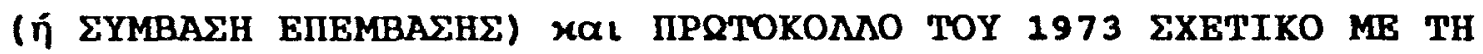

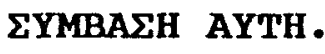

International Convention Relating to Intervention on the High Seas in Cases of oil Pollution Casualties (or Intervention Convention), 1969 and 1973 protocol Relating to the Convention of 1969 .

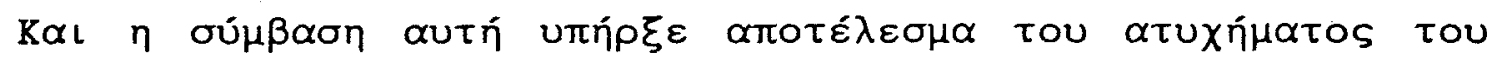

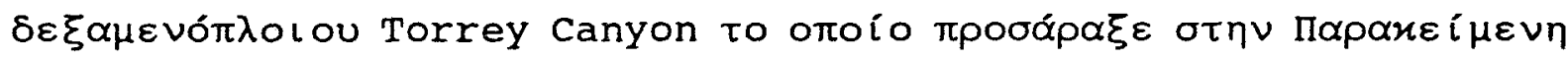

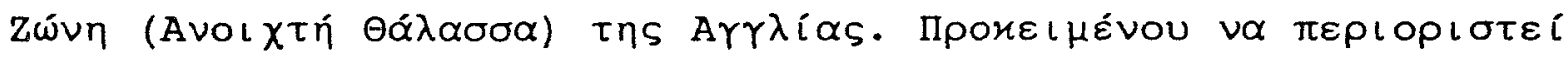

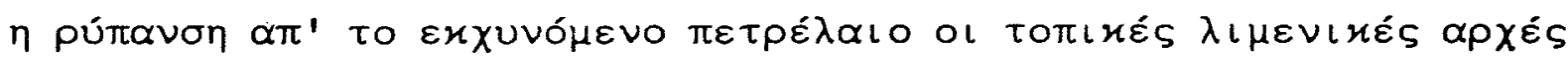

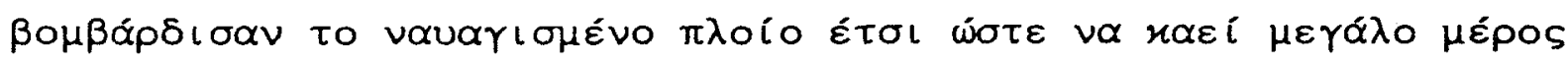
tou poptíou tou.

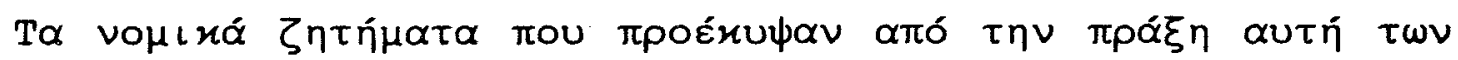

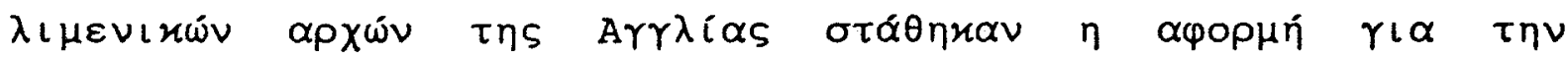

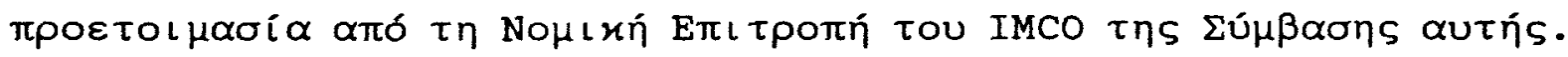

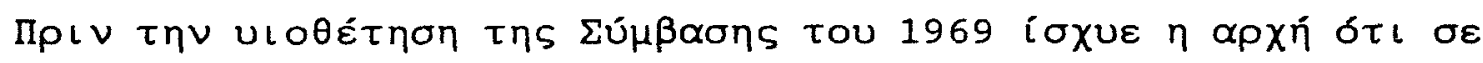

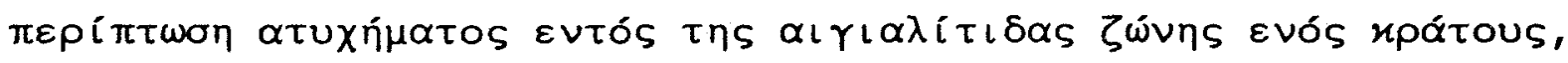

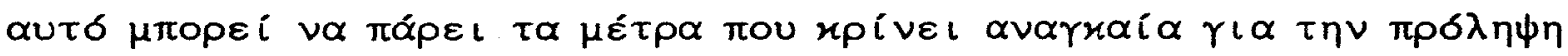




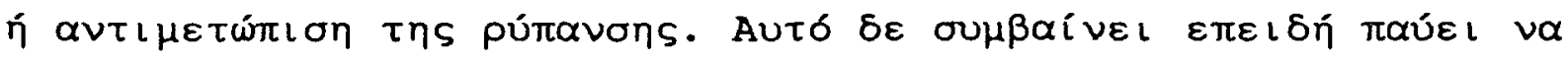

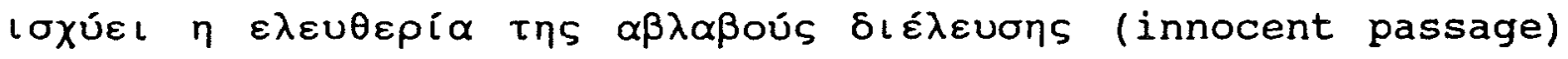
$\varepsilon \varphi \delta ́ \sigma o v ~ t o ~ \pi \lambda o i ́ o ~ \delta \varepsilon \nu ~ \beta p i ́ \sigma K \varepsilon \tau \alpha \iota ~ \varepsilon \nu ~ \pi \lambda \dot{\omega}$.

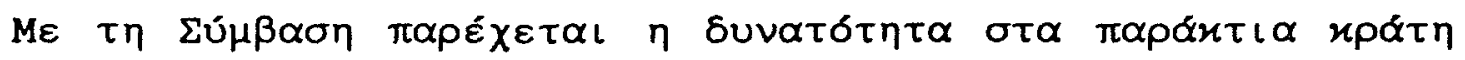

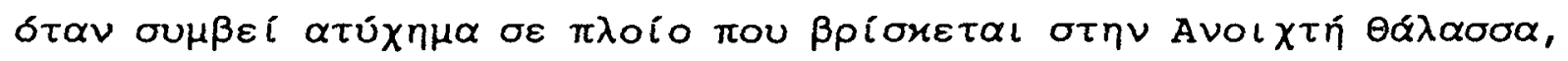

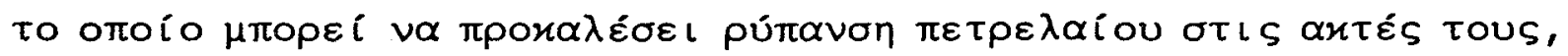

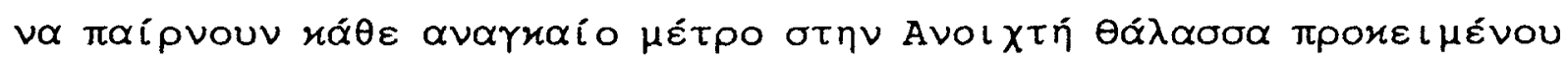

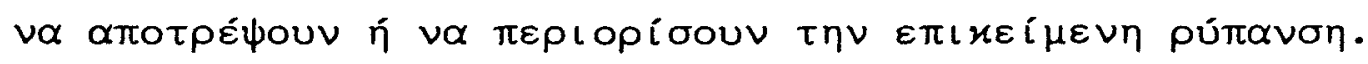

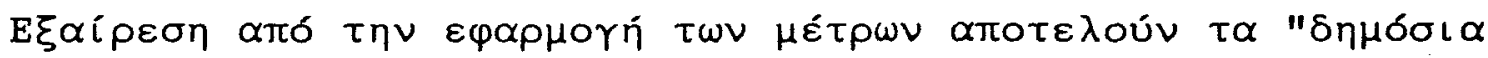

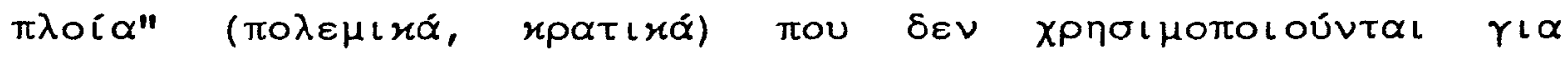
$\varepsilon \mu \pi \circ$ L noús anotoús.

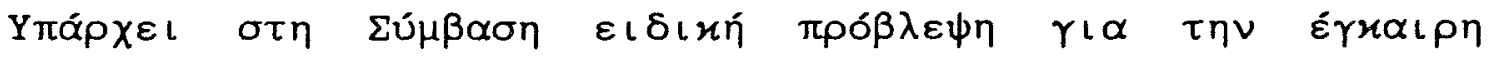

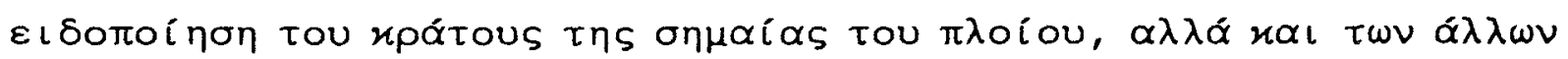

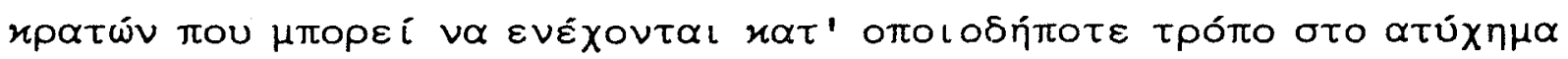

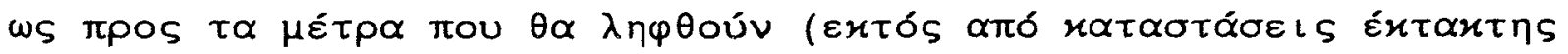
$\alpha \cup \alpha ̛ \gamma \varkappa \eta s)$.

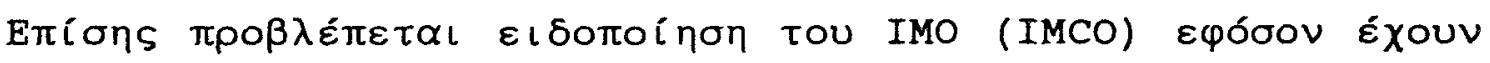

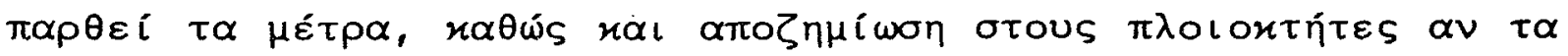

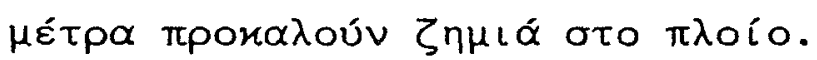

$\Sigma u \mu \pi \lambda \eta \dot{p} \omega \sigma \eta \eta$

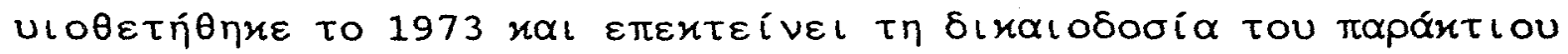

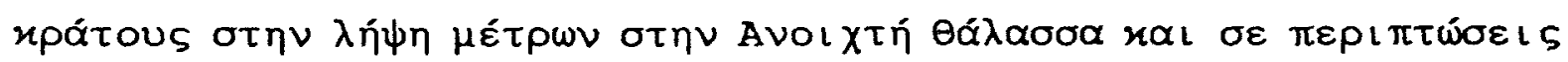




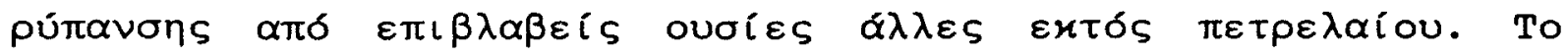

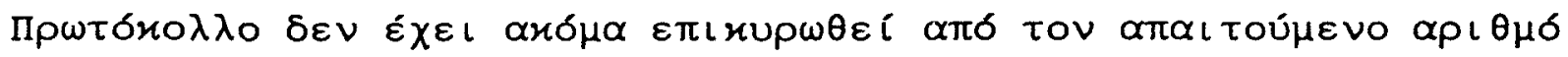

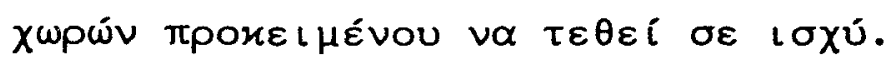

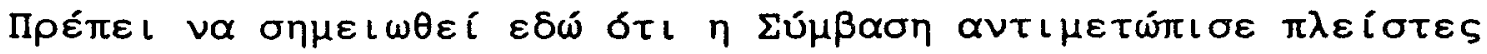

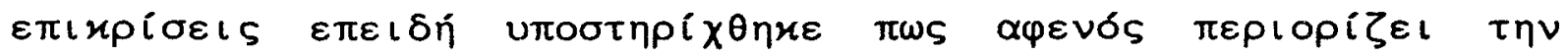

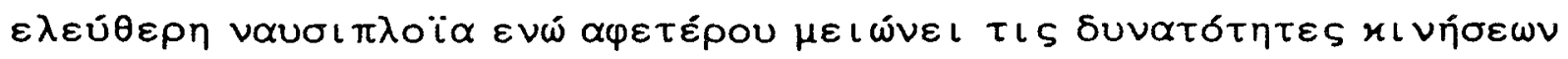

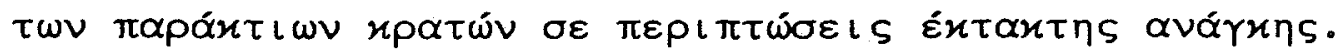




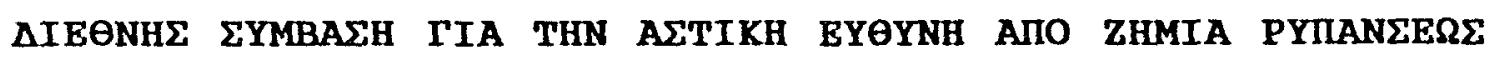
METPENAIOY 1969 í CLC

International convention on civil liability for oil Pollution Damage, 1969.

MPSTOKONAO TOY 1976 ETHN CLC (1976 Protocol to the CLC)

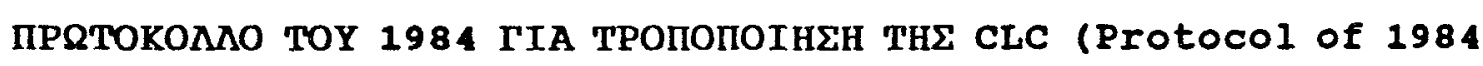
to Amend the (LC).

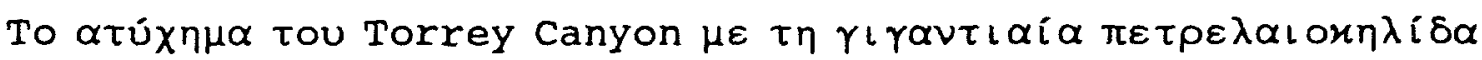

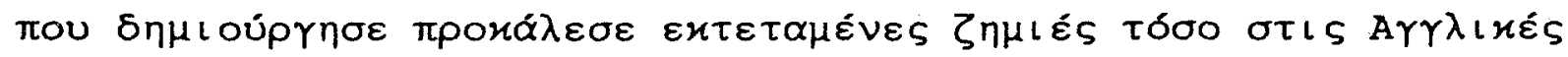

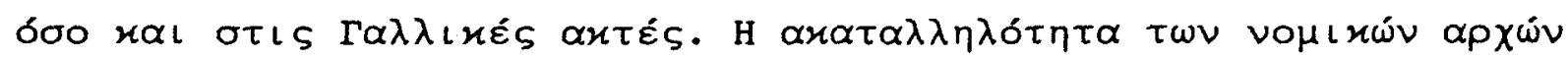

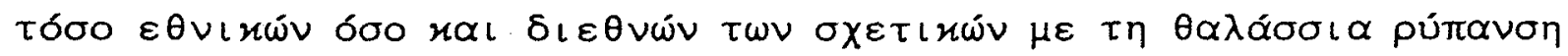

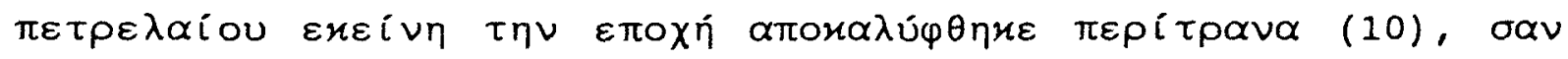

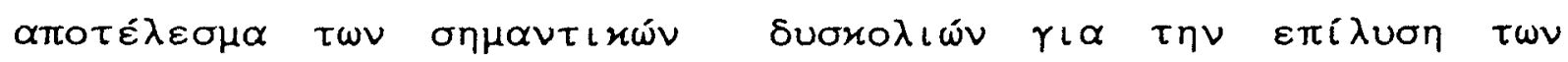

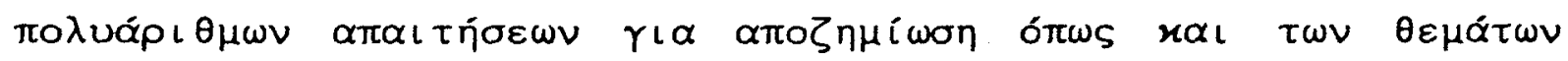

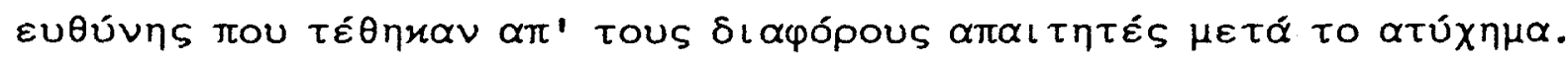

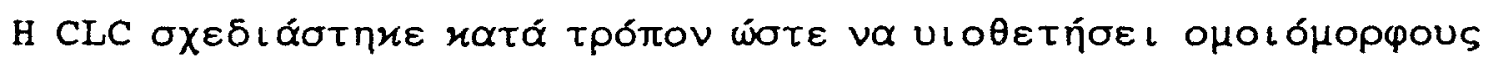

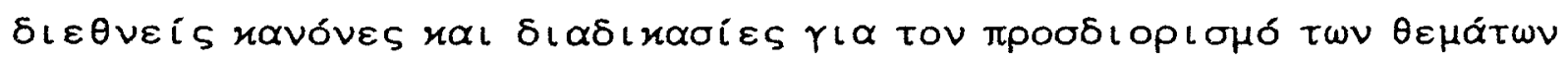

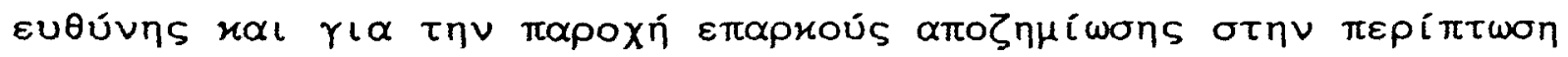

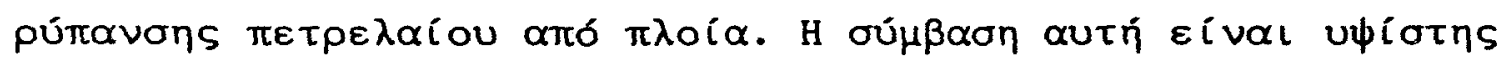




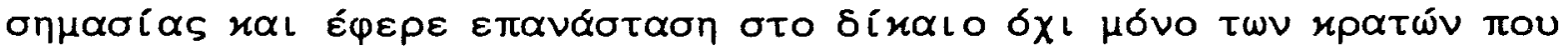

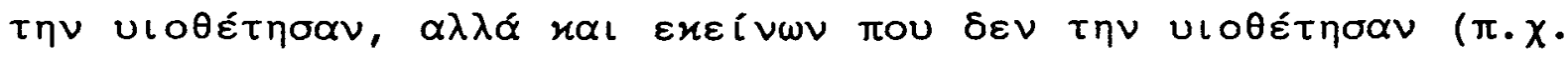

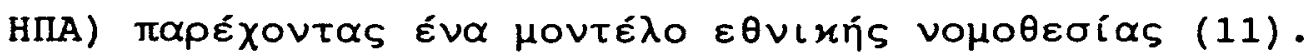

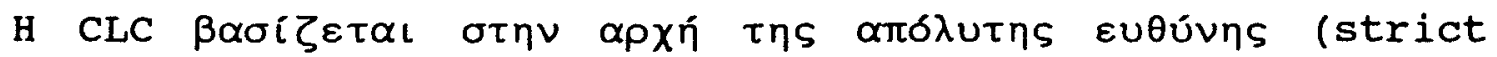

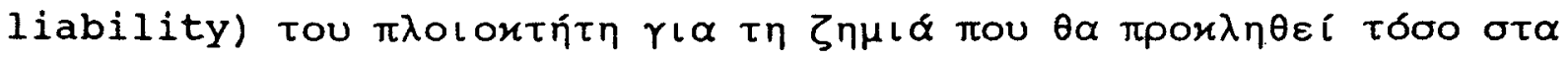

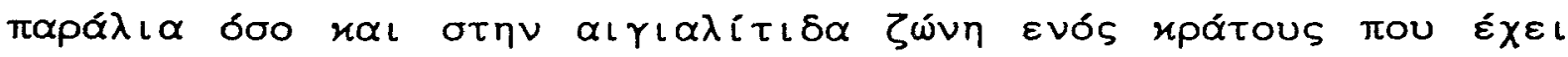

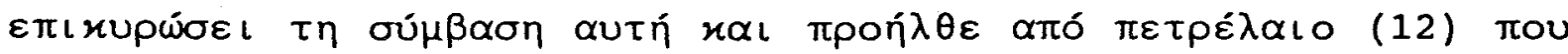

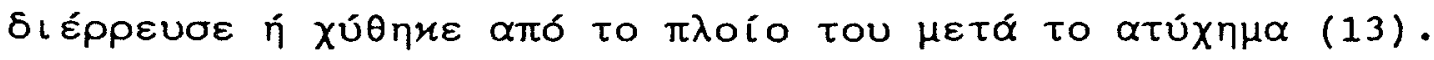

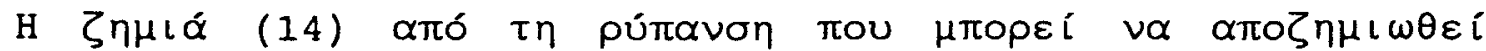

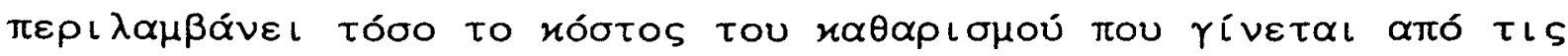

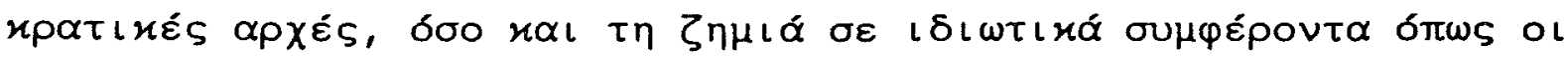

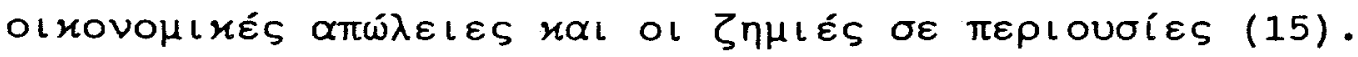

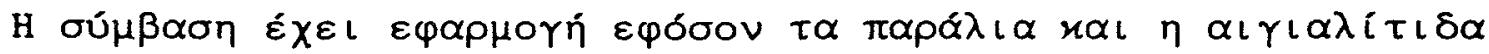

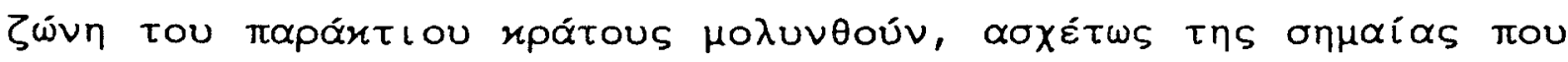

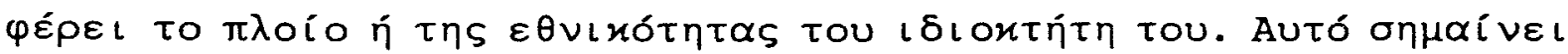

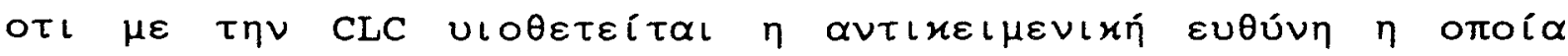

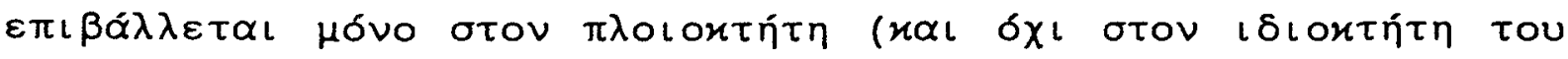

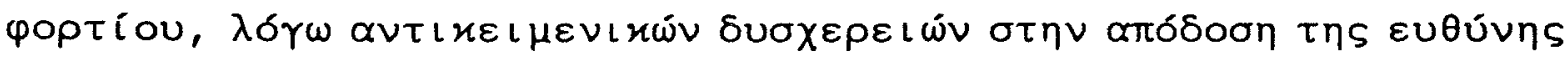

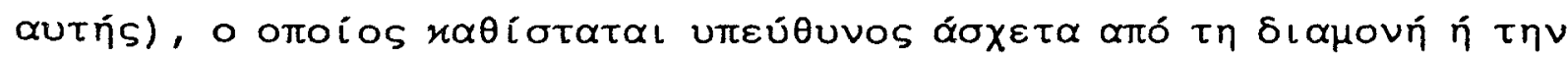

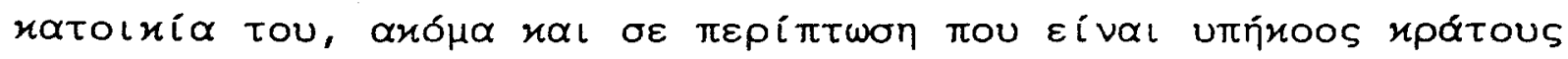

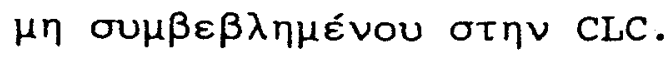




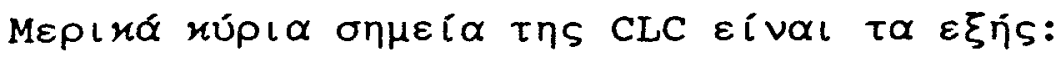

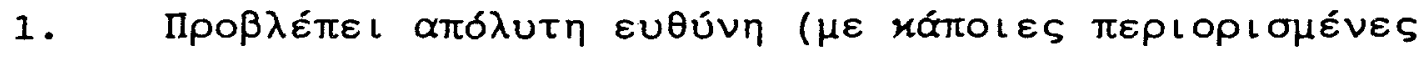

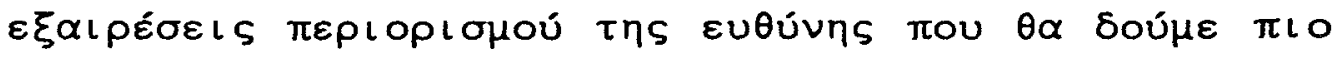
$\varkappa \alpha ̛(\omega)$.

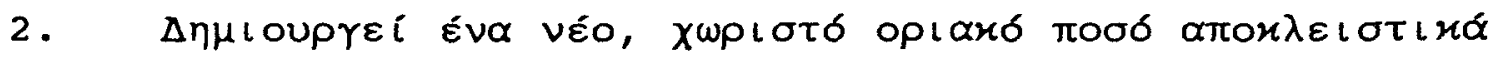

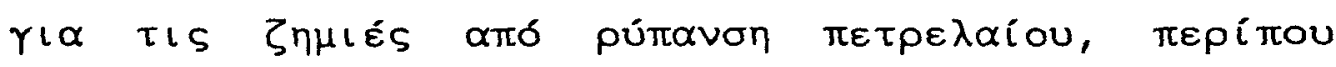

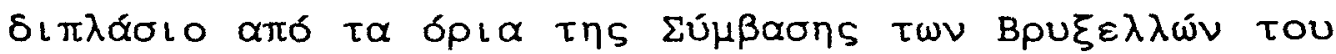
1957.

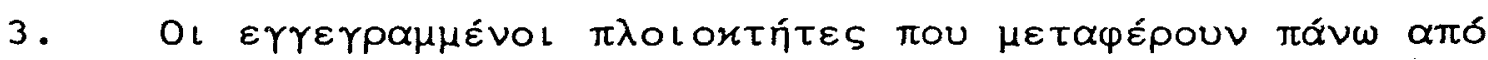

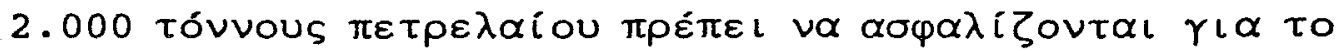

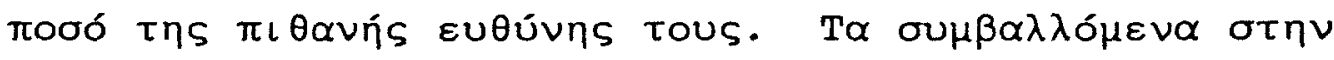

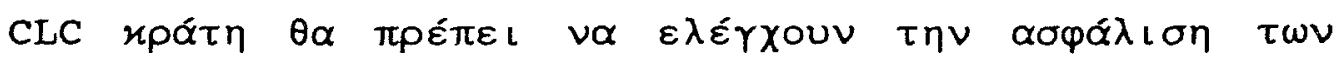

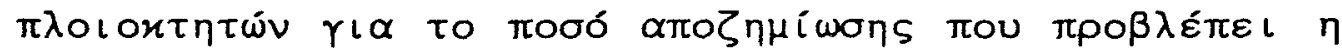

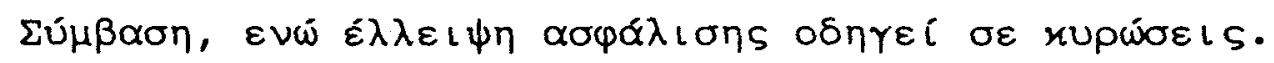

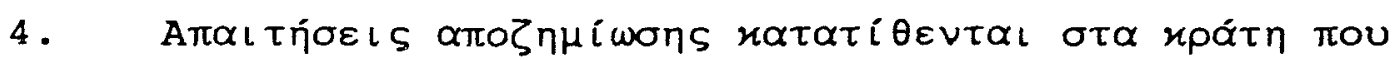

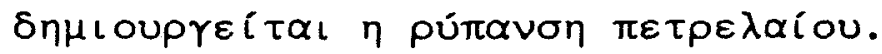

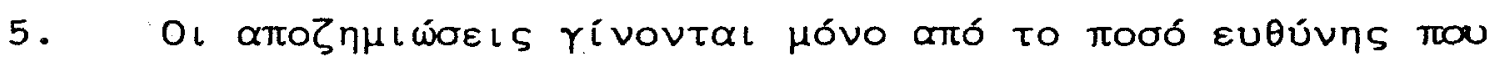

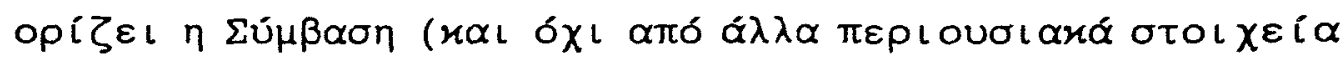

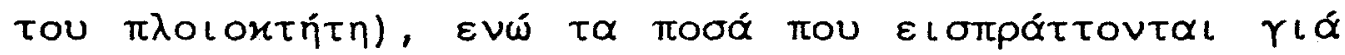

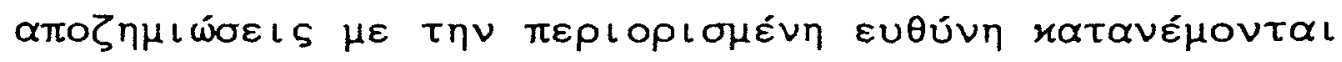

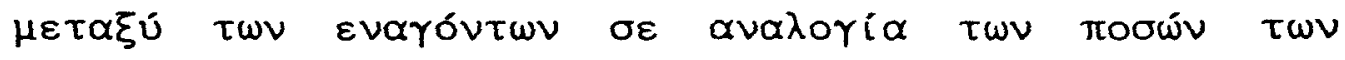

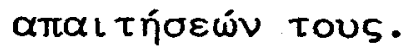




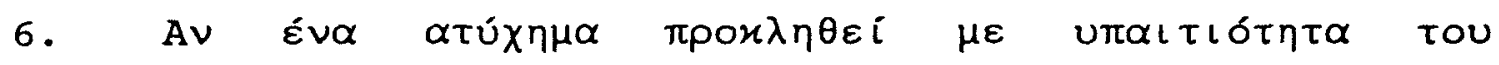

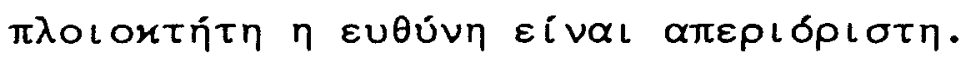

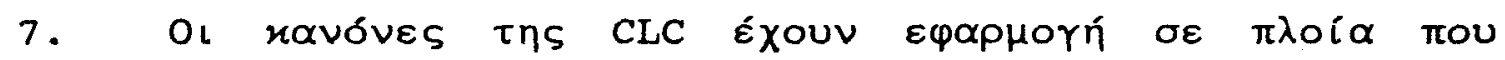

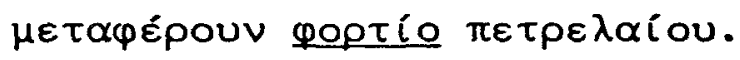

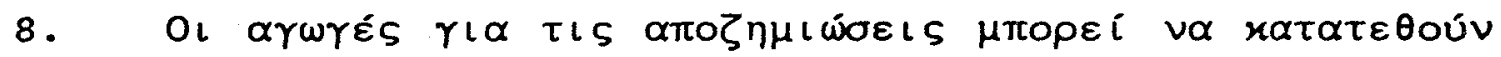

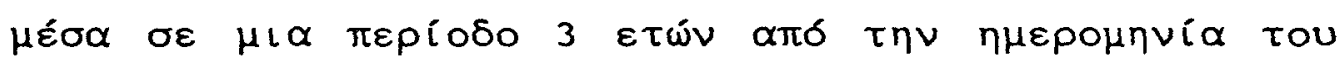

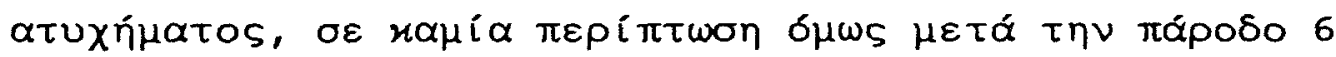

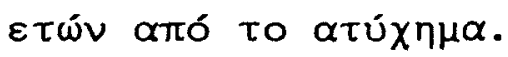

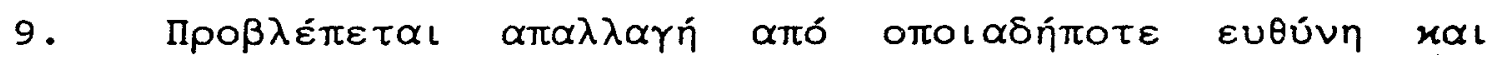

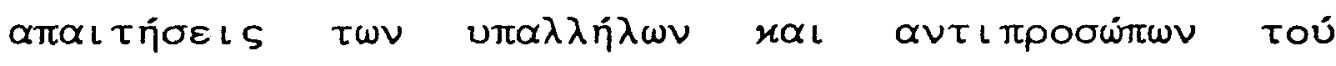

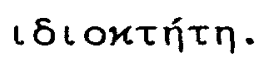

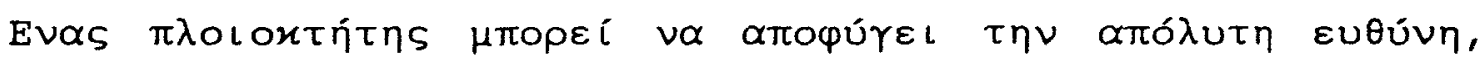

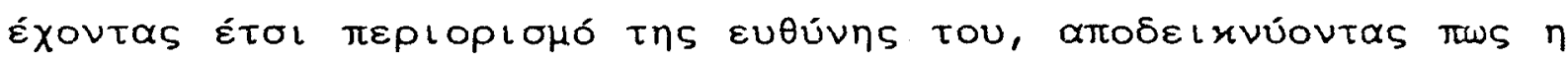

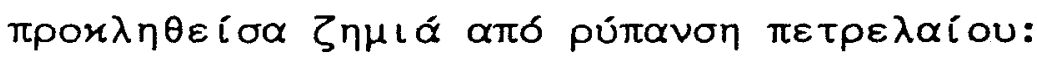

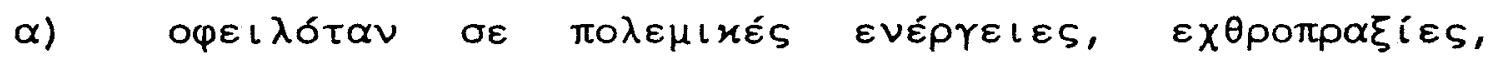

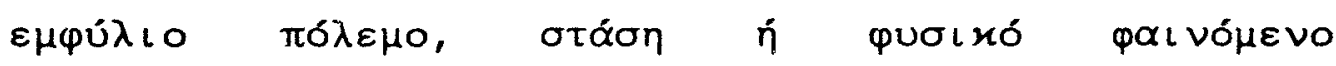

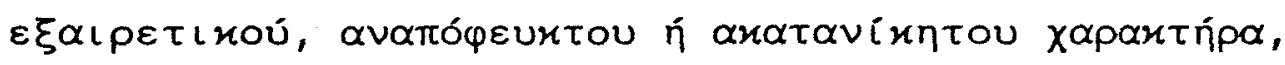

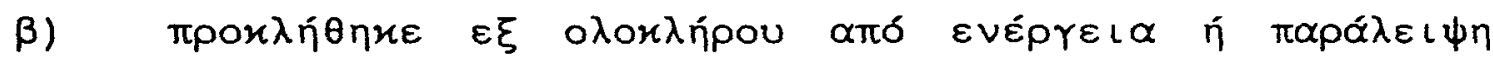

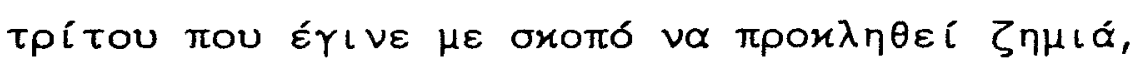

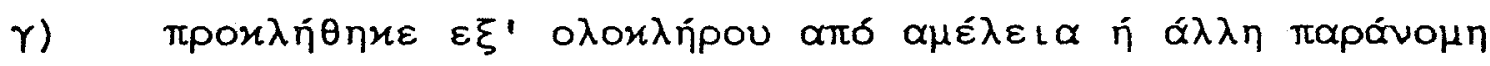

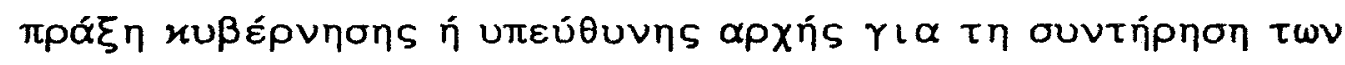

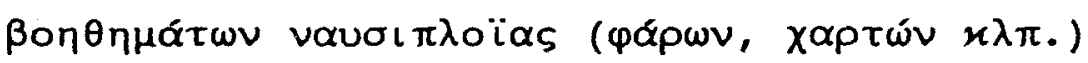




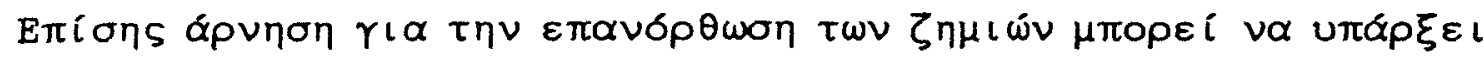
$\varepsilon \varphi \delta \sigma o \nu ~ \circ \pi \lambda \circ \iota$

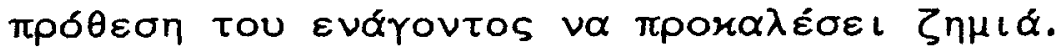

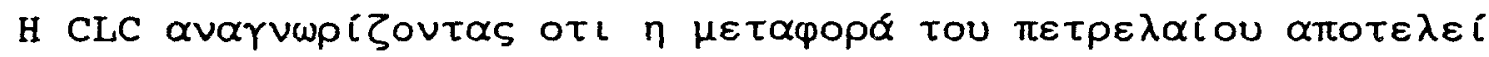

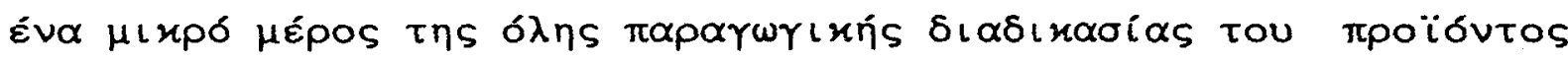

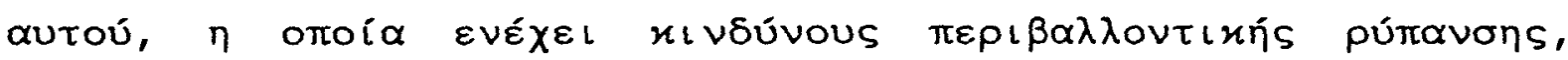

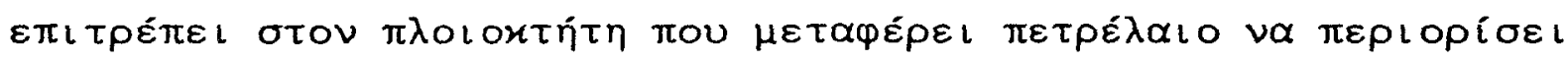

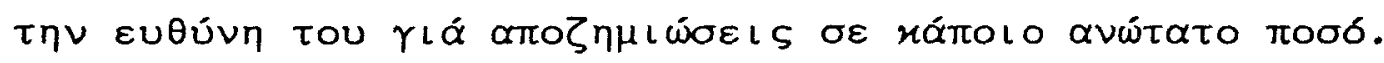

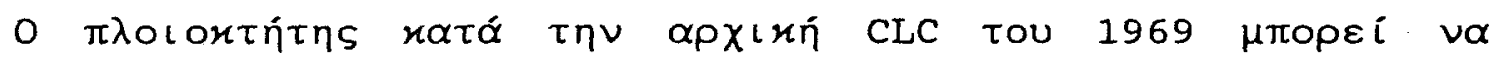

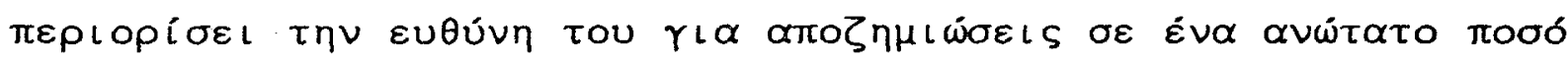

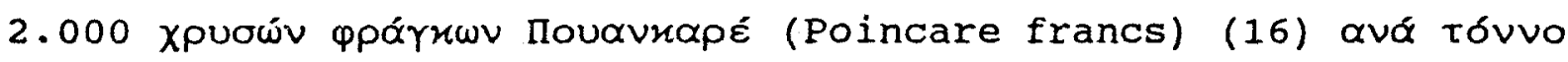

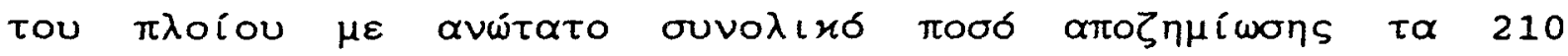

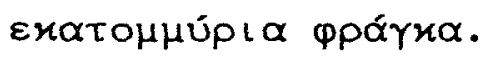

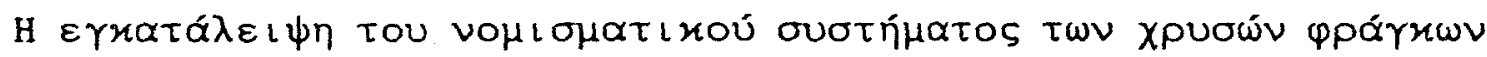

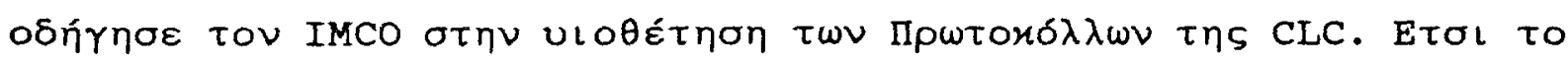

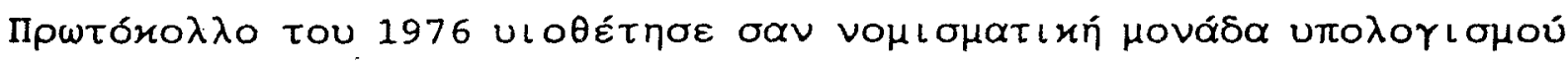

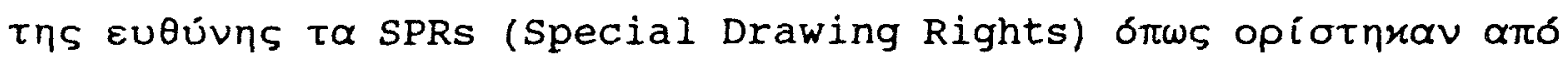

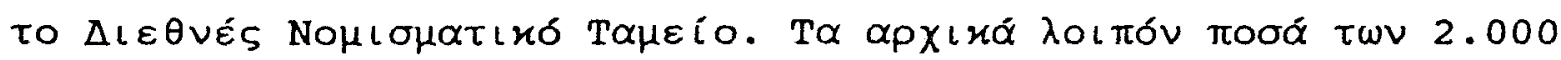

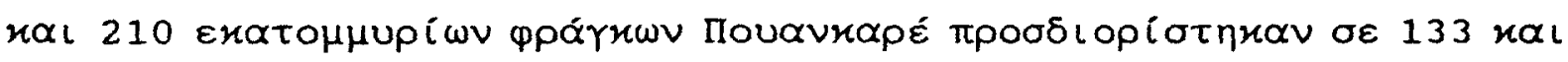

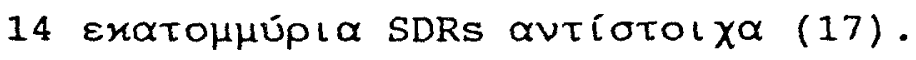

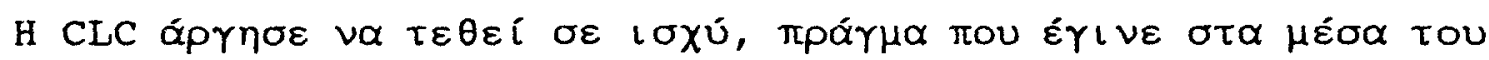
1975. 


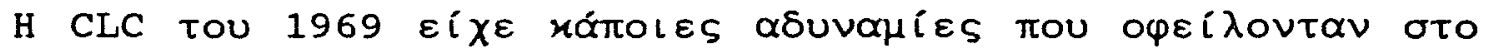

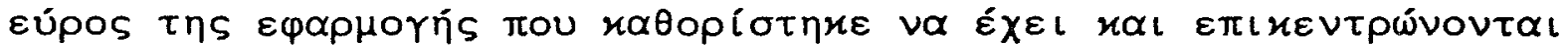

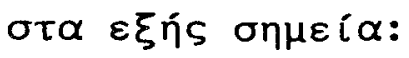

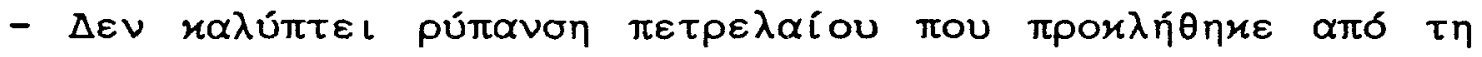

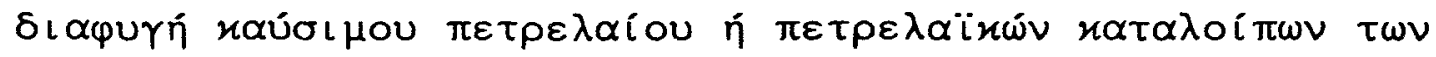

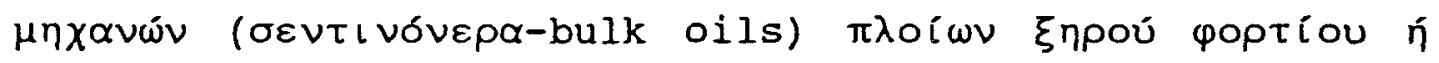

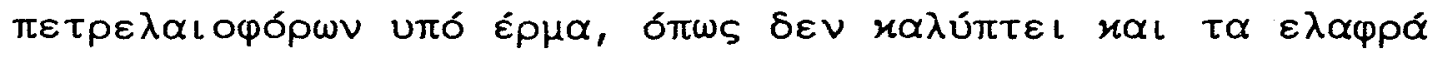
$\pi \varepsilon \tau \rho \varepsilon \lambda \alpha \ddot{\imath} \varkappa \alpha ́ \delta\llcorner\alpha \lambda u ́ \mu \alpha \tau \alpha$.

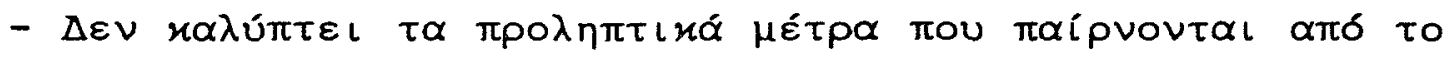

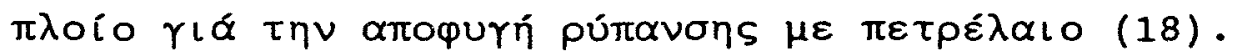

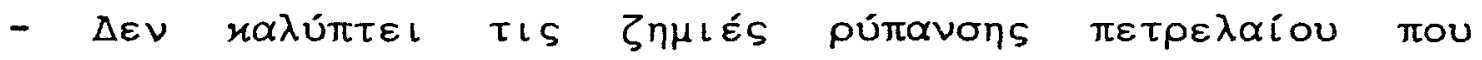

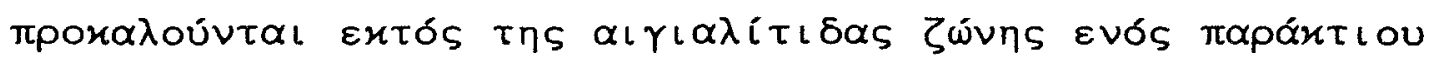

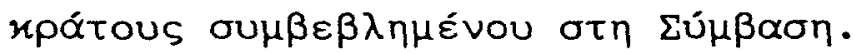

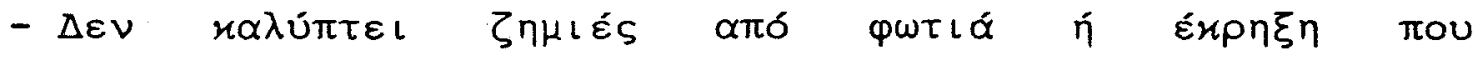

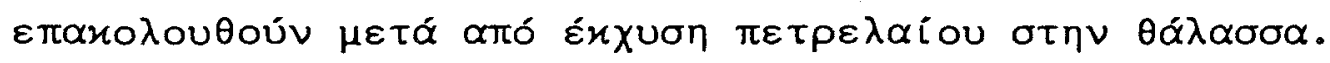

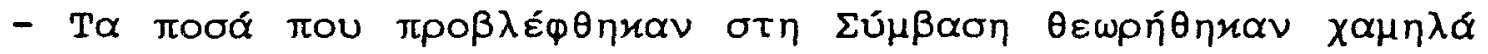

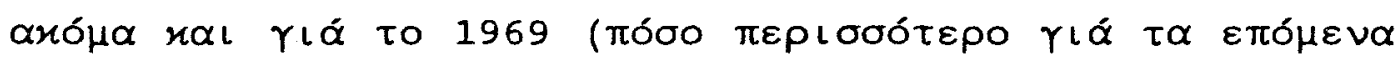
$\varepsilon ́(\eta)$

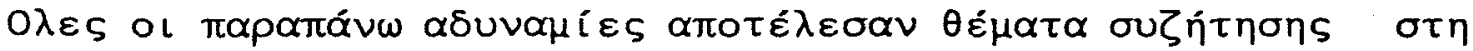

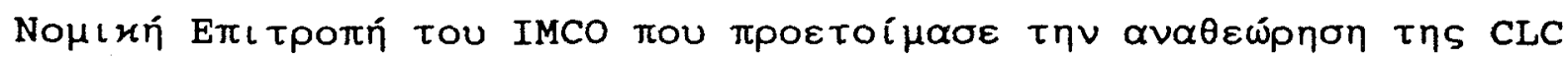

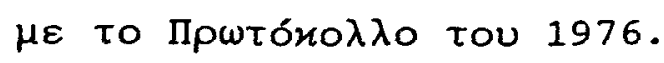




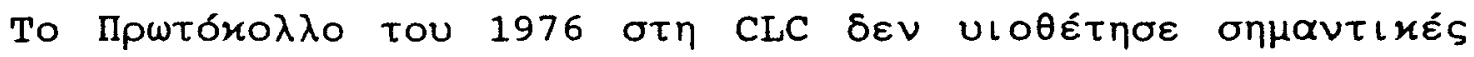

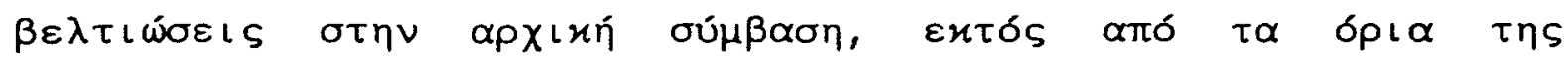

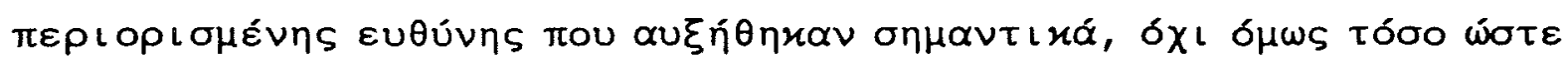

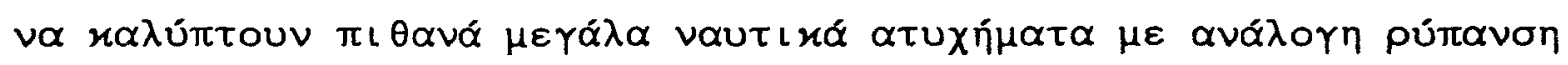

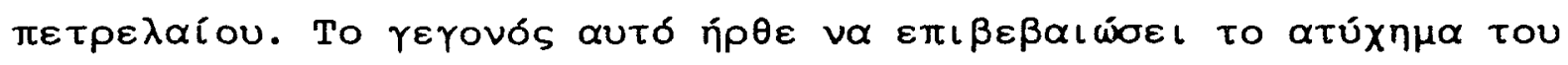

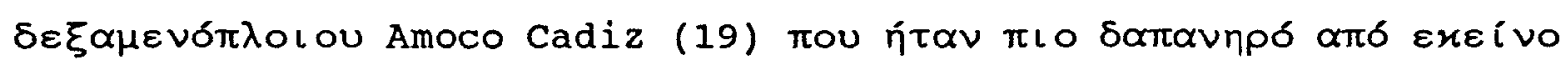

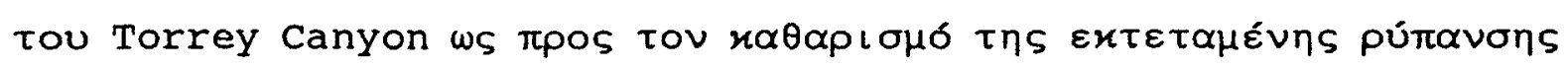

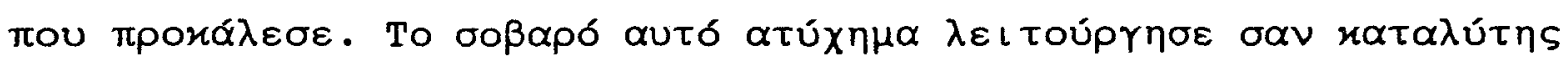

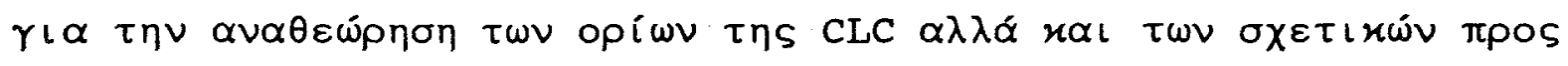

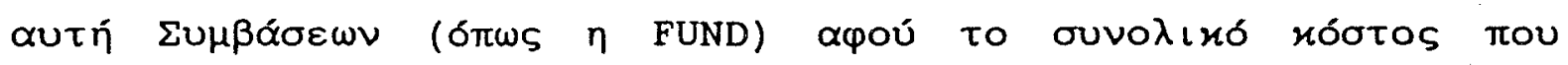

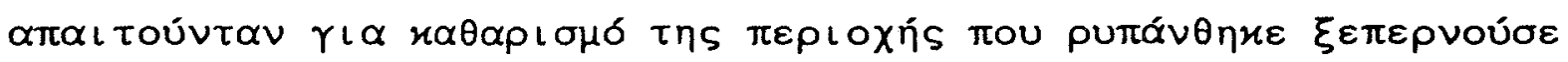

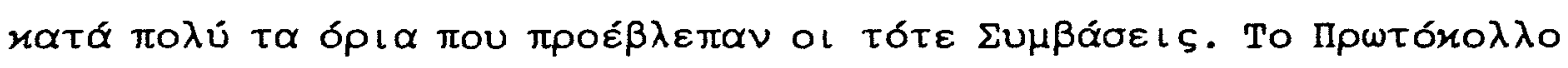

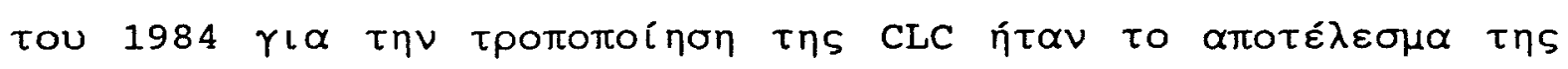

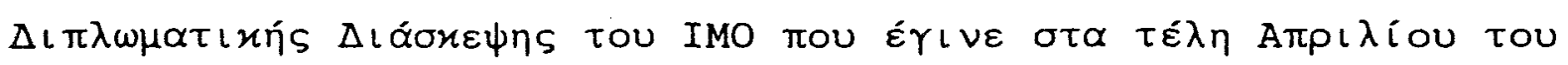

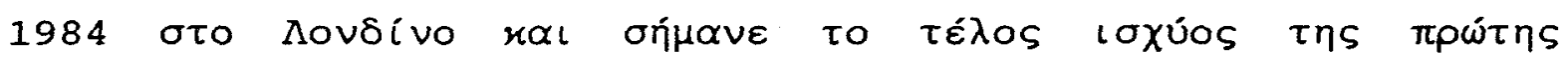

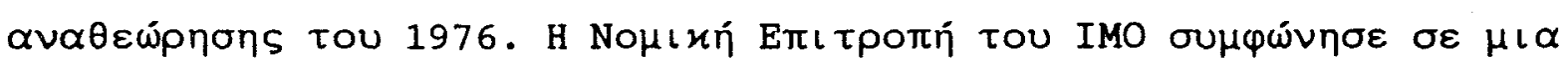

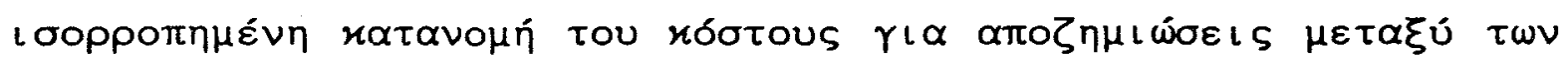

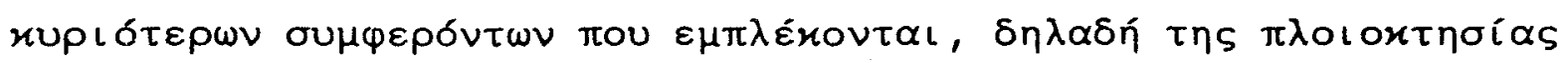

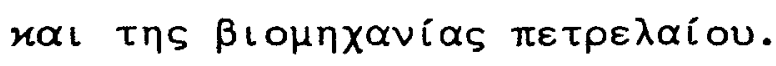

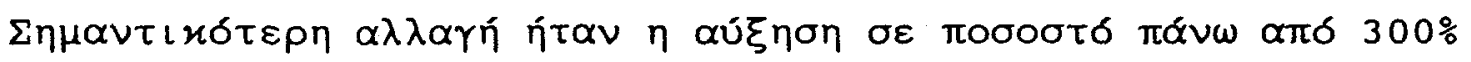

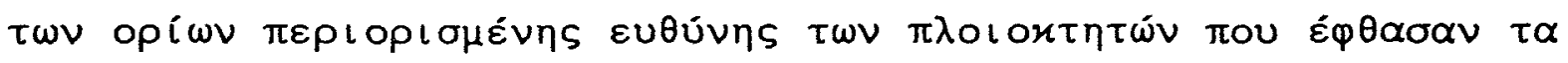

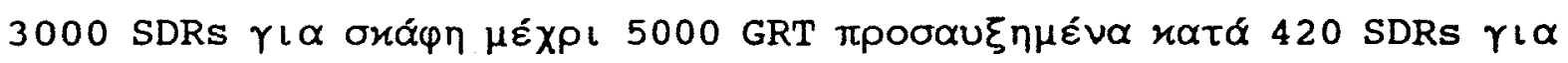




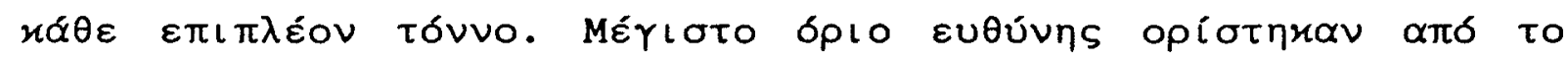

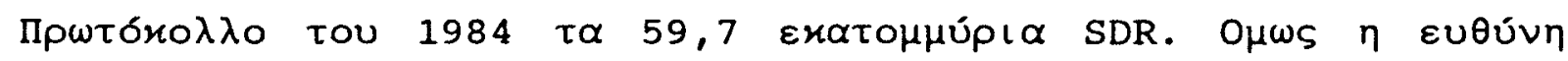

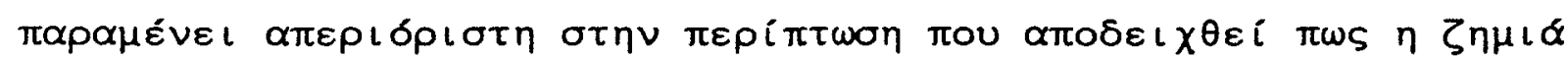

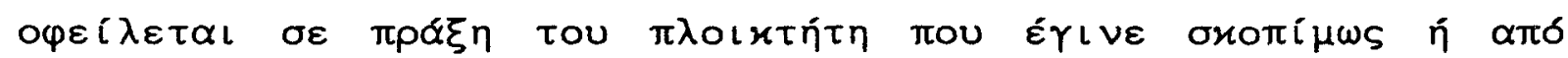
$\alpha \mu \varepsilon \lambda \varepsilon \varepsilon\llcorner\alpha$.

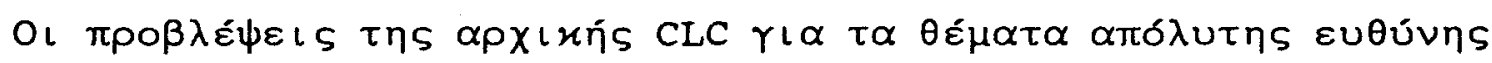

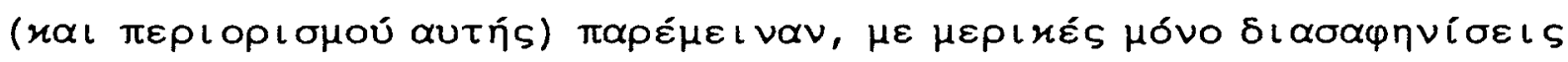

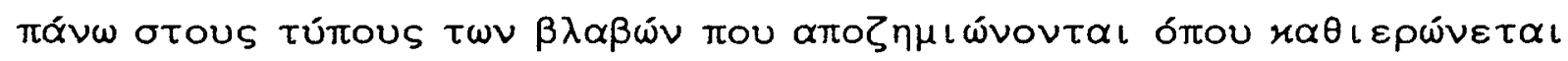

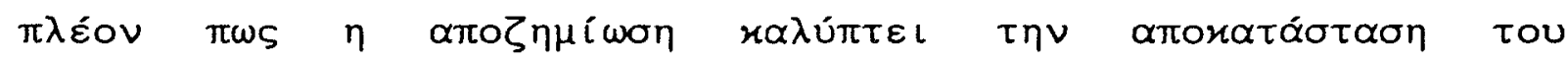

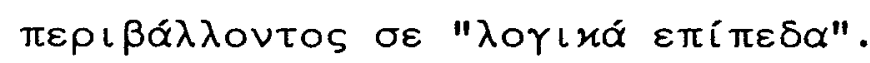

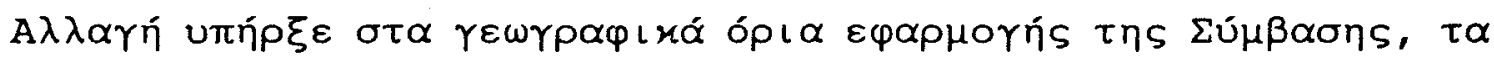

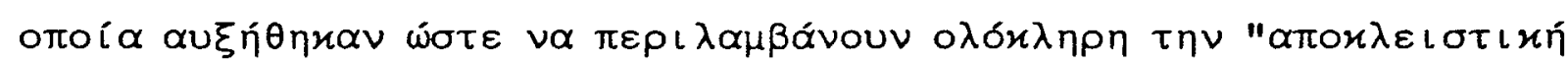

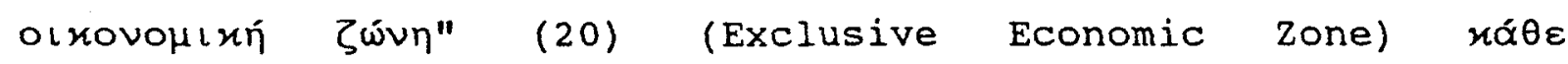

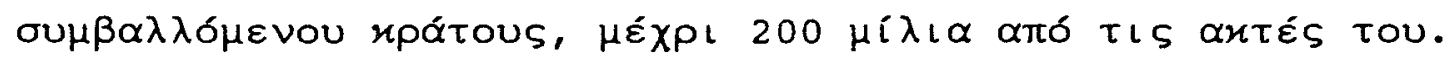

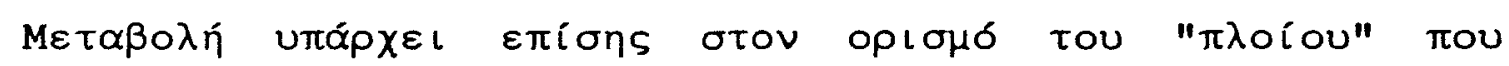

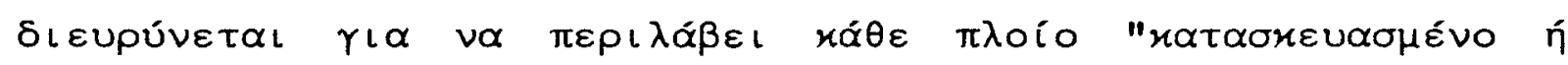

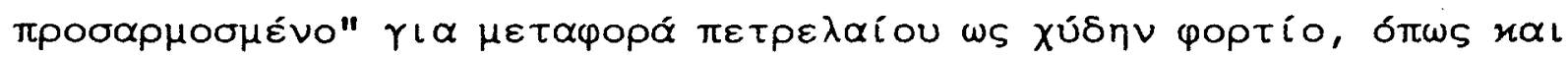

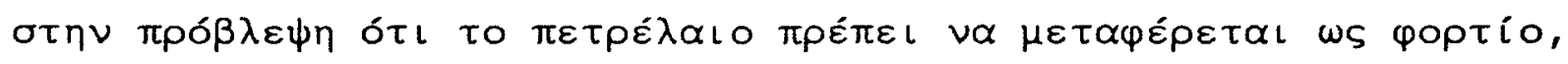

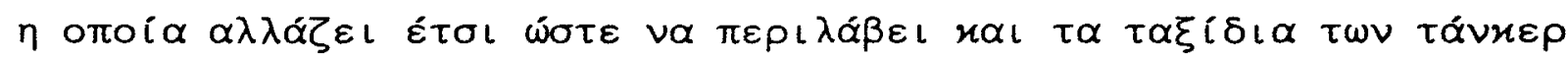

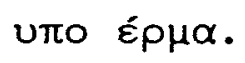

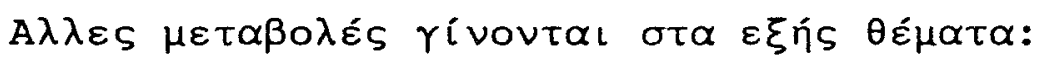




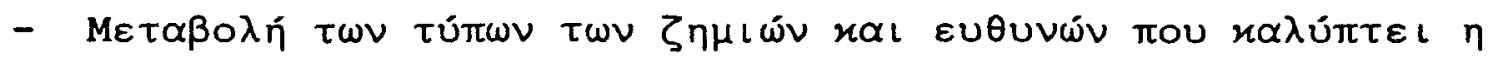
CLC:

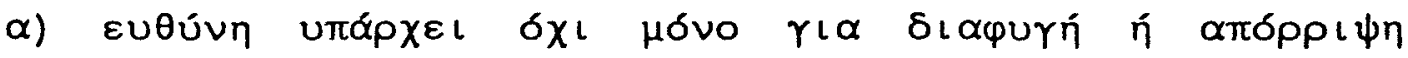

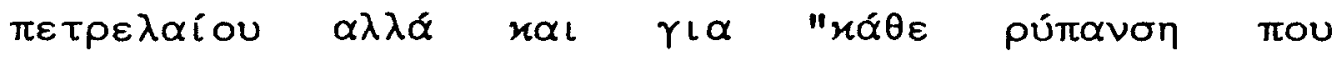

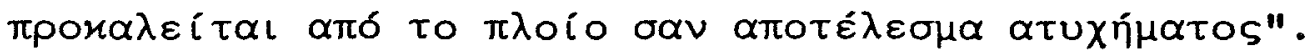

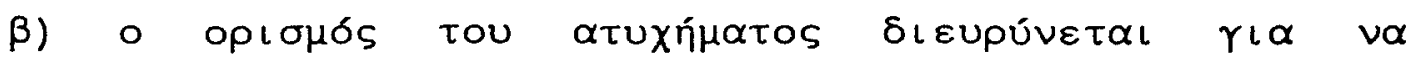

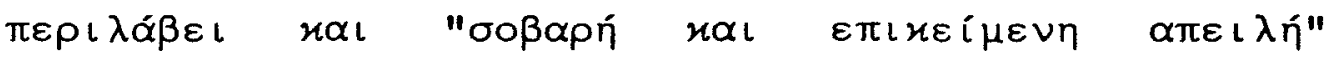

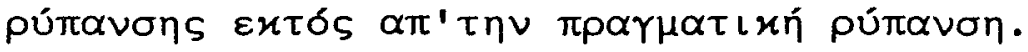

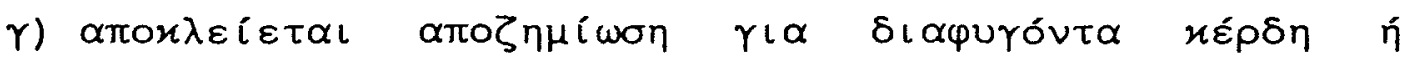

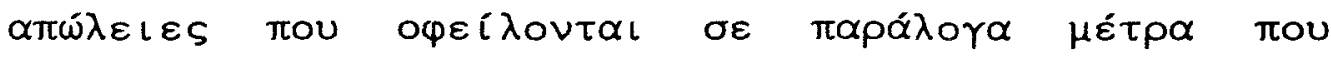

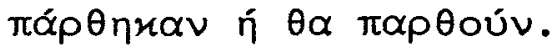

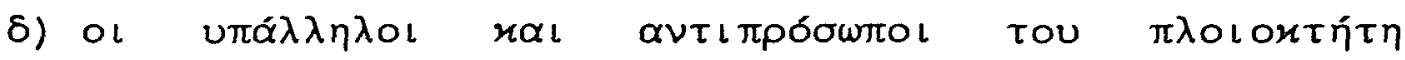

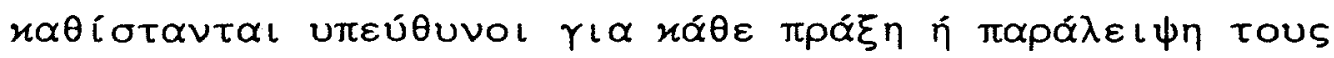

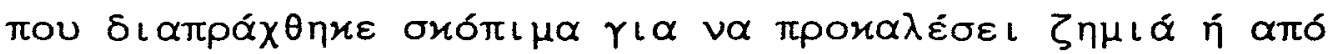

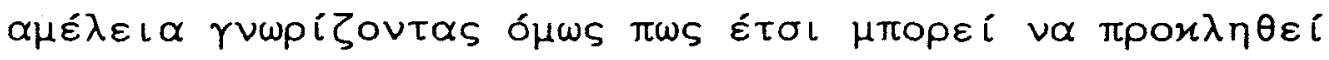
$\zeta \eta \mu\llcorner\dot{\alpha}$.

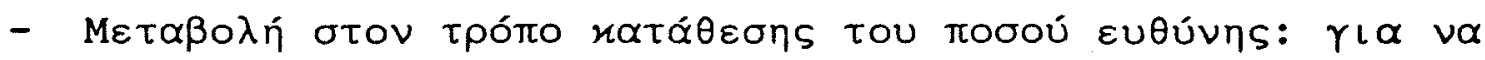

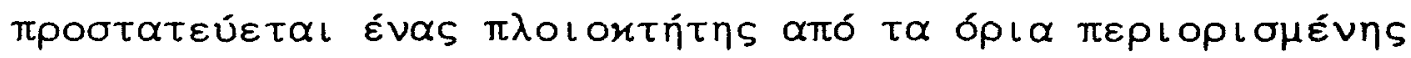

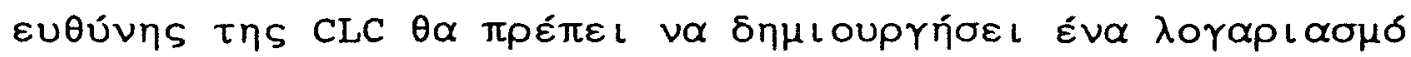

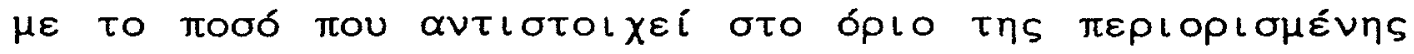

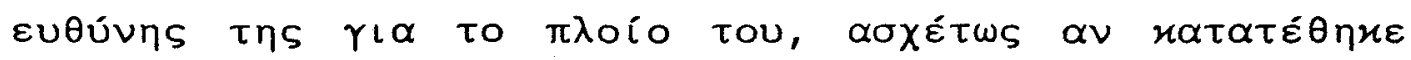

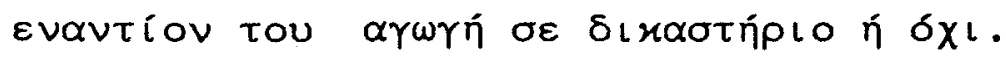




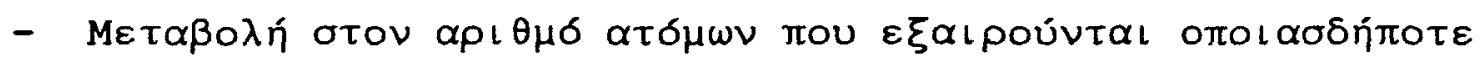

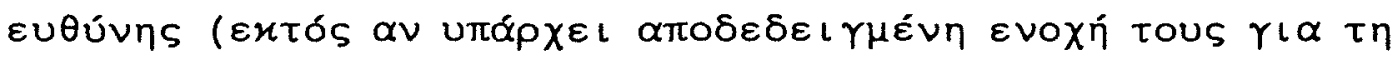

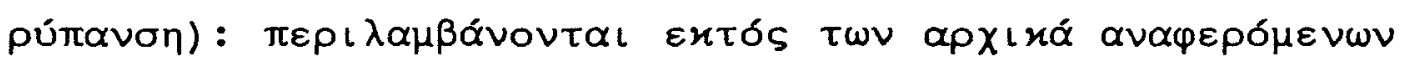

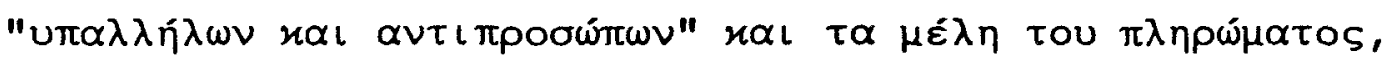

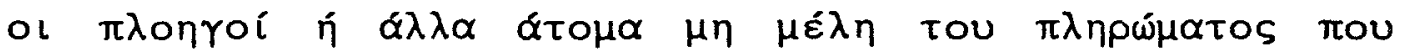

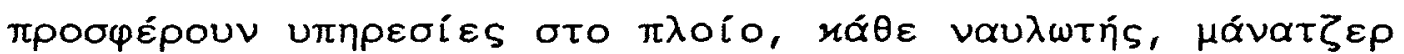

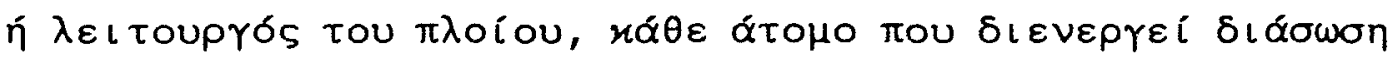

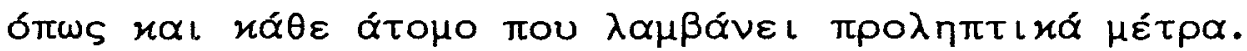

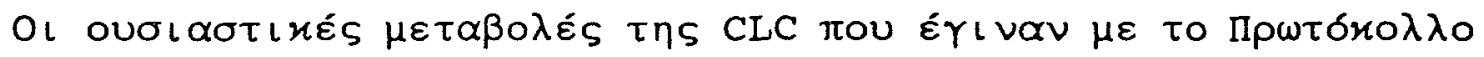

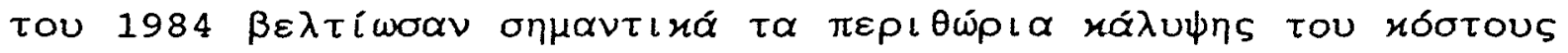

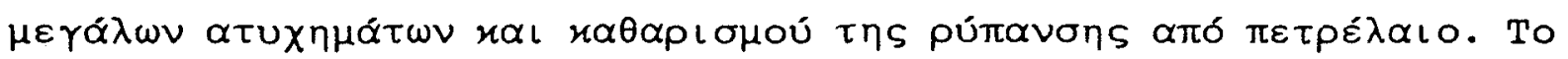

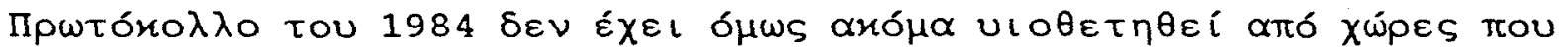

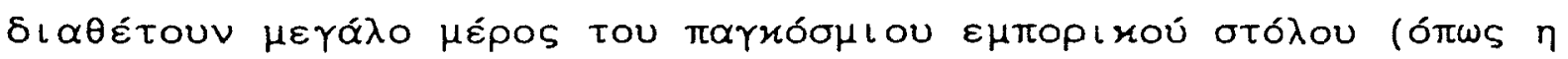

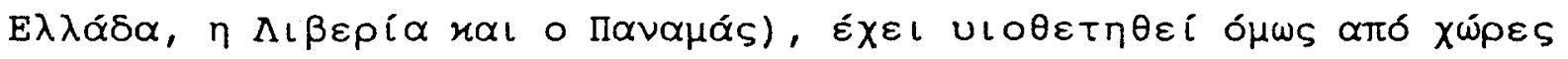

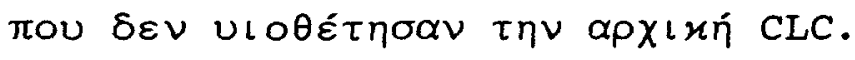




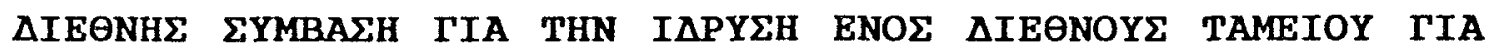

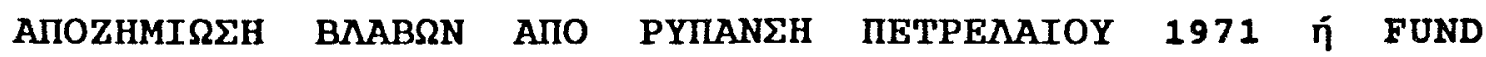
International convention on the Establishment of International Fund for compensation for oil pollution Damage, 1971 (Fund Convention).

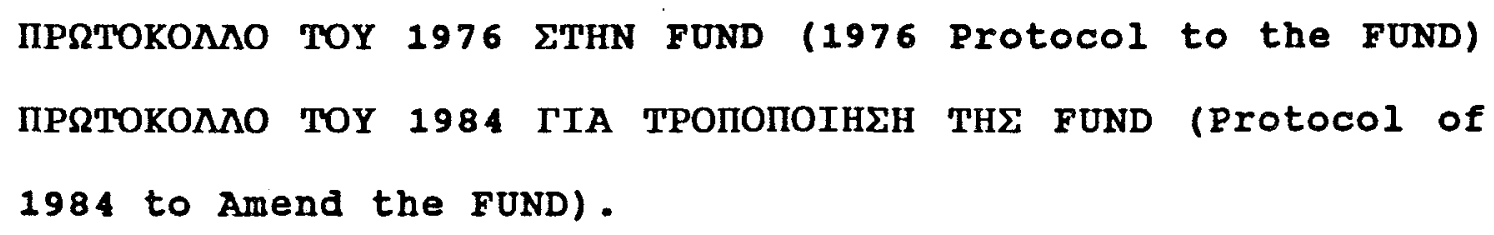

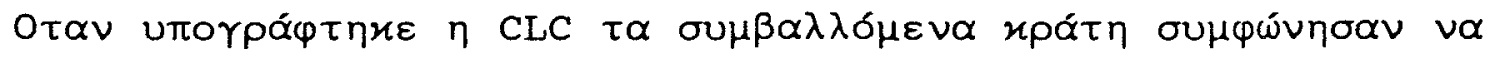

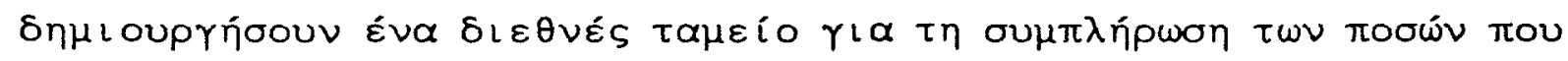

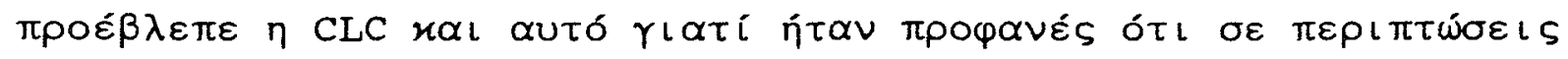

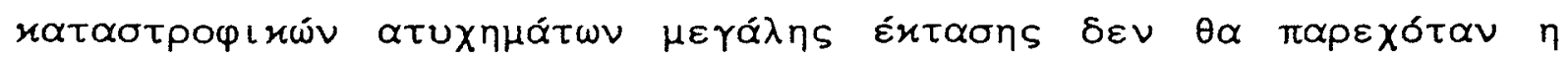

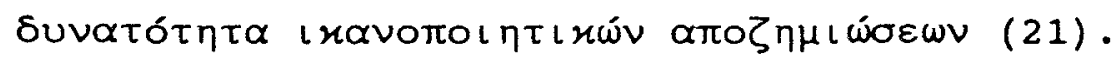

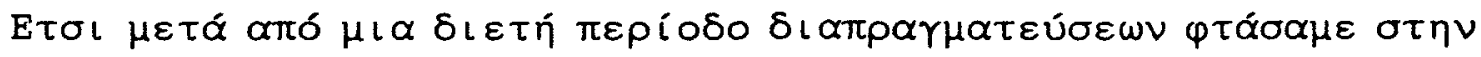

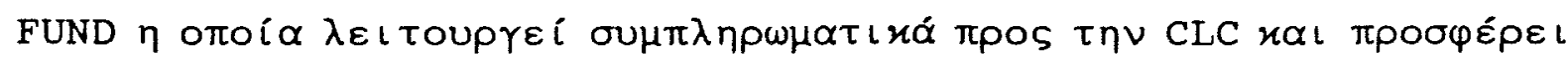

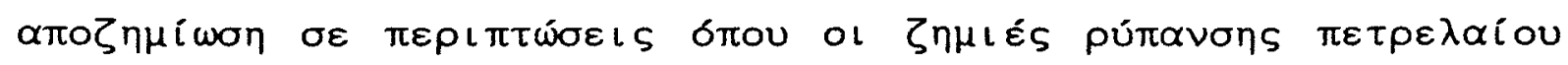

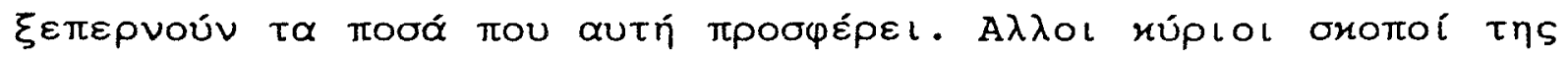

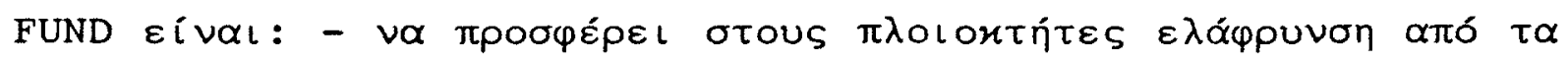

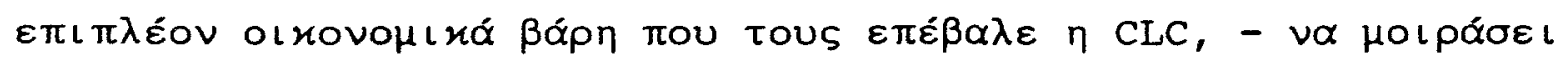




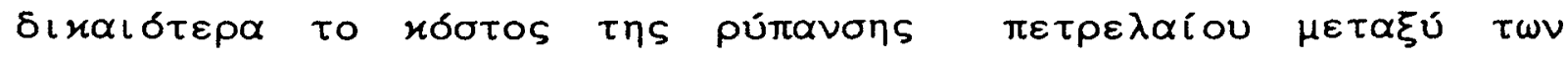

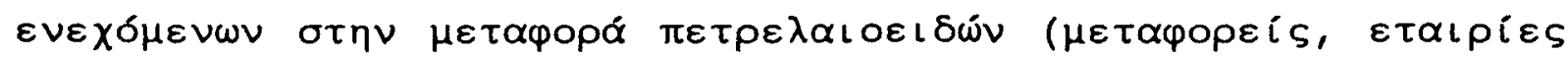
$\pi \varepsilon \tau \rho \varepsilon \lambda \alpha$ iou $\left.x_{.} \lambda . \pi.\right)$.

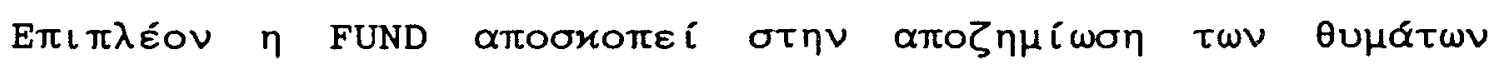

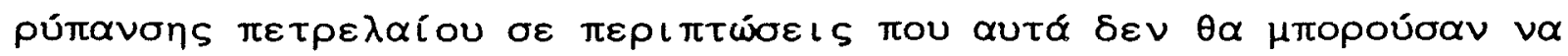

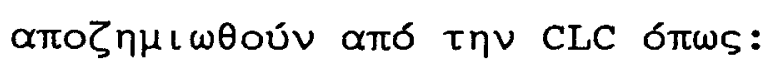

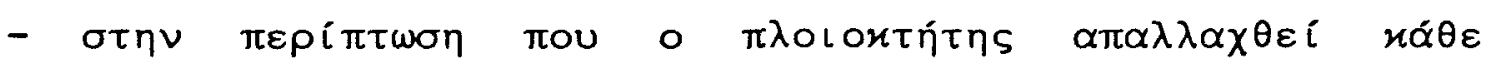

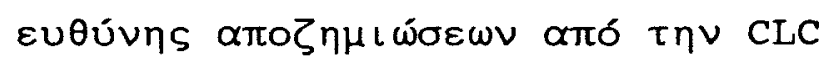

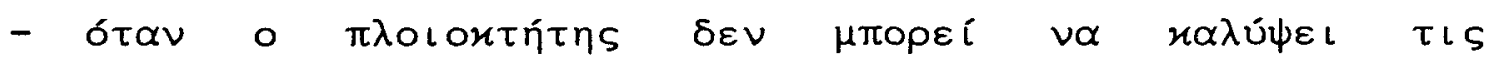

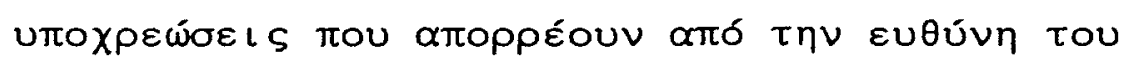

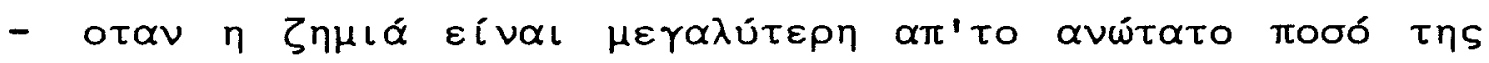

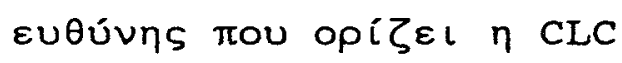

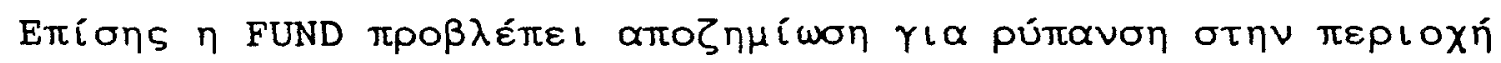

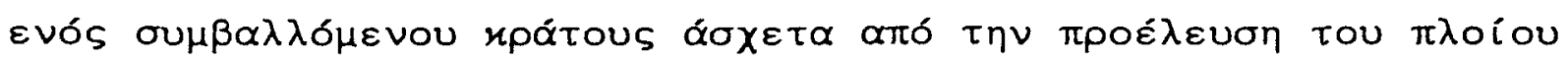

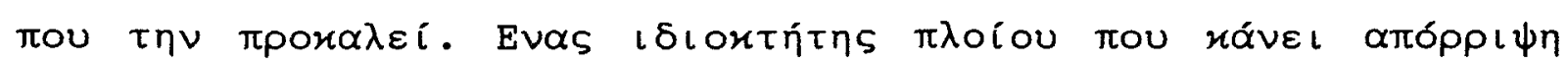

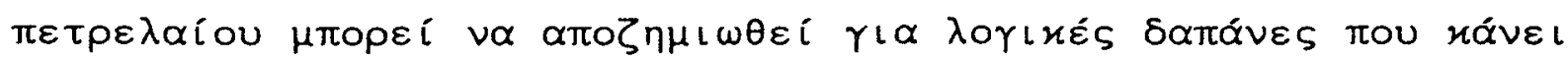

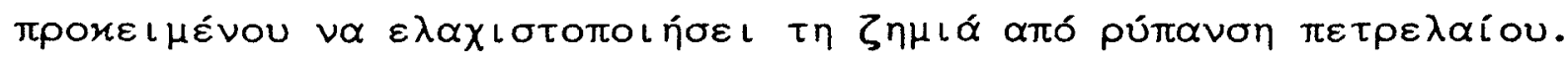

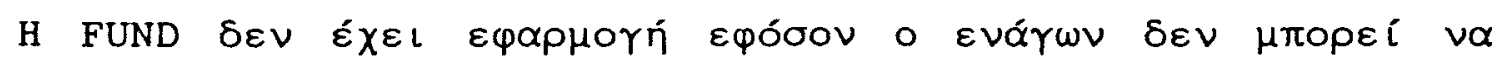

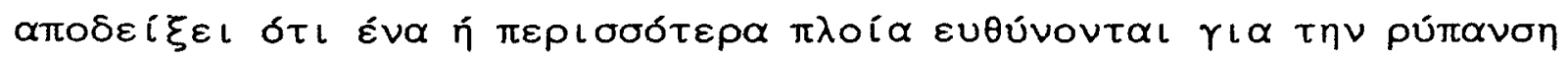

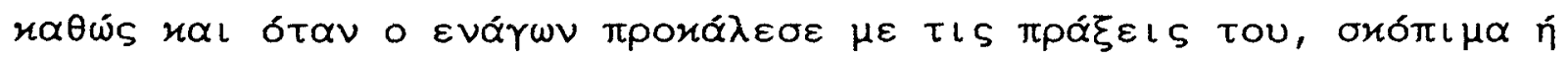

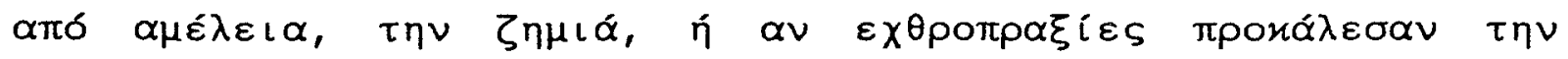
$\pi \varepsilon \tau \rho \varepsilon \lambda \alpha\llcorner$ o $\eta \lambda i \delta \alpha$. 


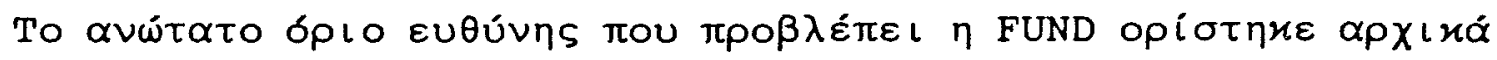

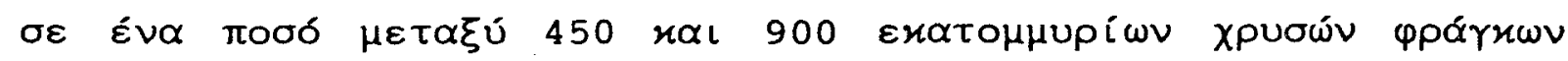

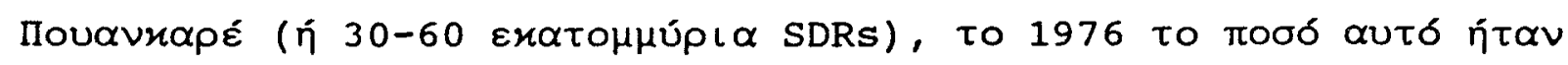

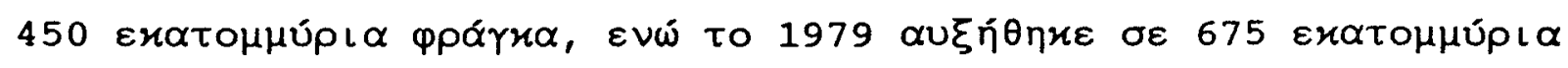

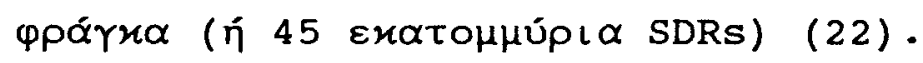

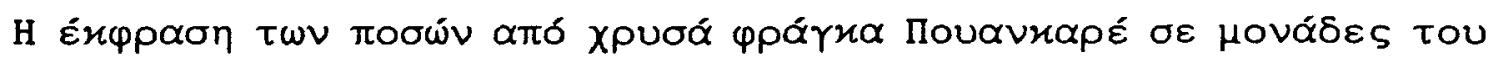

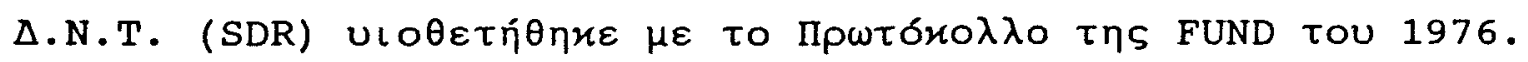

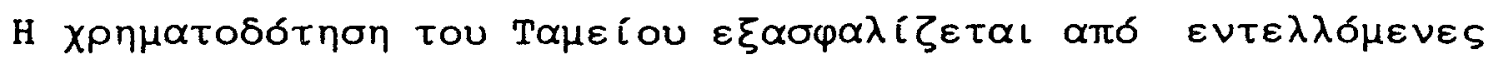

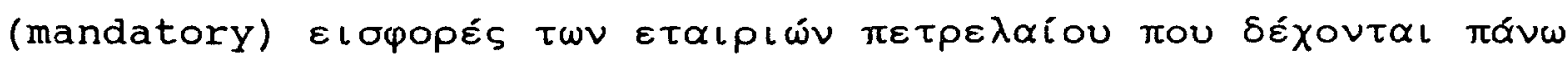

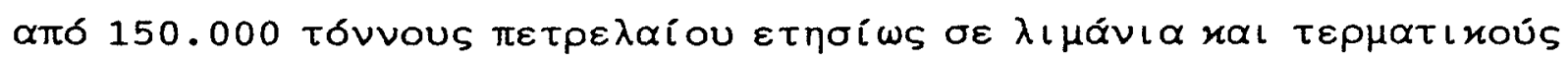

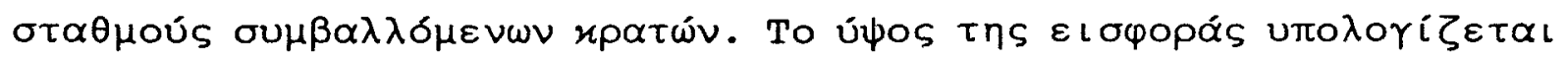

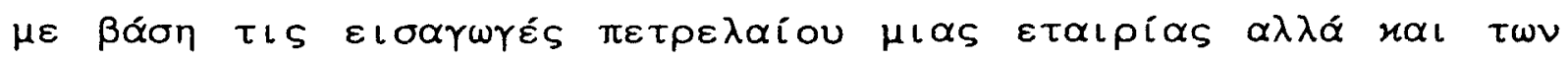

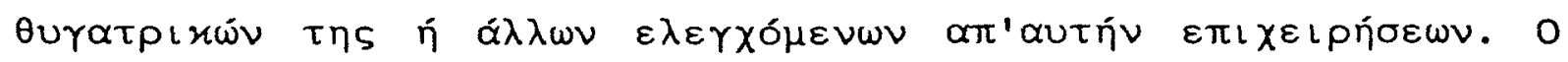

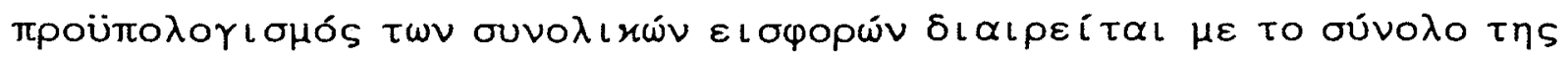

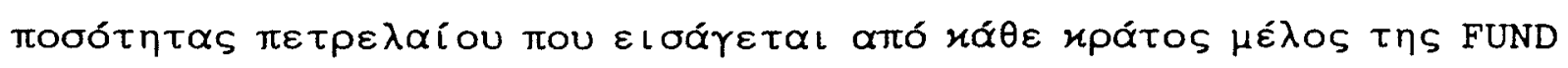

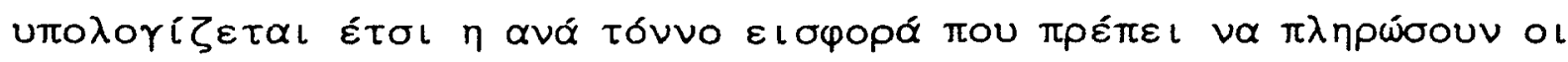

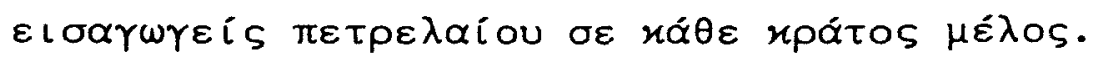

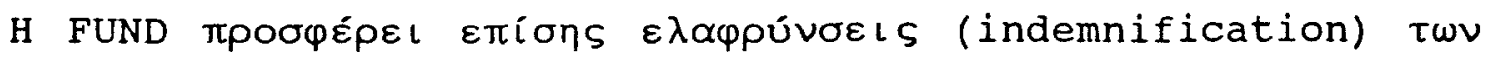

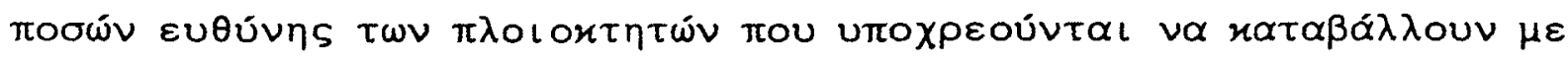

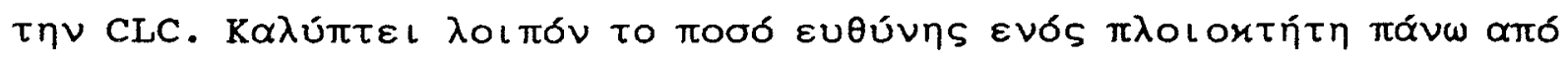

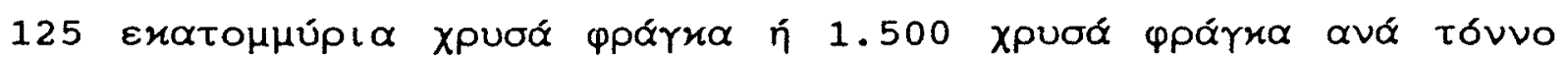

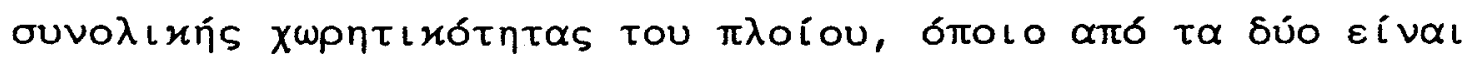




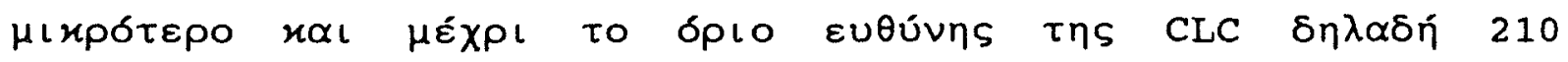

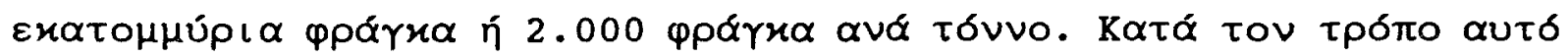

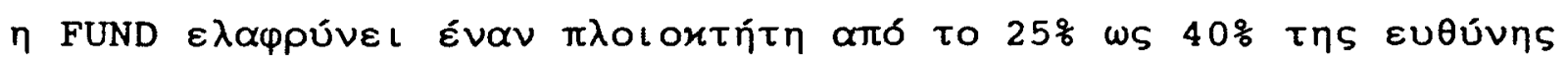

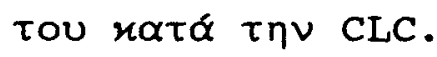

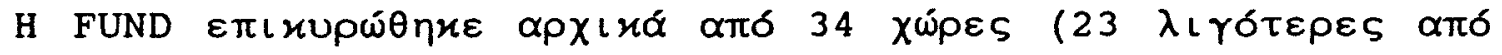

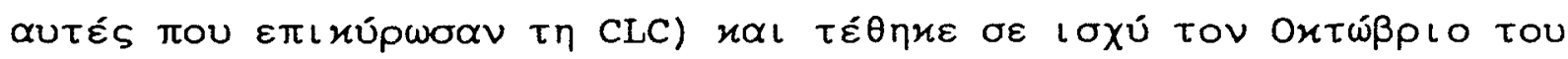

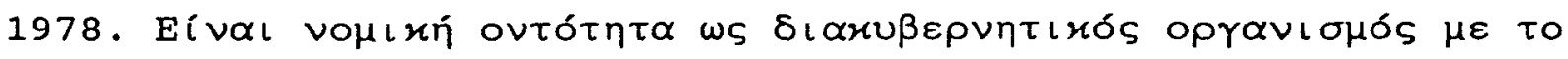

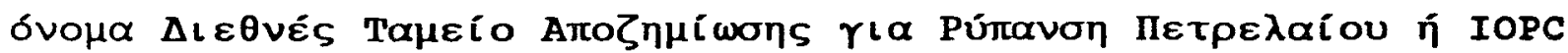
Fund (International oil pollution compensation Fund) $\mu \varepsilon \varepsilon \delta \rho \alpha$ to

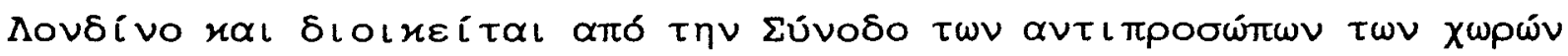

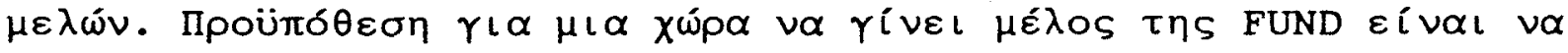

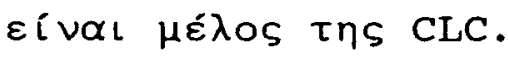

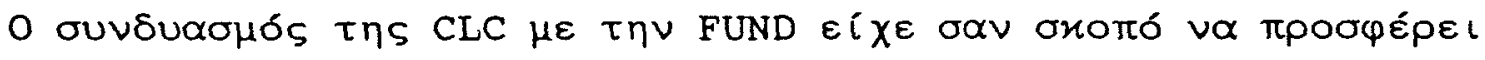

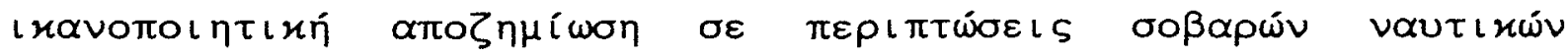

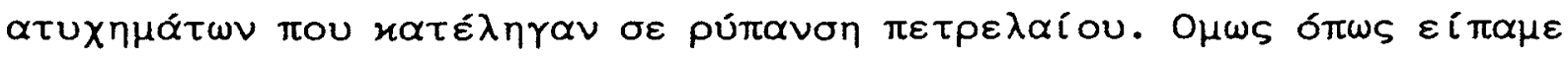

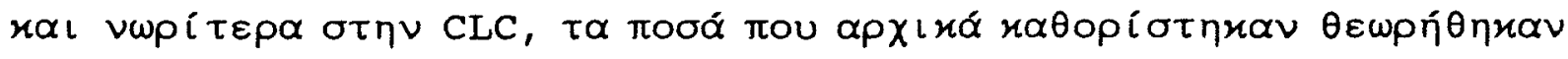

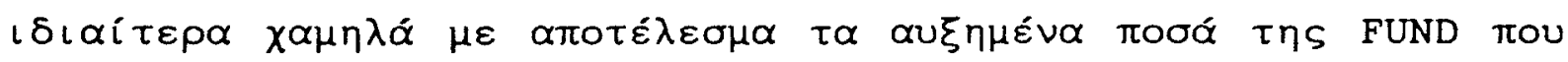

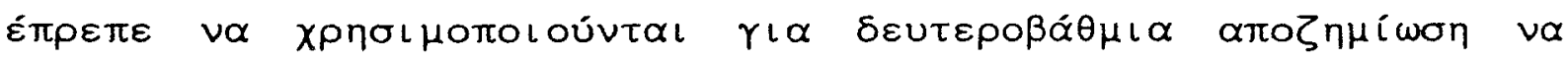

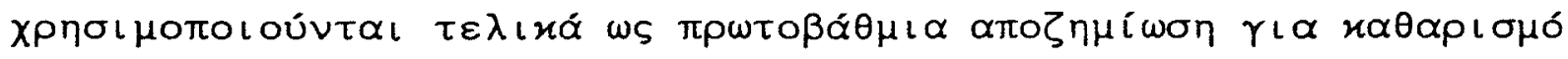

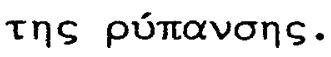

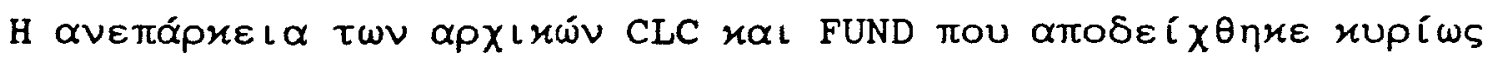

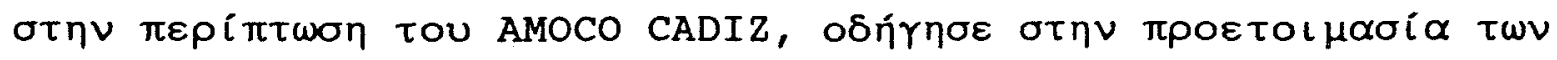




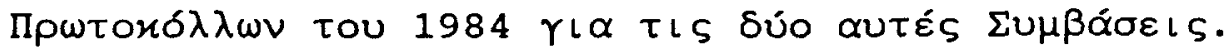

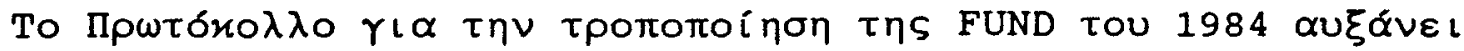

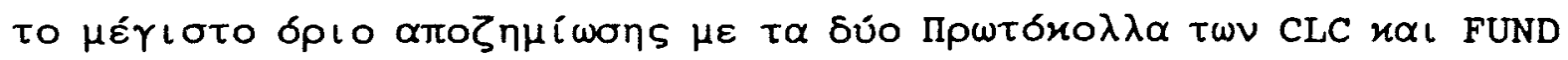

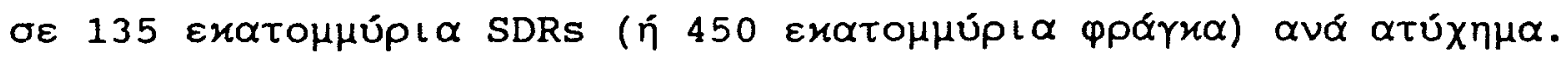

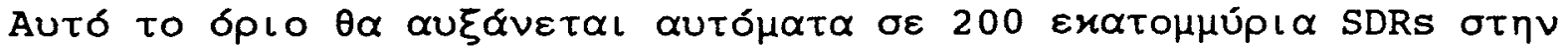

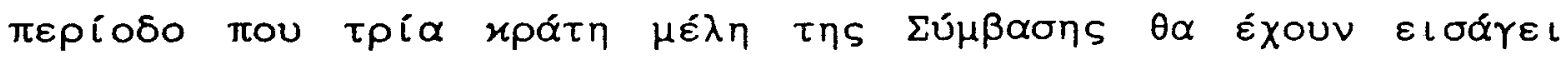

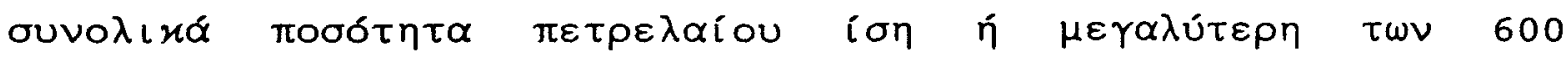

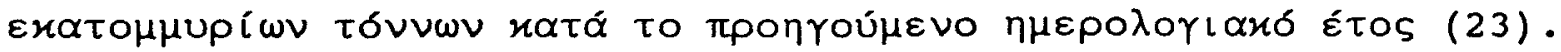

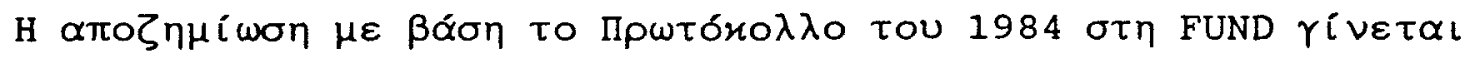

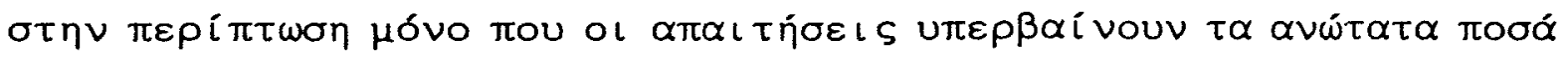

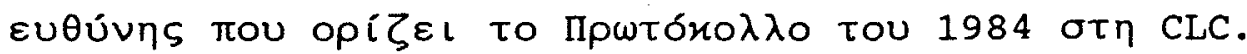

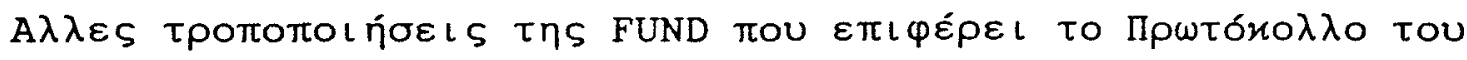
1984 Eival:

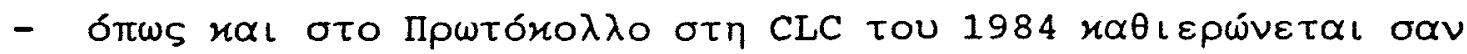

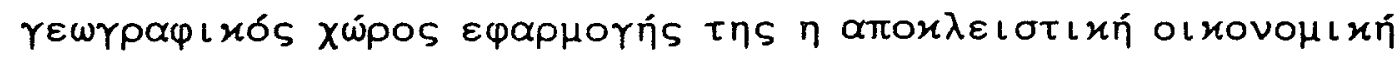

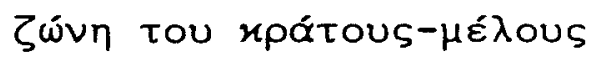

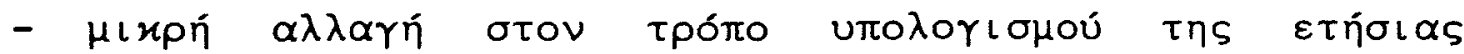

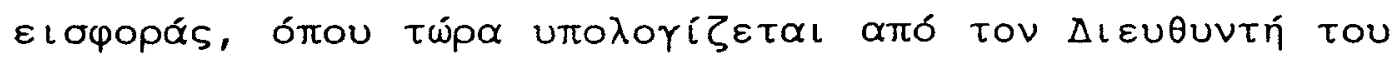

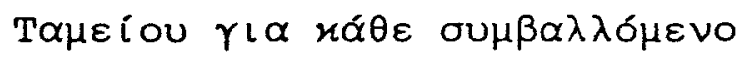

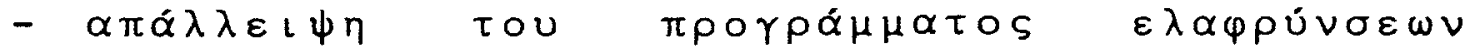
(indemnification) $\gamma \iota \alpha$ tous $\pi \lambda$ oLox

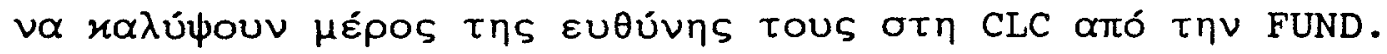




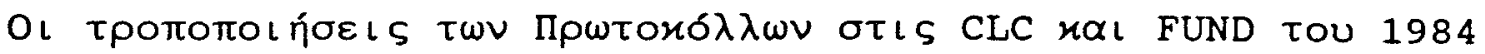

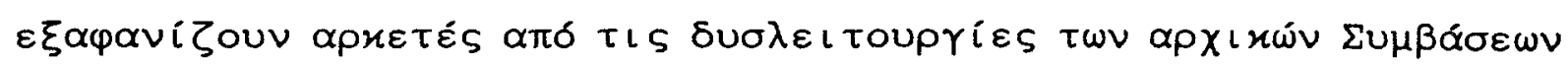

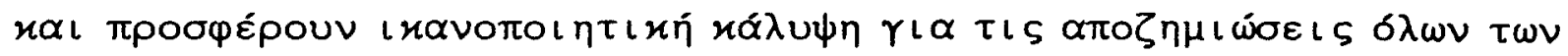

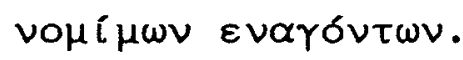




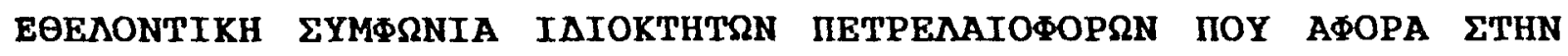
BYOYNH TIA ZHMIA AIO PYIANLH IETPBNAIOY, 1969 í TOVALOP Tanker owners voluntory Agreement Concerning Liability for oil Pollution Damage, 1969

ANAOESPHEH THE TOVALOP TOY 1978 (1978 Revision Of TOVALOP)

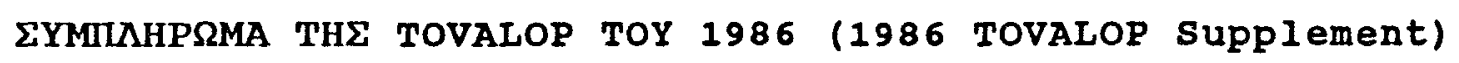

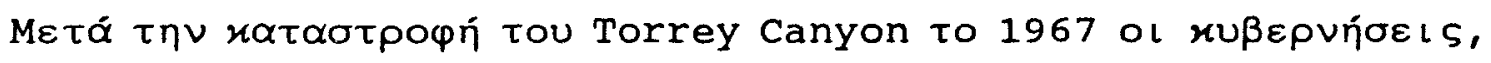

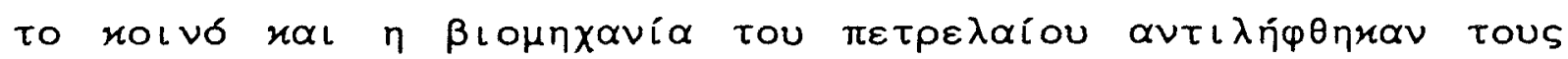

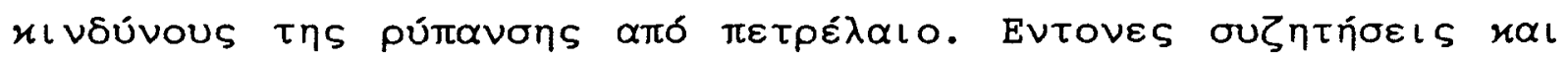

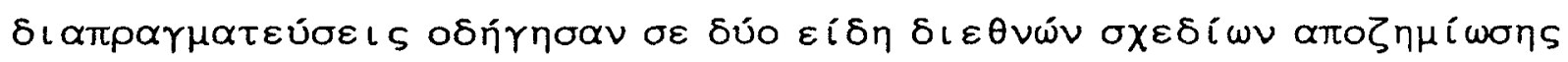

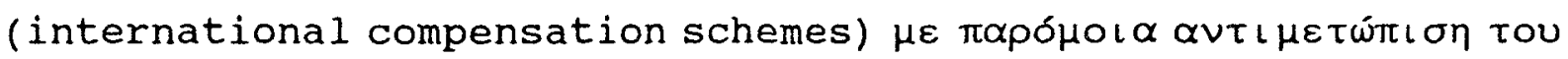

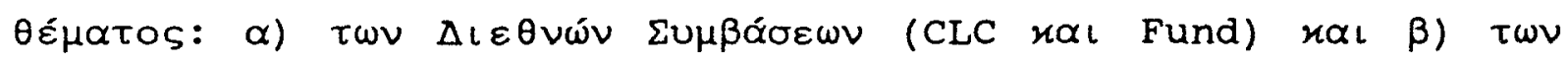

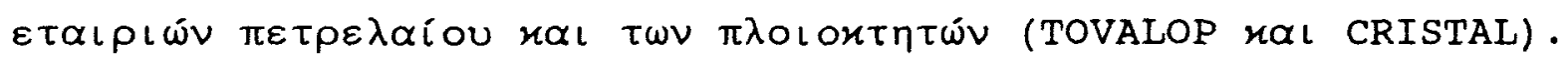

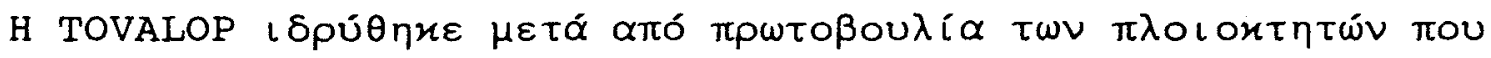

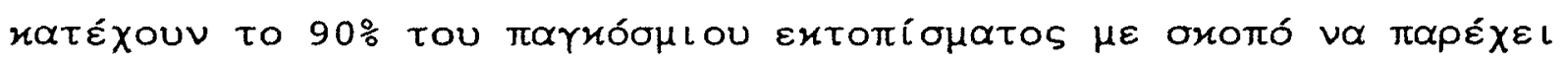

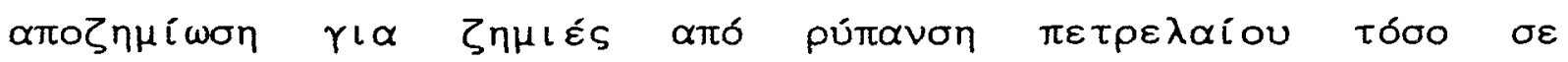

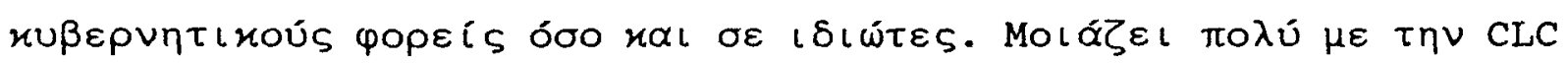

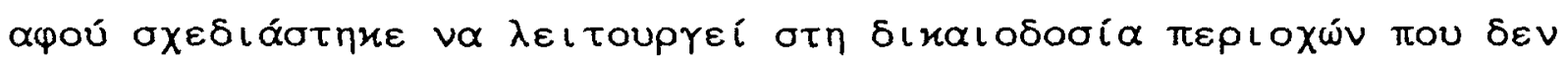

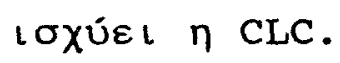




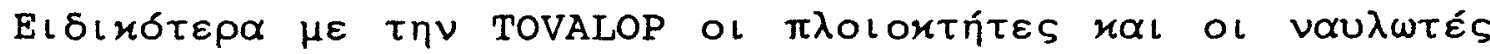

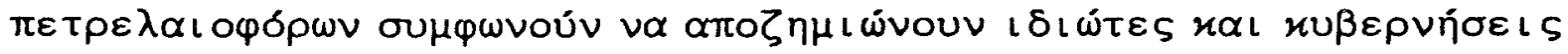

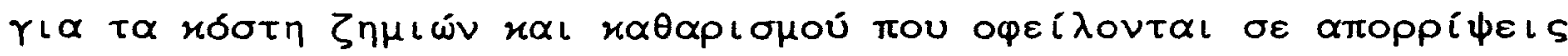

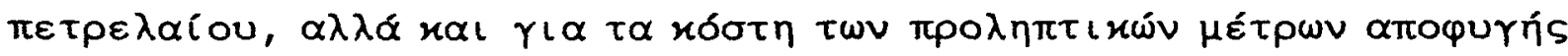

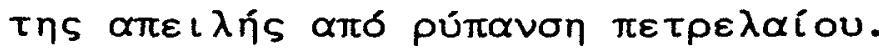

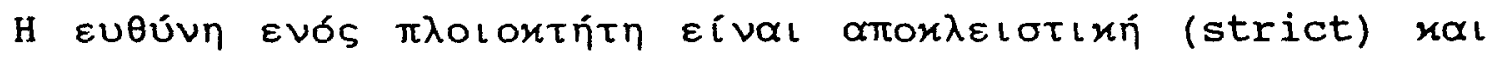

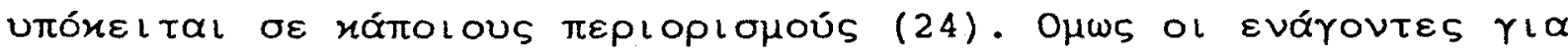

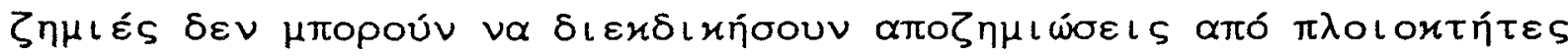

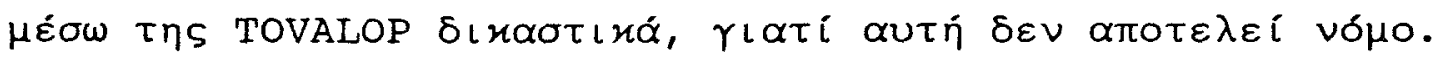

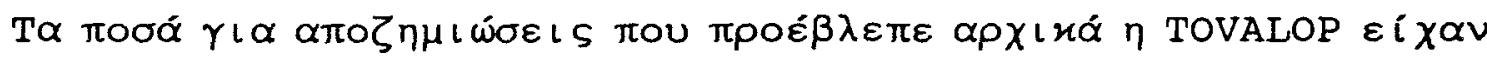

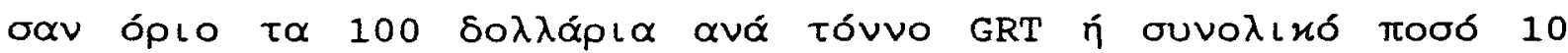

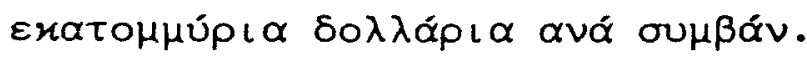

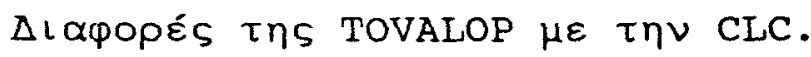

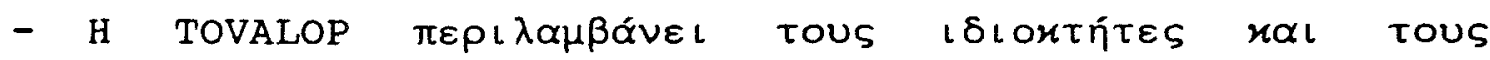

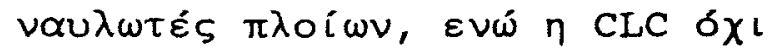

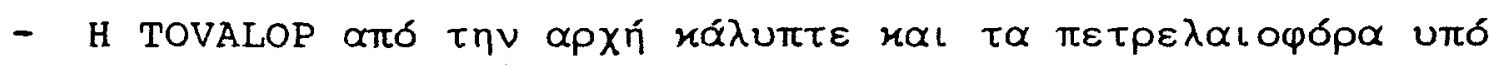

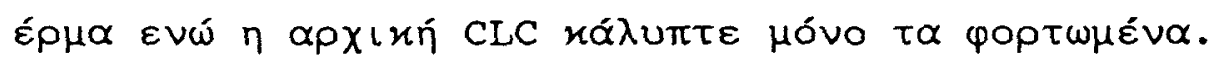

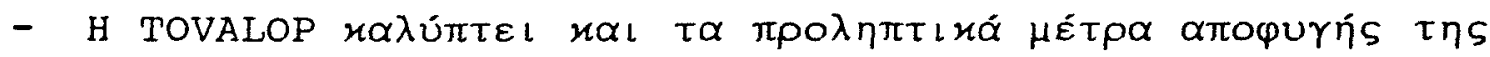

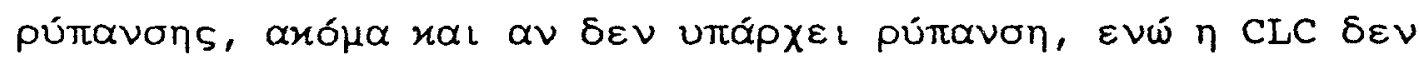

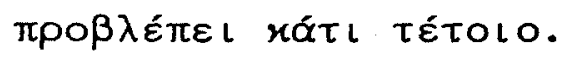




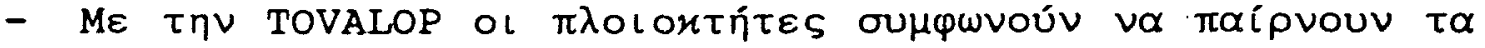

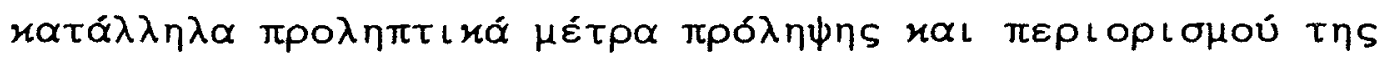

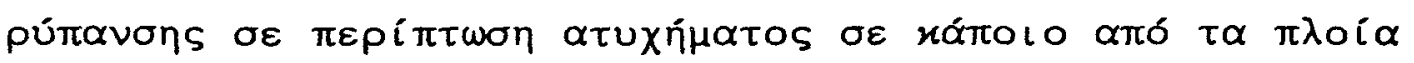

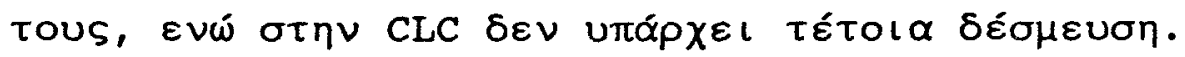

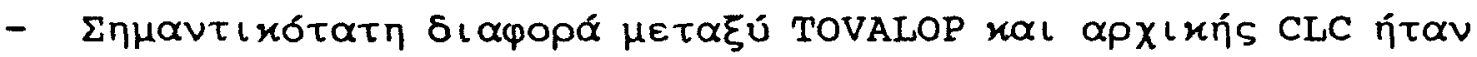

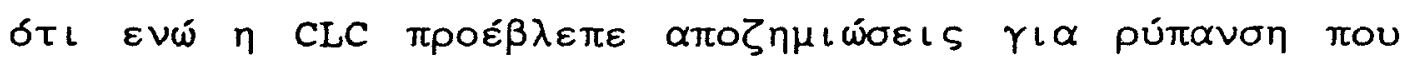

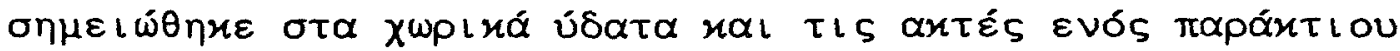

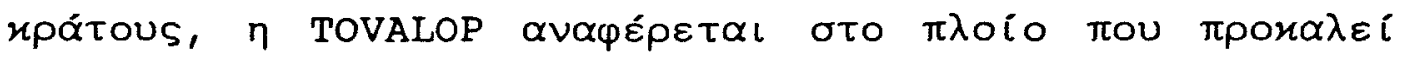

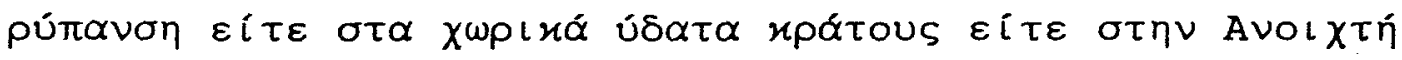
$\theta \alpha ́ \lambda \alpha \sigma \sigma \alpha$.

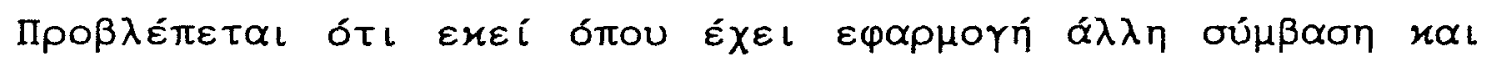

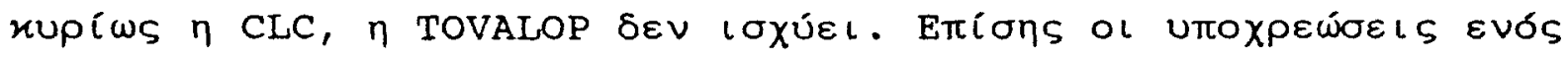

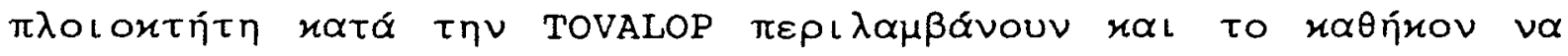

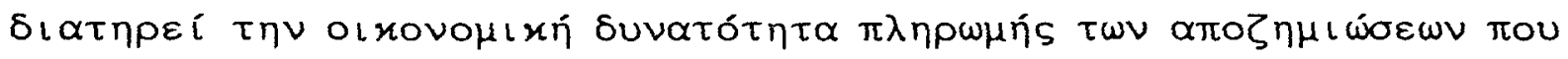

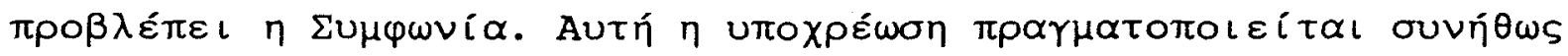

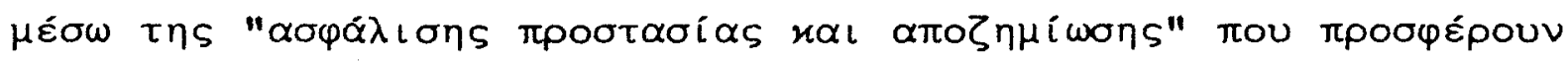

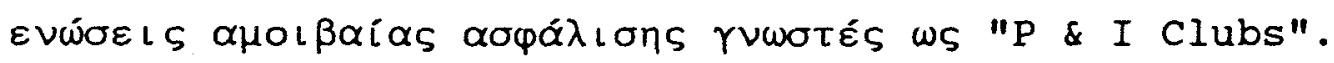

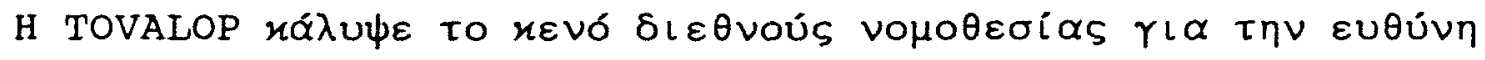

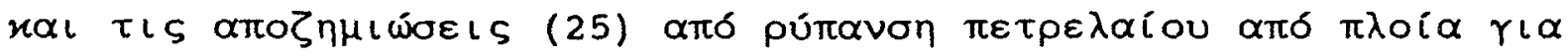

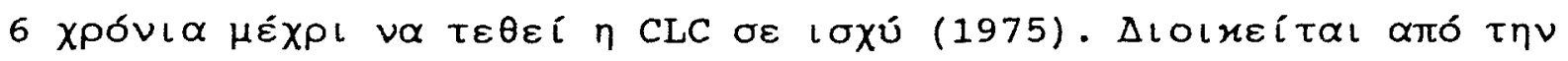

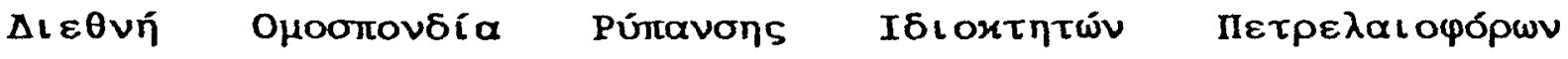
(International Tanker Owners Pollution Federation, Ltd) $\mu \varepsilon \varepsilon \delta \rho \alpha$ To novívo. 


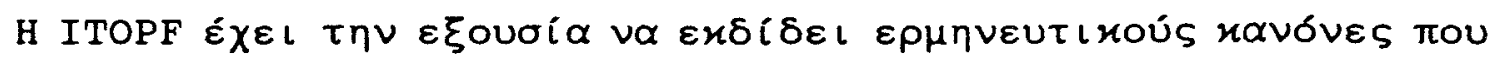

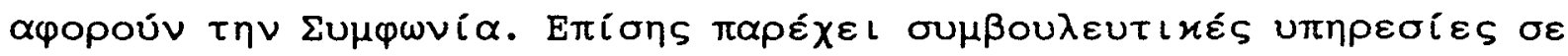

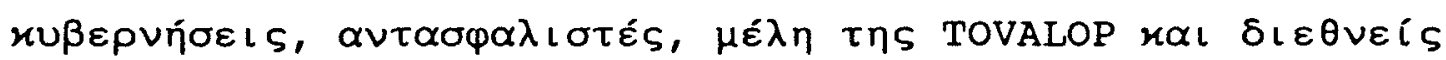

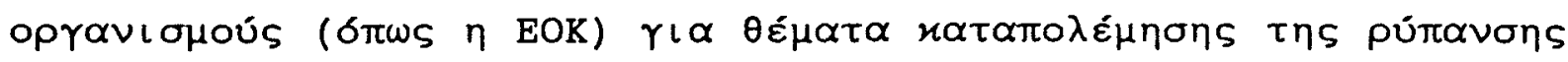
$\pi \varepsilon \tau \rho \varepsilon \lambda \propto$ íou.

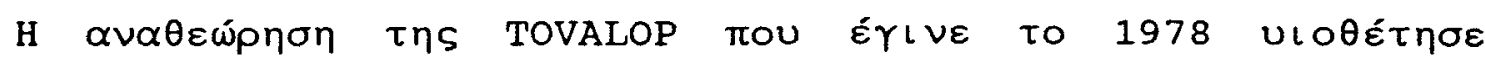

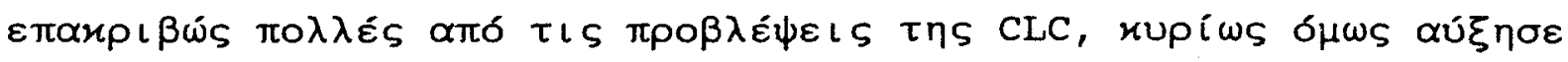

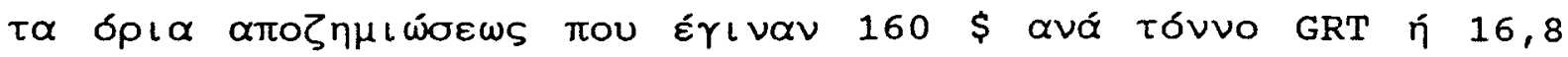

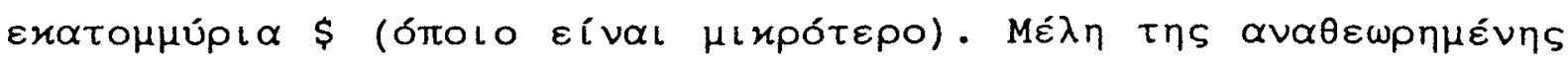

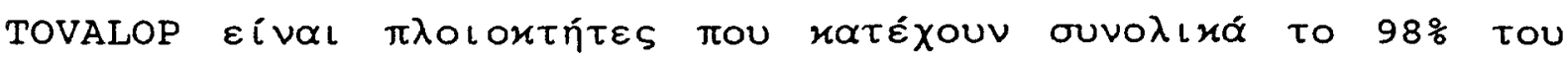

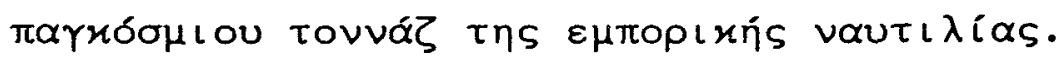

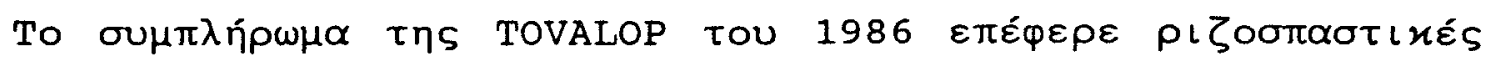

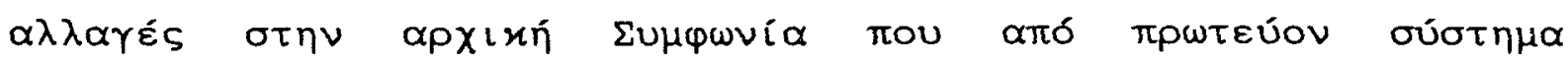

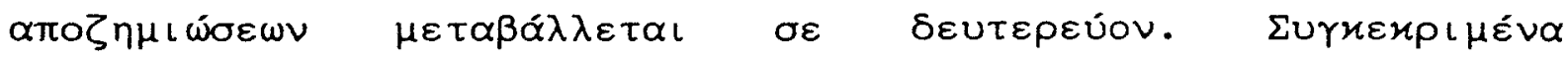

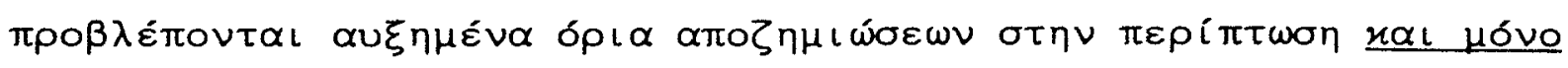

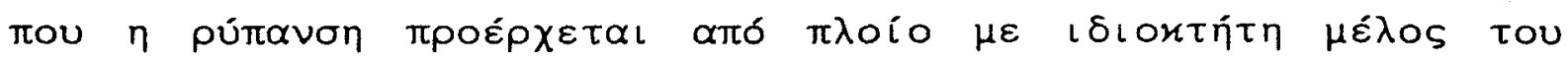

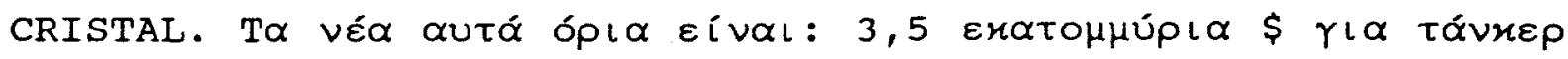

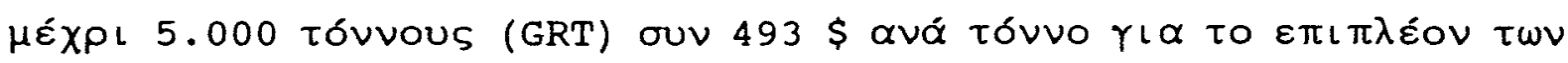

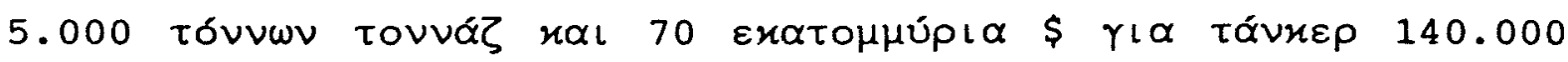

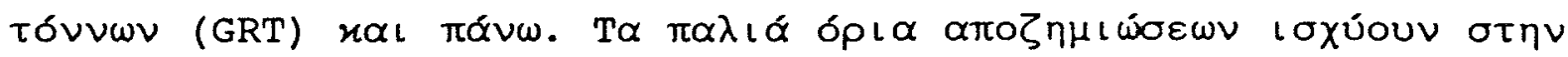

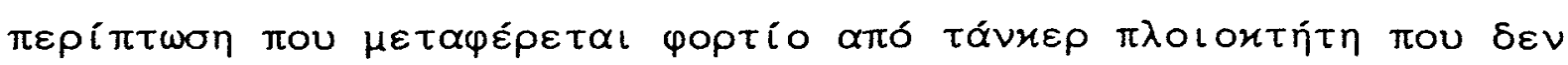

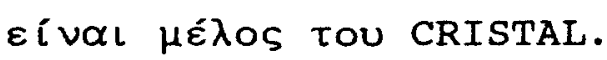




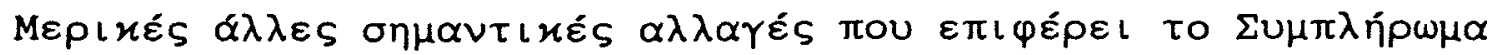
TnS TOVALOP $\varepsilon i$ VQ

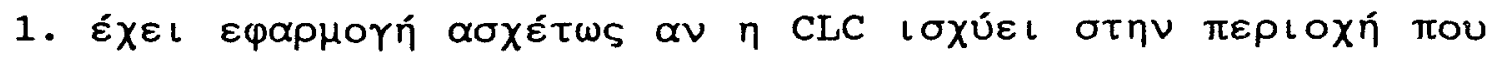
$\delta \eta \mu \iota$ oup

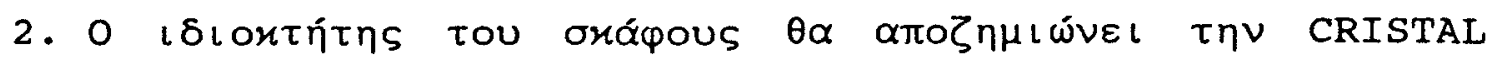

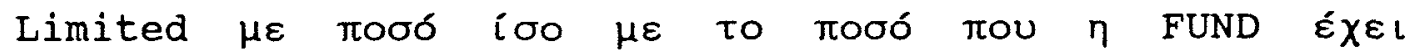

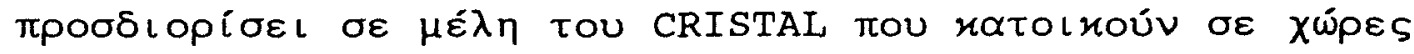

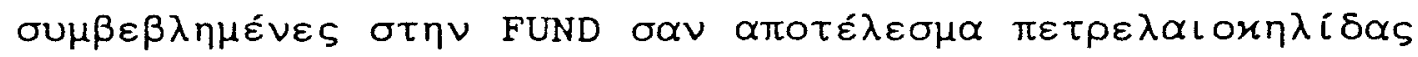

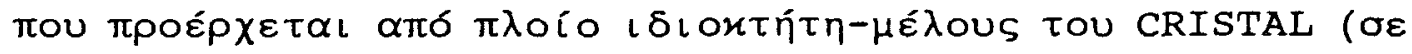

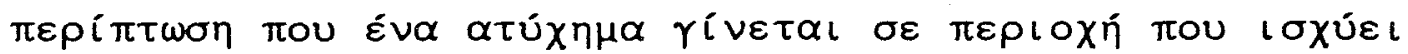
$\eta$ FUND) .

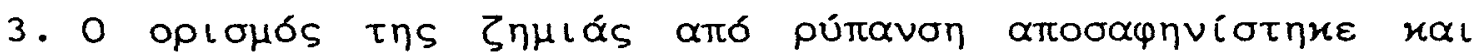

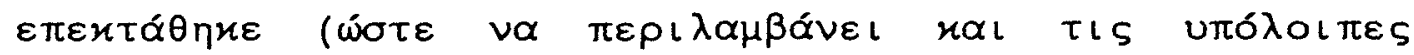

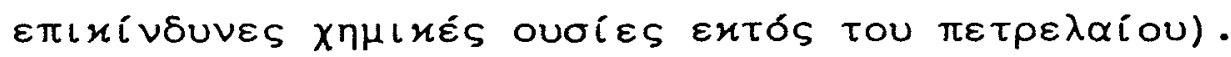

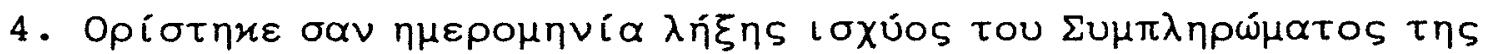

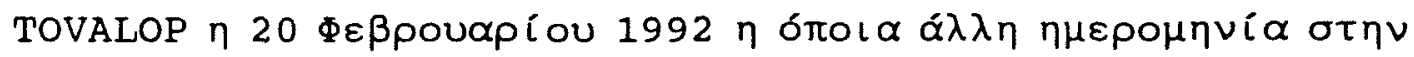

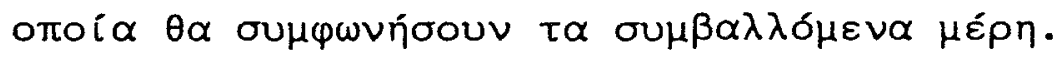




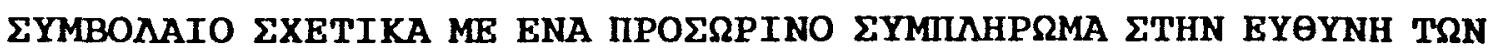
DEEAMENOILOISN TIA ZHMIA AIO PYILNEH IETPENATOY, 1971 í CRISTAL. Contract Regarding an Interim supplement to Tanker Liability for oil Pollution Damage, 1971. ANAEERPHEH TOY CRISTAL TOY 1978 (1978 Revision Of CRISTAL) ANAEERPHEH TOY CRISTAL TOY 1984 (1984 Revision Of CRISTAL)

To CRISTAL $\varepsilon i$ V $\alpha \iota$ ou $\mu \varphi \omega \nu i \alpha \mu \varepsilon \tau \alpha \xi u ́ \varepsilon \pi \iota \chi \varepsilon\llcorner\rho \eta ́ \sigma \varepsilon \omega \nu \pi \varepsilon \tau p \varepsilon \lambda \alpha i$ ou (26)

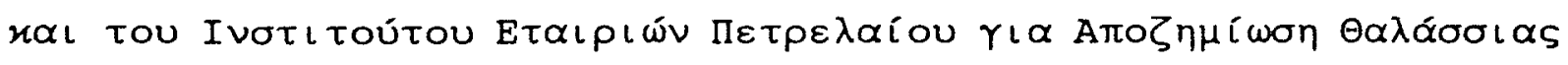
Pútavons (Oil Companies Institute for Marine Pollution

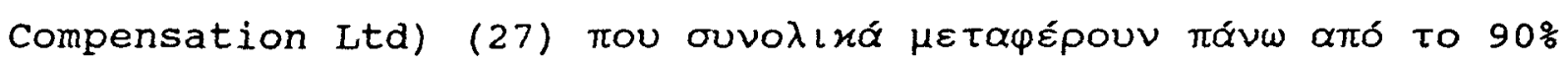

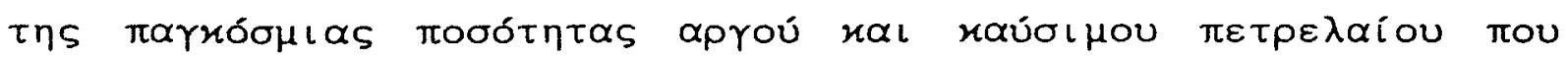

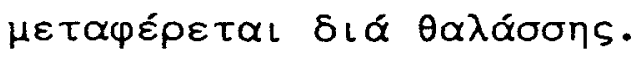

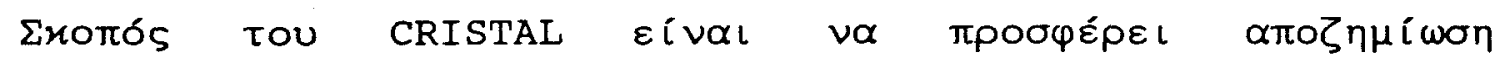

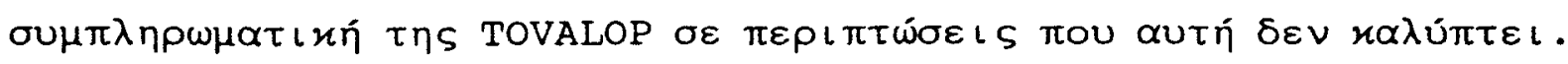

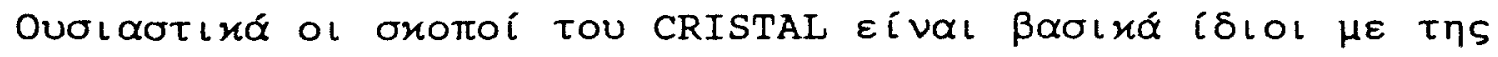

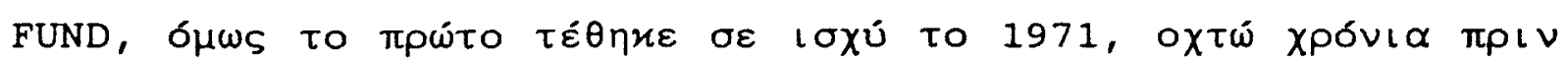

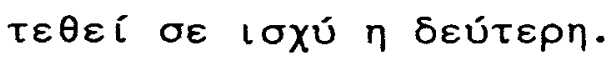

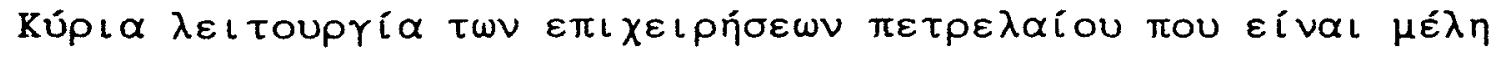

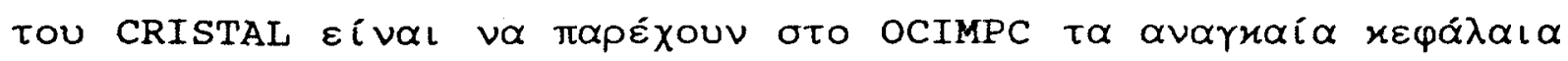

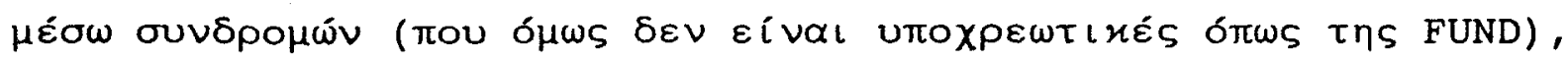




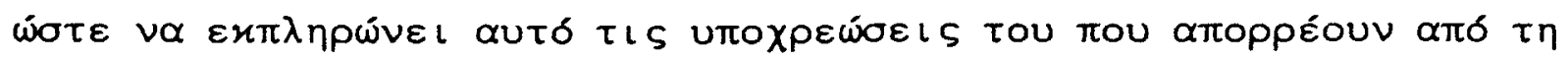

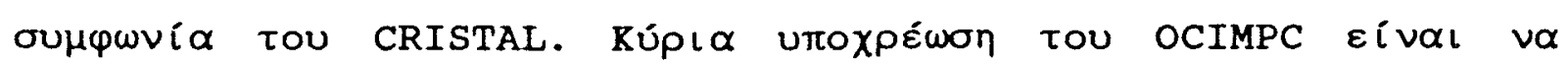

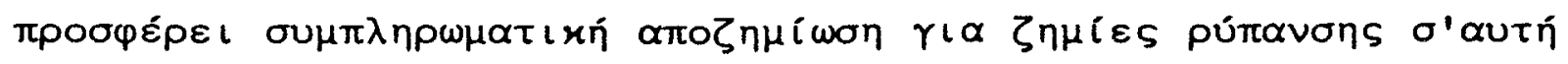

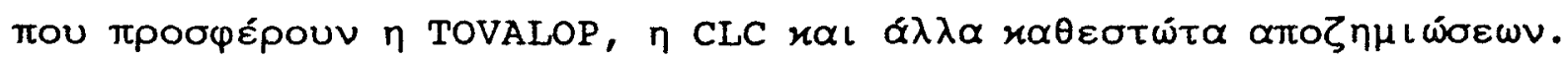

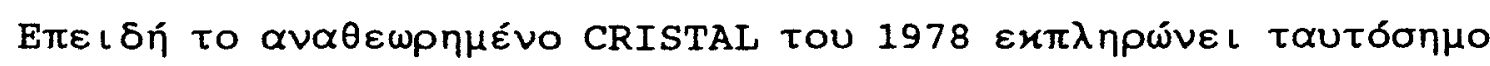

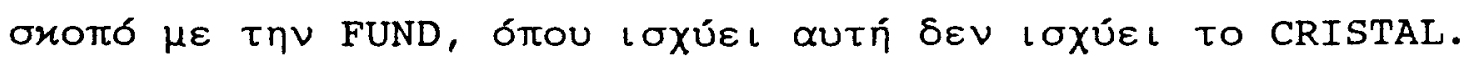

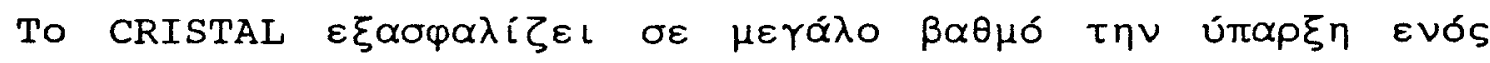

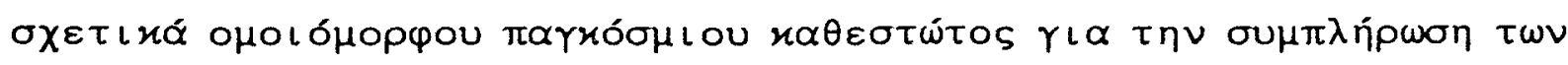

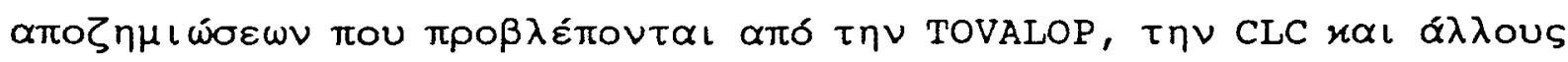

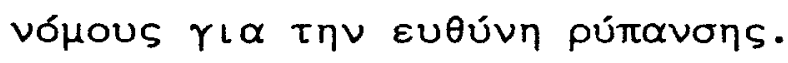

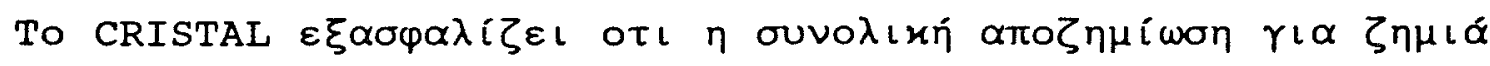

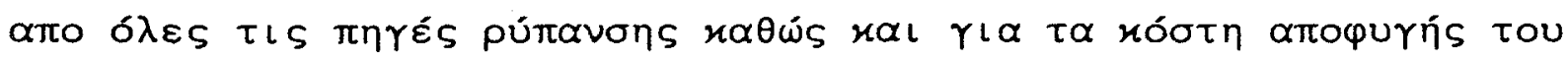

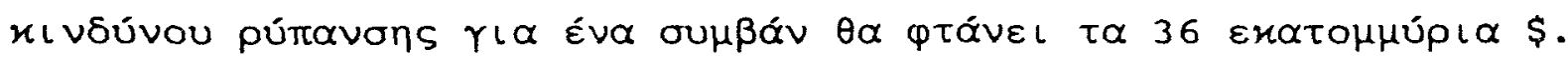

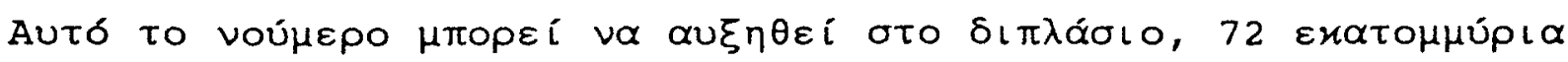

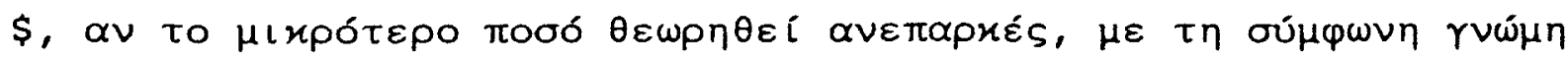

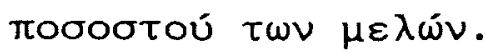

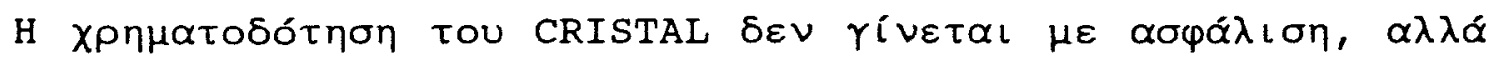

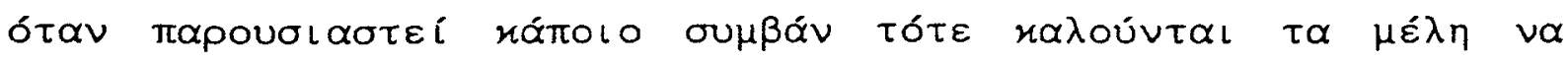

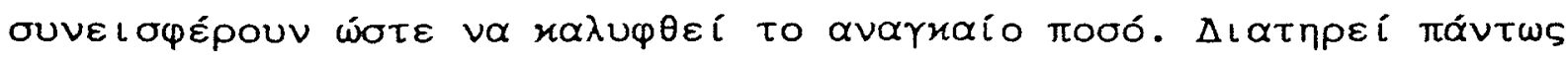

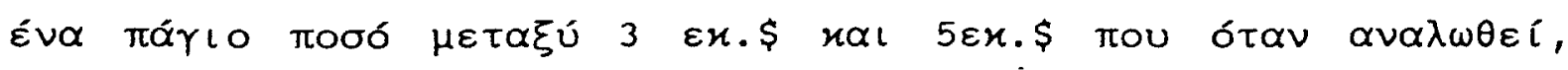

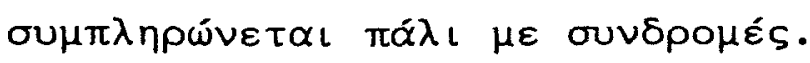

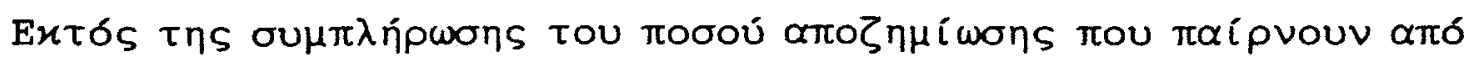




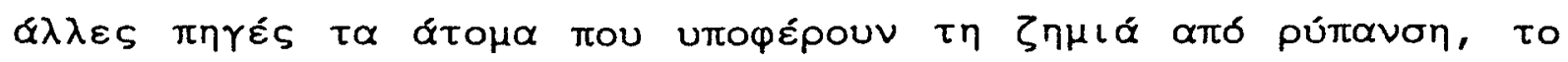

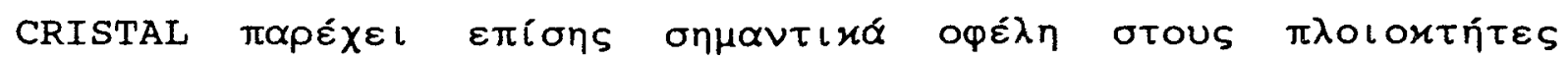

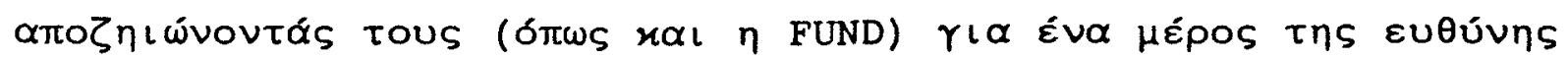

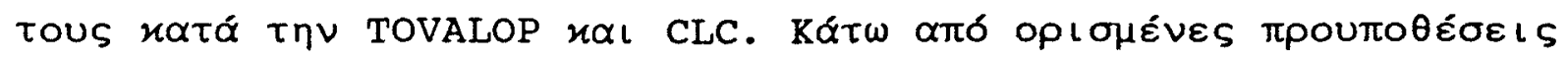

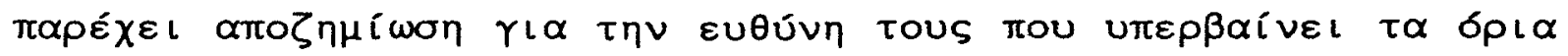

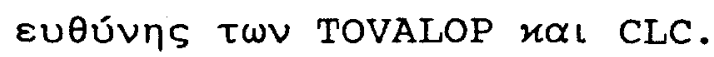

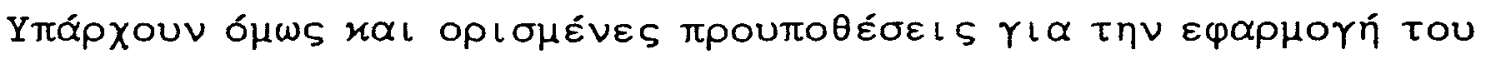

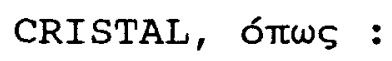

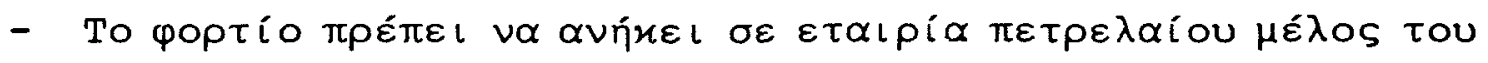

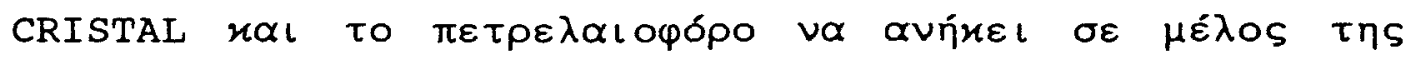

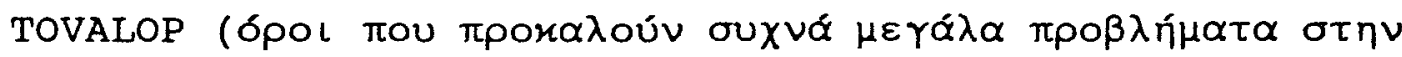

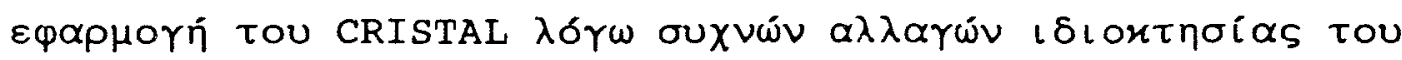
poptíou $\alpha \lambda \lambda \alpha$ xal tou $\pi \lambda \circ i o u)(28)$.

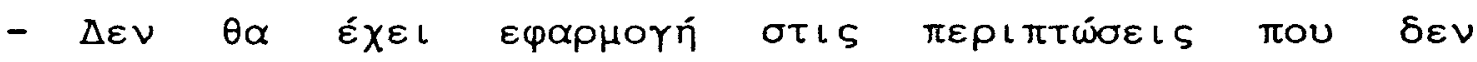

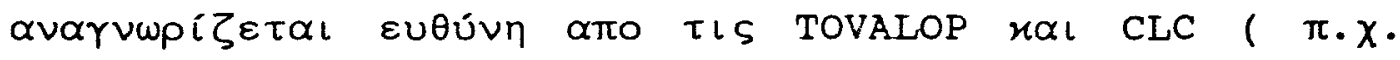

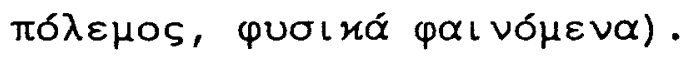

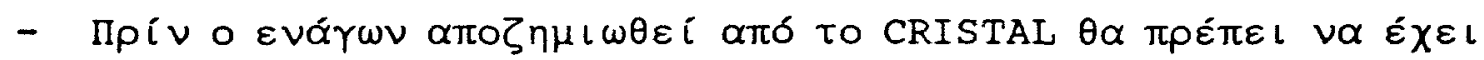

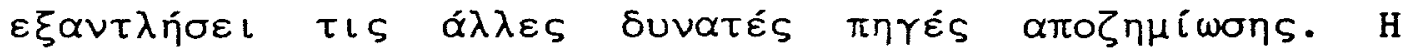

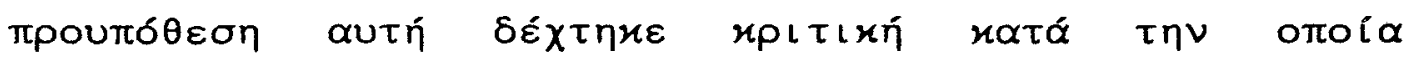

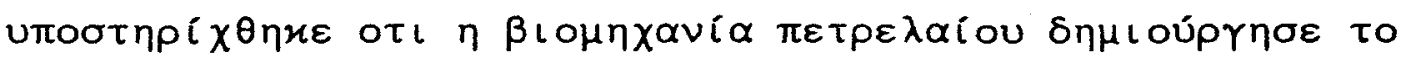




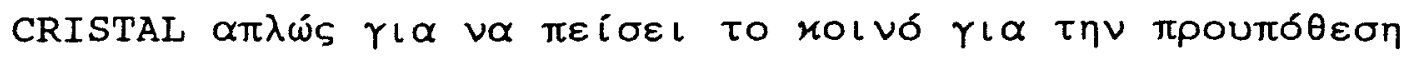

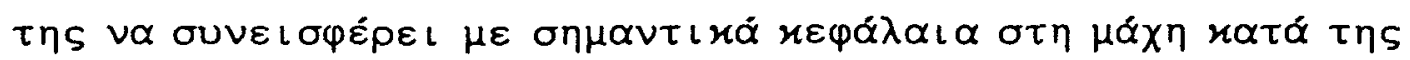
púravons (29).

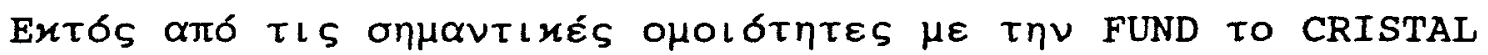

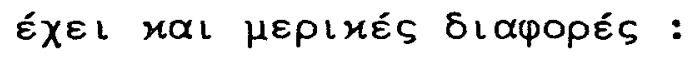

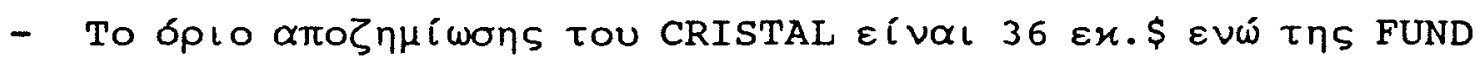
Eíval 50 Ex.\$.

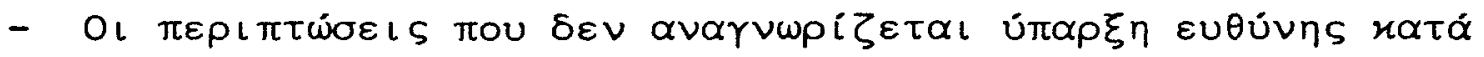

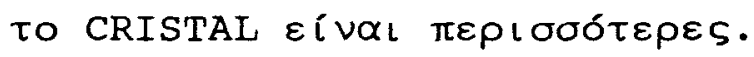

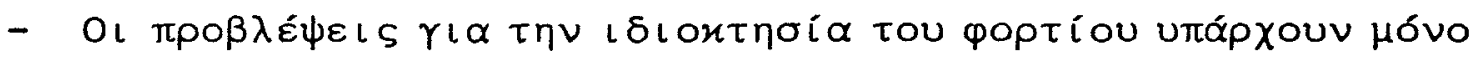
OTO CRISTAL.

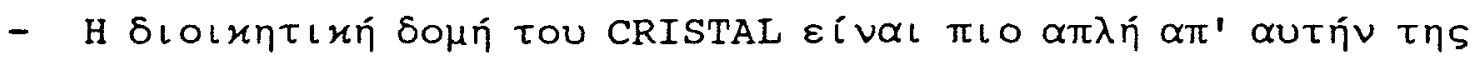
FUND.

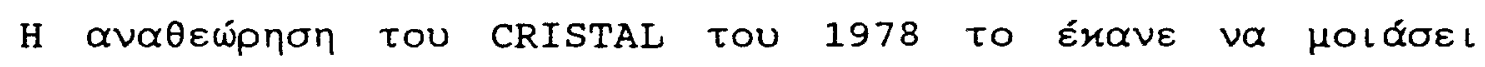

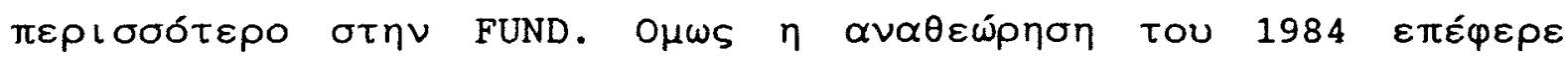

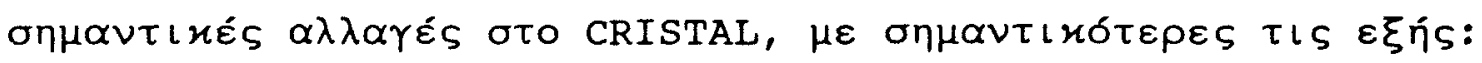

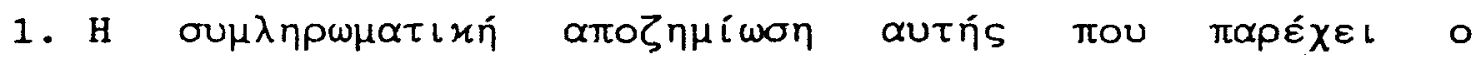

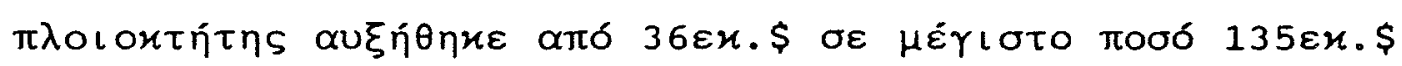

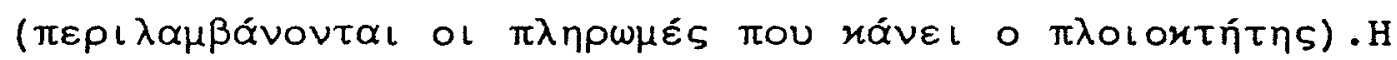




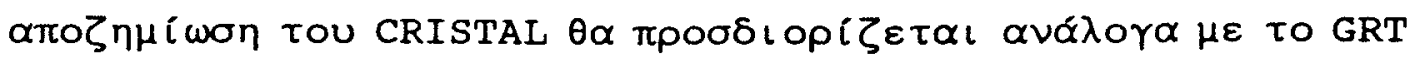

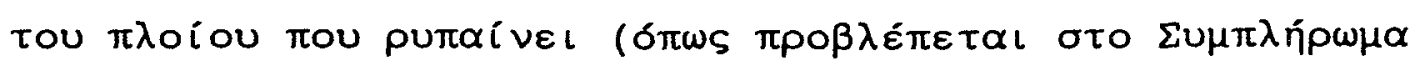
TIS TOVALOP).

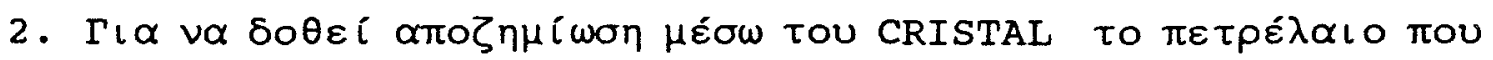

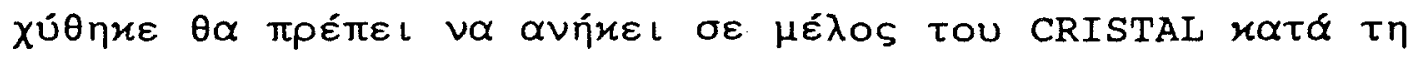

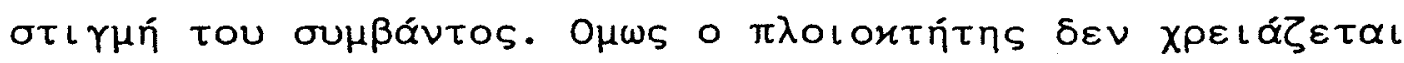

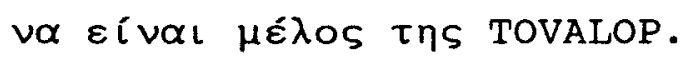

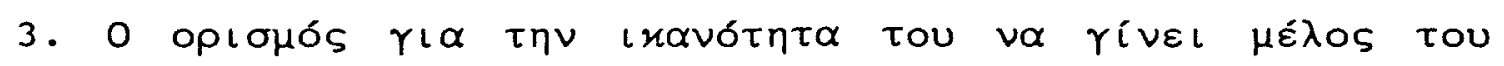

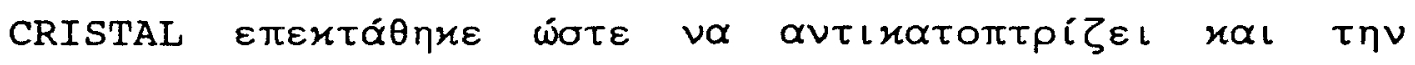
$\varepsilon \pi \alpha \gamma \gamma \varepsilon \lambda \mu \alpha \tau \iota x \eta ́ \varepsilon \quad \varepsilon \mu \pi \varepsilon \iota \rho i \alpha$.

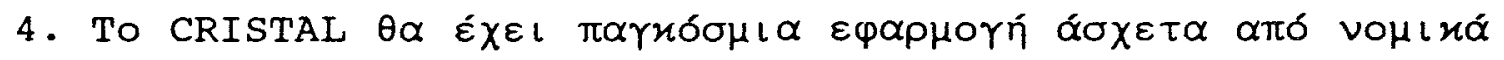
$\varkappa \alpha \theta \varepsilon \sigma \tau \omega ́ \tau \alpha$.

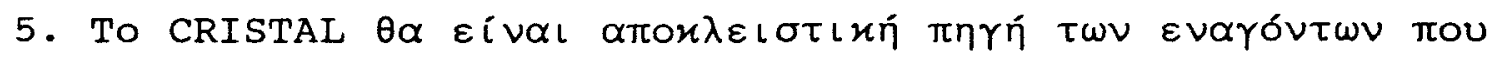

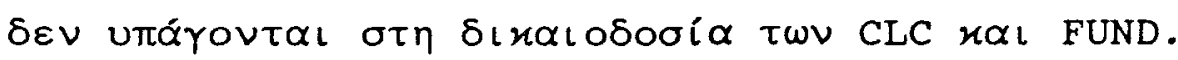

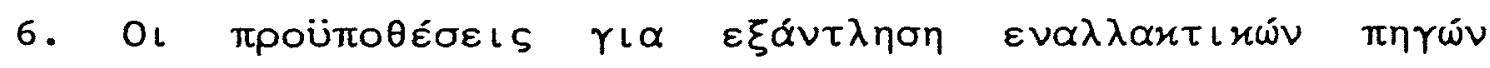

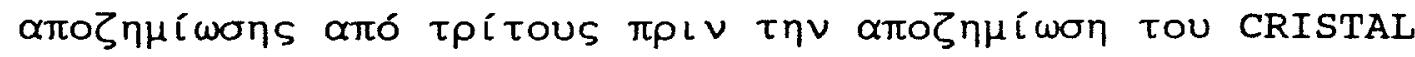
$\chi \alpha \lambda \alpha p \omega \sigma \alpha \nu$. 


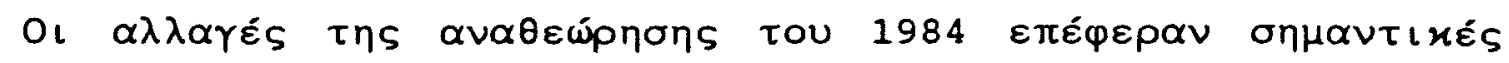

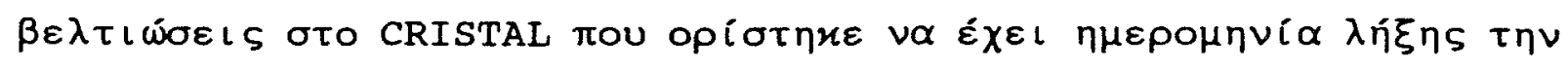

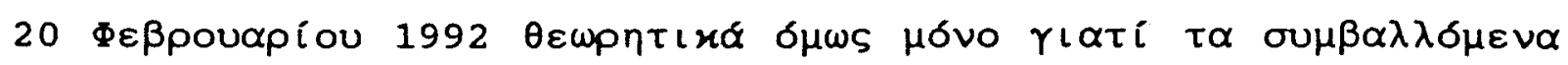

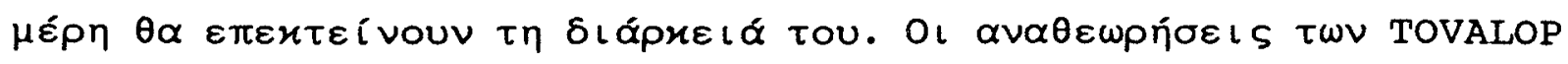

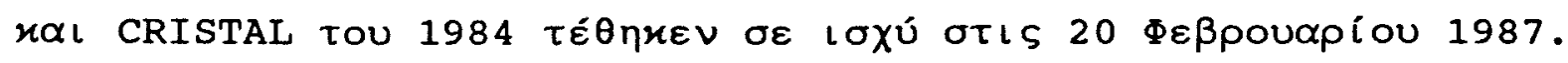




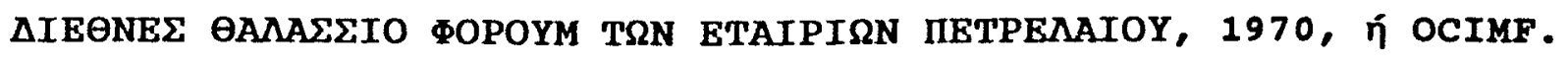
Oil Companies International Marine Forum, 1970

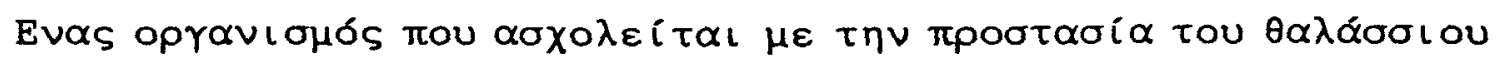

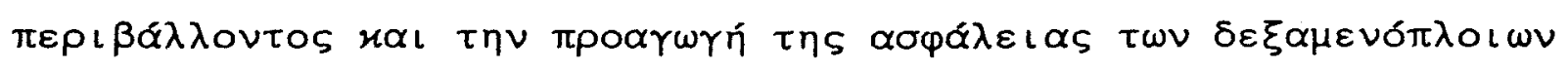

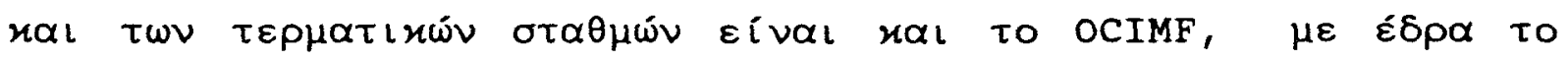

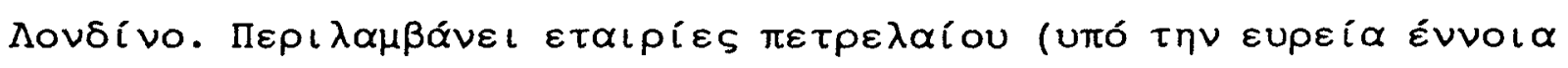

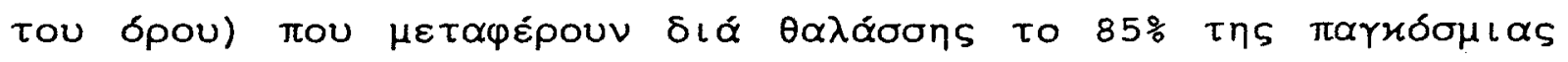

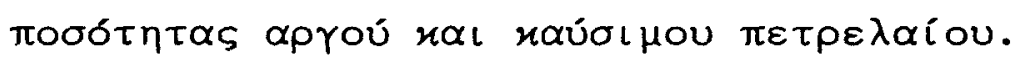

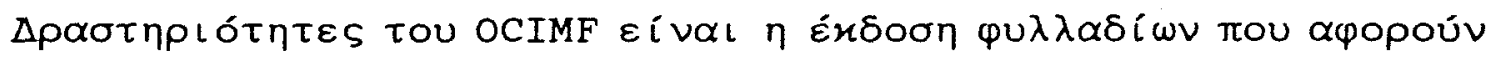

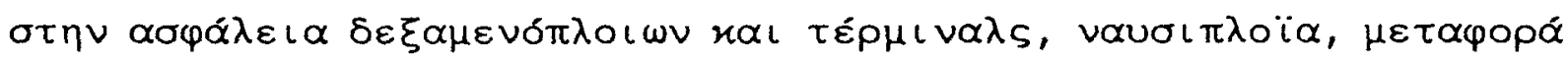

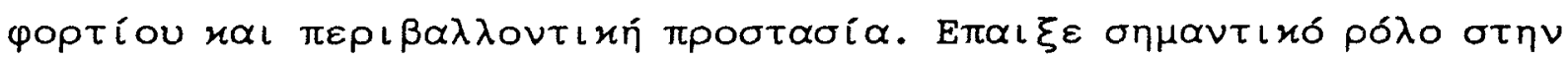

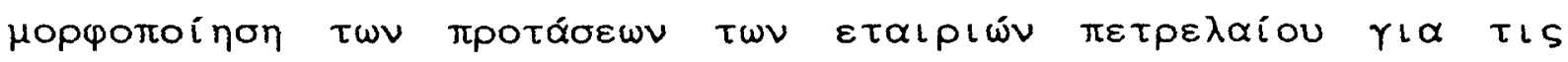

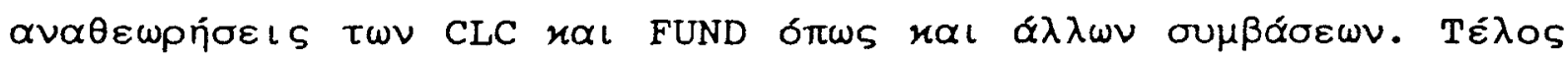

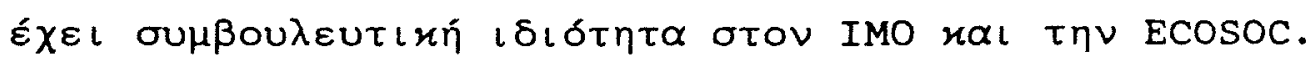




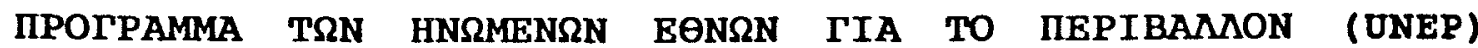 United Nations Environmental Programme}

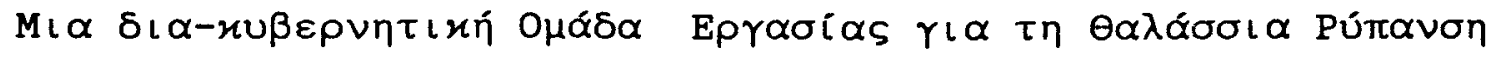

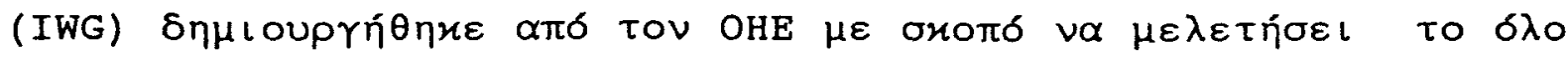

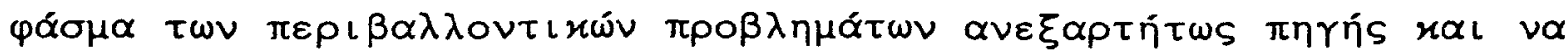

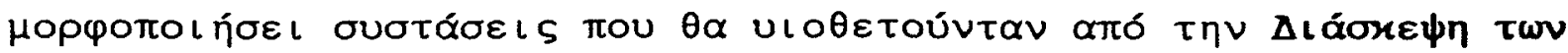

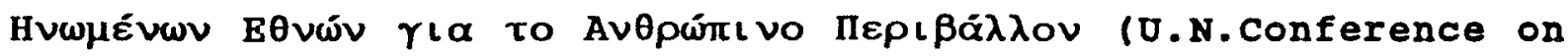

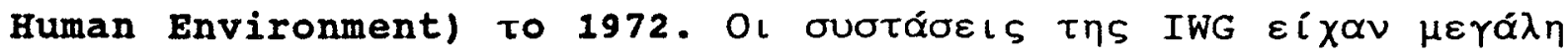

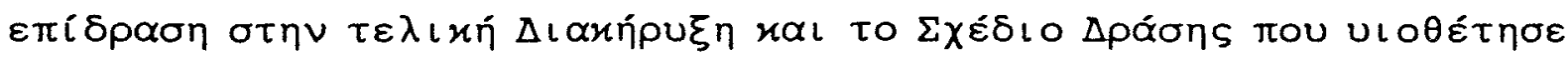

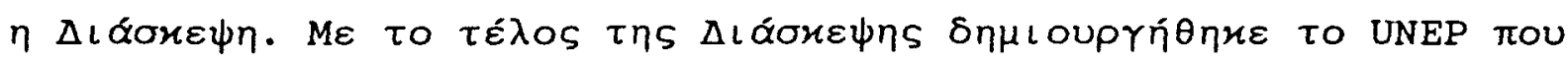

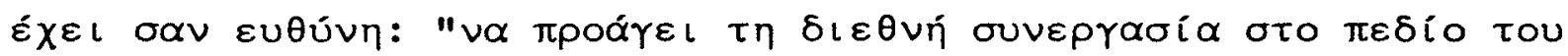

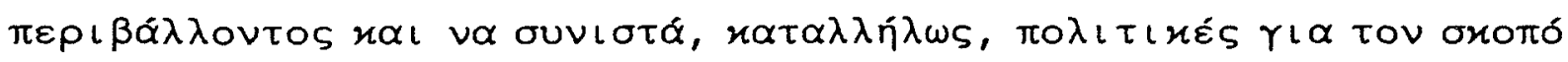

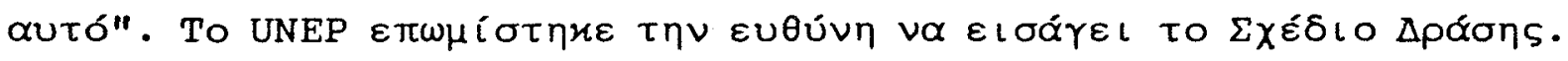

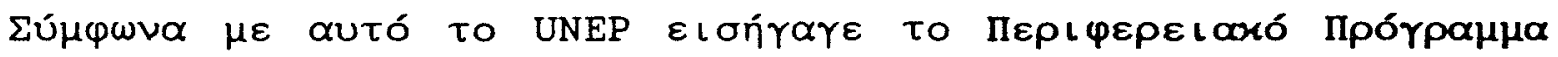

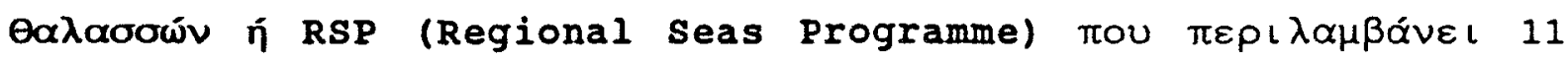

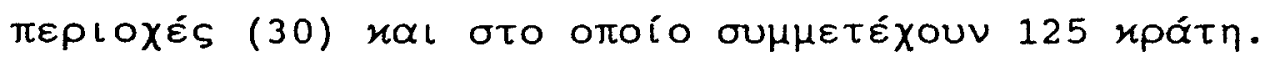

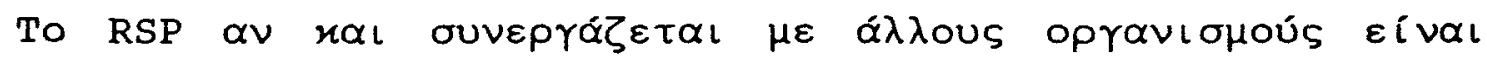

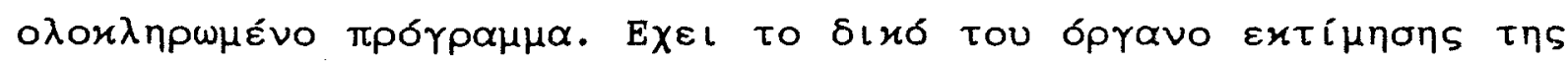

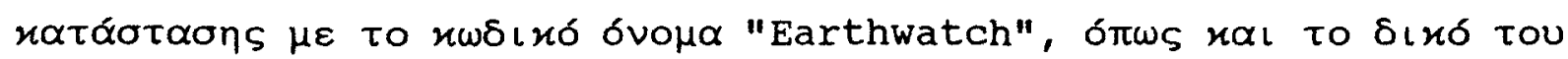

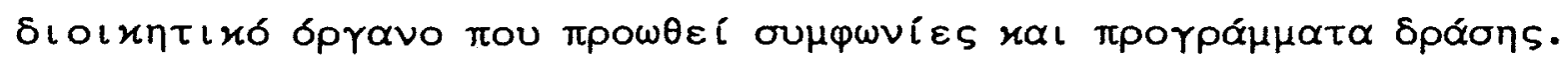




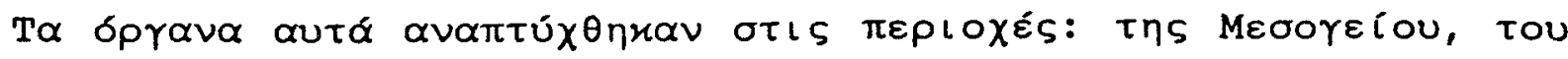

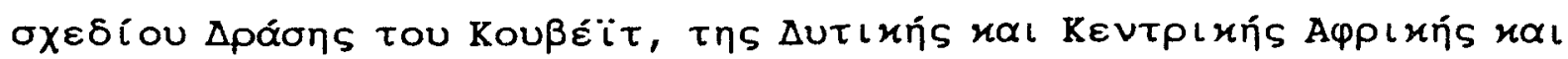

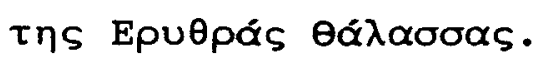

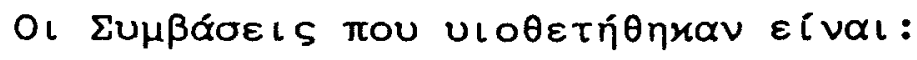

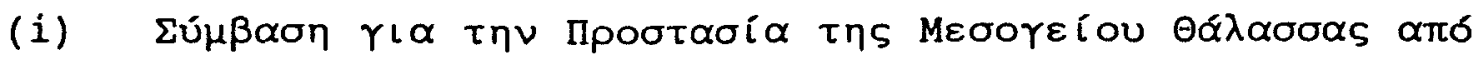

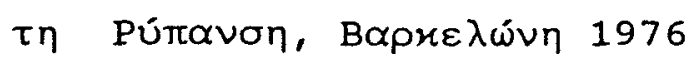

Convention for the Protection of the Mediterranean Sea Against Pollution, Barcelona, 1976

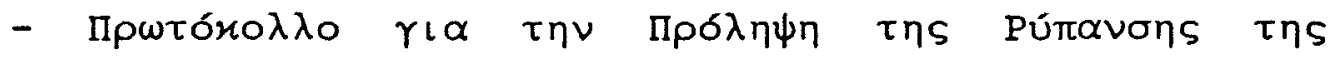

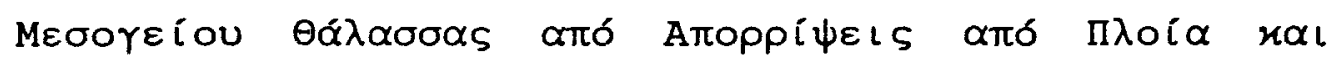
Aвpooหর́ $\varphi \eta, 1976$

Protocol for the Prevention of Pollution of the Mediterranean sea by Dumping from ships and Aircrafts, 1976

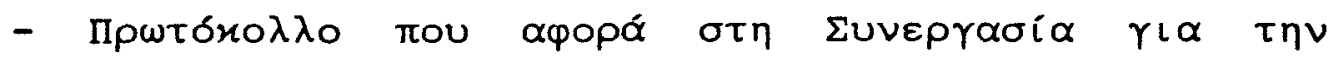

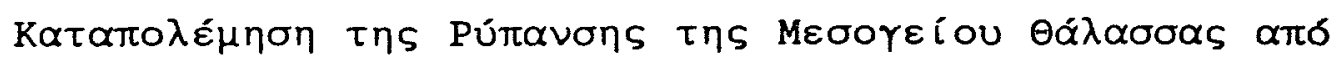

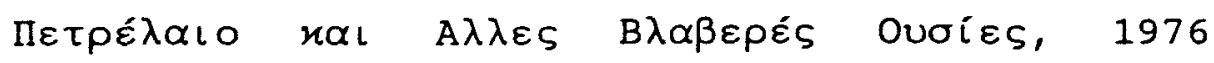

Protocol Concerning Cooperation in combating Pollution of the Mediterranean sea by oil and other Harmful substances. 


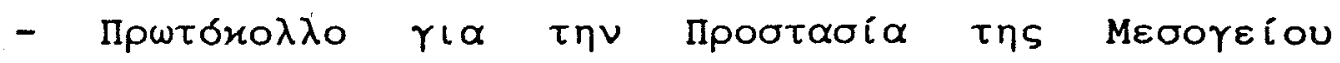

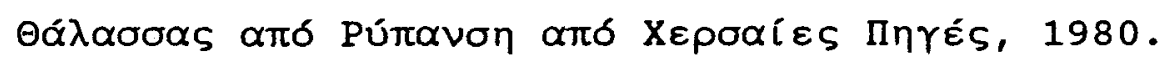
Protocol for the Protection of the Mediterranean Sea Against Pollution from Land Based Sources, 1980.

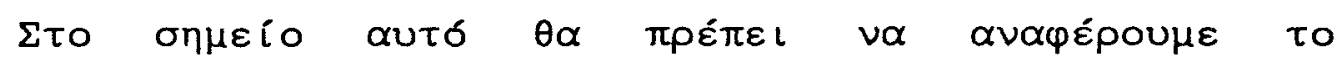
Meooreı

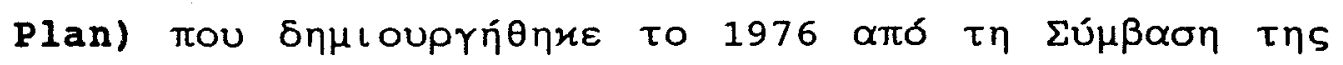

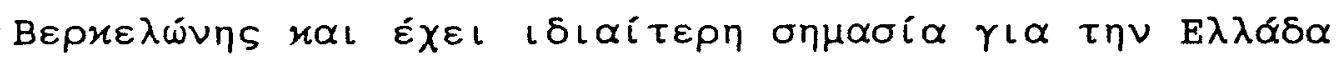

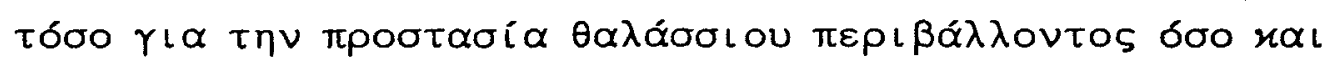

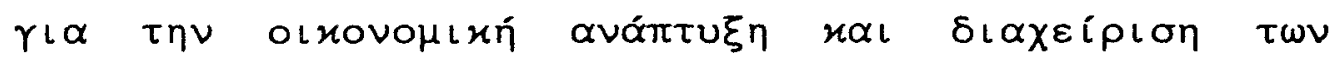

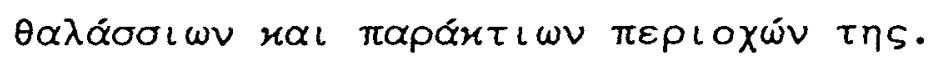

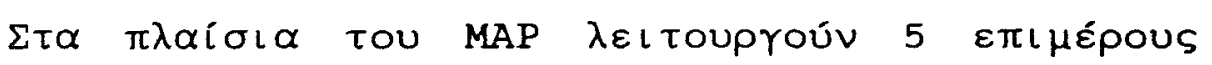

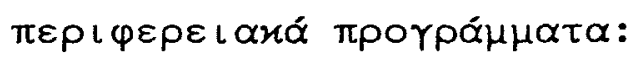

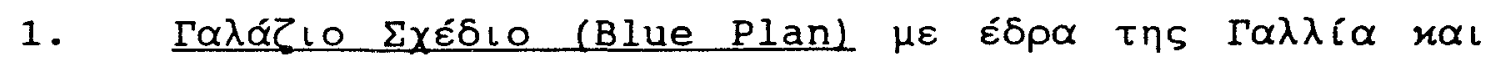

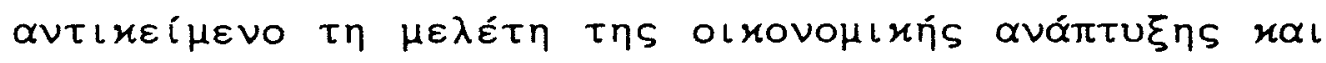

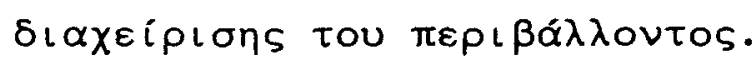

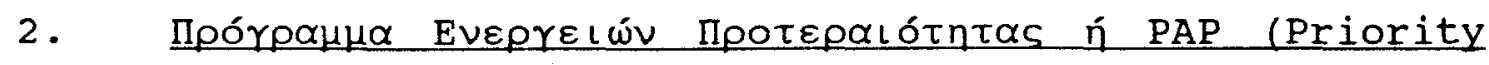

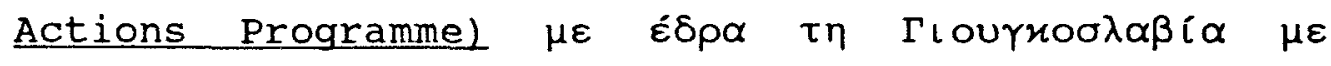

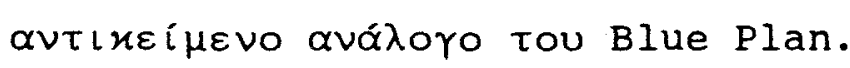

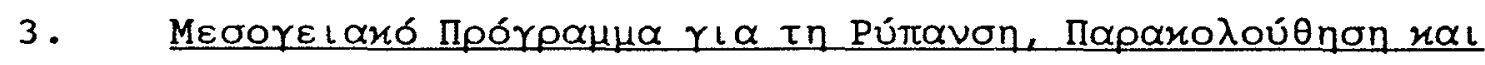

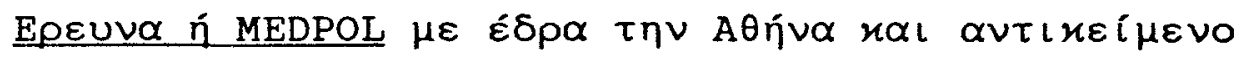




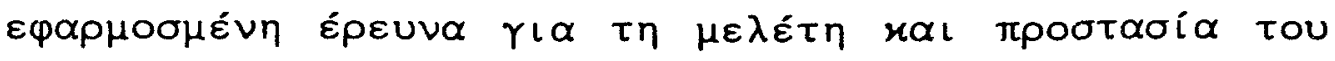

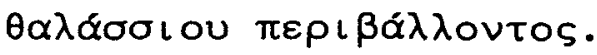

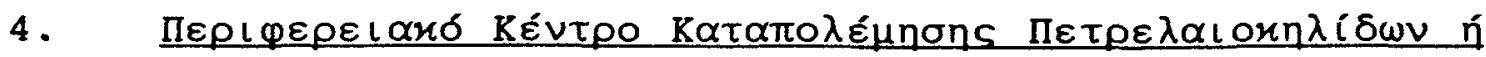

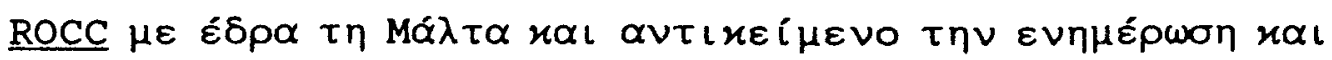

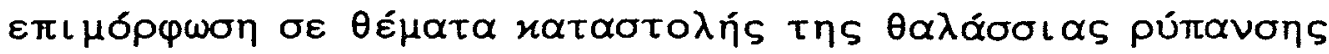

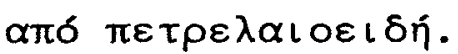

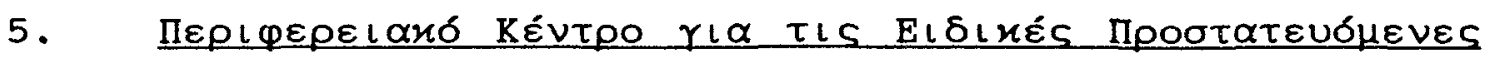

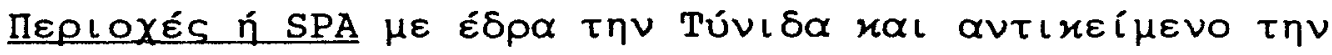

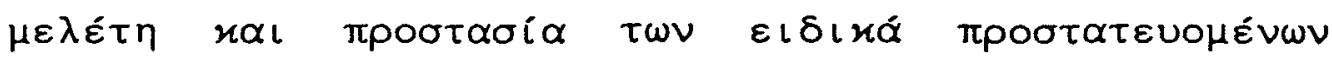

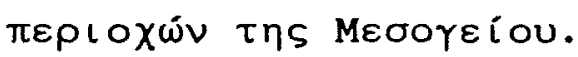

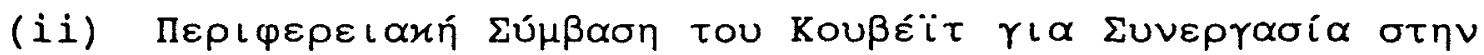

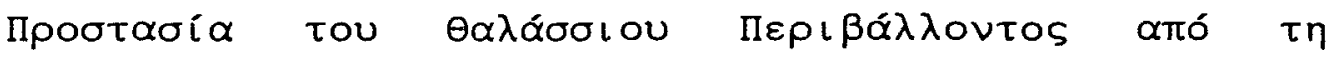
Púravon, 1978

Kuwait Regional Convention for cooperation on the Protection of the Marine Environment from Pollution, 1978

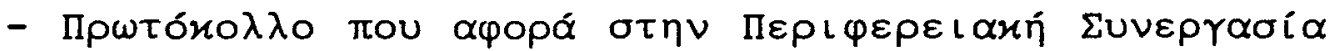

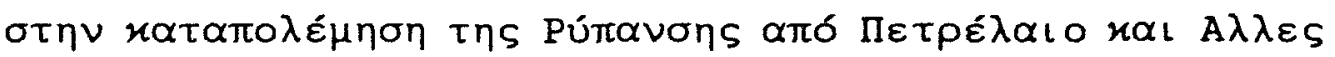

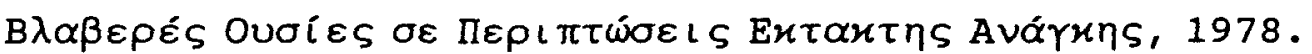
Protocol Concerning Regional cooperation in Combating Pollution by oil and other Harmful substances in Cases of Emergency, 1978 . 


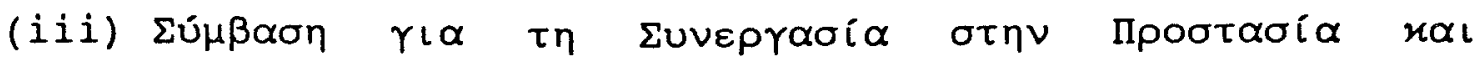

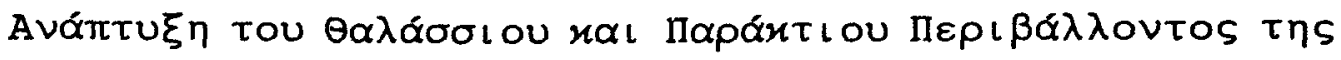

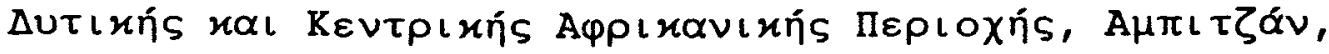
1981

Convention for the cooperation in the Protection and Development of the Marine and Coastal Environment of the West and Central African Region, Abidjan, 1981

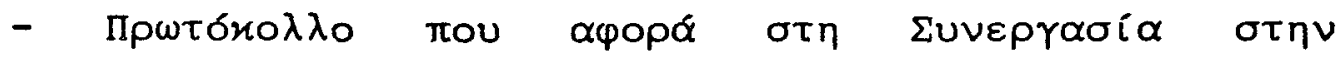

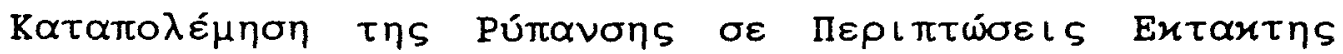
Avárหns, 1981.

Protocol Concerning cooperation in combating Pollution in Cases of Emergency, 1981

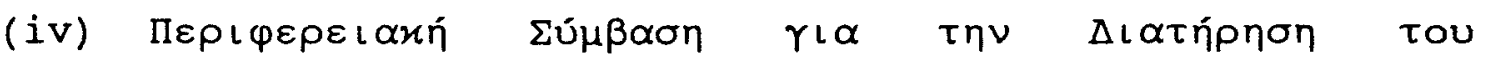

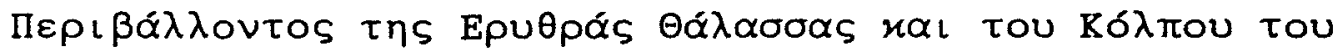
A $\nu \tau \varepsilon \nu, T \zeta \varepsilon ์ \nu \tau \alpha, 1981$.

Regional Convention for the Conservation of the Red sea and Gulf of Aden Environment, Jeddah, 1981.

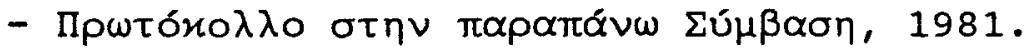

Protocol to the Regional convention for the Conservation of the Red Sea and the Gulf of Aden Environment, 1981. 


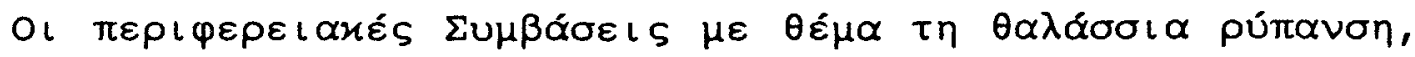

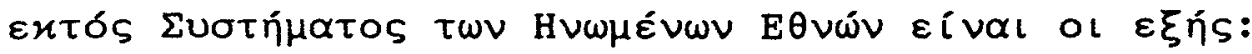

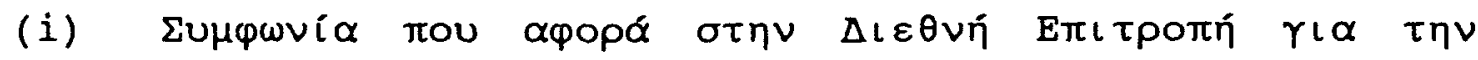

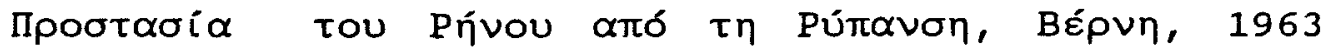
Agreement concerning the International Commission for the Protection of the Rhine against Pollution, Bern, 1963

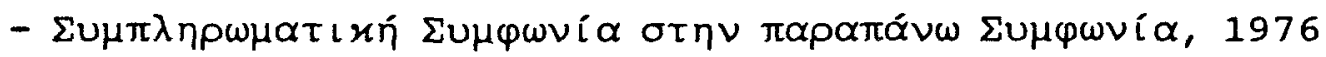
Supplementary Agreement to the 1963 Agreement on the International Commission for the Protection of the Rhine against Pollution, Bonn, 1976

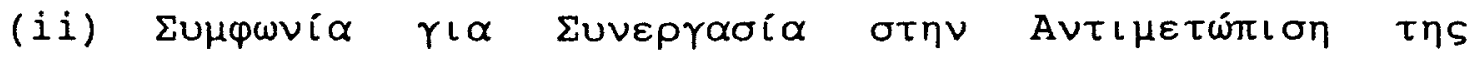

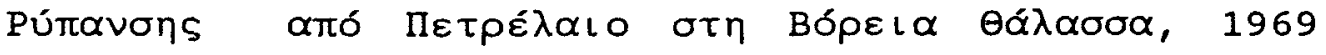
Agreement for Cooperation in Dealing with Pollution of the North Sea by Oil, Bonn, 1969

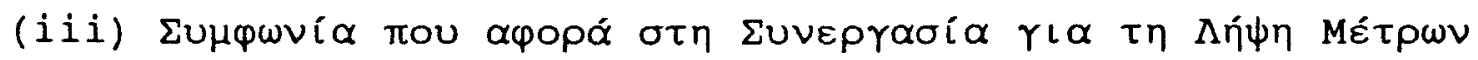

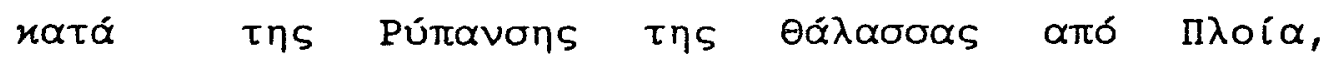

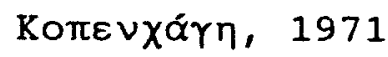

Agreement Concerning Cooperation in Taking Measures Against Pollution of the Sea by oil, Copenhagen, 1971 


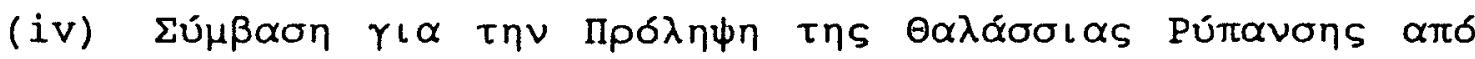

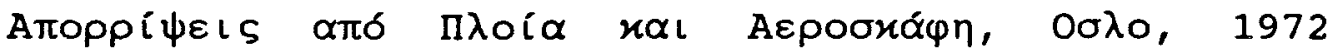
Convention for the Prevention of Marine Pollution by Dumping from Ships and Aircrafts, Oslo, 1972

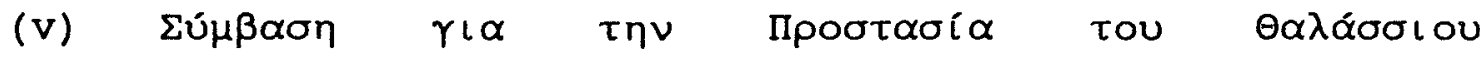

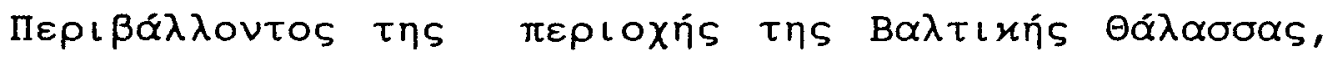
EגoivKL, 1974

Convention on the Protection of Marine Environment of the Baltic Sea Area, Helsinki, 1974

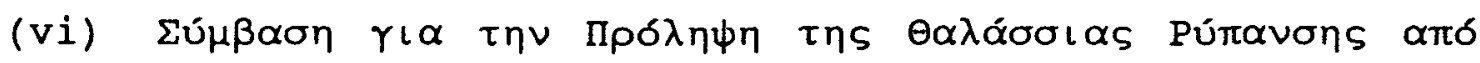

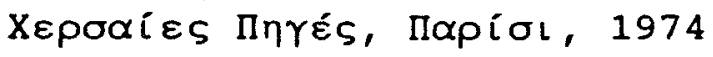

Convention for the Prevention of Marine Pollution from Land Based Resources, Paris, 1974

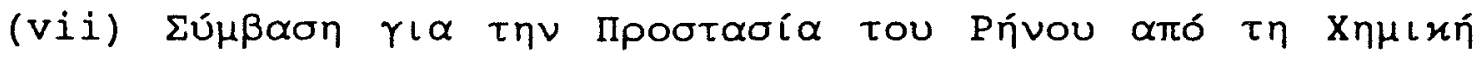
Pútavơ, Bóvvi, 1976

Convention for the Protection of the Rhine Against Chemical Pollution, Bonn, 1976

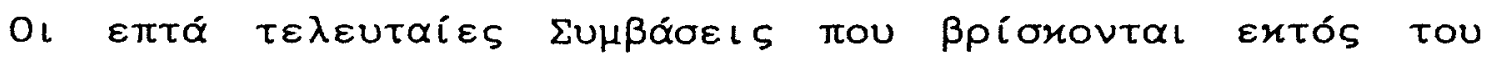

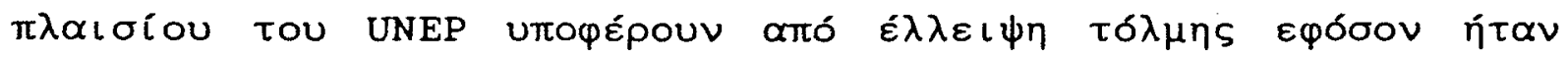

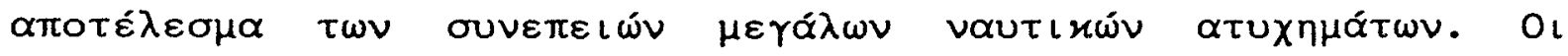

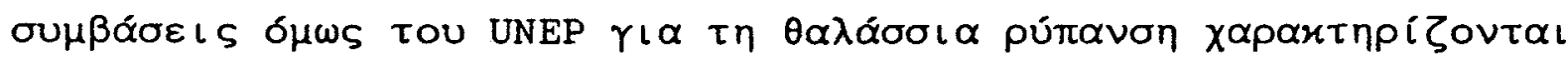




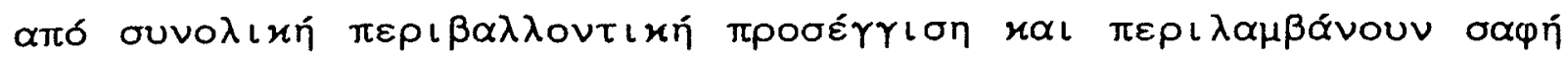

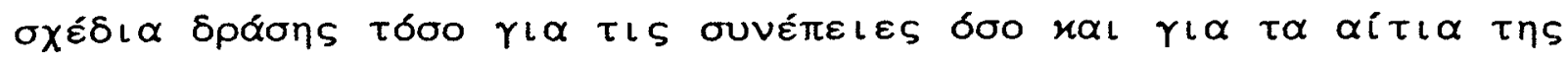

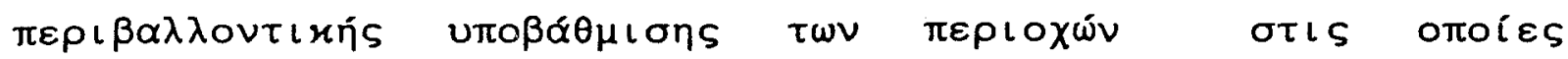
$\alpha \vee \alpha \varphi \varepsilon ́ p o v \tau \alpha \iota$.

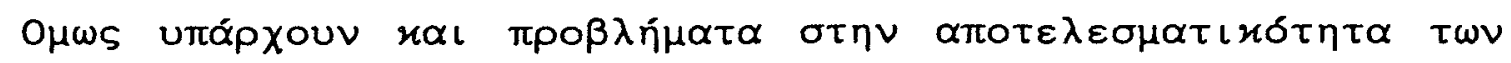

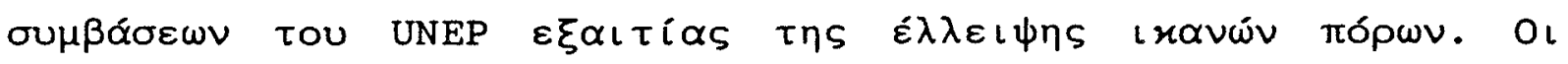

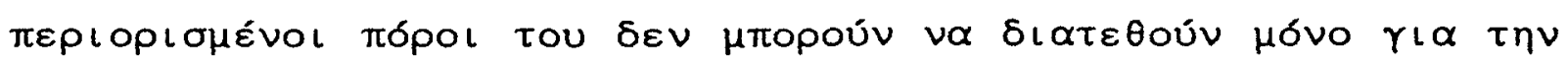

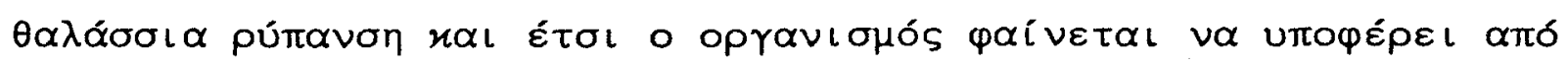

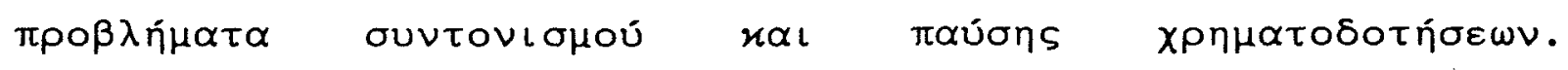

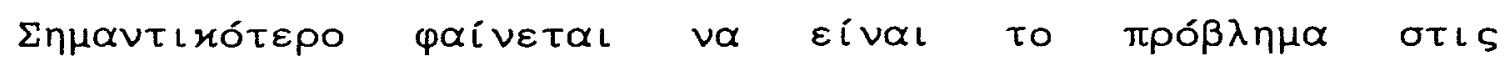

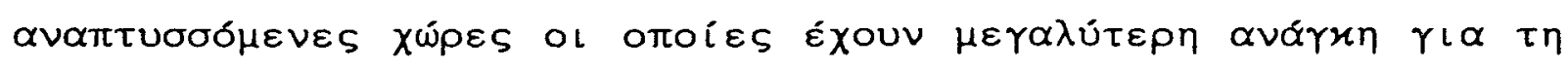

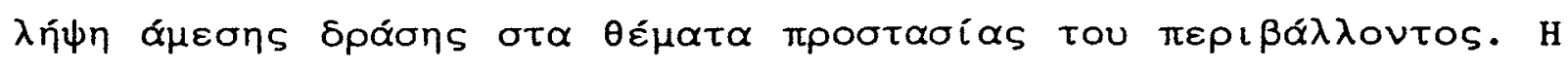

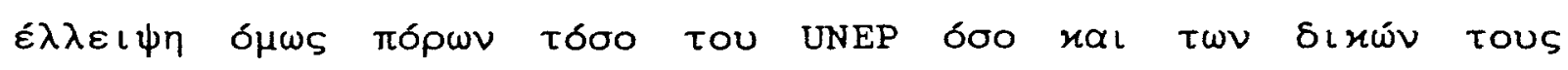

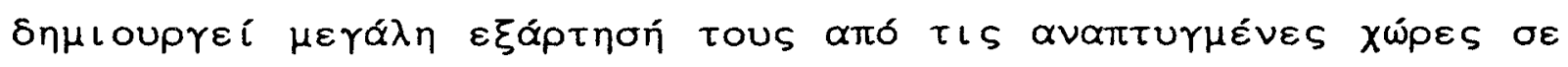

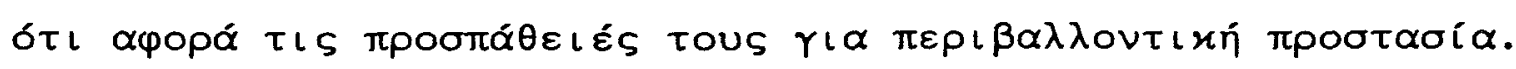




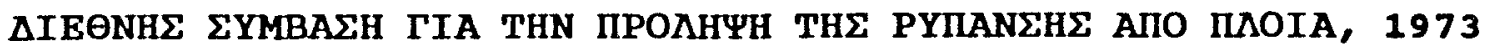
ń MARPOL

International Convention for the Prevention of Pollution from Ships (MARPOL), 1973

IIPSTOKONAO TOY 1978 ń MARPOL $73 / 78$ (Protocol of 1978 or MARPOL $73 / 78$ )

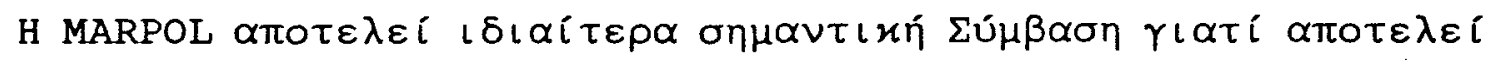

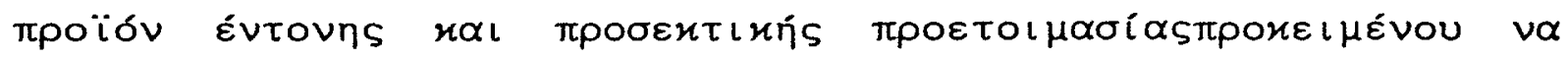

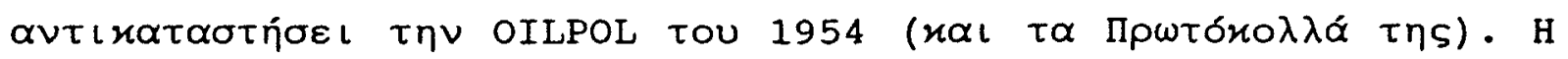

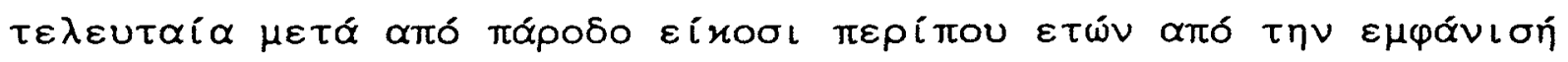

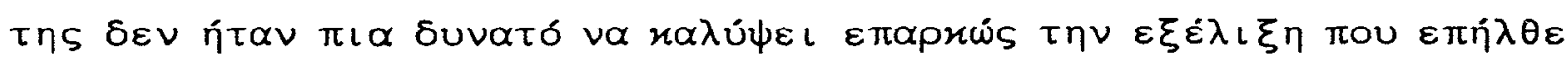

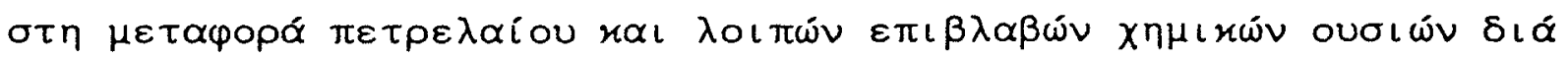
$\theta \alpha \lambda \alpha ́ \sigma \sigma \eta s$.

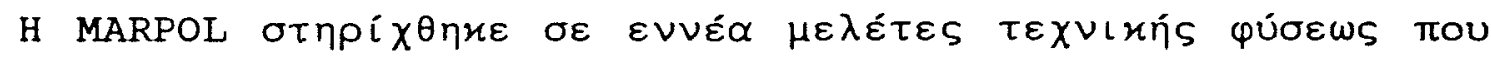

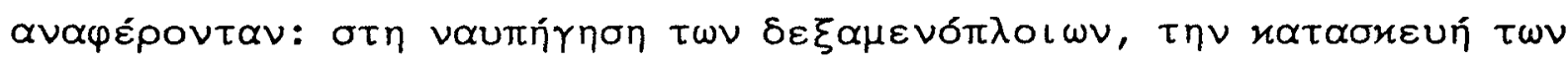

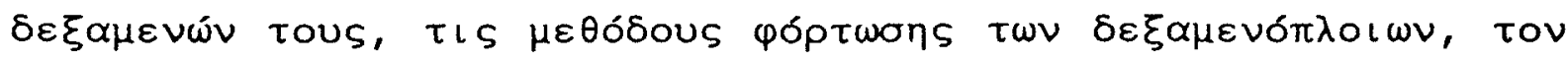

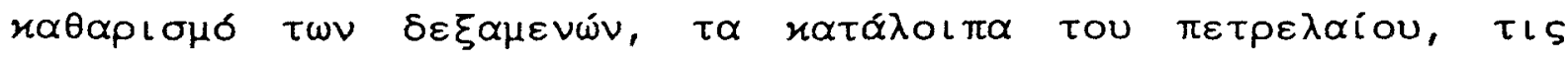

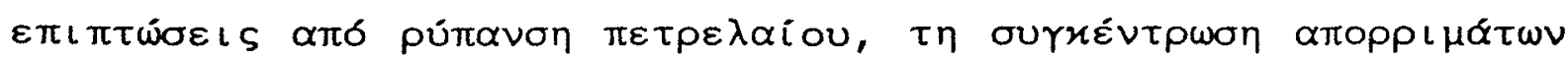

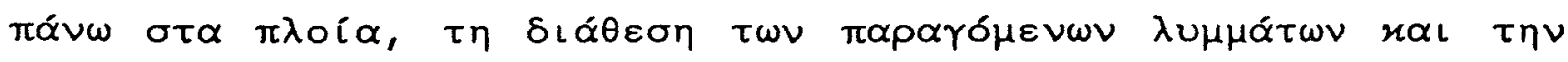

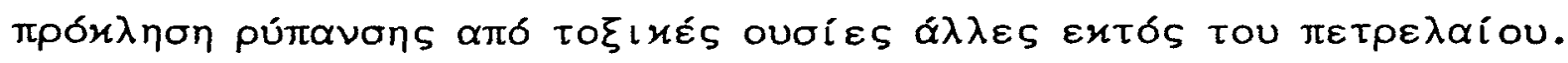




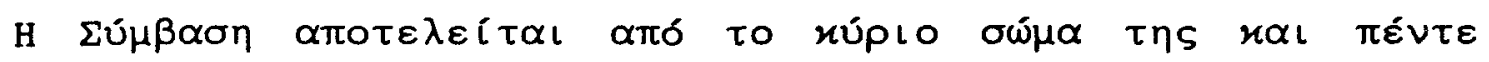

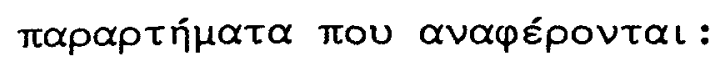

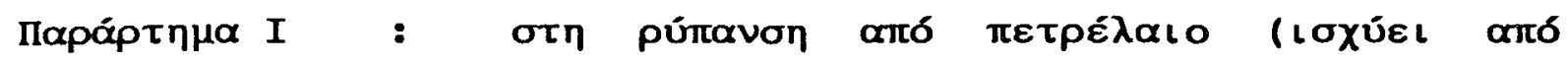
$2 / 10 / 1983)$

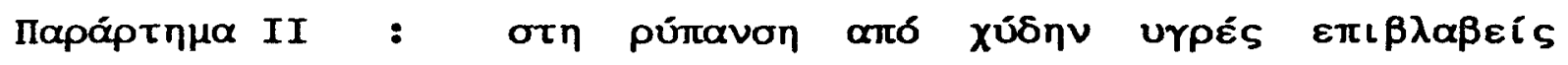

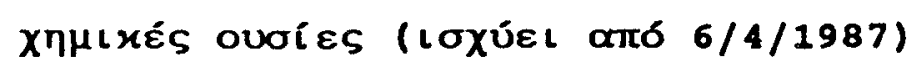

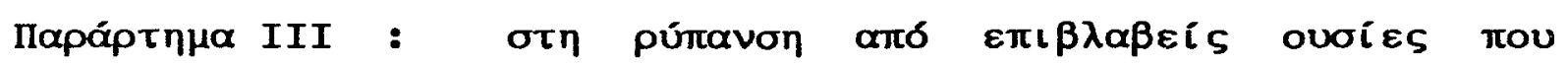

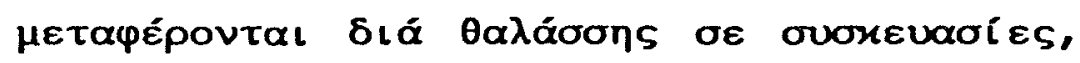

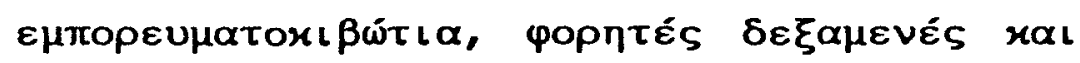

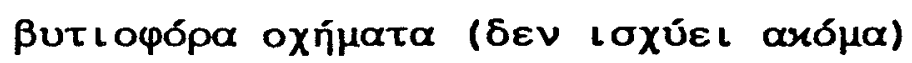

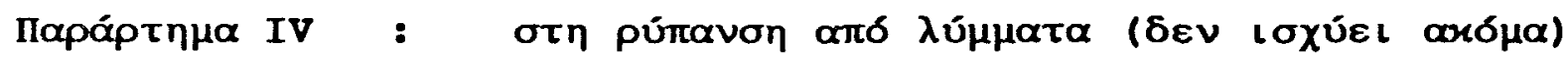

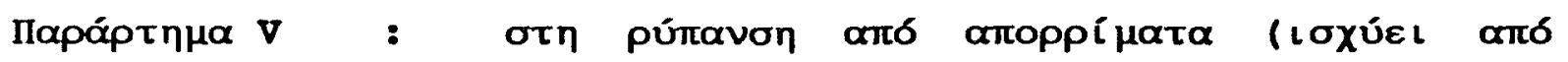
$31 / 12 / 1988)$

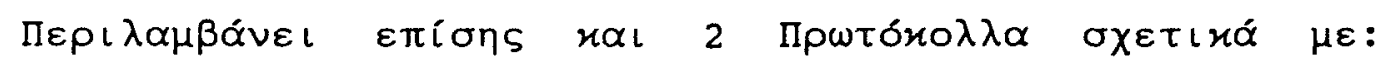

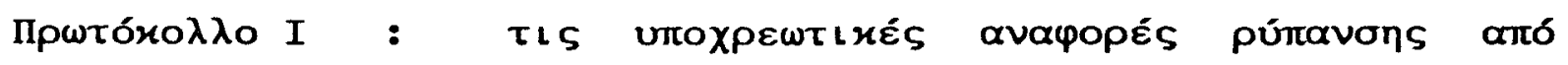
$\varepsilon \pi\llcorner\beta \lambda \alpha \beta \varepsilon i ́ s$ ouoís

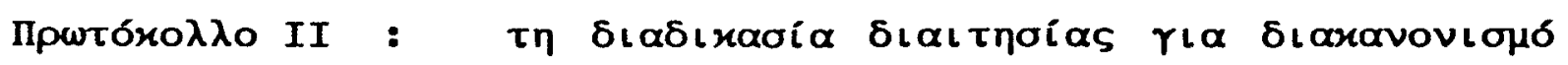
$\delta\llcorner\alpha \varphi \omega \vee\llcorner\omega ์$

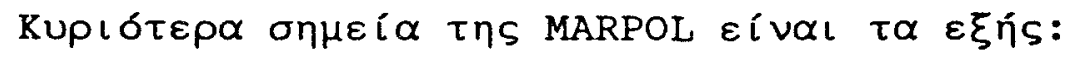

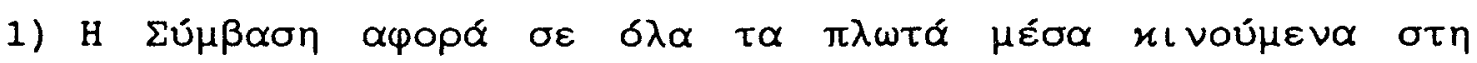

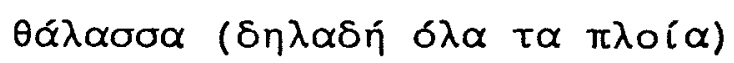




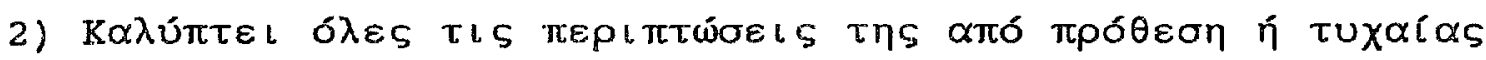

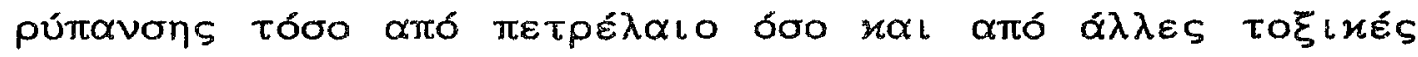

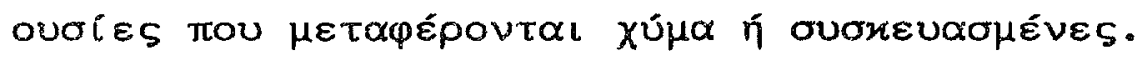

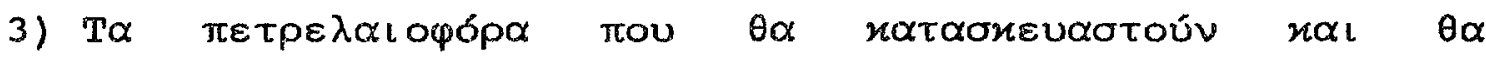

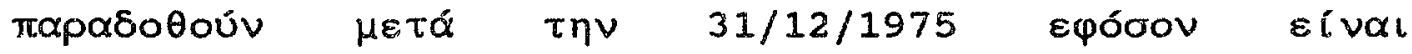

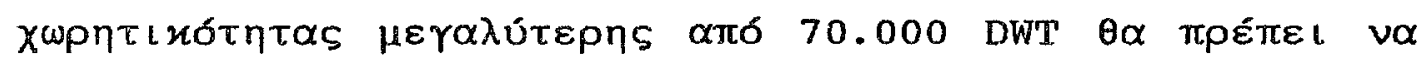

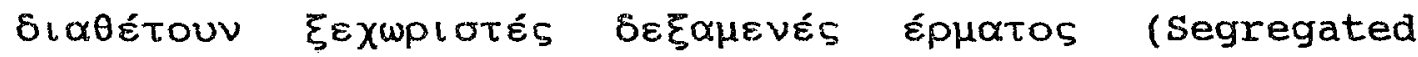

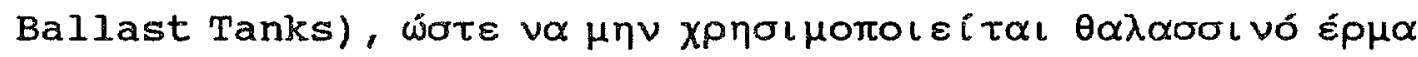

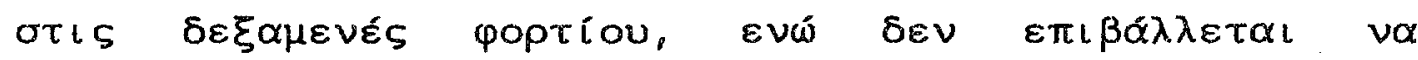

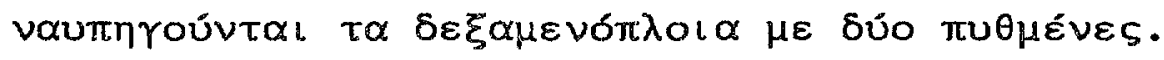

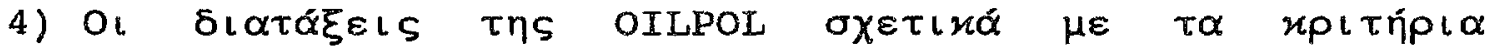

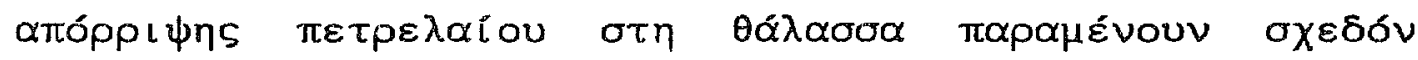

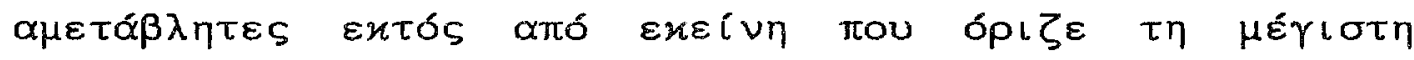

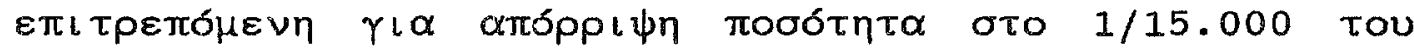

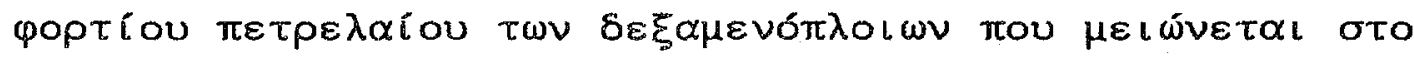
$1 / 30.000$.

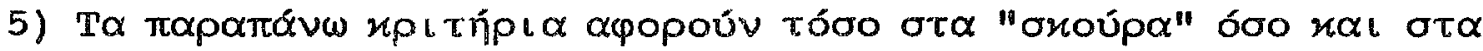

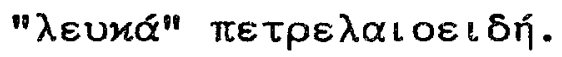

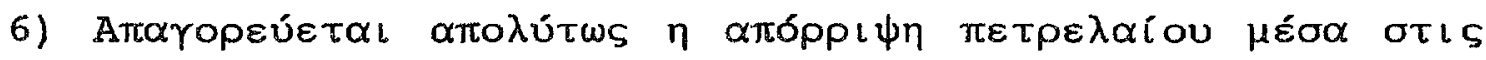

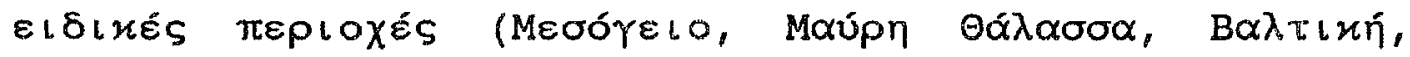

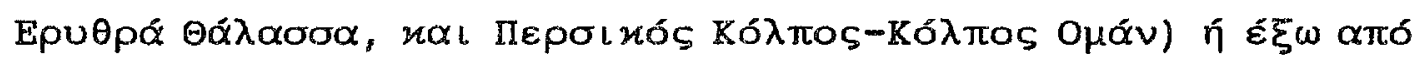

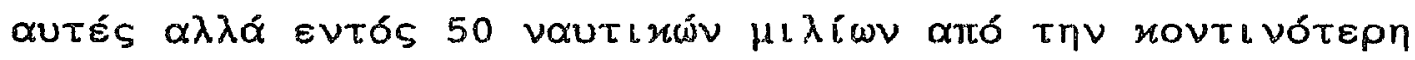

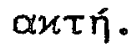




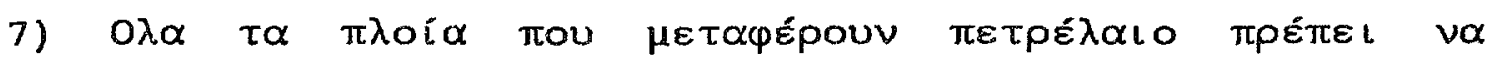

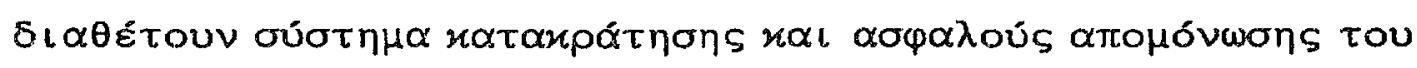

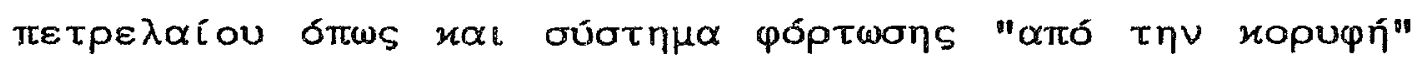
(Load on Top) rla va hropoúv va hóvouv tis

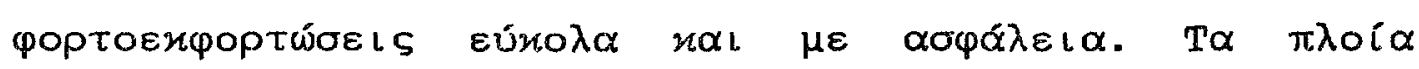

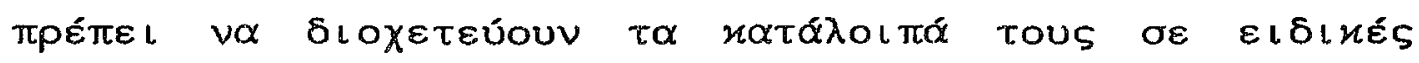

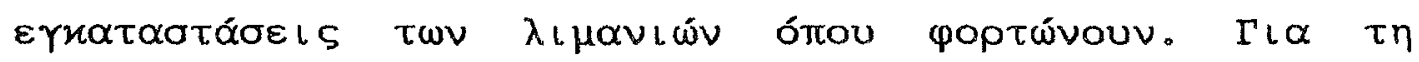

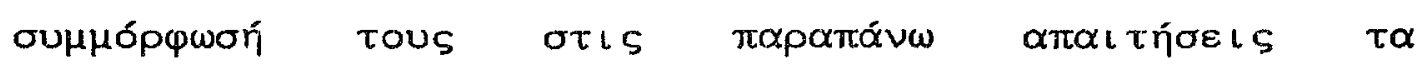

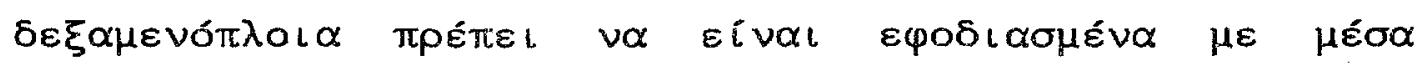

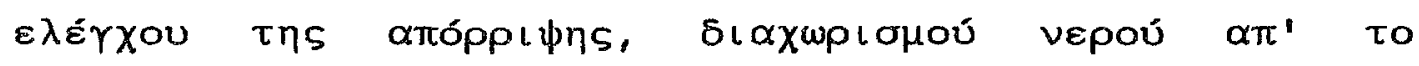
$\pi \varepsilon \tau \rho \varepsilon ́ \lambda \alpha \iota$ ○

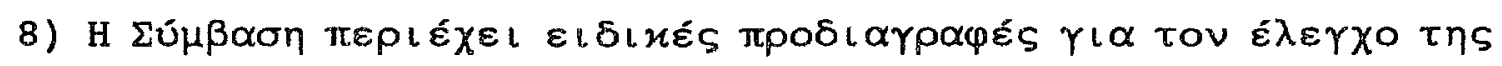

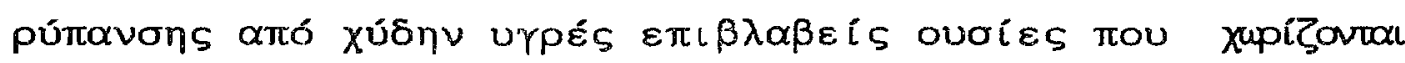

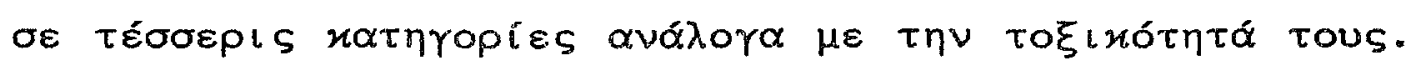

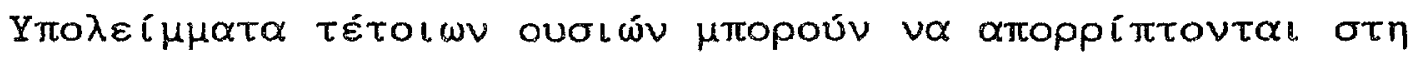

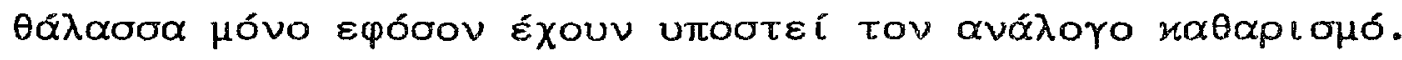

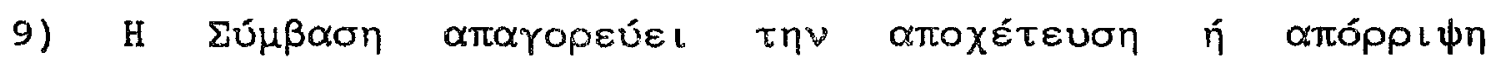

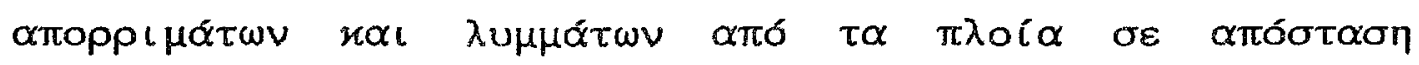

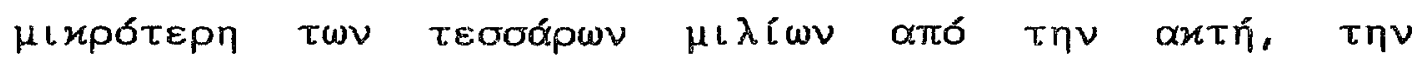

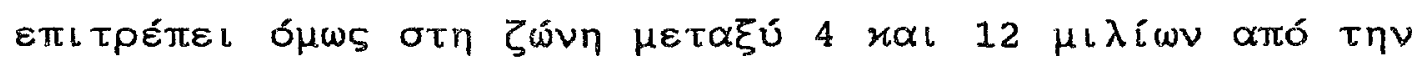

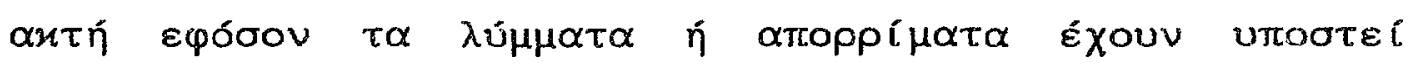

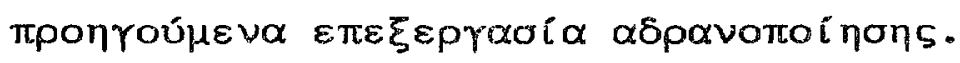




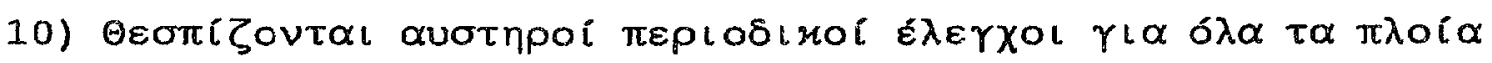

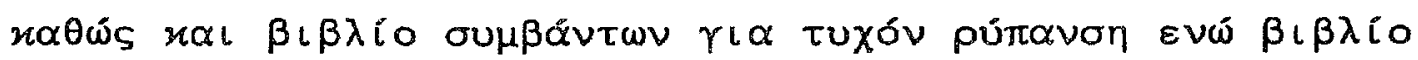

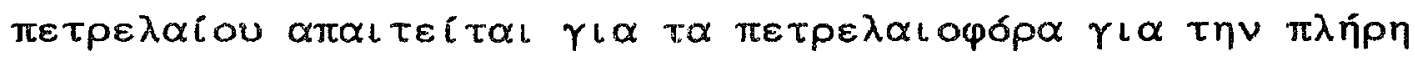

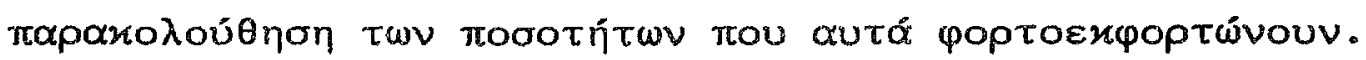

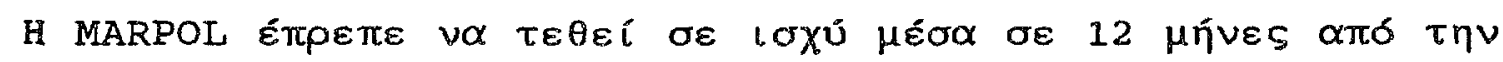

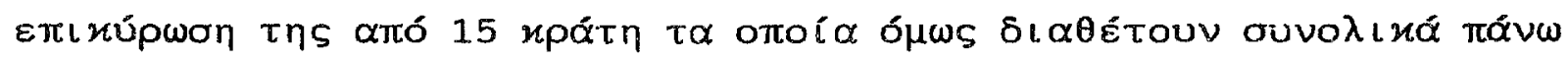

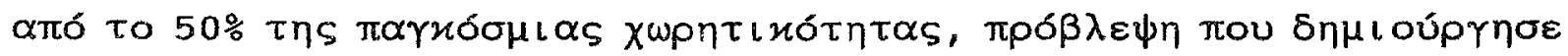

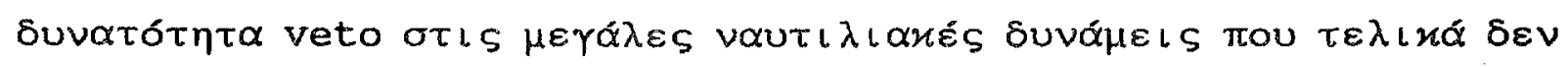

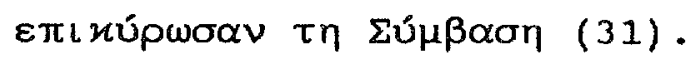

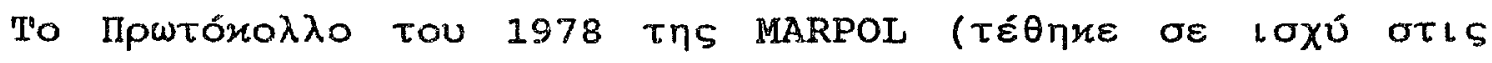

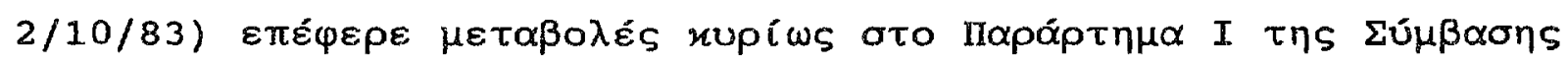

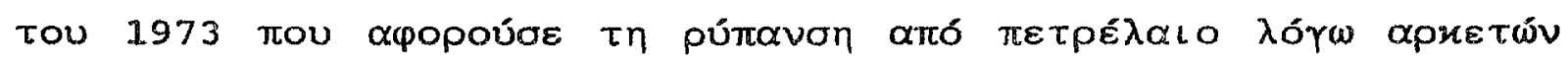

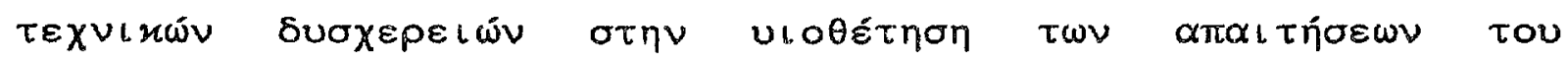

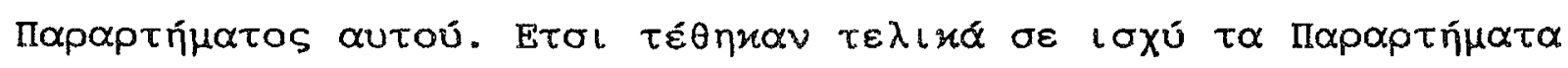

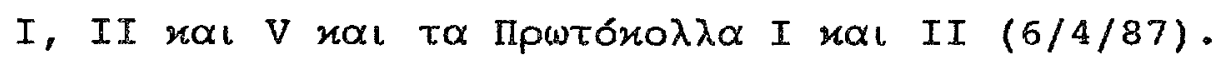




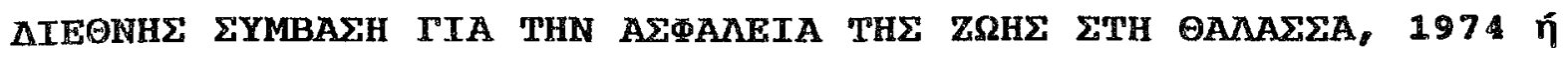
sOLAS.

International conventionn for the safety of Life at sea, 1974. IIPSTOKONAO tou 1978 (1978 Protocol) TPOMOIOIHEETE TOY 1983 (1983 Amendments)

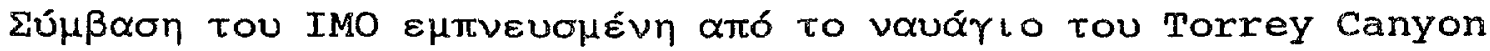

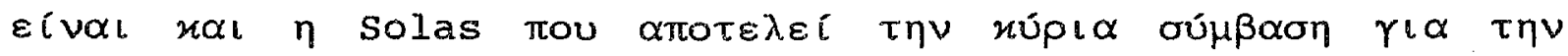

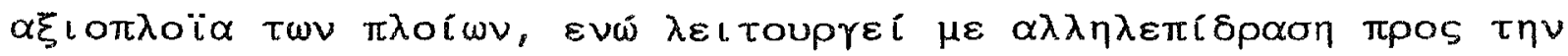

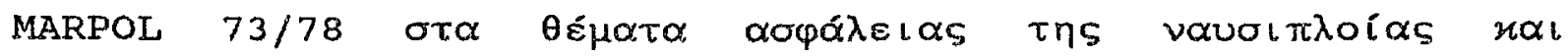

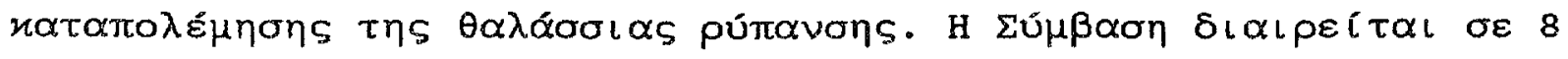

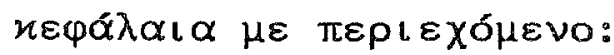

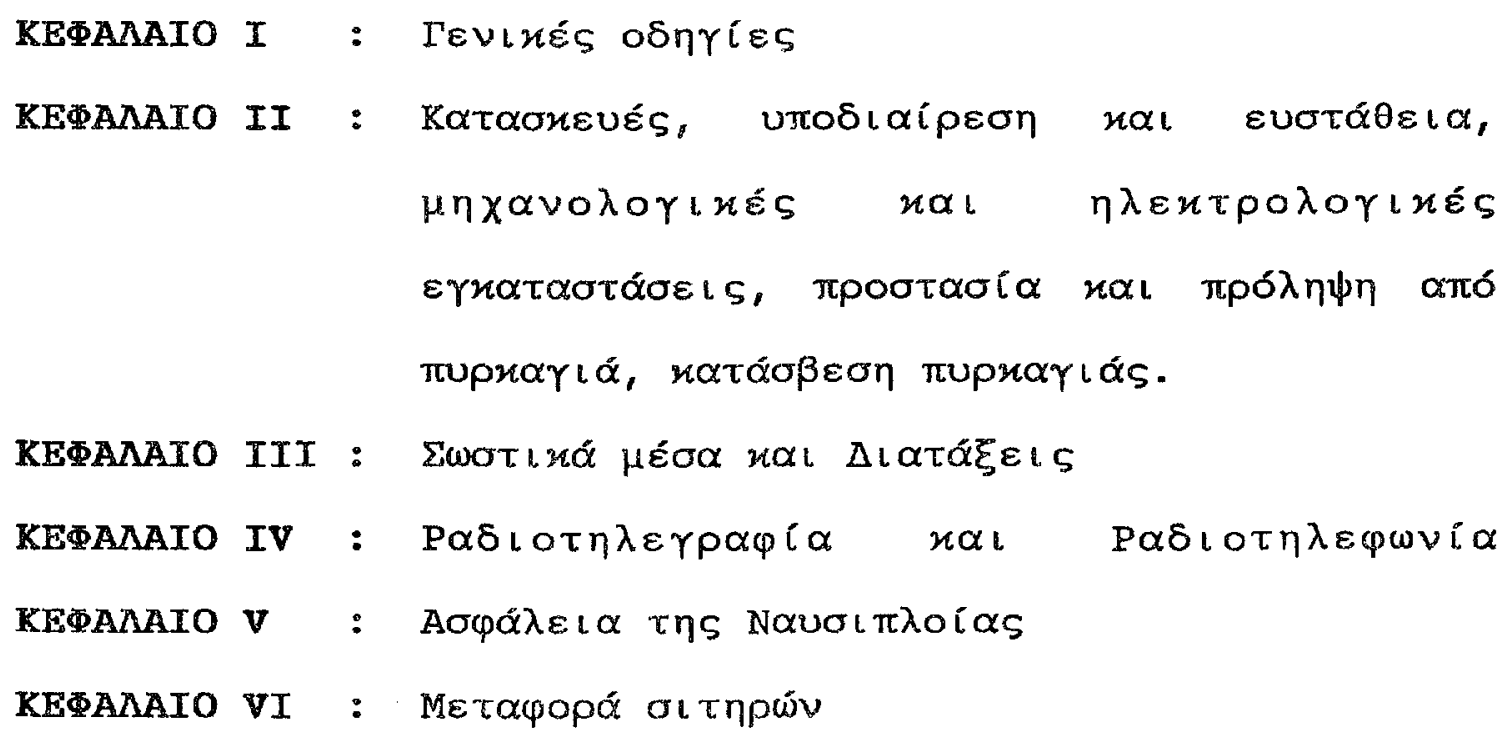




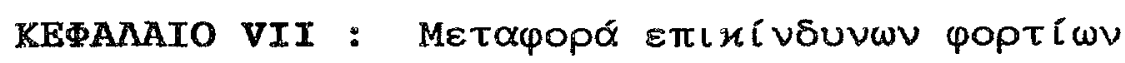

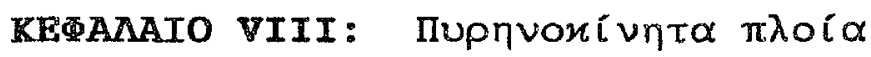

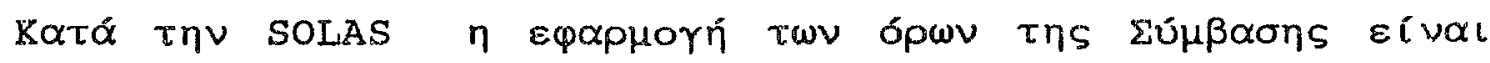

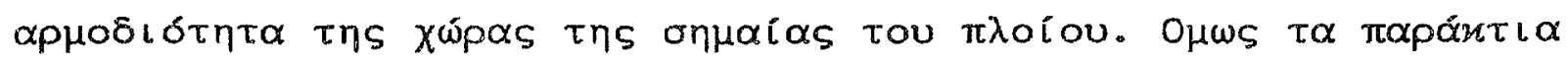

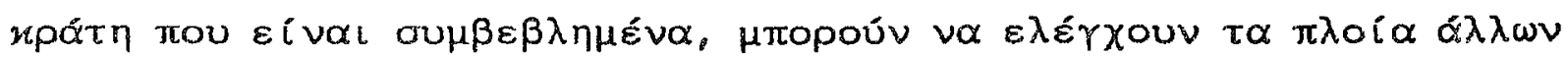

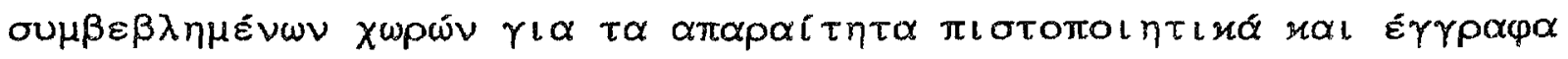

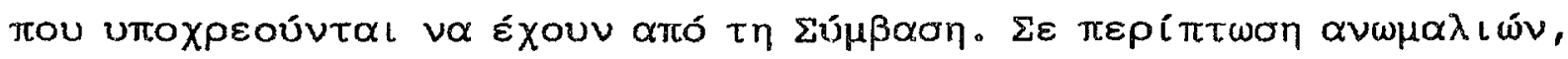

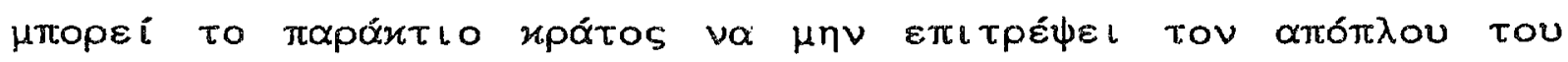

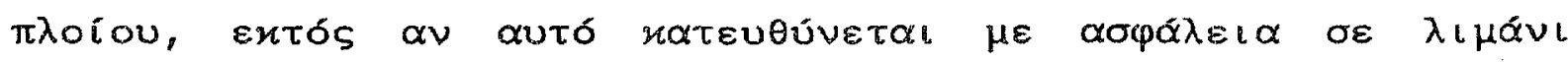

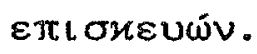

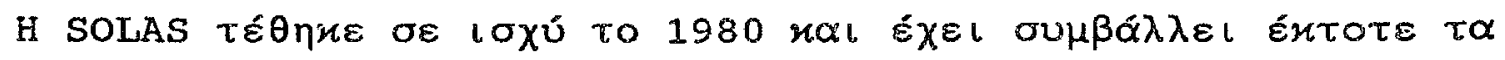

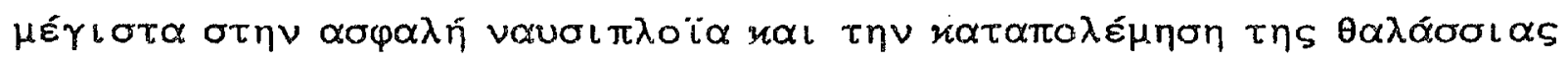
púmavợร.

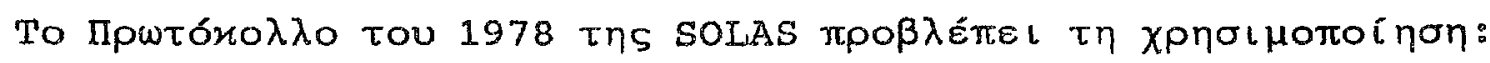

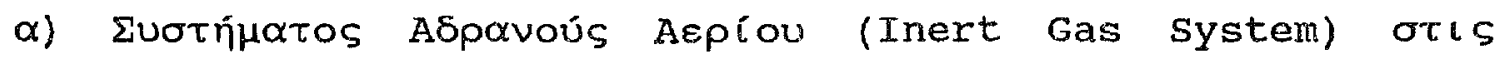

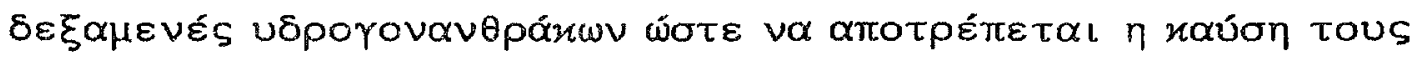
( $\lambda$ ó

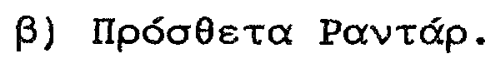

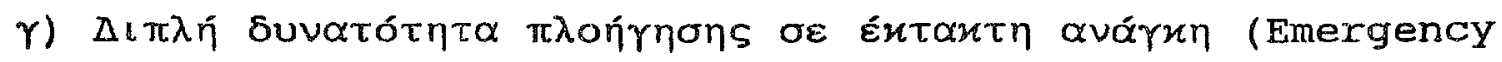
steering Gear).

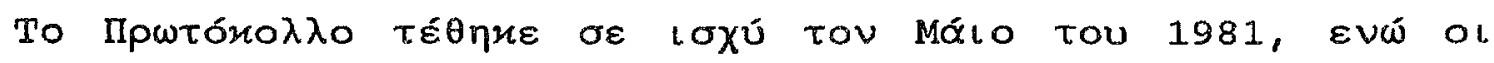

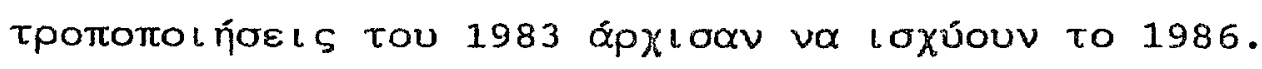




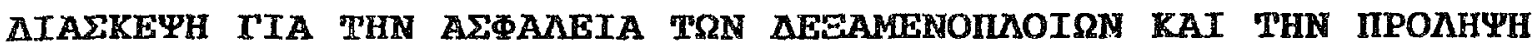

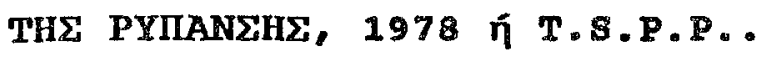 \\ Conference on Tanker Safety and pollution Prevention, 1978.}

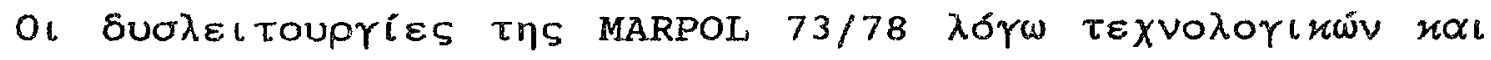

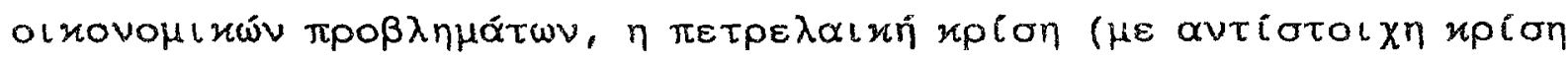

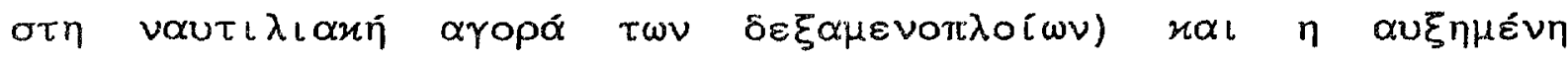

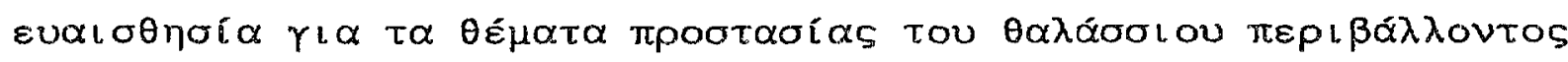

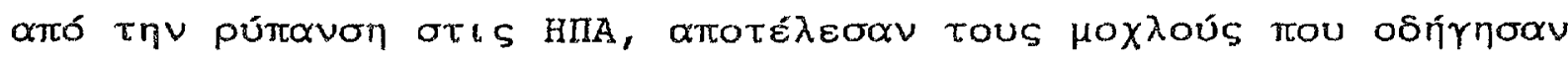

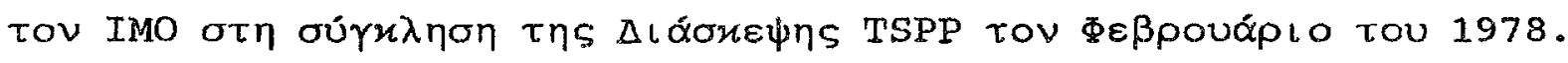

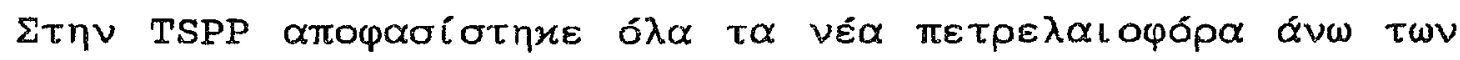

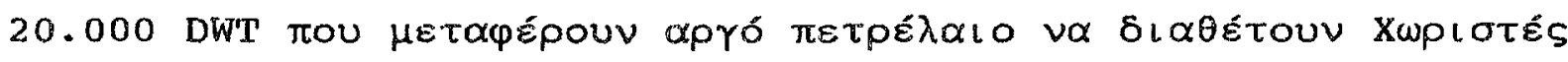

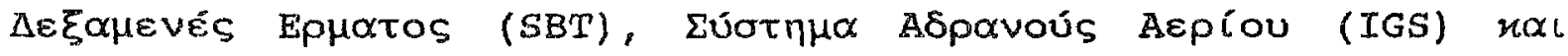

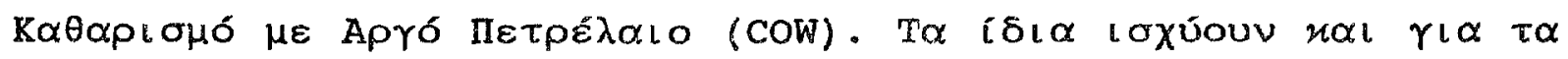

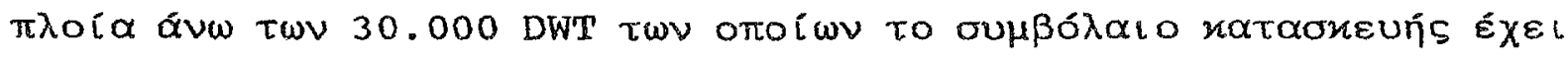

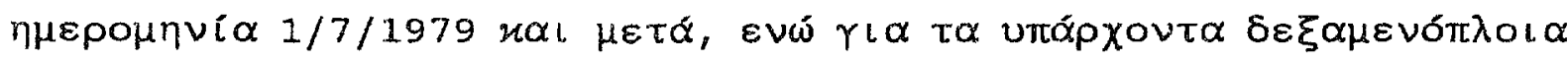

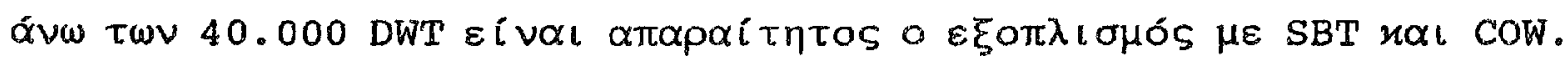

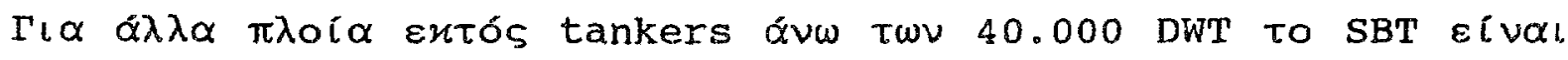

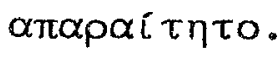

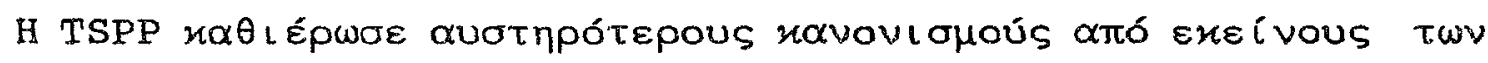

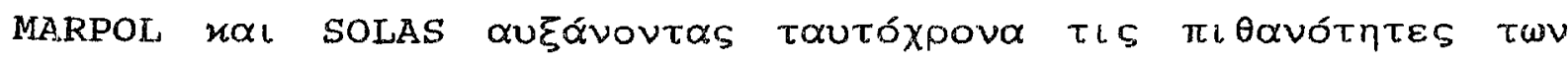

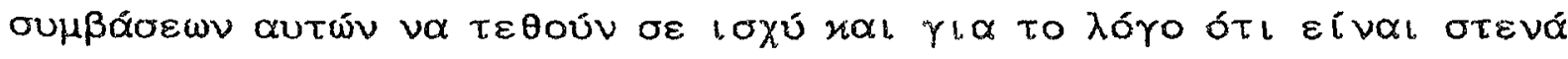

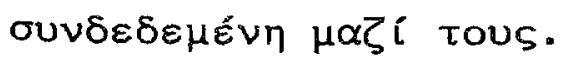




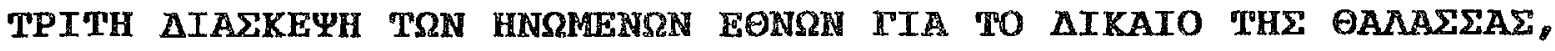
1982 ท́ UNCLOS III。

Third united Nations confsrence on the waw of the sea,1982

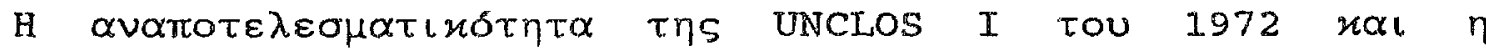

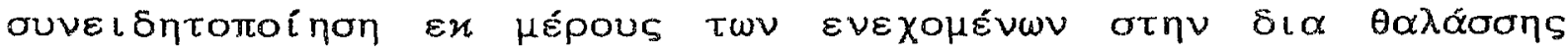

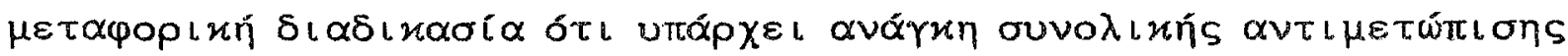

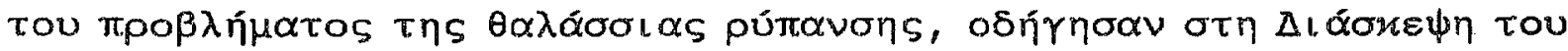

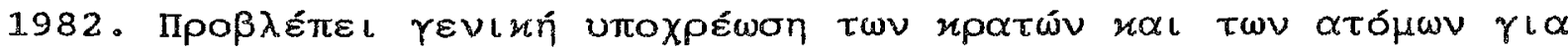

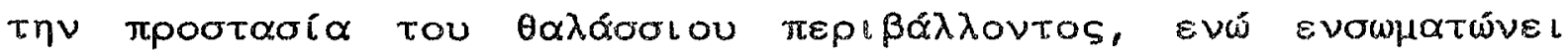

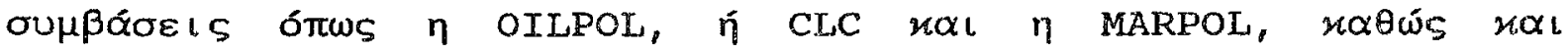

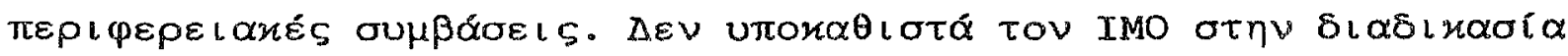

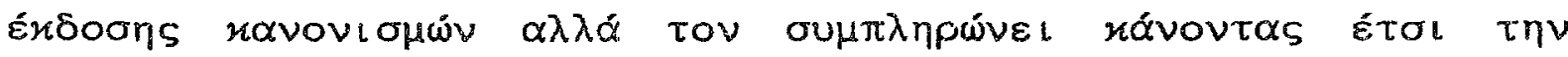

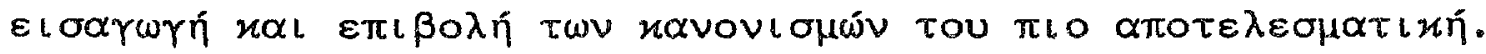

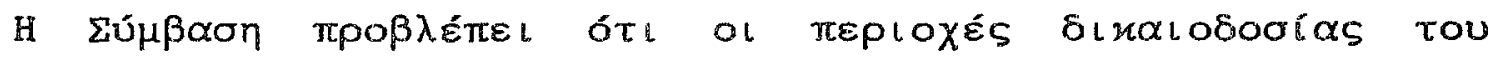

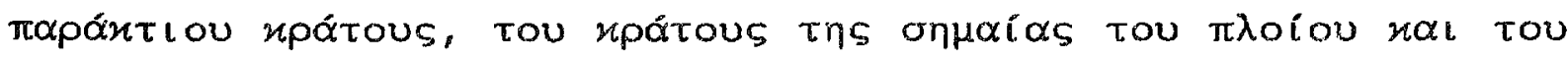

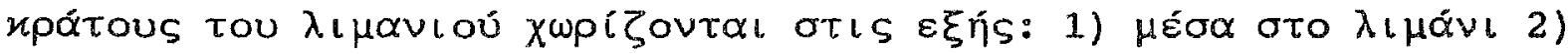

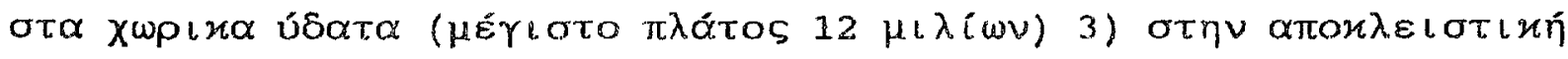

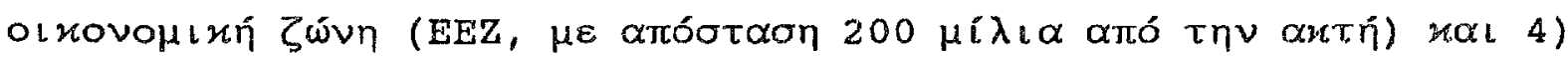

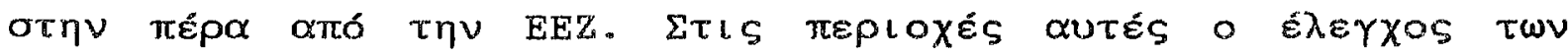

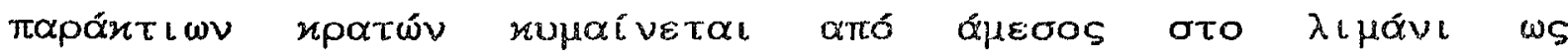

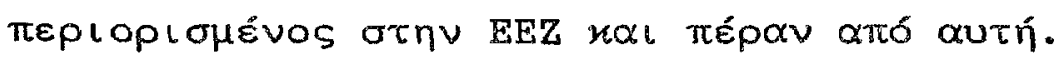




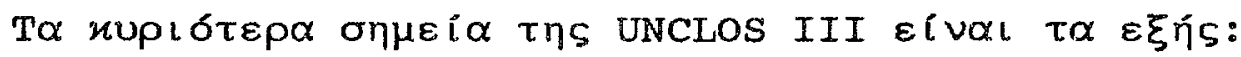

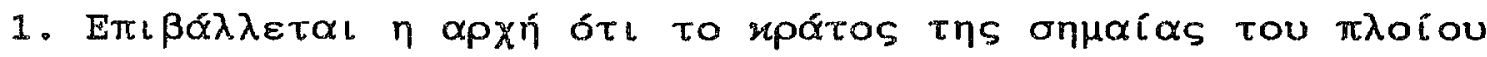

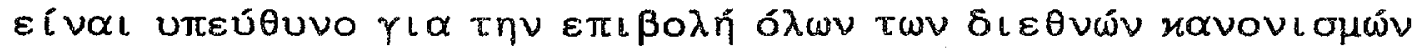

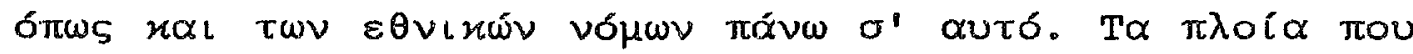

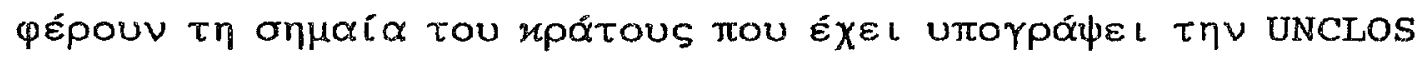

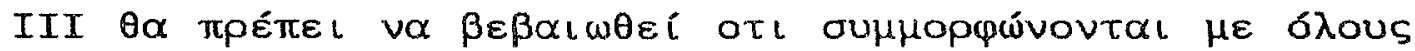

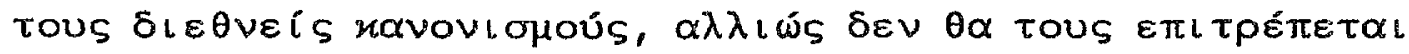

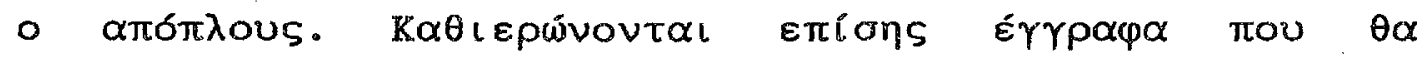

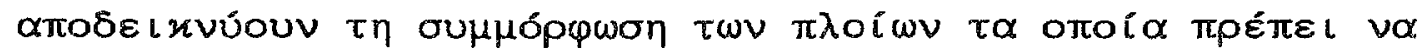

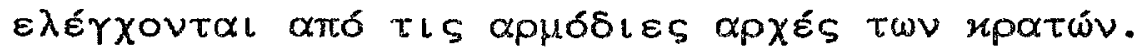

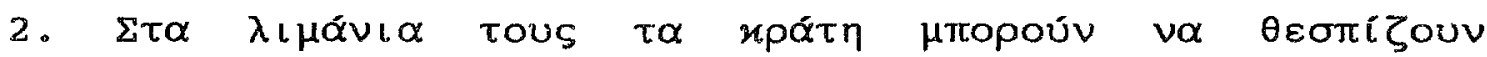

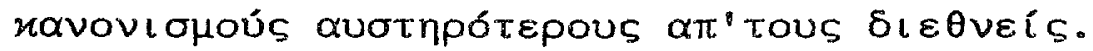

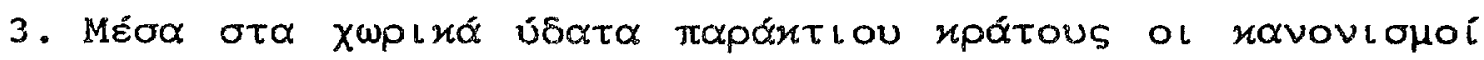

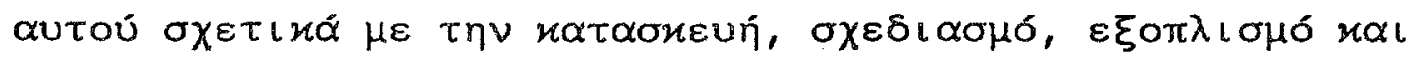

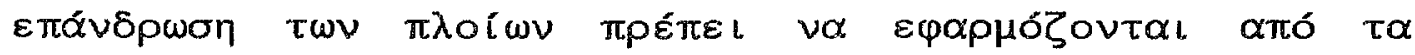

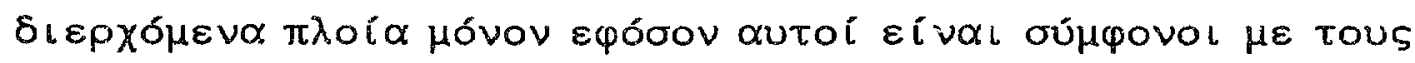

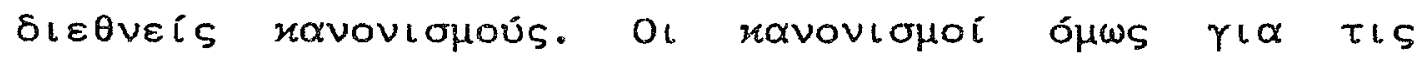

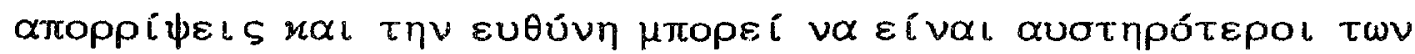

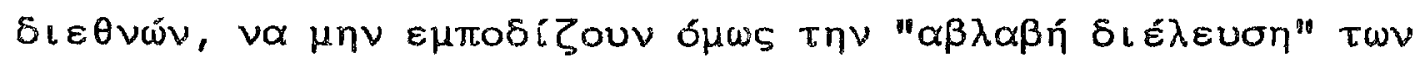
$\pi \lambda \circ i \omega \nu$. 


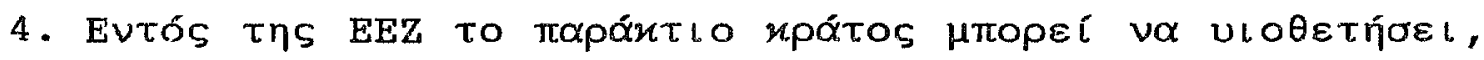

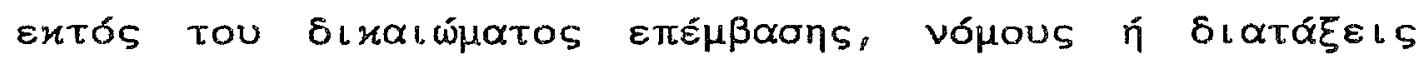

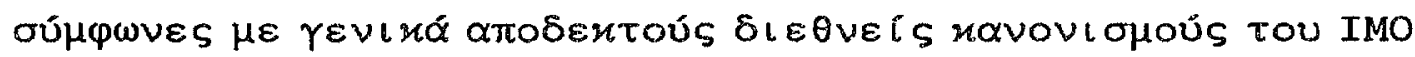

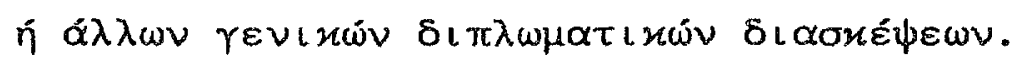

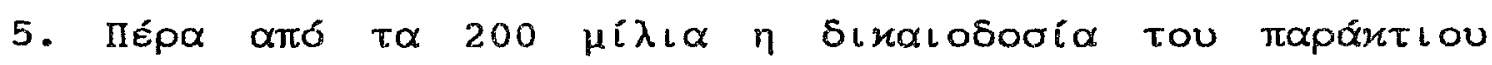

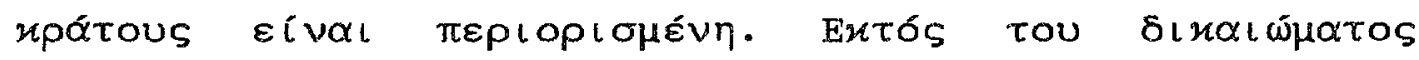

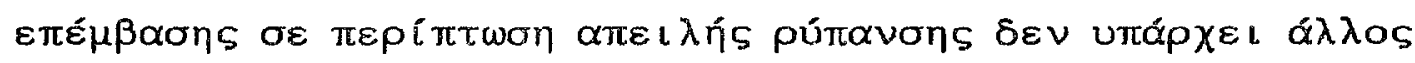

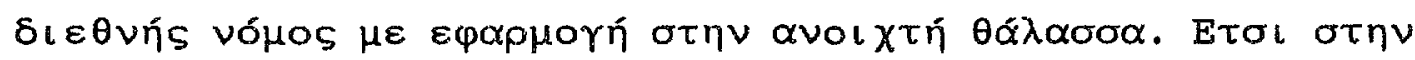

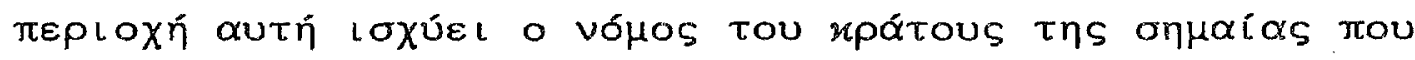

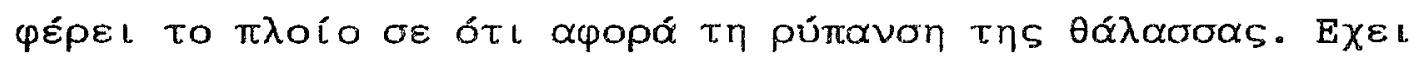

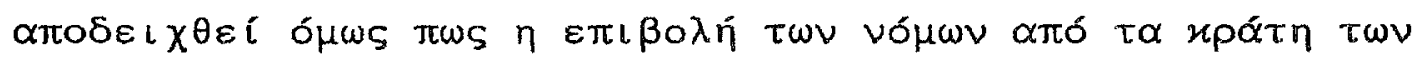

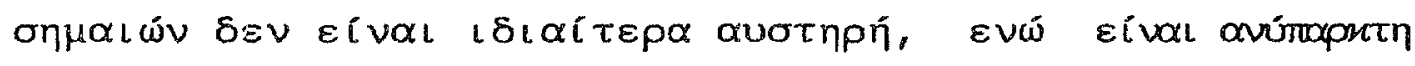

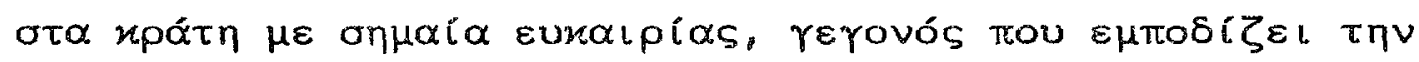

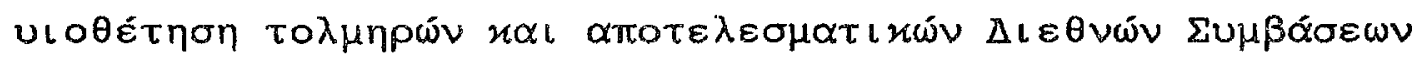
(32).

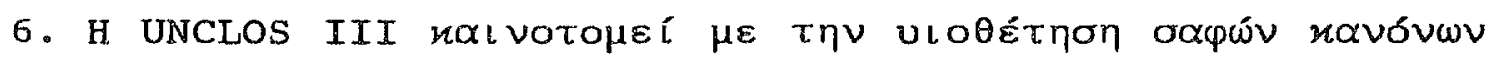

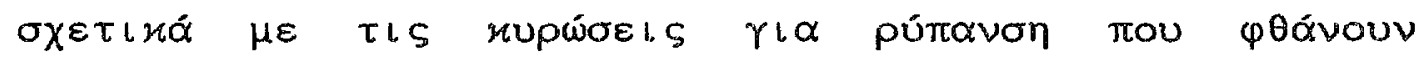

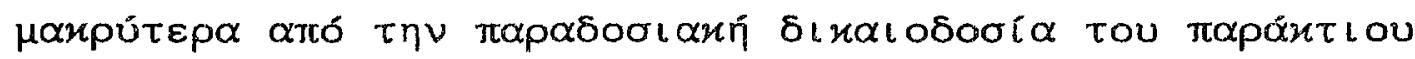

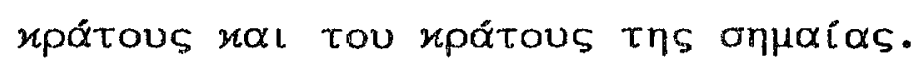

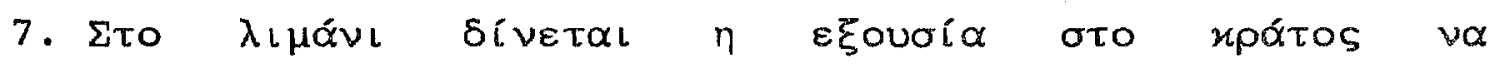

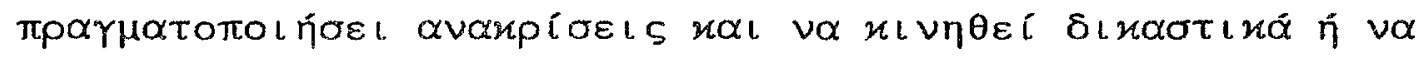

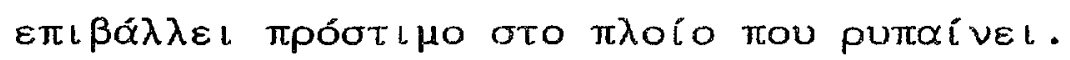




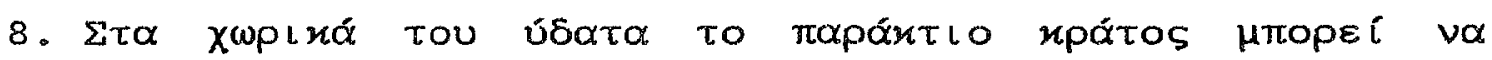

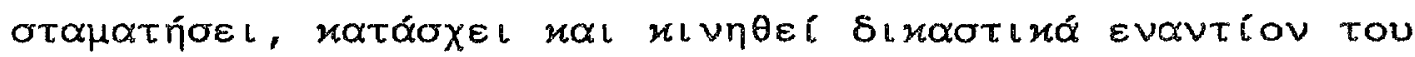

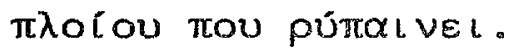

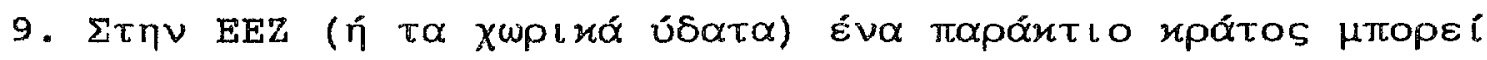

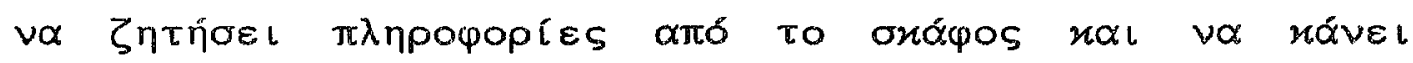

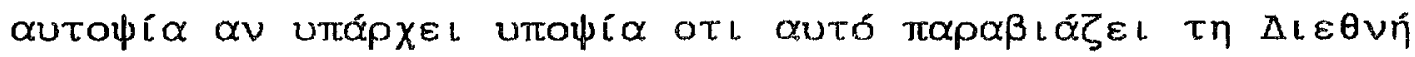

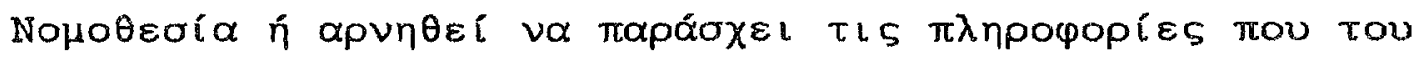

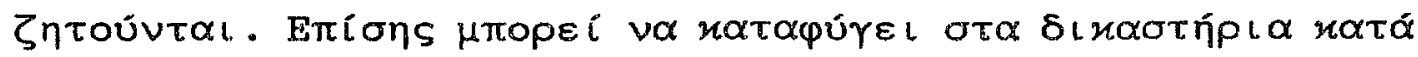

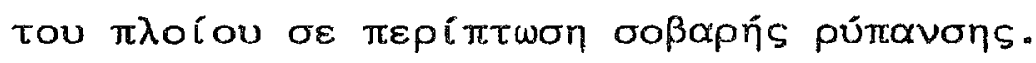

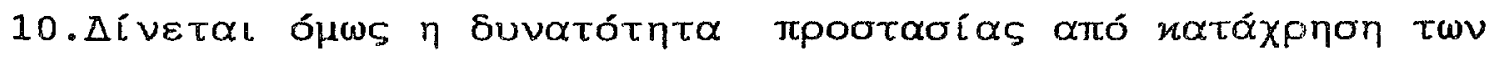

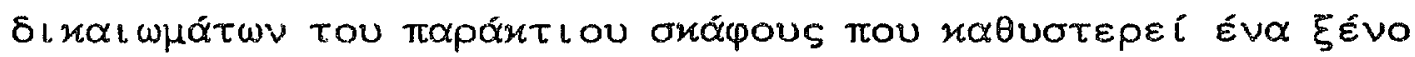
oxর์ழos.

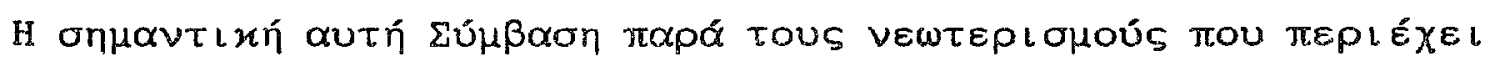

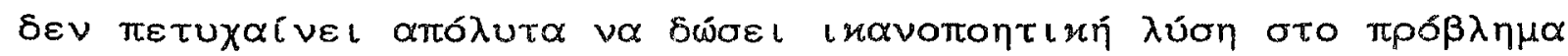

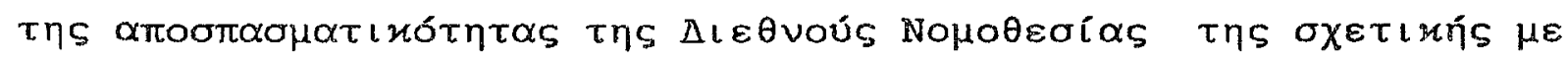

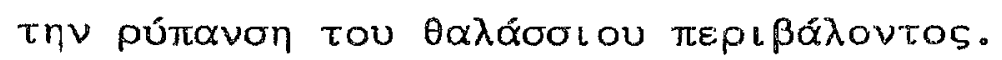




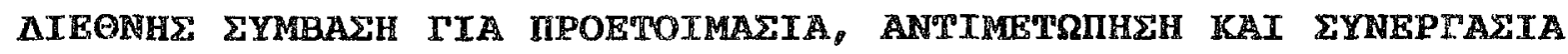
KATA THE PYMANEHE ANO METPENATO, 990. Interntional convention on oil Pollution Pesponse and Comoperation, London, 1990.

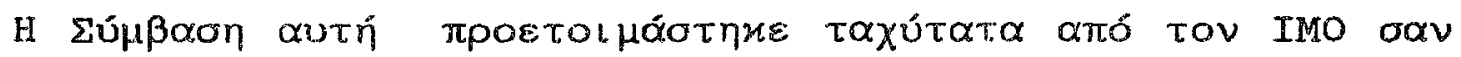

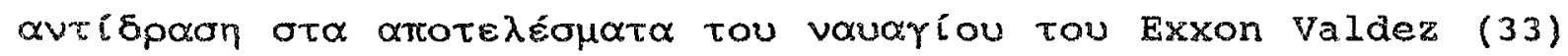

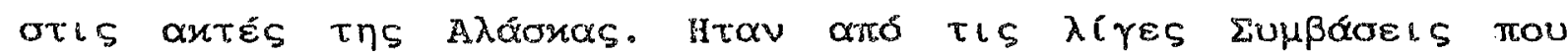

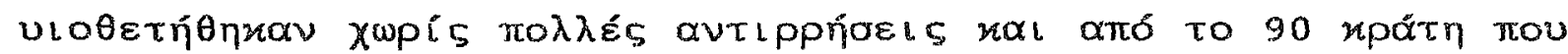

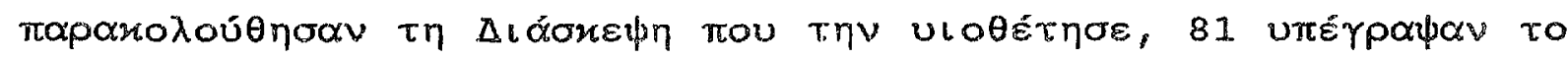

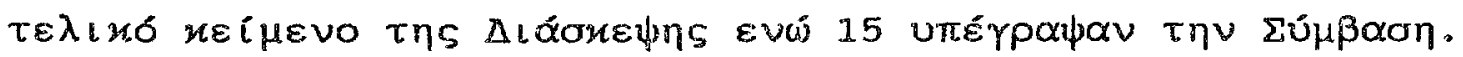

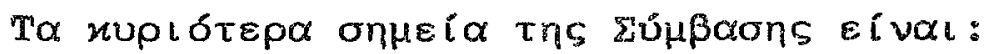

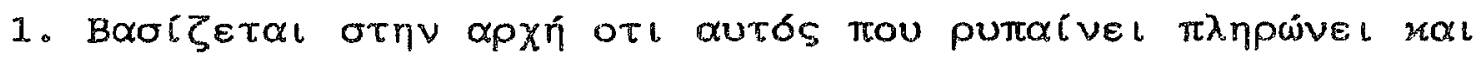

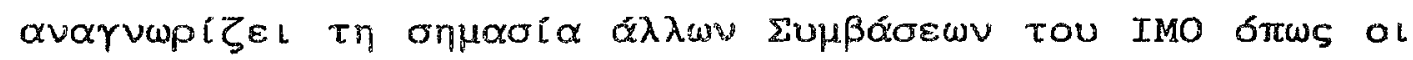
SOLAS, MARPOL, CCLC,FUND hoOẂs haL t7S UNCLOS III.

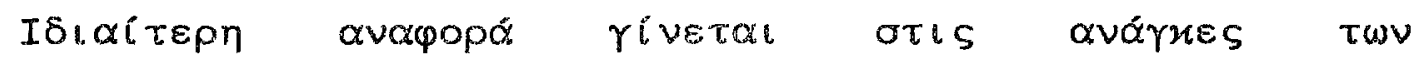

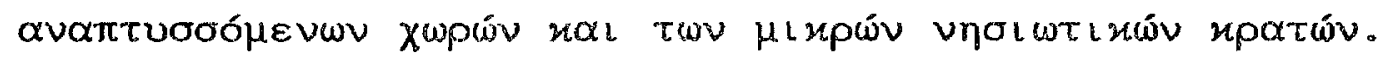

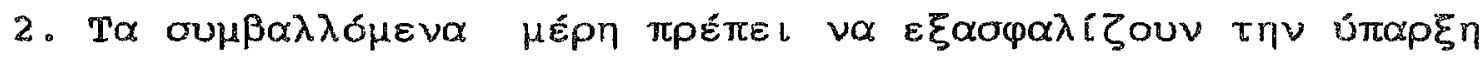

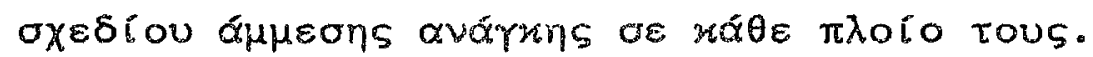

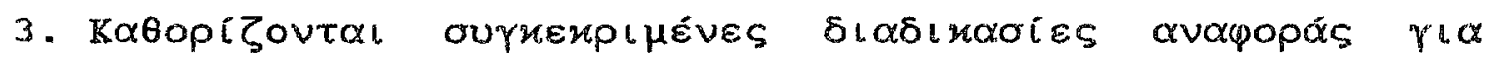

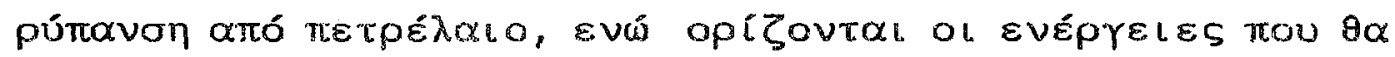




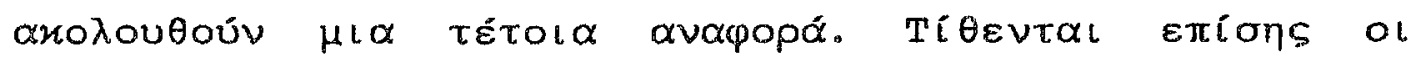

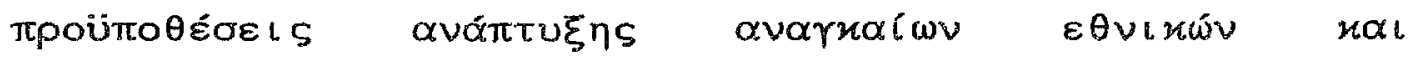

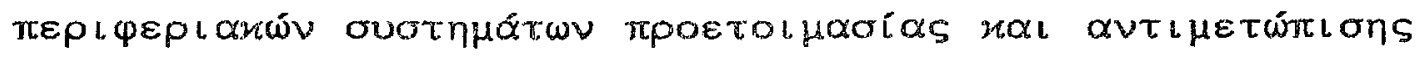

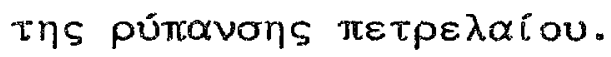

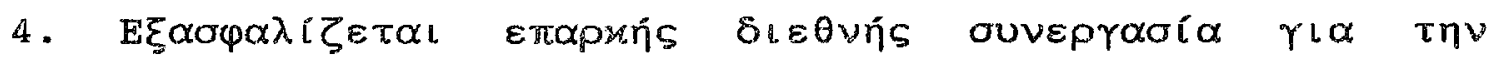

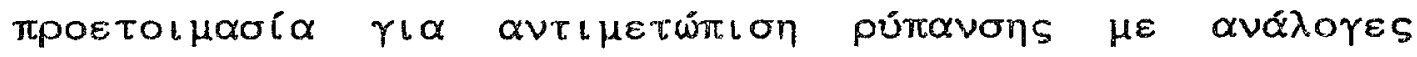

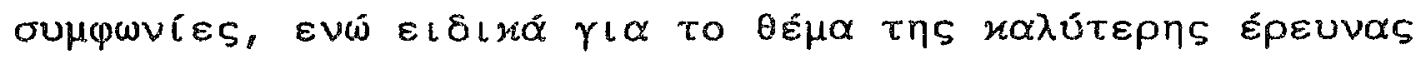

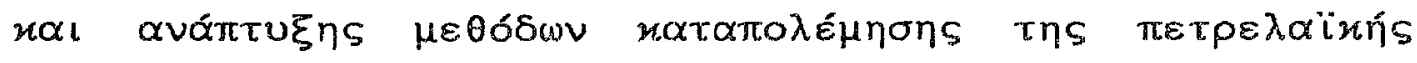

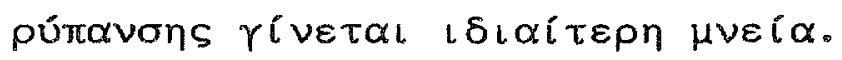

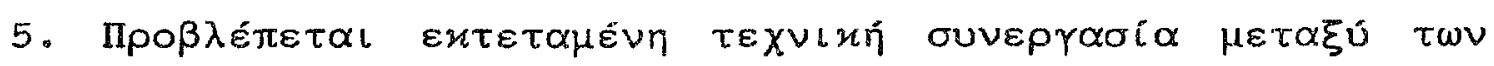

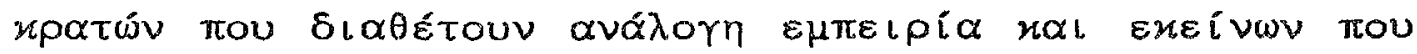

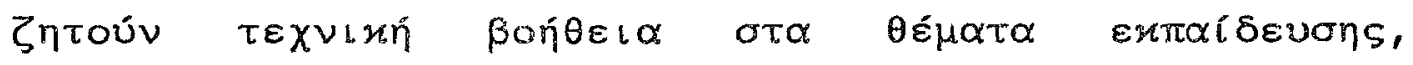

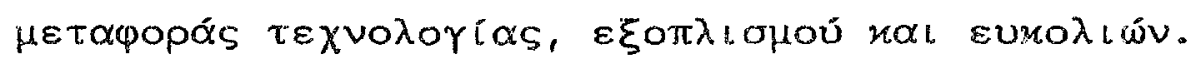

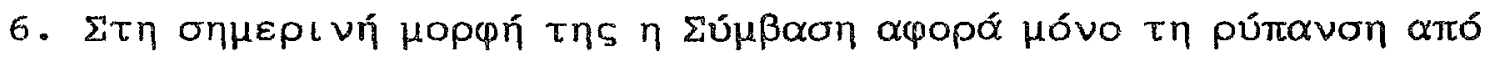

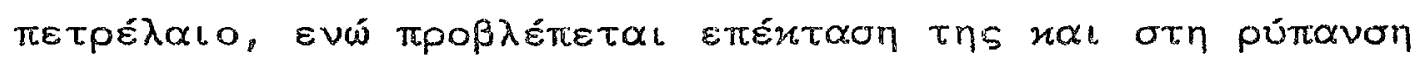

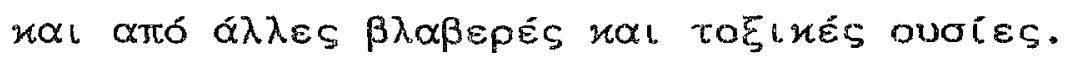

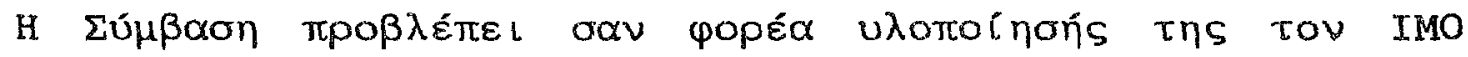

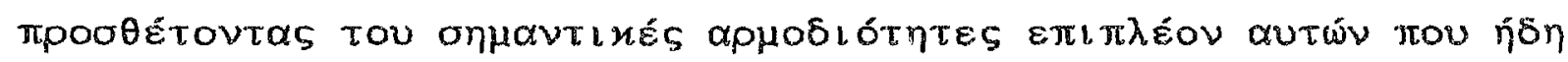

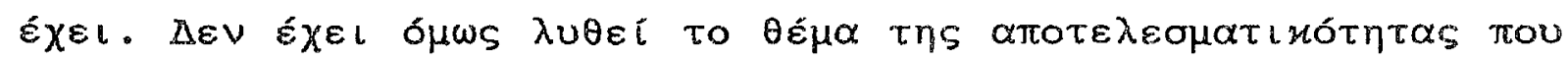

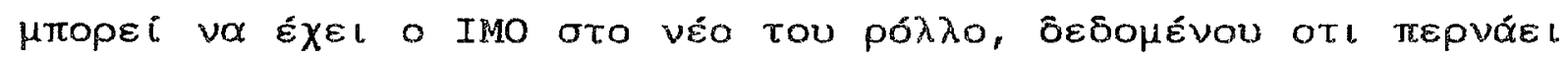

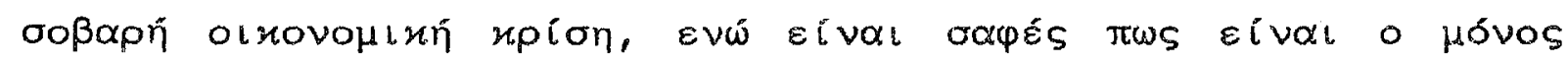

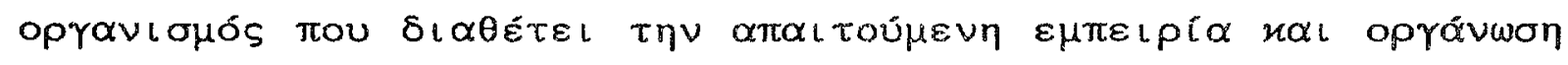
(34). 


\section{SHMEIREEIE}

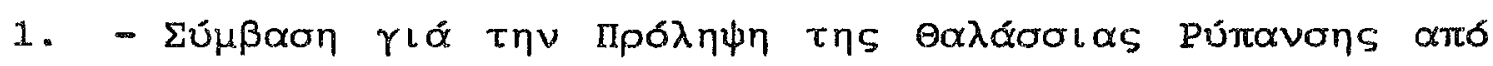

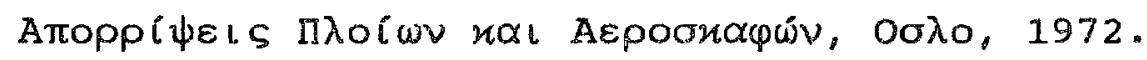

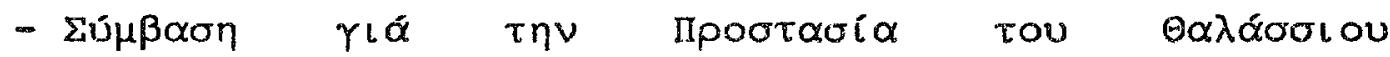

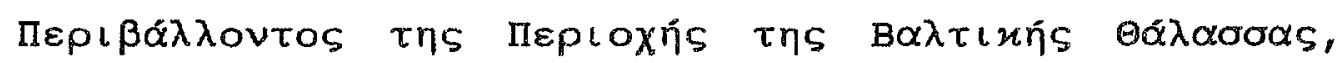
Eגoívหl, 1974.

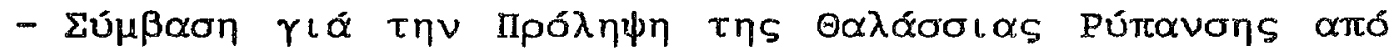

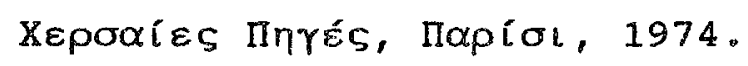

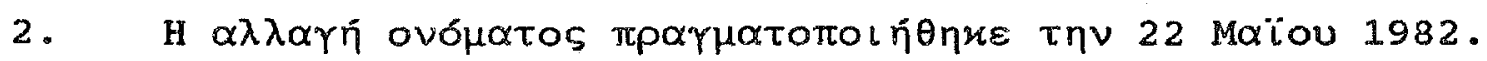

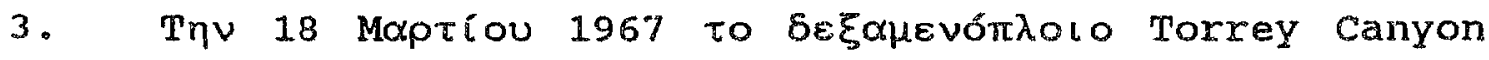

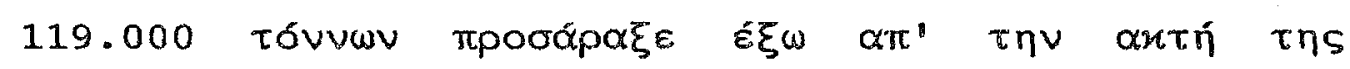

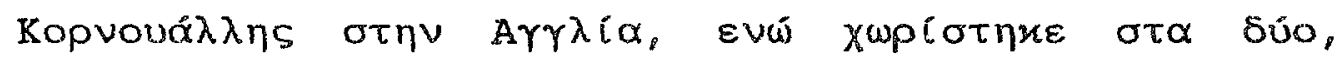

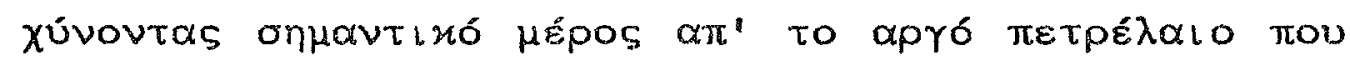

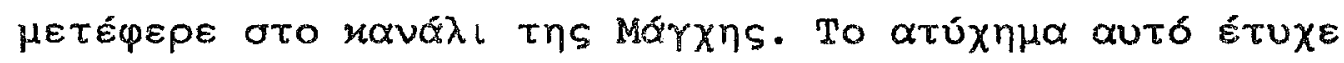

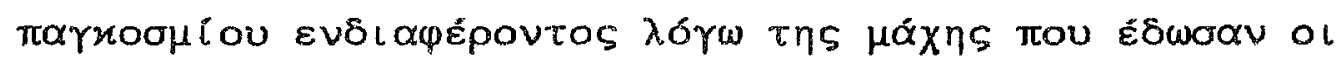

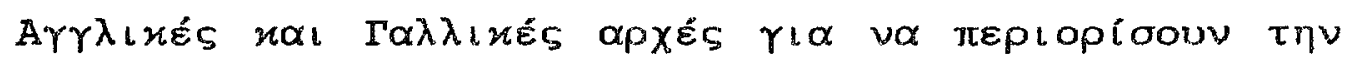
$\tau \varepsilon \rho \alpha ́ \alpha \sigma \iota \alpha \pi \varepsilon \tau \rho \varepsilon \lambda \alpha\llcorner\circ \varkappa \eta \lambda\lceil\delta \alpha$. 


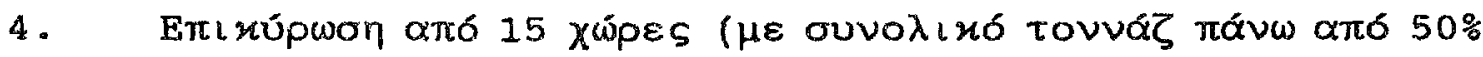

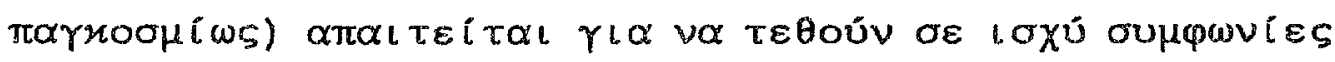

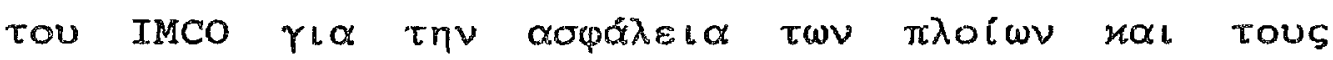
kavovionoús ratá ths pútravons.

5. K. Ramakrishna, Environmental Concerns and the New Law of the Sea, J.M.I.C. TEúxos 16 No1. 0.9, Iav. 1985.

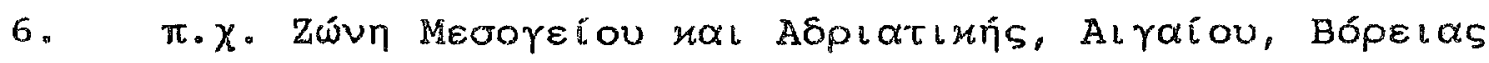

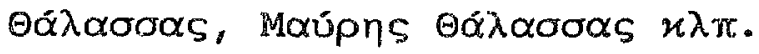

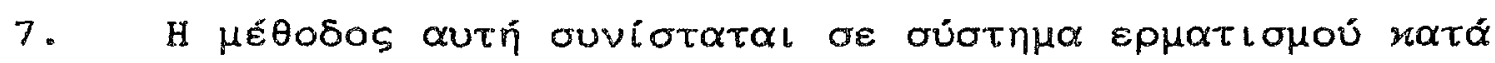

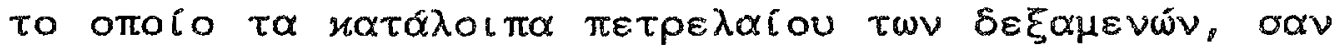

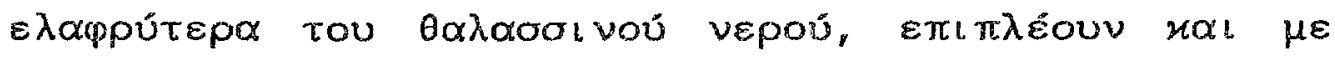

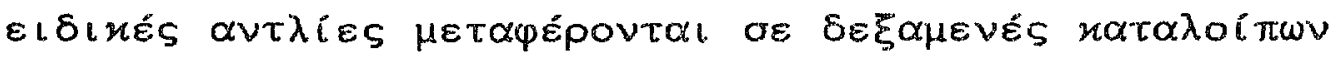

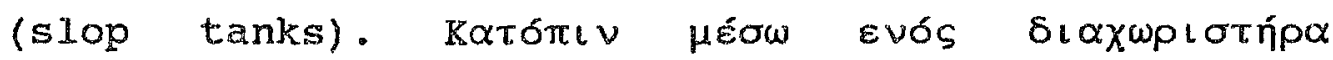

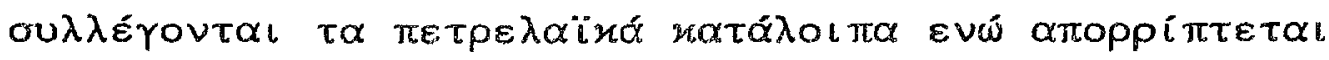

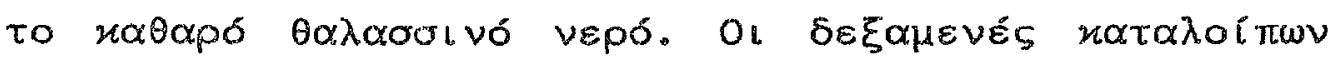

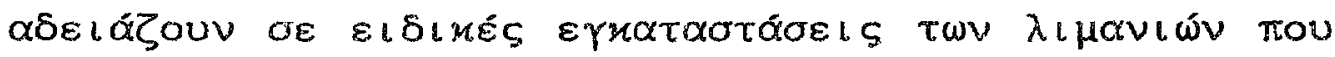

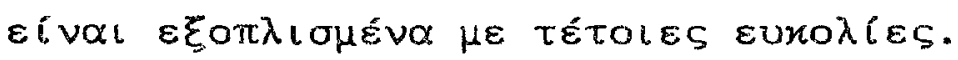

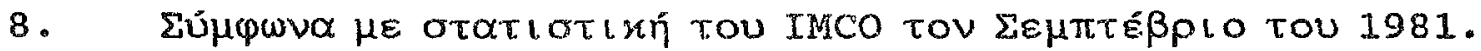


9. Dr. David Abecassis: "IMO and liability for oil pollution from ships:a retrospective", Lloyd's Maritime and Commercial Law Quarterly, $0.46, \Phi \varepsilon \beta .1983$.

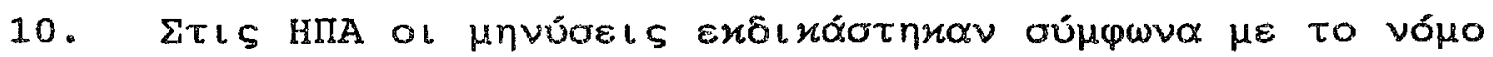

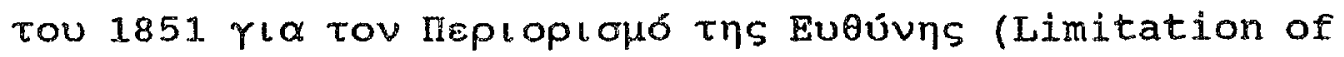

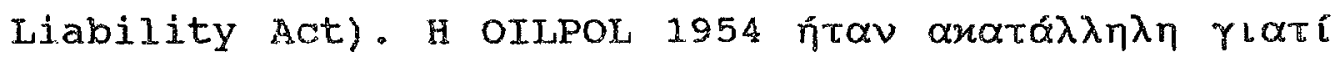

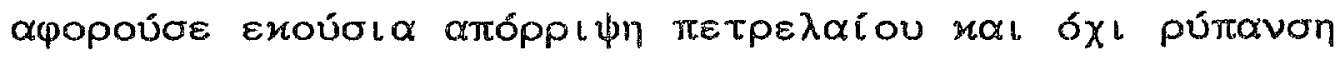

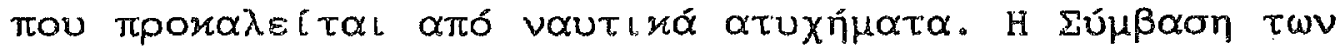

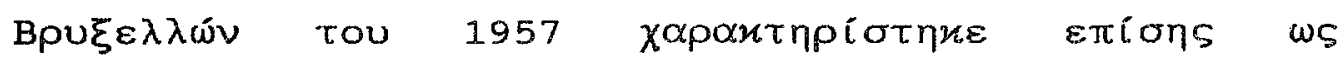
$\alpha \varkappa \alpha \tau \alpha \lambda \lambda \eta \lambda \eta$.

11. Dr David Abecassis: "TMO and liabiljty for oil pollution from ships: a retrospective". Lloyd's Maritime and Commercial Law Quarterly, $0.46, \Phi \varepsilon \beta .1983$.

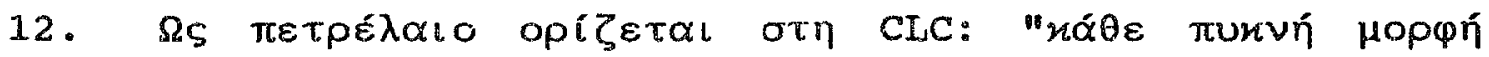

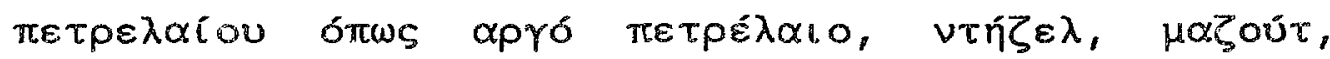

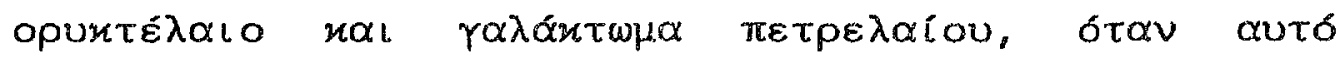

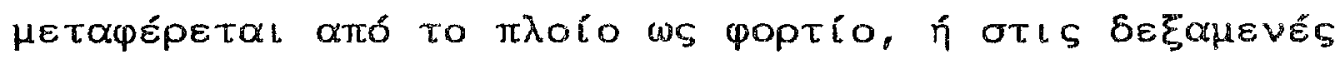

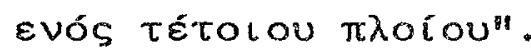




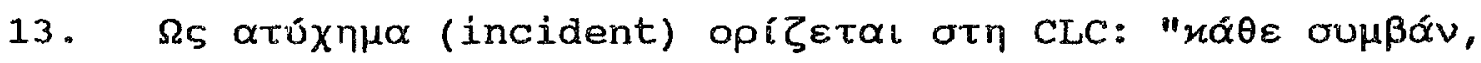

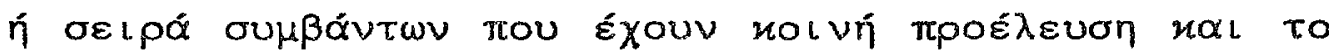

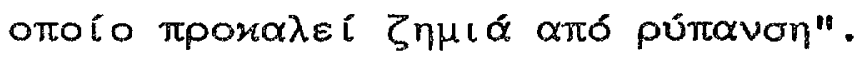

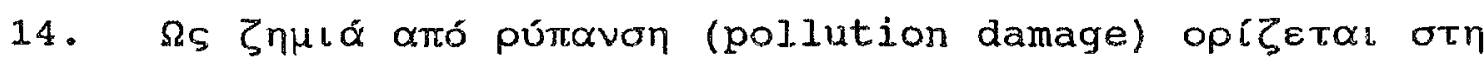

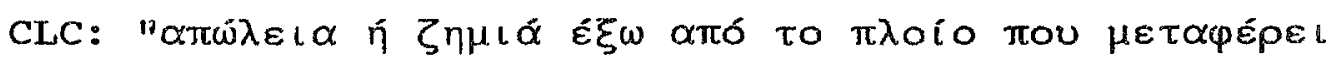

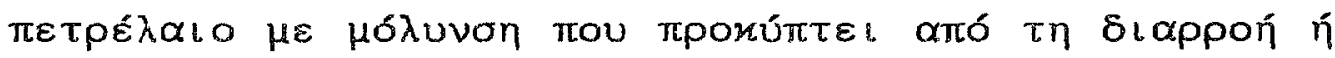

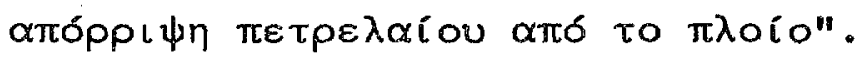

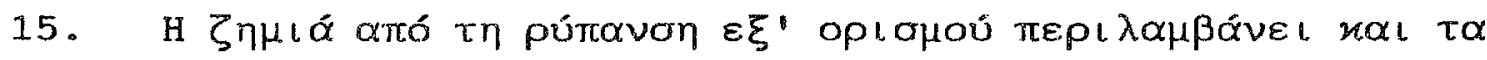

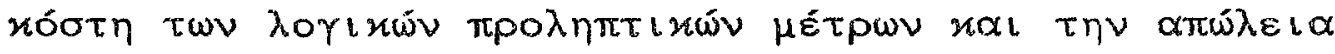

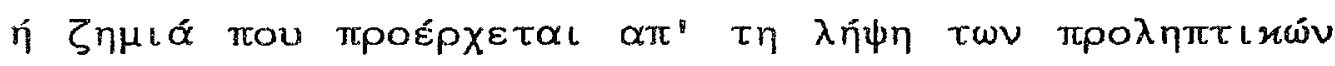

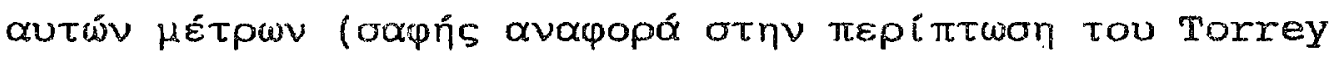
Canyon).

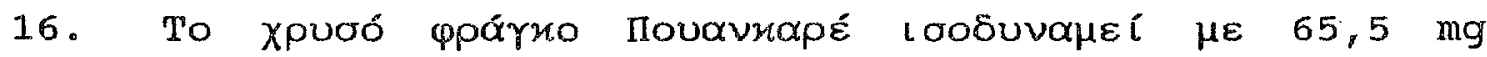

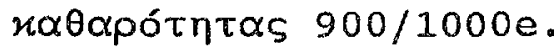

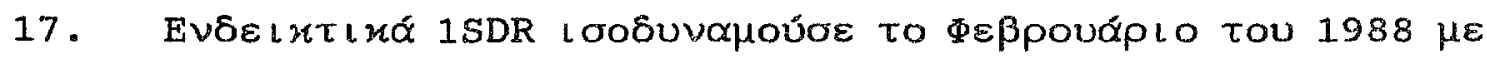

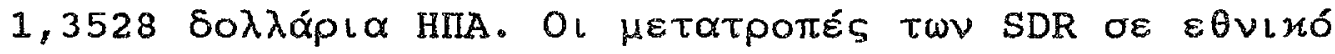

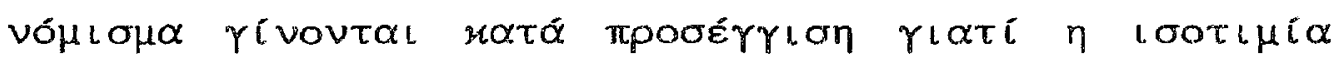

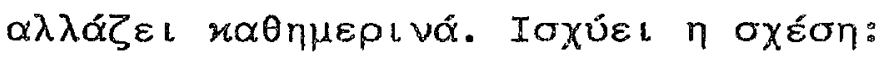

1 Poincare franc $<1$ SDR. 


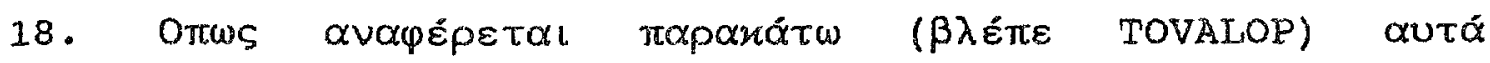

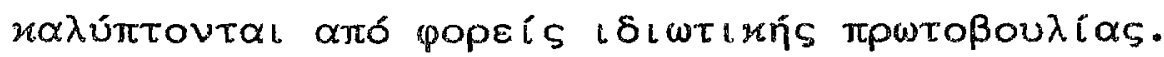

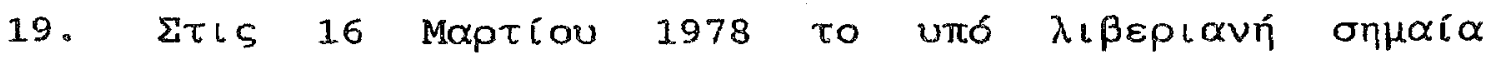

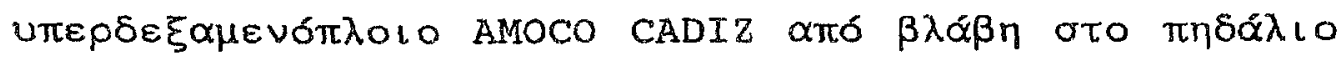

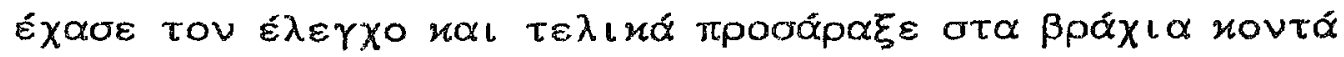

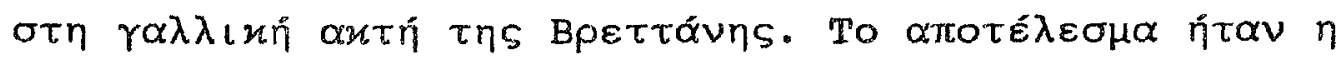

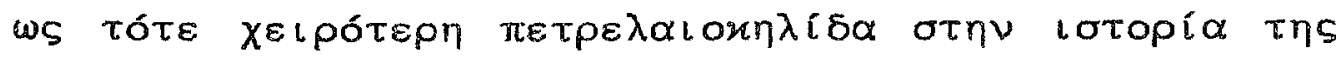

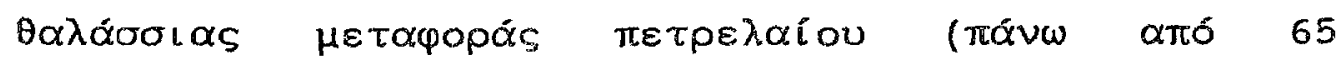

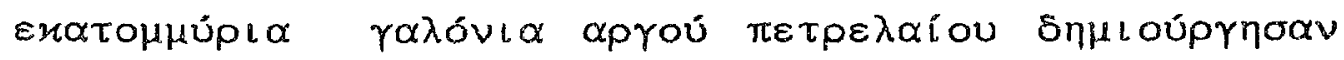

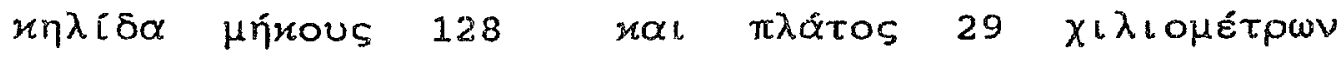

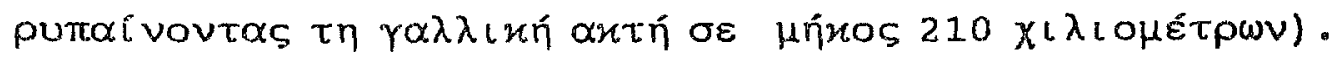

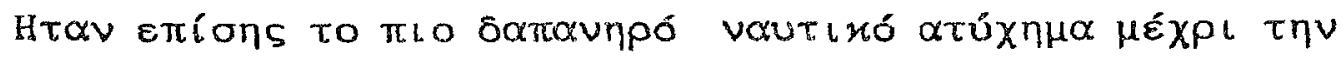

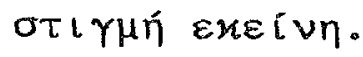

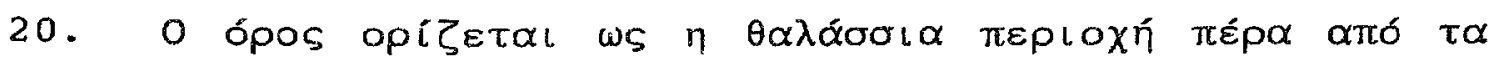

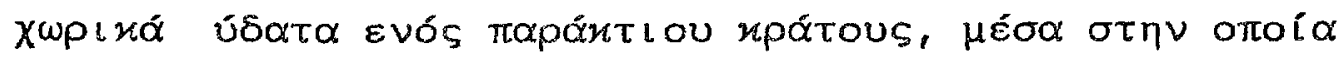

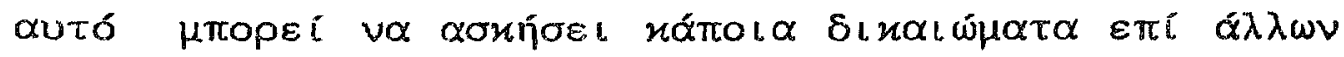

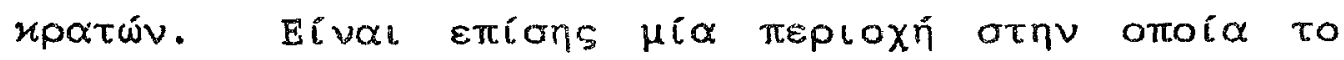

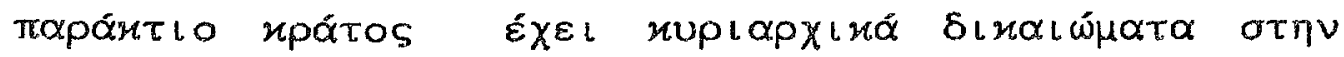

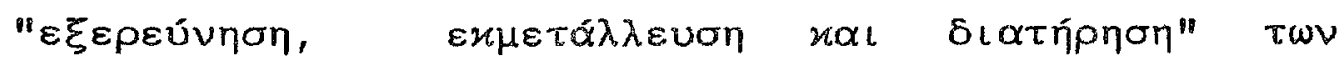

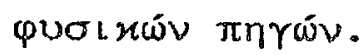




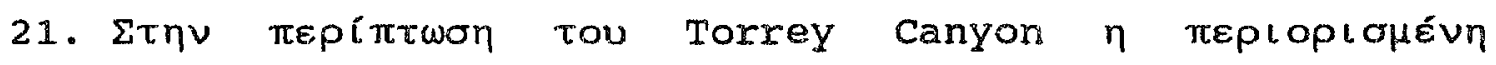

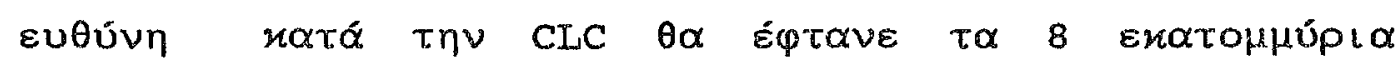

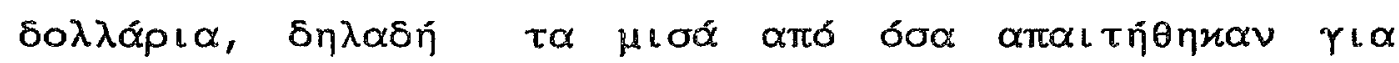

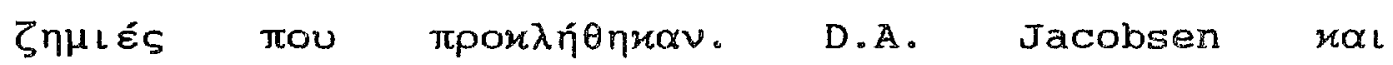
J.D.Yellen: "Oil Pollution: The 1984 London Protocols and the Amoco CADIZ". Journal of Maritime Law and commerce, Teúxos 15 No $4,0.474$, Oxtúßplos 1984.

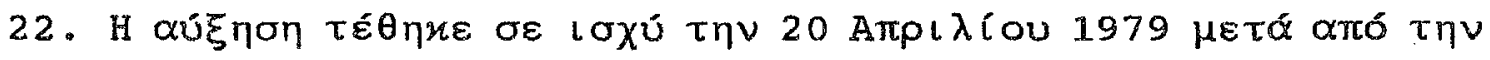

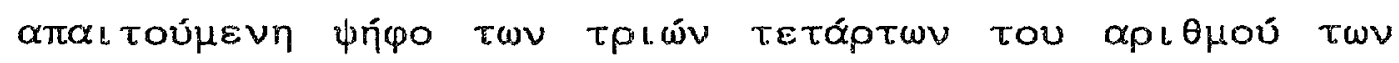

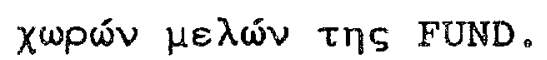

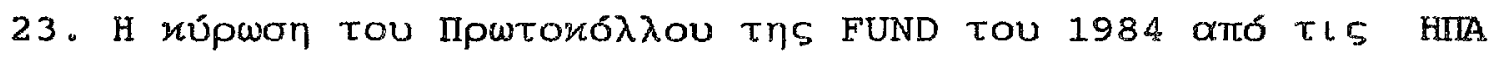

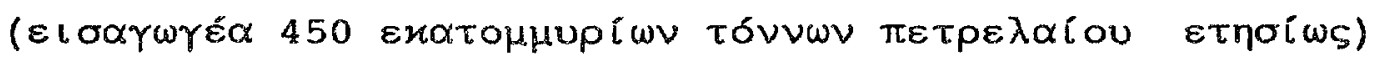

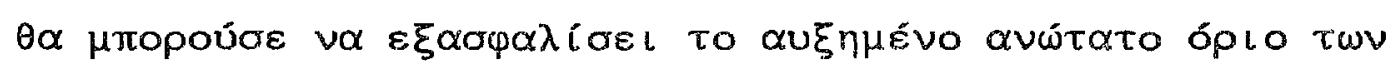
$200 \varepsilon \varkappa$. SDRs. Beth Van Hanswyk: "The 1984 Protocols to the International. Convention on civil Liability for oil pollution Damages and the International Fund for Compensation for oil pollution Damages: An option for Needed Reform in United States Law". The International

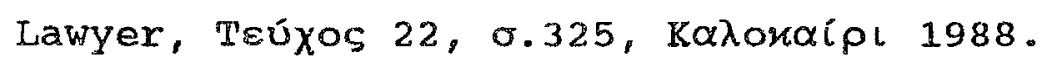




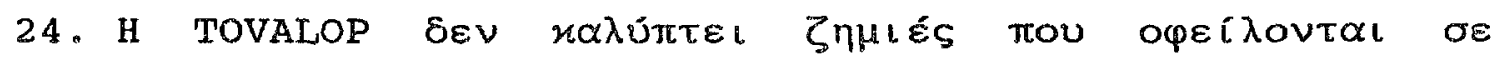

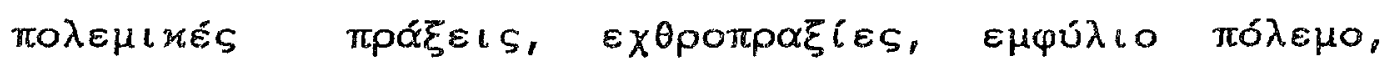

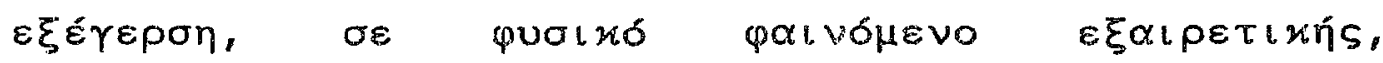

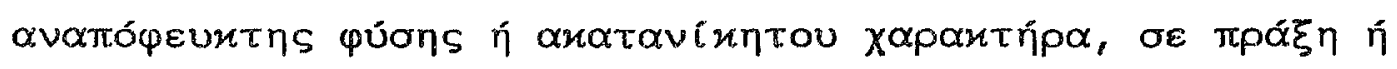

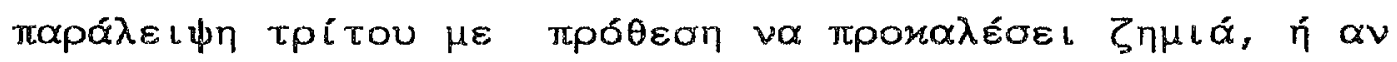

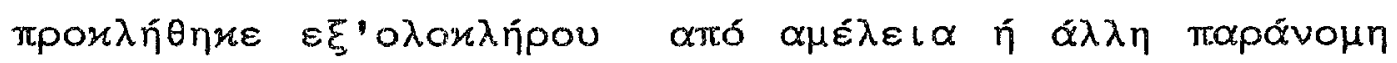

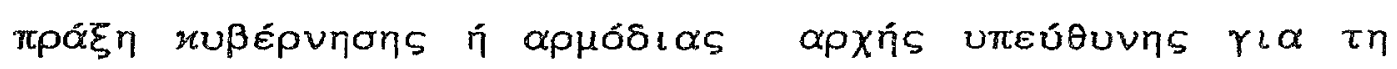

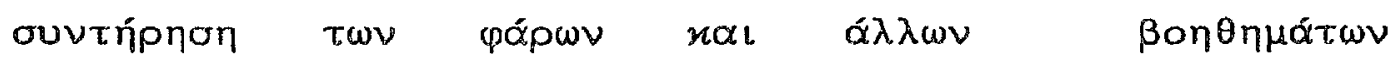

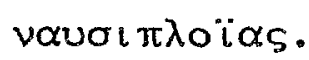

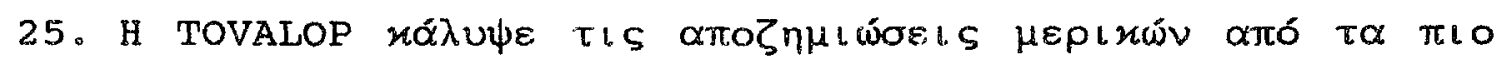

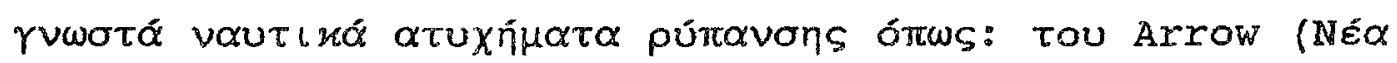

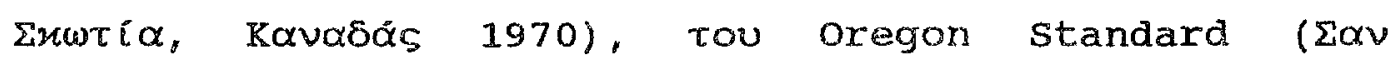

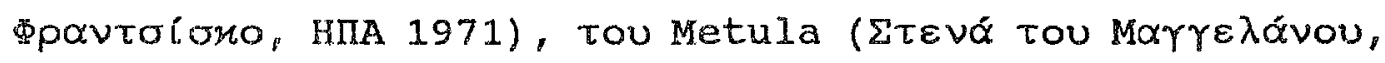

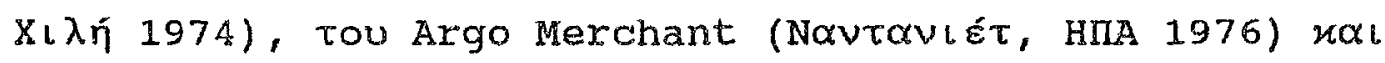

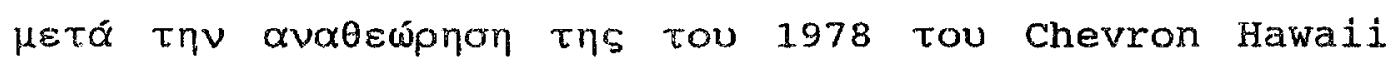
(Xıoúotov, HIA 1979).

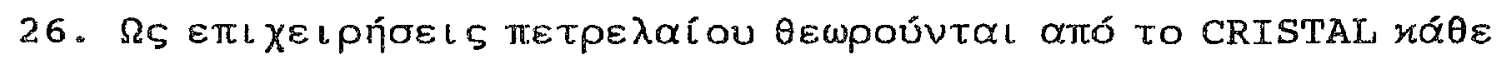

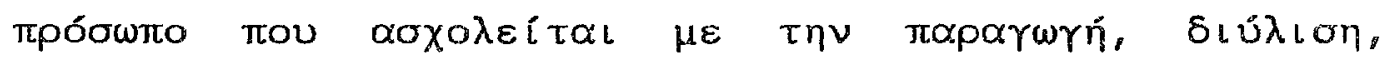

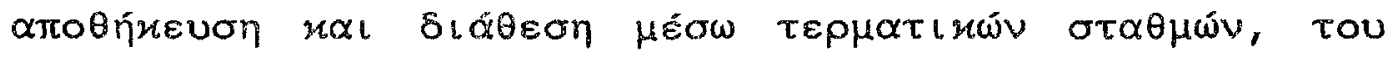

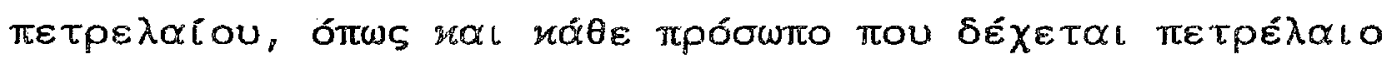

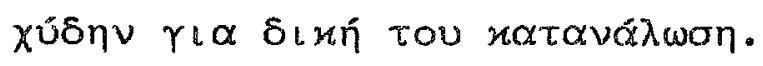




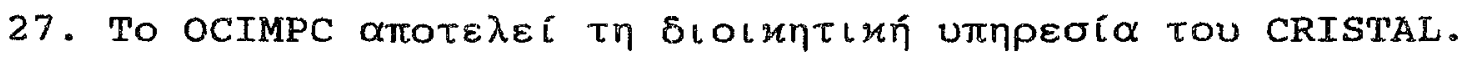

28. Lawrence G.Cohen: "Revisions of TOVALOP and CRISTAL: Strong ships for stormy seas" J.M.L.C., teúxos 18 No 4 , б. 530, Ontúßplos 1987 .

29. Amy McKaig: "Liability for oil Tanker spills".

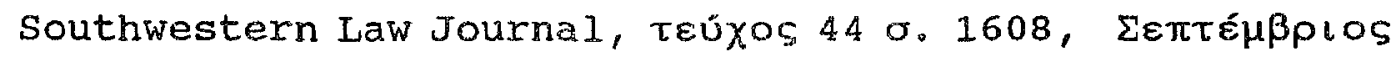
1991.

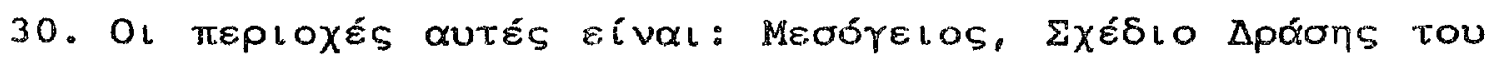

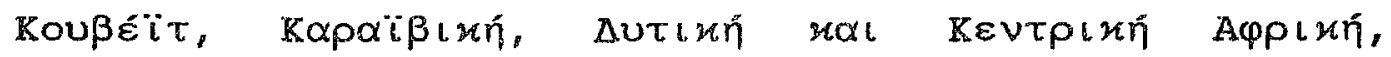

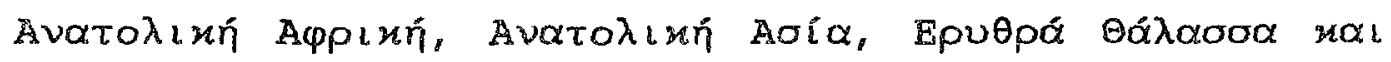
Kóxtros tou Autev, Notlóvtixós Elpqulxós,

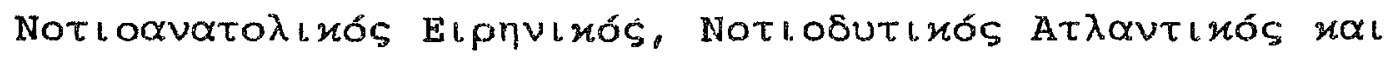

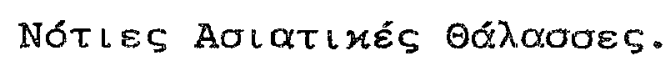

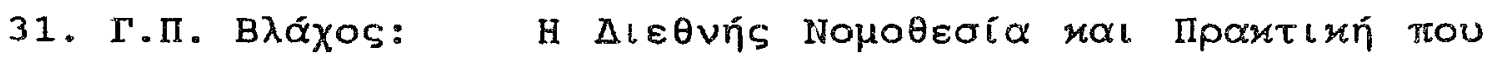

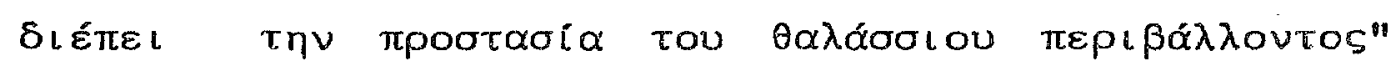

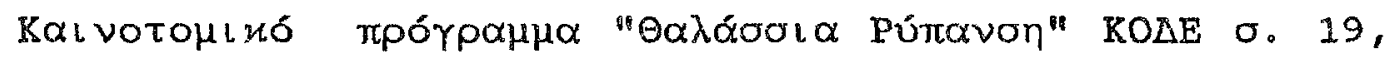
Mớlos 1991. 
32. Sally A. Meese: "When Jurisdictional Interests Collide: International, Domestic and State Effors to Prevent Vessel Source oil Pollution". Ocean Development and International Law, тعúxos 12 o. 91,

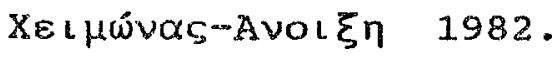

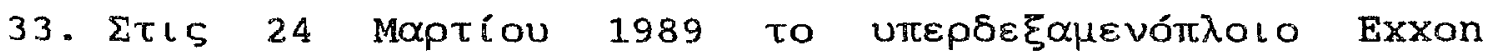

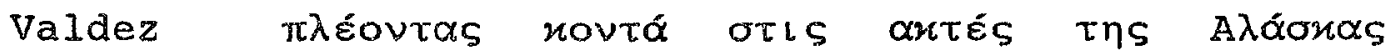

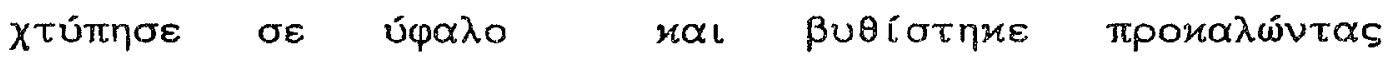

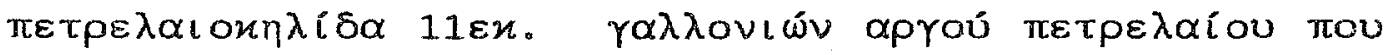

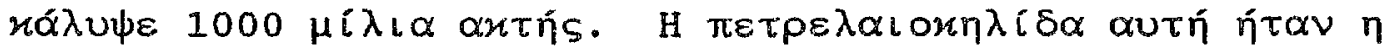

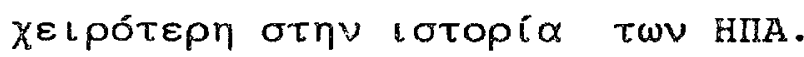

34. Prof.Edgar Gold:"International Convention on oil Pollution Preparedmess, Response and Co-Cperation, 1990 Report". J.M.L.C, tยúxos 22 No 2 o. 343, Ampìlos 1991. 


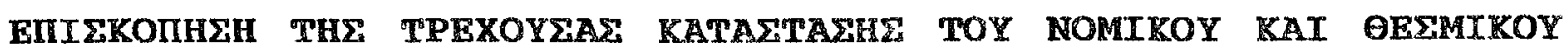

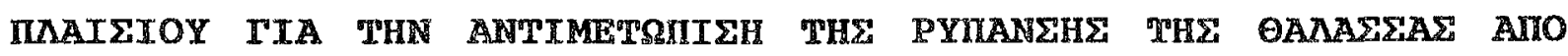 IETPBNATO MOY IIPORPXETAT AIO ILOIA.}

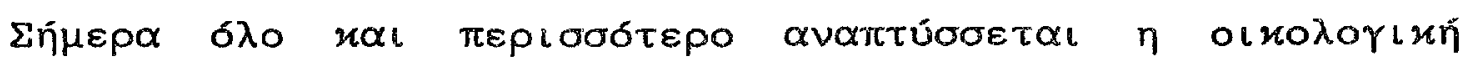

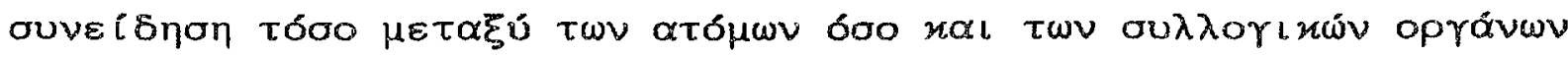

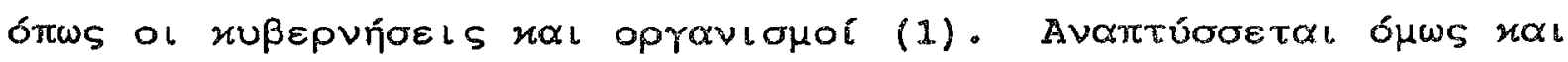

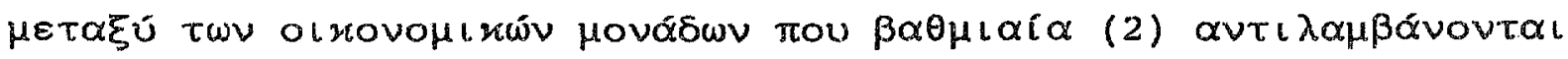

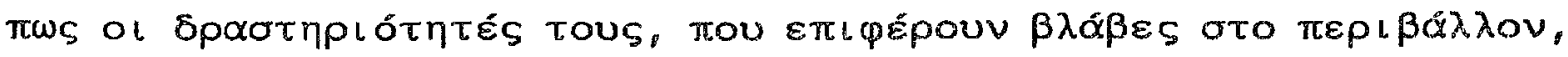

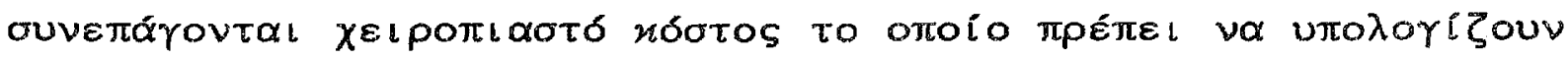

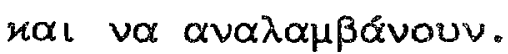

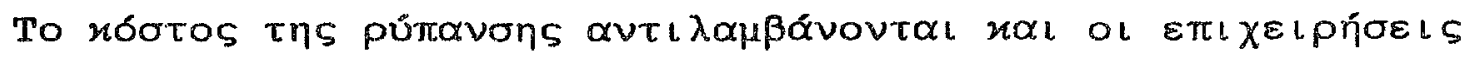

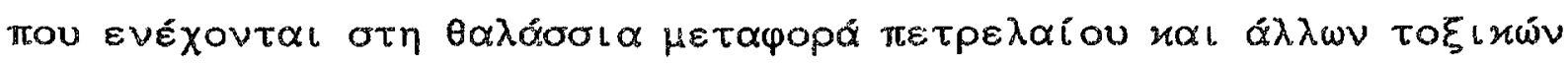

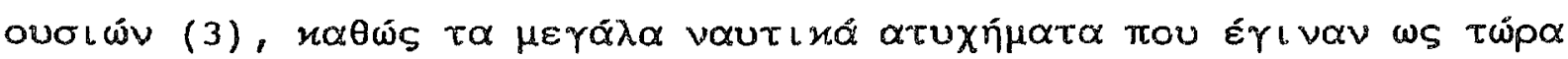

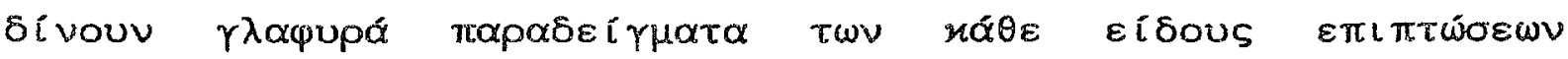

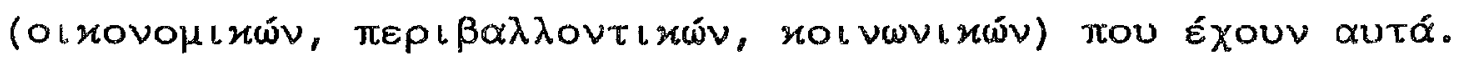

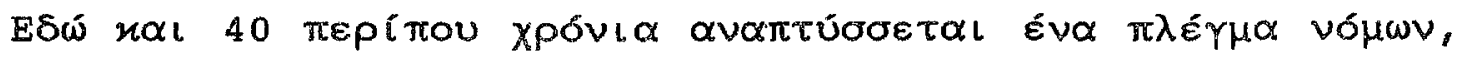

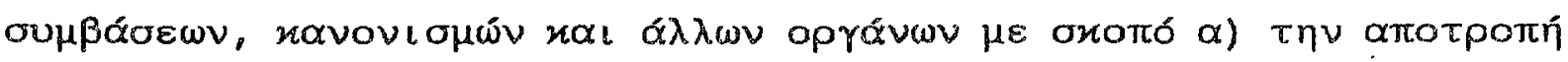

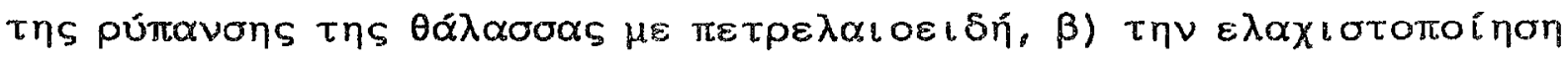

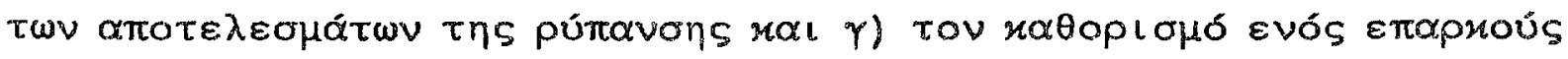

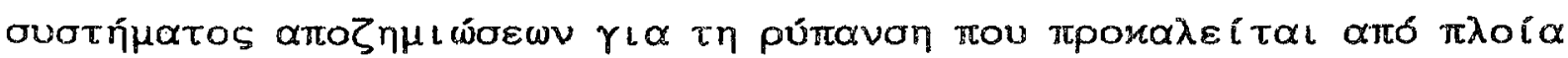

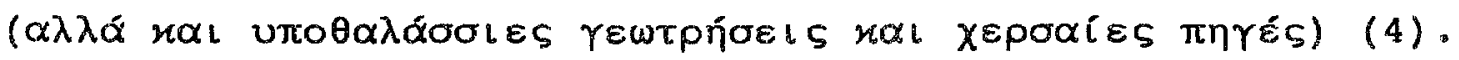




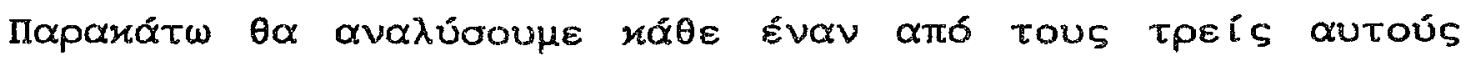

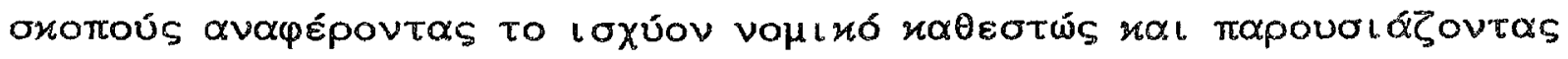

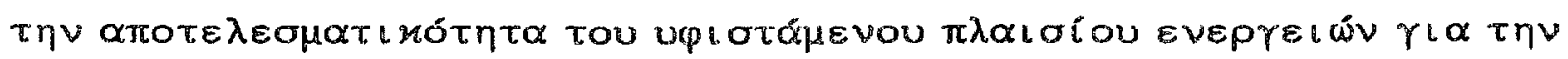

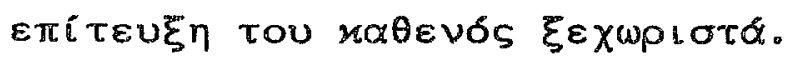




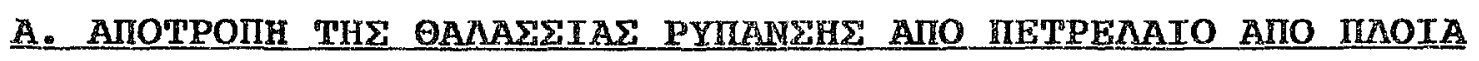

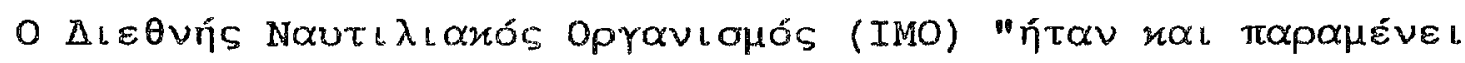

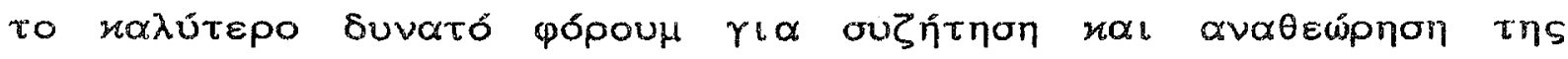

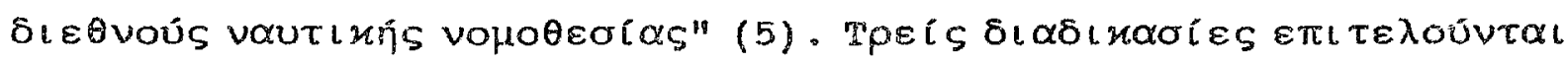

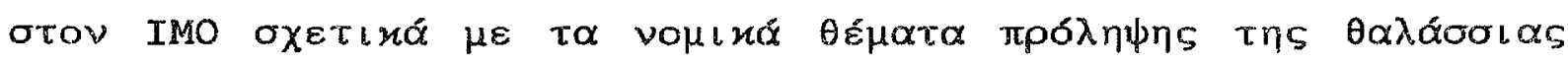

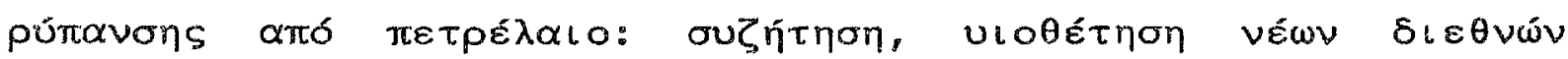

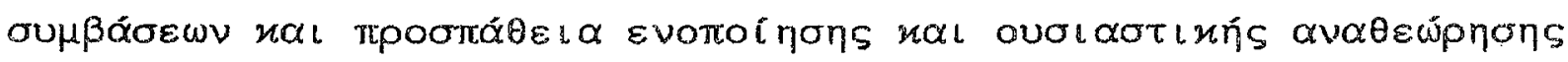

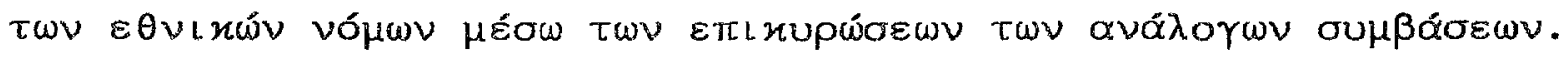

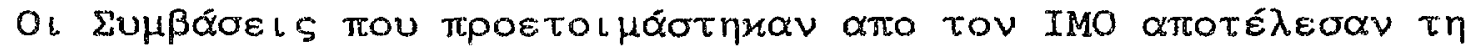

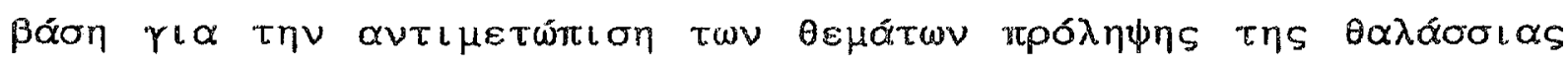

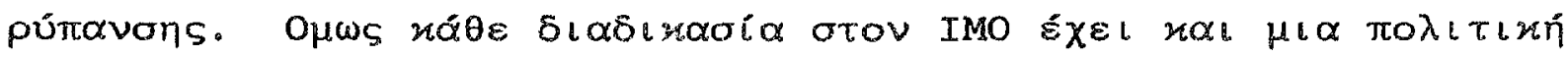

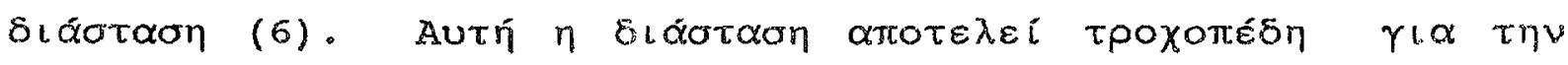

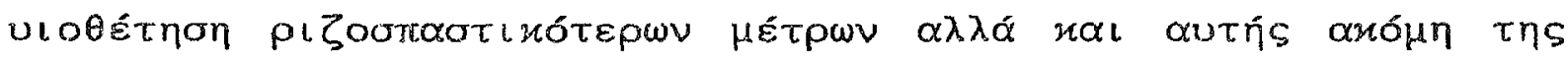

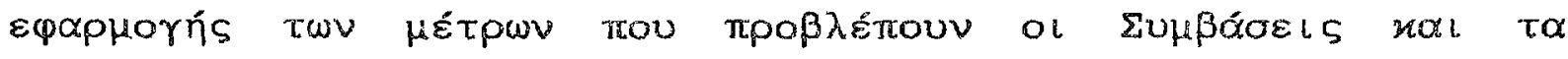

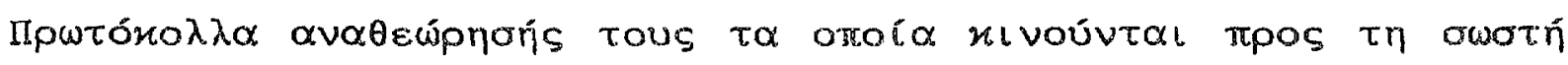

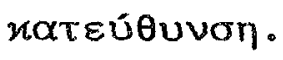

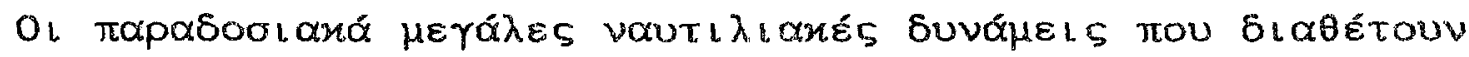

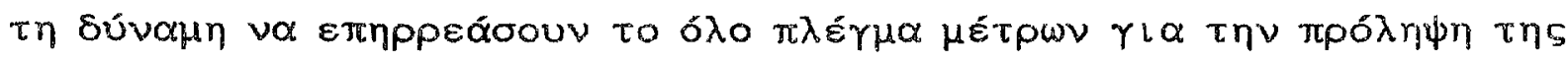

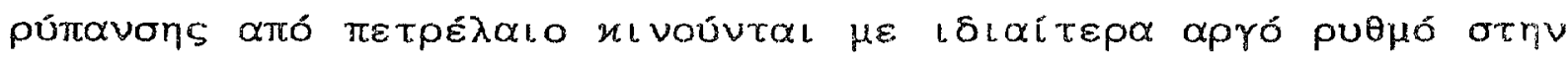

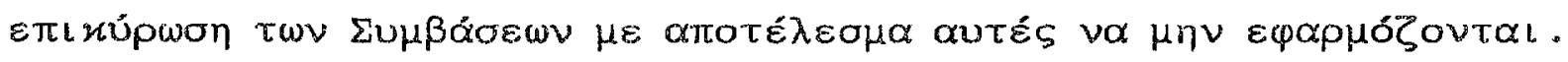




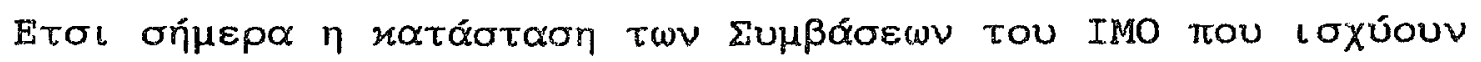
$\varepsilon \varepsilon_{1}$ ws $\varepsilon \xi \tilde{\zeta} 5:$

1) OILPOL 1954 Té

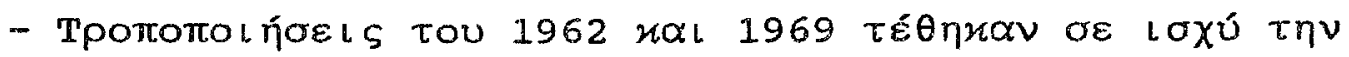

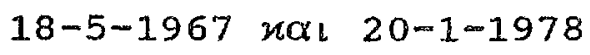

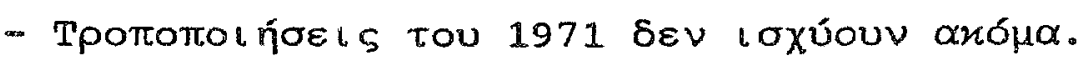

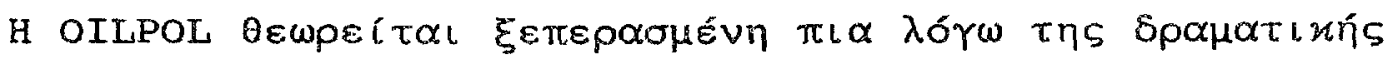

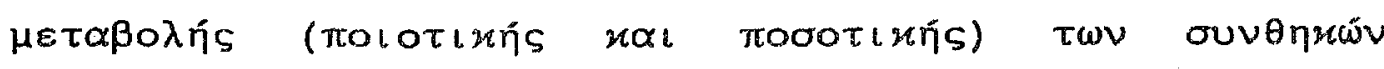

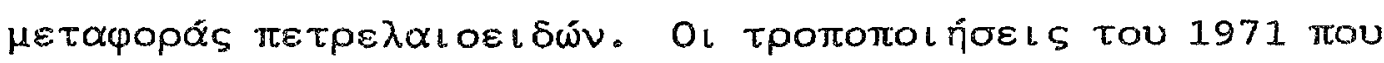

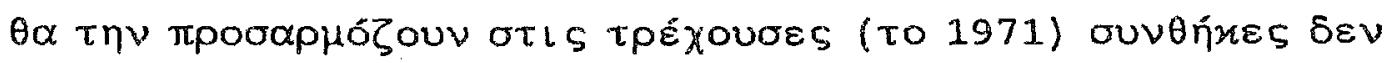

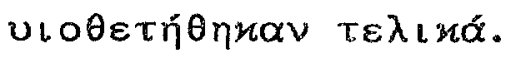

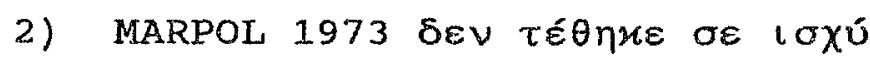

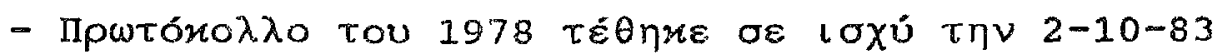

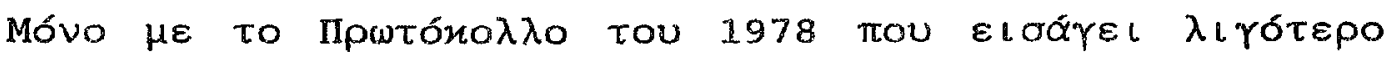

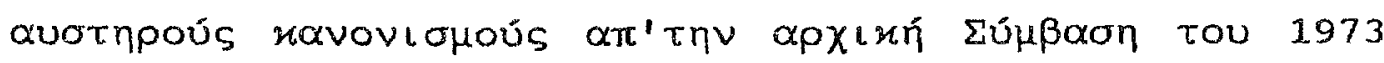

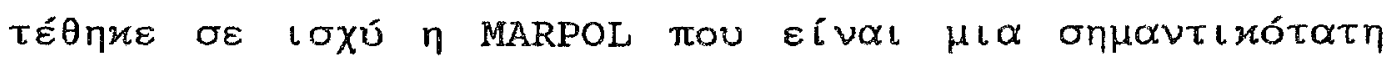

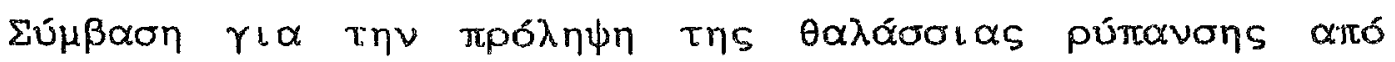

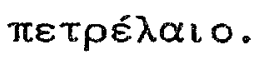

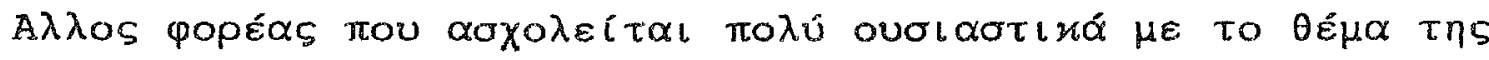

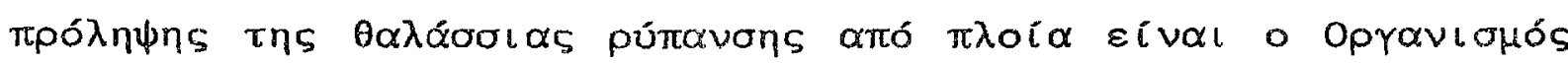

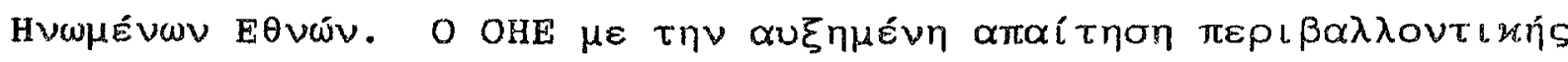

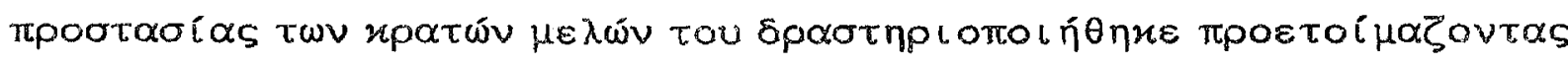




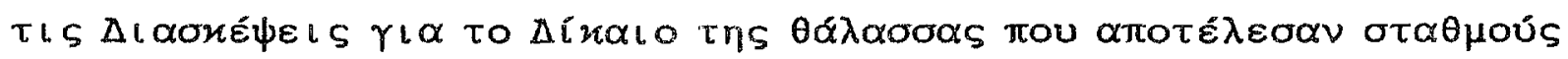

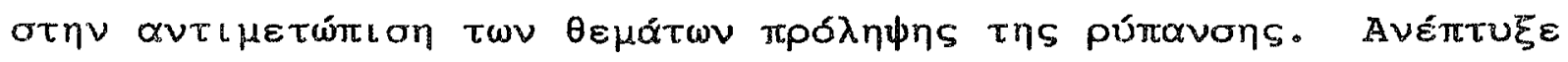

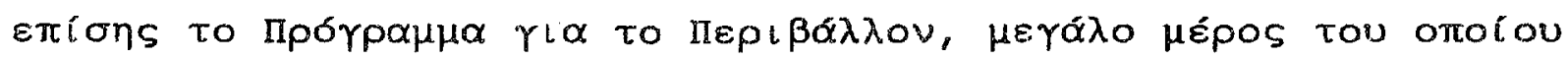

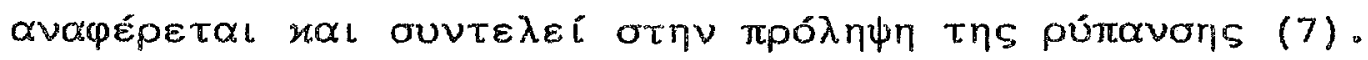

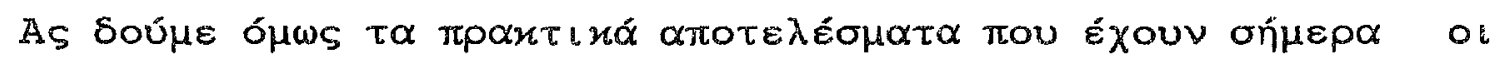

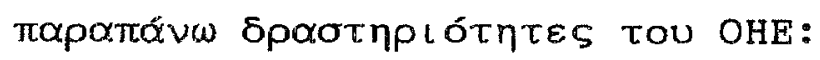

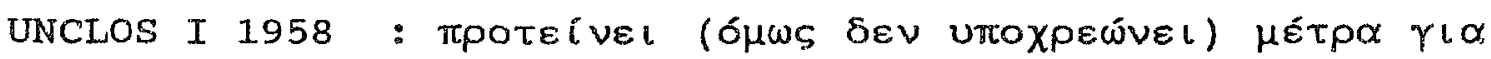

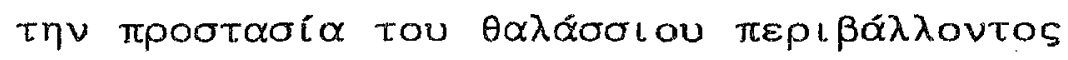

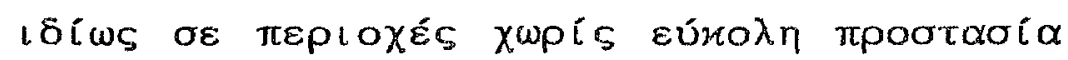

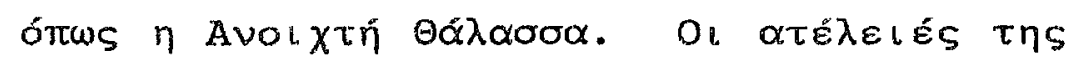

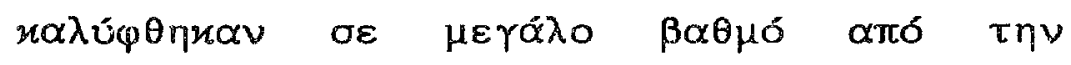
Eாó

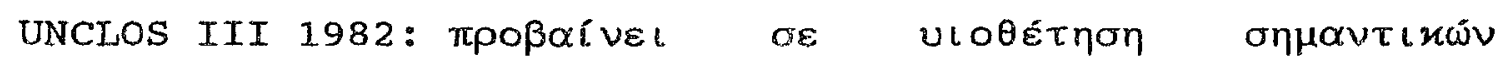

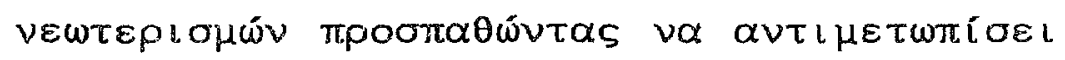

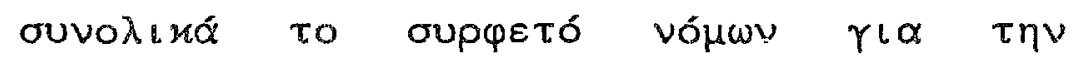

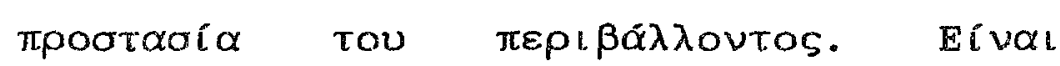

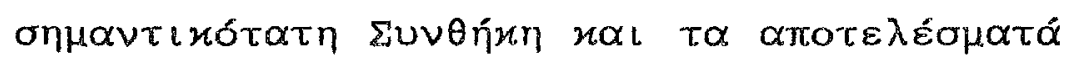

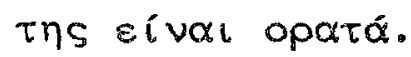


UNEP

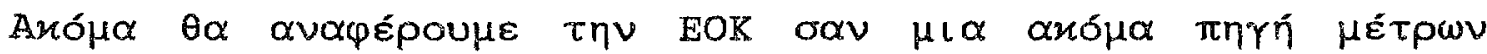

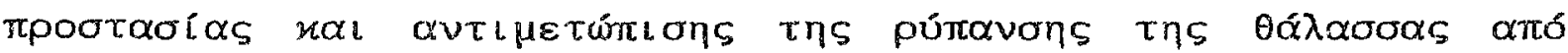

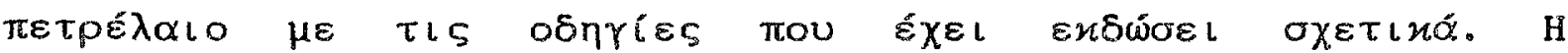

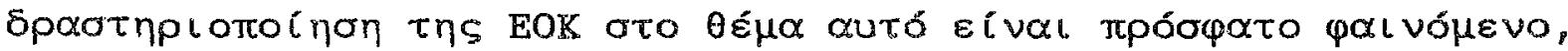

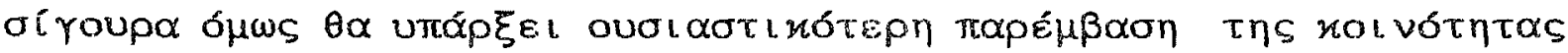

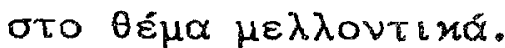

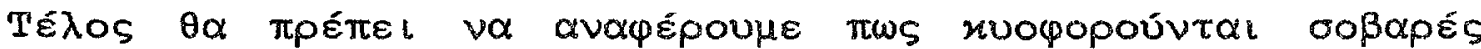

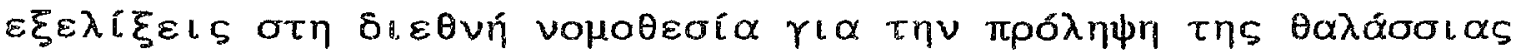




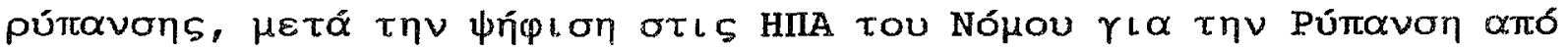

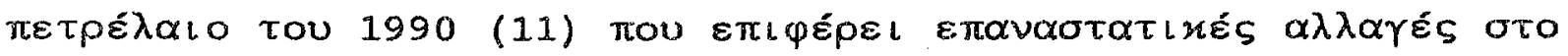

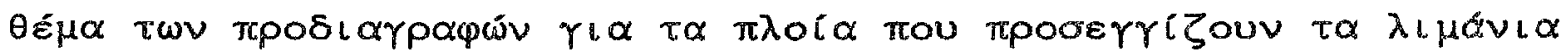

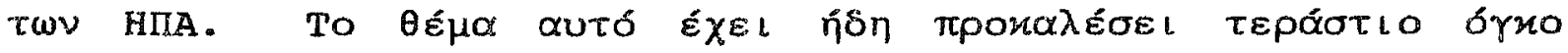

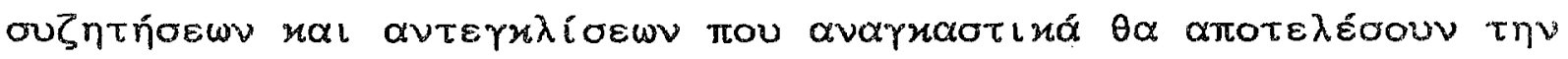

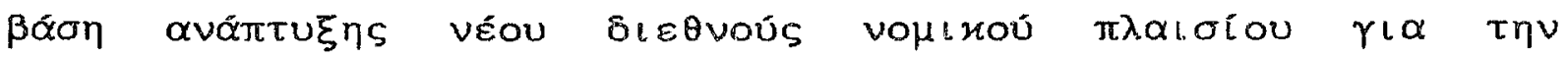

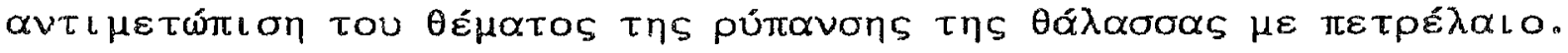




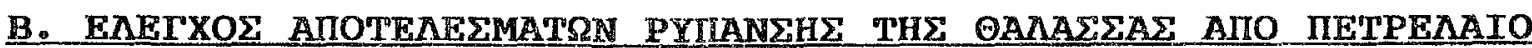

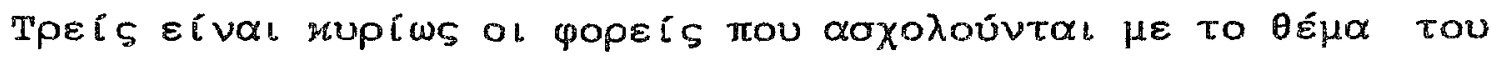

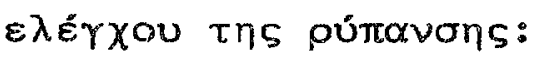

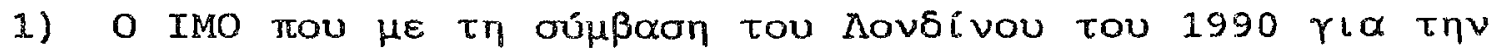

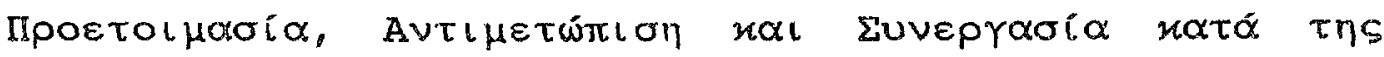

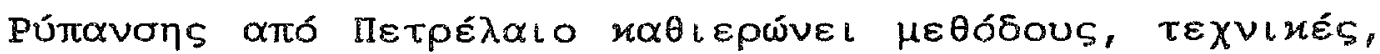

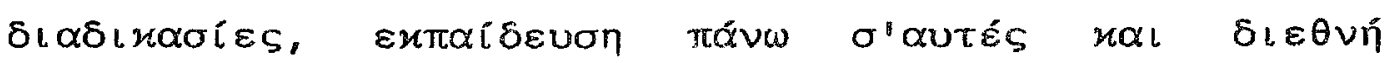

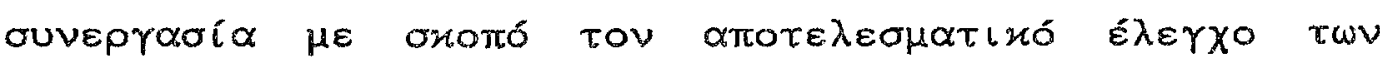

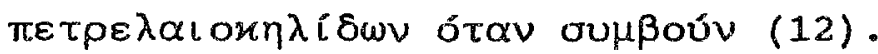

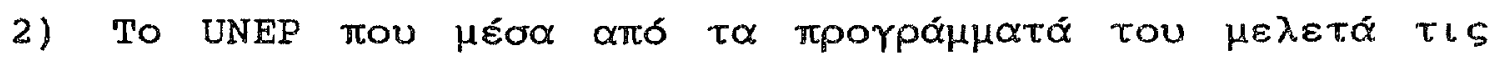

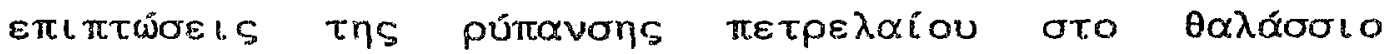

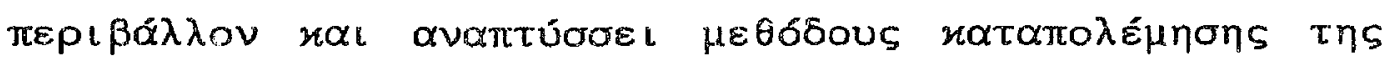

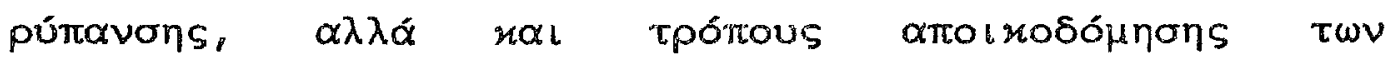

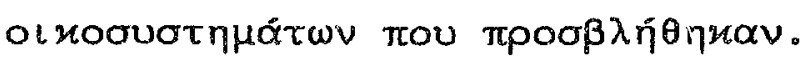

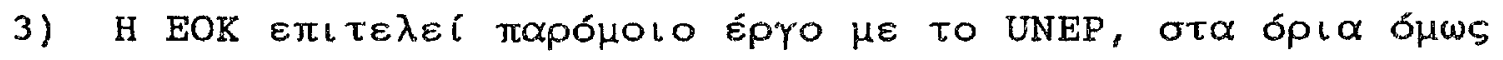

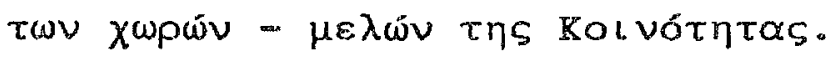




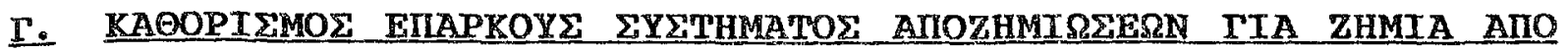 PYIIANEH METPENAIOY.}

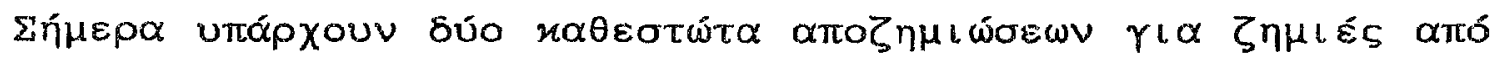

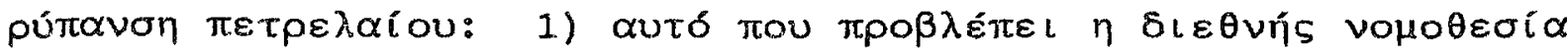

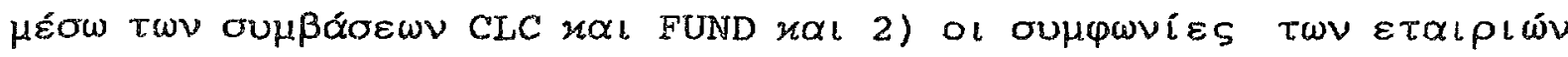

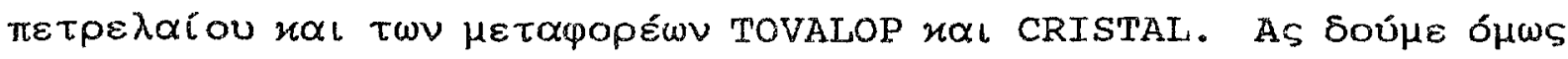

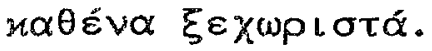

\section{1) CLC KL FUND}

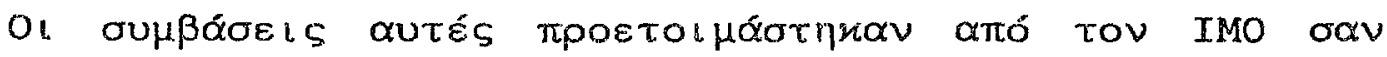

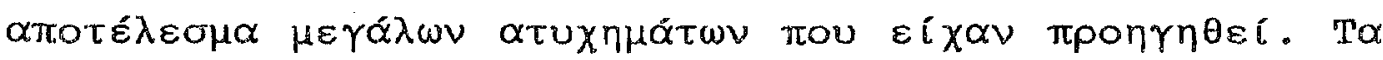

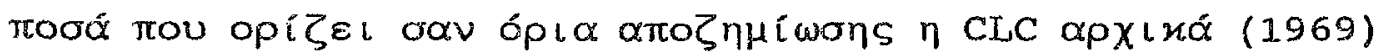

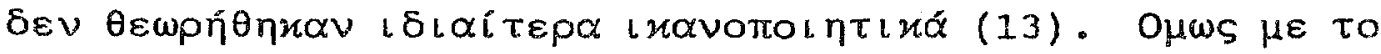

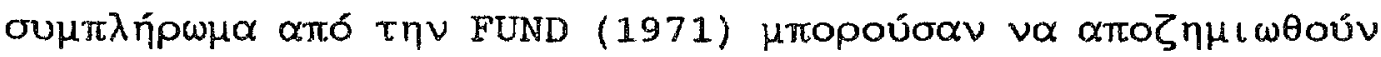

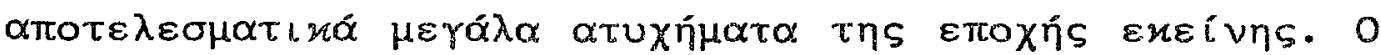

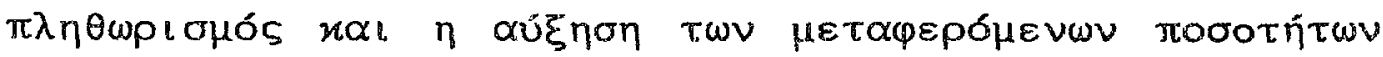

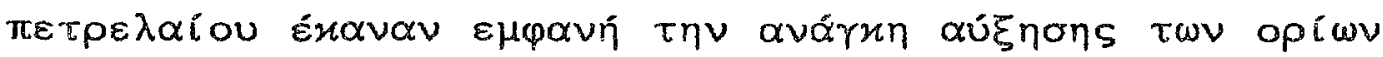

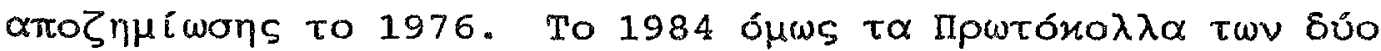

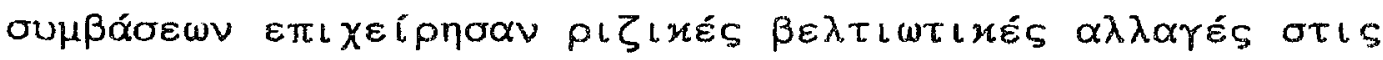

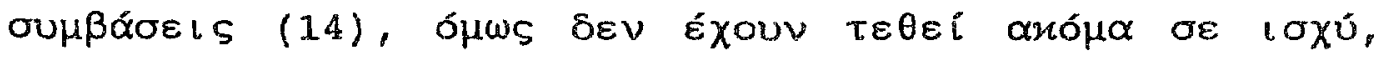

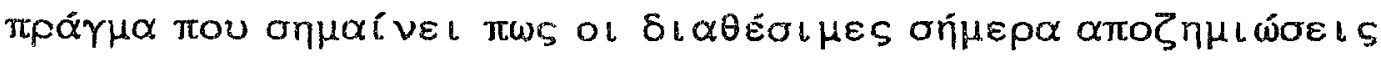

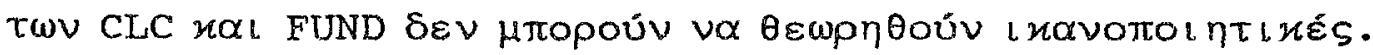




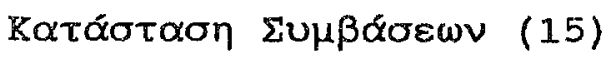

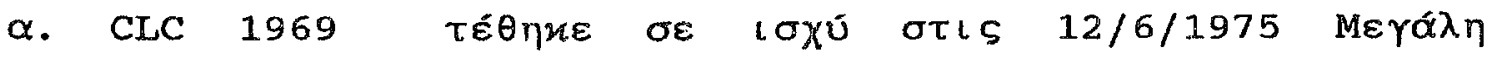

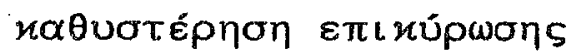

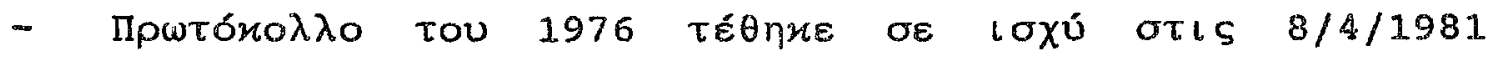

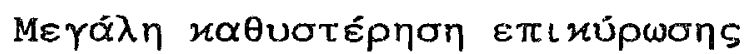

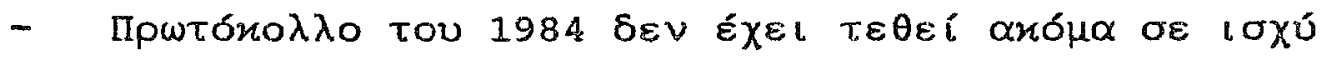

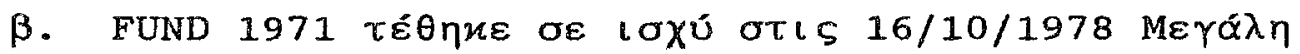

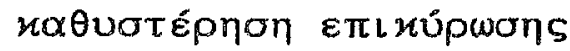

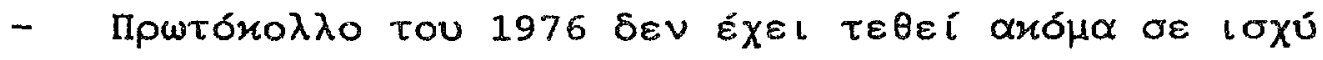

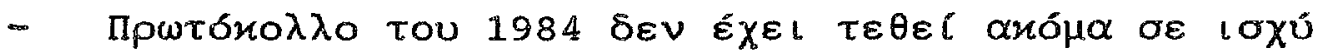

2) TOVALOP KQ CRISTAL

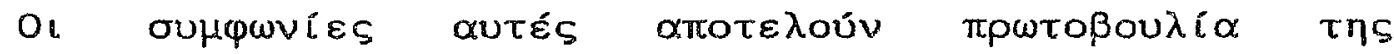

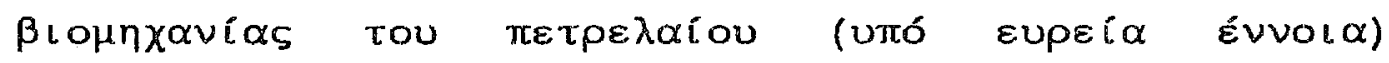

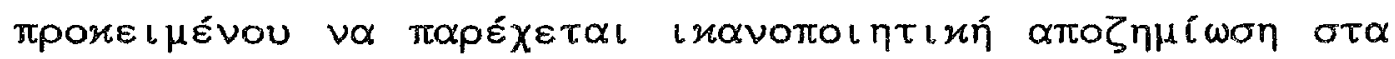

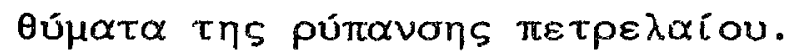

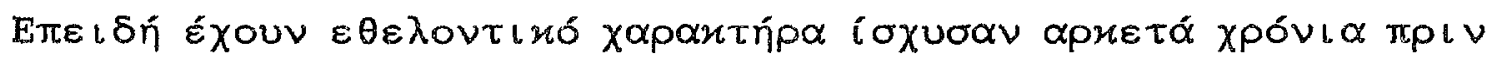

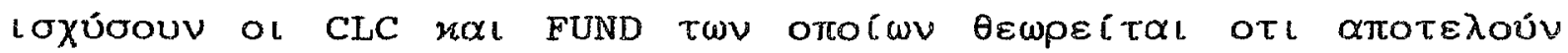

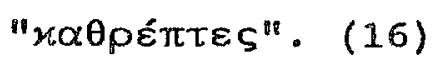




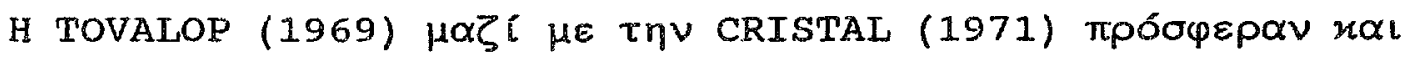

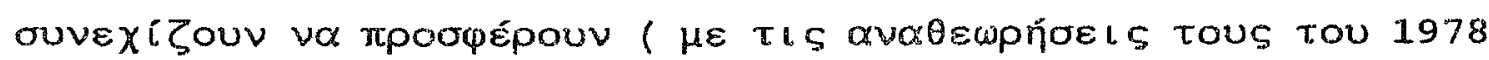

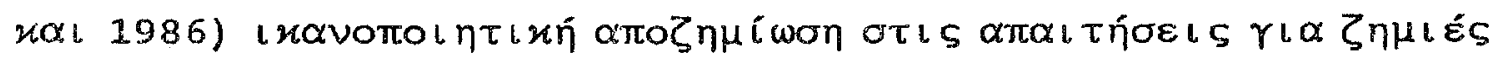

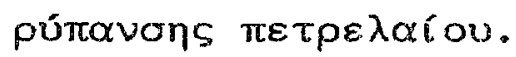

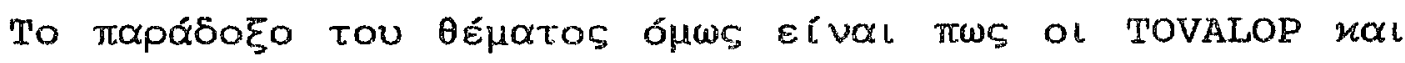

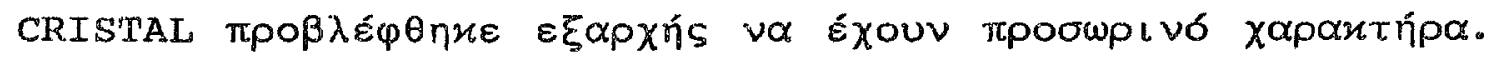

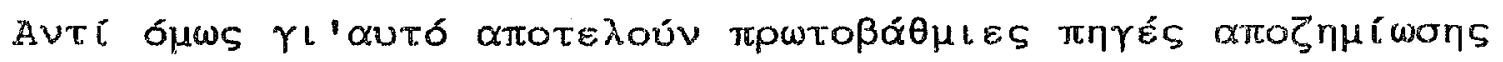

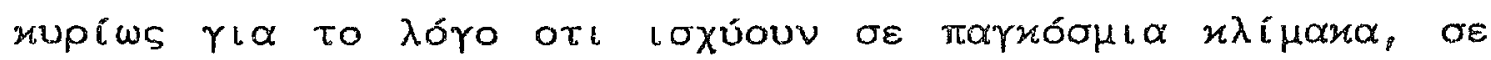

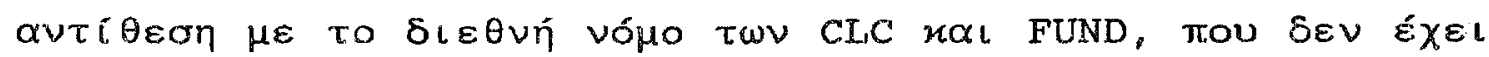

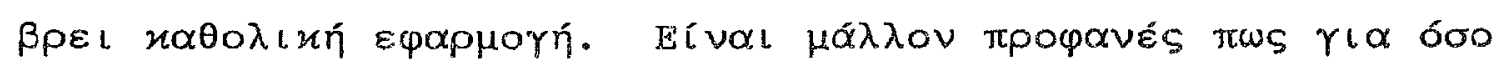

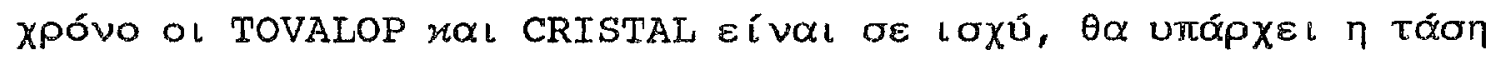

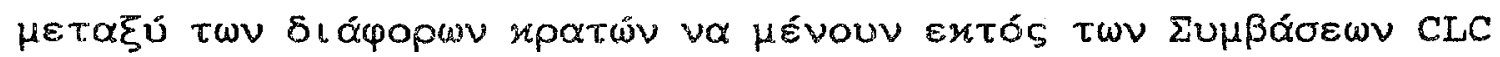

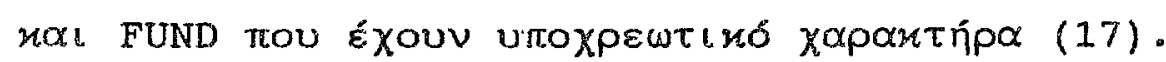

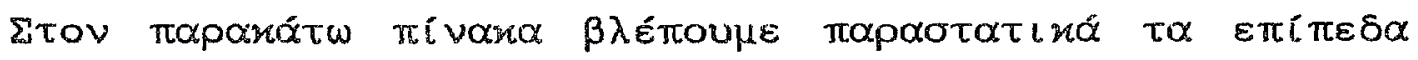

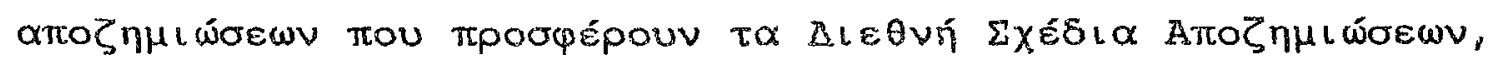
ótrov paivetal halapá twW ol TOVALOP hal CRISTAL

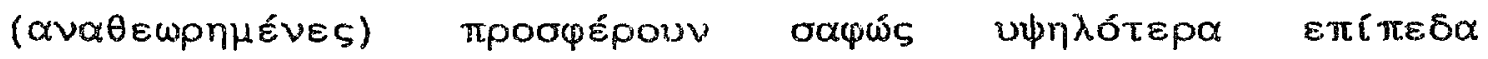

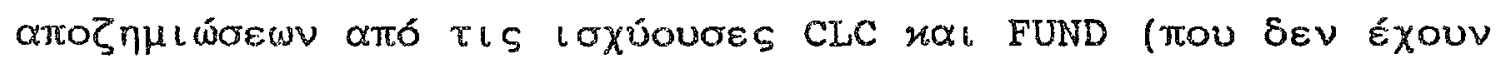

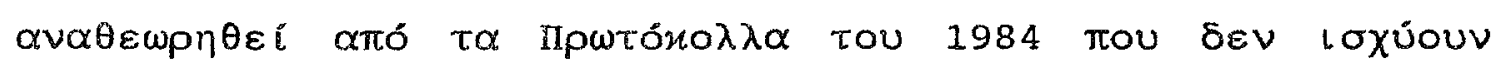
aró $\mu \alpha)$. 


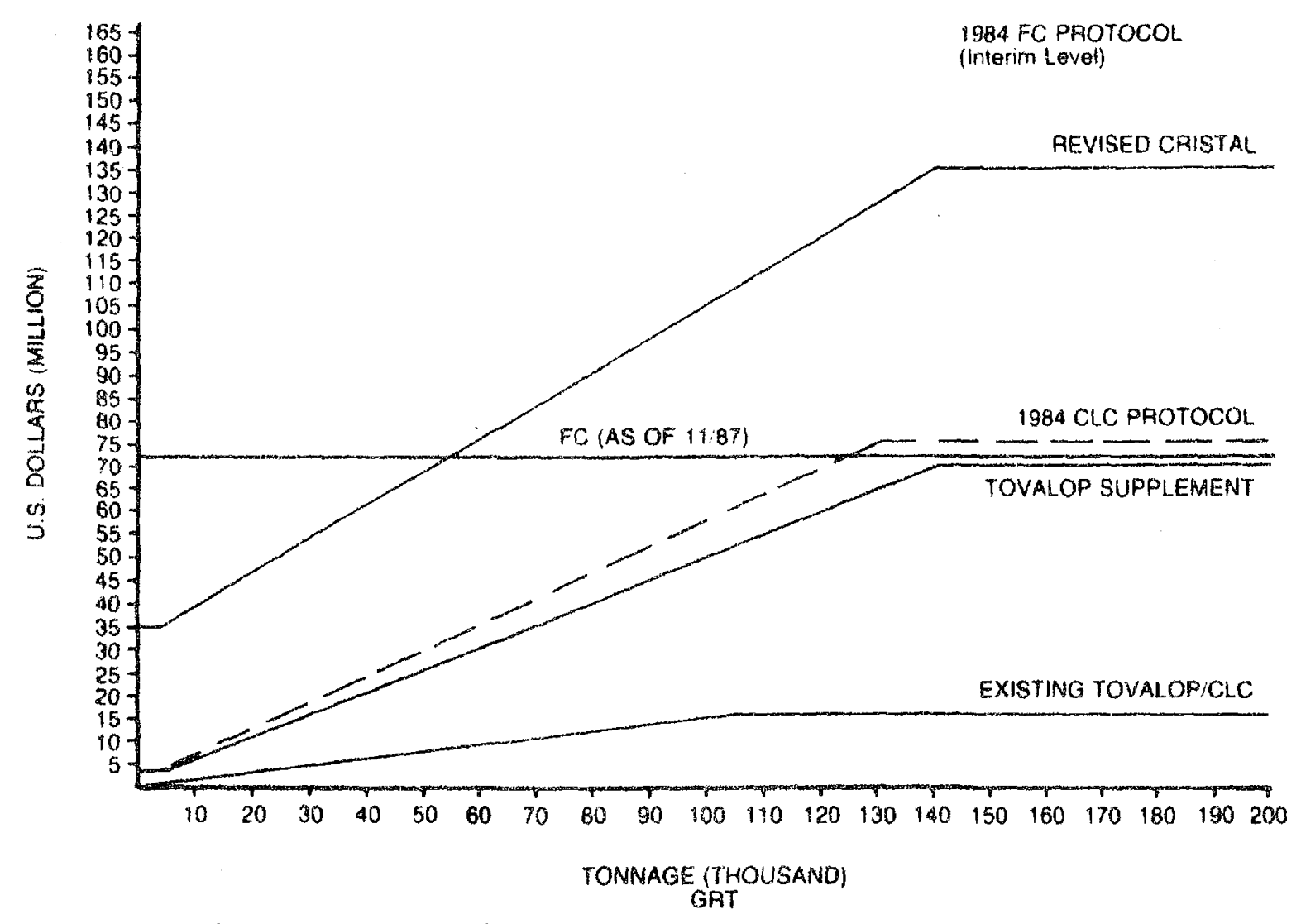

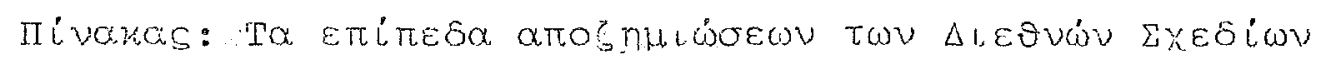

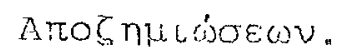




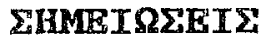

1. Edgar Gold "International Convention on oil Pollution Preparedness, Response and Co-Operation, 1990 Report".

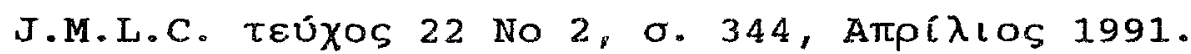

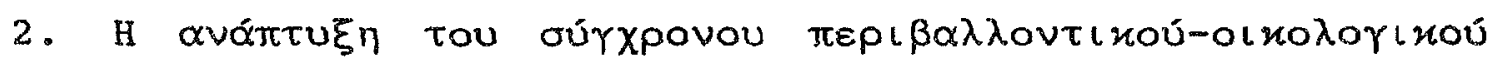

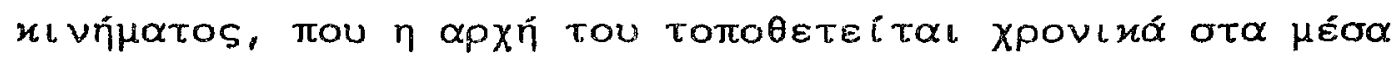

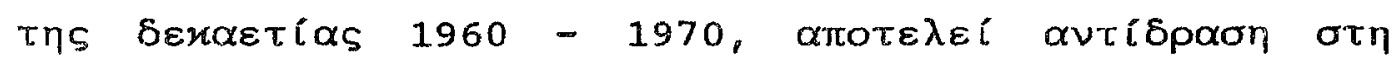

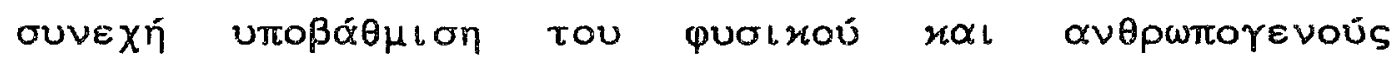

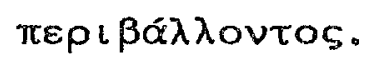

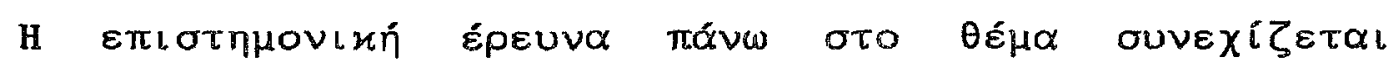

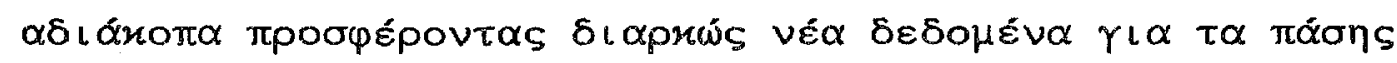

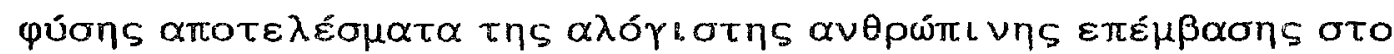
$\pi \varepsilon \rho\llcorner\beta \alpha ́ \lambda \lambda$ ov.

3. Aline F.M. De Bievre: "Liability and Compensation for Damage in Connection with the Carriage of Hazardous and

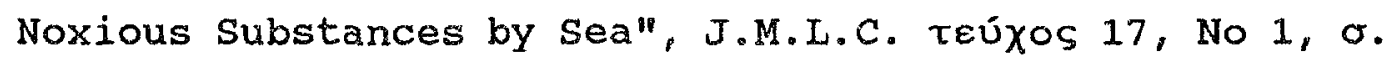
61 - 62, Iavouóplos 1986. 
4. David D. Caron: "Liability for Transnational Pollution Arising from offshore oil Development: A Methodological Approach". Ecology Law Quarterly, teúxos 10, 0. 641 -

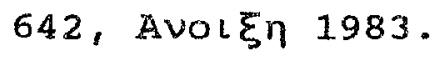

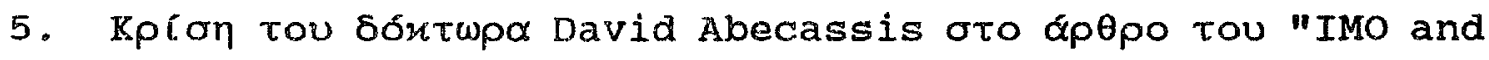
Liability for oil Pollution from ships: a retrospective"

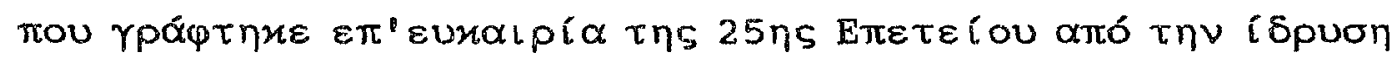
TOU IMO.

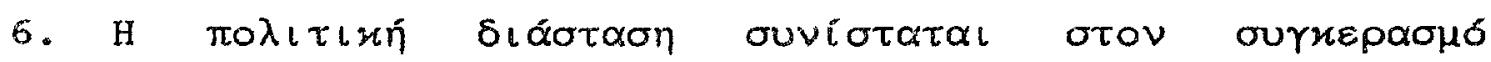

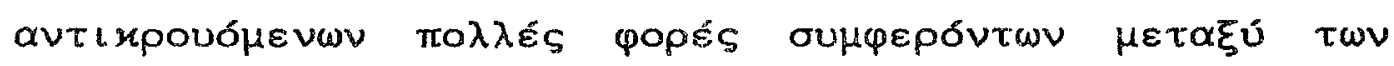

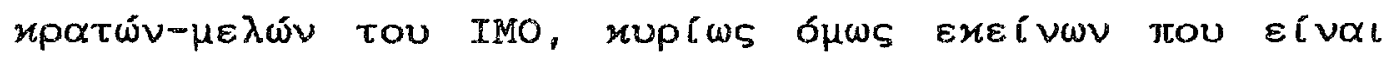

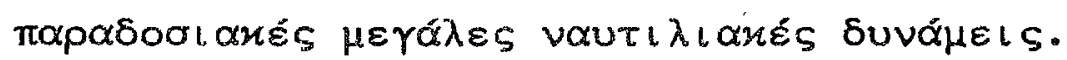

7. K. Ramakrishna: "Environmental Concerns and the New Law

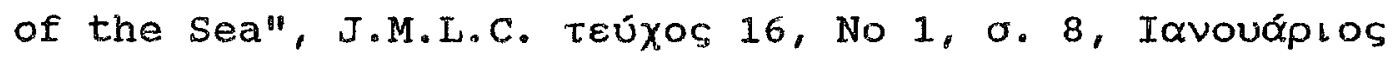
1985.

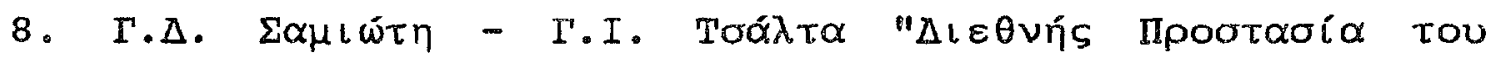

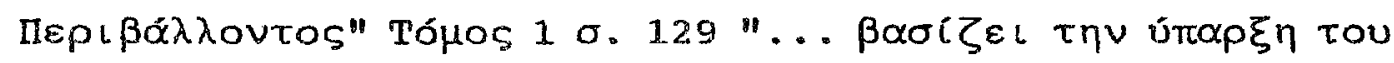

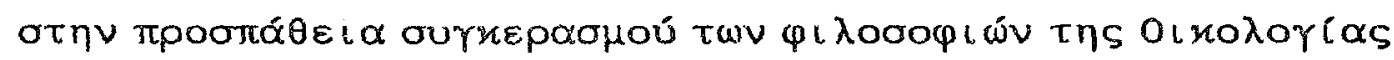

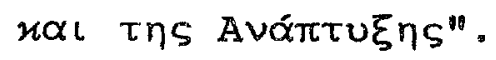




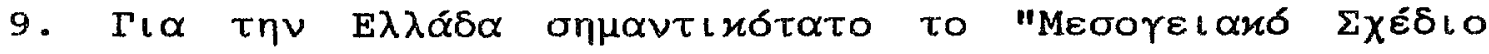

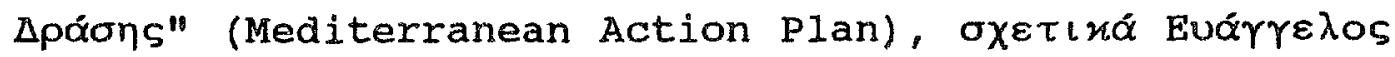
r. Poprótroulos: "The Mediterranean Action Plan in a Functional Perspective: a quest for law and policy". MAP Technical Reports Series No 25.

10. K. Ramakrishna óm. $\pi .0 .16$.

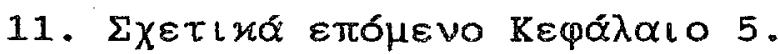

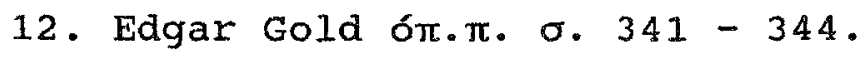

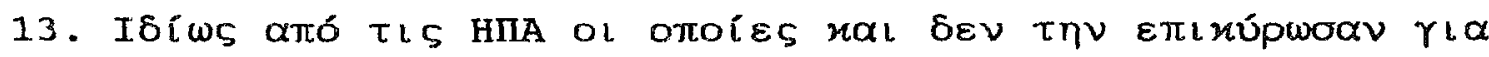

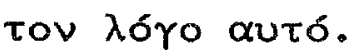

14. Beth Van Hanswyk: "The 1984 Protocols to the International convention on civil Liability for oil Pollution Damages and the International Fund for Compensation for oil pollution Damages: An option for Needed Reform in United States Law". The International

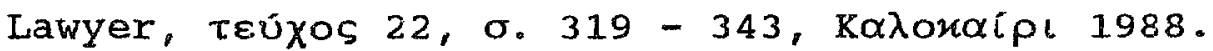




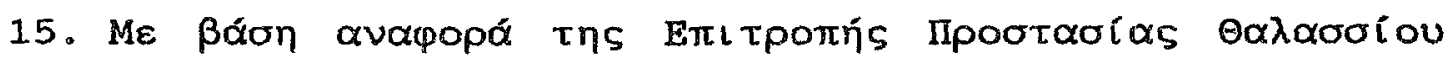

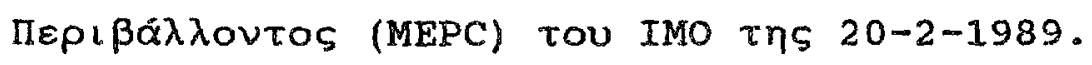

16. Lawrence G. Cohen: "Revisions of TOVALOP and CRISTAL: "strong ships for stormy seas". J.M.L.C. TEúxos 18, No

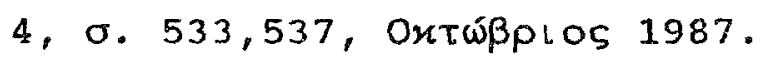

17. Gordon L. Becker: "Acronyms and Compensation for oil Pollution Damage from Tankers". Texas International Law

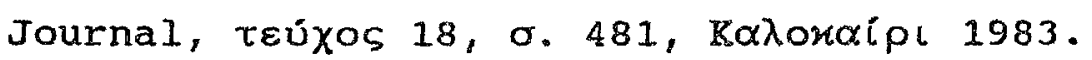




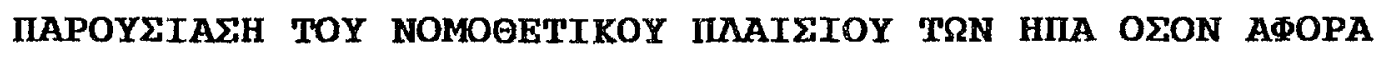 THN GYOYNH TIA PYIIANSH THE OANAEEAS AIO IINOIA}

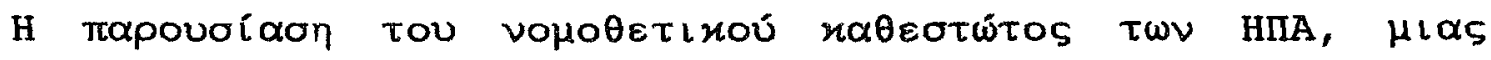

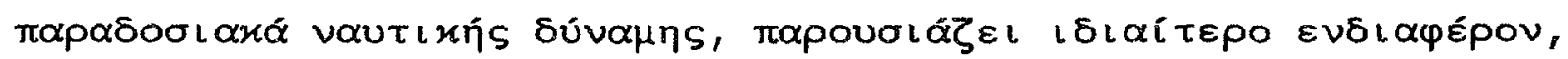

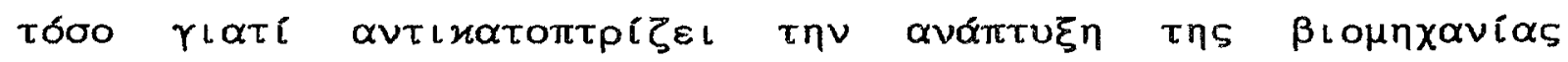

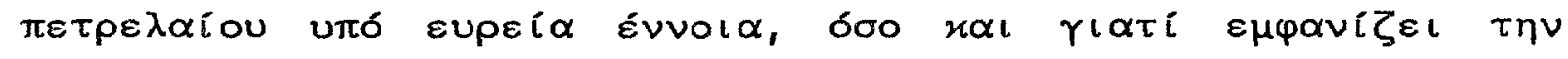

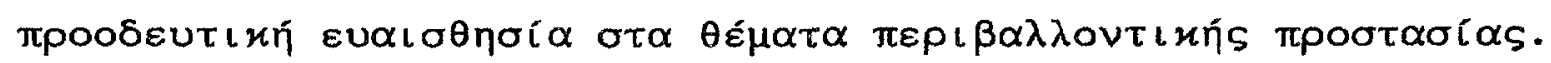

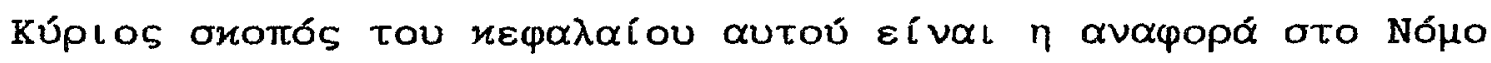

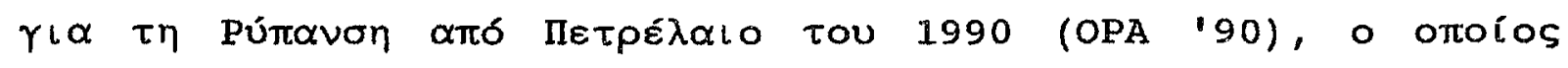

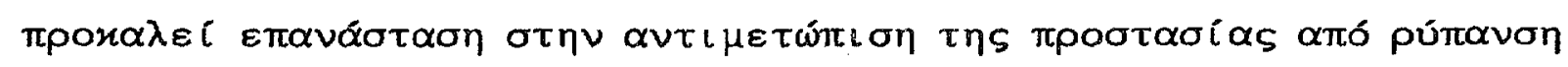

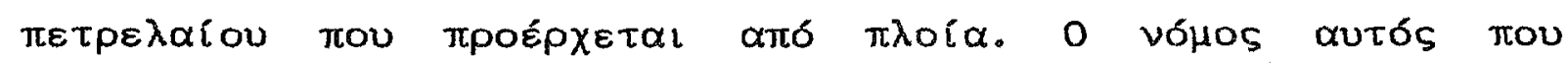

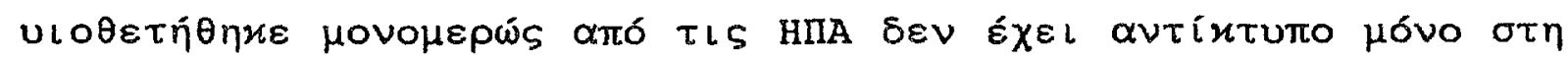

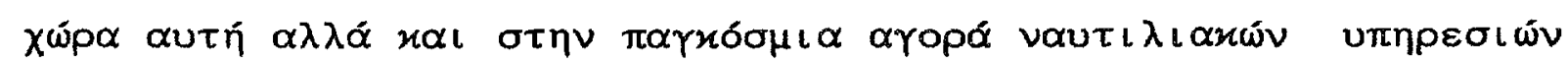

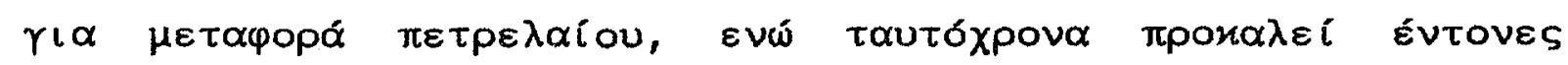

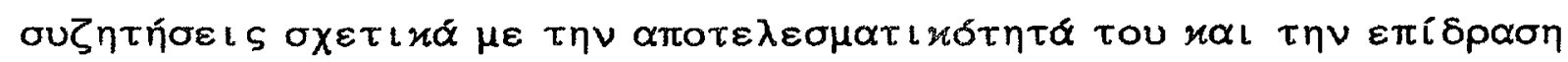

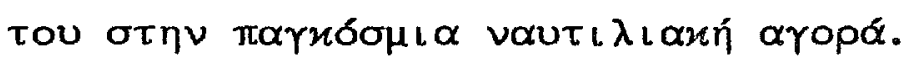

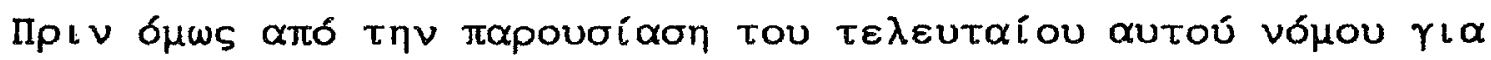

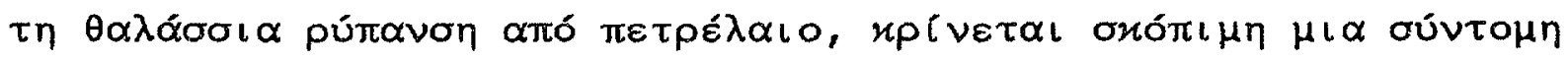

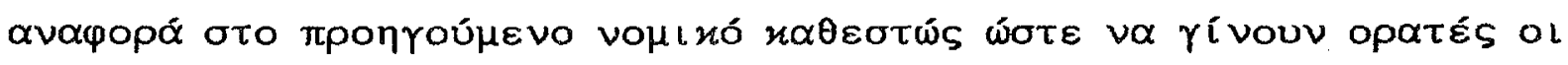

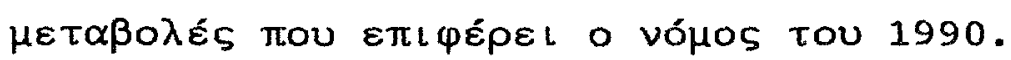




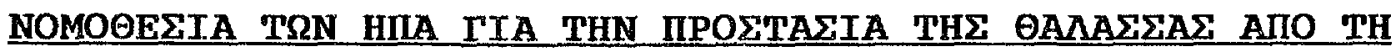

\section{PYIANEH MEXPI TO 1990}

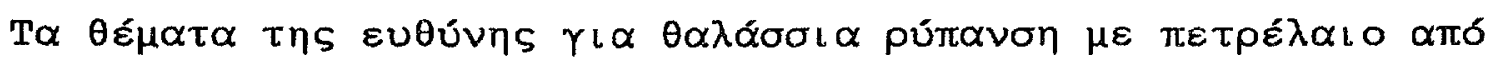

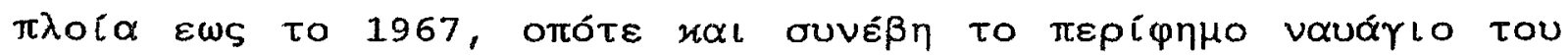

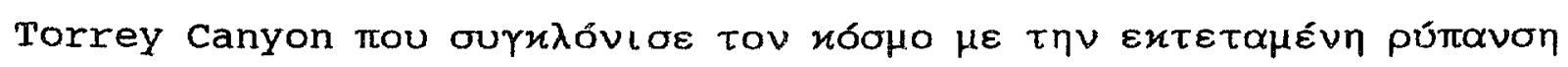

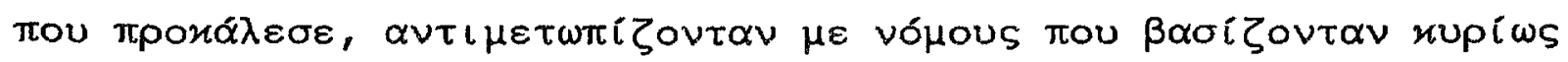

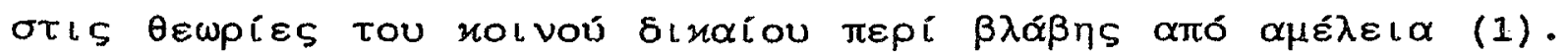

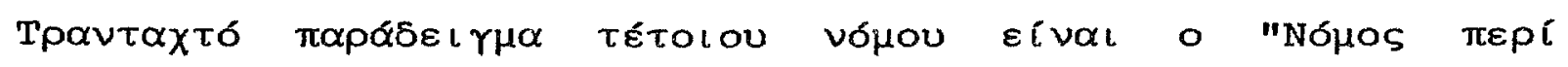

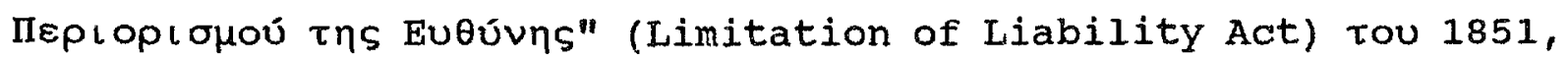

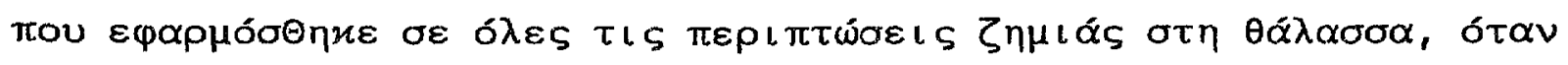

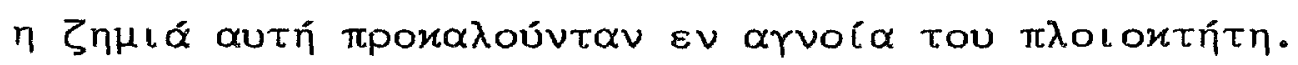

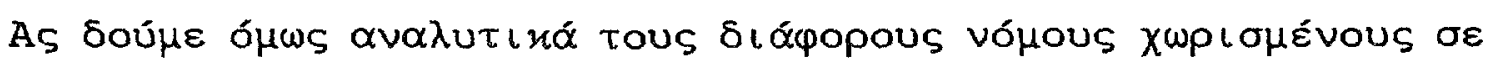

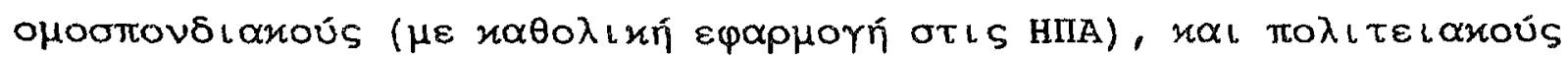

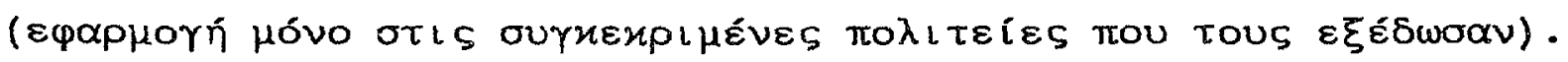




\section{A) OMOEIIONAIAKH NOMOOEEIA TSN H.П.A.}

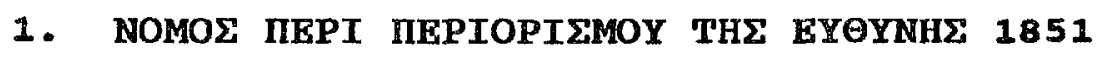

\section{(Limitation of Liability Aet)}

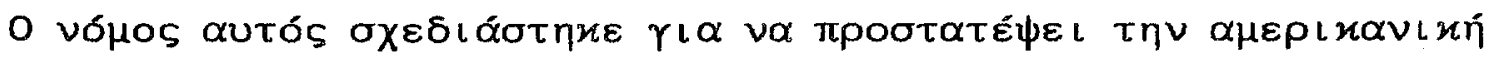

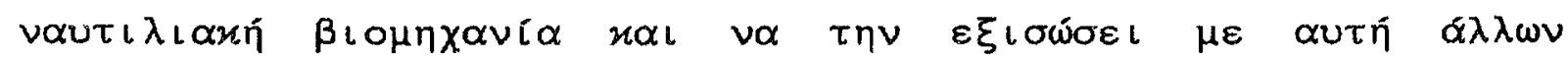

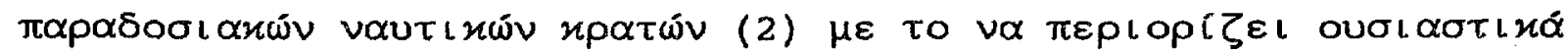

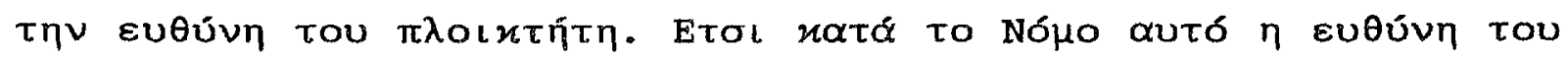

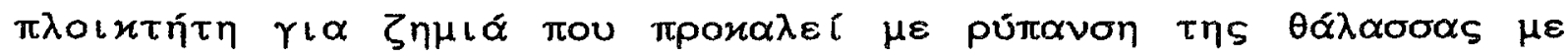

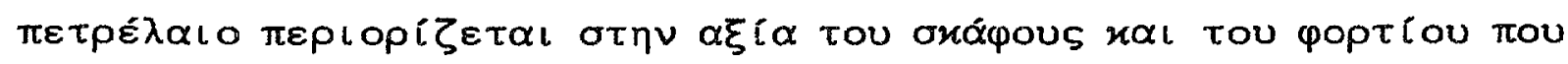

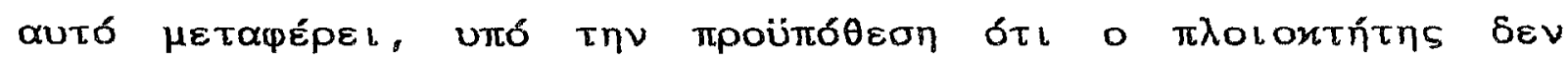

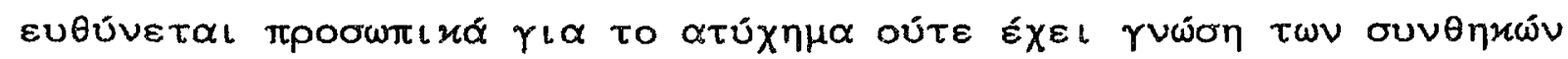

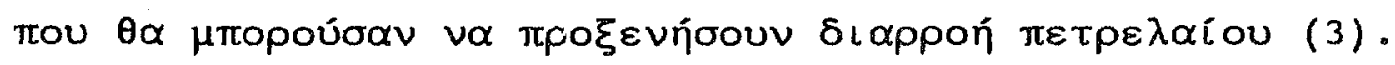

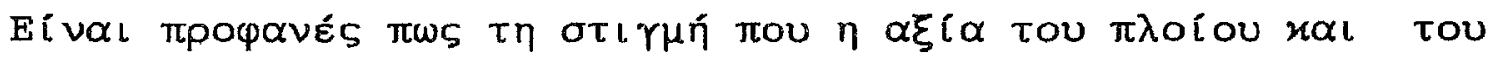

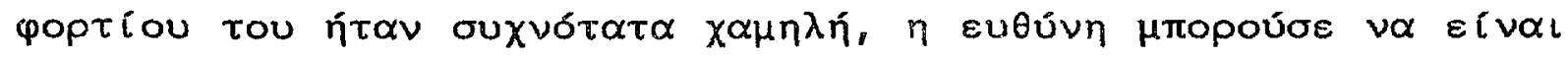

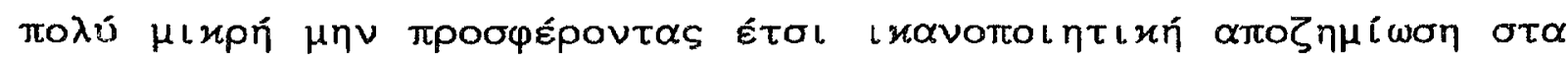

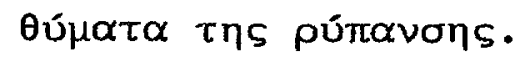

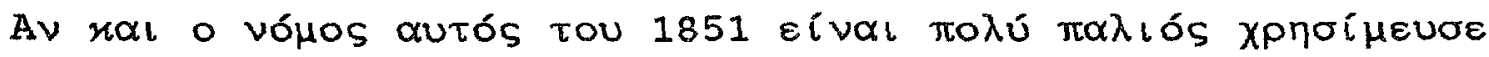

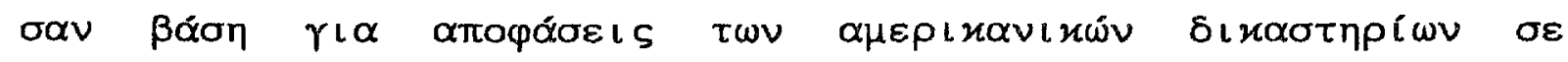

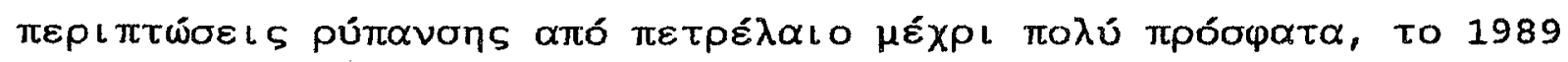
(4). 
2. "NOMOE IEPI IOTAMSN KAI AIMENRN (Rivers and Harbors Act)

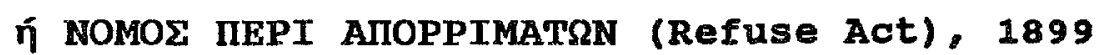

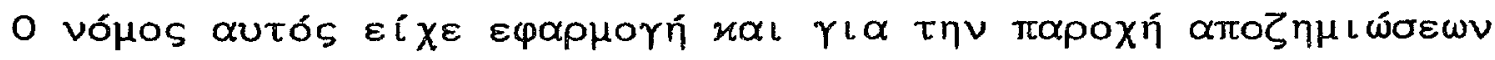

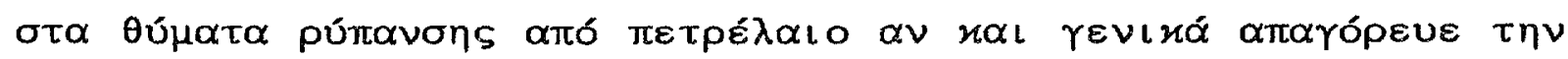
$\alpha \pi o ́ p \rho เ \psi \eta$ oтo

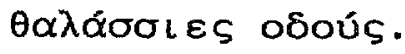

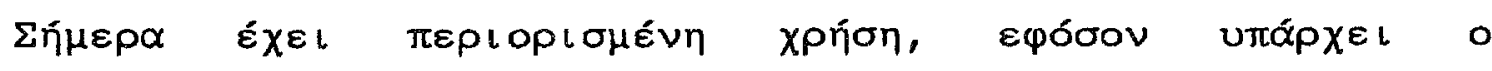

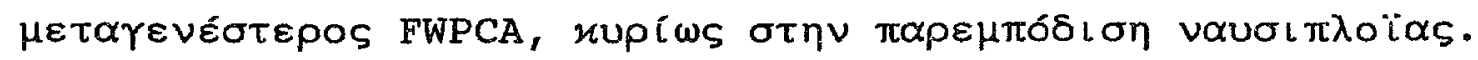

\section{NOMO} (Intervention on the High Seas Act) 1969

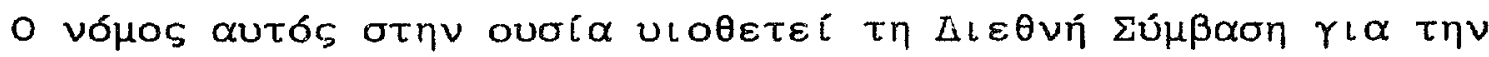

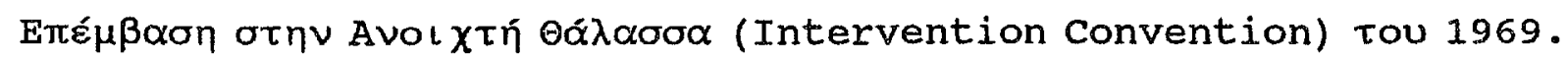

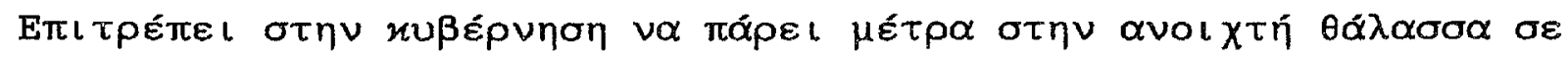

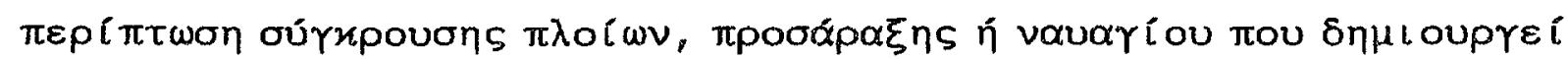

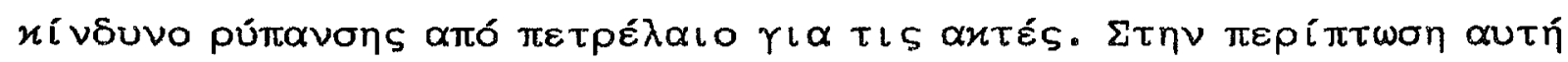

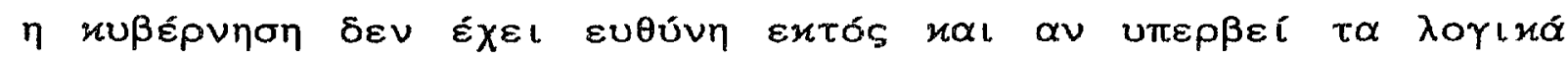

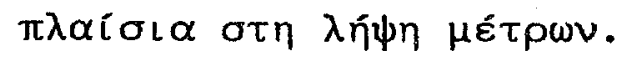


4. NOMOE TIA TH PYIIANEH AIO metpenaIo (Oil Pollution Act) 1924

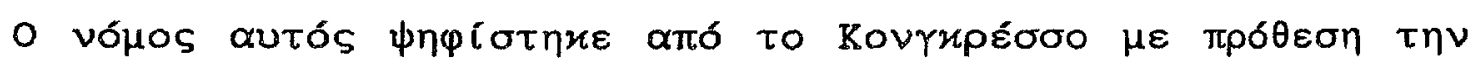

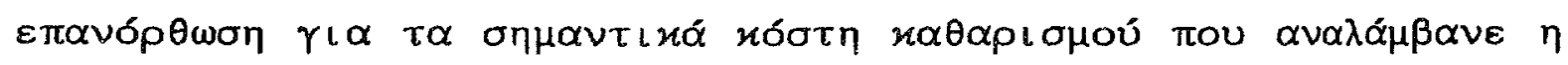

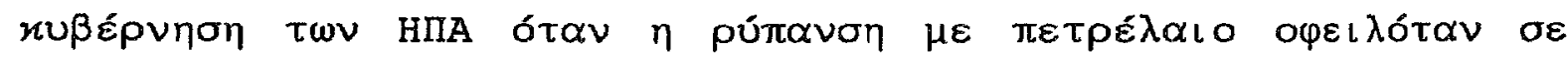

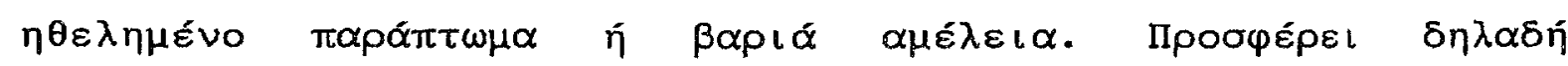

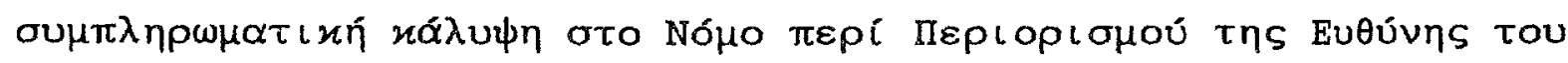
1851.

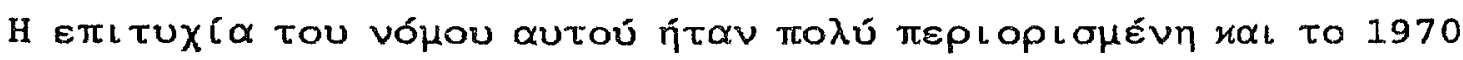

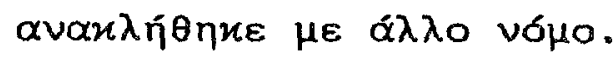


5. OMOEMONAIAKOE NOMOE TIA TON EAETXO THE PYIANSHE TSN YAATSN í FWPCA 1948 (Federal Water Pollution Control Act)

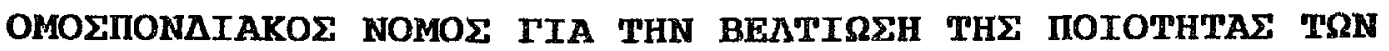
YAATSN ń

WQIA 1970 (Federal water Quality Improvement Act)

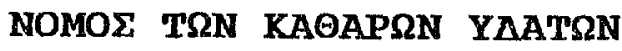

ர் CWA 1977 (Clean Water Act)

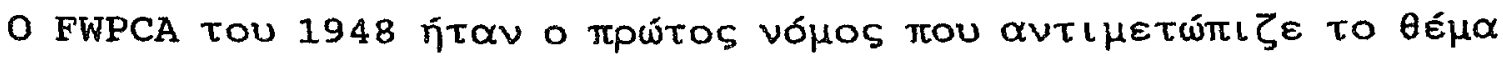

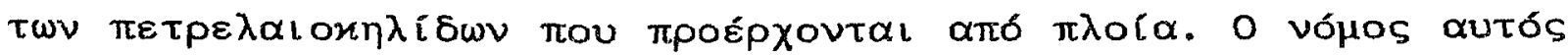

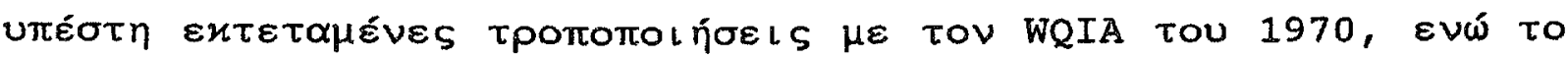

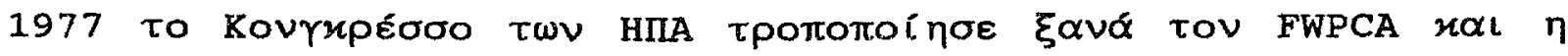

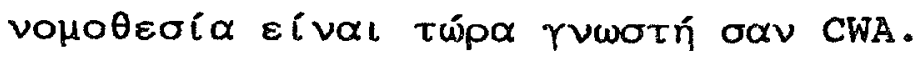

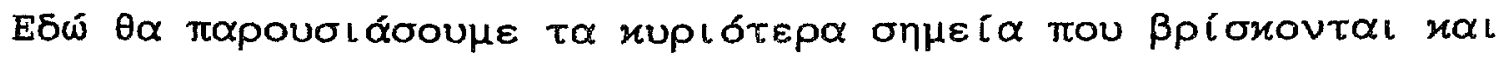

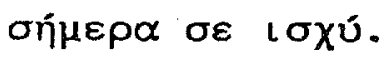

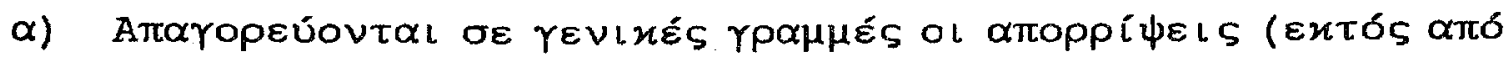

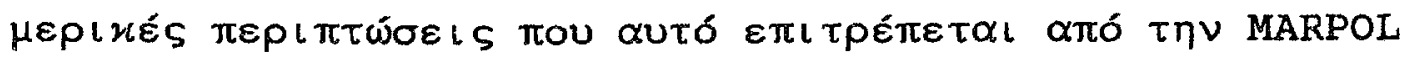

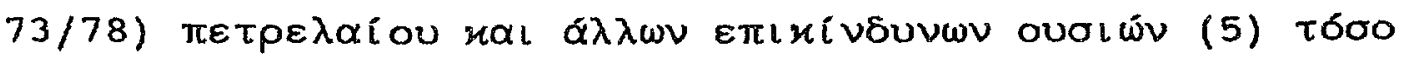

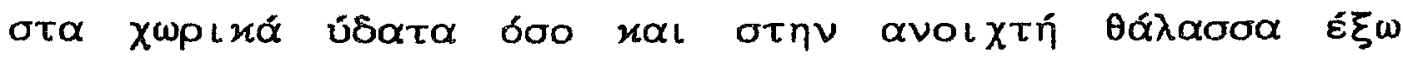

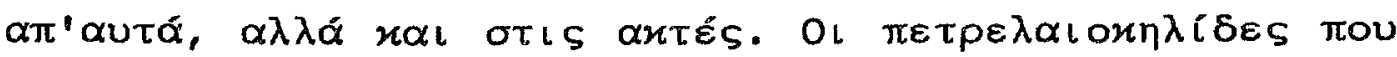

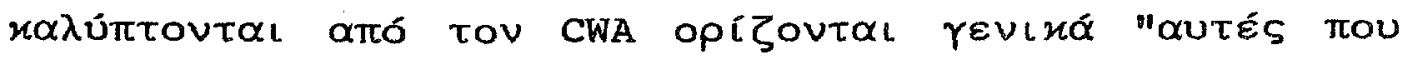

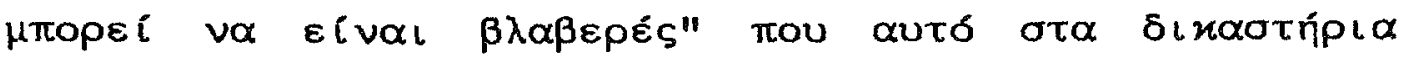

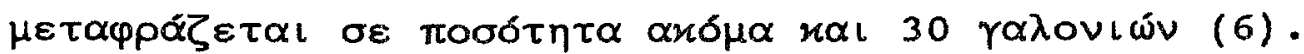




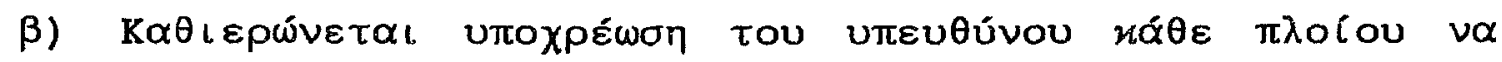

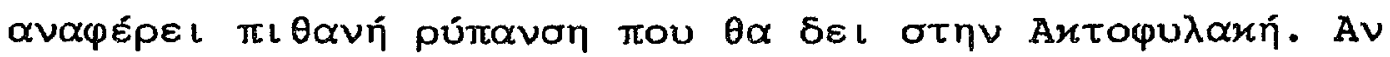

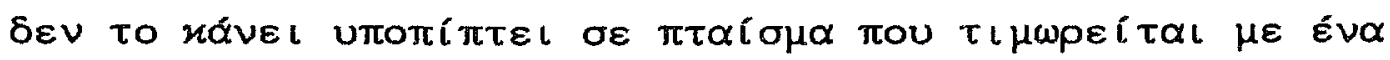

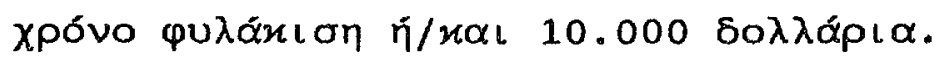

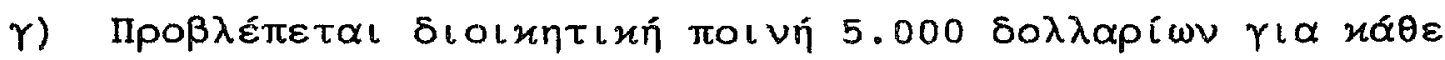

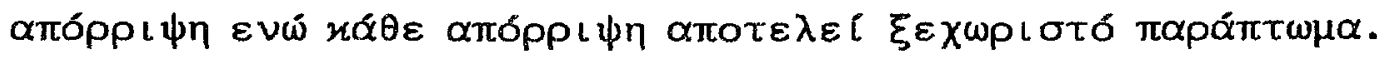

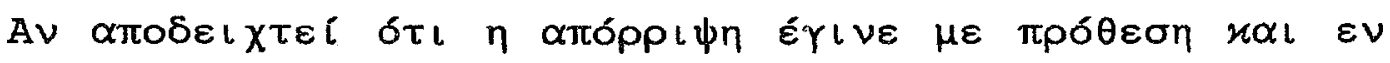

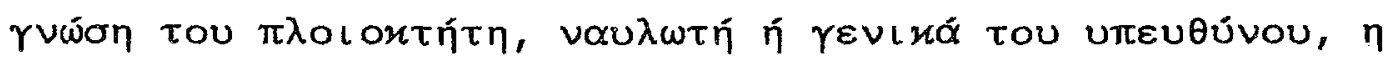

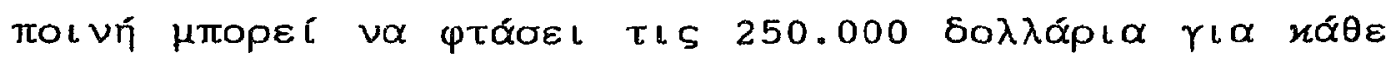
$\alpha \delta$ in $и \mu \alpha$.

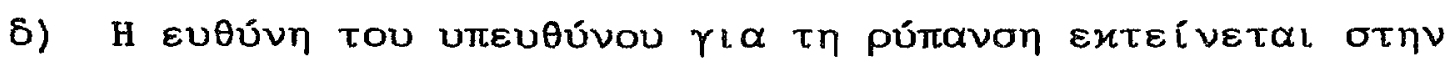

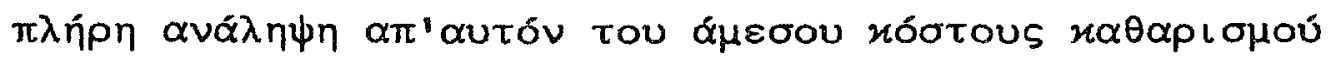

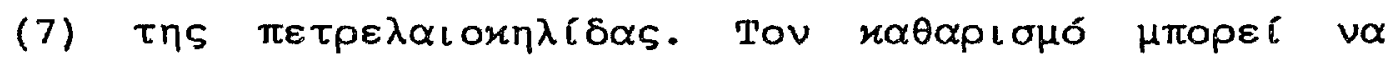

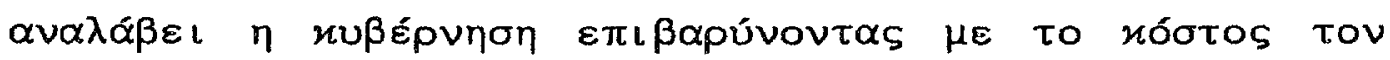

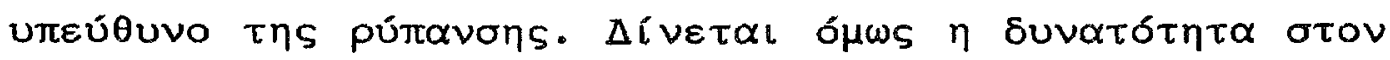

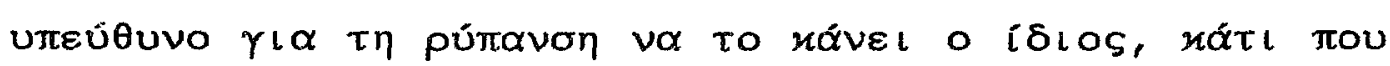

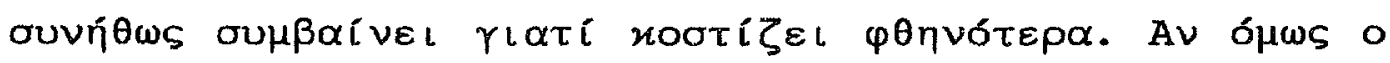

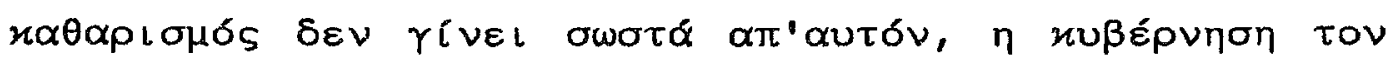

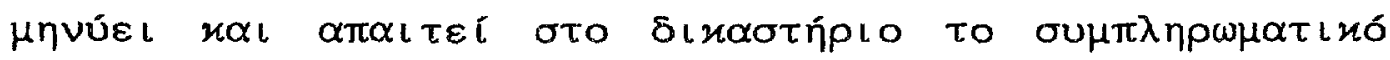

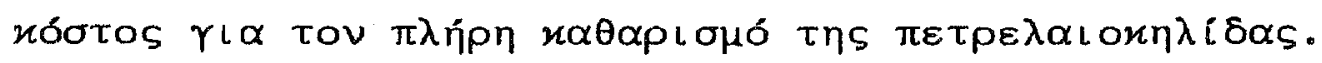




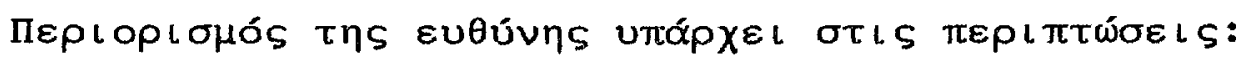

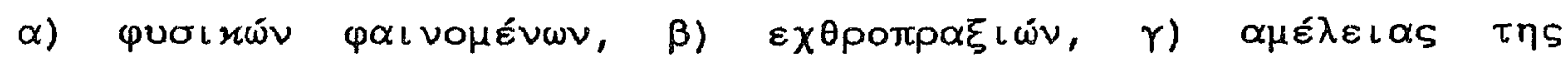

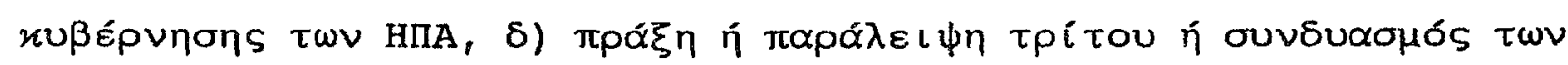

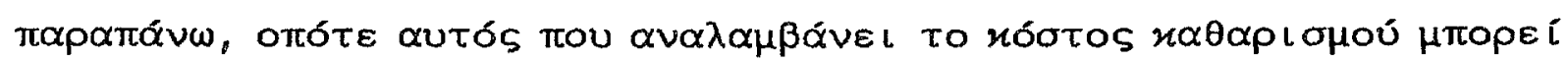

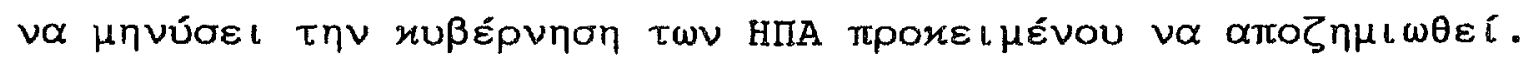

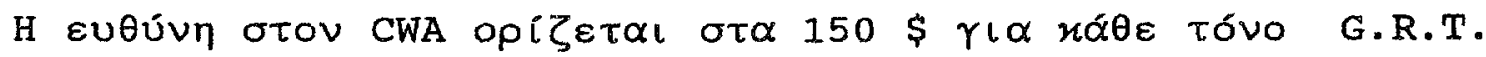

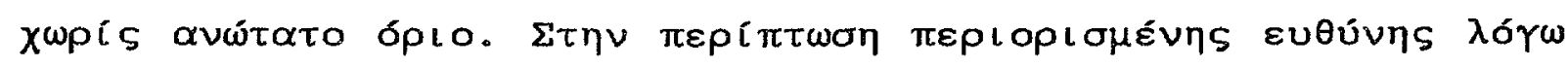

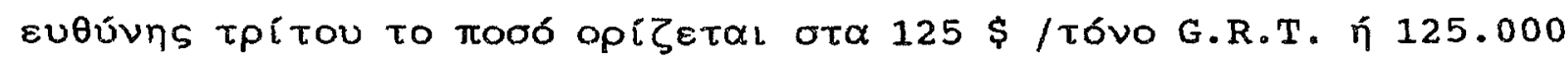

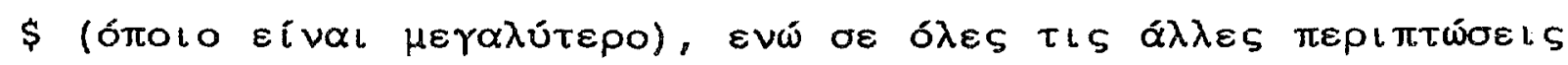

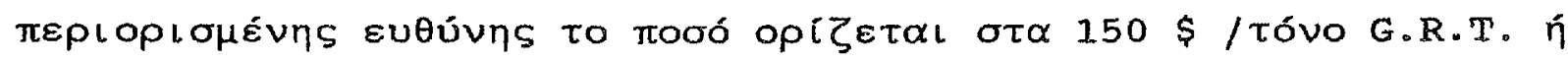

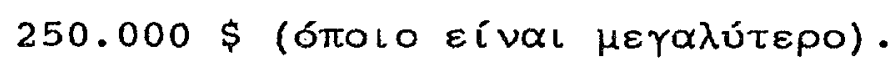

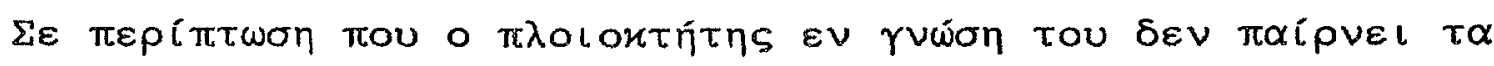

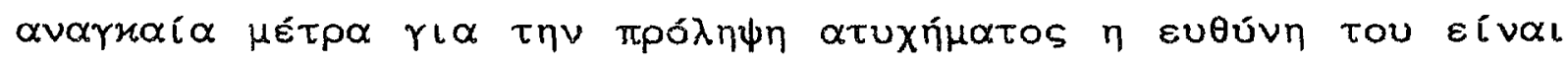
$\alpha \pi \varepsilon \rho \iota o ́ p \iota \sigma \tau \eta$.

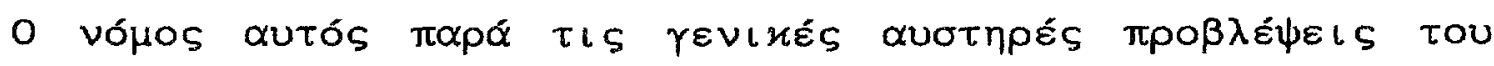

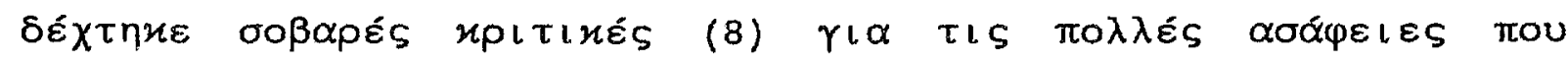

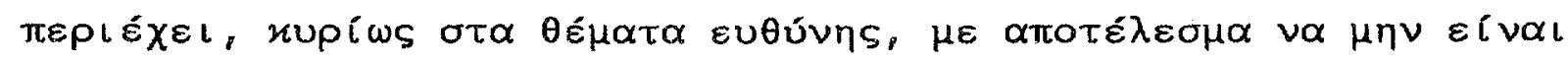

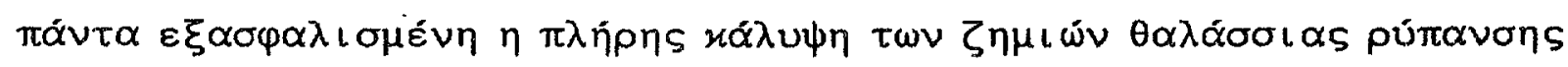
$\mu \varepsilon \pi \varepsilon \tau \rho \varepsilon \dot{\lambda} \alpha \iota \mathrm{o}$. 


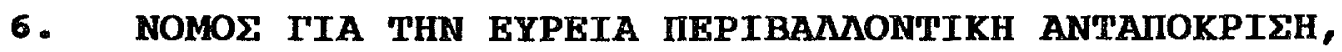
AIOZHMISEH KAI BYOYNH ń

CERCLA ñ Superfund, 1980 (Comprehensive Environmental Response, Compensation and Liability Act)

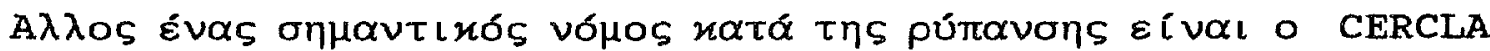

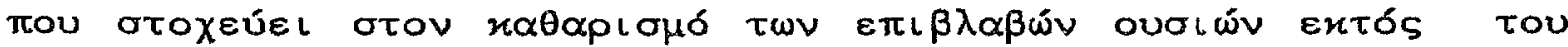

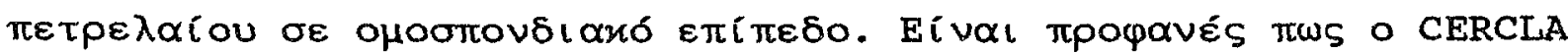

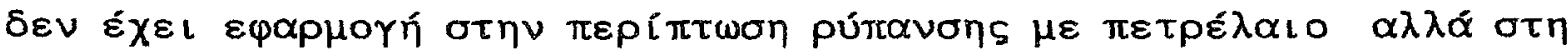

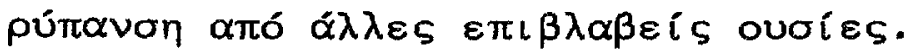

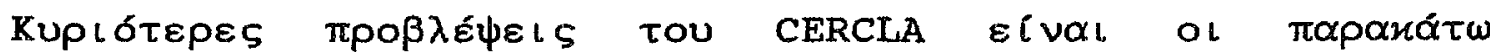

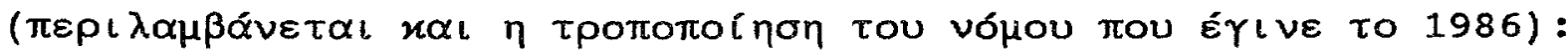

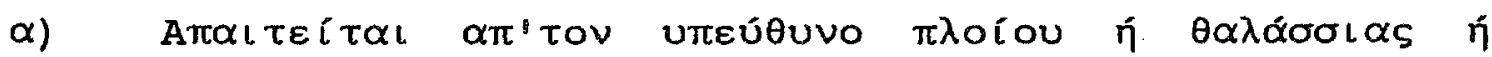

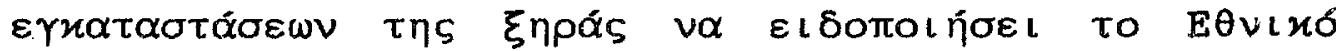

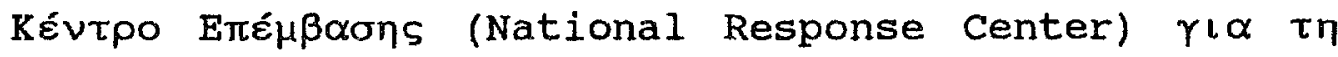

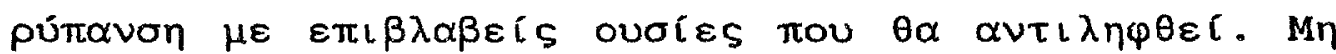

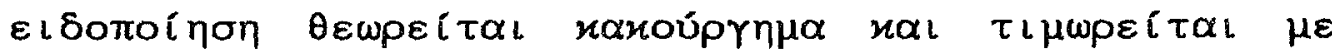

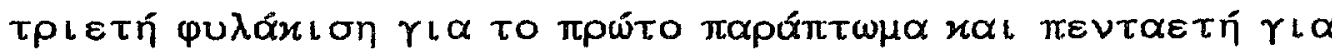
$\varepsilon \pi \alpha \nu \varepsilon\llcorner\lambda \eta \mu \mu \varepsilon \varepsilon \vee \alpha \pi \alpha \rho \pi \tau \omega \mu \alpha \tau \alpha$. 


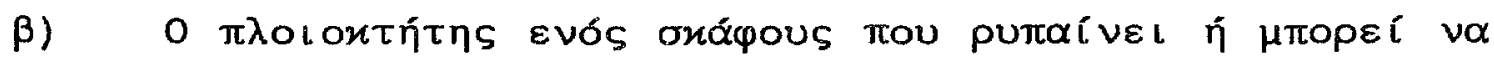

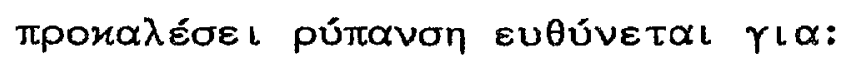

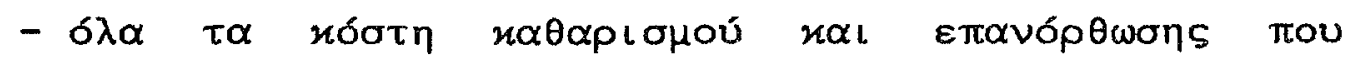

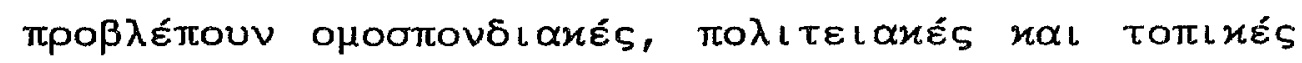
$\alpha \rho \chi \varepsilon ́ s$.

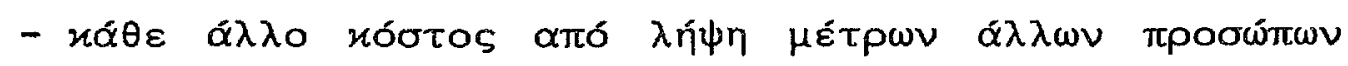

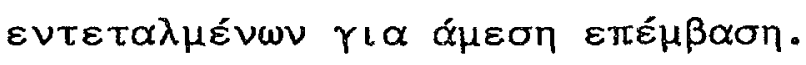

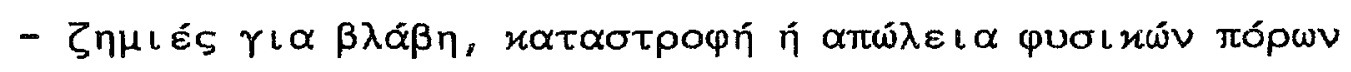
(9)

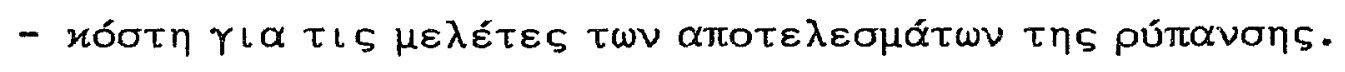

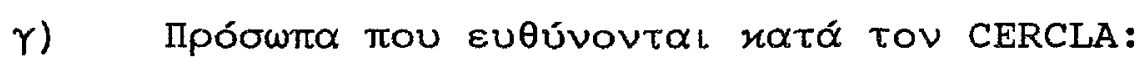

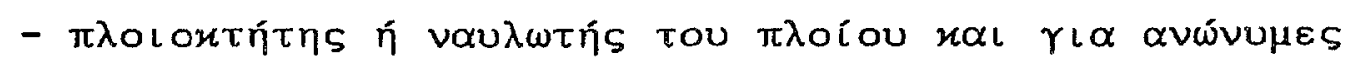

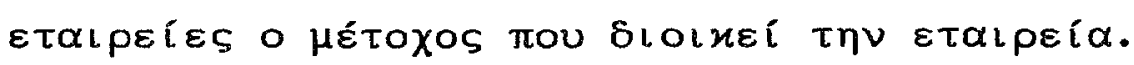

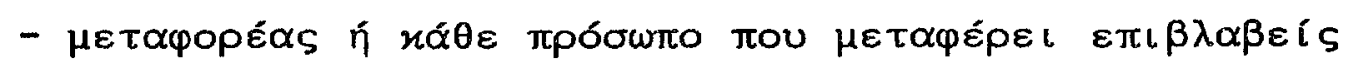
ou

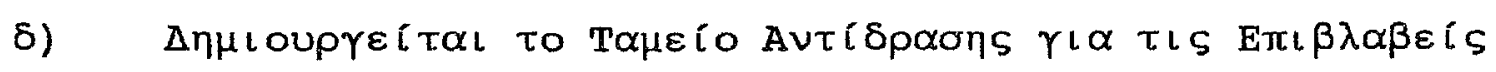
Ovoís (Hazardous Substances Trust Fund) $\varepsilon \xi '$ ou भal $\eta$

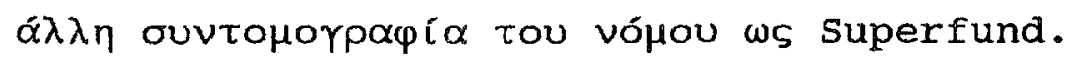

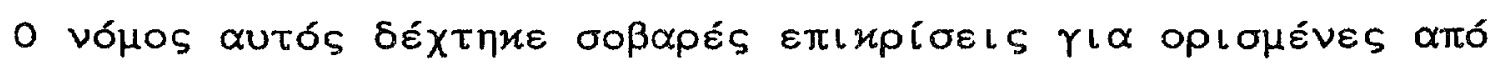

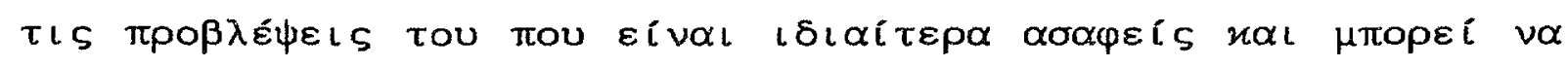

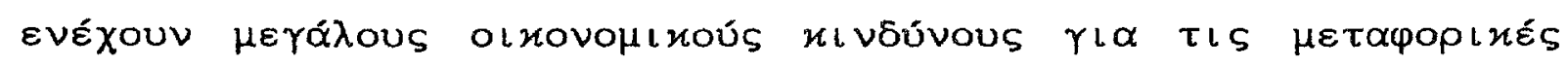
$\varepsilon \pi \iota \chi \varepsilon\llcorner\rho \eta ́ \sigma \varepsilon \iota \varsigma(10)$. 


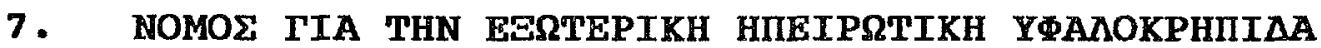
í OCSLA, 1953 (Outer Continental shelf Lands Act)

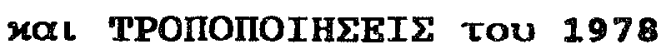

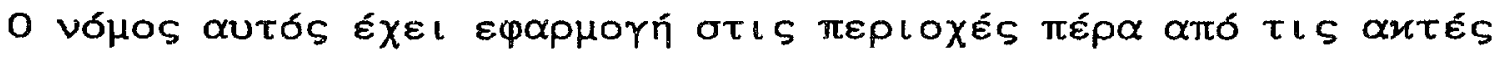

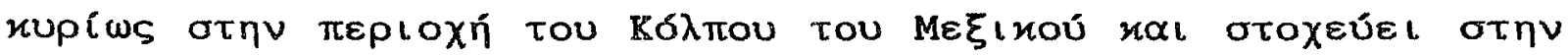

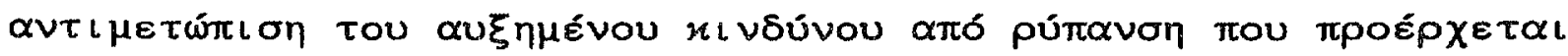

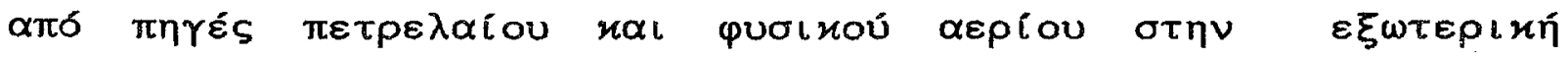

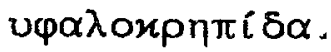

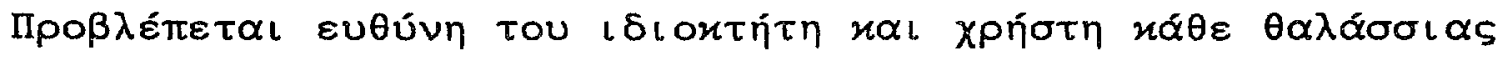

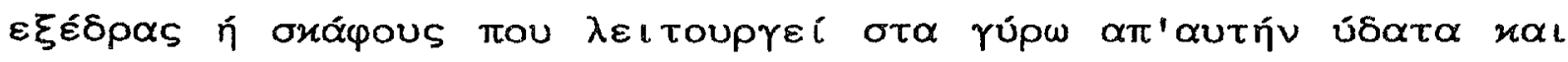

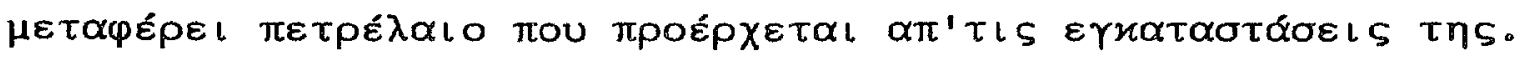

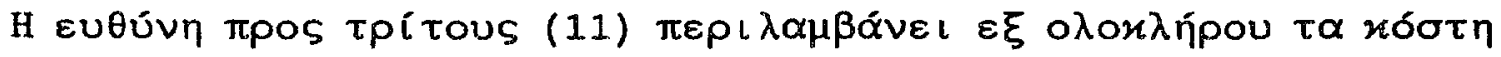

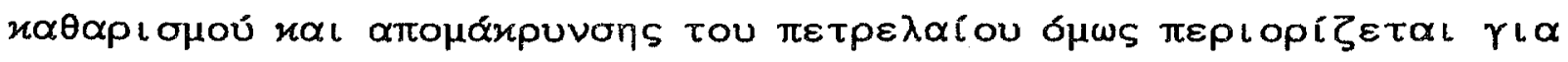

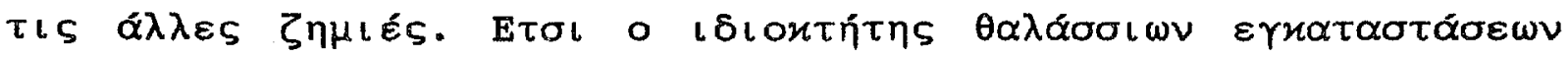

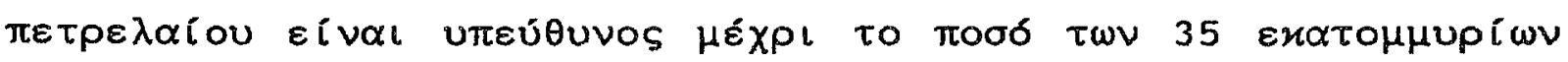

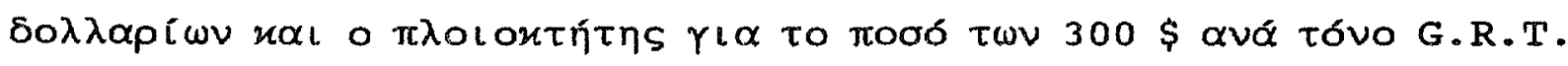

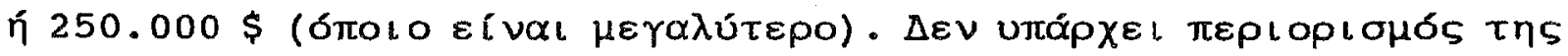

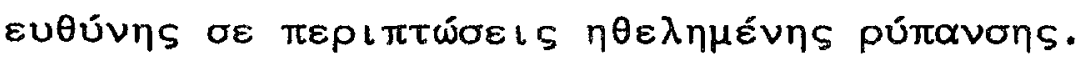




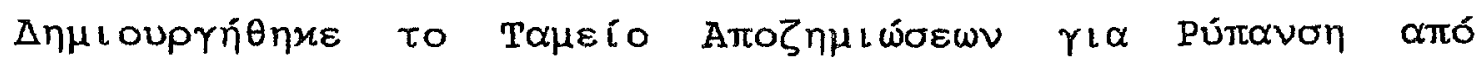

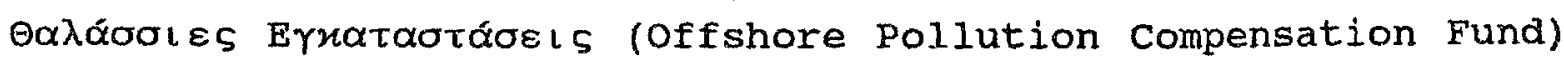

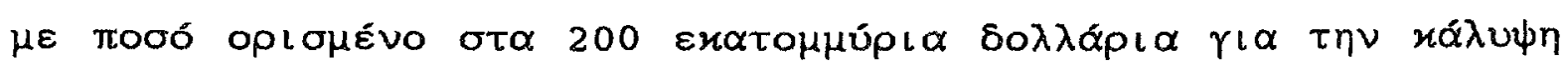

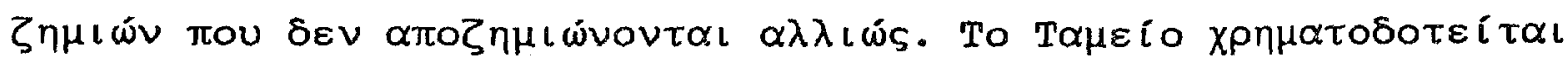

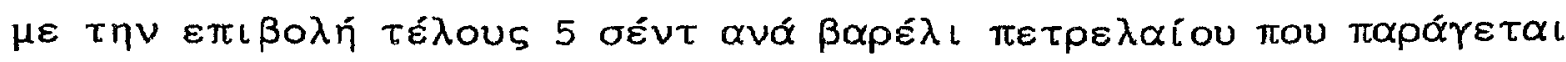

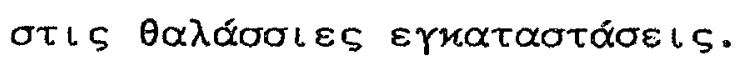

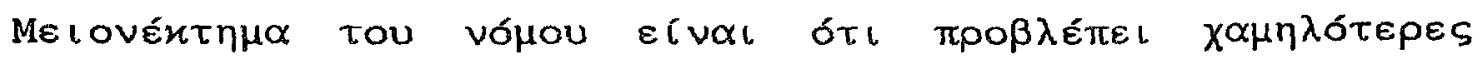

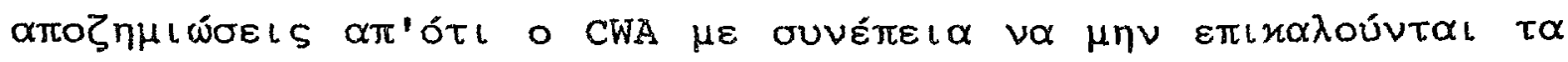

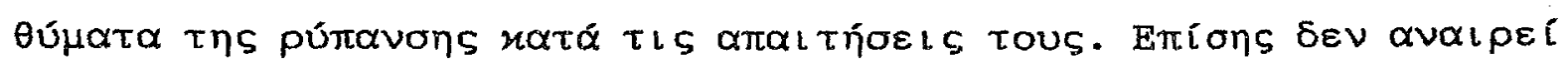

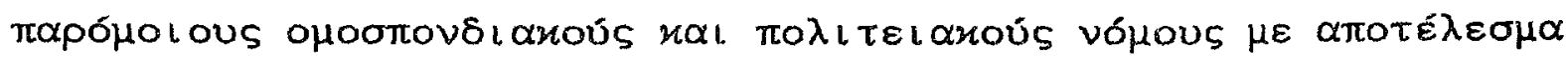

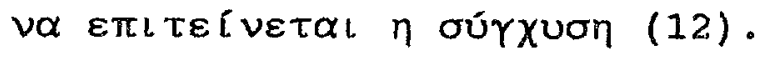




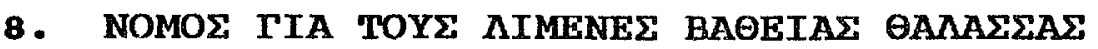

\section{í DPA, 1974 (Deepwater Port Act)}

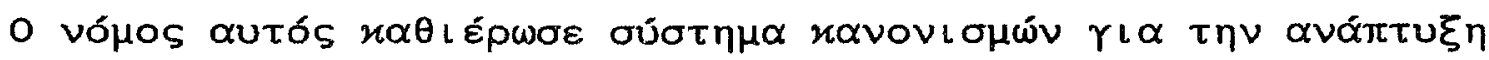

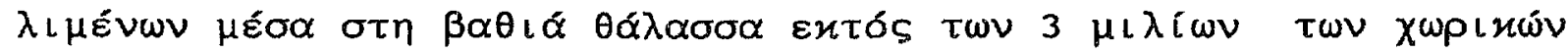

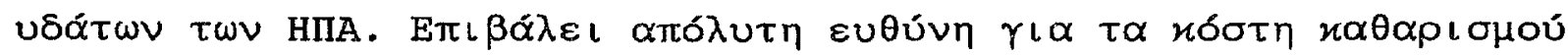

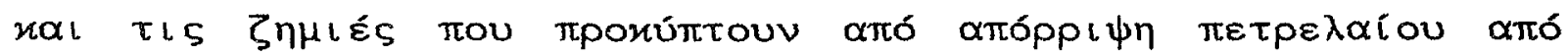

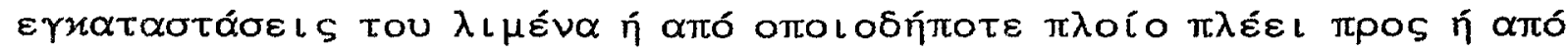

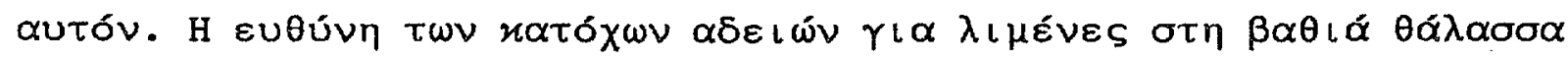

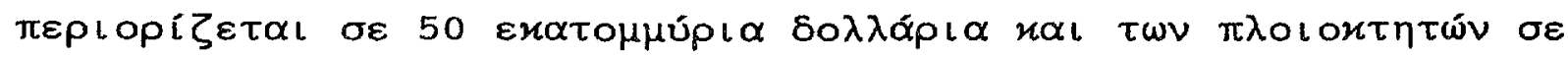

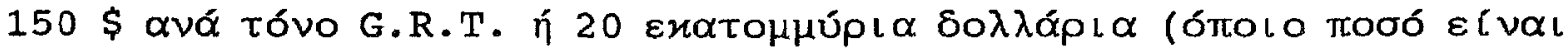
$\mu(\varkappa \rho \delta ́ \tau \varepsilon \rho \circ)$.

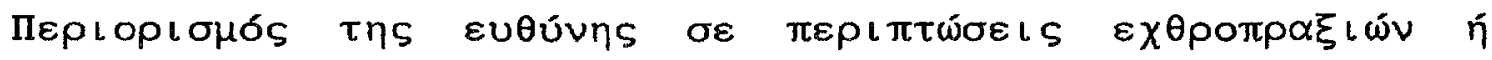

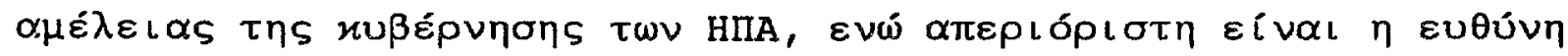
$\gamma \iota \alpha \beta \alpha \rho \iota \alpha ́ \alpha \mu \varepsilon \lambda \varepsilon \iota \alpha, \pi \rho \delta \theta \varepsilon \sigma \eta \eta$ $\lambda\llcorner\mu \varepsilon ́ v \alpha$.

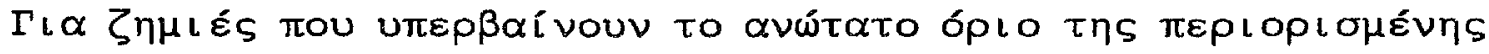

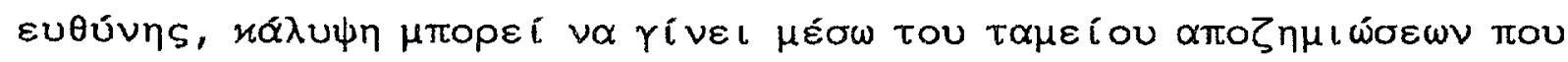

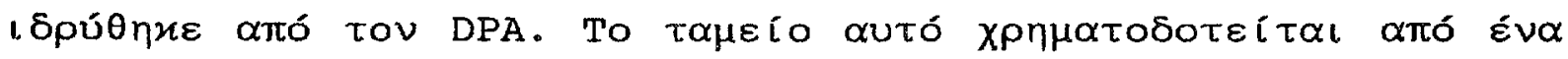

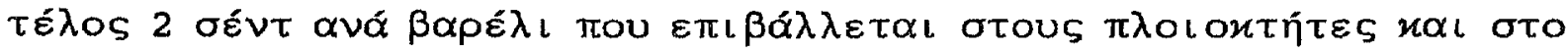

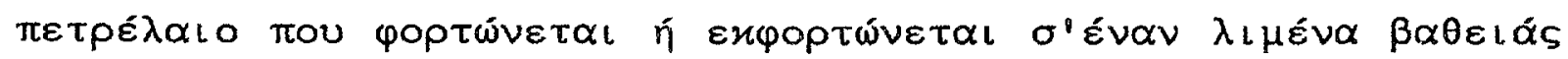

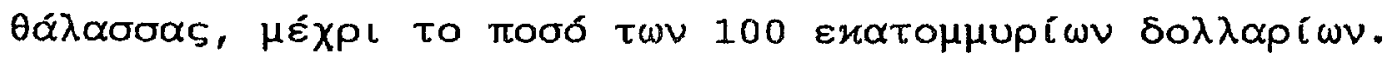

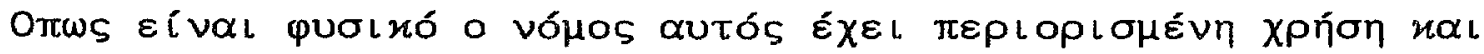

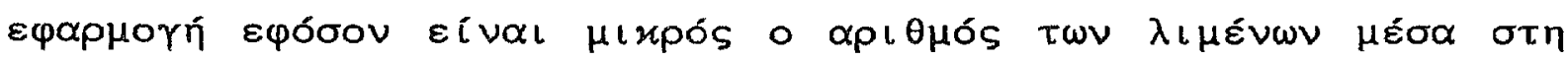
$\beta \alpha \theta \varepsilon\llcorner\alpha$ $\theta \alpha \alpha \lambda \alpha \sigma \sigma \alpha$. 


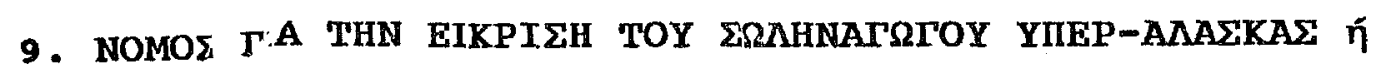 TAPP 1973 (Trans-Alaska Pipeline Authorization ACt)}

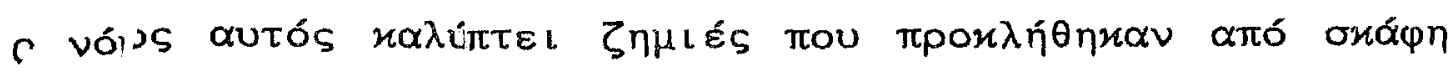

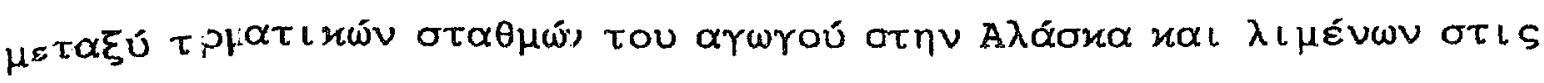

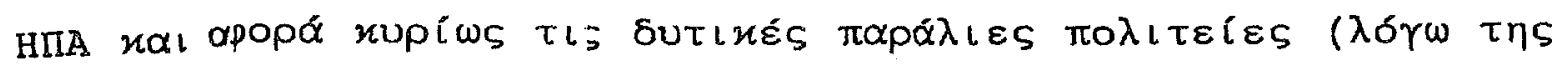

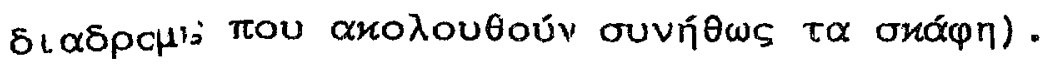

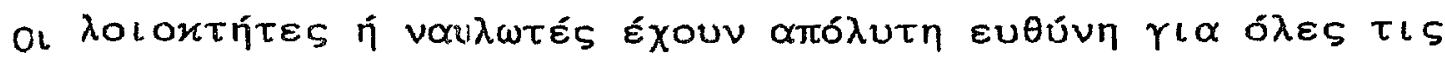

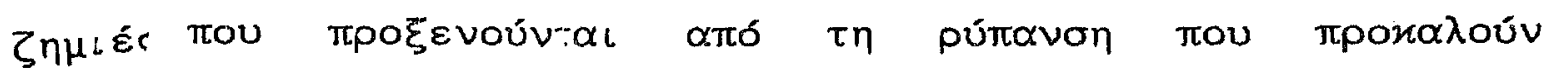

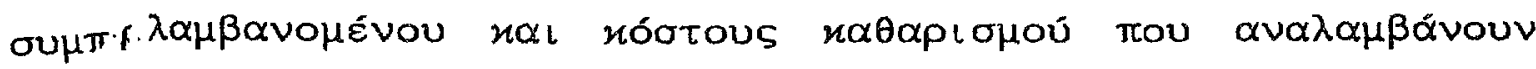

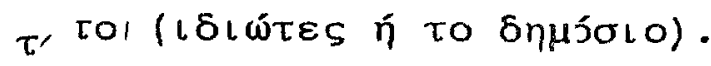

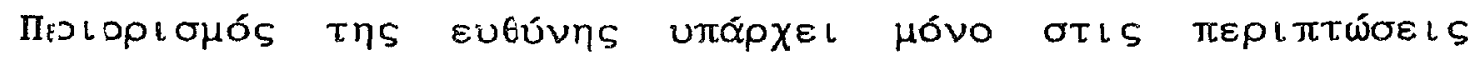

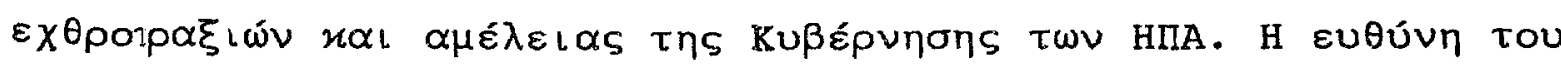

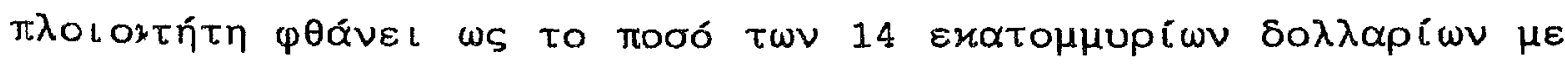

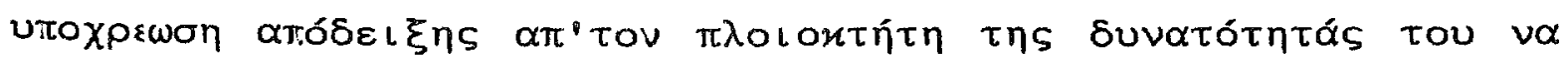

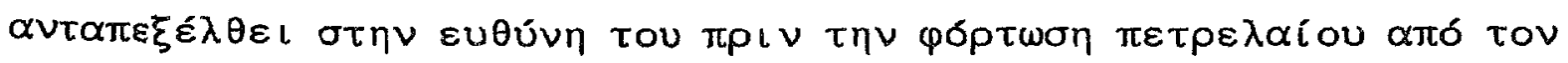
arworó.

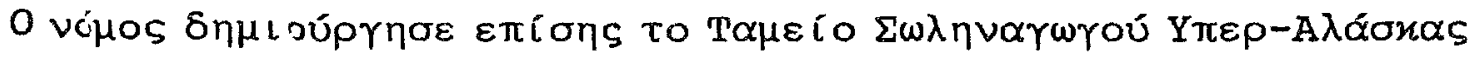

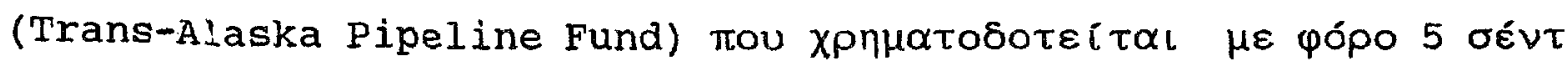

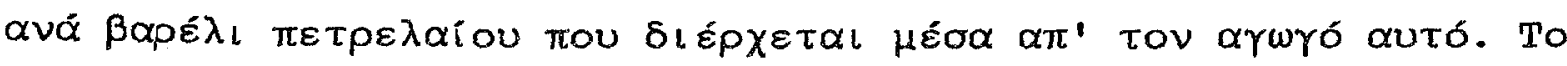

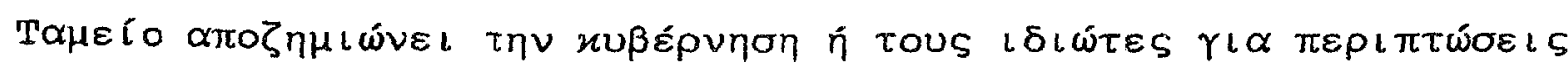

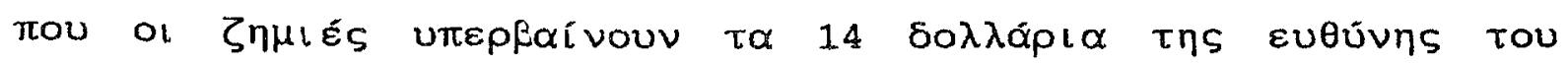

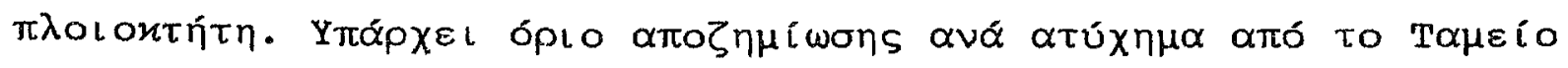

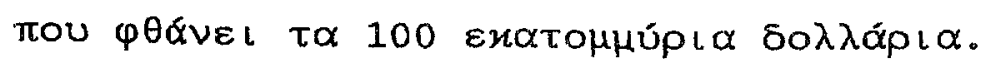




\section{B. MOAITEIAKH NOMOOEEIA TSN HILA}

\section{TENIKA}

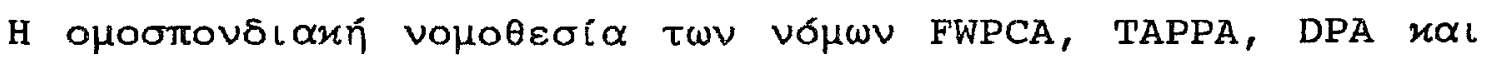

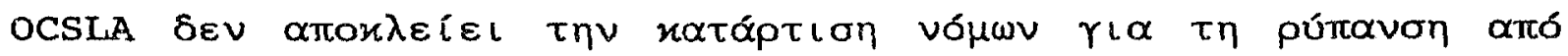

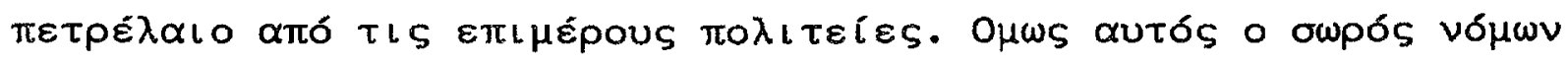

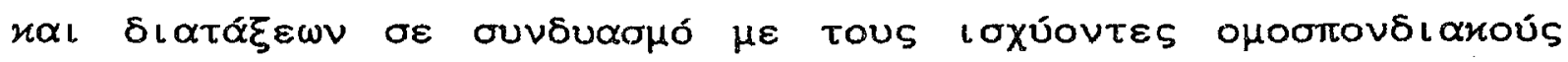

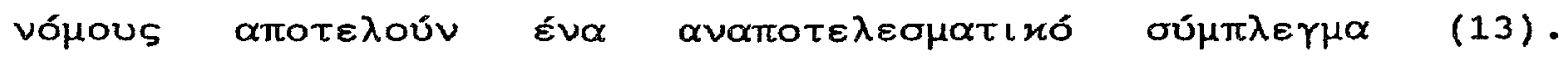

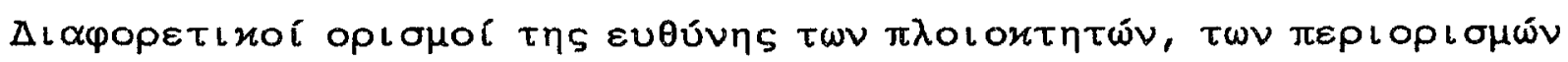

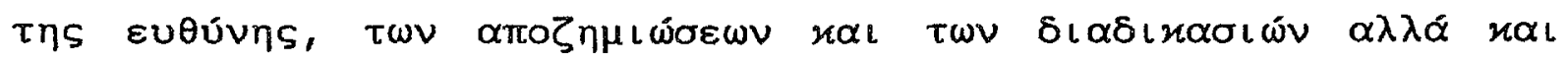

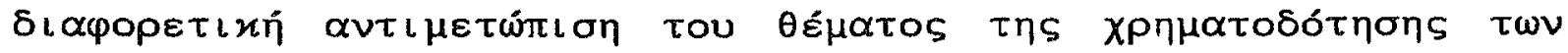

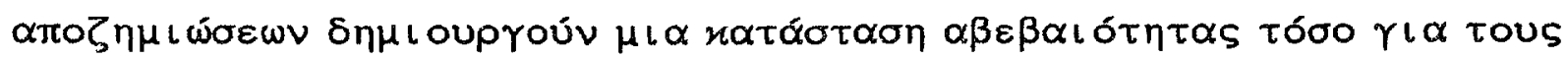

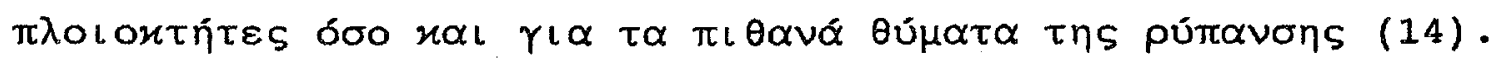




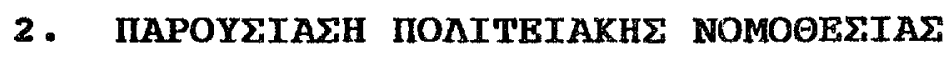

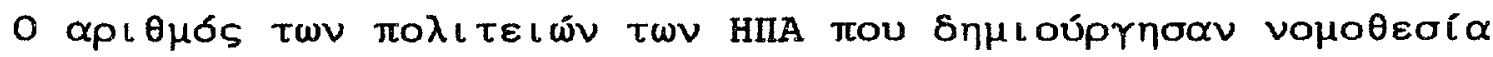

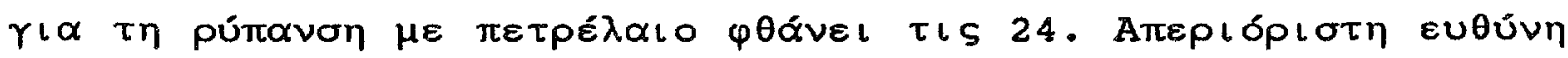

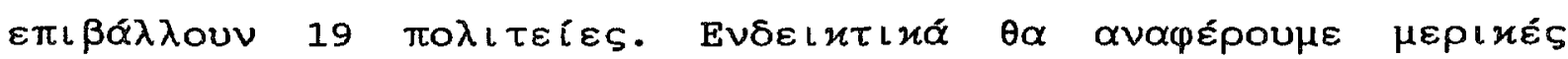

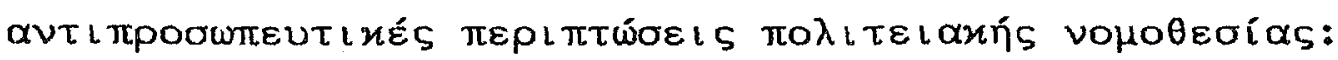

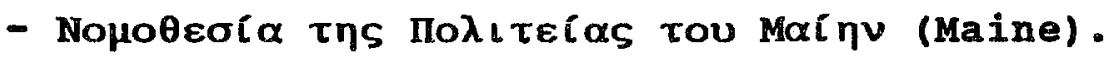

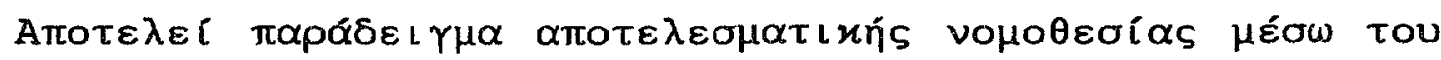

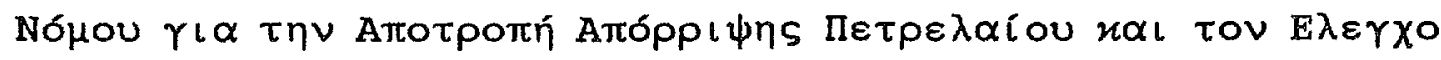

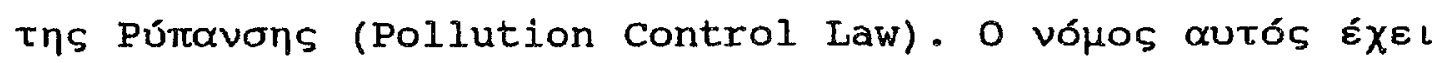

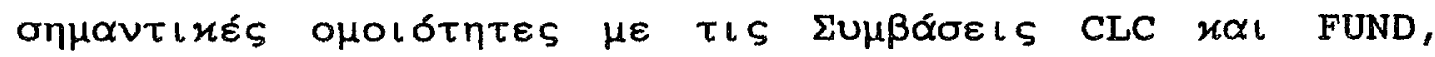

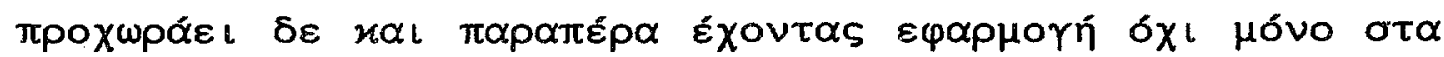

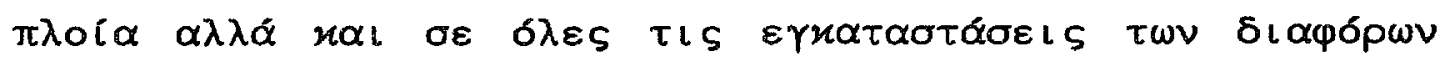

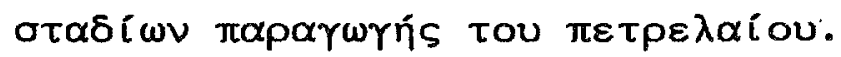

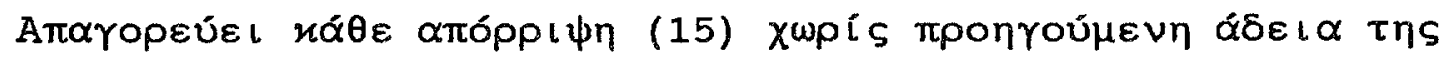

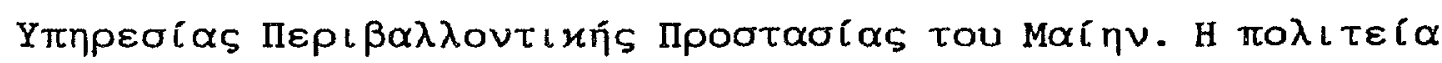

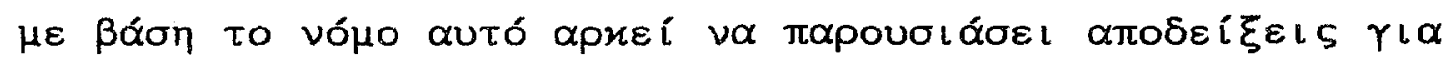

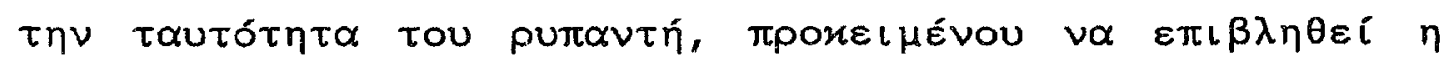

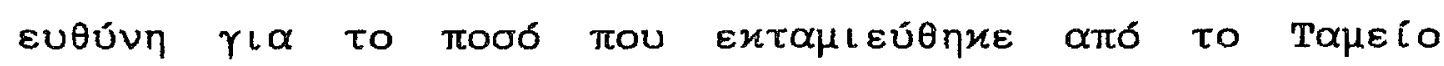

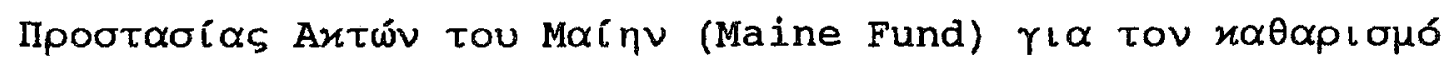
тทร pútravans. 


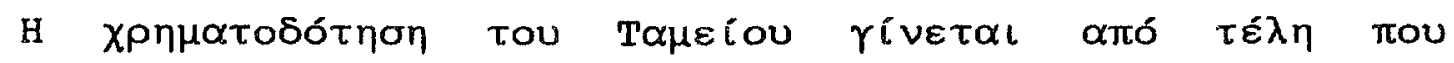

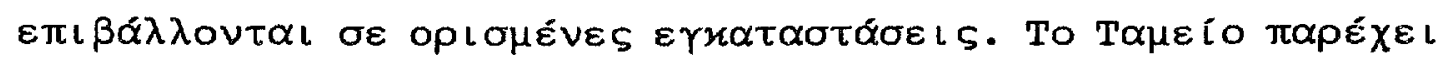

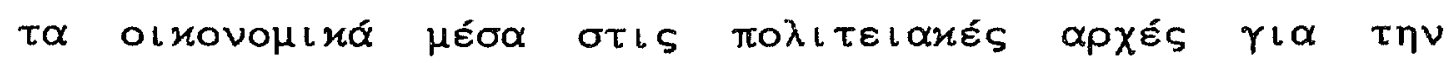

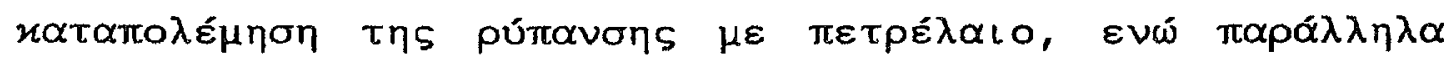

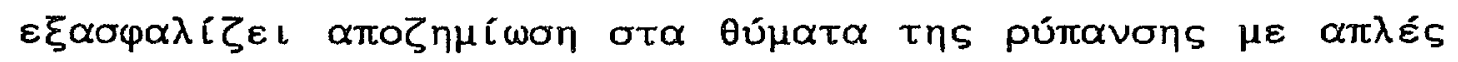
$\delta \iota \alpha \delta \imath \varkappa \alpha \sigma i \varepsilon s$.

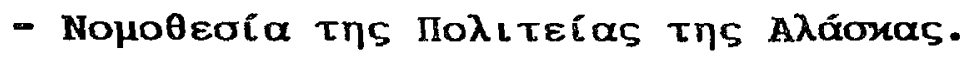

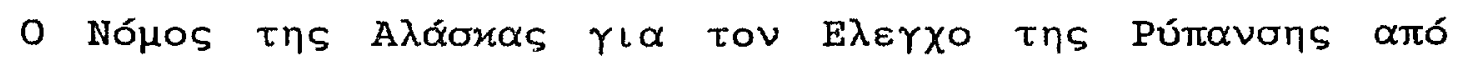

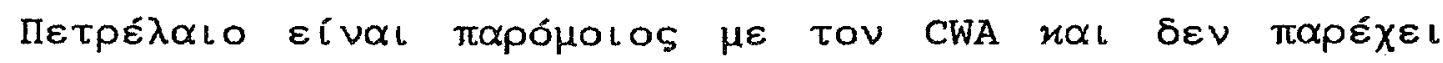

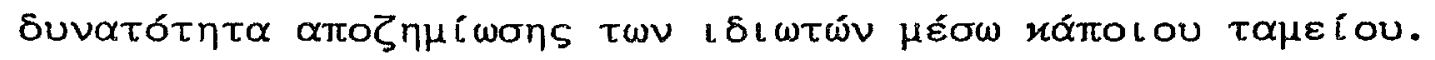

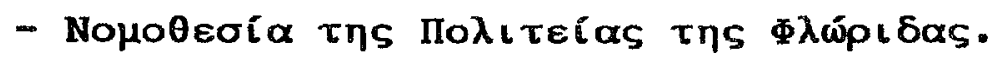

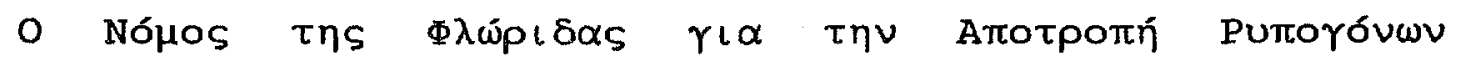

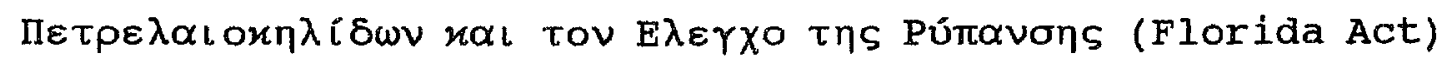

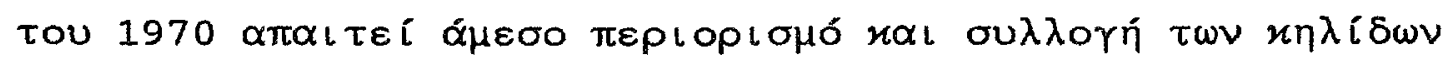

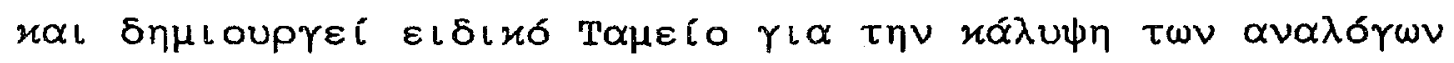

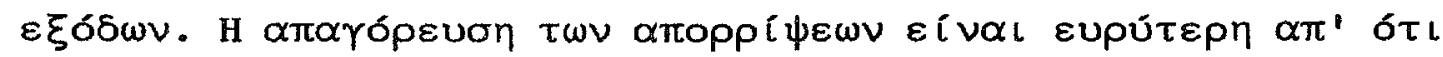
oTov CWA.

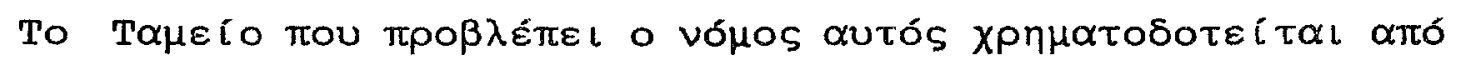

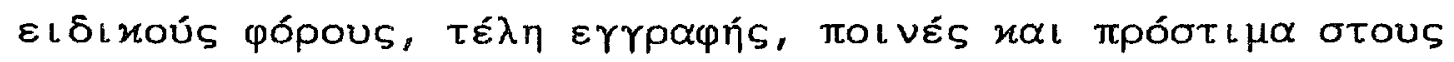

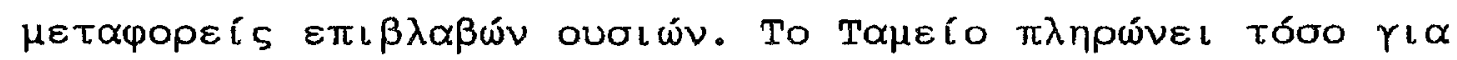

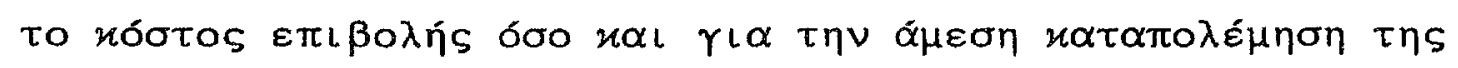
púmavons. 


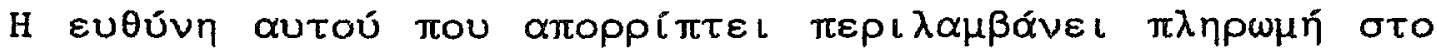

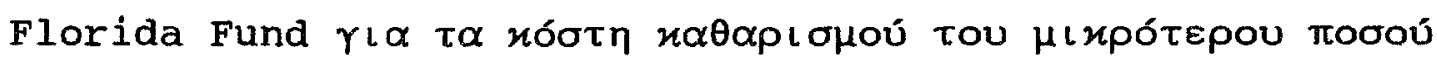

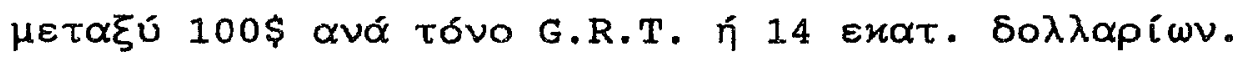

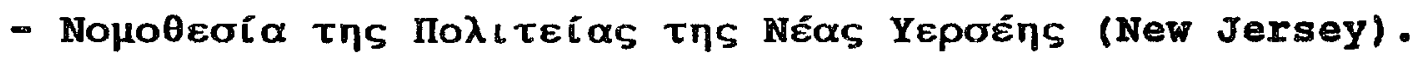

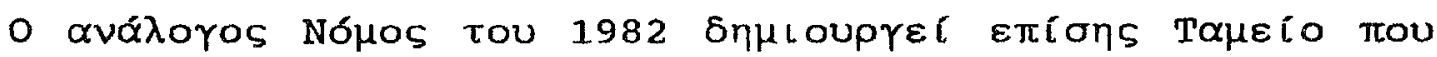

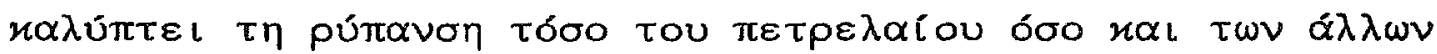

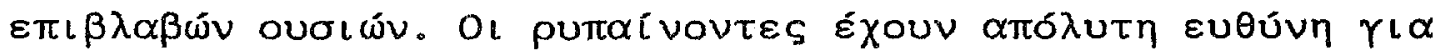

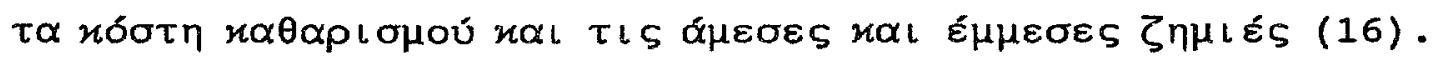

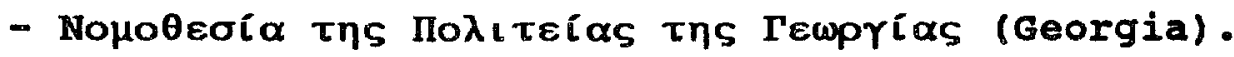

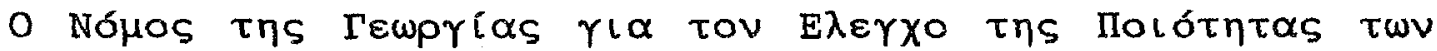

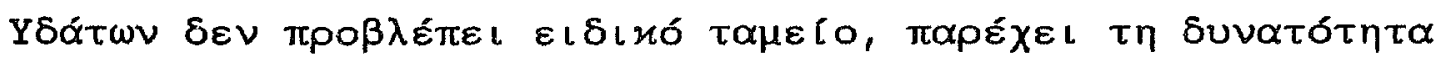

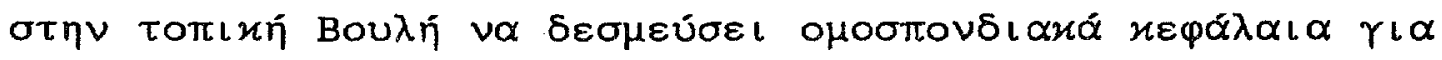

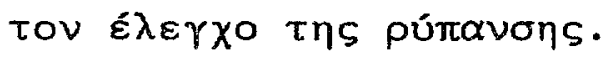

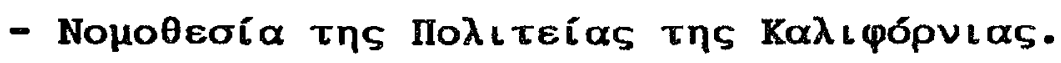

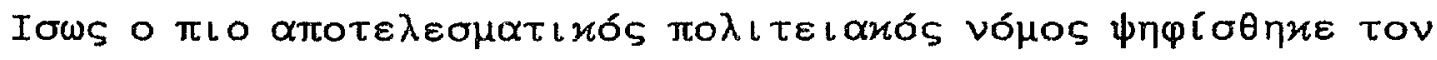

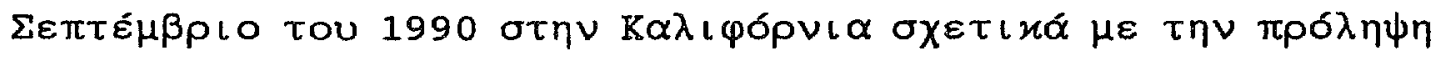

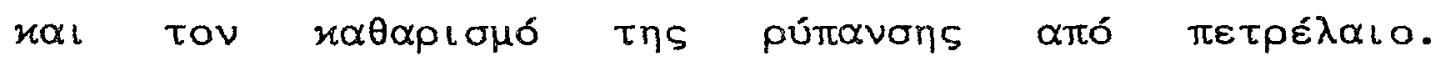

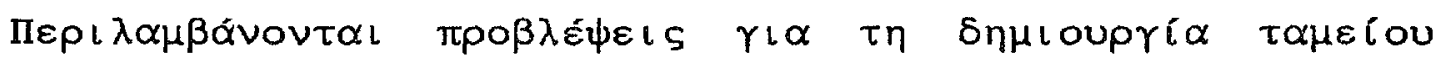

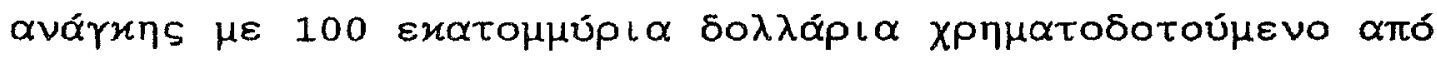

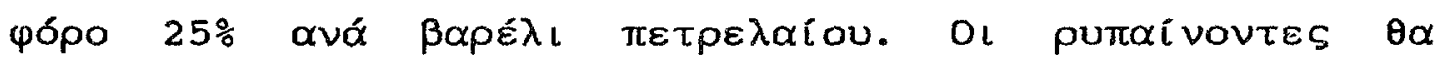

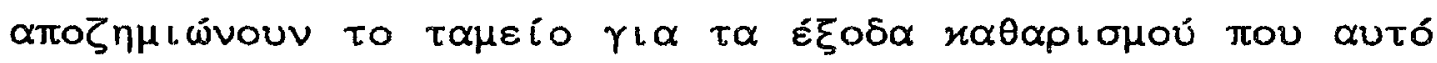

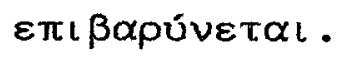




\section{NOMOE TIA THN PYMANSH AMO METPEAATO ń OPA '90, 1990}

\section{Coil Polution Act)}

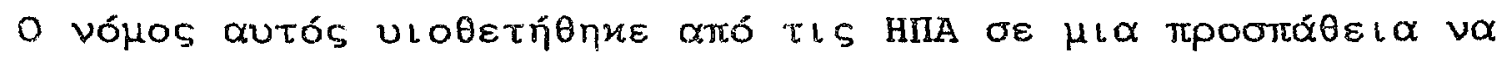

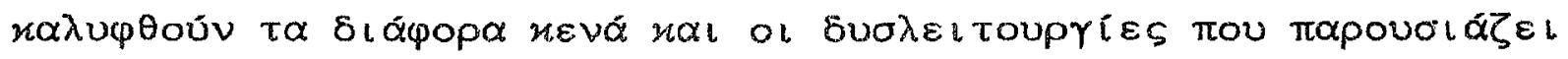

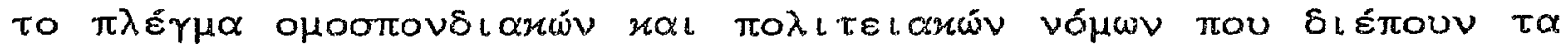

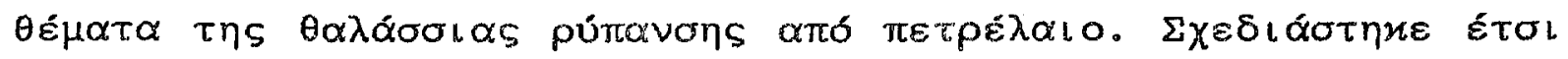

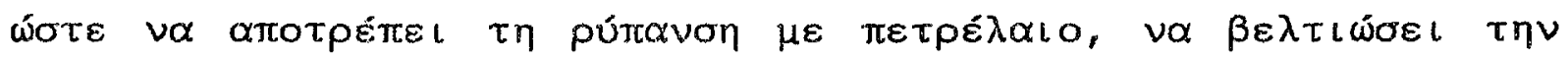

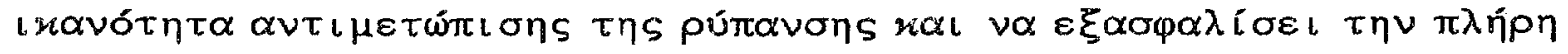

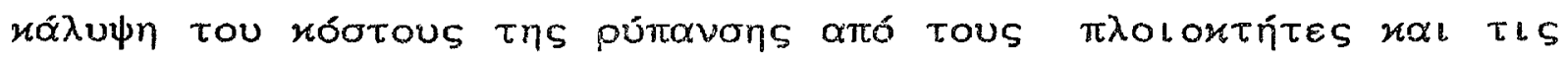
$\varepsilon \tau \alpha \iota \rho \varepsilon[\varepsilon \varsigma \pi \varepsilon \tau \rho \varepsilon \lambda \alpha i$ ou (17).

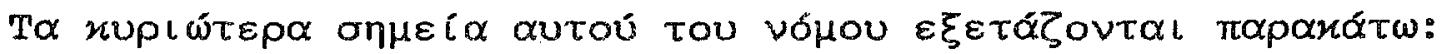

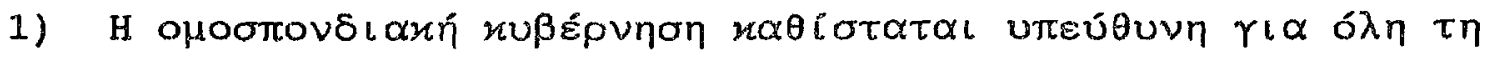

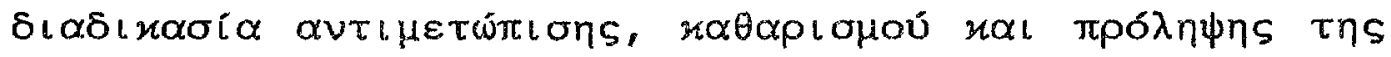
pútravons.

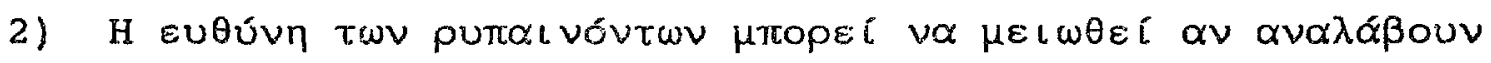

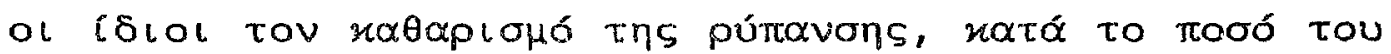

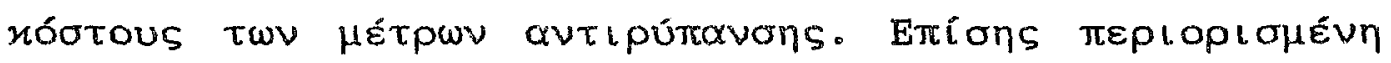

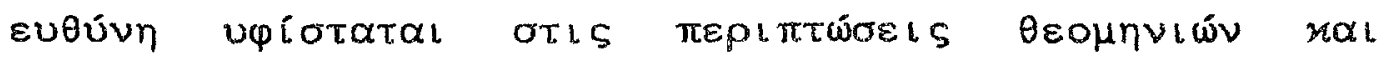
$\varepsilon \chi \theta \rho \circ \pi \rho \alpha \xi\llcorner\omega$.

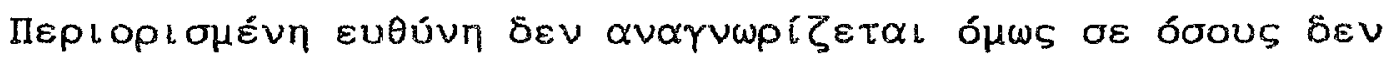

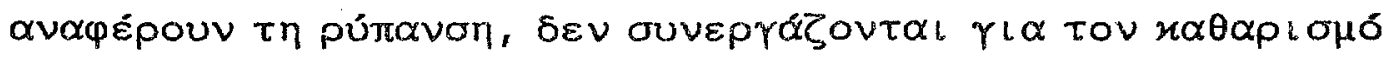

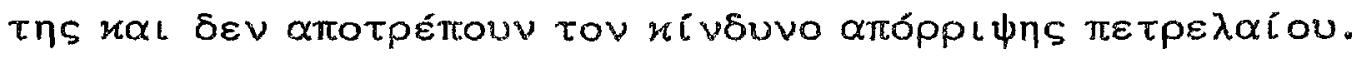


3) H ع

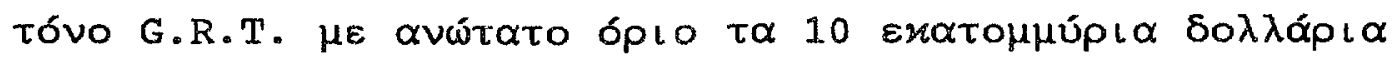

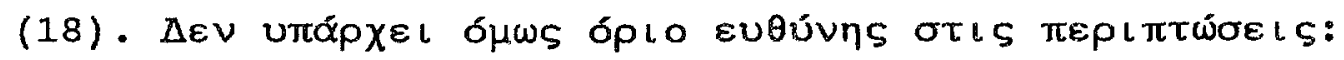

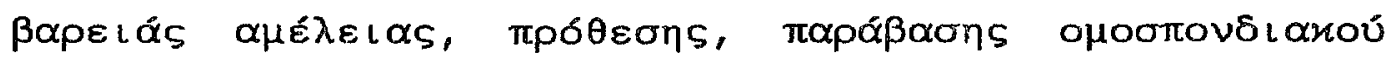

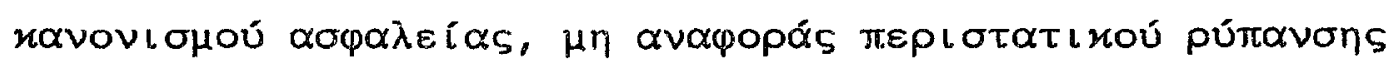

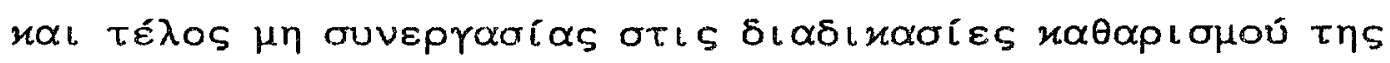
pürovons.

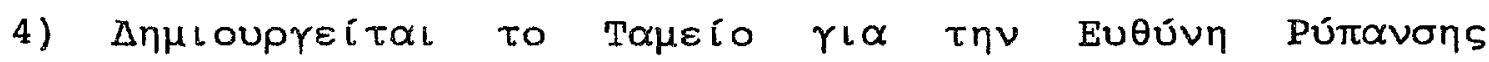

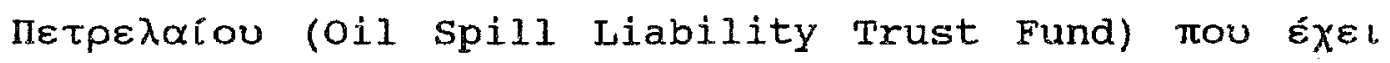

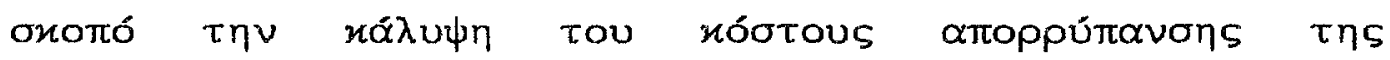

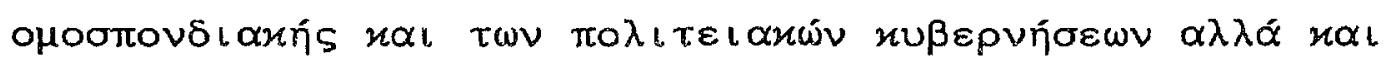

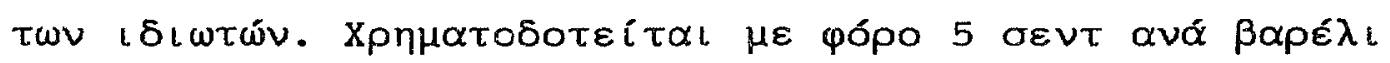

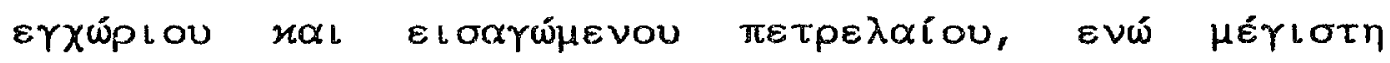

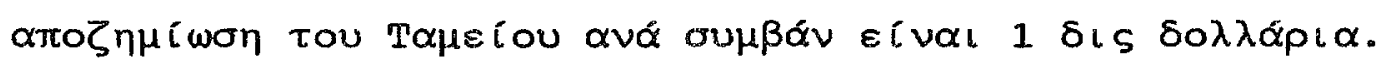

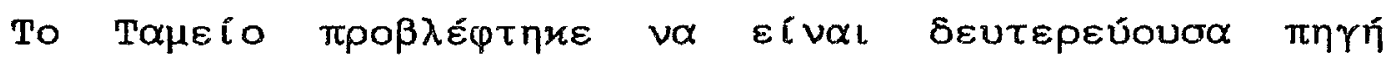

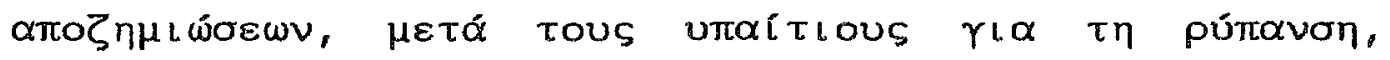

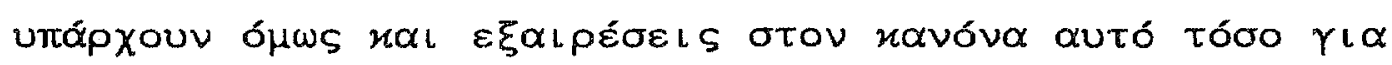

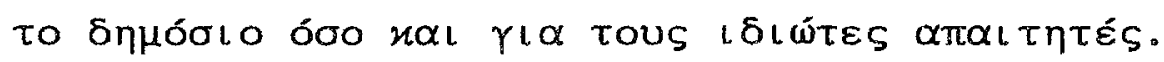

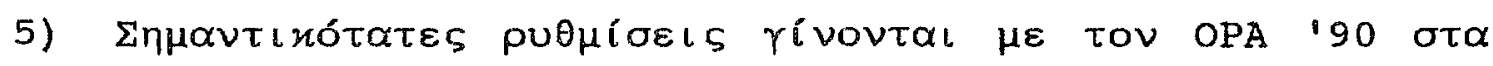

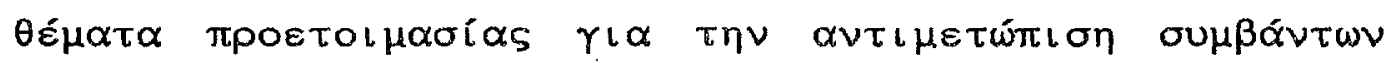

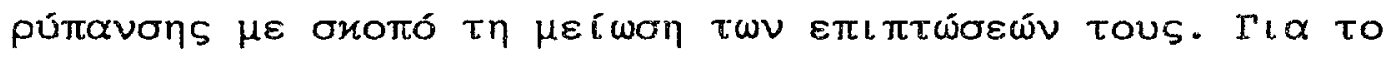

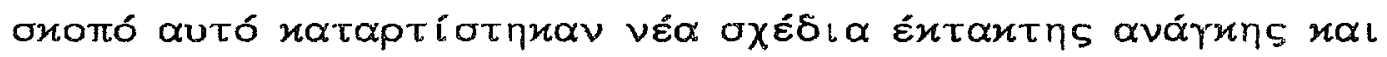

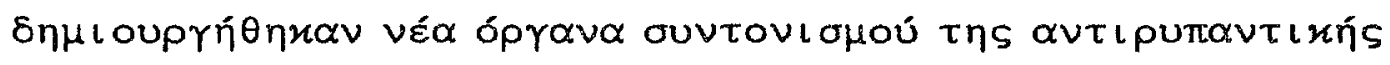

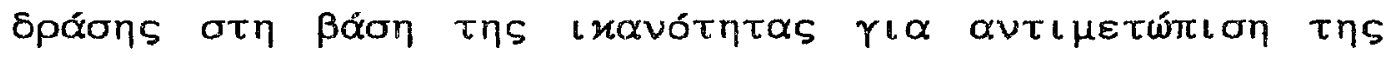

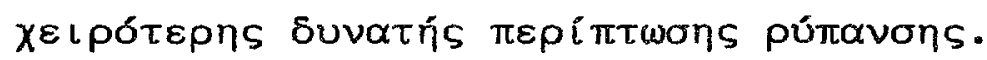




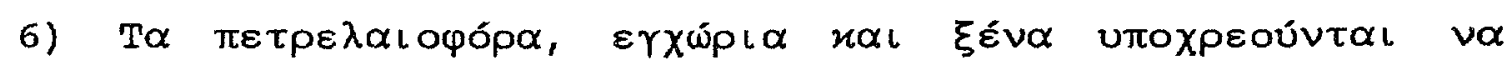

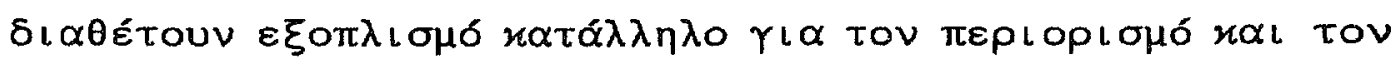

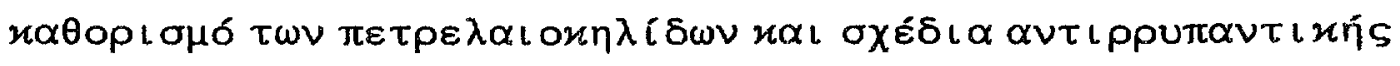

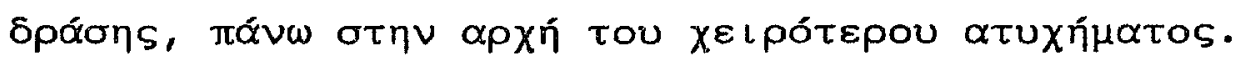

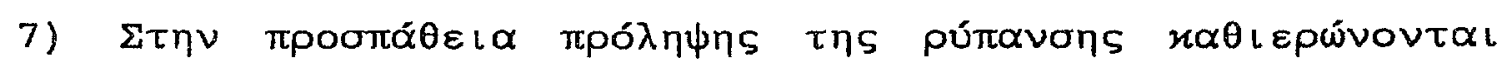

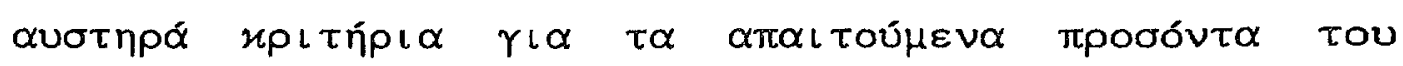

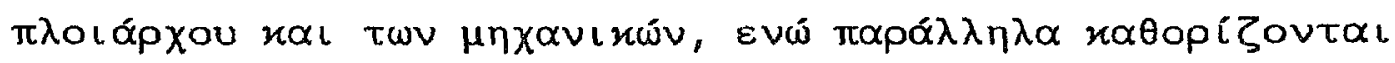

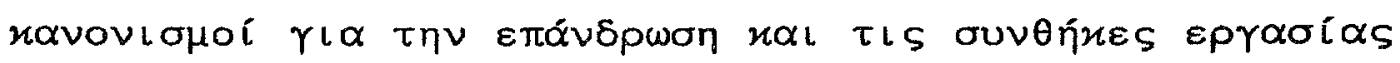

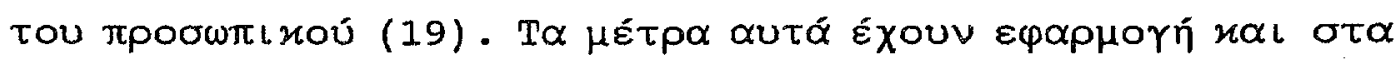

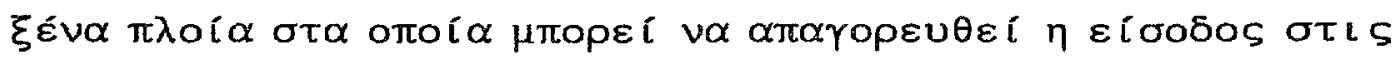

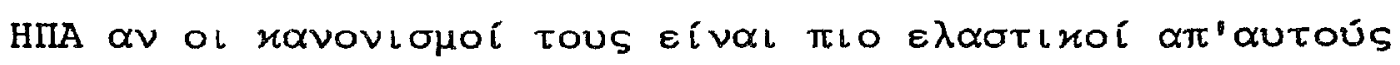

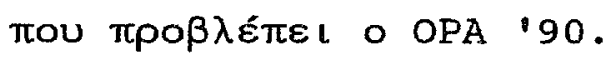

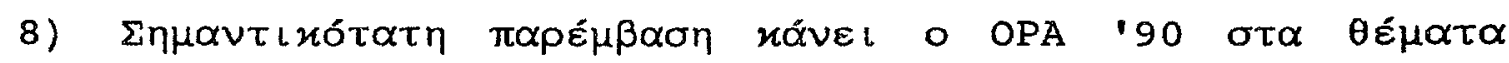

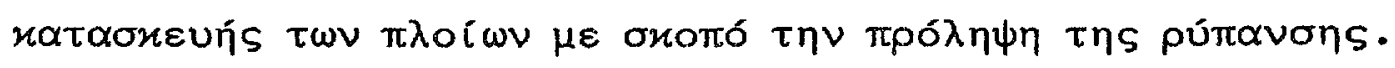

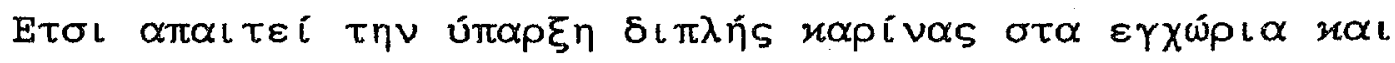

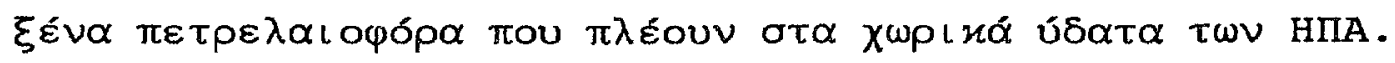

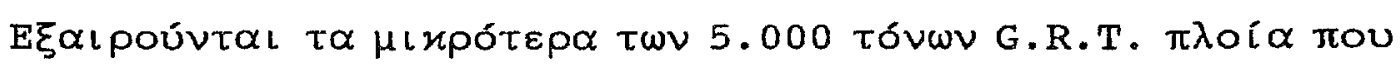

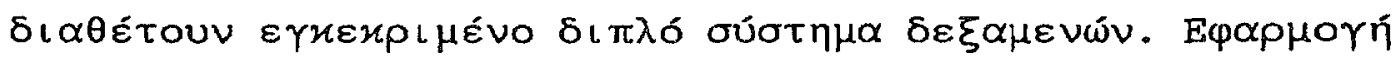

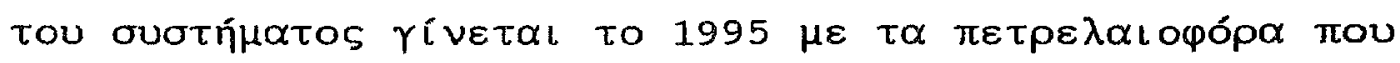

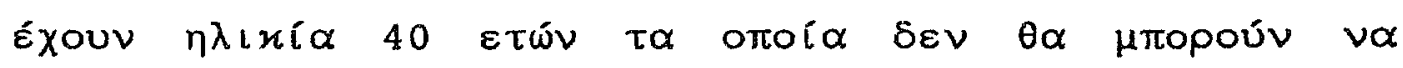

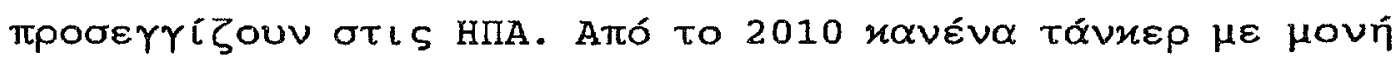

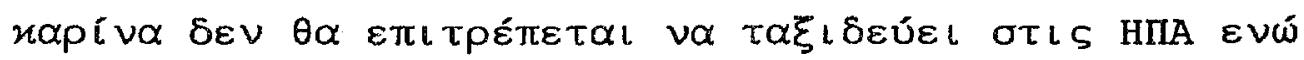

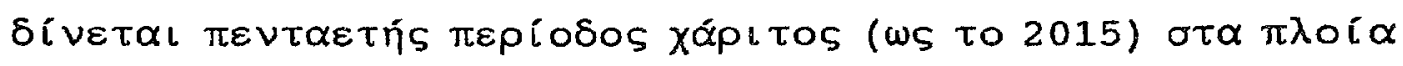

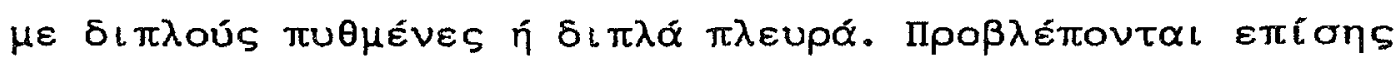




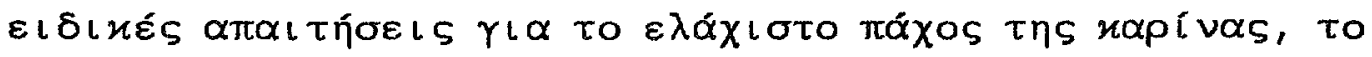

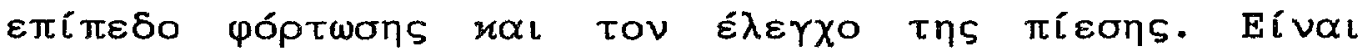

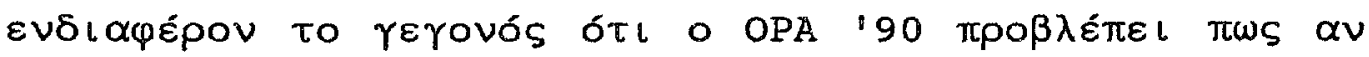

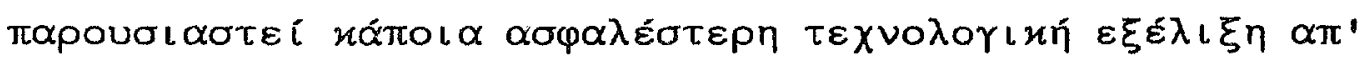

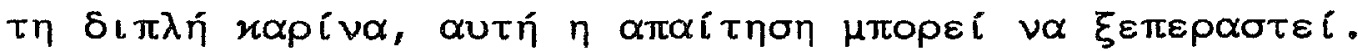

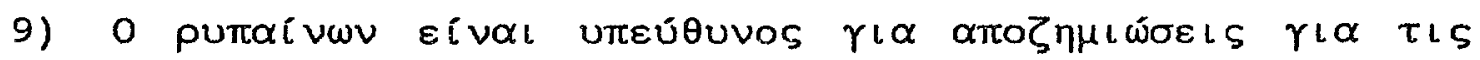

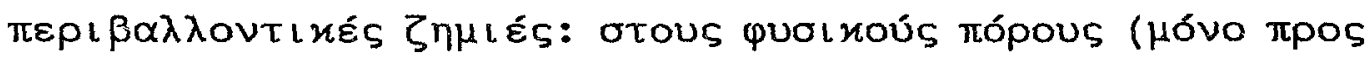

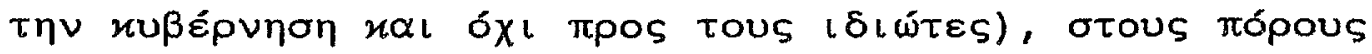

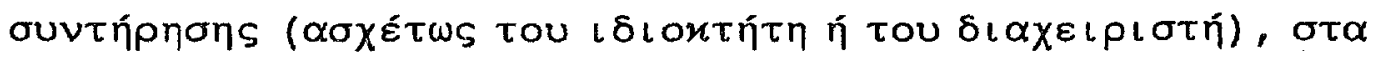

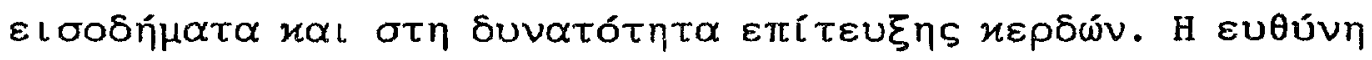

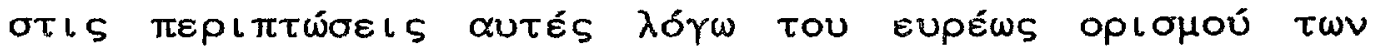

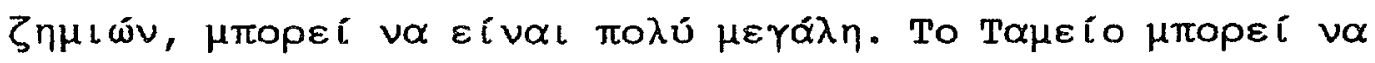

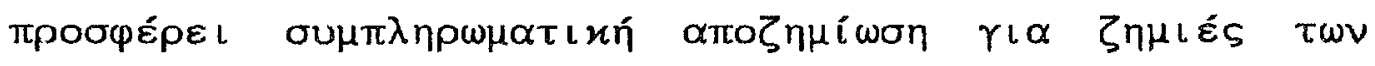

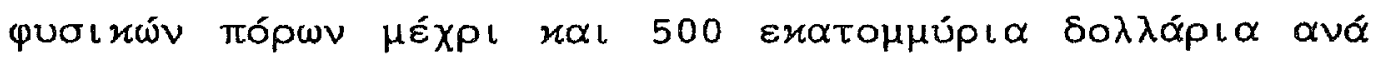

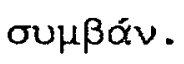

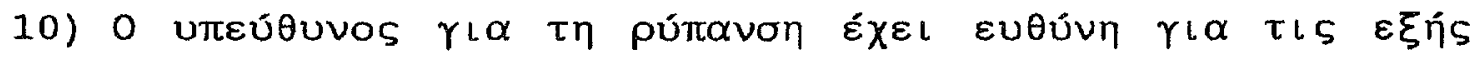

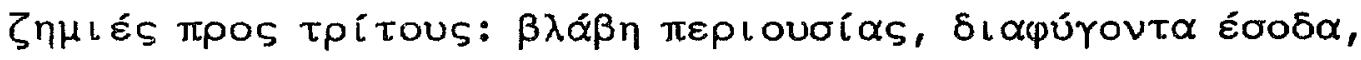

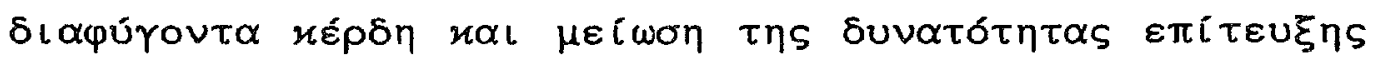

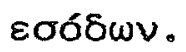

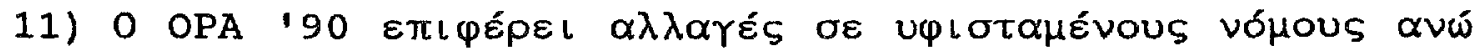

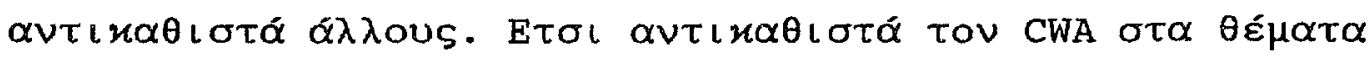

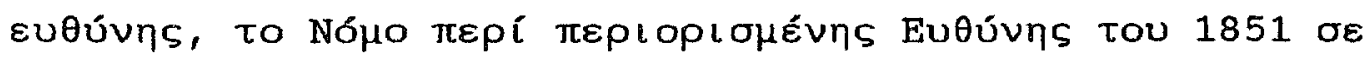

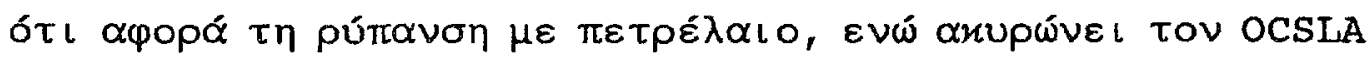
naL TOV TAPPA. 


\section{EIIXEIPHMATA YIIEP TOY OPA ' 90}

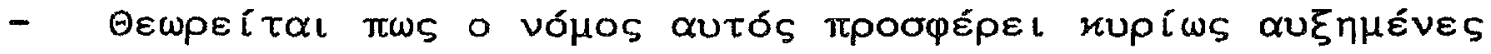

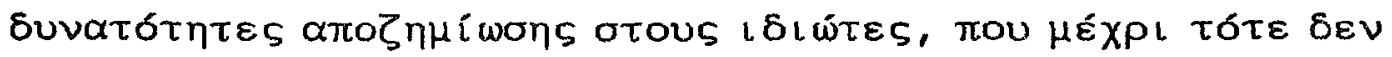

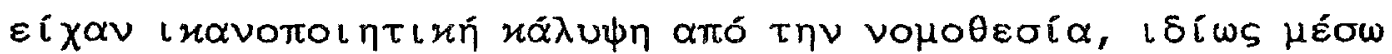

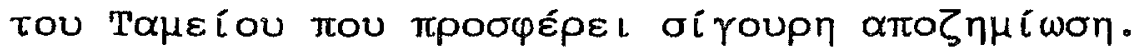

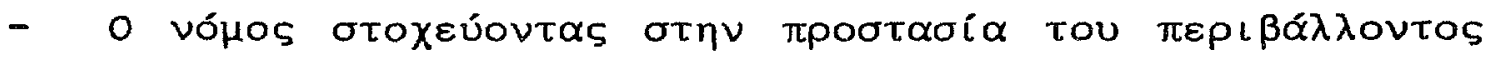

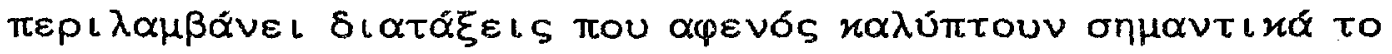

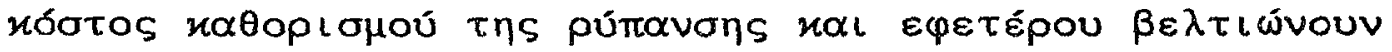

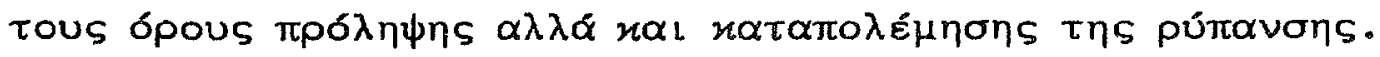


EIIXEIPHMATA KATA TOY OPA 190

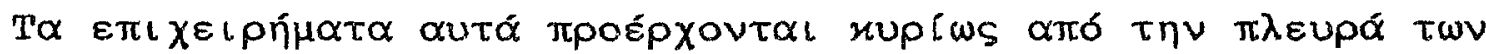

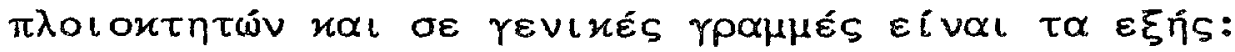

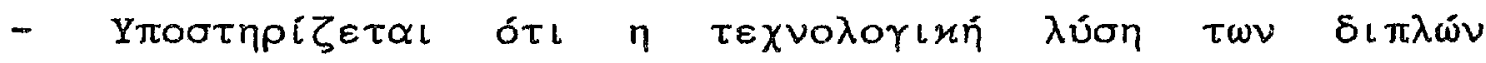

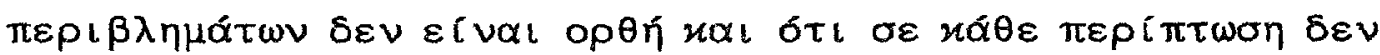

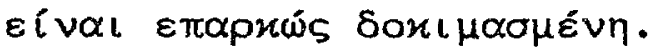

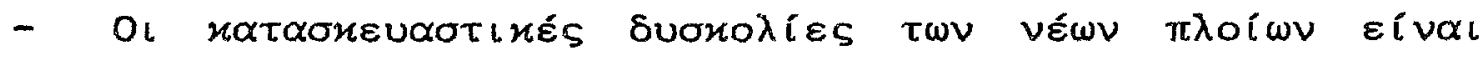

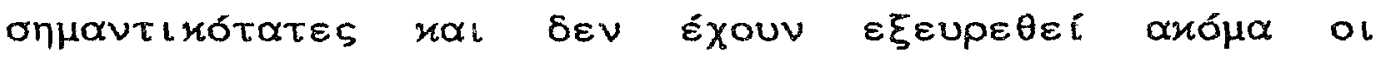

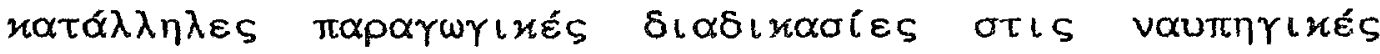
$\varepsilon \pi \iota \chi \varepsilon \iota p \eta ́ \sigma \varepsilon \iota s$.

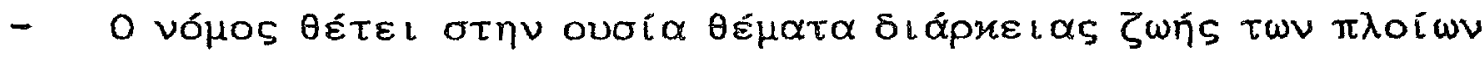

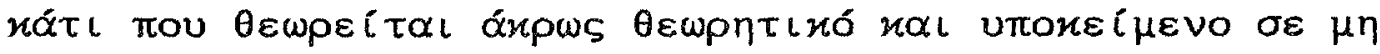

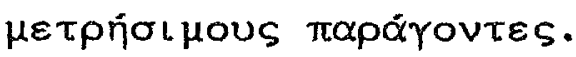

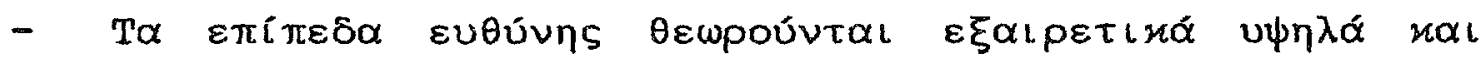

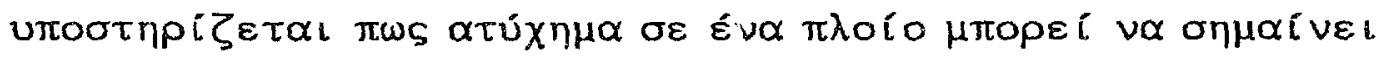

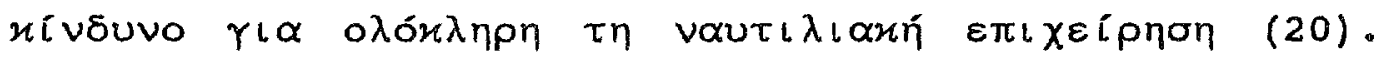

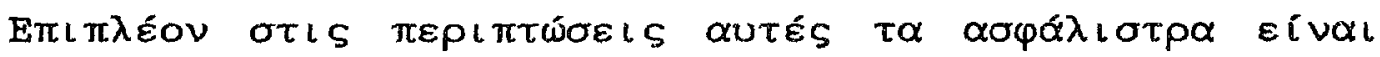

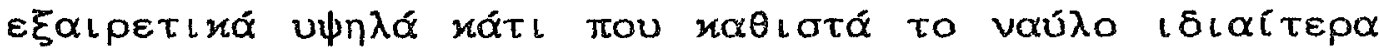

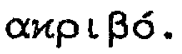




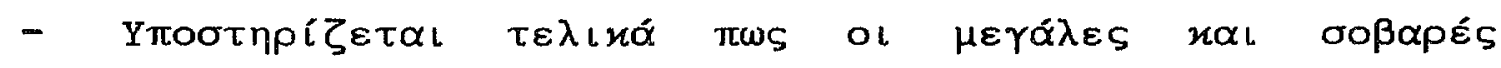

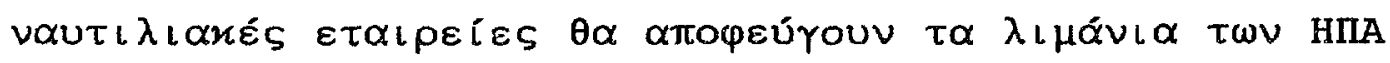

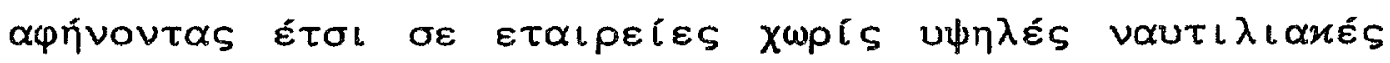

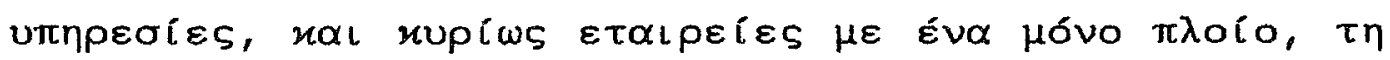

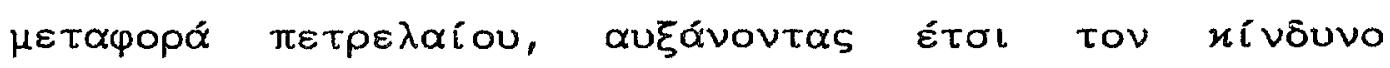
pútavaๆs.

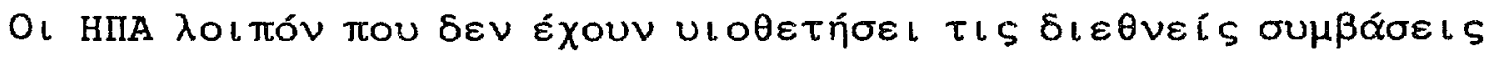

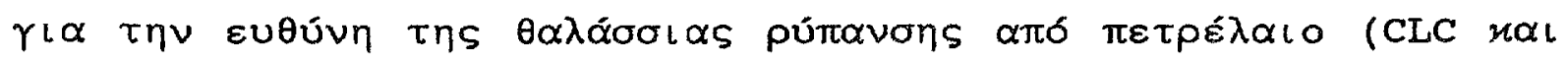

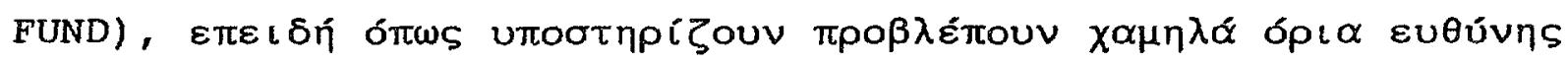

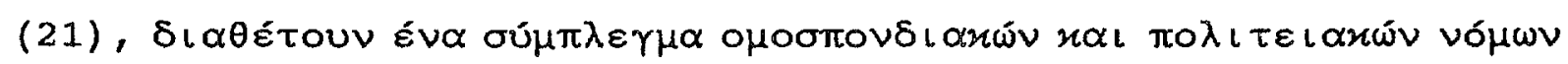

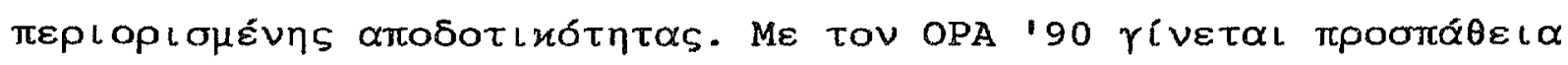

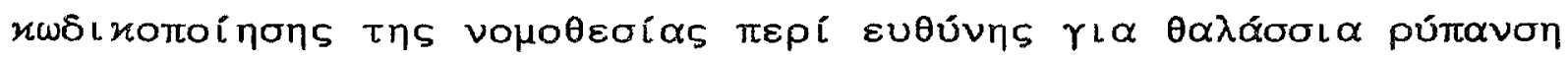

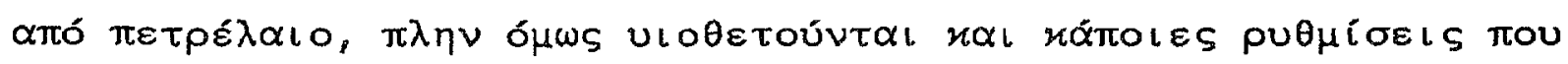

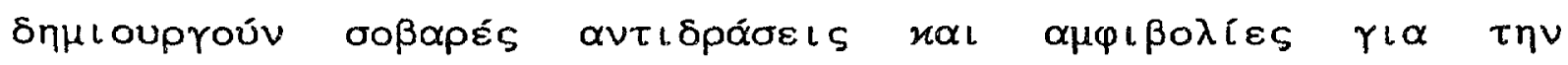

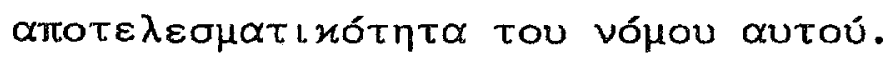




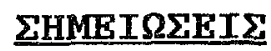

(1) Amy McKaig : "Liability for oil Tanker Spills"

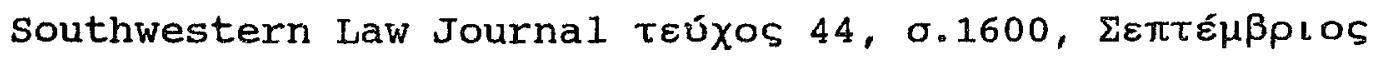
1991.

(2) Beth Van Hanswyk oл. $\pi .0 .330$.

(3) Judith R. Eaton : "Oil Spill liability and compensation: time to clean up the law" George Weshington Journal of

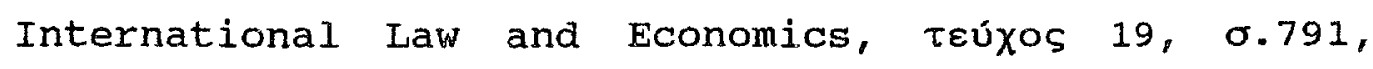

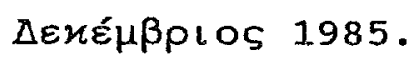

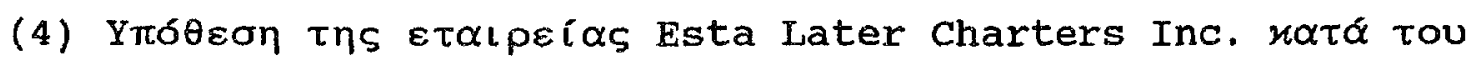

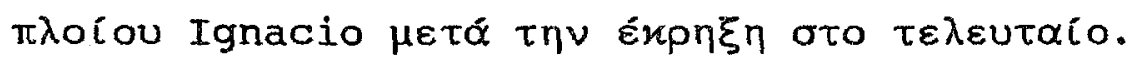

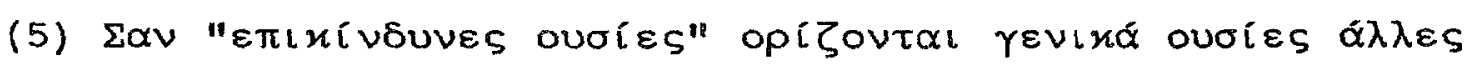

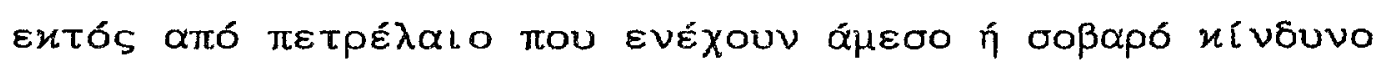

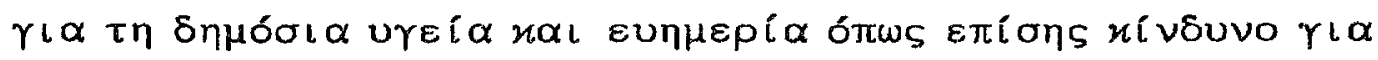

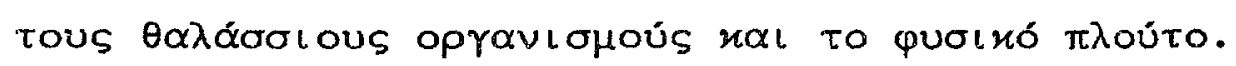

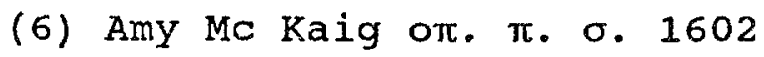


(7) To óf

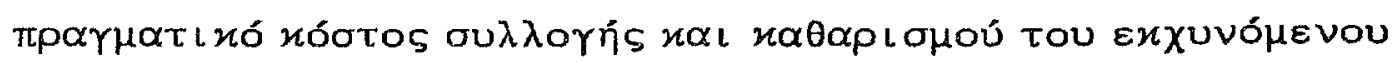

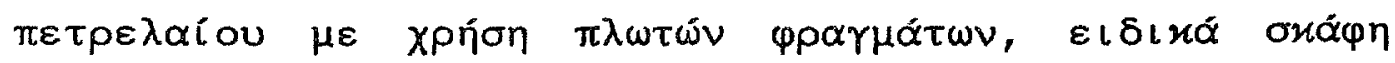

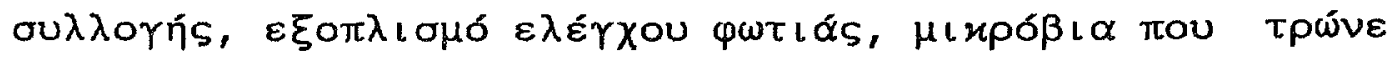

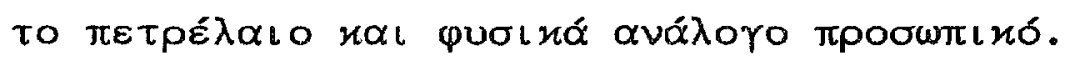

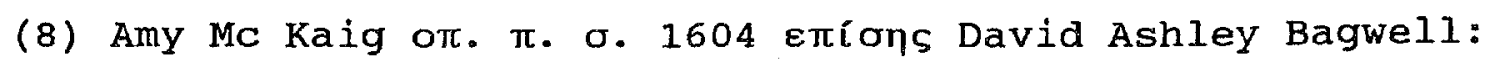
"Liability under United States law for spills of oil or

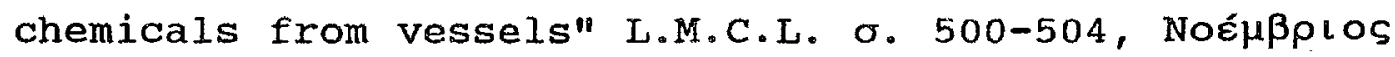
1987.

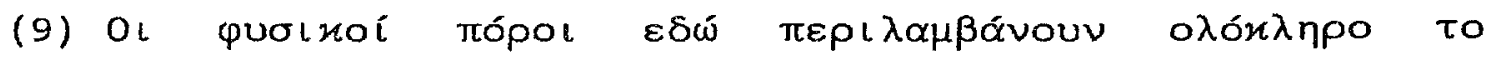

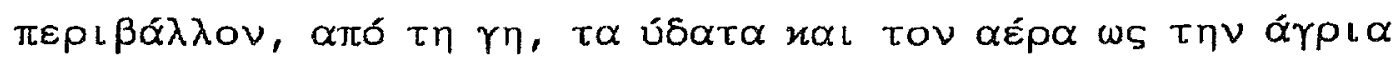

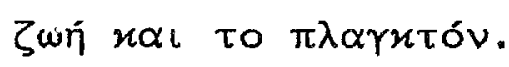

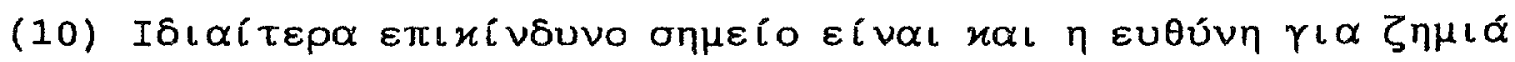

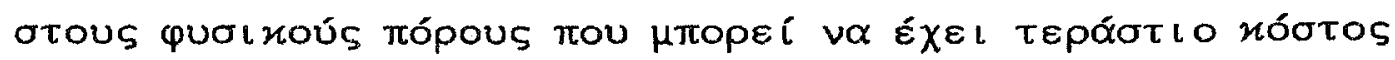

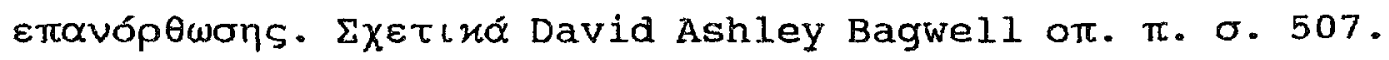

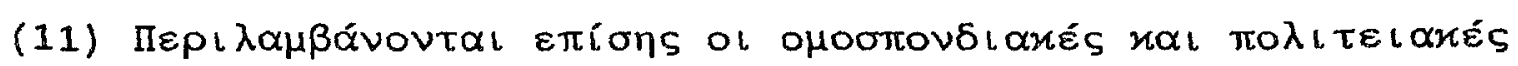

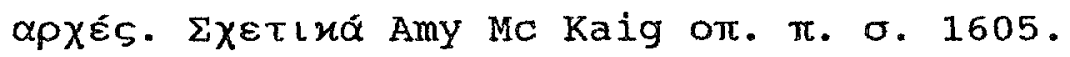

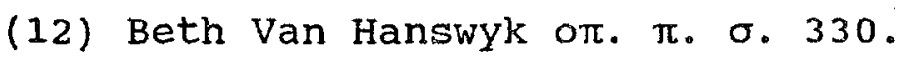




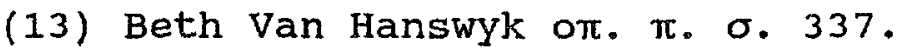

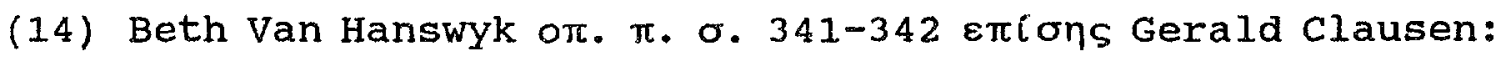
"Liability for High Seas Oil Pollution Clean up costs: Domestic and International Provisions". Hastings International and Comparative Law Review teúxos 3 No 3 $0.474-476$, Avol $\xi \eta 1980$.

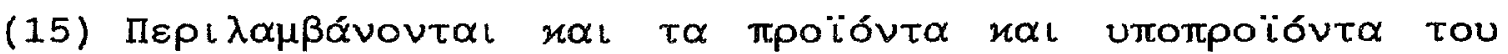

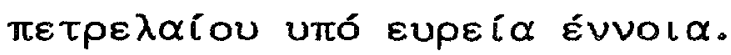

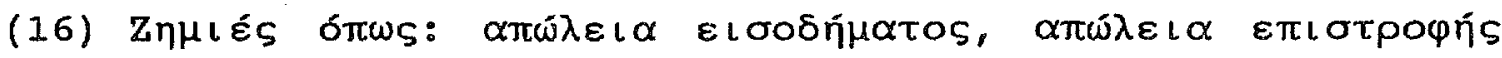

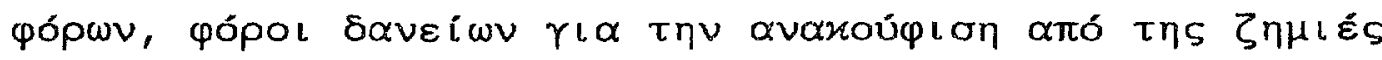

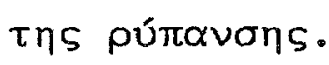

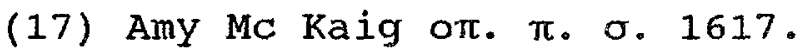

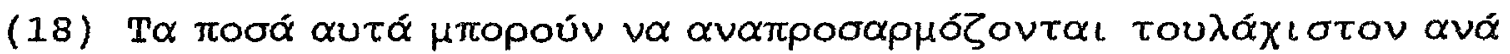

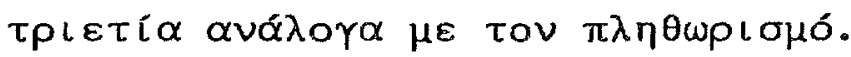

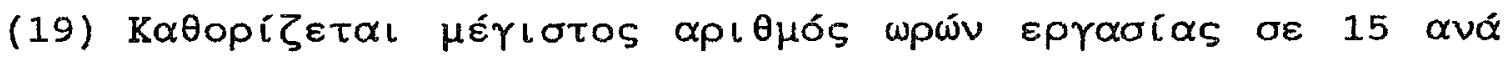

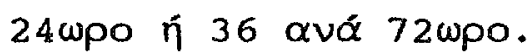




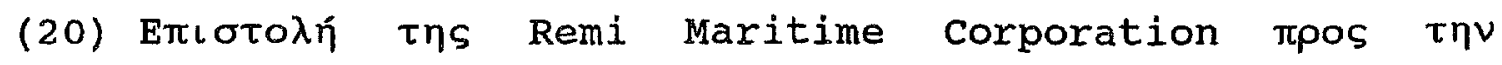

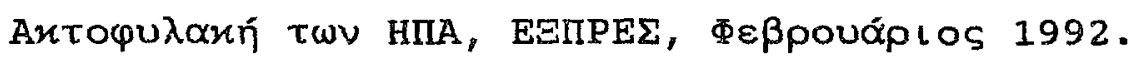

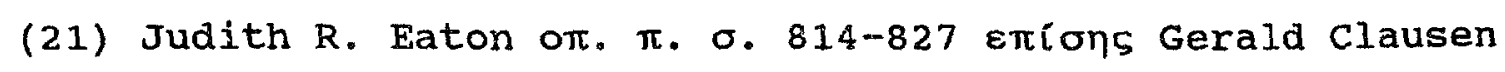

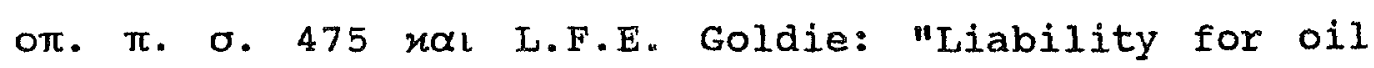
pollution disasters: international law and the delimitation of competences in a federal policy" JMLC

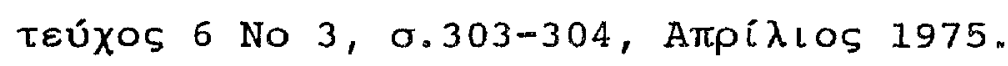




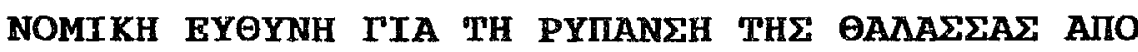

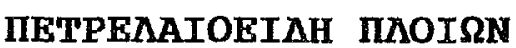

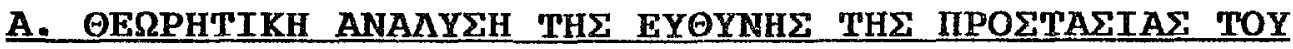 IIEPIBAA NONTOE}

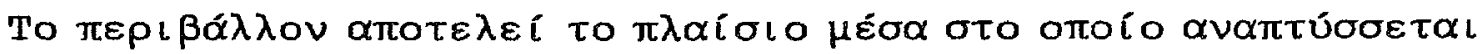

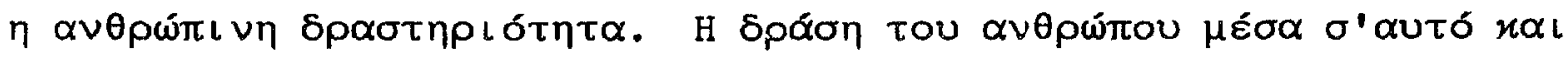

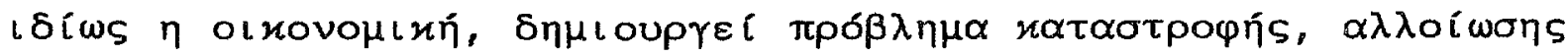

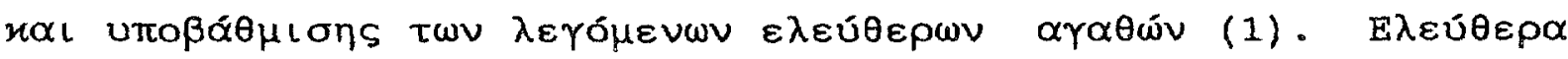

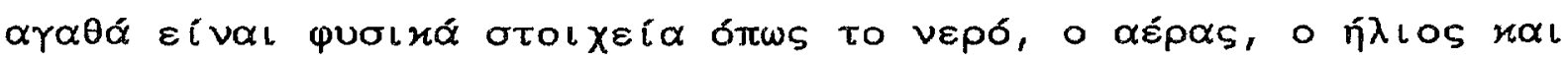

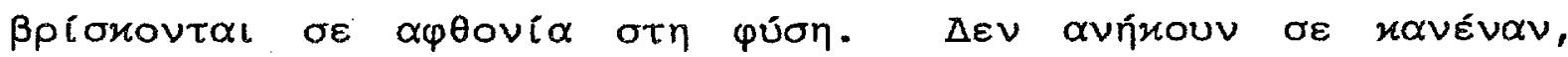

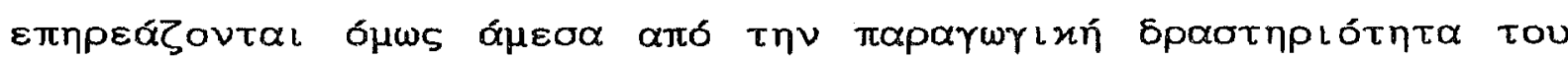

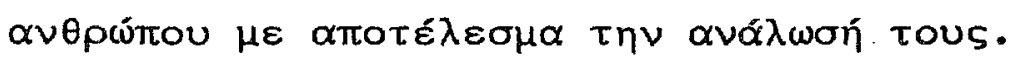

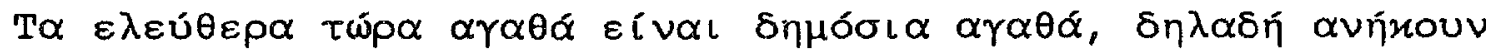

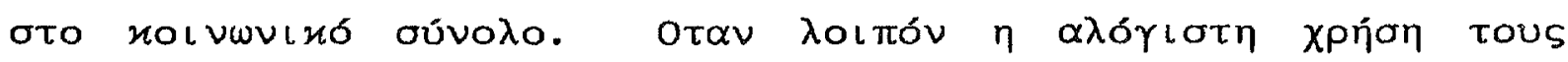

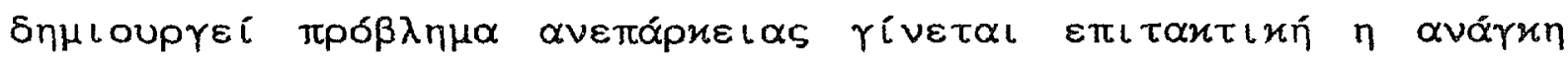

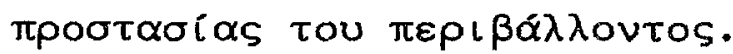

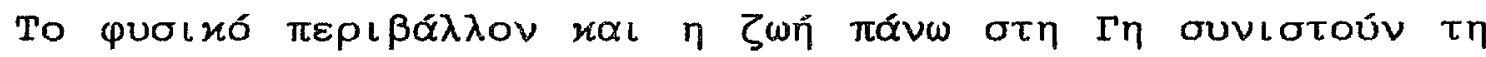

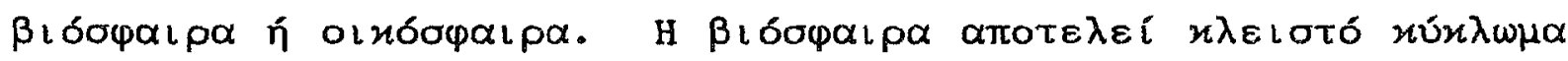

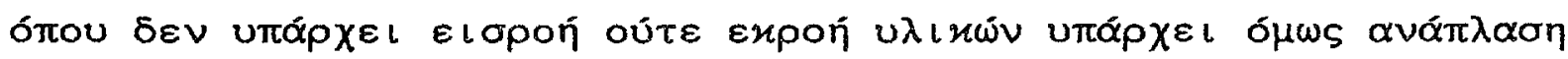

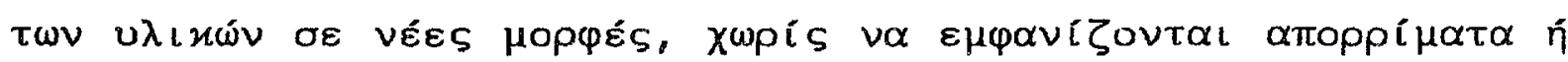

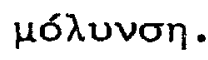




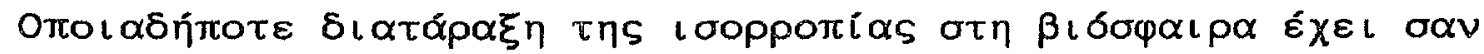

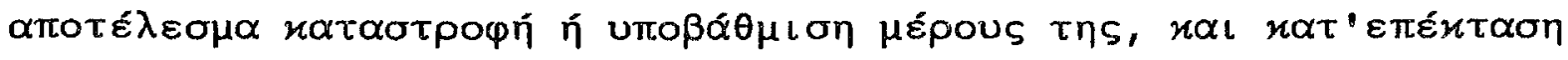

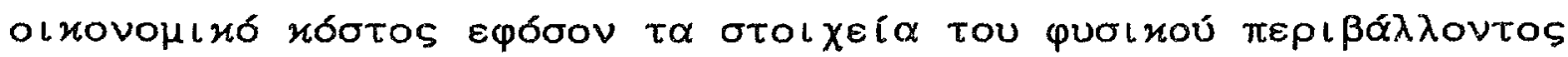

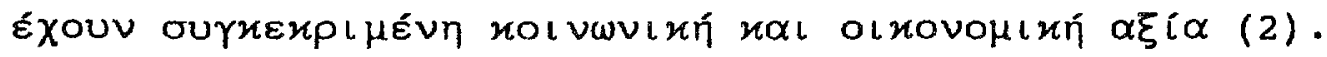

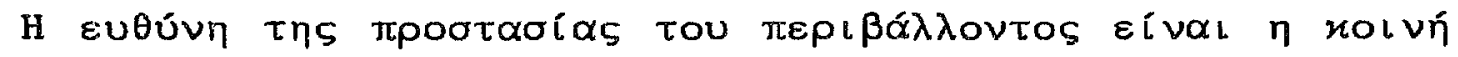

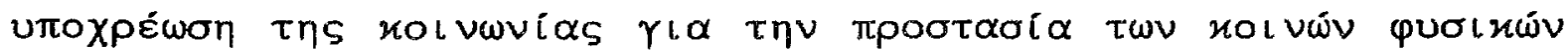

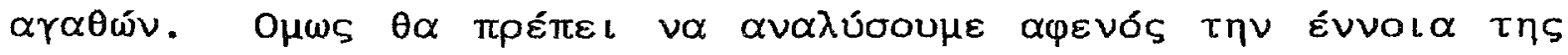

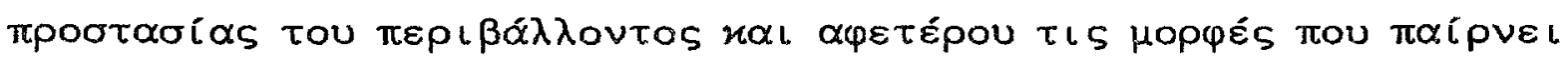

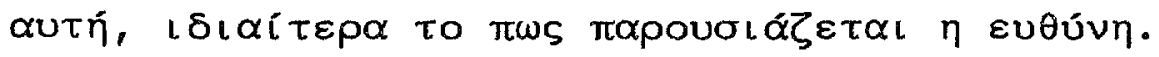

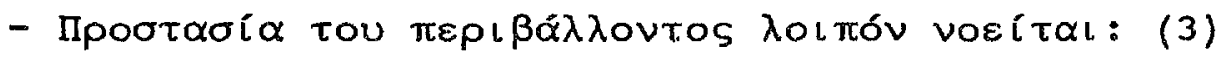

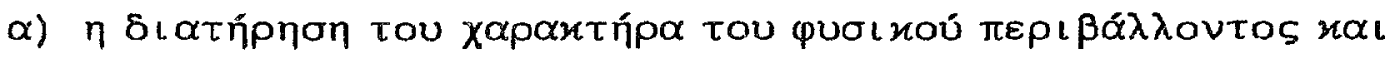

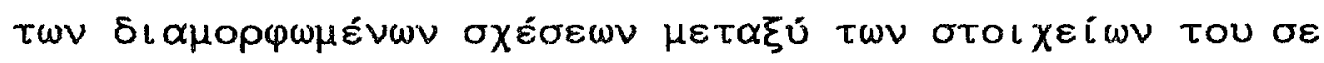

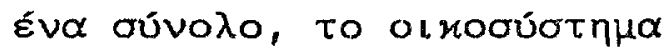

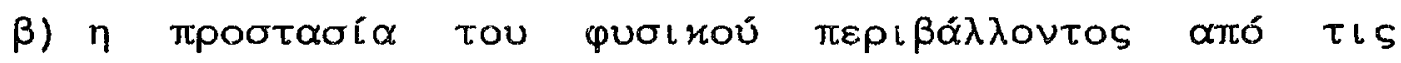

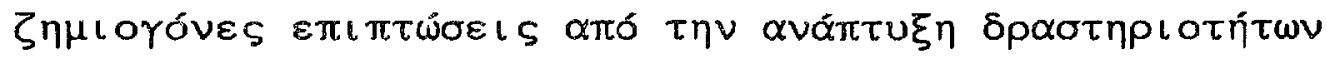

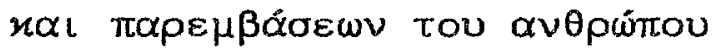

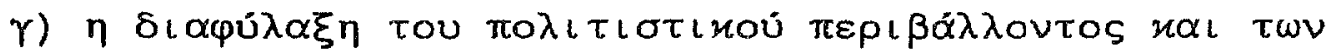

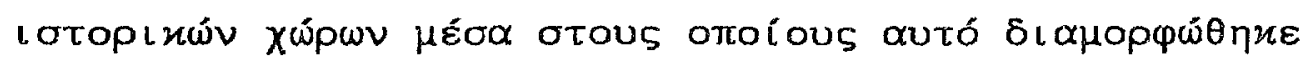




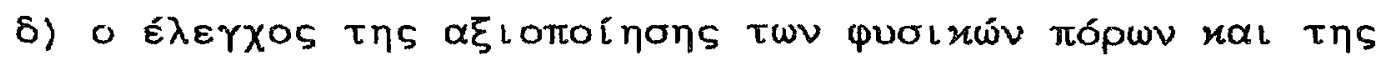

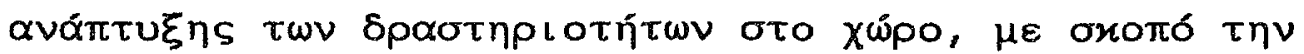

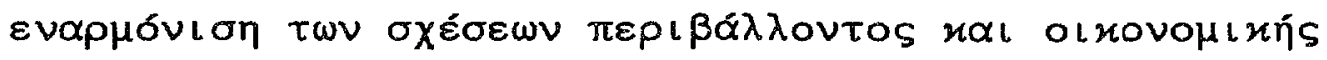

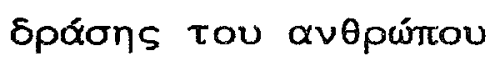

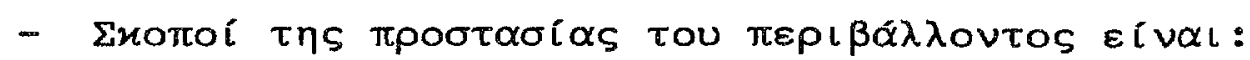

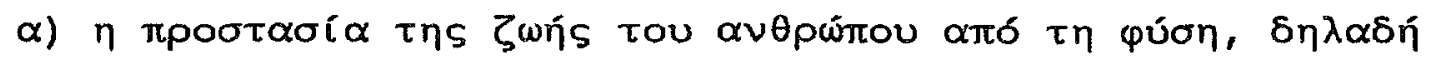

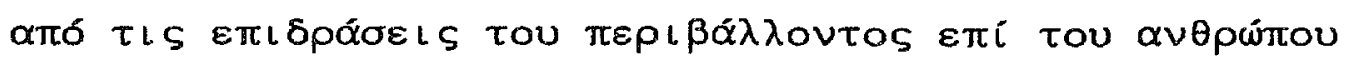

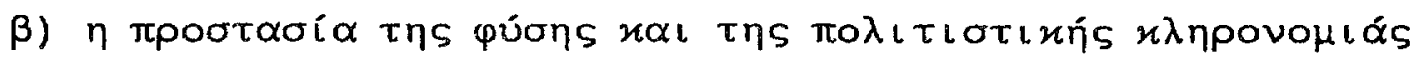

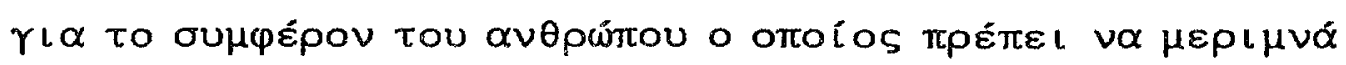

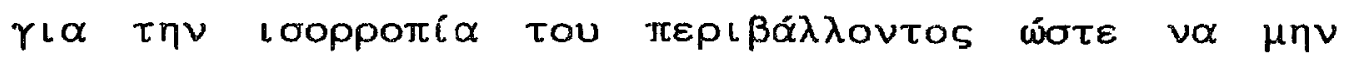

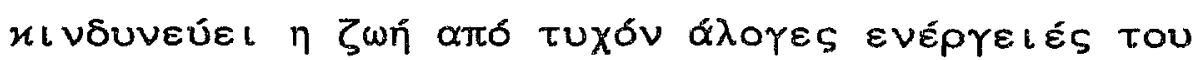

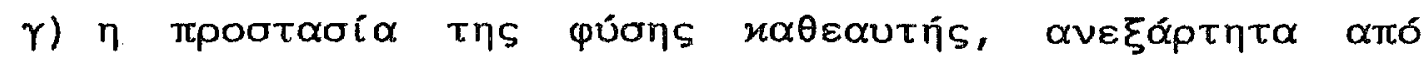

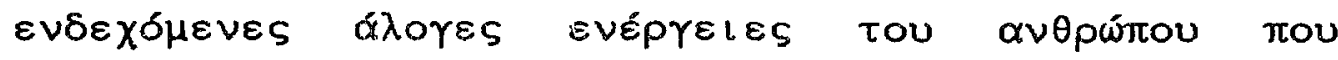

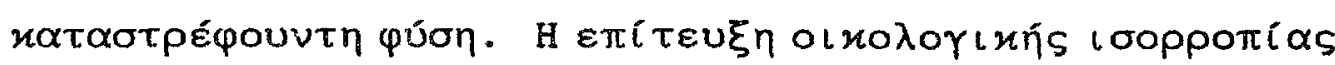

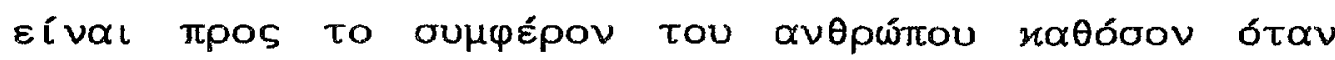

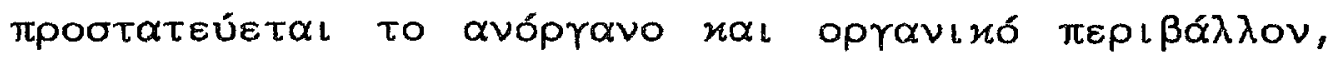

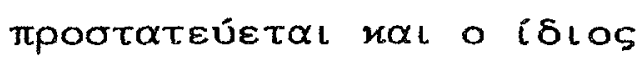




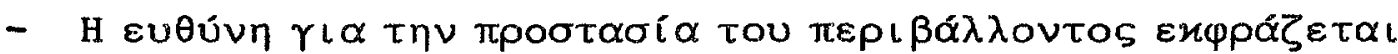

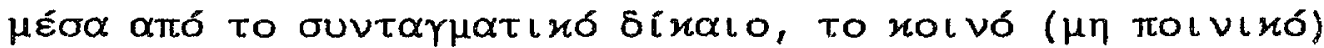

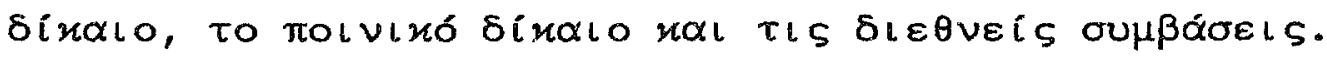

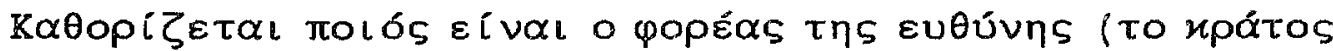

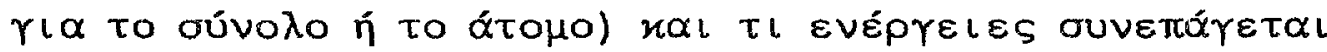

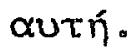

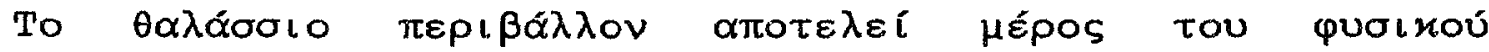

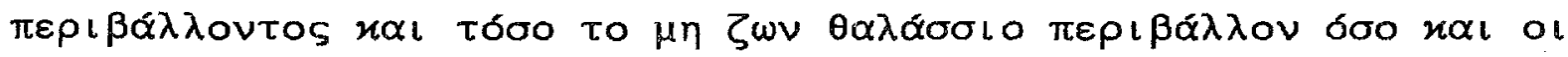

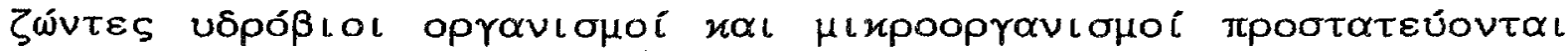

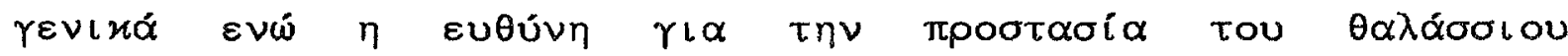

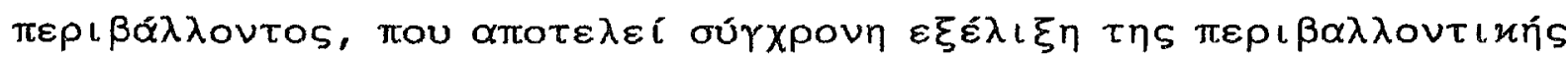

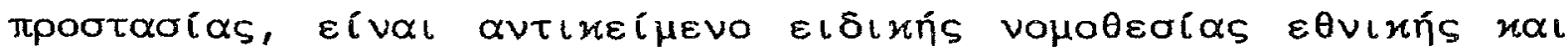

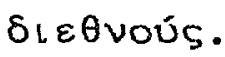




\section{B. EKTASH THE EYOYNHE TIA THN IPOETASTA TOY MEPIBAANONTOE}

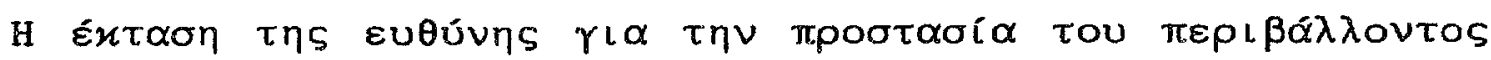

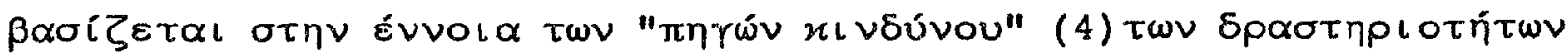

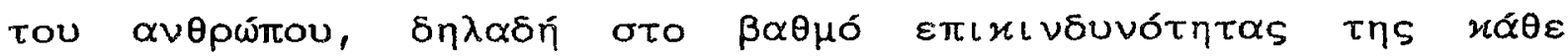

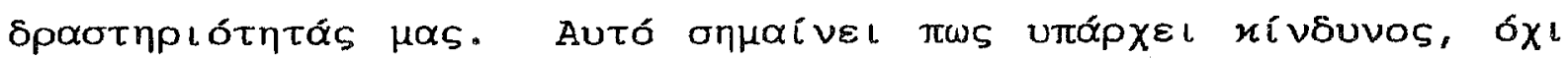

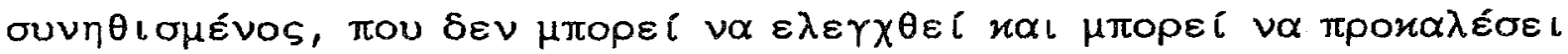

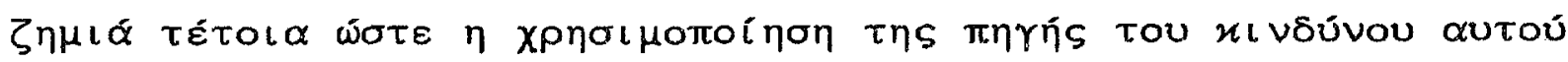

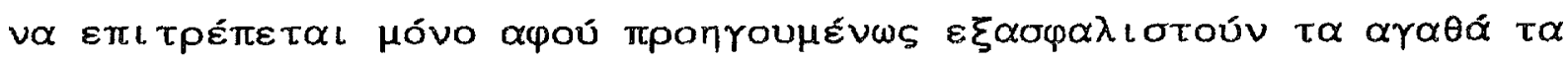

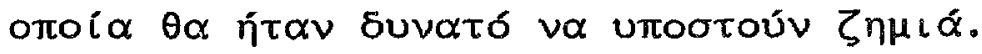

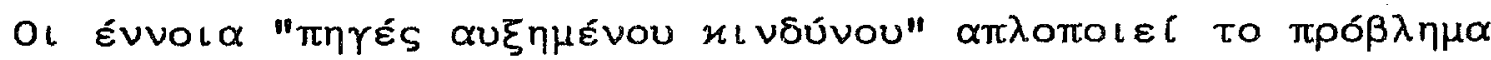

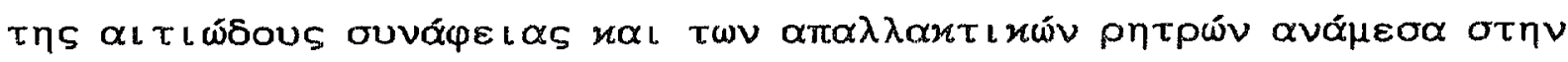

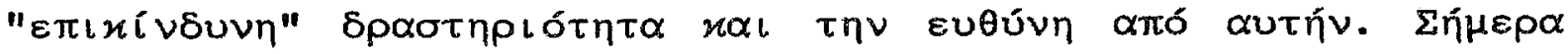

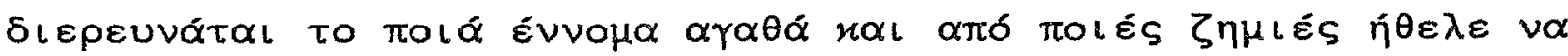

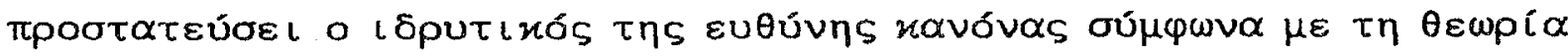

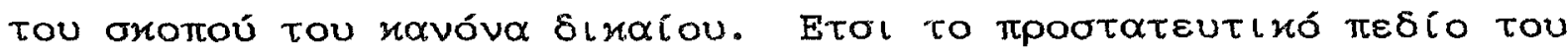

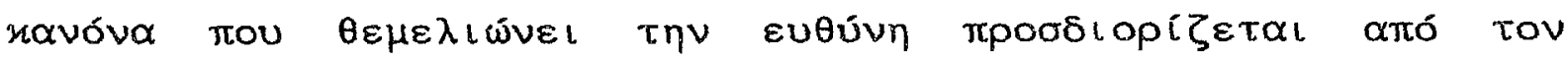

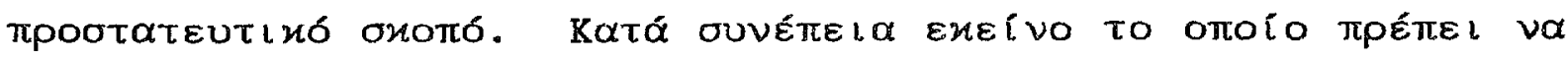

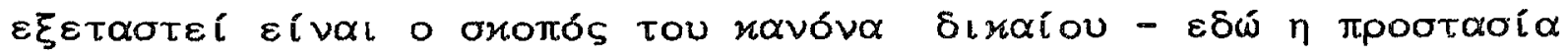

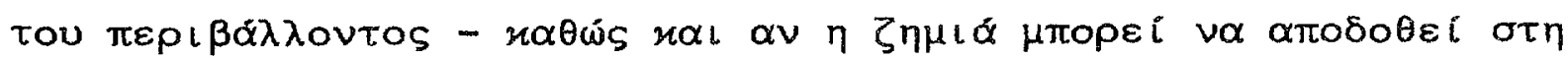

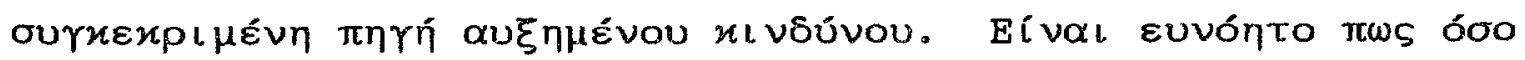




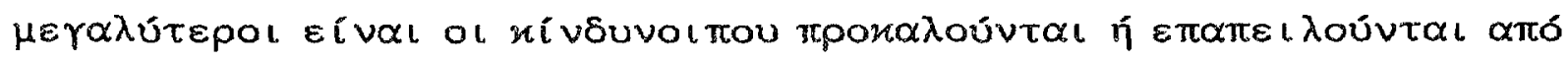

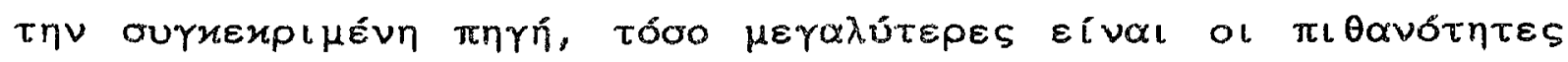

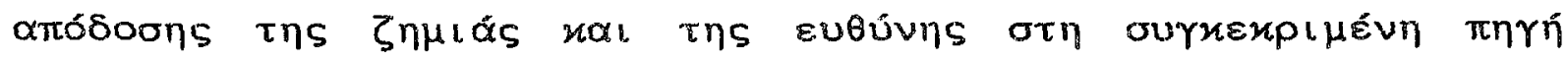
xเทธ์บ์

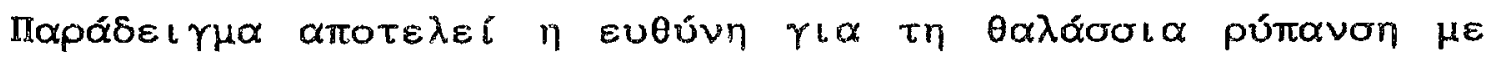

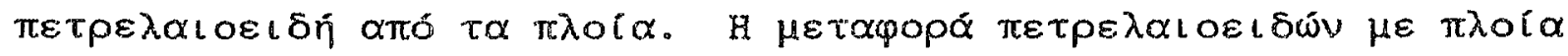

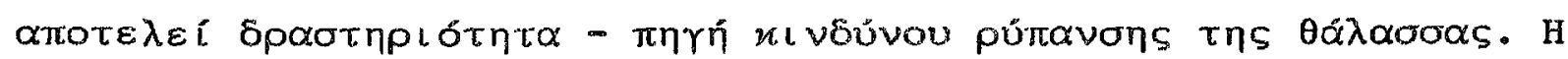

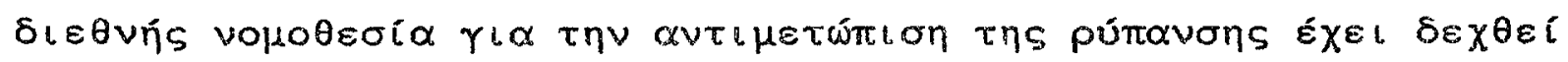

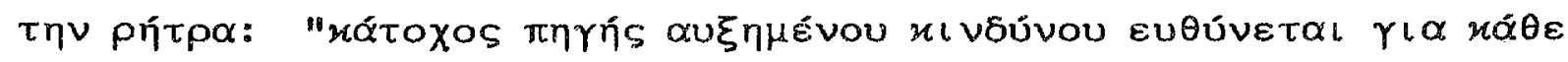

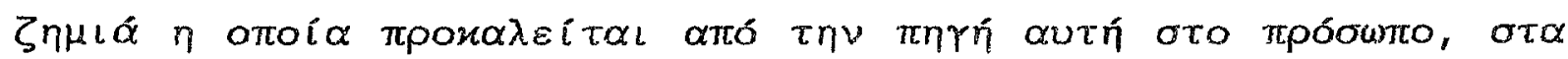

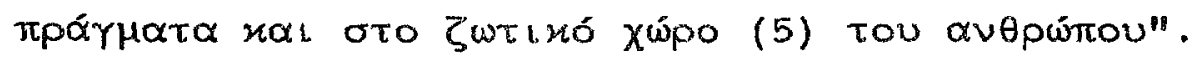

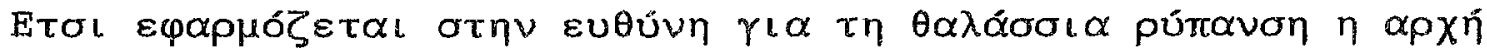

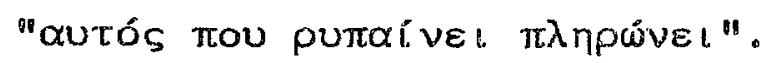

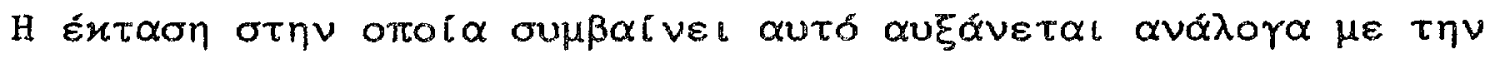

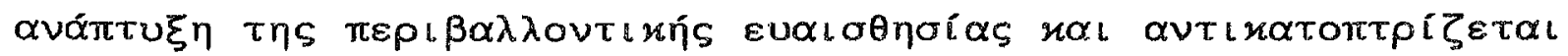

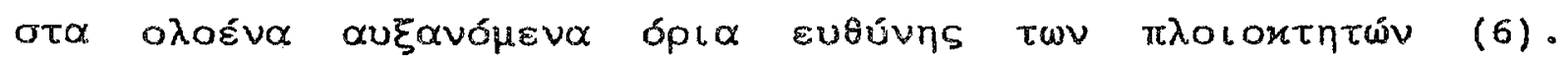

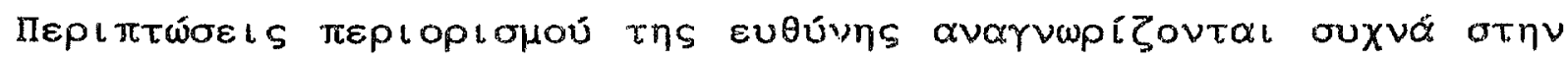

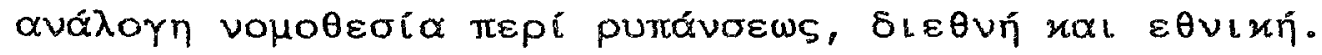




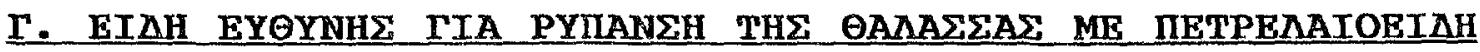 AחO חПOTA}

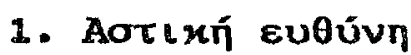

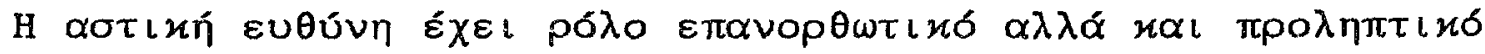

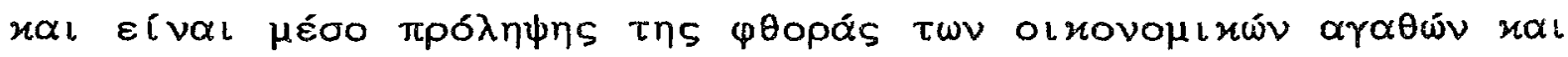

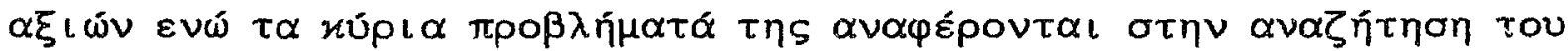

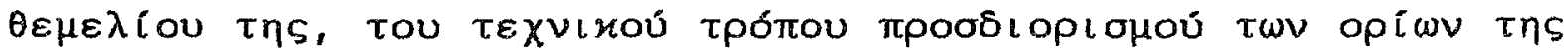

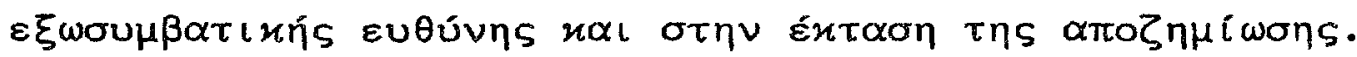

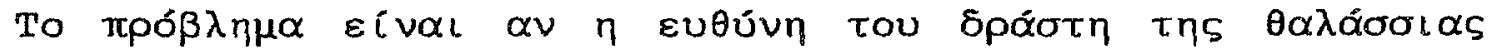

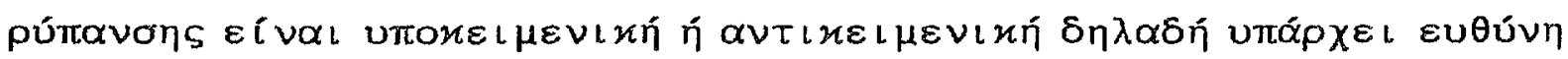

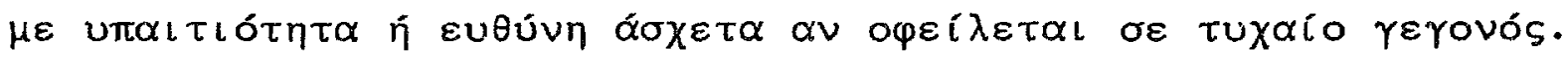

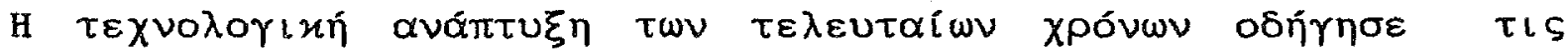

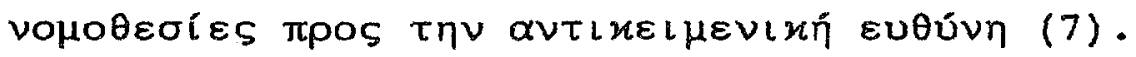

Kúplos tóros tou rerovótos autoú eíval to xpltíplo tou

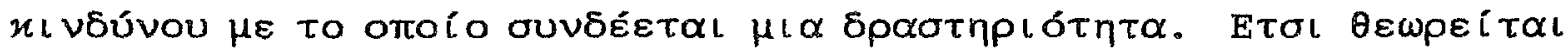

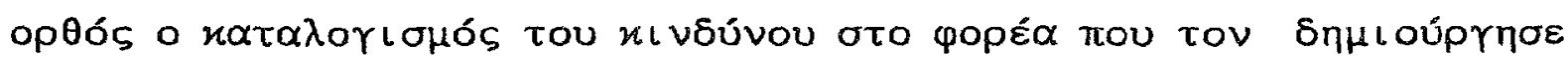

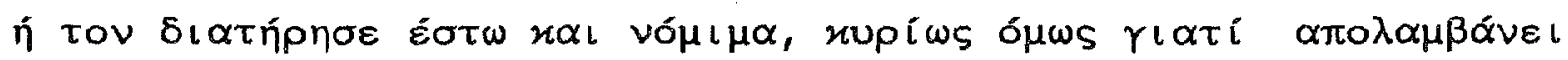

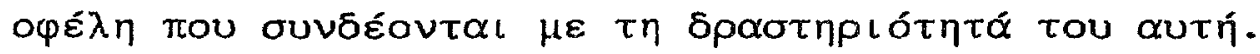




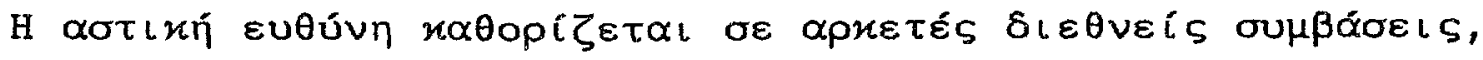

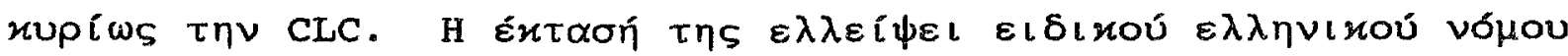

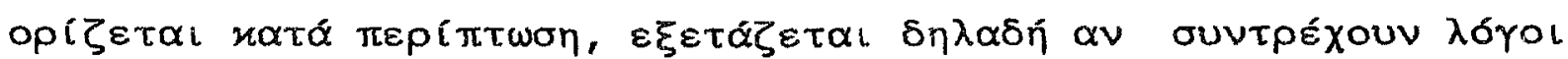

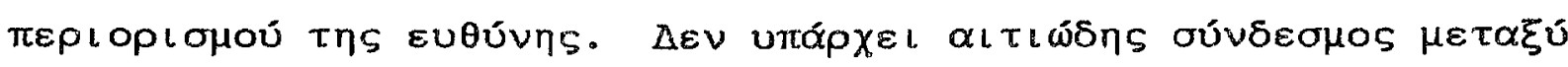

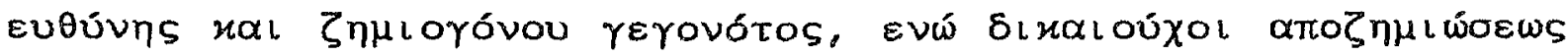

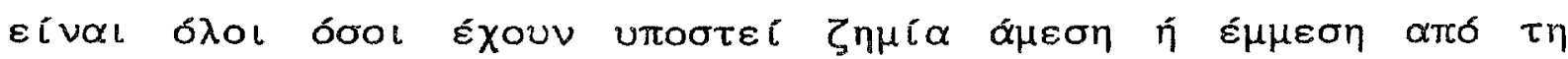
púravon.

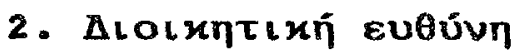

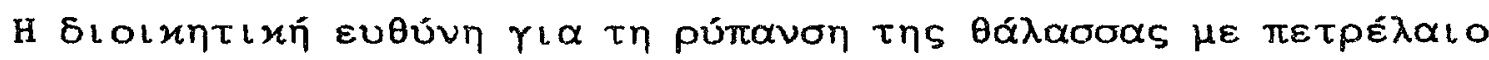

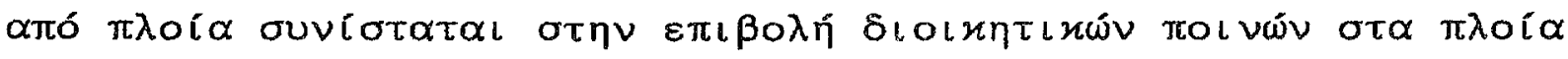

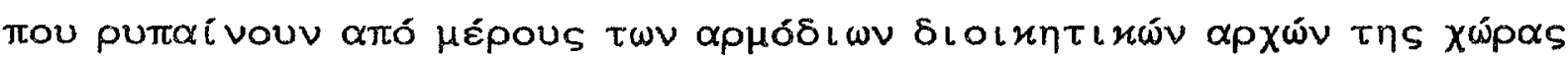

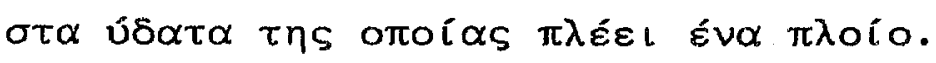

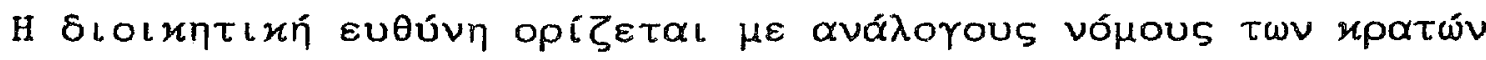

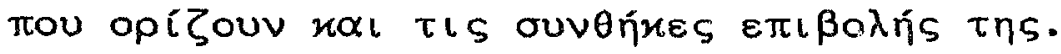

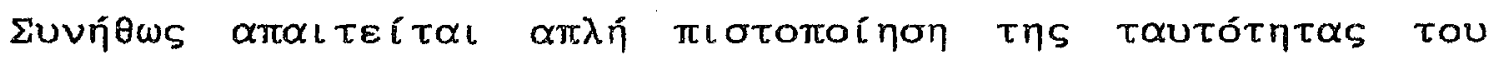

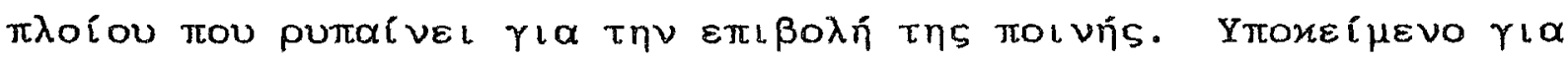

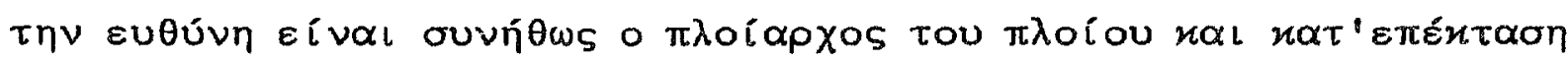

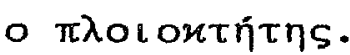

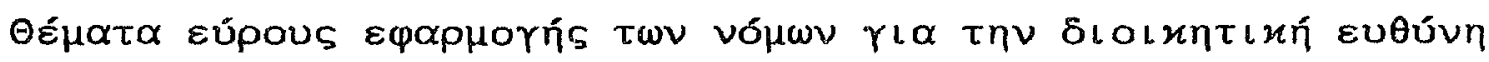

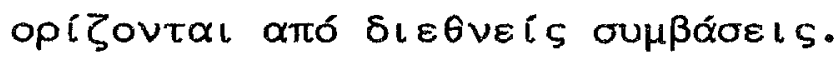




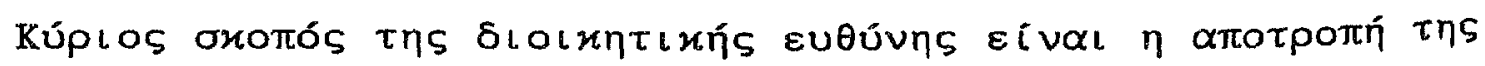

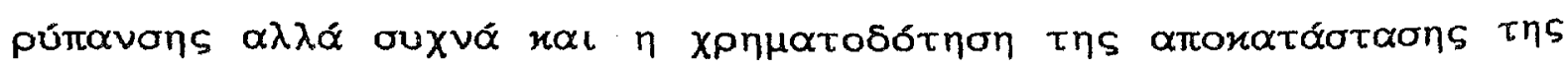

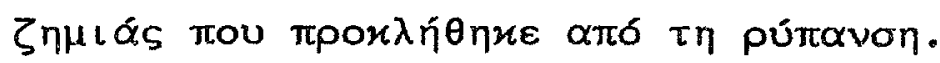

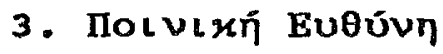

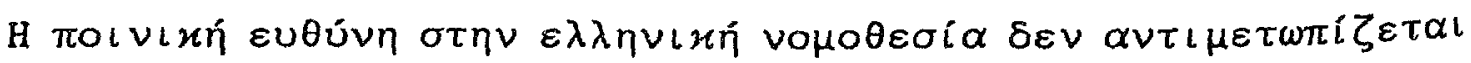

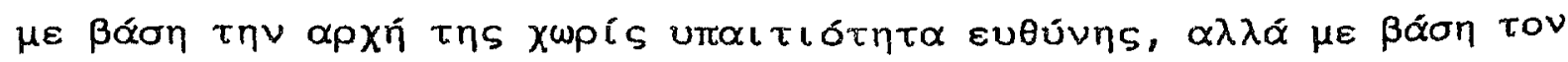

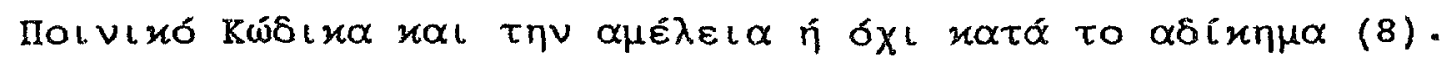

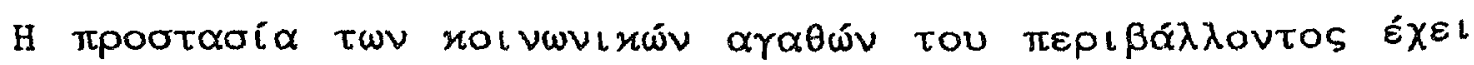

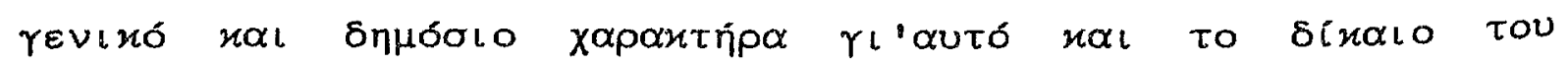

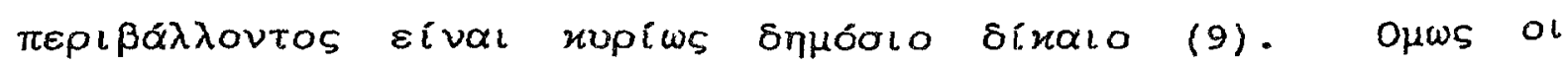

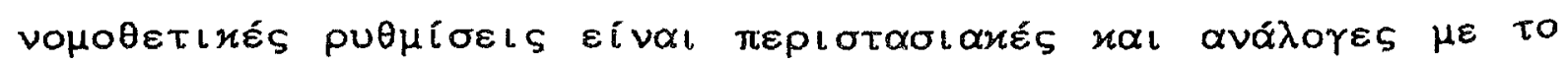

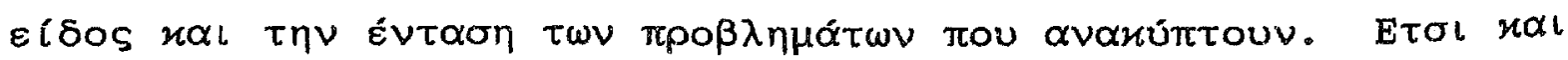

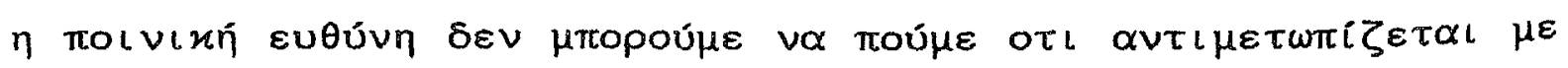
Eviaío tpómo (10). 


\section{SHMEIRSETS}

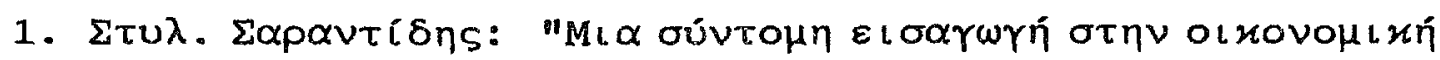

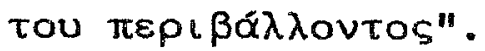

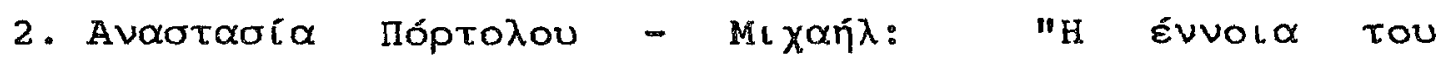

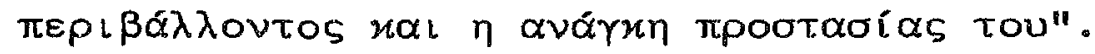

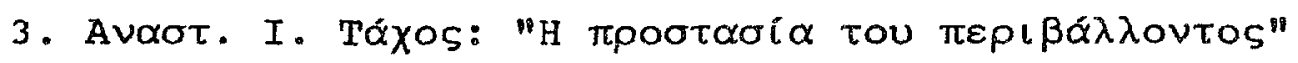

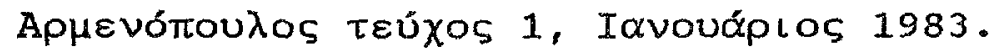

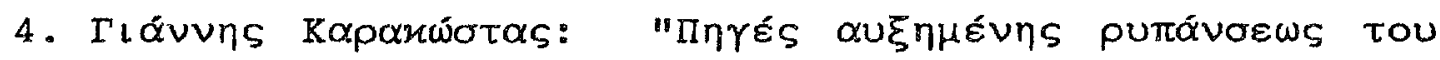

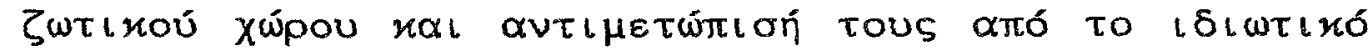

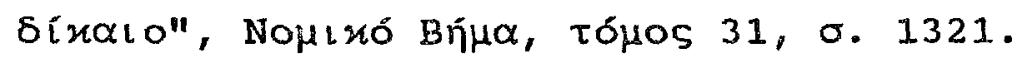

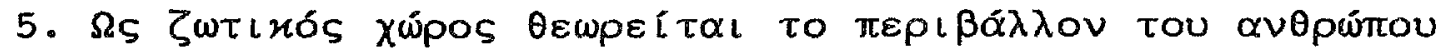
uTó eupeía Évขol $\alpha$.

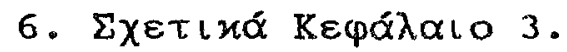

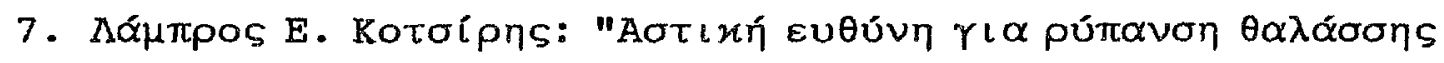
$\alpha$ đTó $\pi \varepsilon \tau \rho \varepsilon \lambda \alpha\left\llcorner\circ \varepsilon\left\llcorner\varepsilon \eta^{\prime \prime}\right.\right.$ б. 507. 


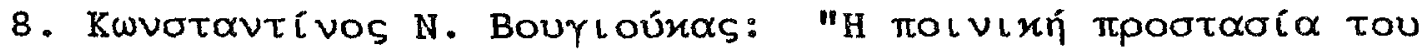

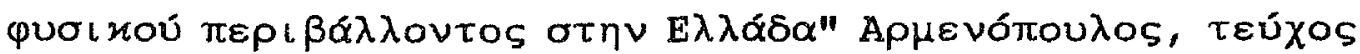

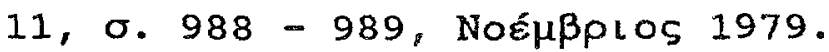

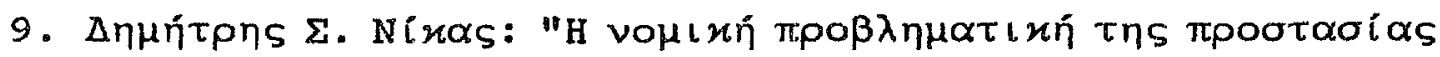

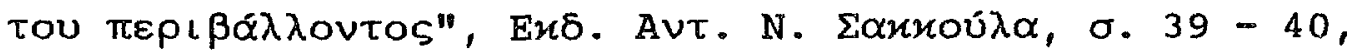

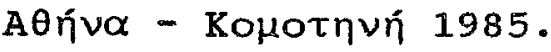

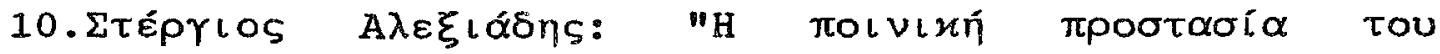

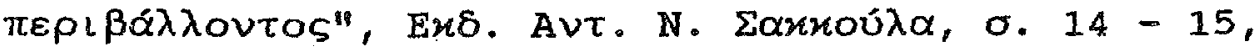

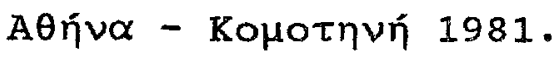




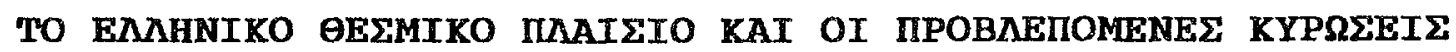

\section{EYNTATMATIKH KATOXYPREH}

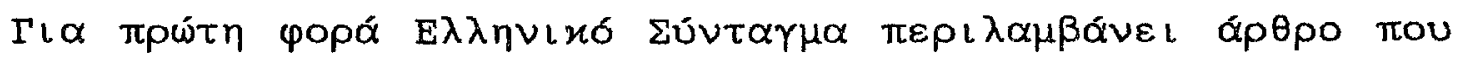

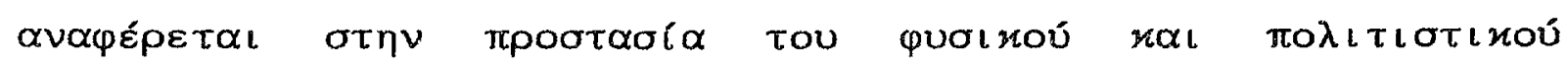

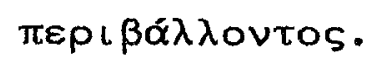

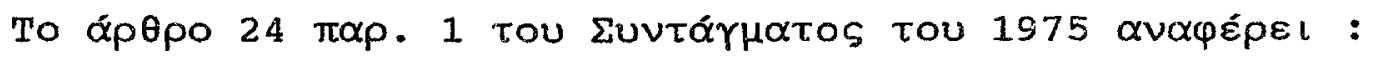

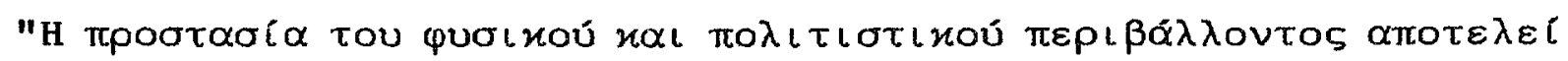

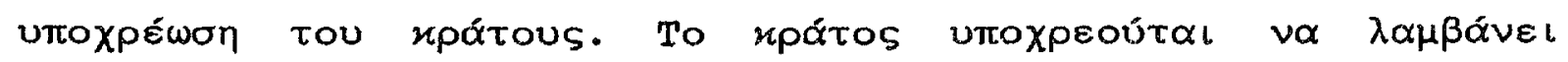

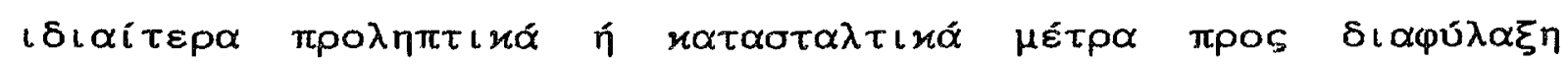
auтớ..."

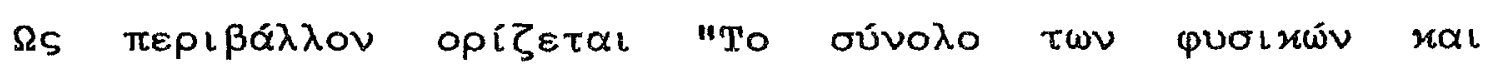

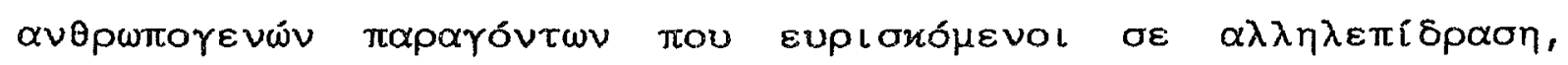

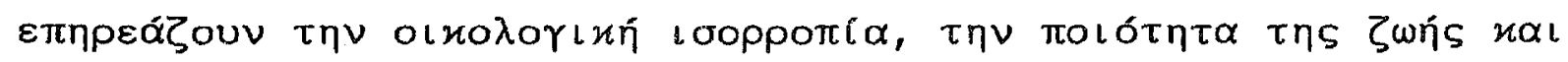

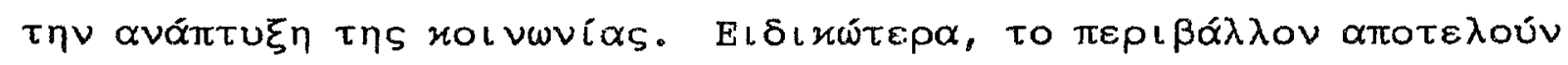

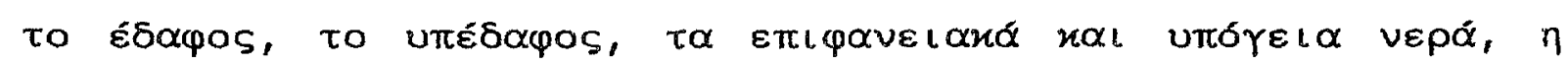

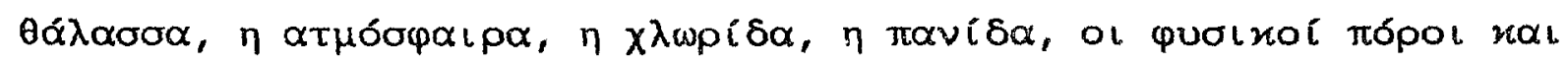

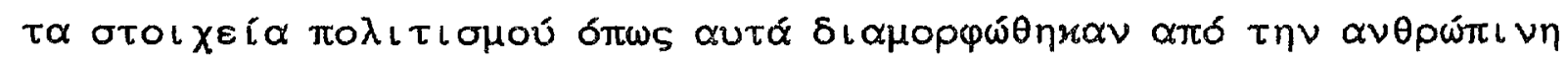

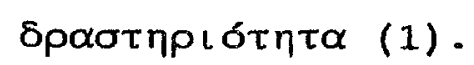




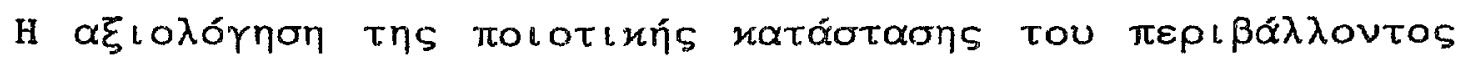

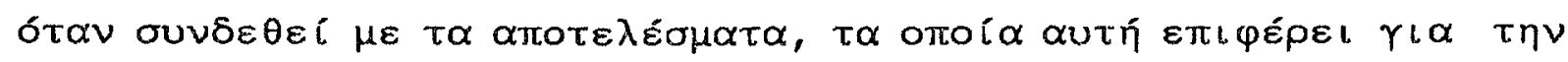

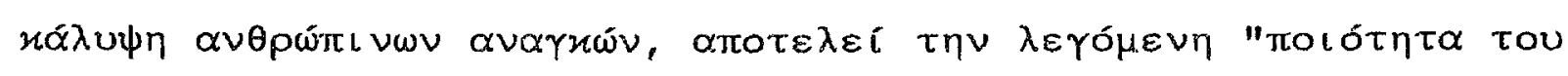

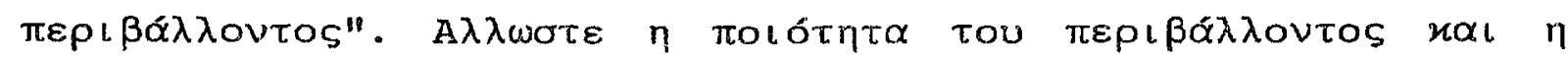

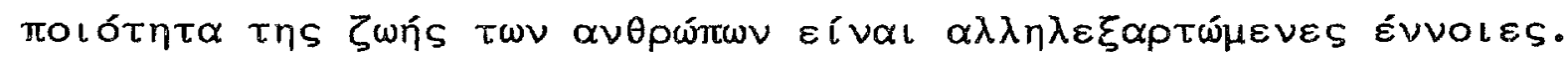

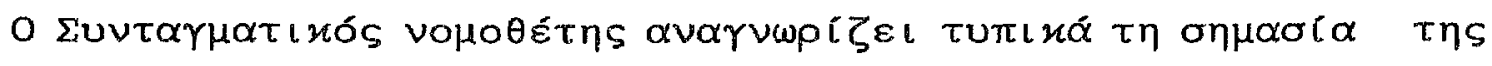

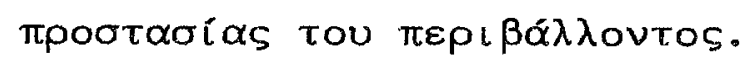

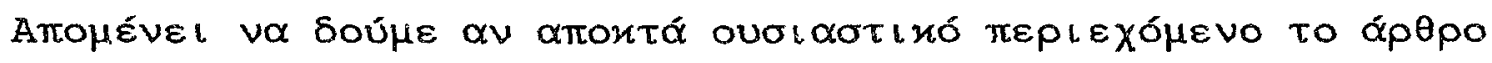

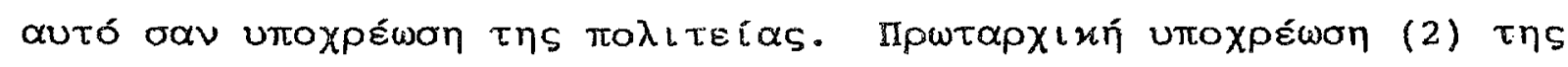

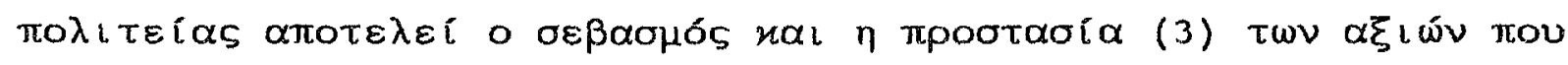

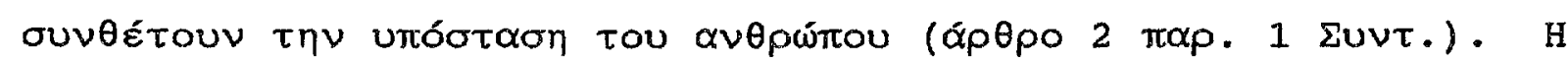

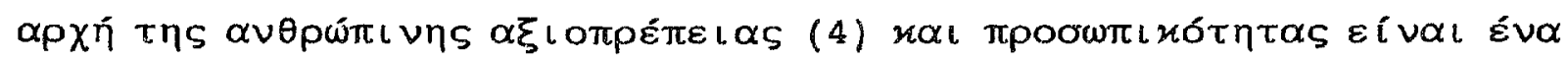

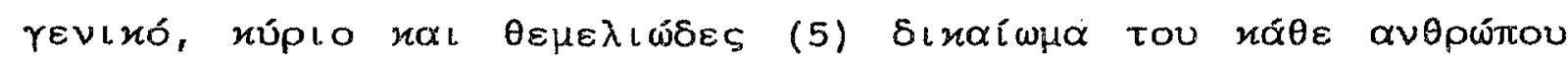

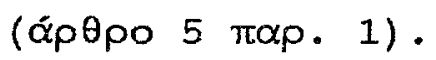

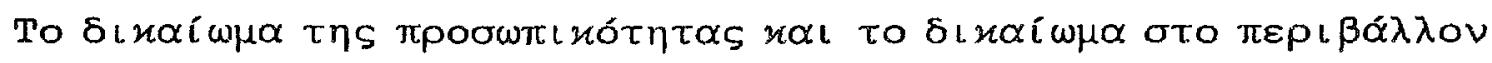

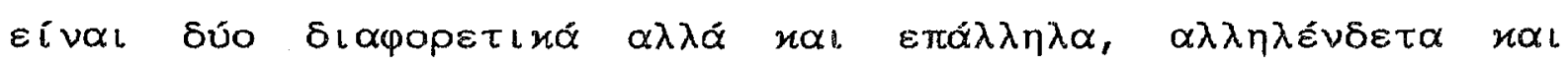

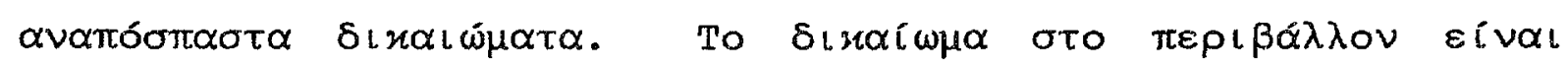

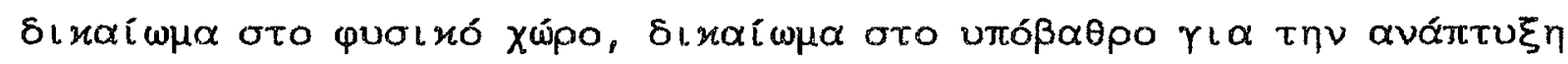

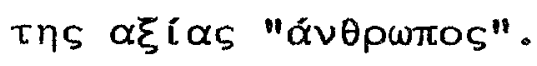

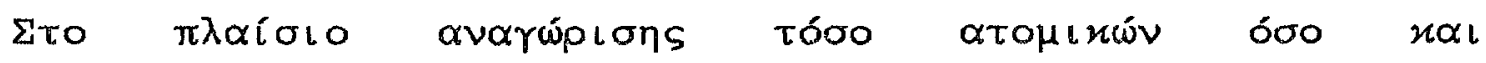

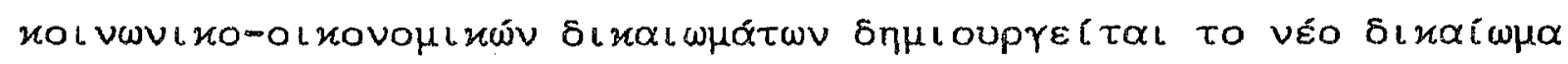

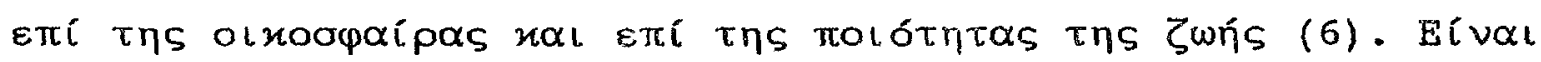




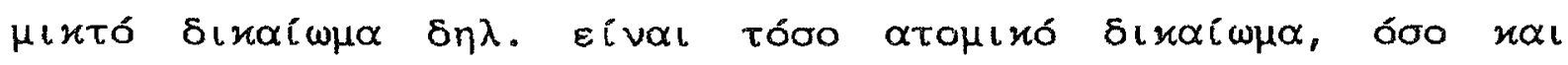

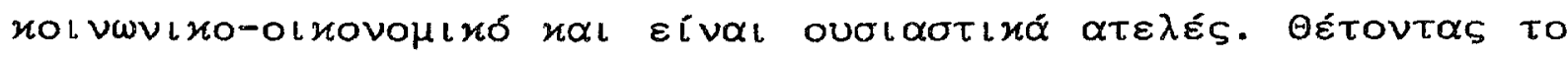

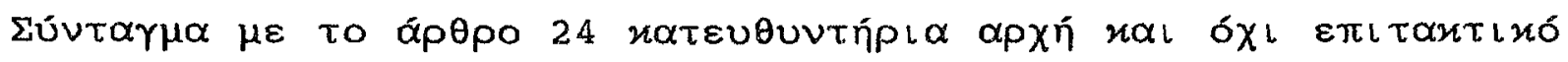

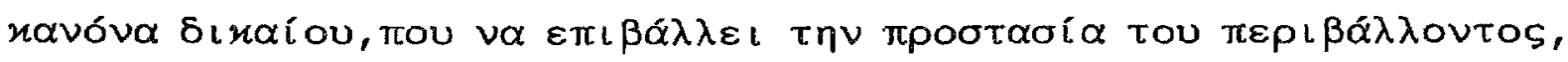

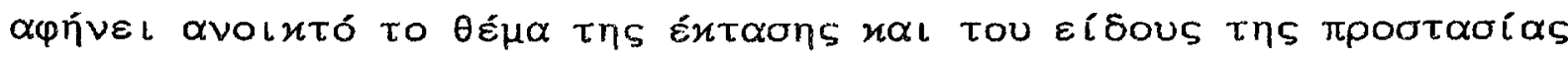

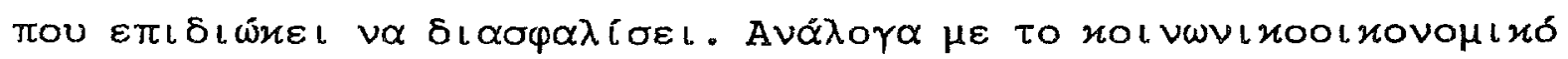

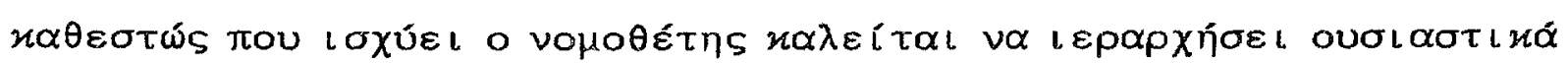

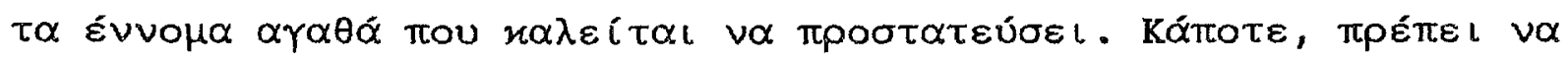

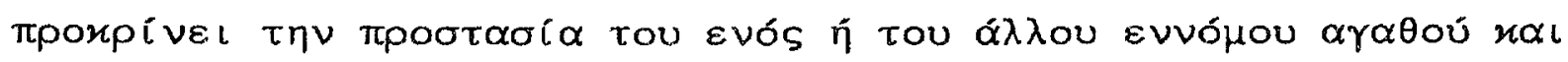

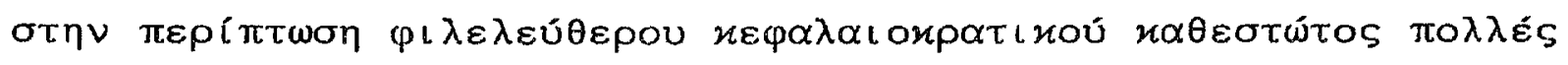

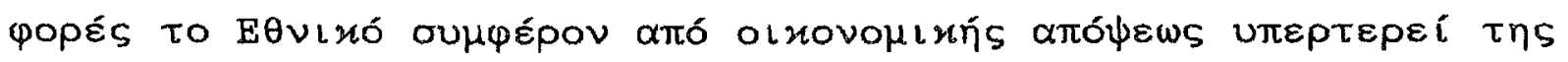

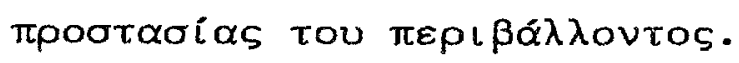

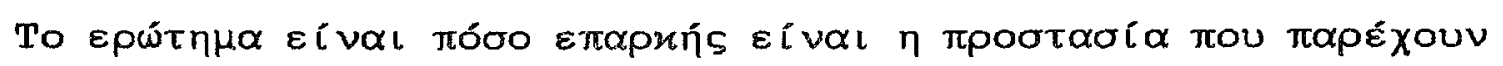

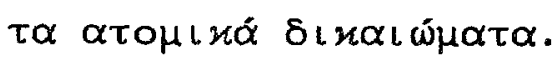

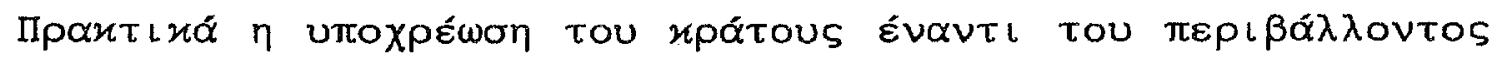

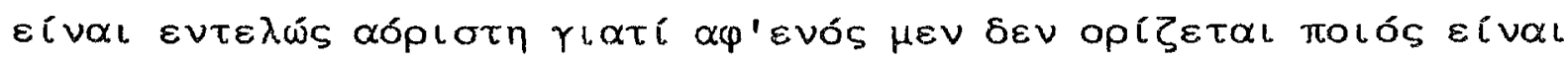

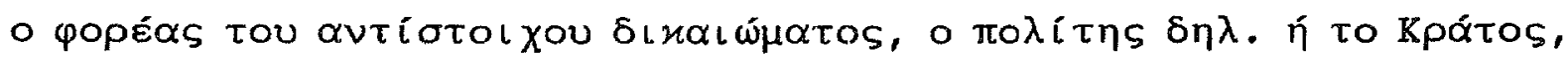

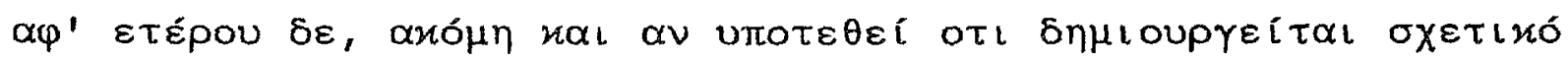

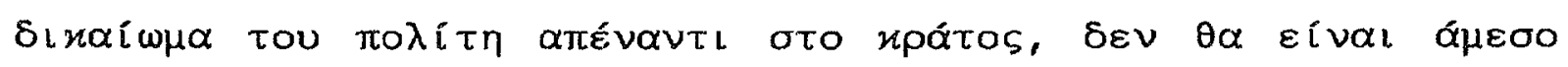

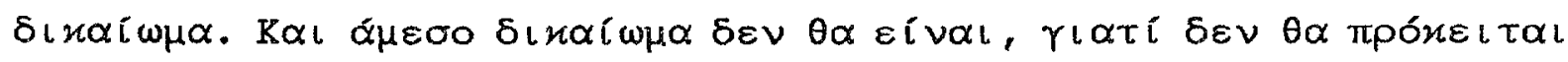

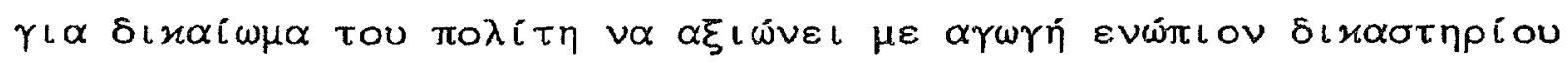

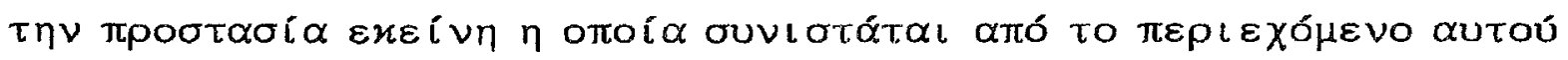




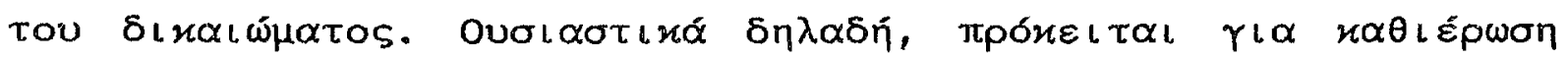

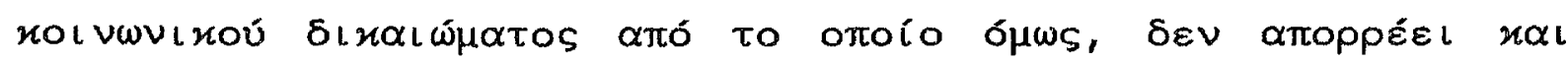

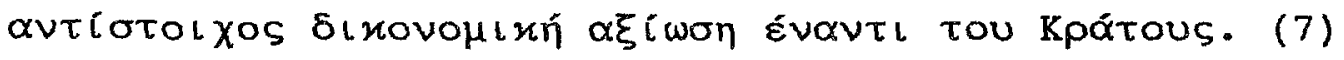

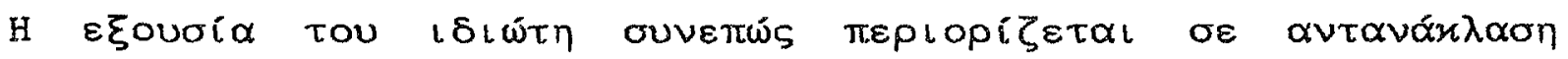

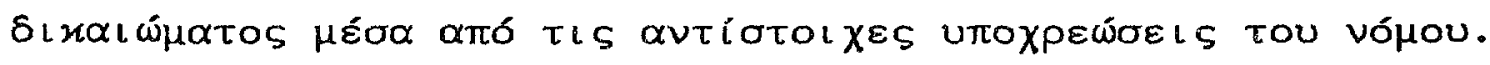

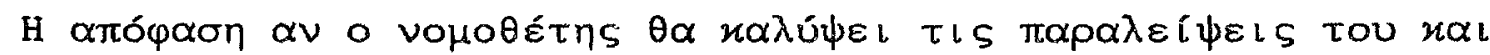

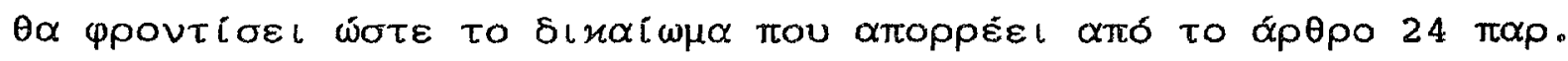

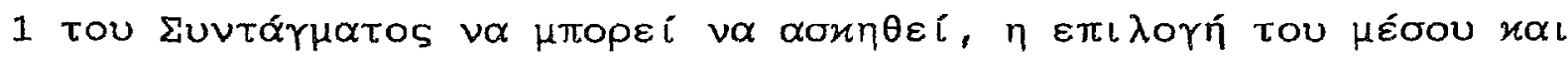

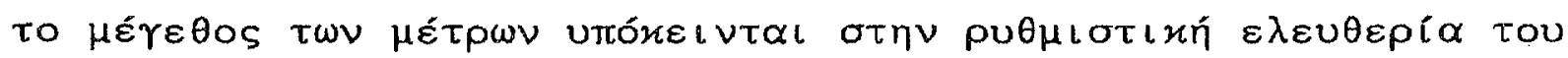

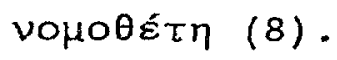

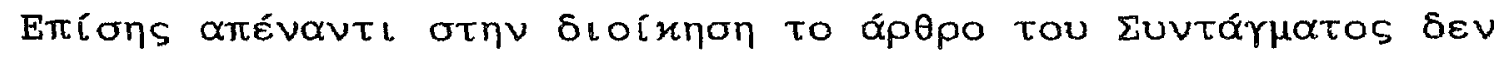

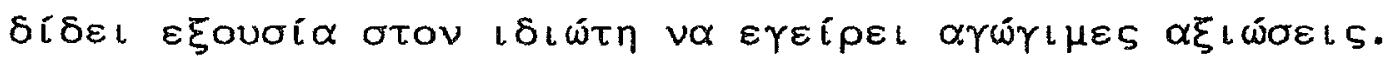

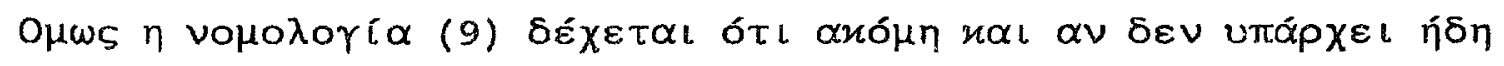

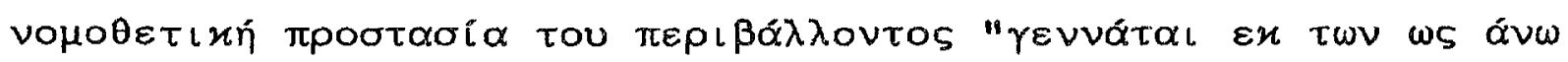

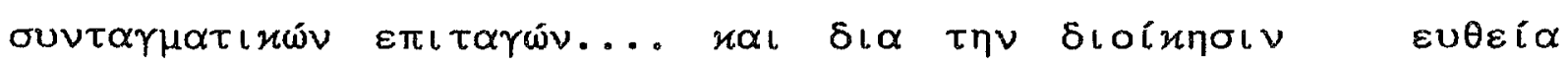

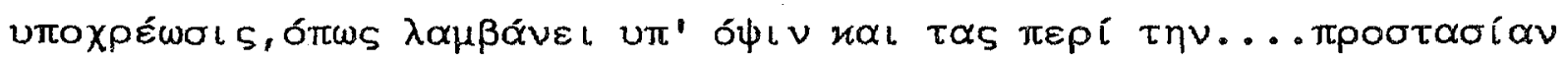

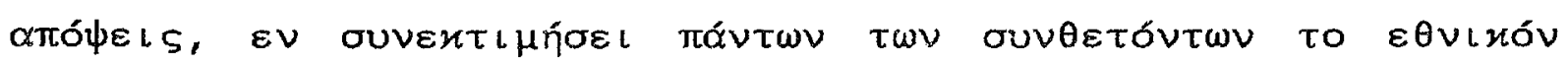

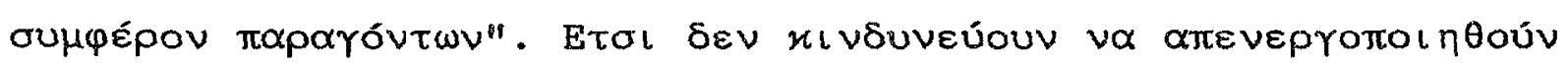

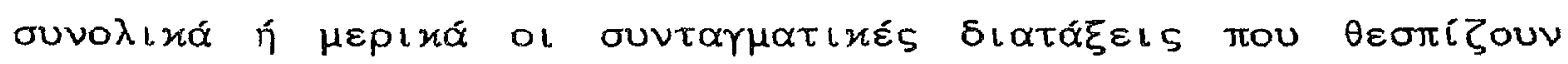

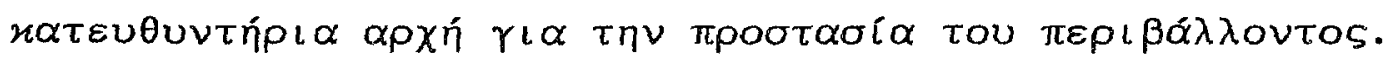

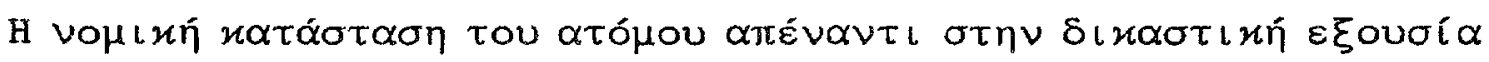

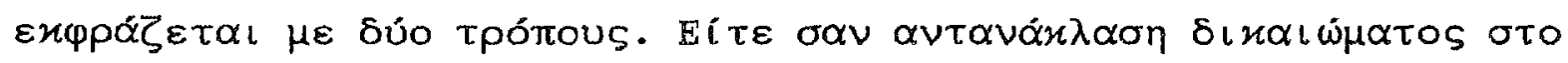




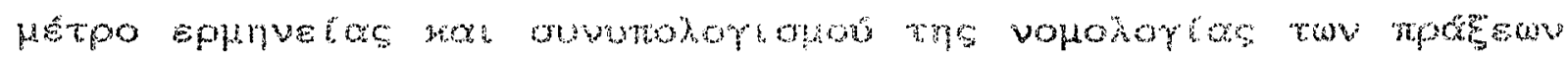

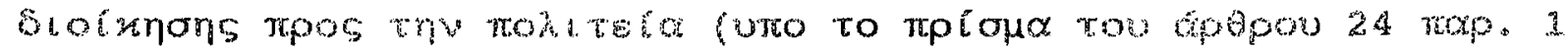

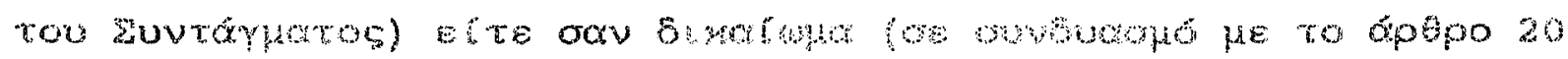

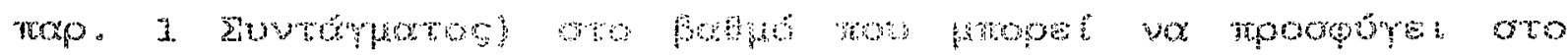

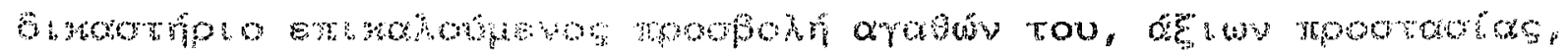

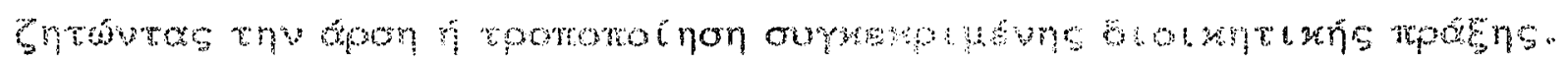

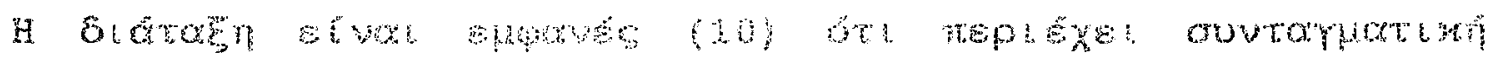

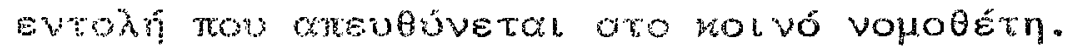

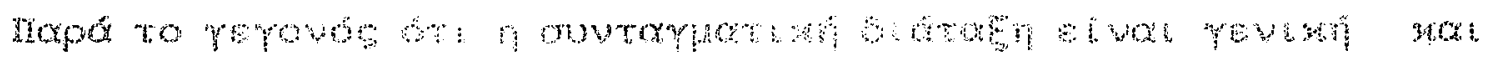

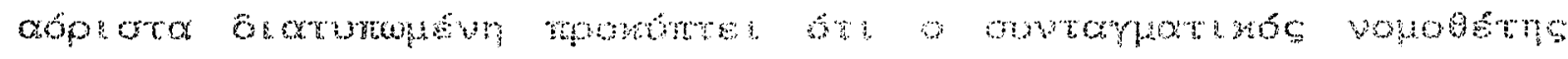

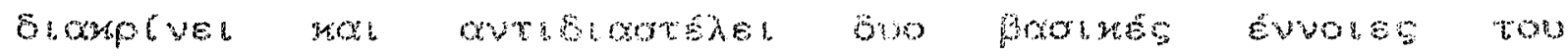

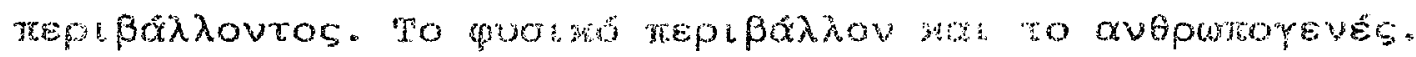

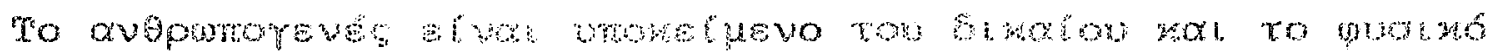

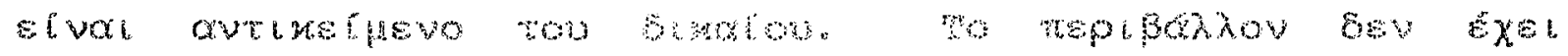

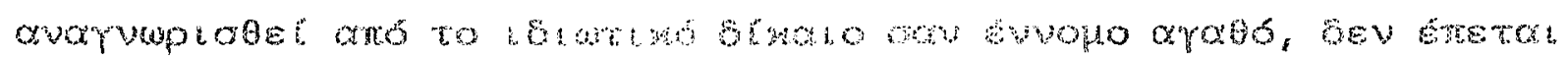

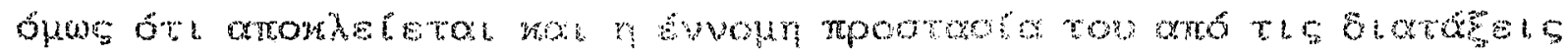

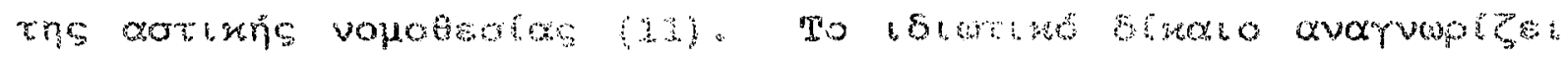

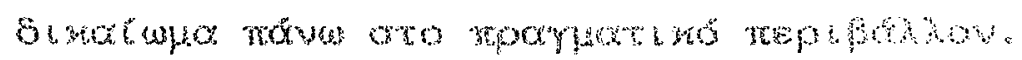

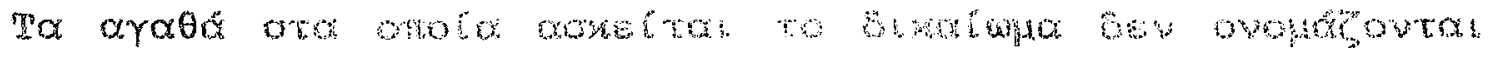

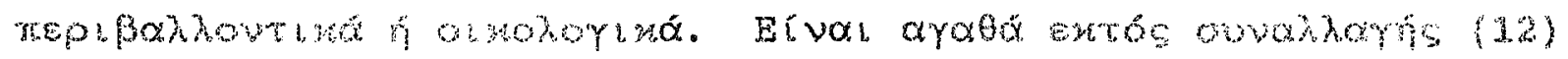

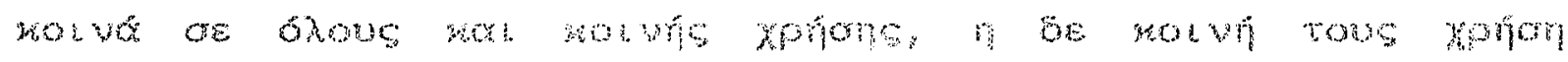

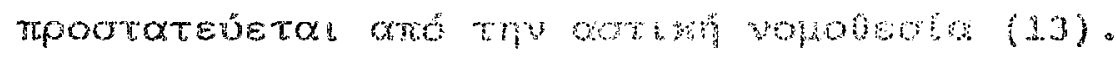




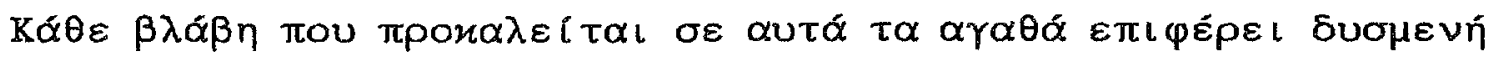

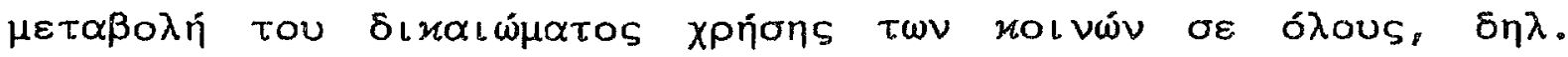

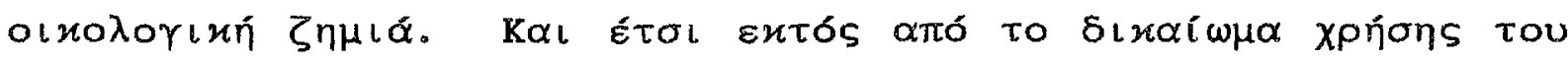

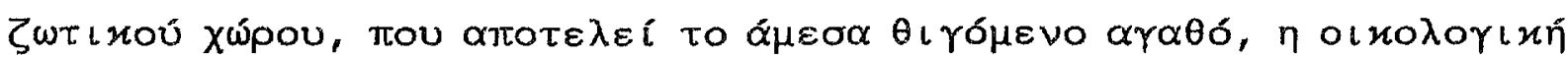

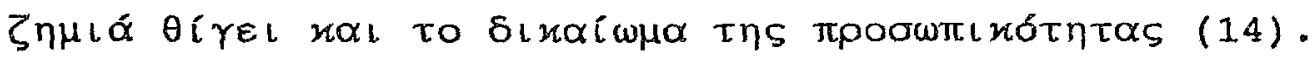

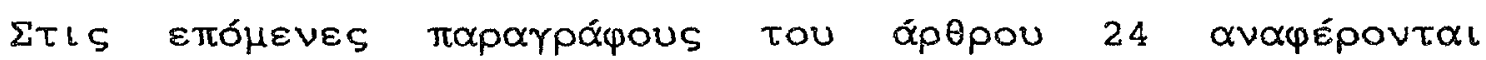

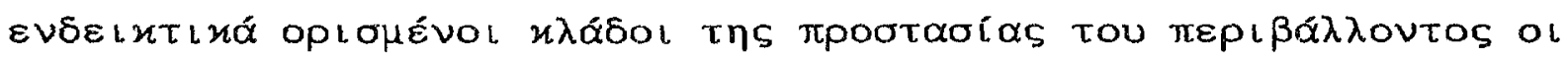

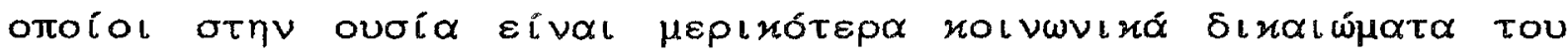

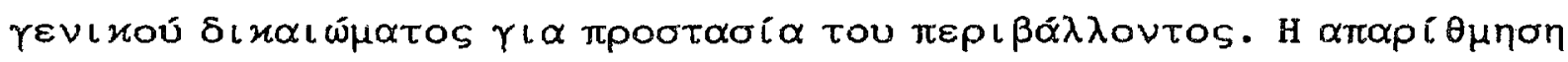

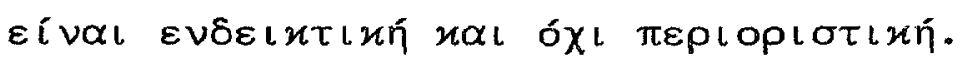

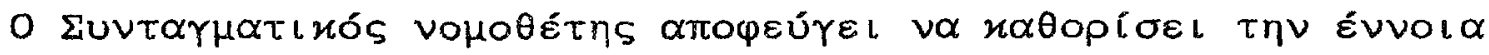

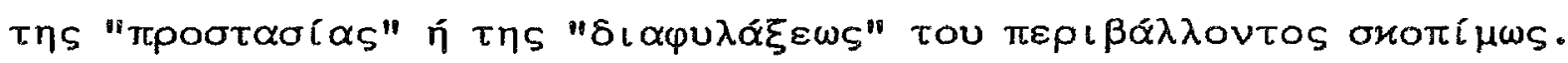

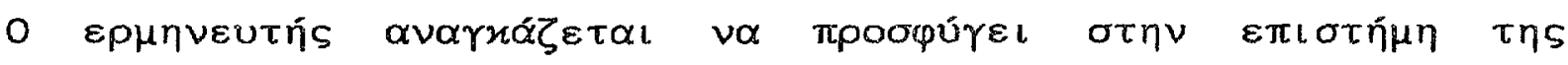

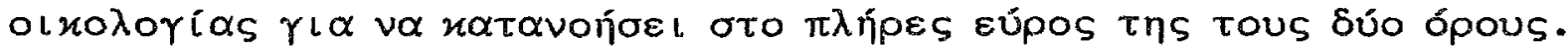

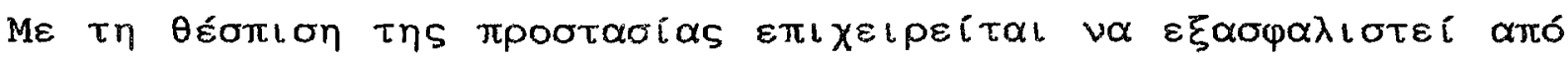

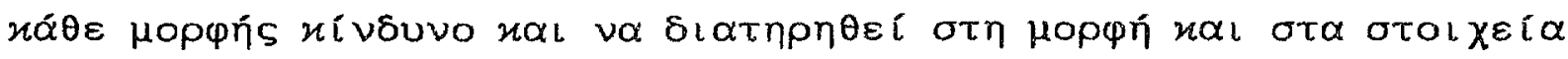

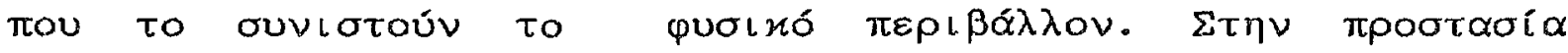

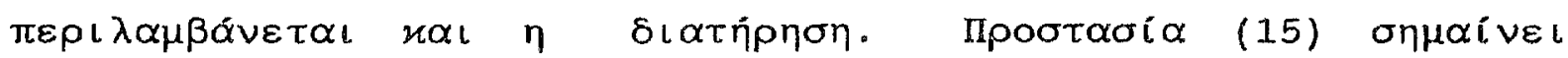

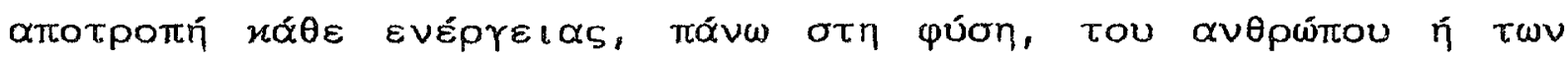

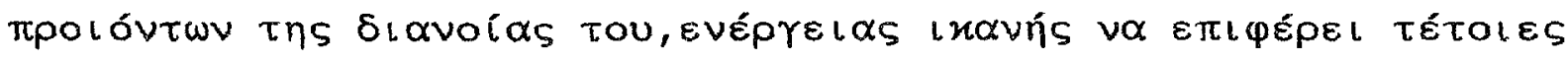
$\alpha \lambda \lambda \operatorname{lin}$

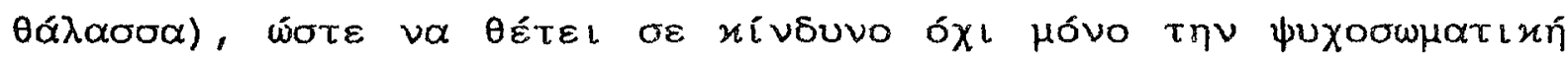

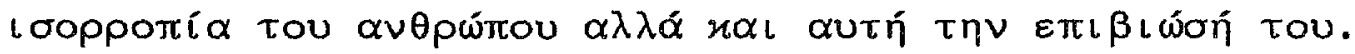




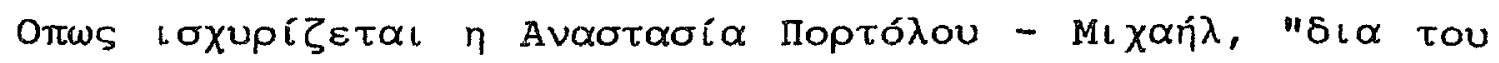

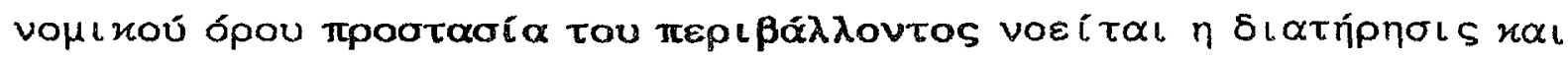

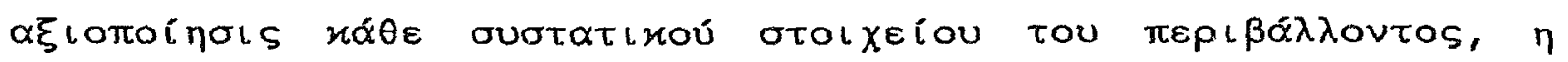

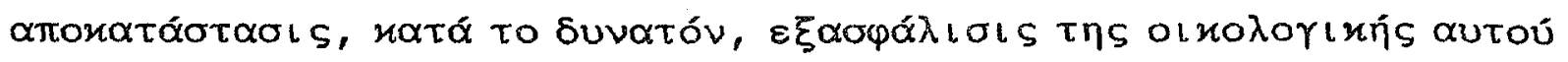

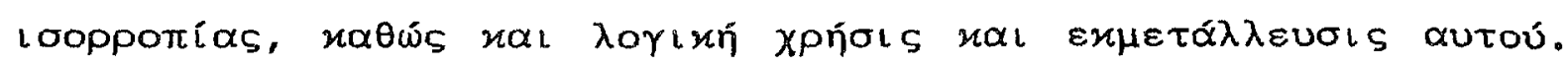

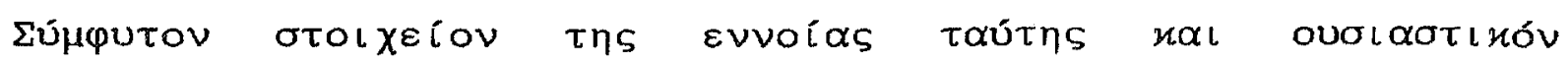

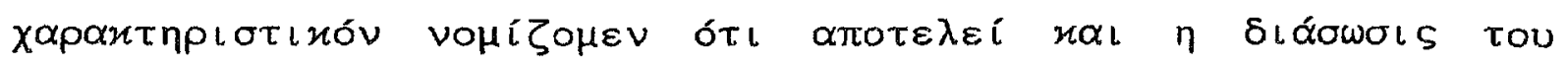

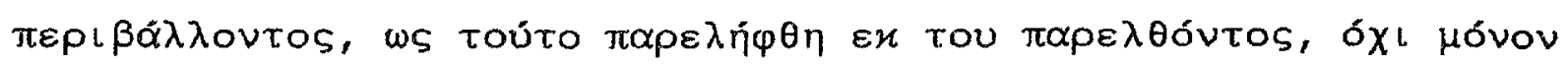

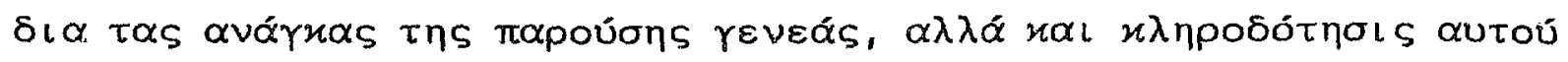

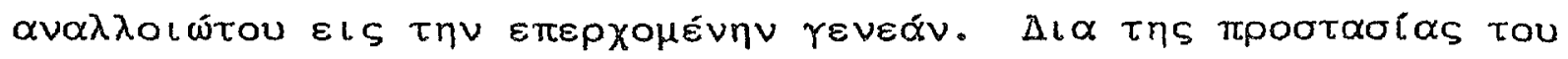

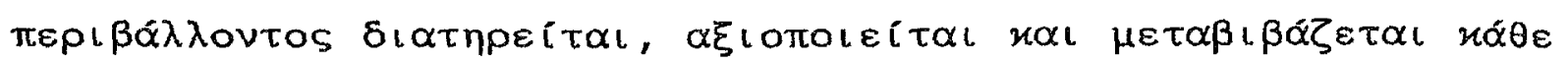

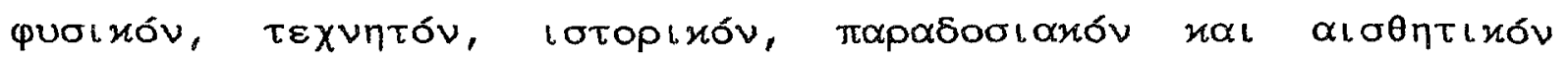

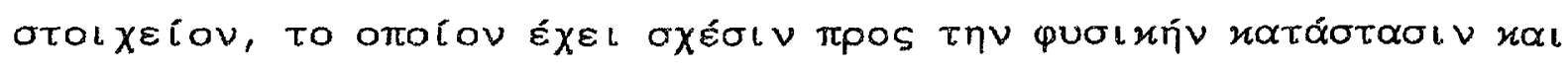

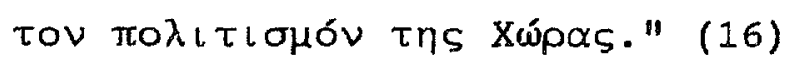




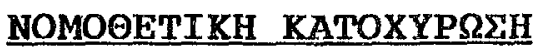

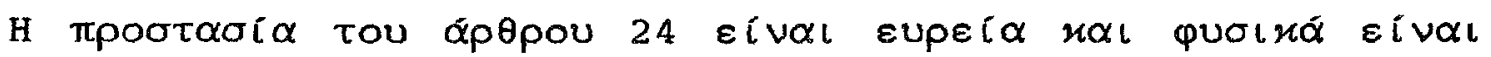

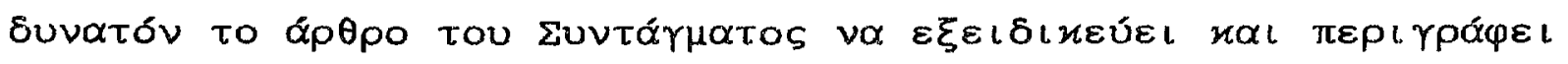

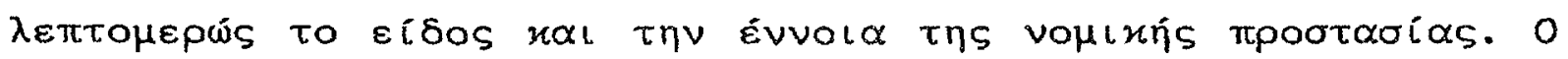

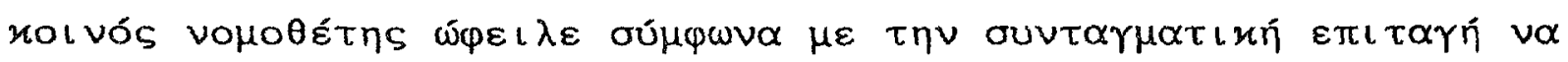

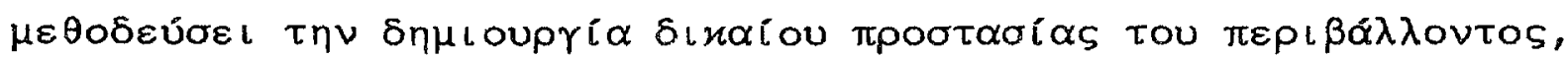

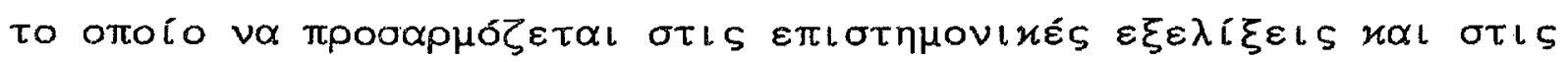

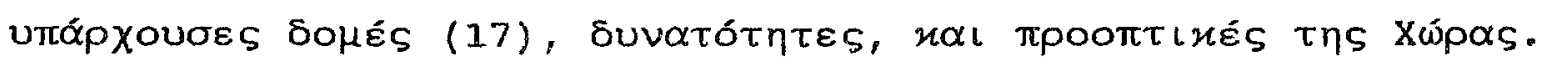

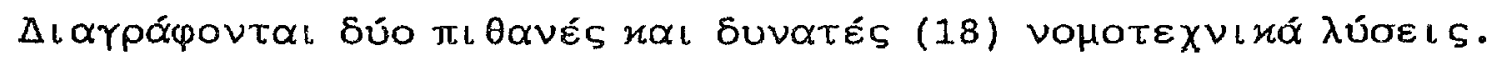

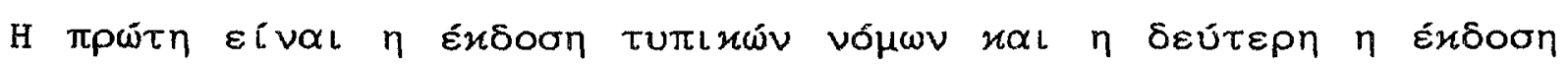

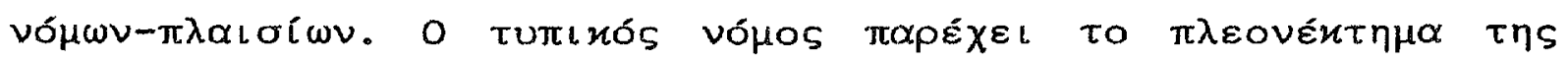

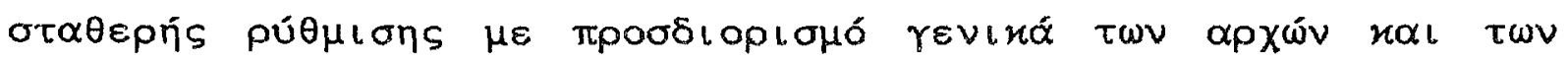

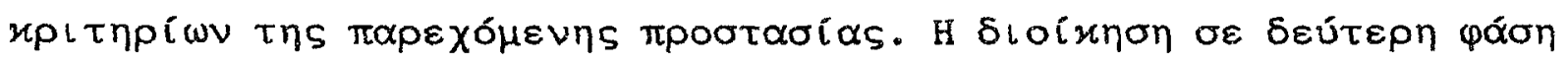

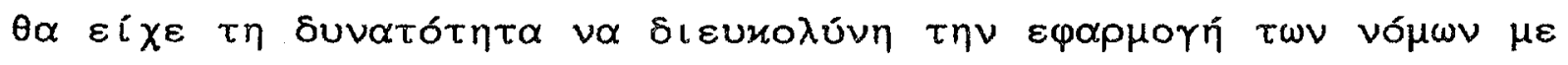

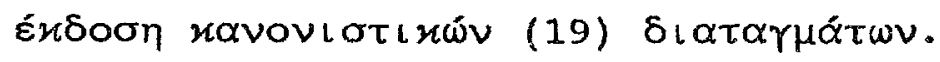

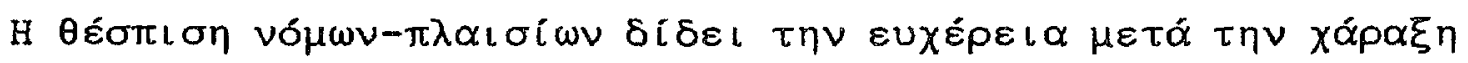

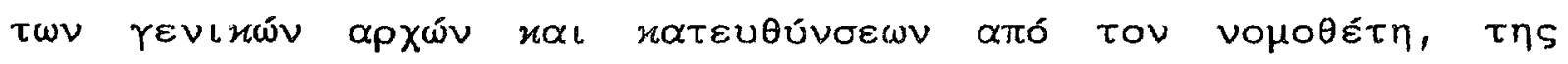

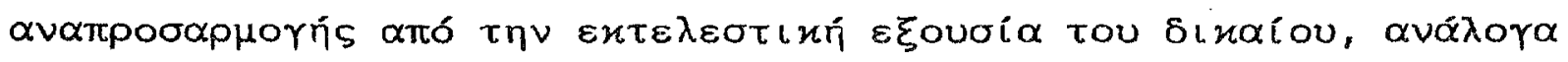

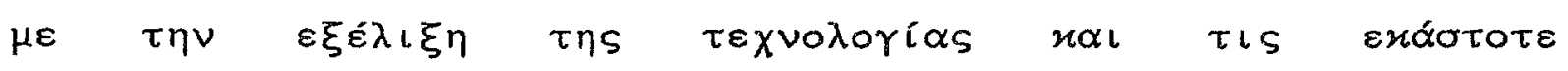

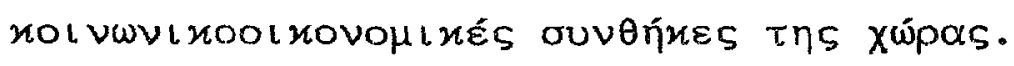




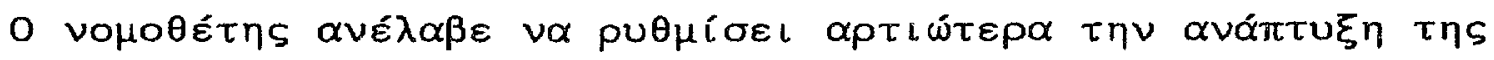

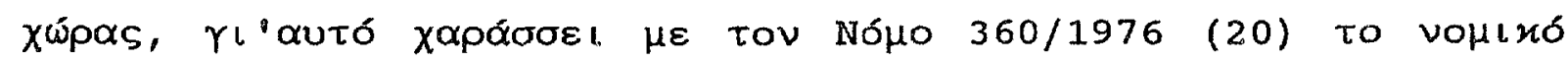

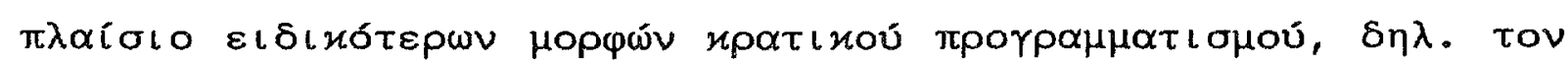

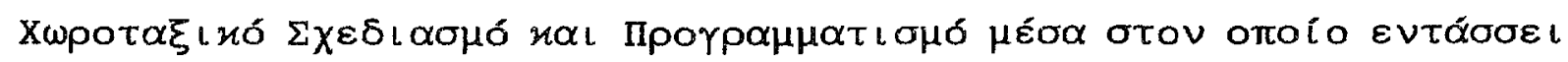

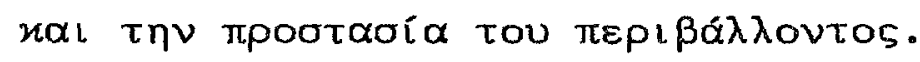

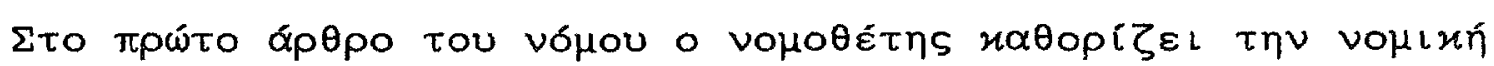

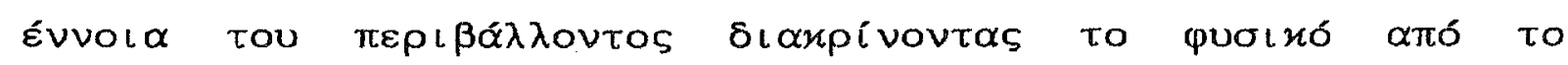

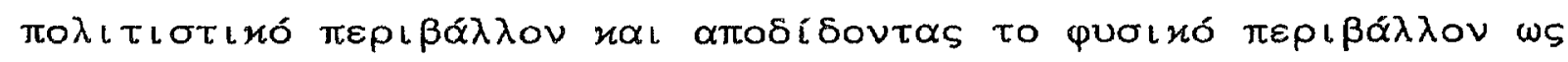

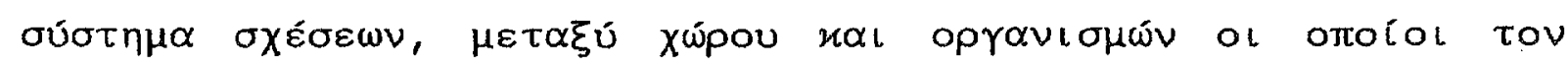

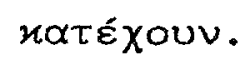

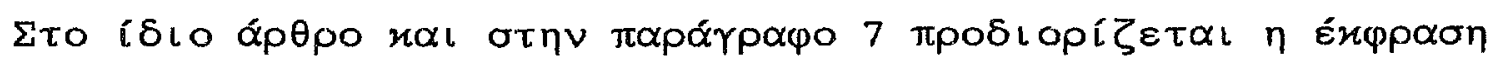

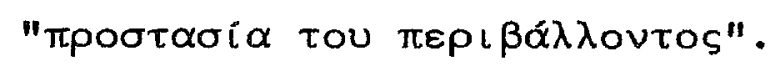

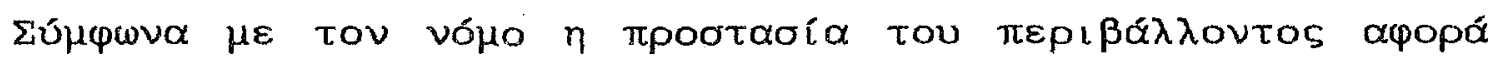

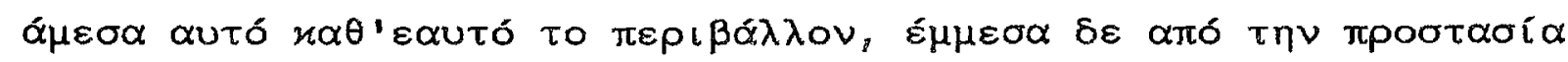

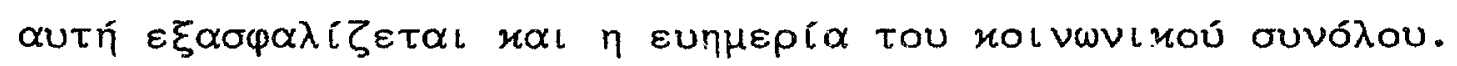

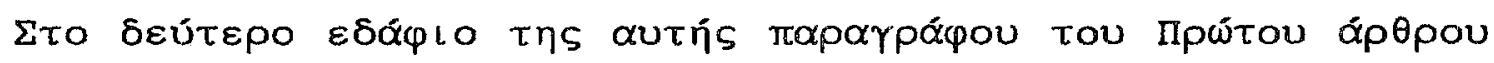

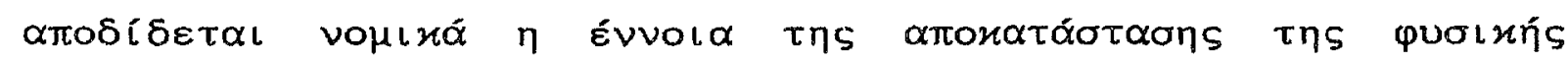

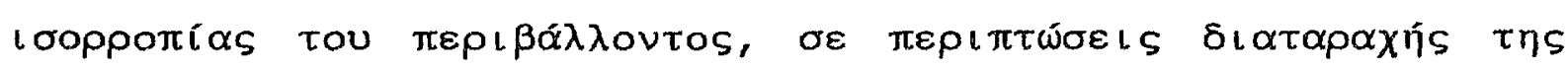

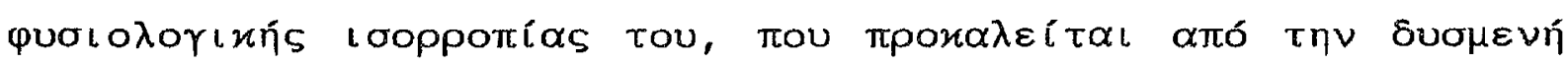

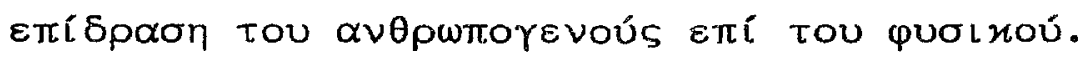

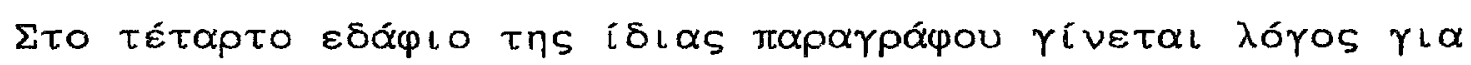

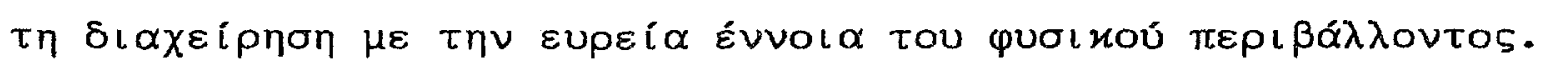




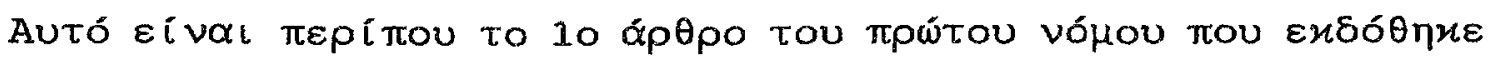

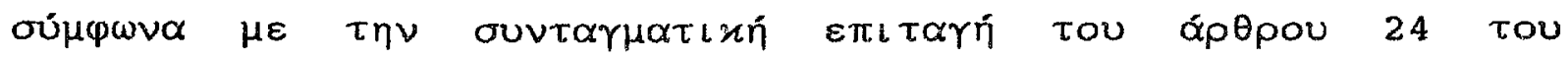

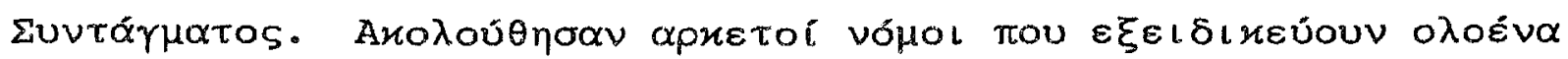

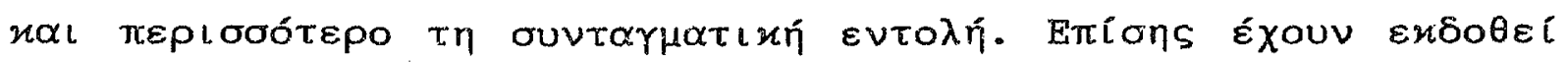

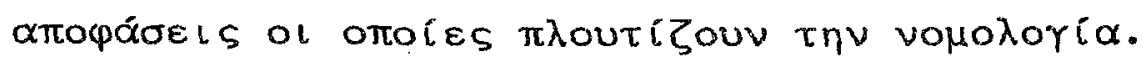

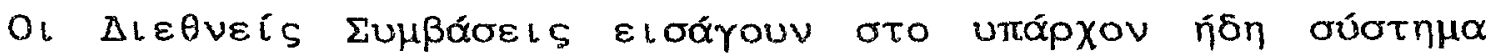

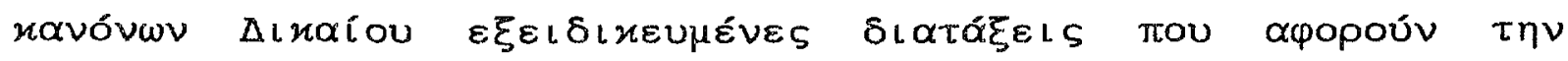

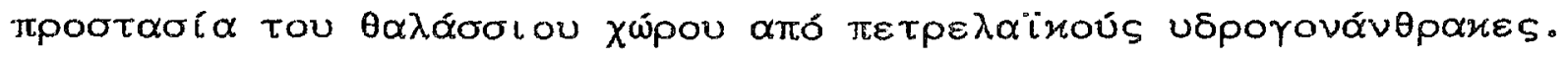

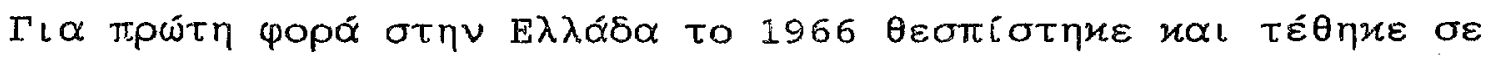

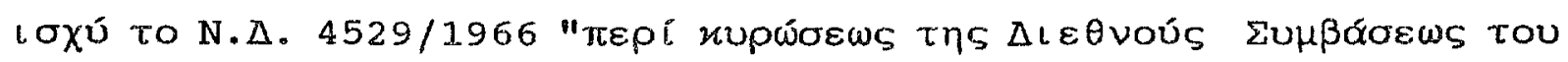

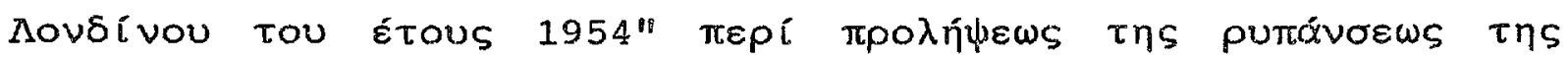

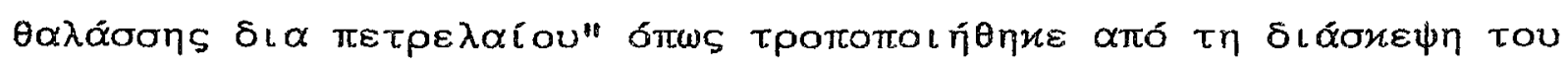
novó́ vou tou Étous 1962 .

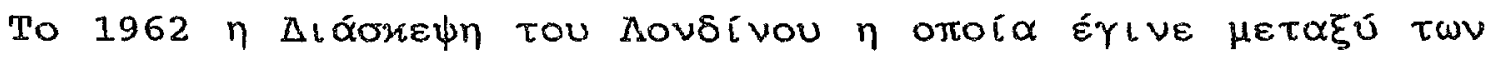

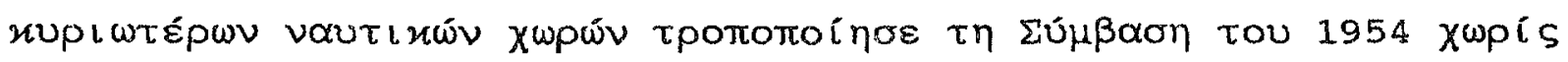

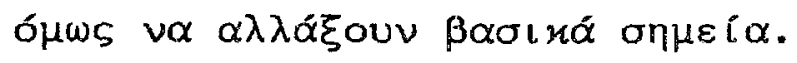

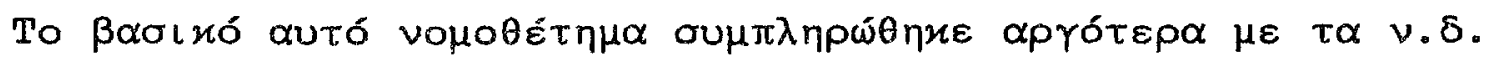

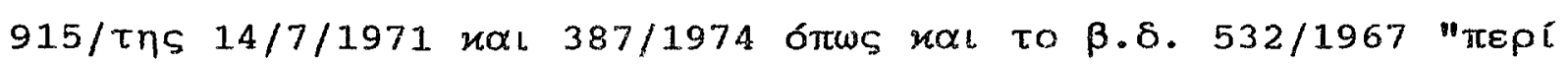

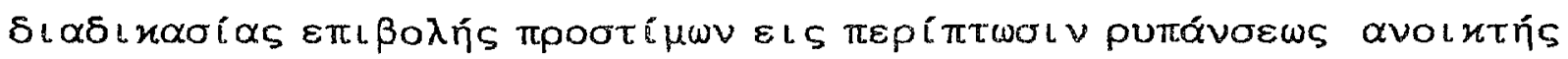

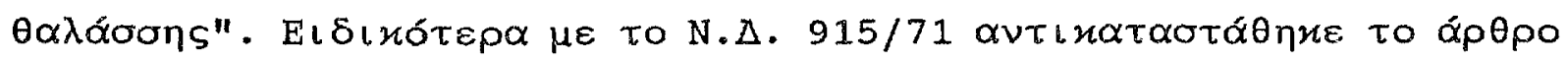

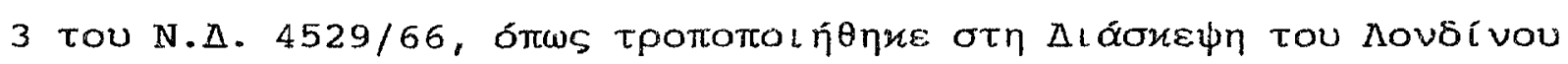

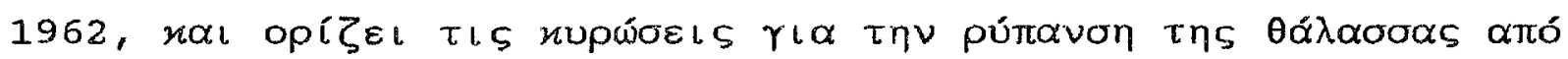

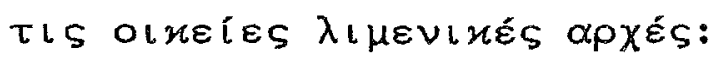




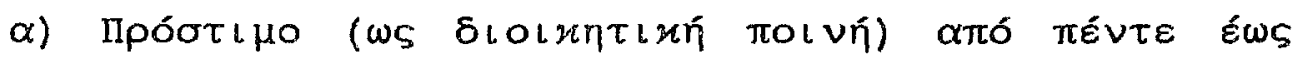

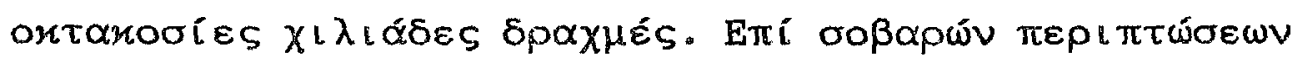

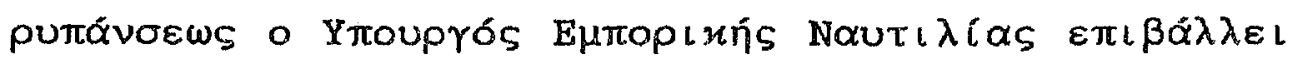

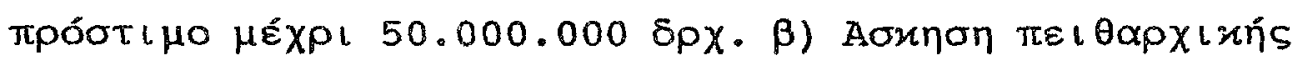

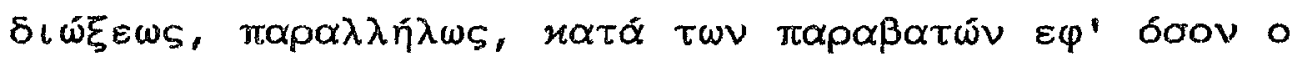

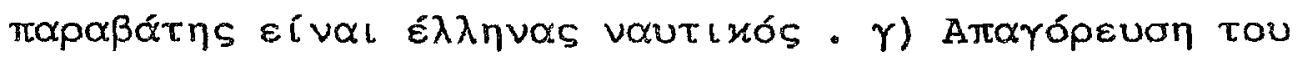

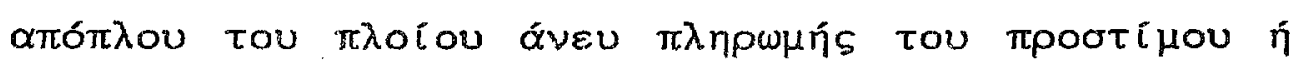

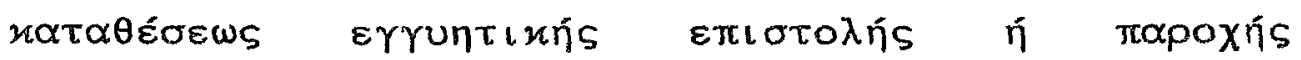

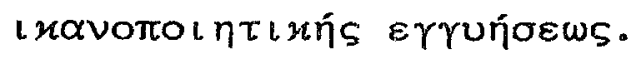

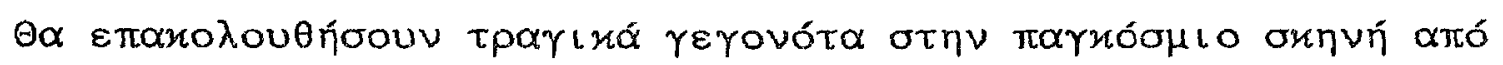

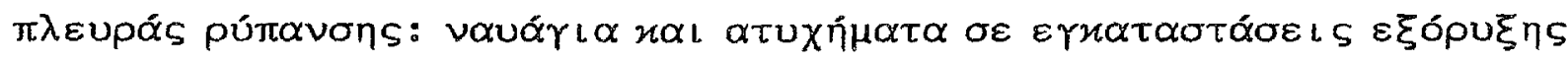

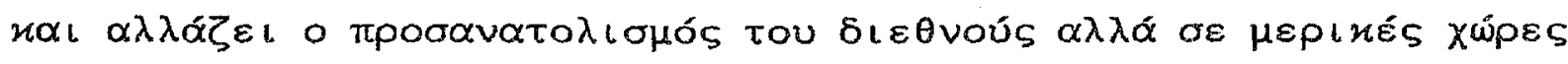

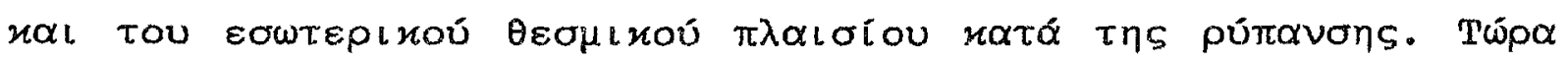

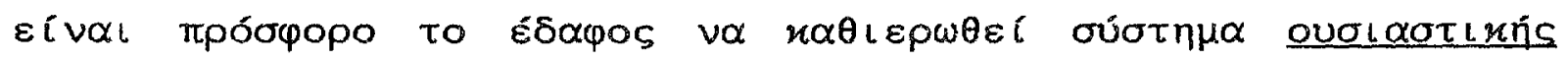

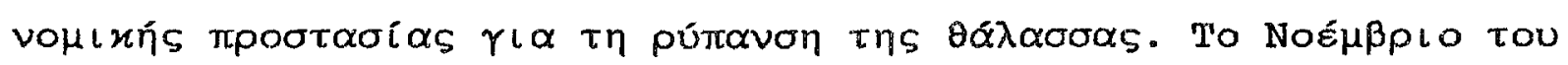

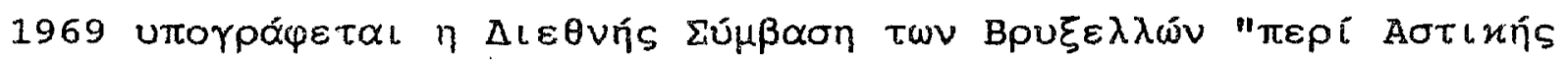

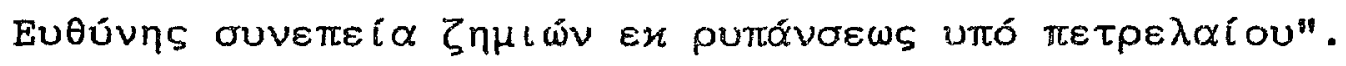

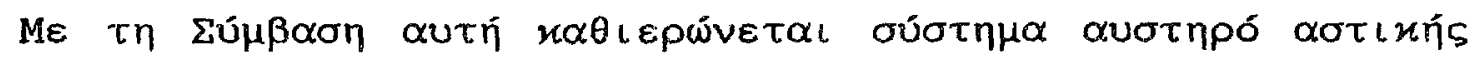

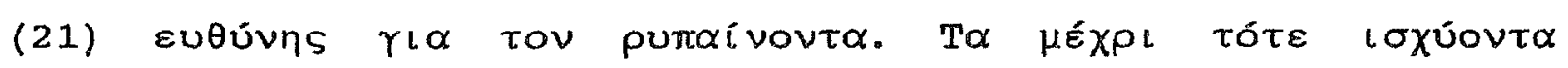

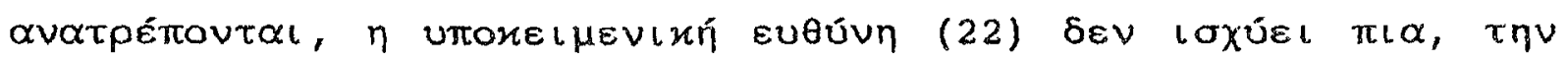

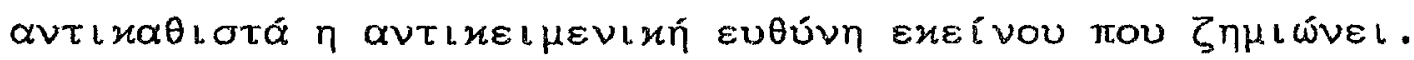




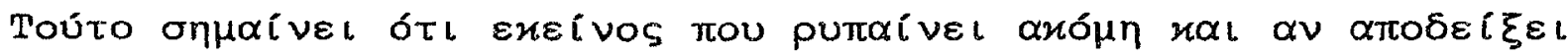

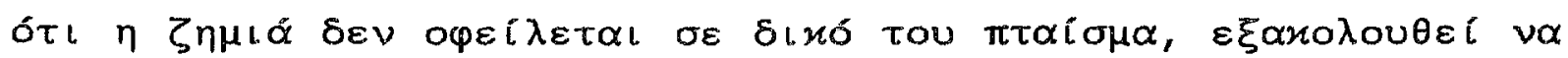

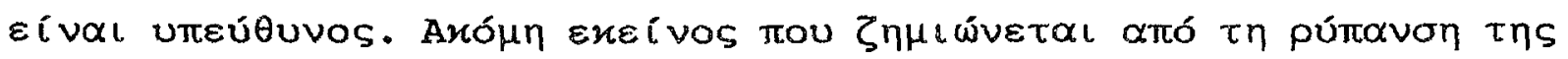

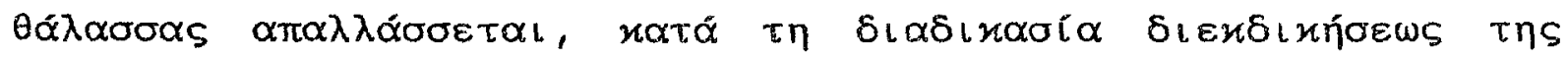

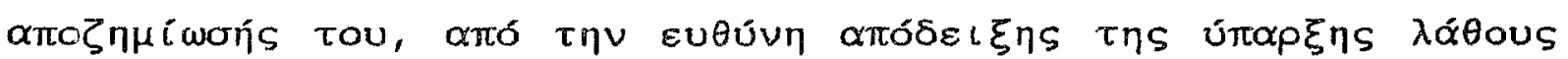

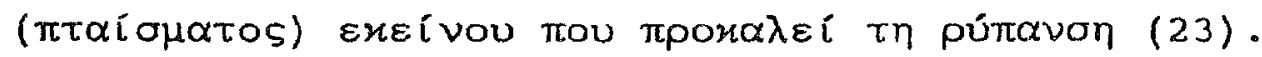

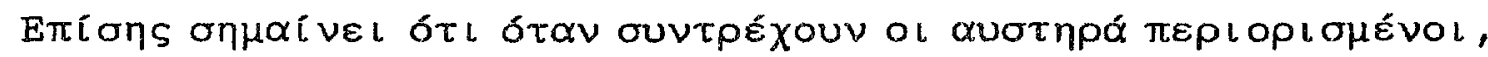

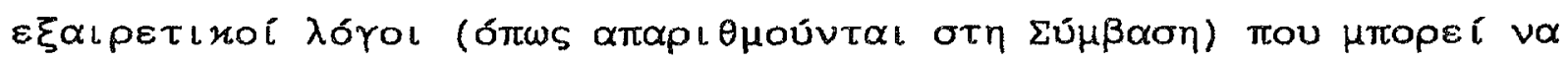

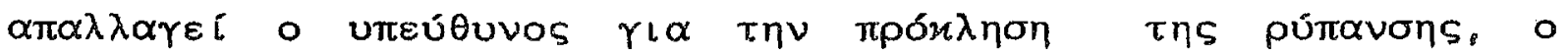

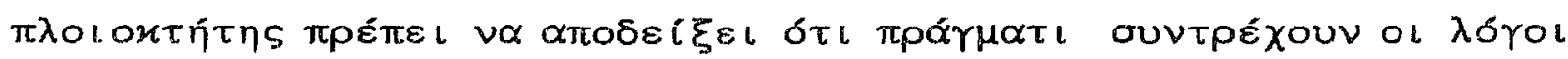

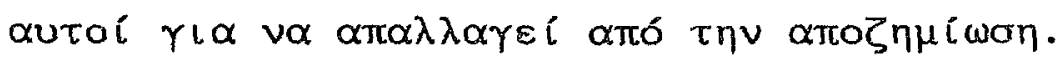

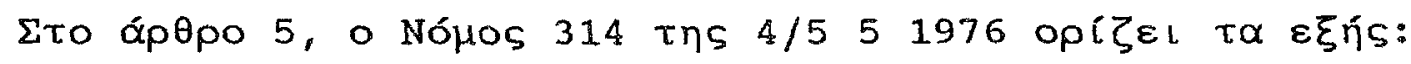

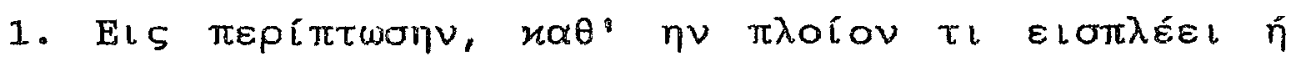

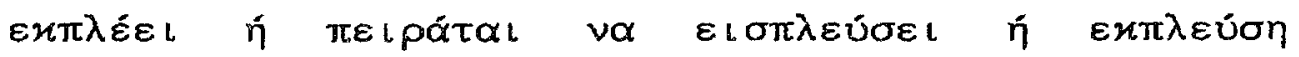

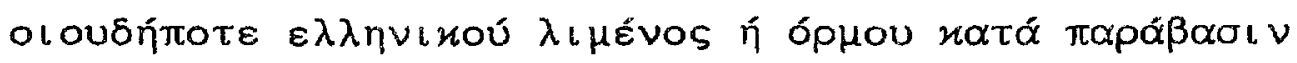

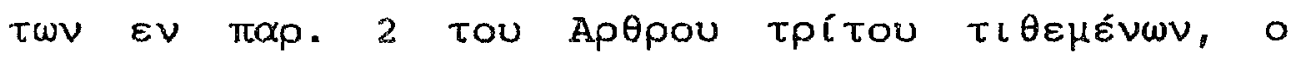

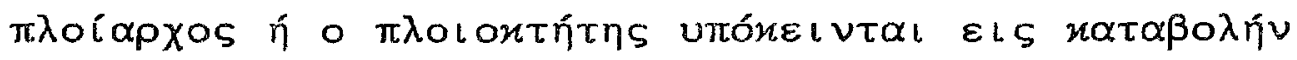

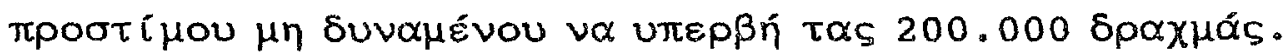

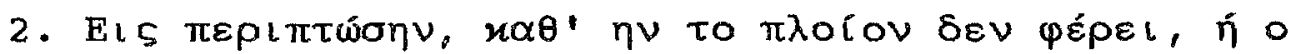

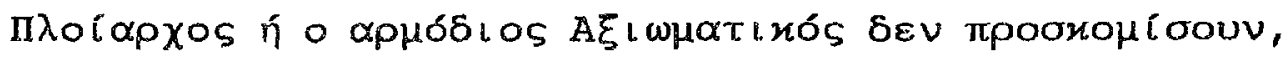

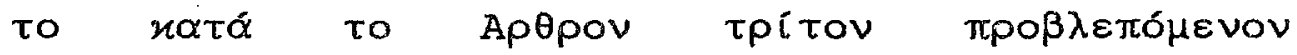

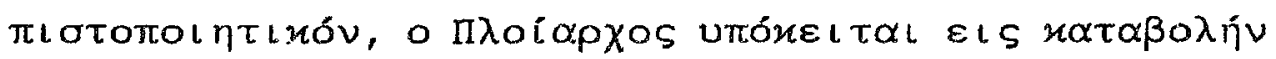




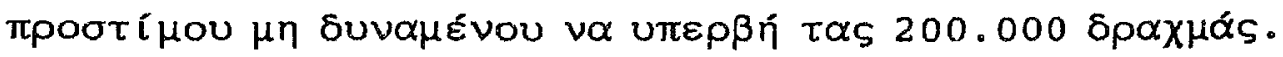

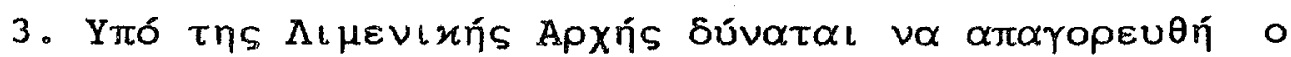

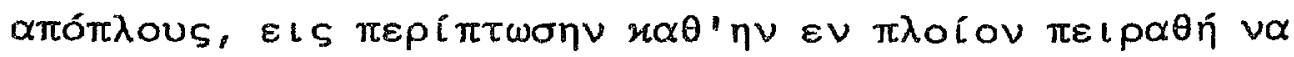

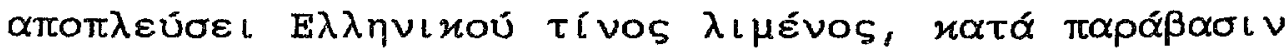

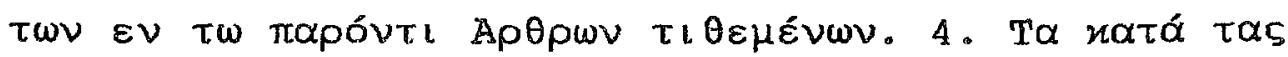

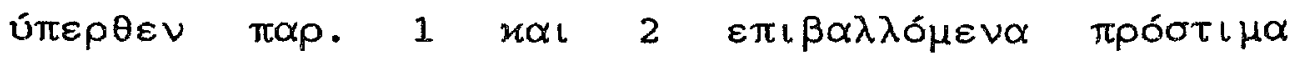

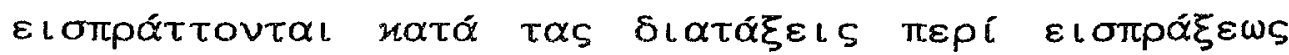

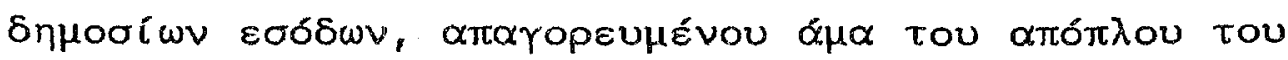

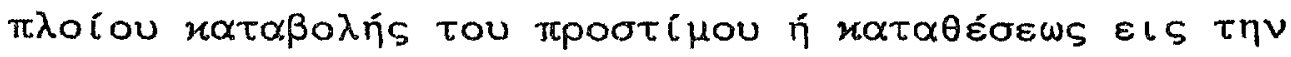

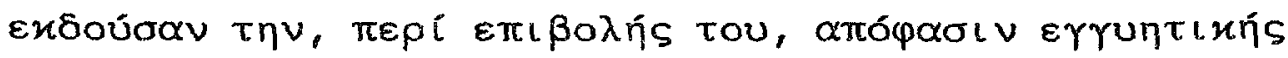

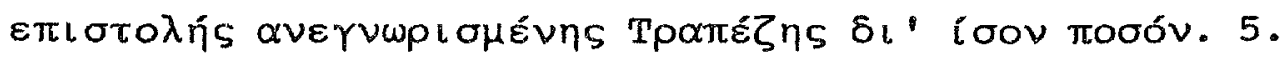

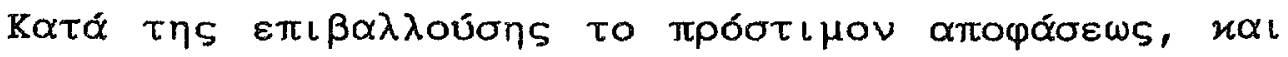

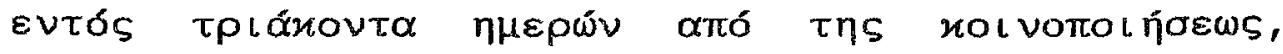

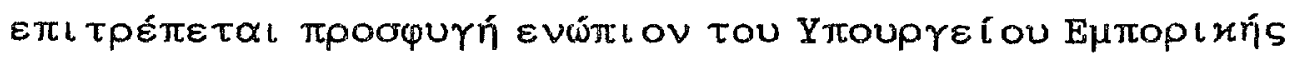

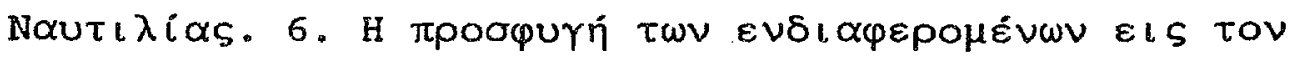

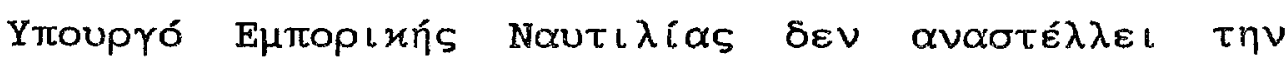

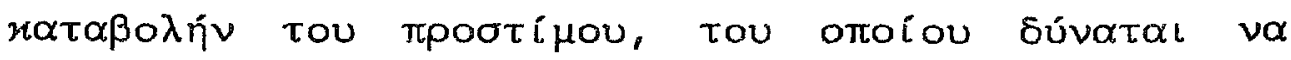

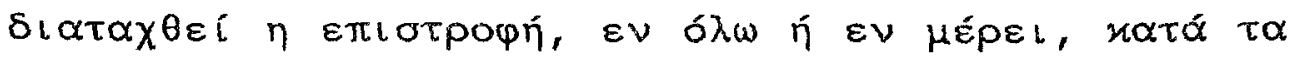

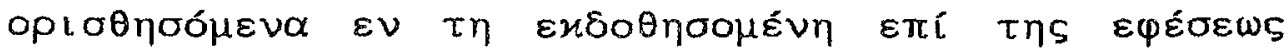
$\alpha \pi \circ \varphi \alpha ́ \sigma \varepsilon l "$.

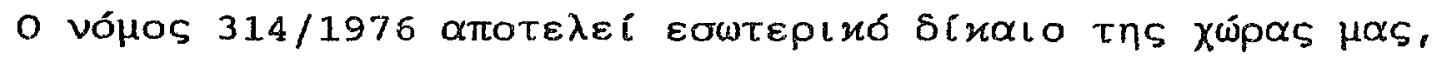

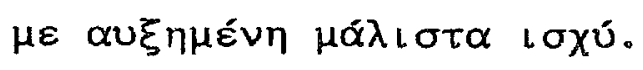




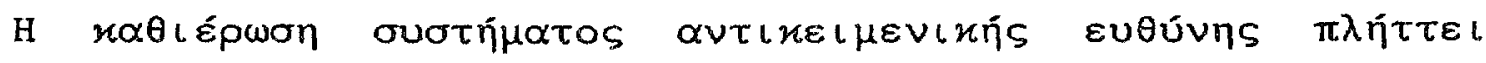

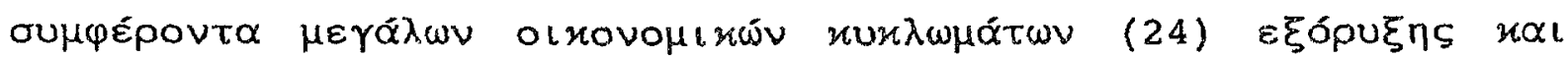

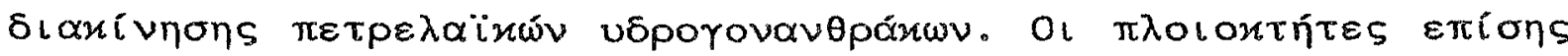

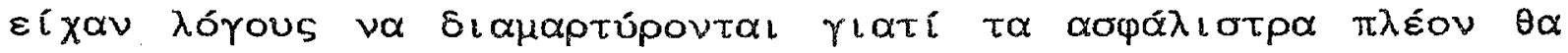

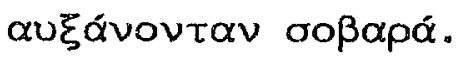

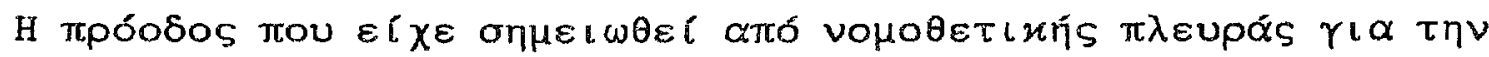

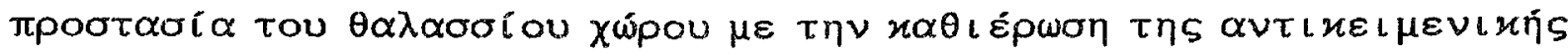

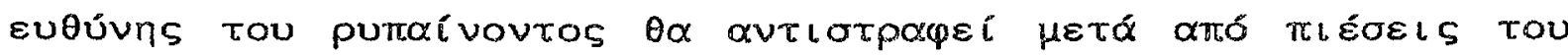

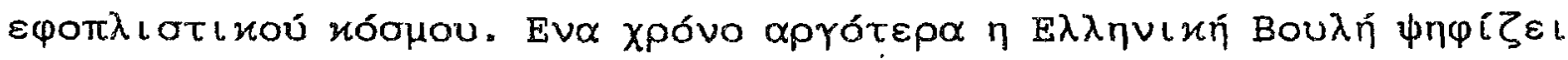

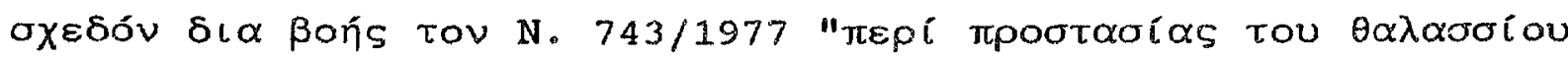

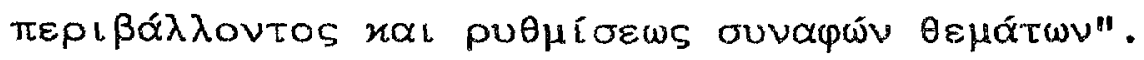

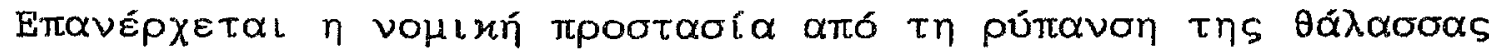

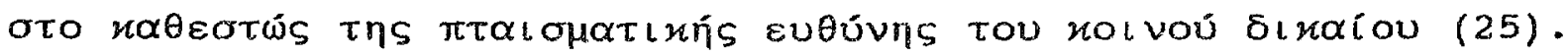

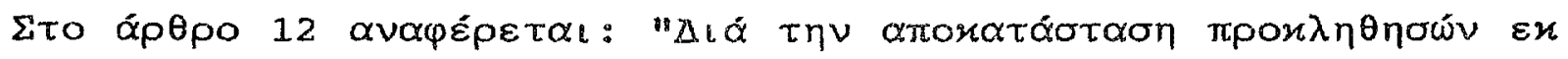

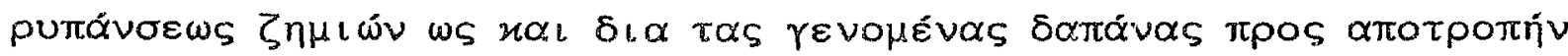

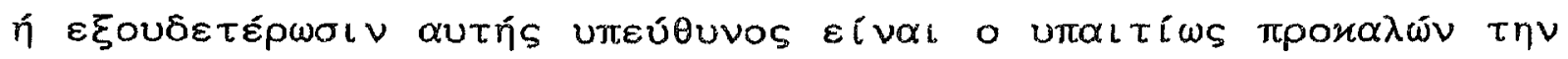
púTavolv".

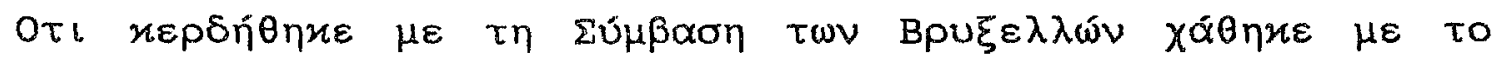
N. $743 / 1977$.

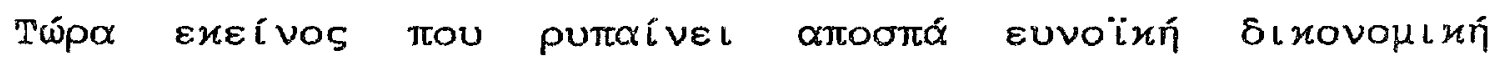

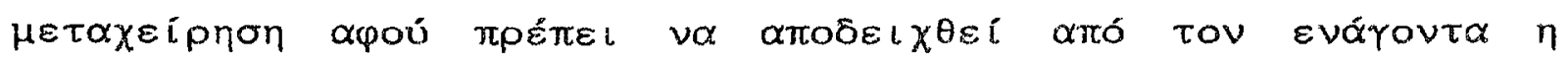

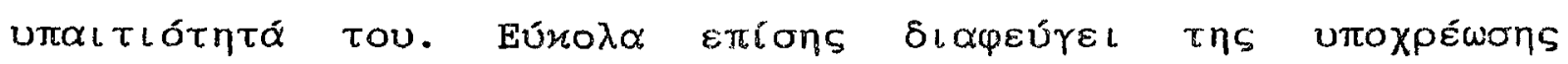

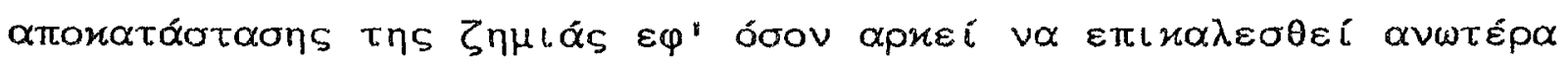

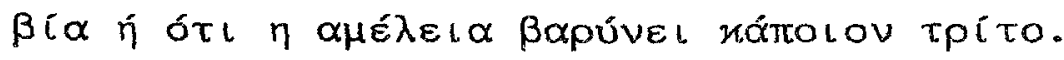




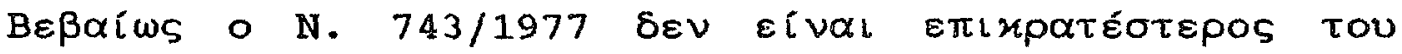

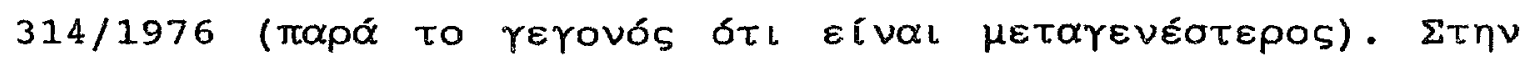

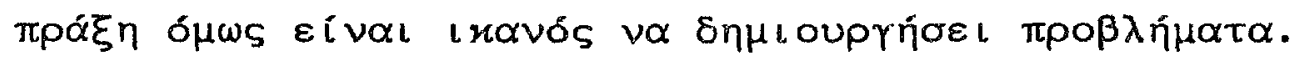

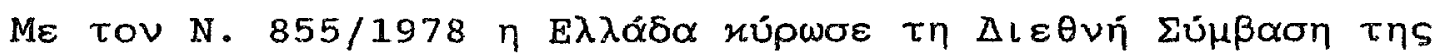

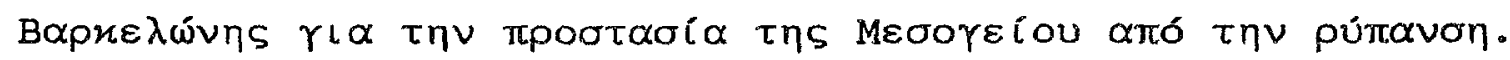

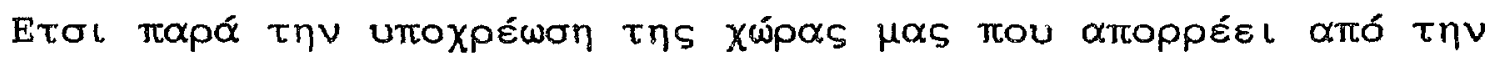

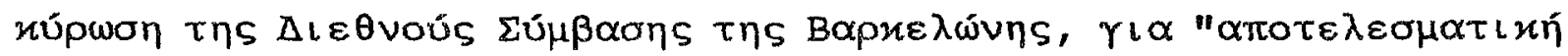

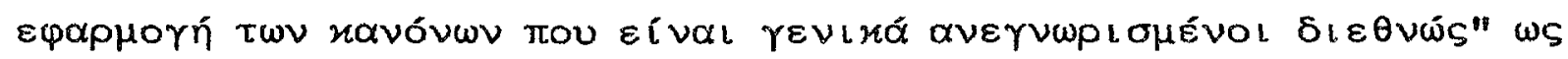

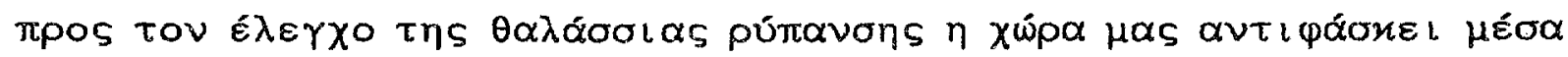

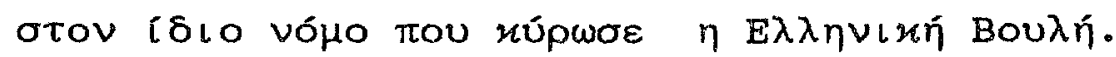

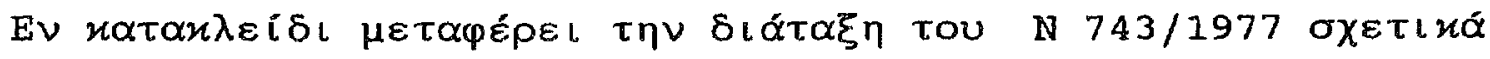

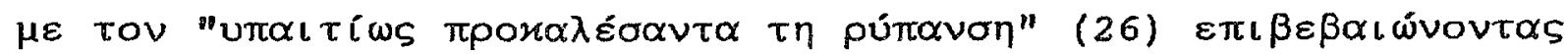

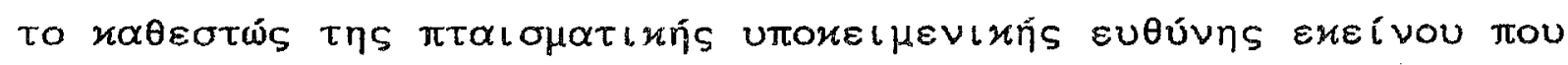

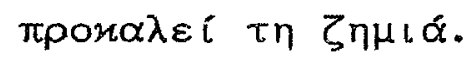




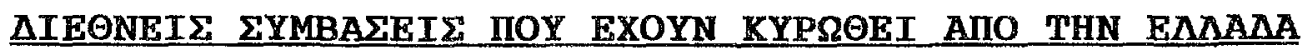

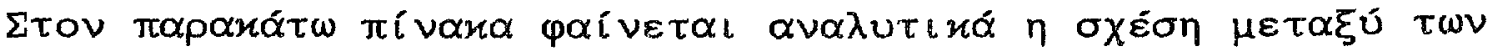

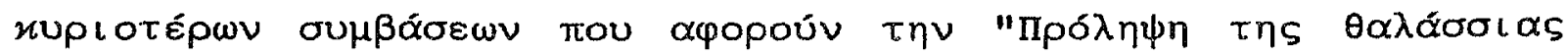

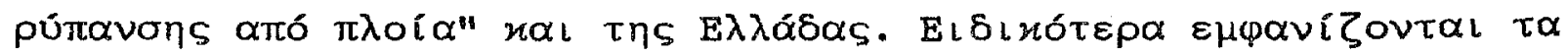

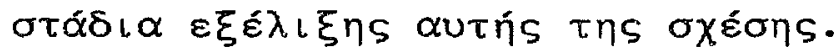

\begin{tabular}{|c|c|}
\hline & 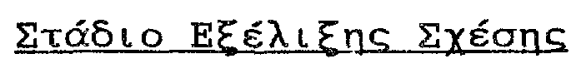 \\
\hline SOLAAS 174 & 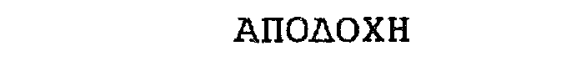 \\
\hline PROT & EN $\Omega \Sigma H$ \\
\hline INMARSA'T & 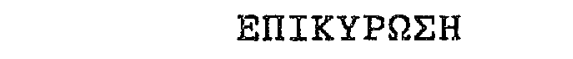 \\
\hline INMARSAT & ҮПОГРАФН \\
\hline S.T.C.W. & 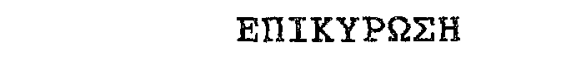 \\
\hline S.A.R. & 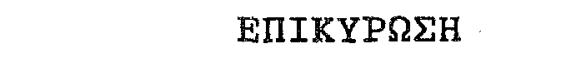 \\
\hline FUND $\quad 171$ & $\mathrm{EN} \Omega \Sigma \mathrm{H}$ \\
\hline L.D.C. 72 & 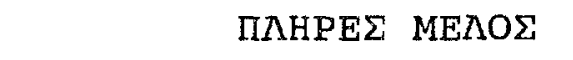 \\
\hline INTERVENTION ' 69 & П \\
\hline C.L.C. 169 & 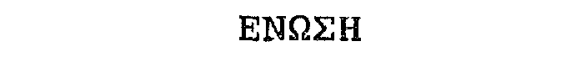 \\
\hline PROTOCOL & 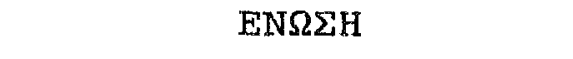 \\
\hline MARPOL $\cdot 78$ & $\mathrm{EN} \Omega \Sigma \mathrm{H}$ \\
\hline
\end{tabular}

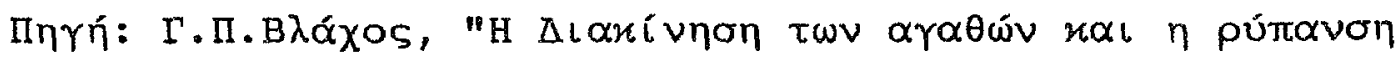

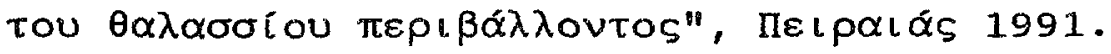




\section{RYPREEIE}

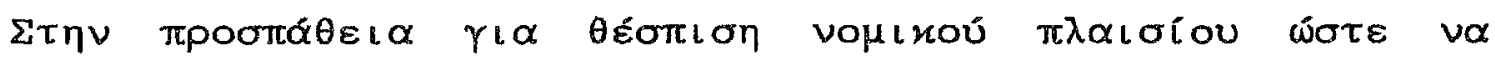

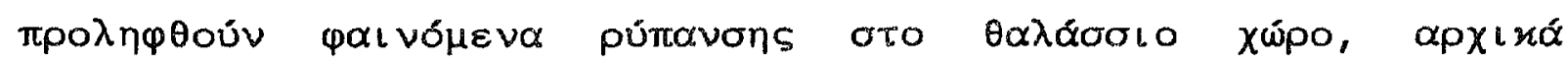

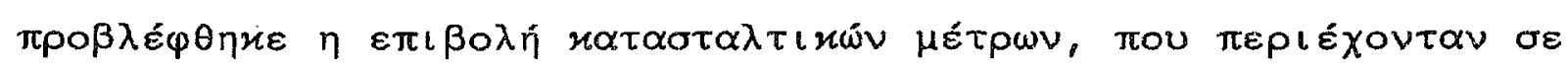

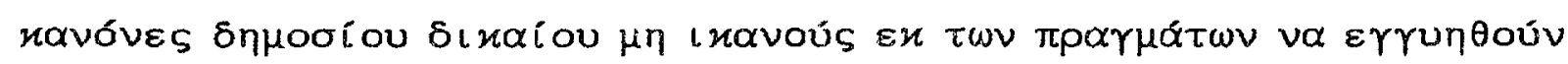

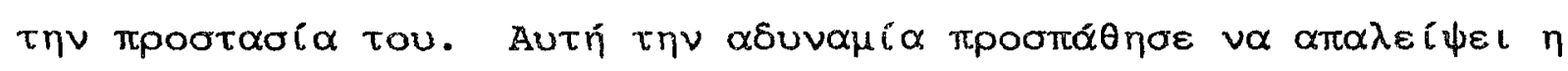

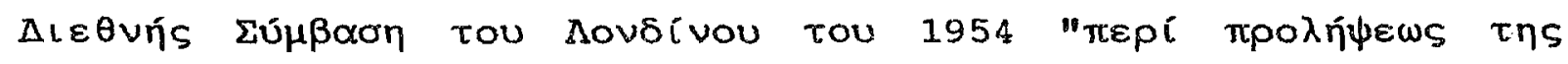

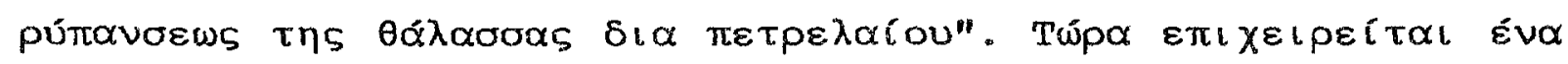

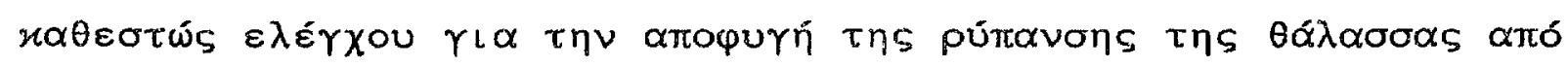

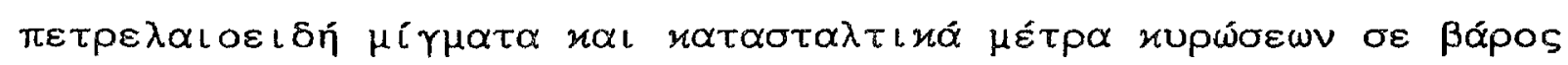

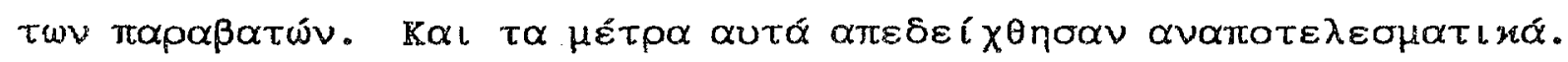

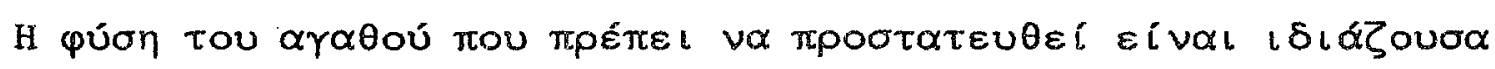

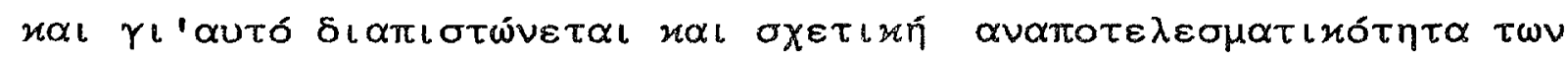

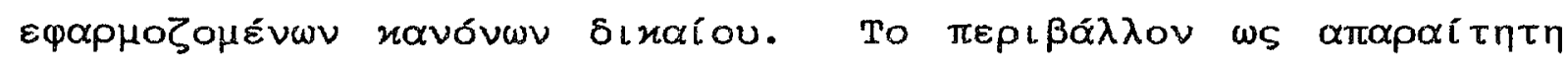

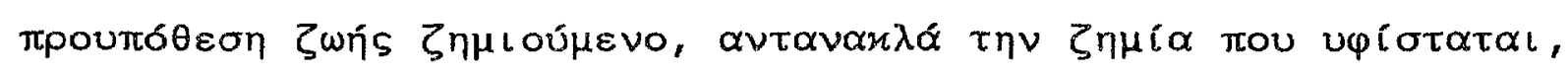

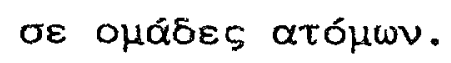

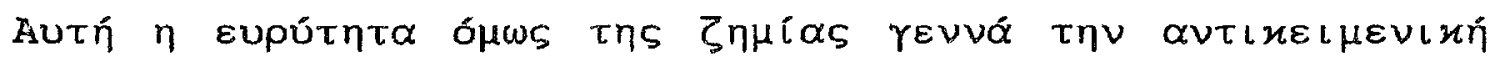

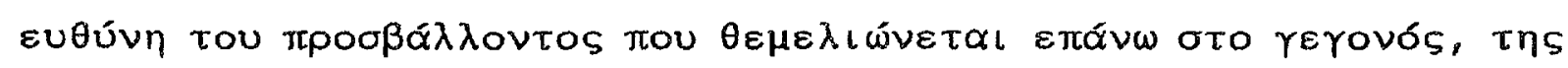

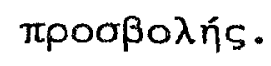




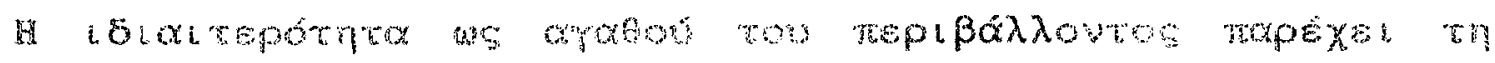

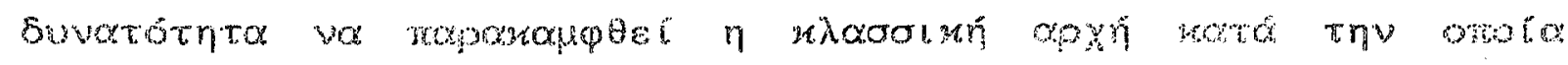

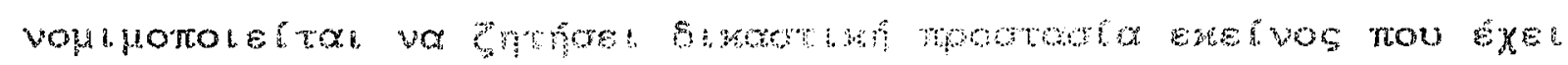

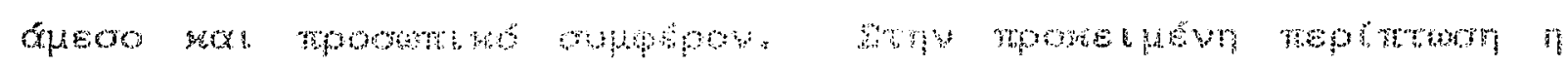

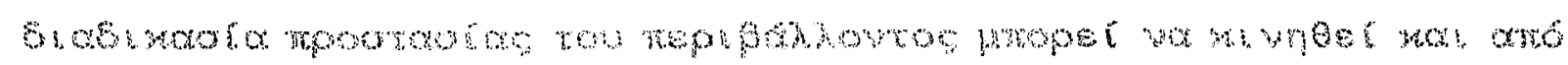

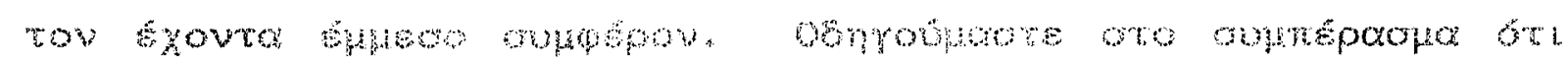

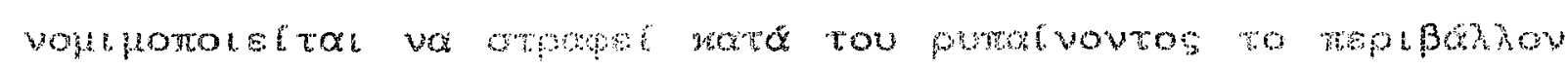

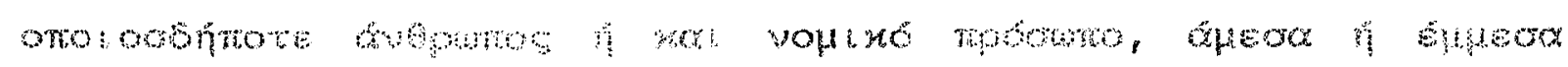

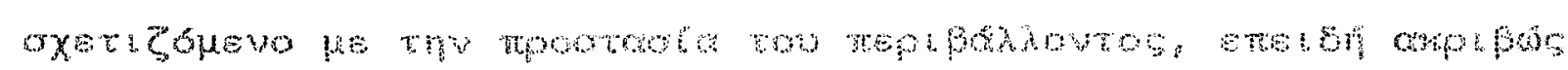

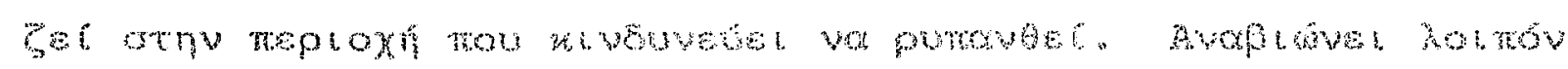

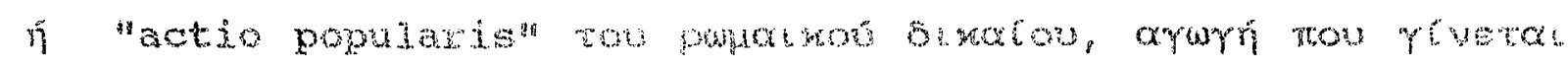

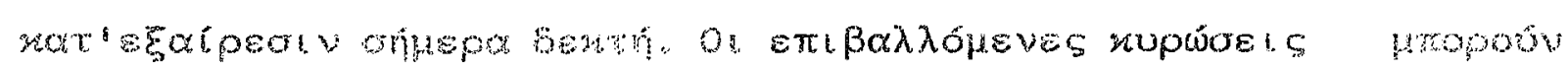

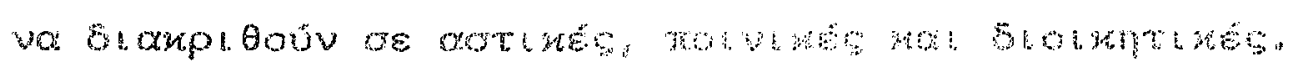

\section{AOTLRES}

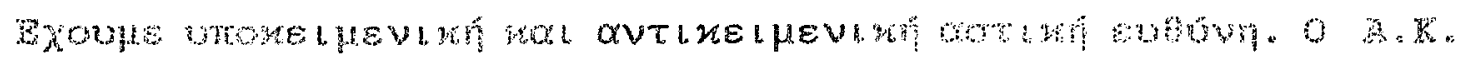

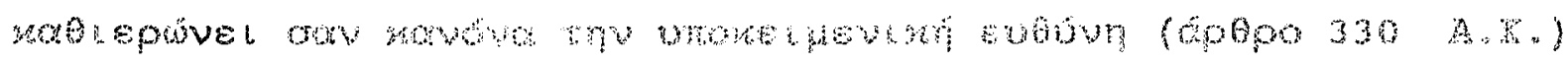

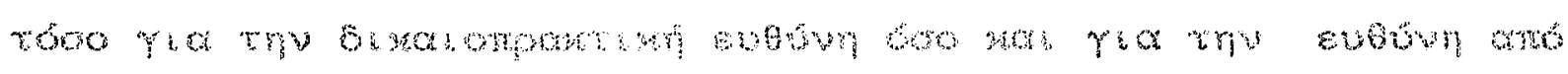

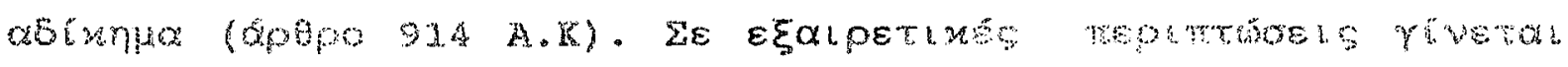

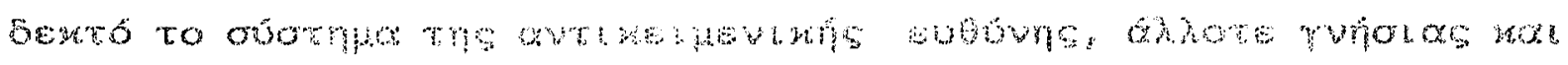

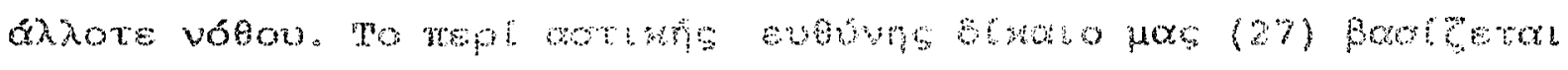

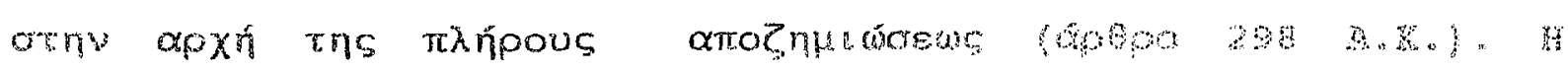

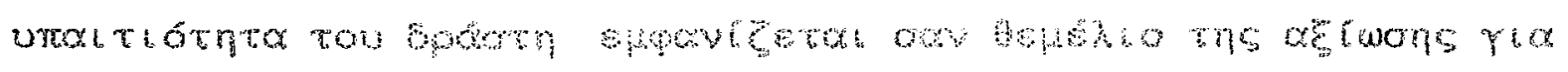




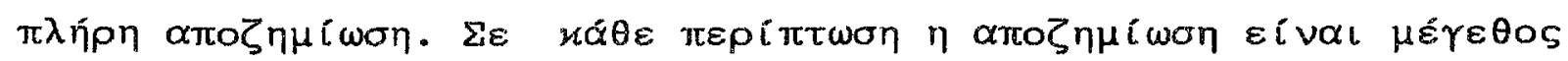

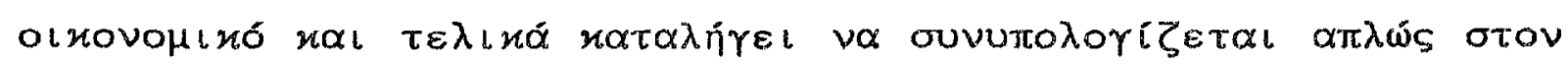

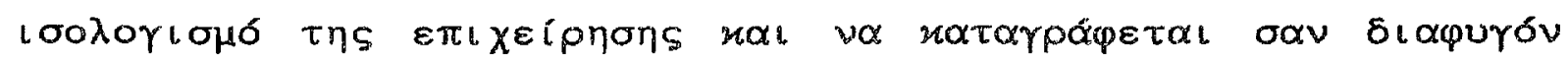

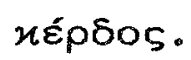

\section{חоㄴำés}

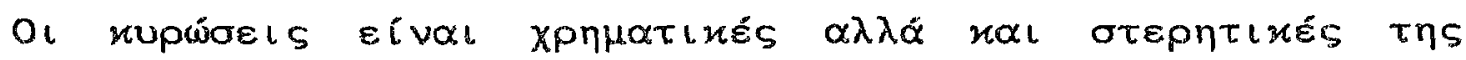

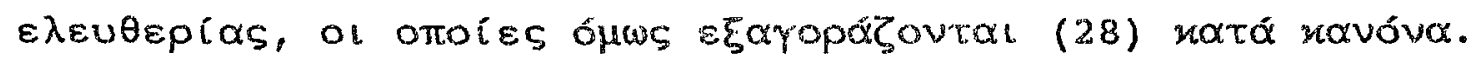

\section{DLOLKnTlués}

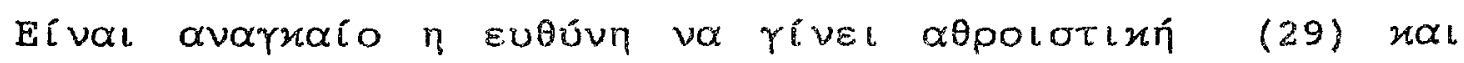

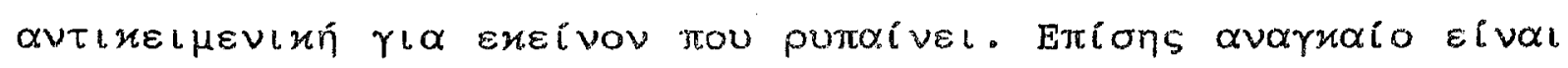

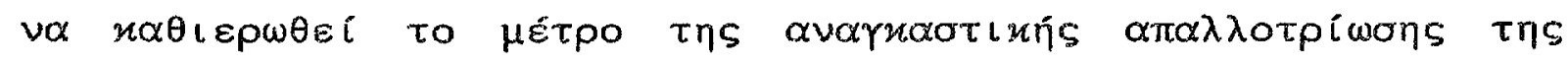

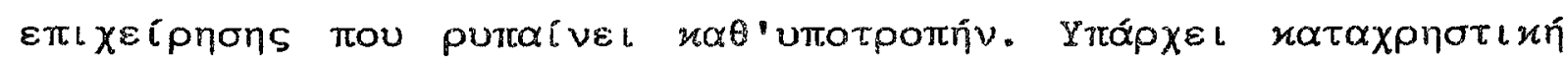

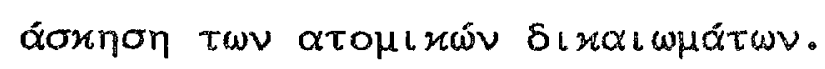




\section{SHMEIREEIS}

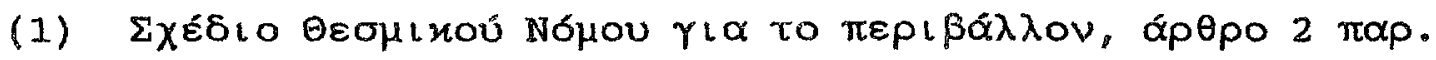

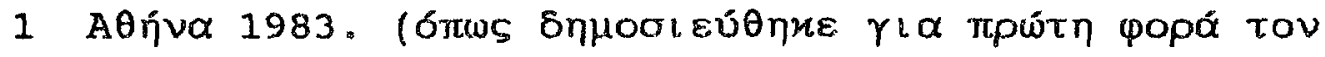

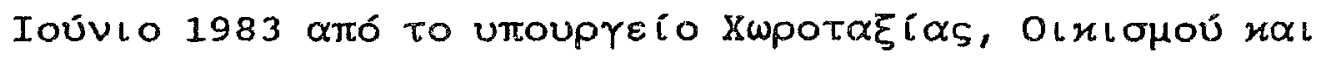
$\Pi \varepsilon p(\beta \alpha ́ \lambda \lambda \circ \vee \tau \circ \varsigma)$.

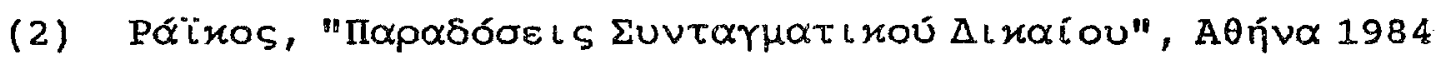
( Tónos B' teúxos $\mathrm{A}^{\prime}$ ).

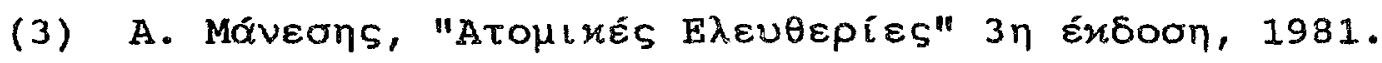

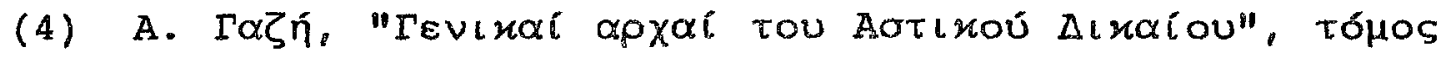
BI, 1973 .

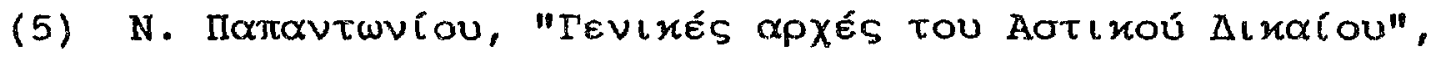
1983.

(6) Aтó 


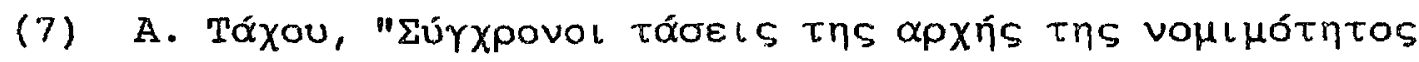

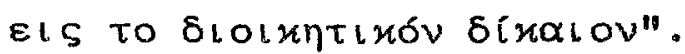

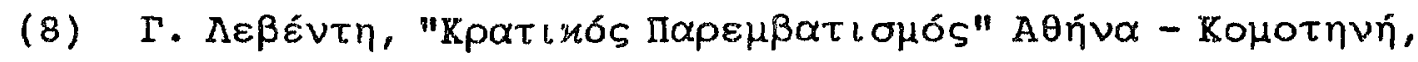
1982 .

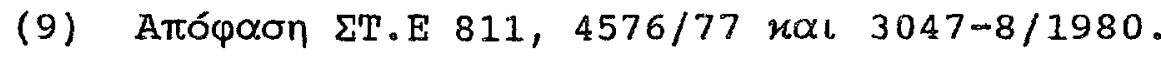

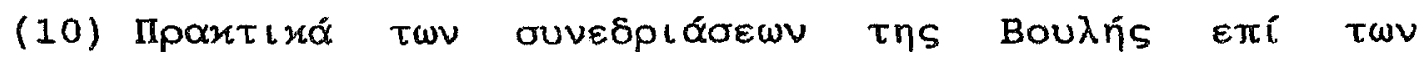

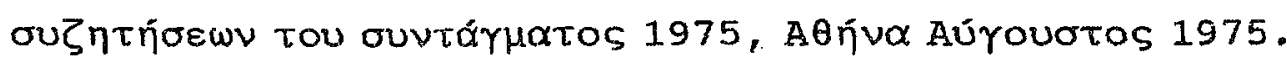

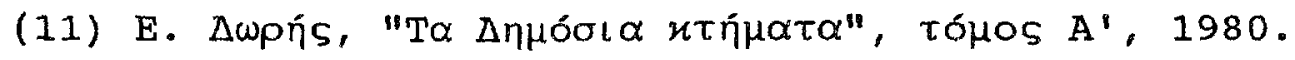

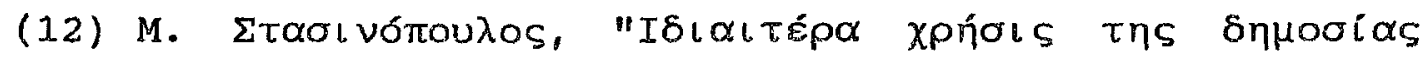

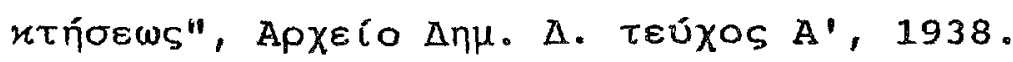

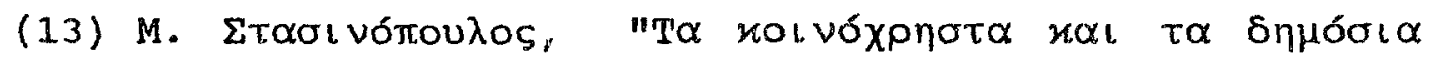

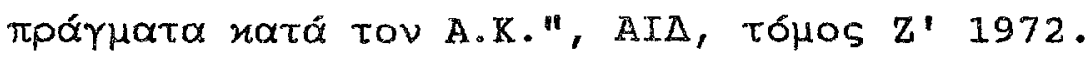

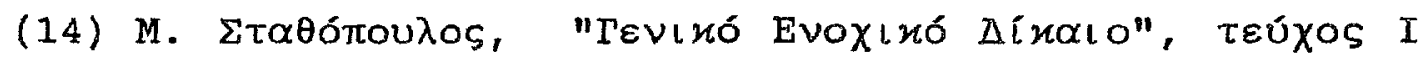
1978, teúxOS II 1983. 


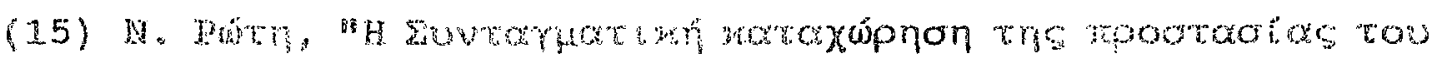

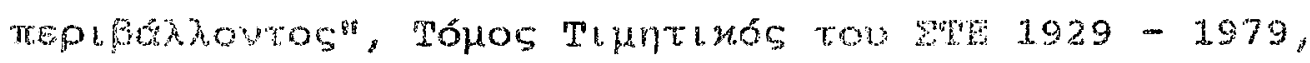

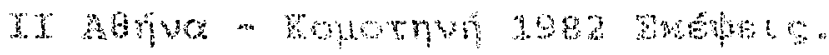

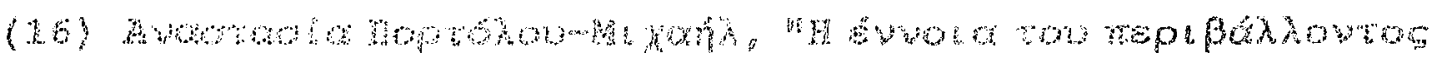

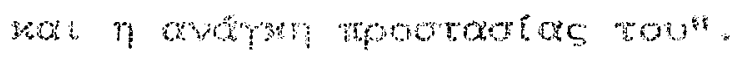

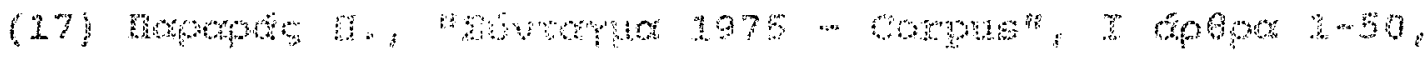
sh

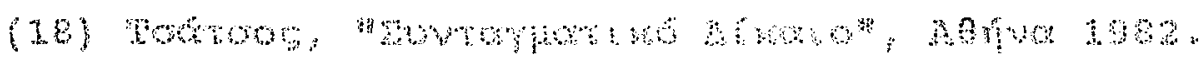

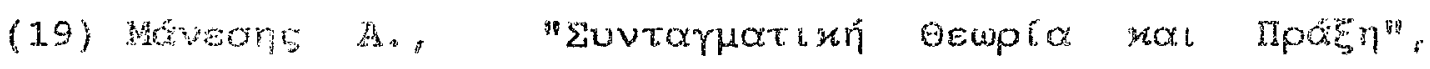
exoctionten 1980

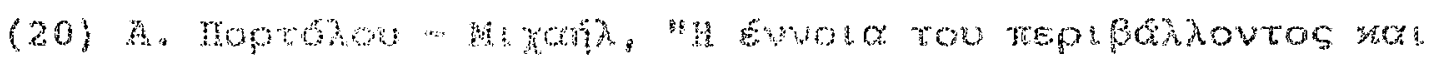
1 owhm Tporachs rov"

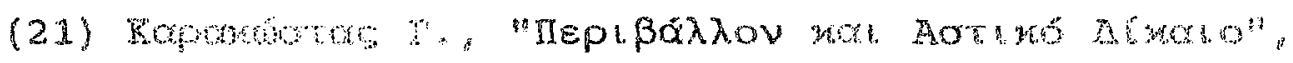

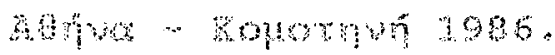

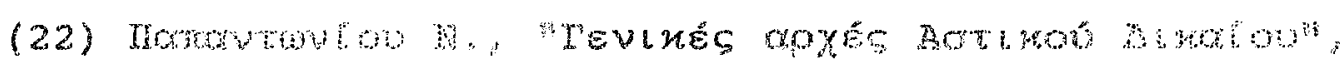
Agvo 1800 


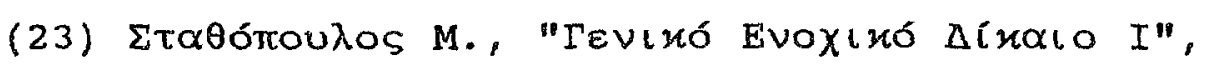
A日ñua 1982 .

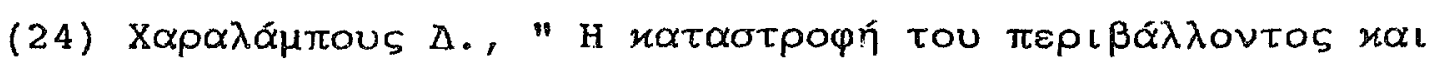

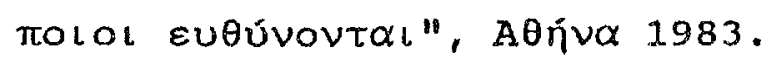

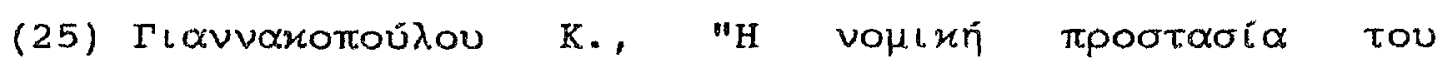

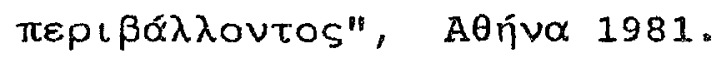

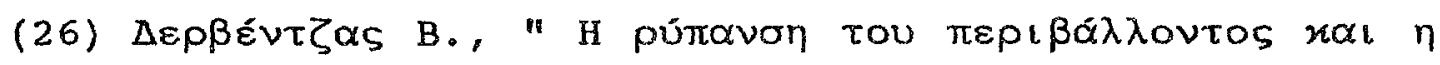

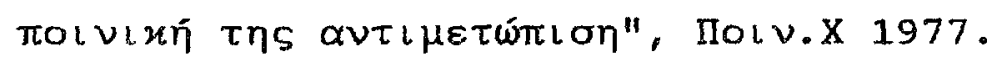

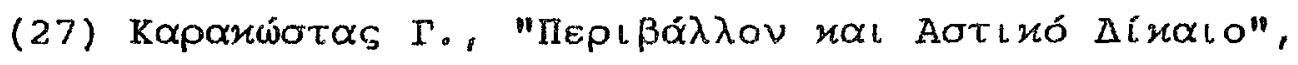

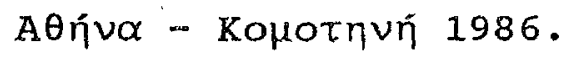

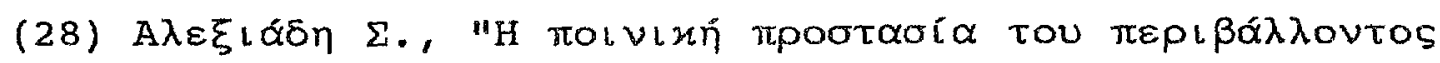

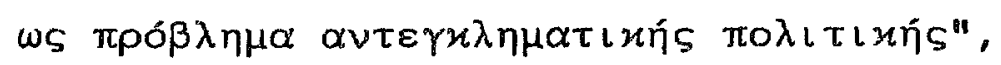

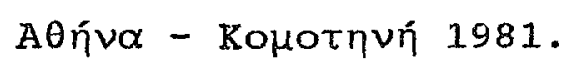

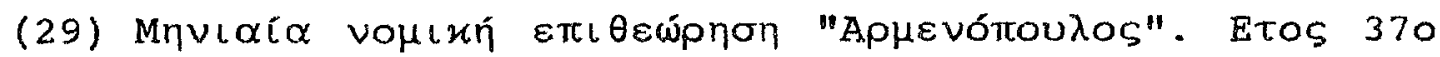
TEúxos Iavoudpíou 1991. 
EYMIIEPASMATA - MPOTASEIE

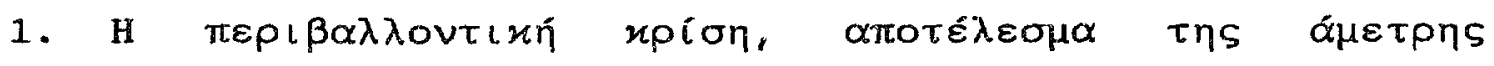

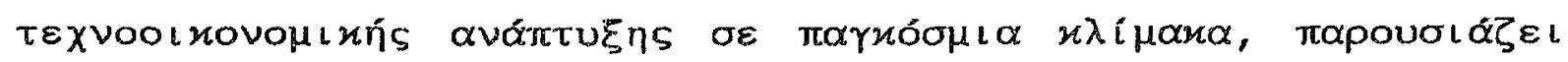

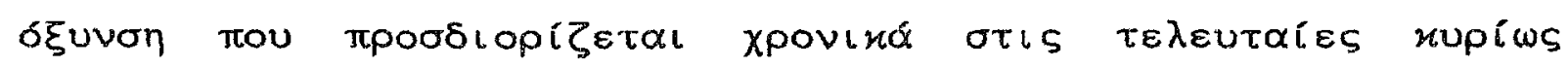

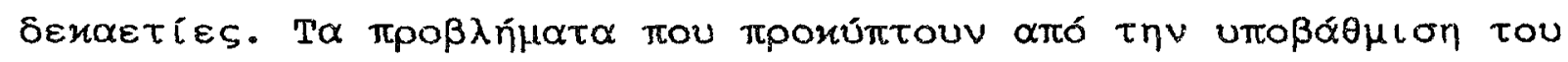

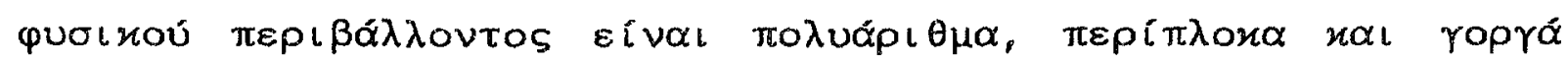

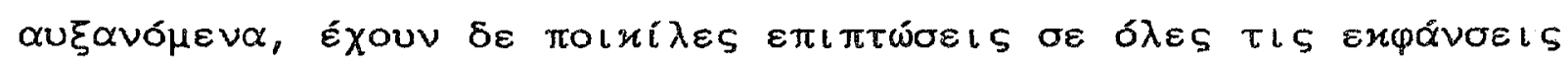

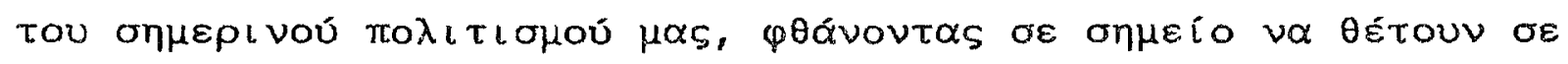

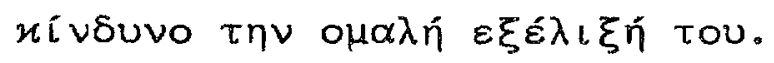

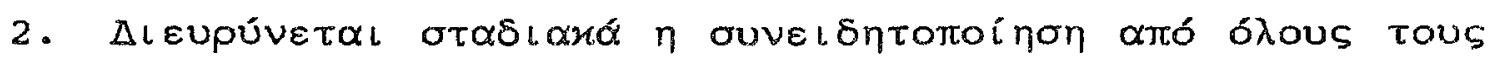

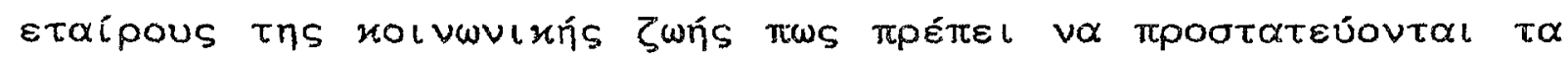

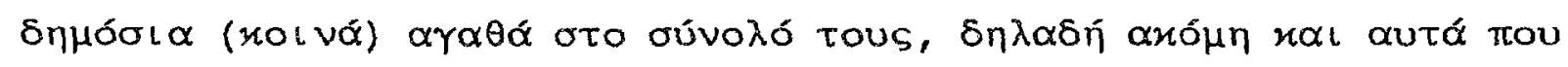

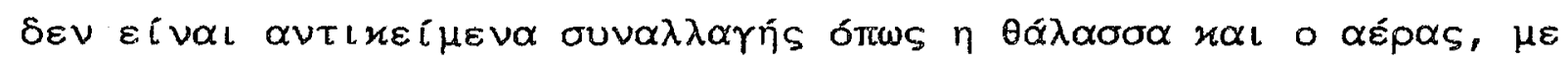

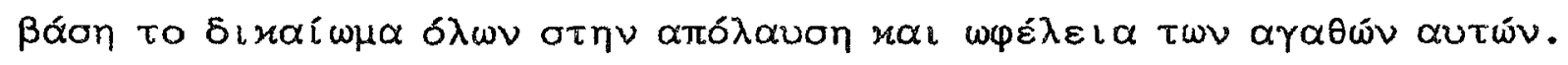

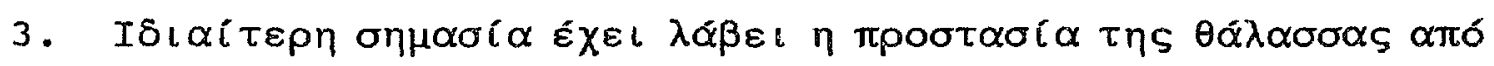

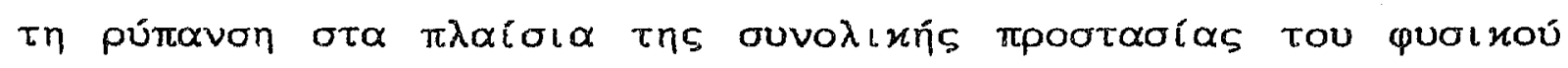

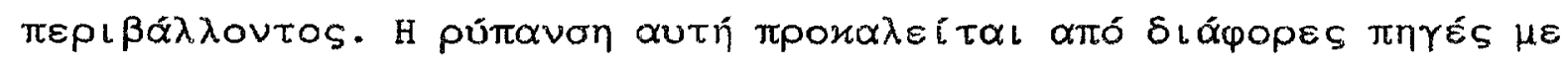




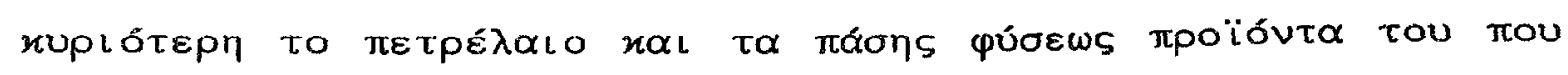

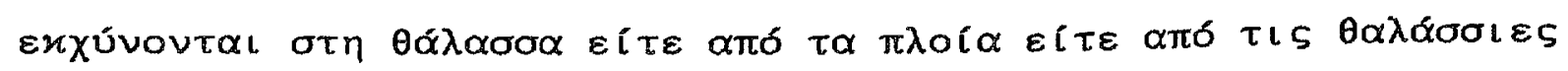

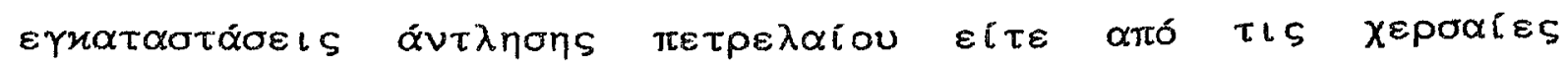

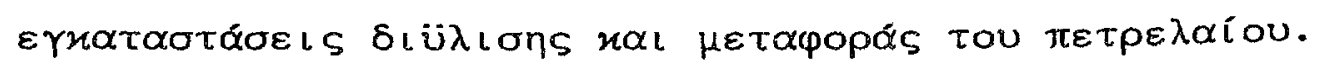

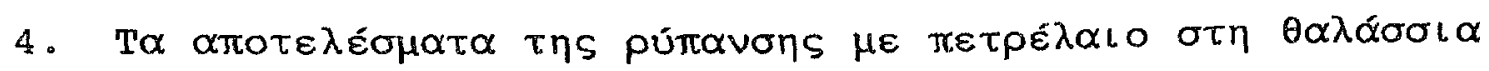

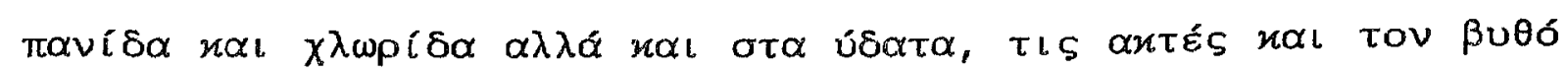

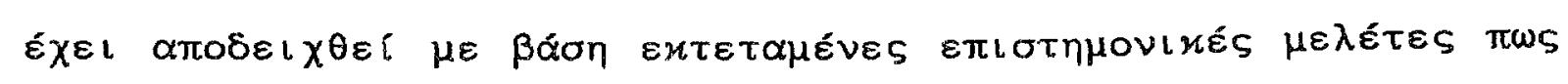

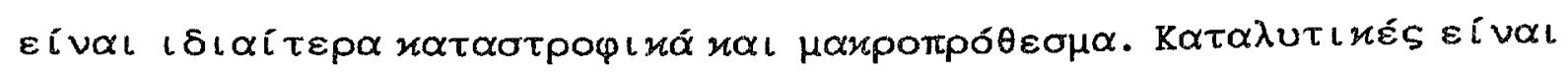

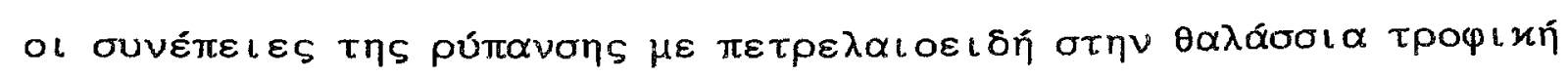

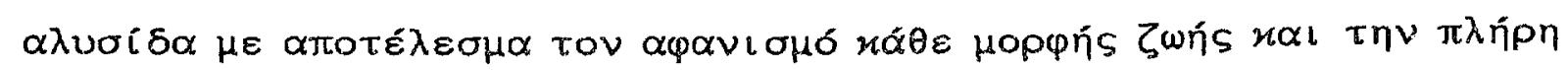

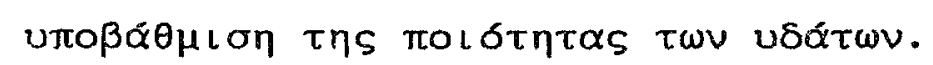

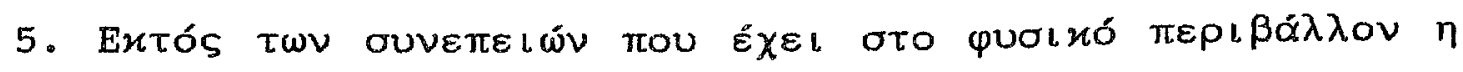

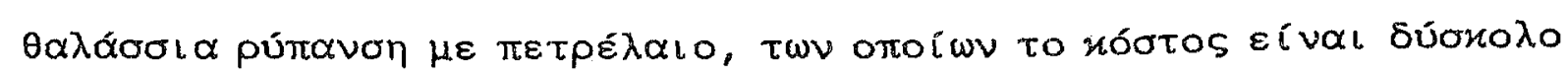

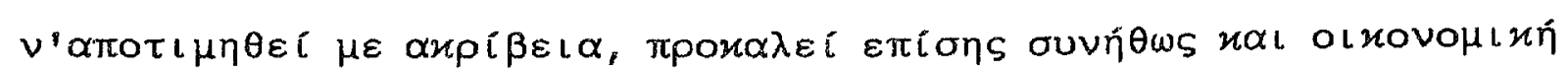

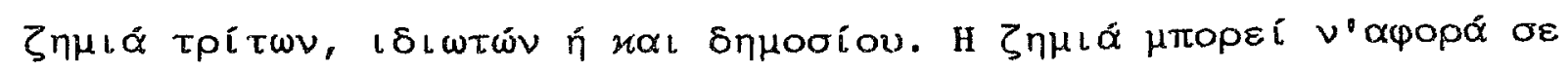

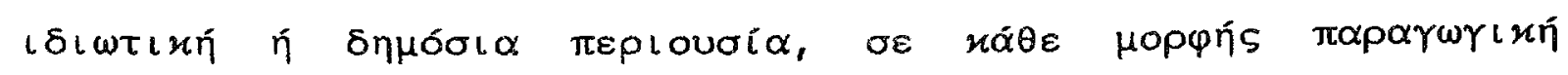

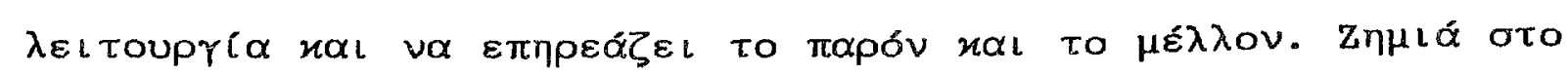

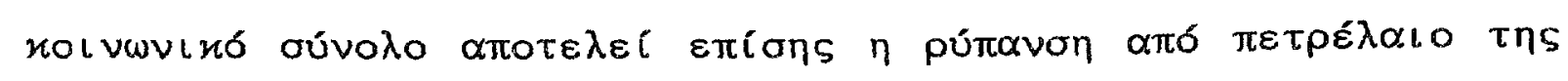

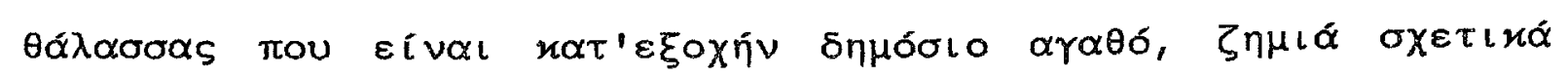

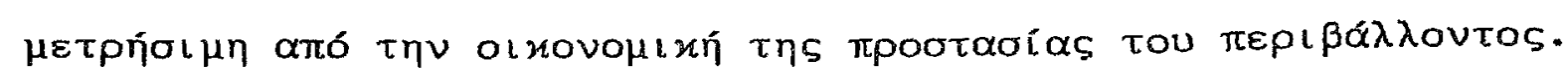




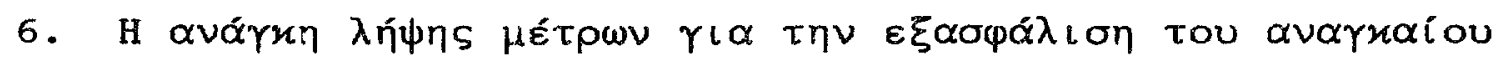

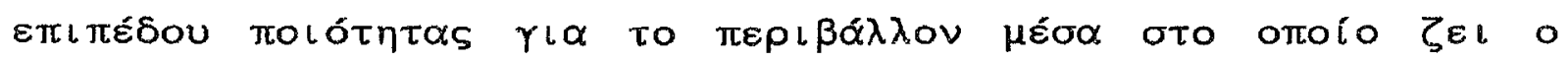

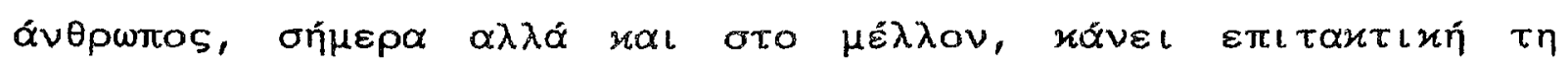

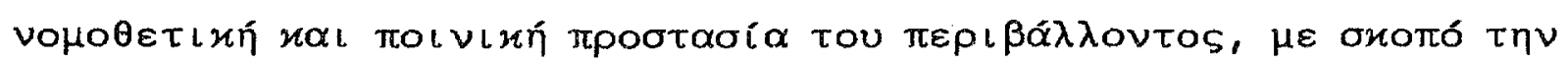

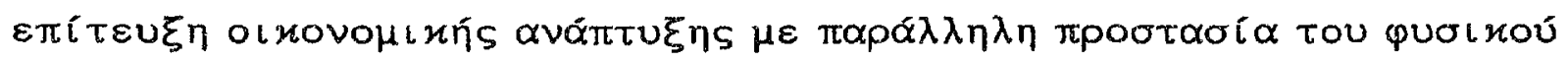

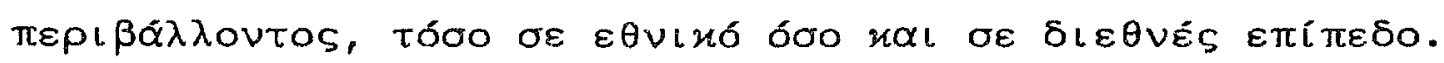

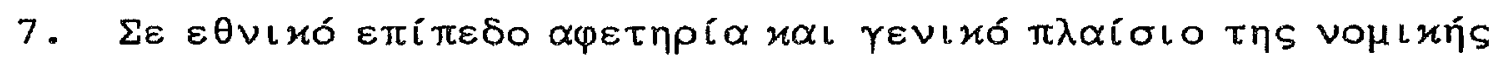

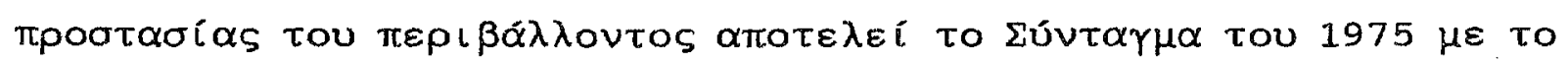

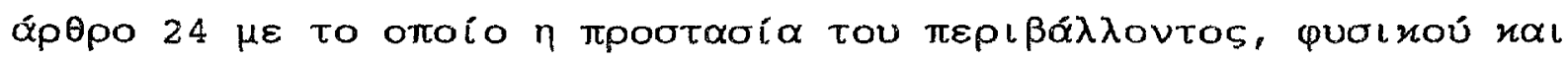

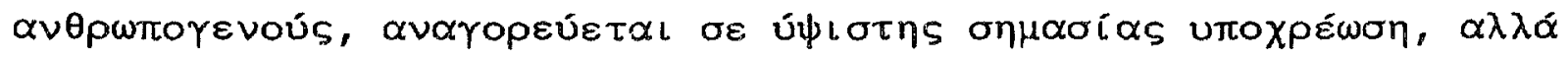

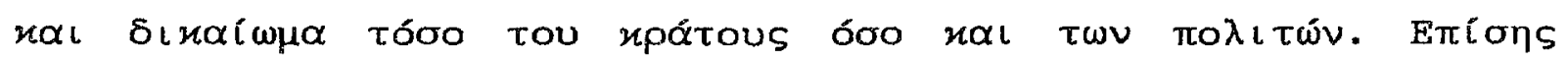

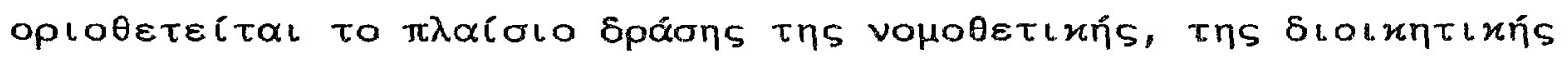

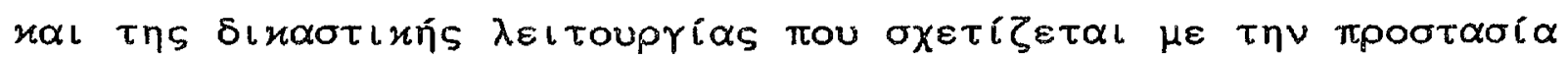

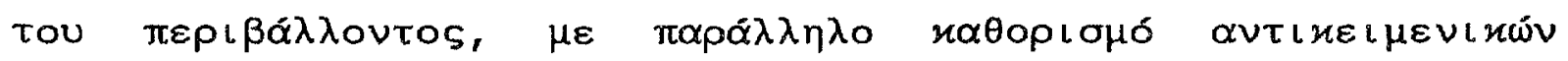

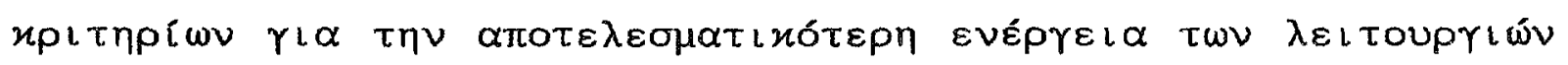

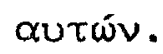

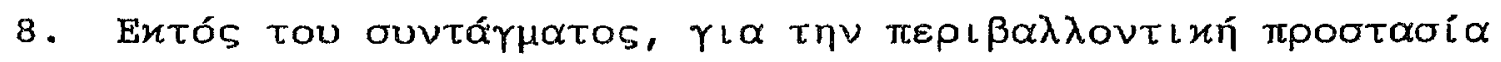

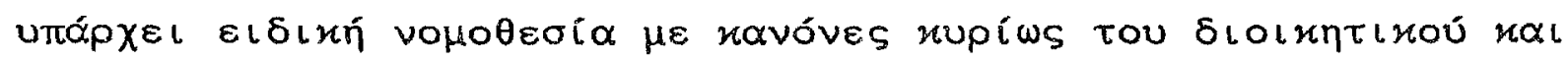

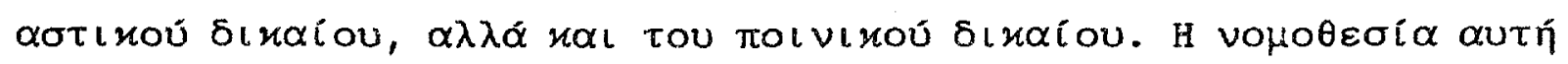

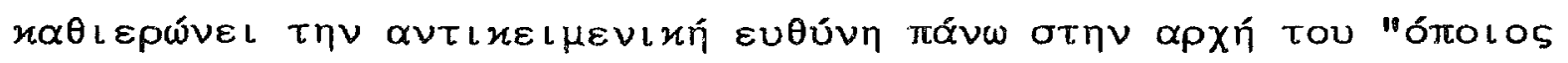




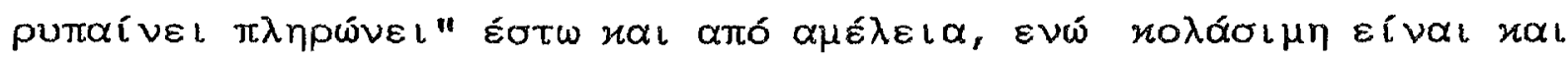

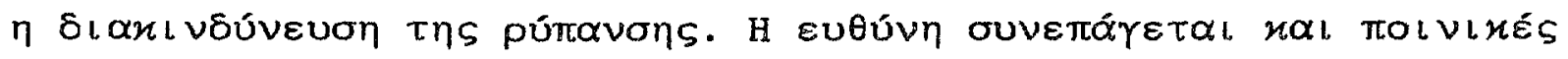

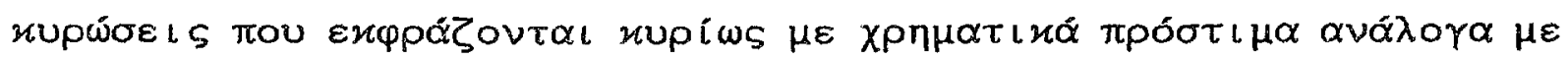

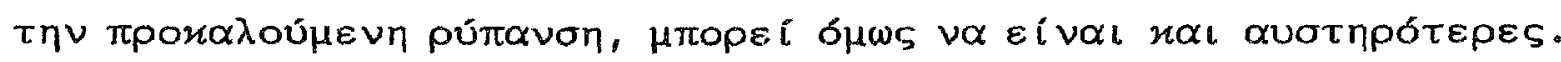

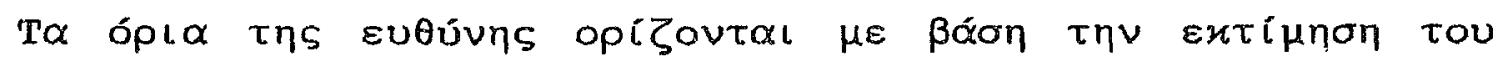

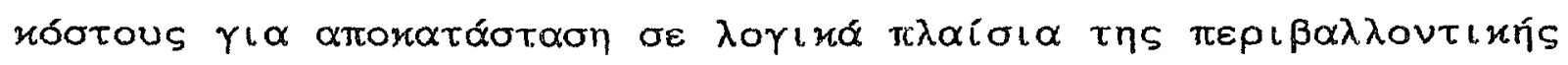

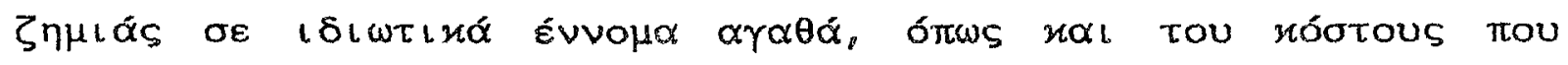

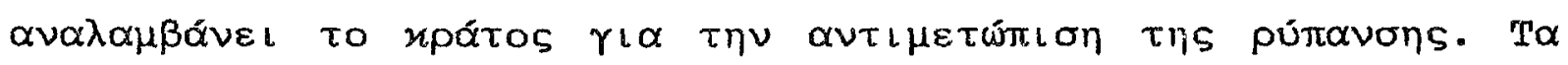

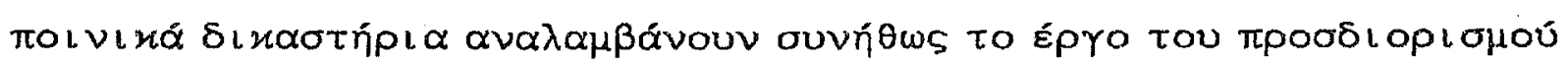

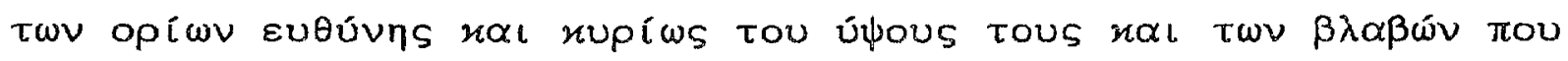

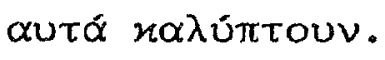

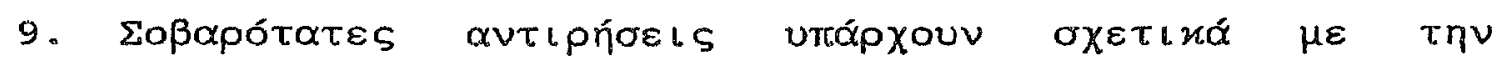

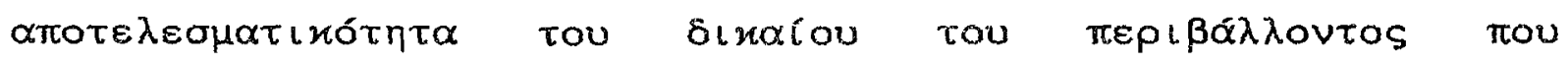

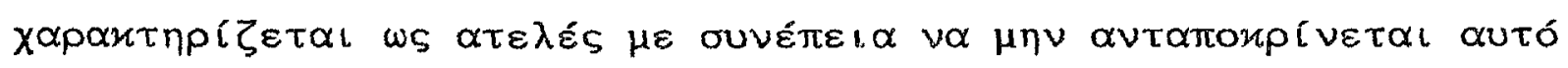

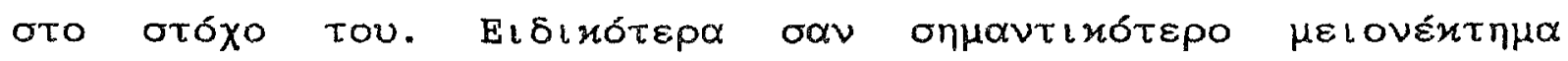

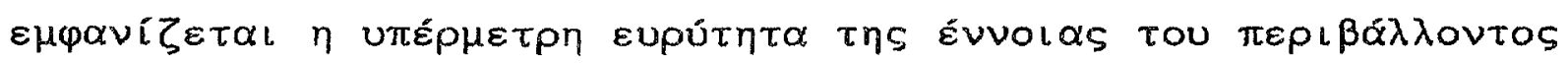

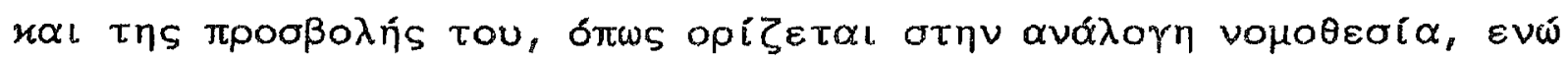

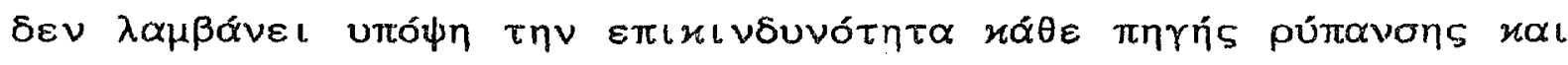

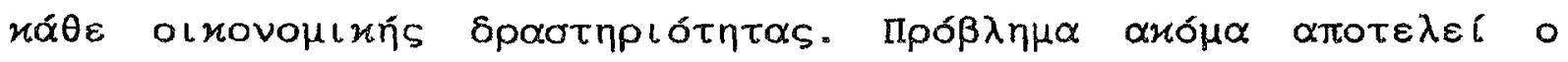

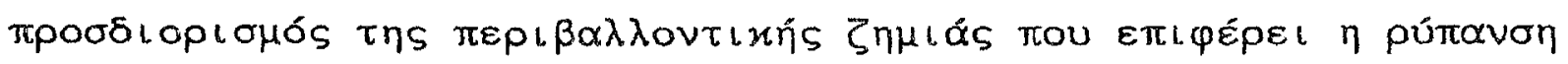

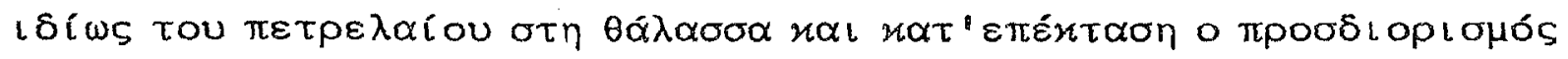




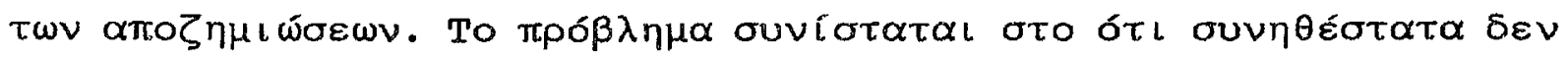

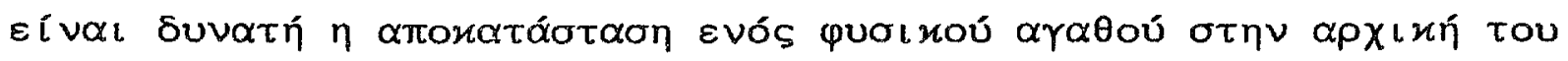

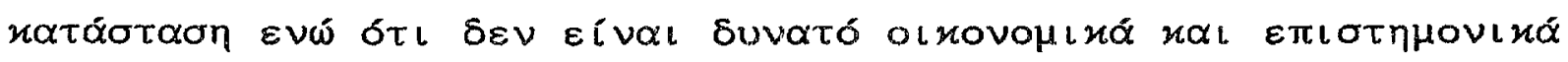

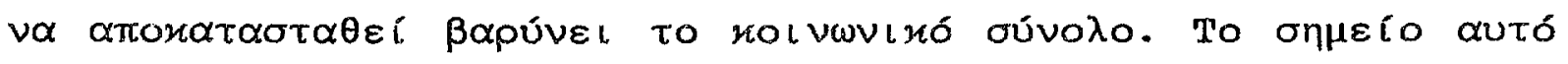

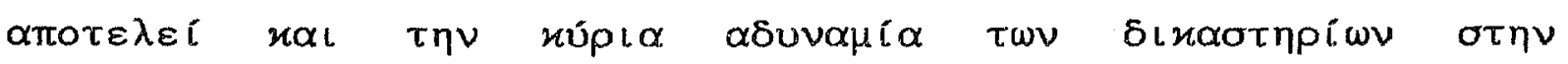

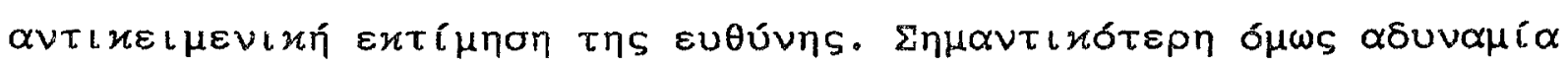

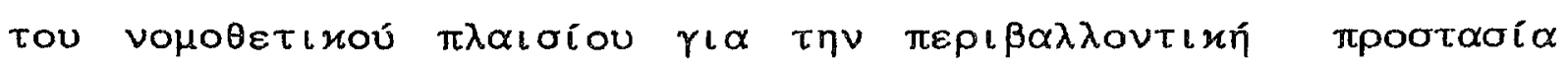

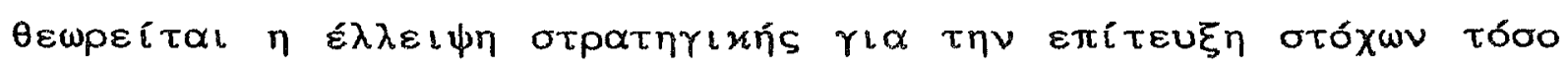

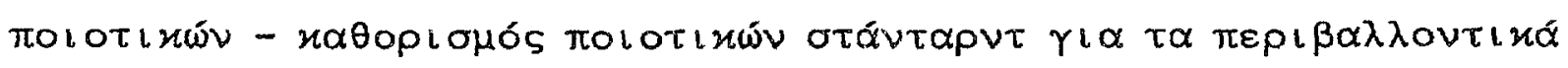

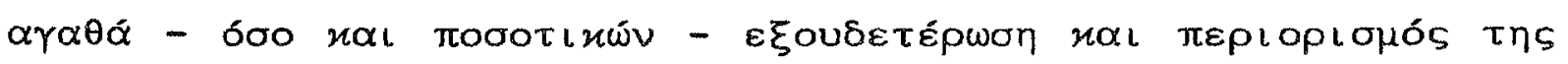

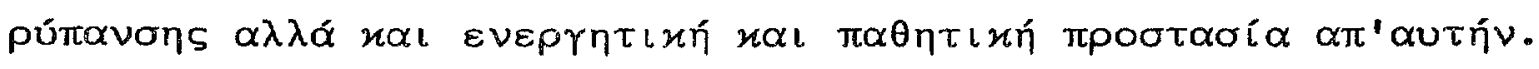

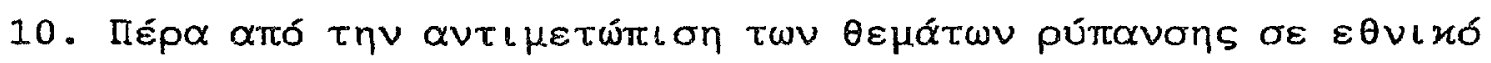

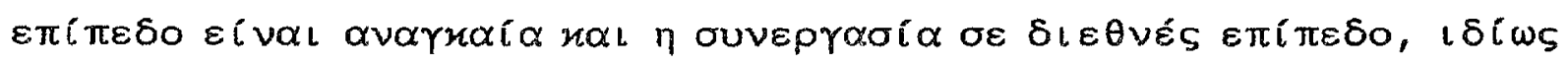

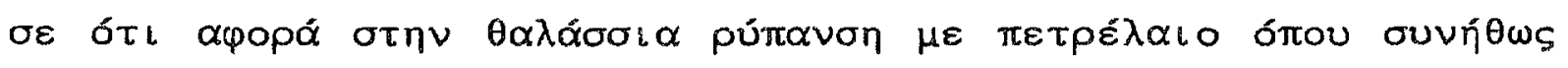

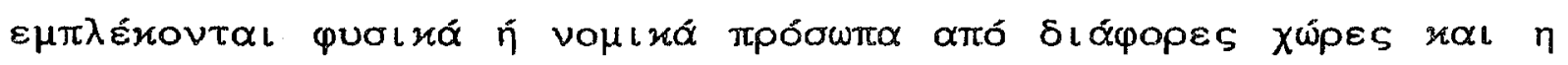

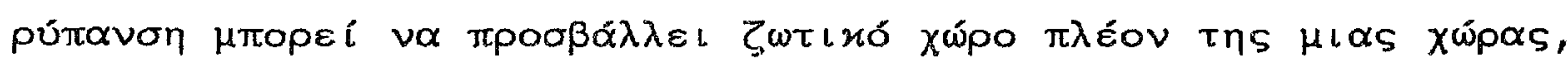

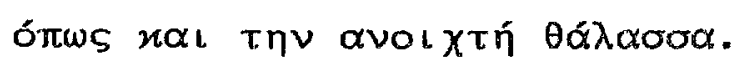

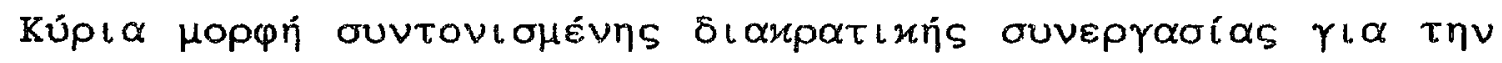

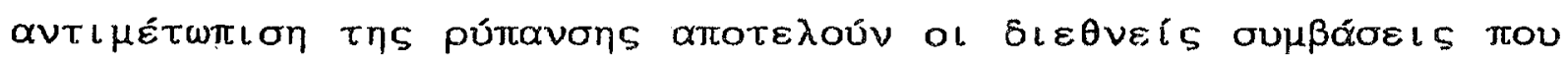

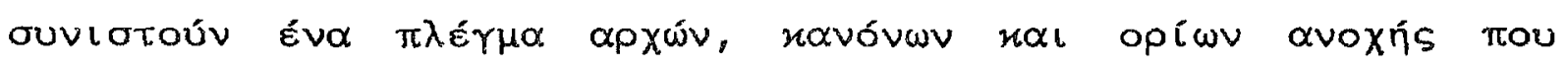

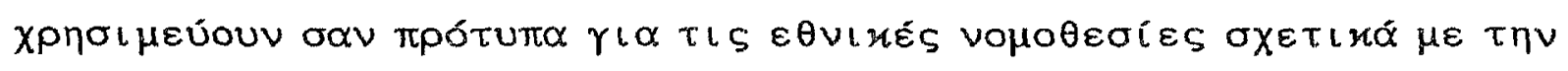

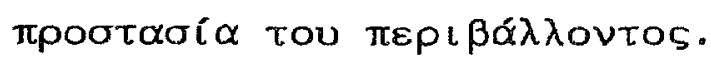




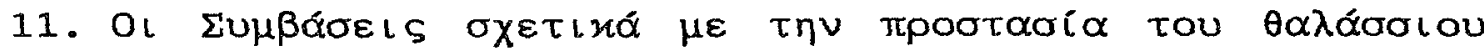

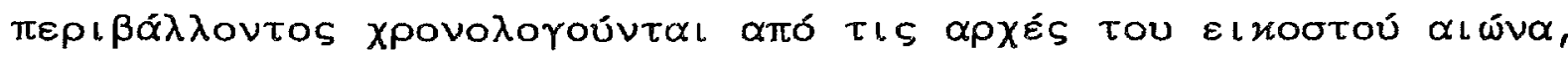

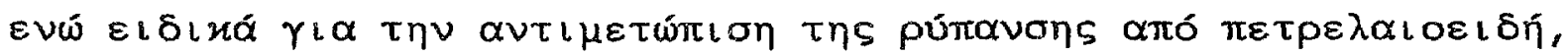

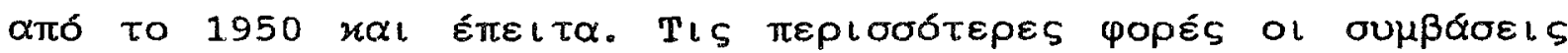

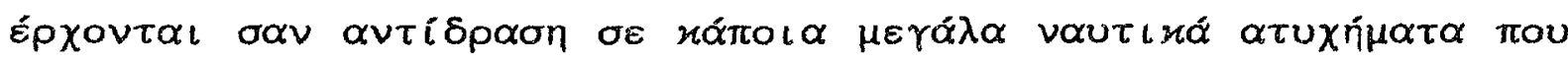

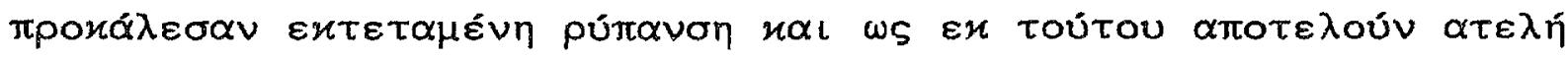

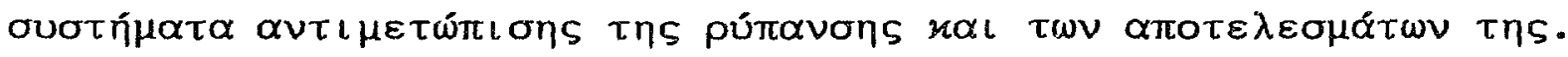

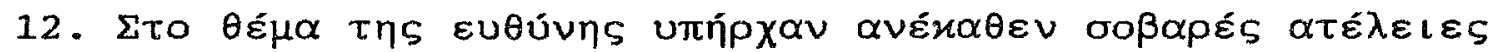

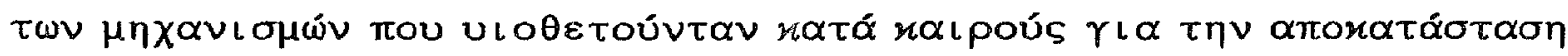

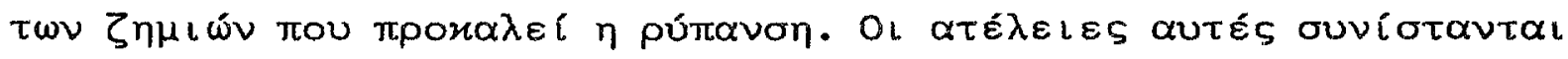

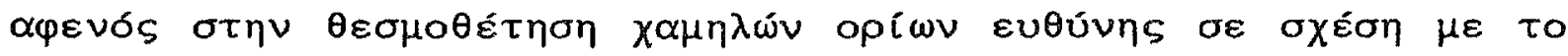

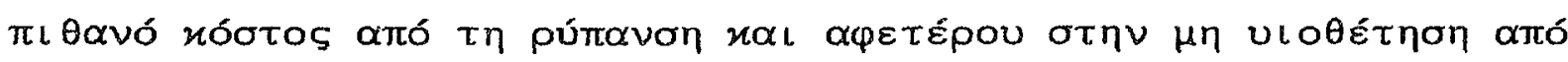

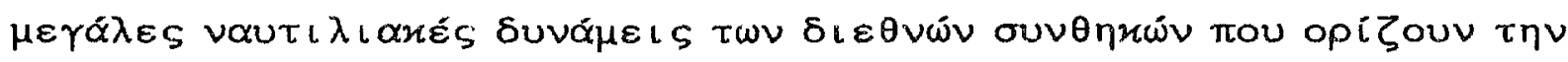

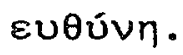

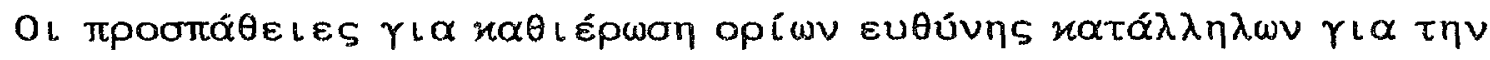

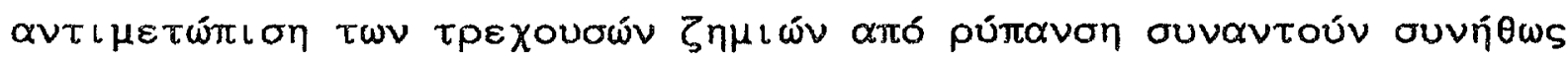

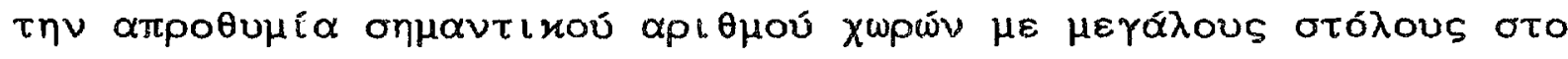

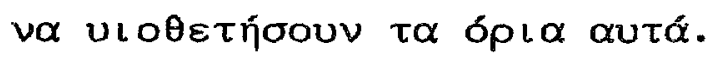

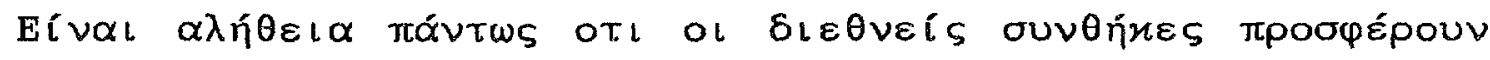

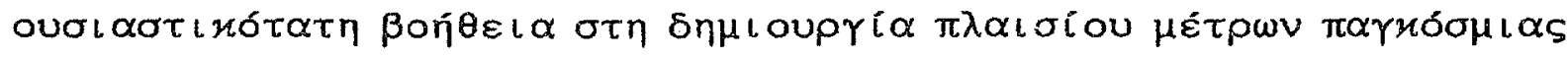

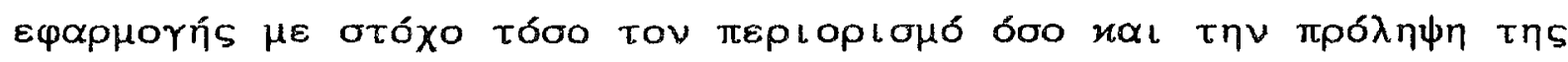

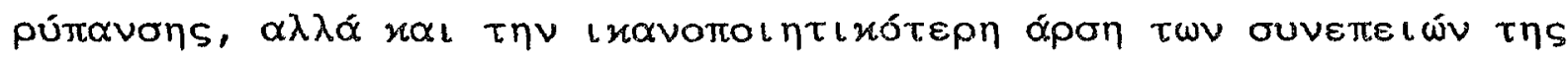

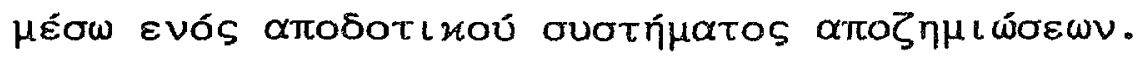




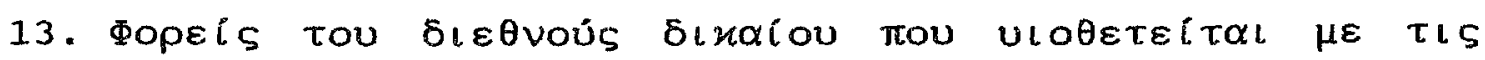

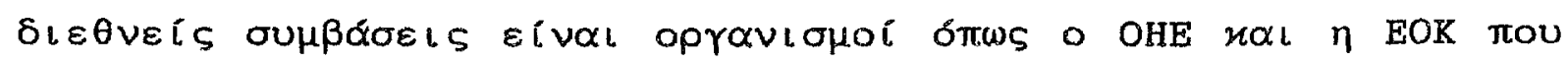

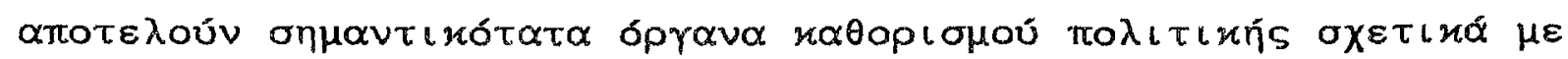

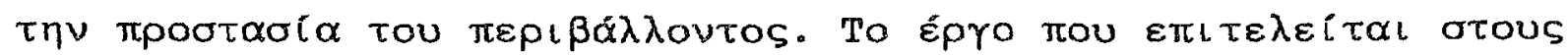

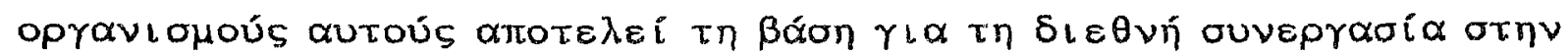

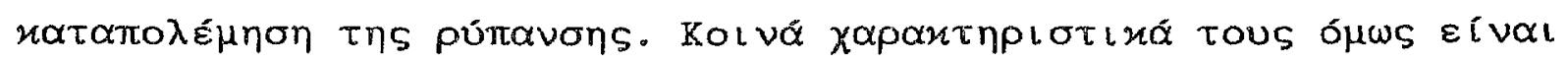

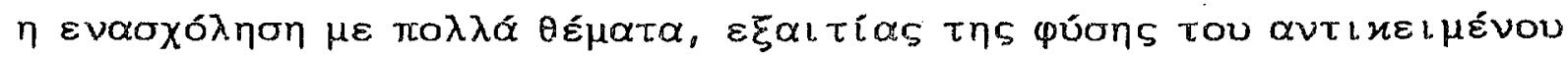

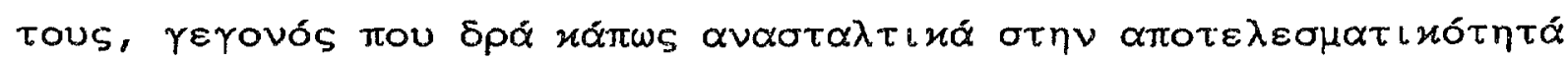

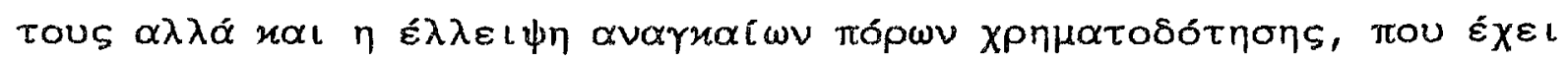
$\alpha \cup \alpha ́ \lambda \circ \gamma \alpha \alpha \pi \circ \tau \varepsilon \varepsilon \varepsilon \sigma \mu \alpha \tau \alpha$.

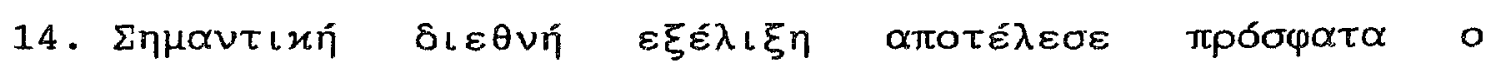

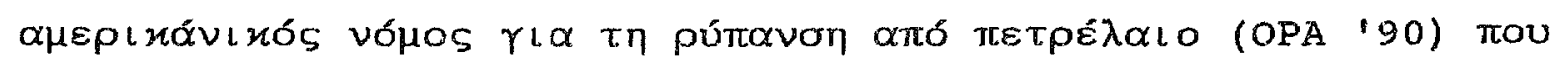

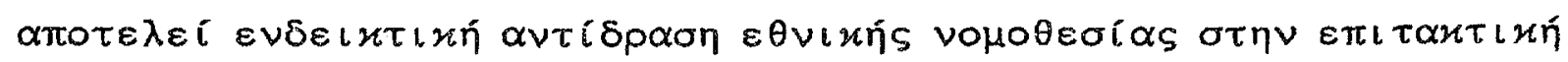

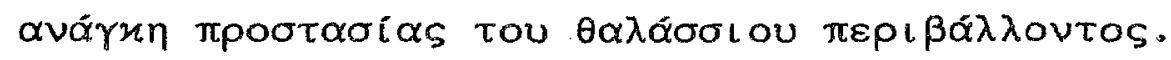

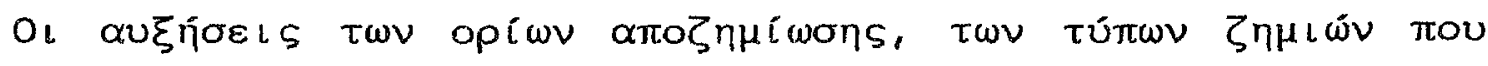

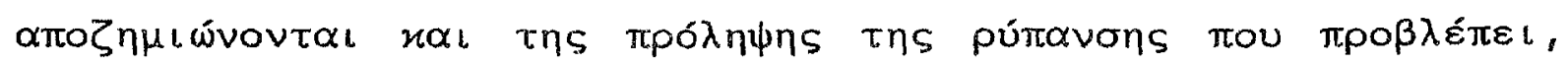

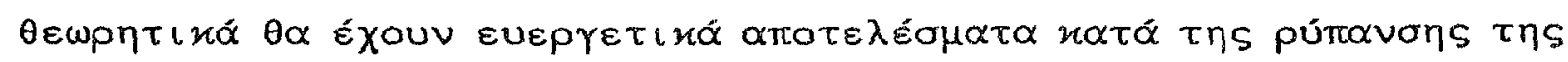

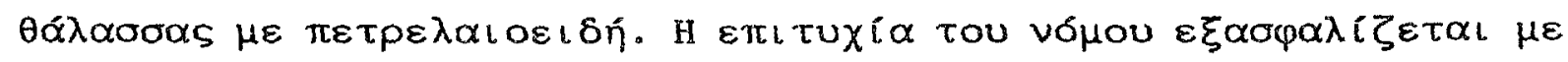

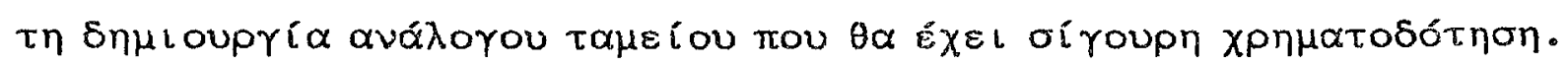

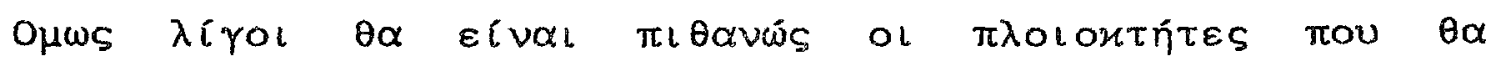

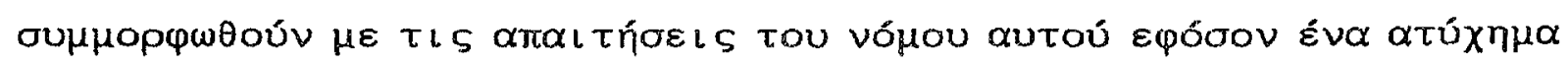




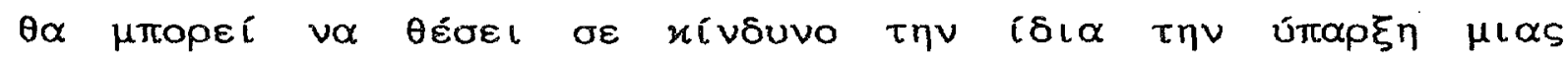

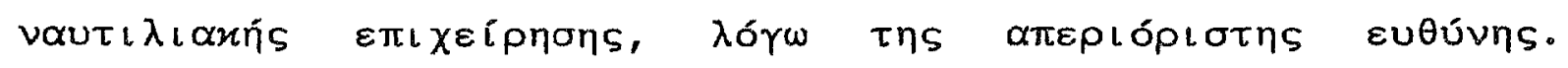

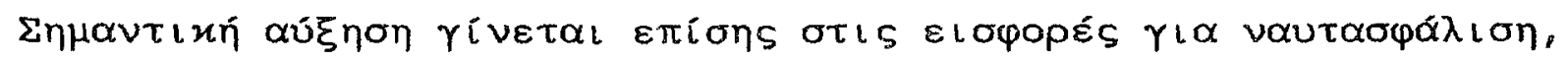

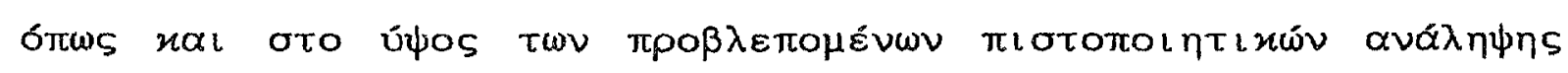

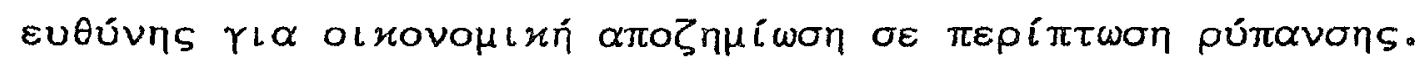

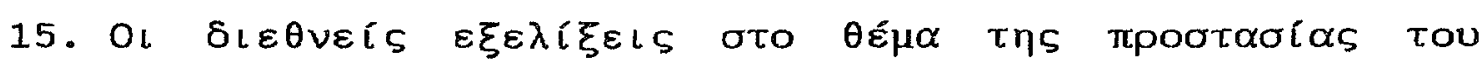

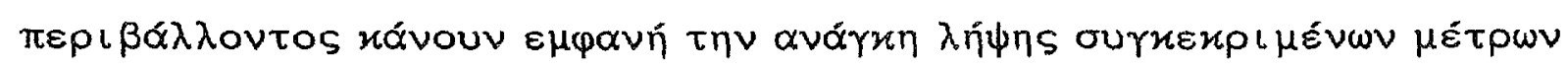

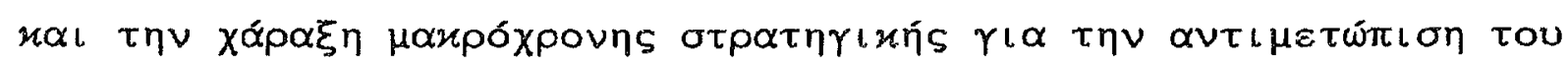

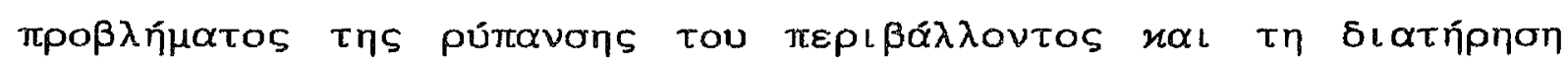

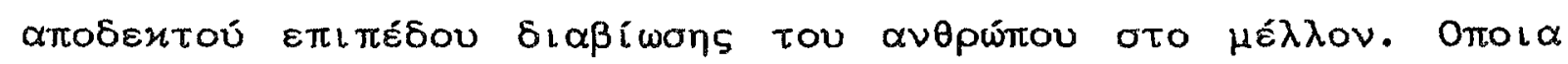

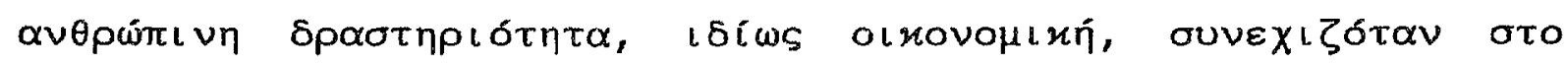

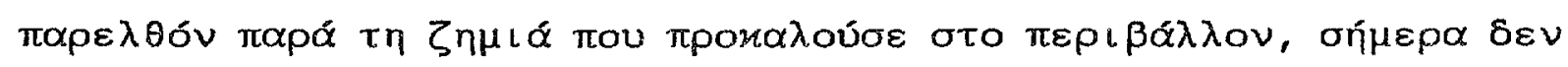

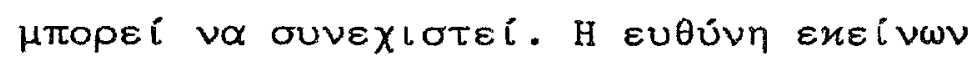

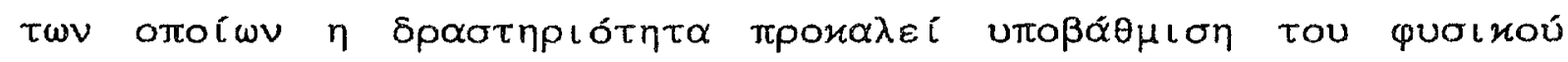

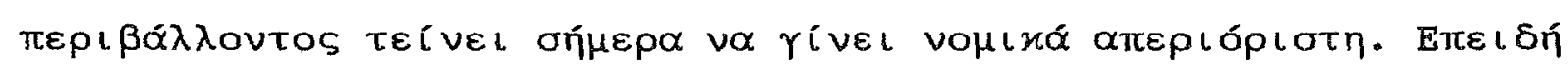

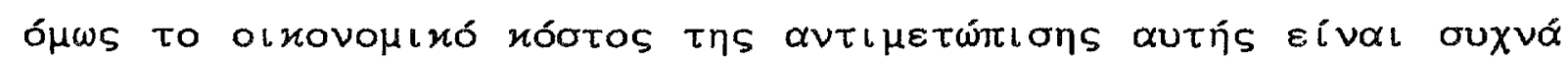

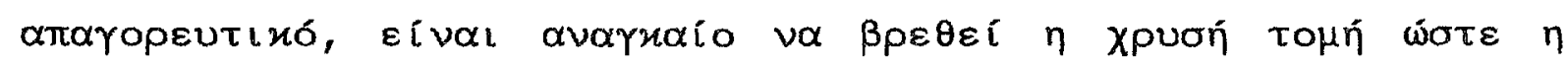

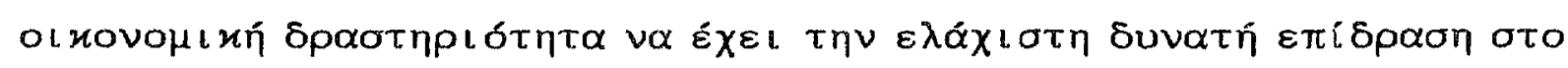

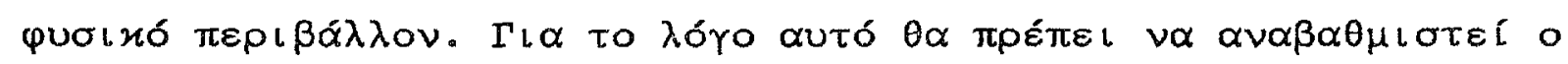

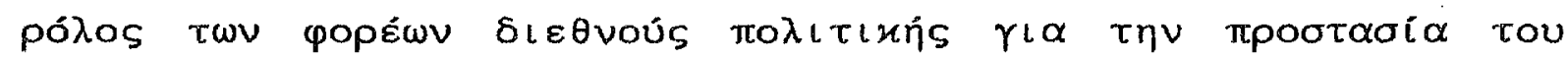

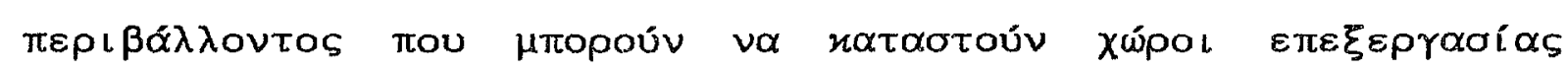

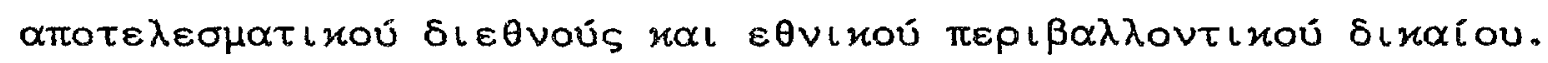




\section{EAAHNIKH BIBATOTPAФIA}

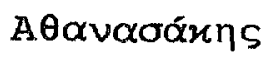

Kouooúpns $\theta$.

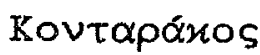

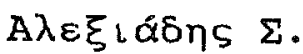

Avarvwotótrouגos A.

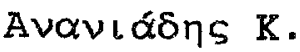

Avavıóôns K.

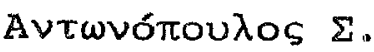

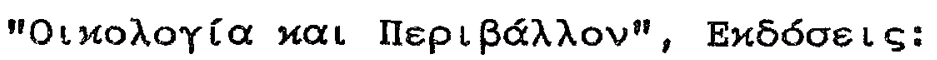

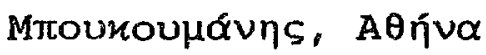

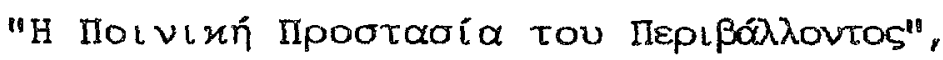
A $\theta \tilde{v} v \alpha 1981$.

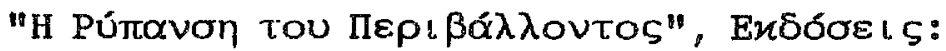

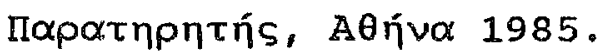

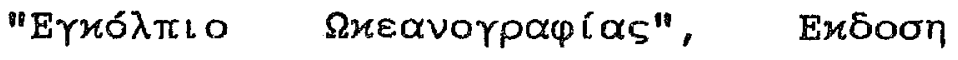

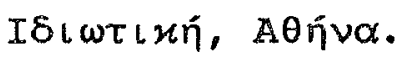

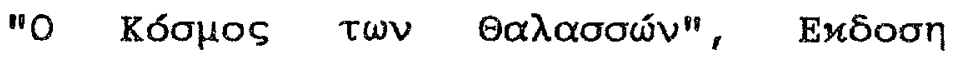

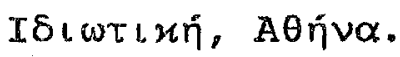

"Aitia nal Moppés Pútavons

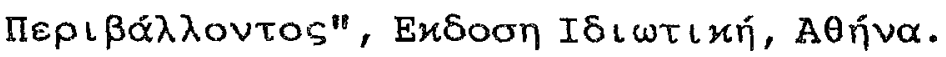




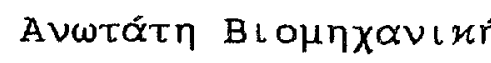

$\Sigma \chi \circ \lambda \eta ́ ~ \Pi \varepsilon\llcorner\rho \alpha \iota \omega ́ s$

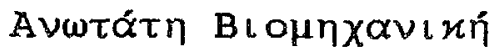

$\Sigma \chi \circ \lambda \eta َ ~ \Pi \varepsilon \iota p \alpha \iota \omega ́ s$

Aprupláōns A.

$B \alpha \sigma: \lambda\llcorner\varkappa \iota \omega ́ \tau \eta ⿻ \Sigma . \Gamma$.

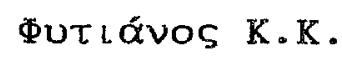

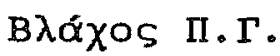

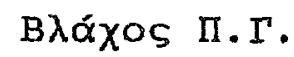

Bopóóxas B.X.

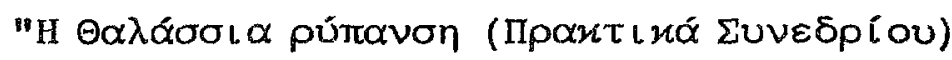

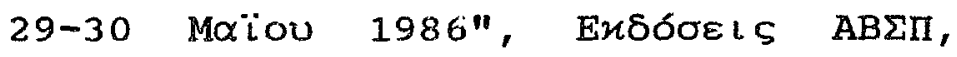

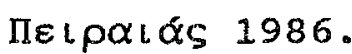

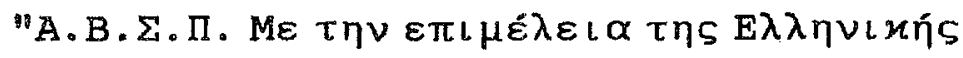

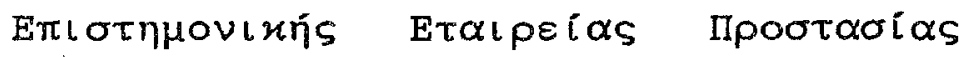

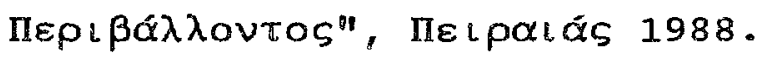

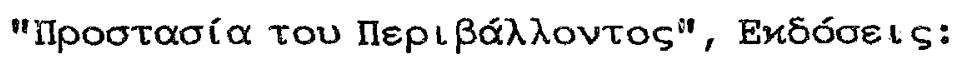

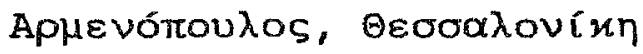

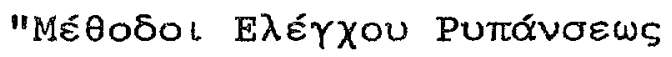

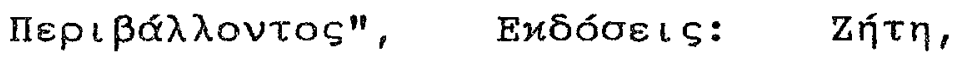

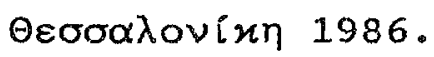

"H $\Delta \iota \alpha \varkappa[$ L

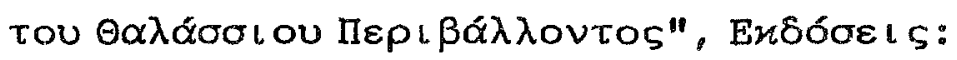

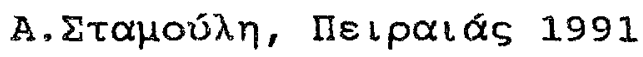

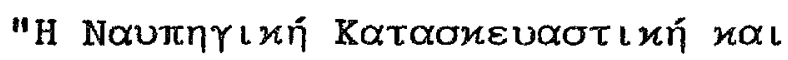

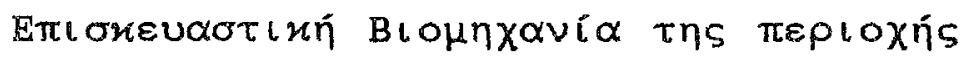

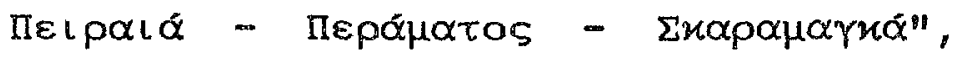

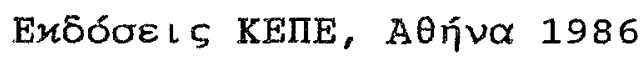




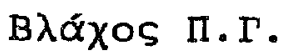

Bosquet $M$.

Bótons IIÉtpos

Bourtoúnas $\mathrm{K}$.

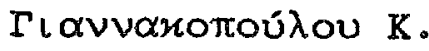

Crawford M.

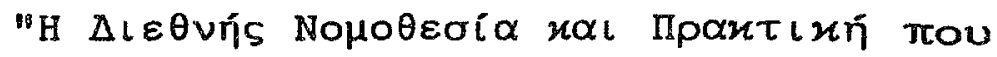

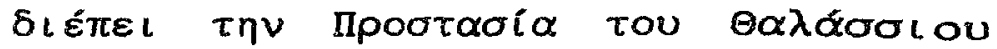

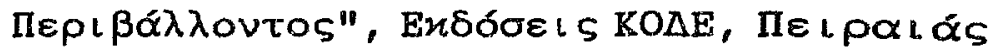
1991.

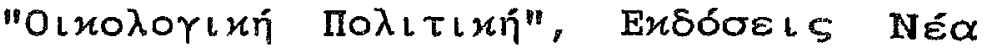

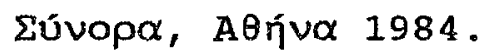

"Olnokoría Mónuvon nal Púmavan tou

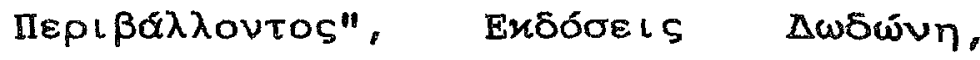

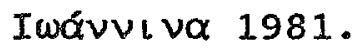

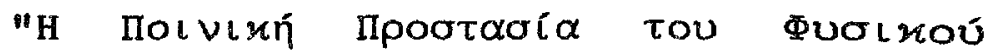

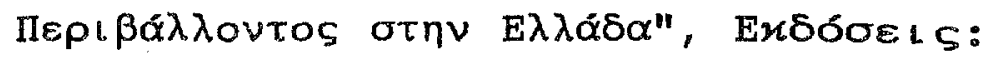

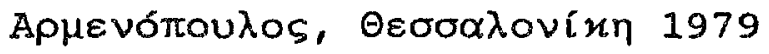

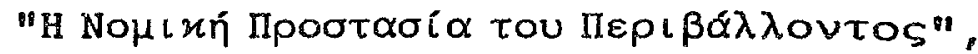
Aeñua 1981

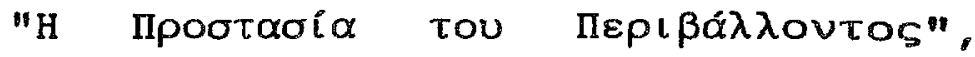

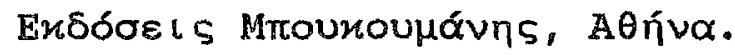


$\Delta \varepsilon \lambda$ oúnas A.N.

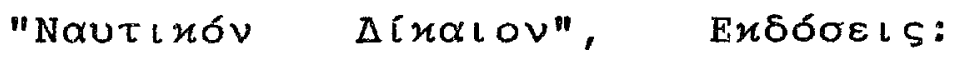

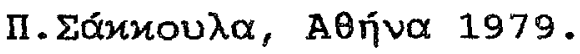

$\triangle \varepsilon \rho \beta \varepsilon ́ v \tau \zeta \propto S B$.

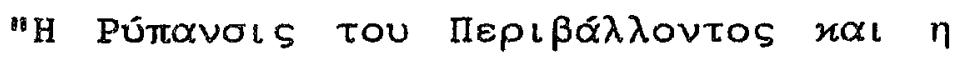

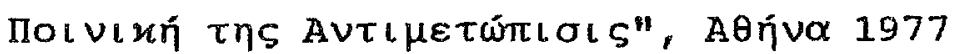

Ecologist

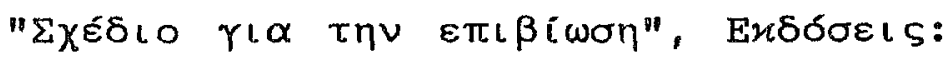

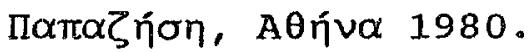

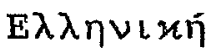

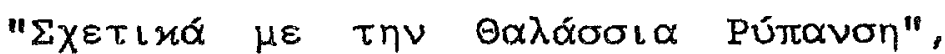

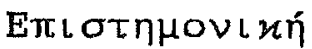

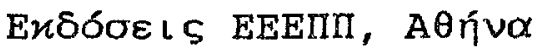

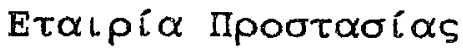

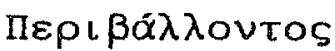

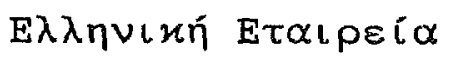

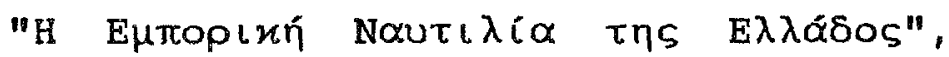

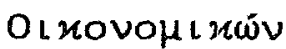

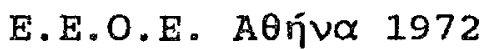

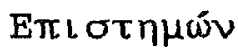

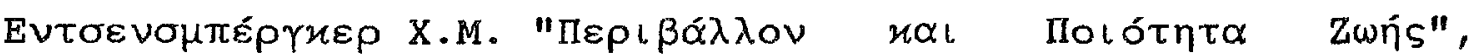

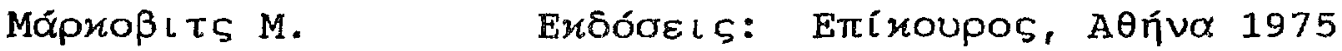

Гหорт $\mathrm{A}$. 
E.P.Y.E.A.

E.P.Y.E.A.

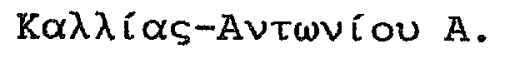

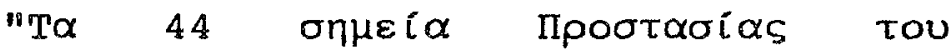

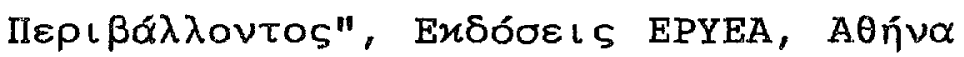
1977.

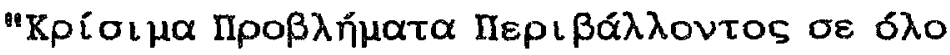

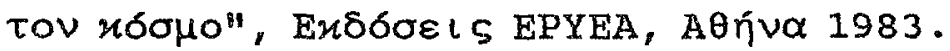

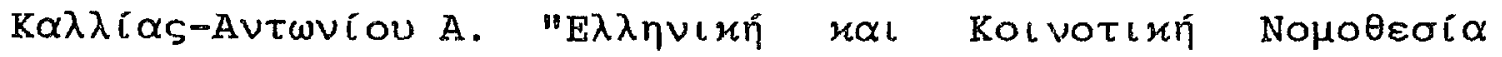

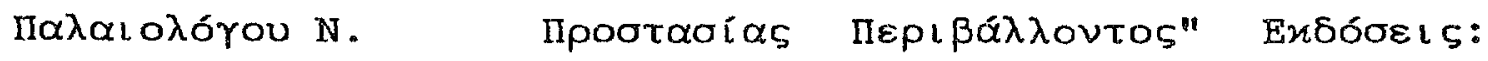

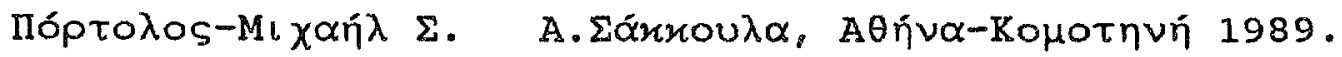

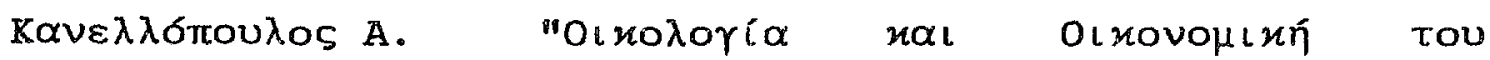

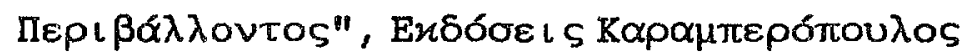

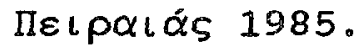

Kopoxẃotas I.

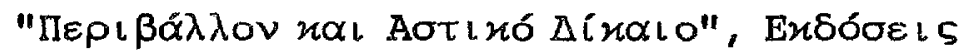

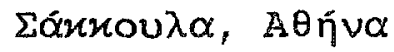




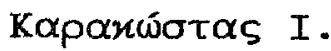

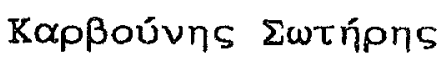

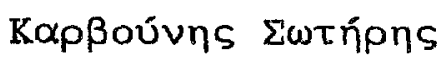

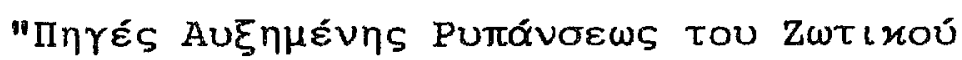

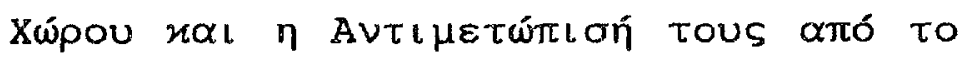

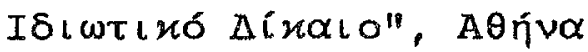

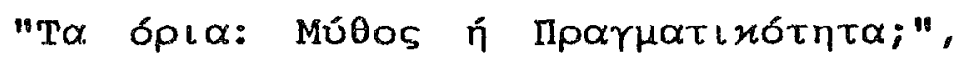

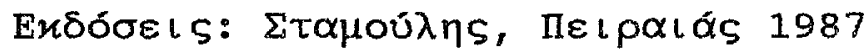

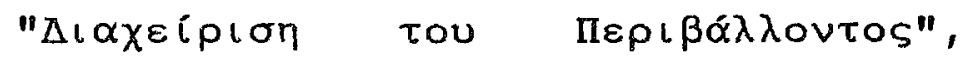

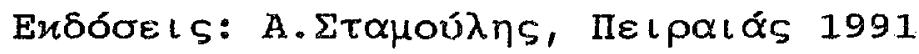

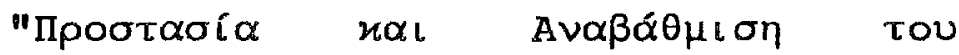

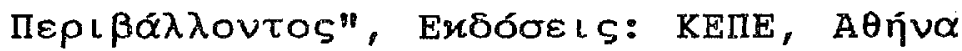
1986

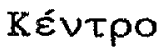

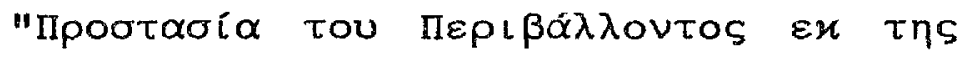

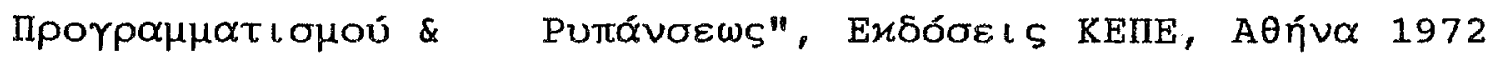

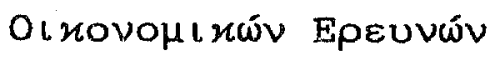

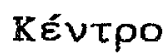

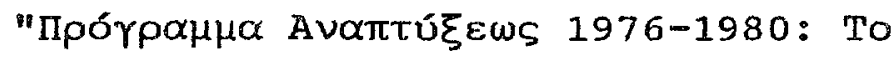

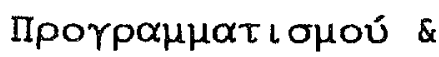

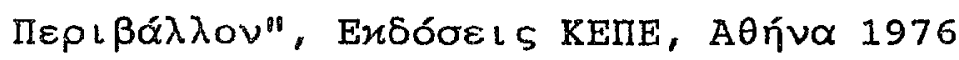

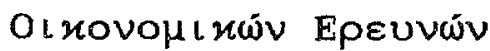


$\mathrm{K} \iota \lambda \iota x i \delta \eta \Sigma s$.

Kóvт l $\Lambda$ óoupa

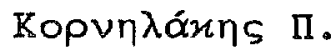

Kotoípns $\lambda$.

Kovïนて̧́s B.

KwotótTouגos B.

$\mathrm{K} \omega \tau \operatorname{too} \beta$ ívos $\mathrm{N}$.

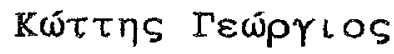

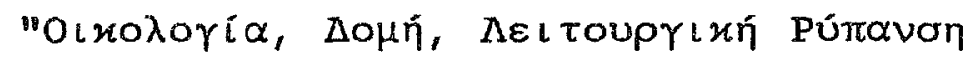

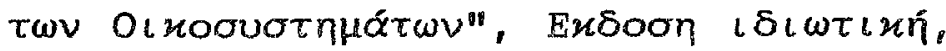
A日ñva 1979.

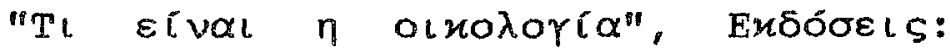
Aotépl, Aดñva 1979.

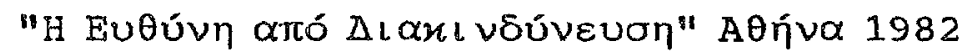

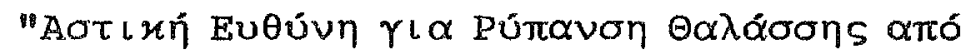

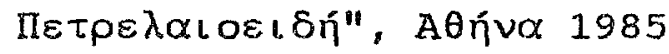

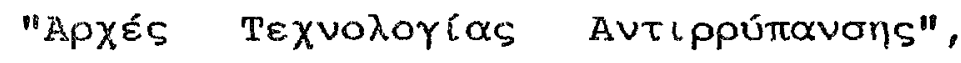

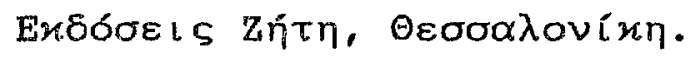

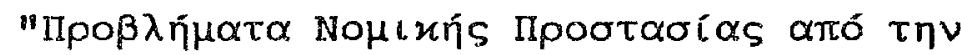

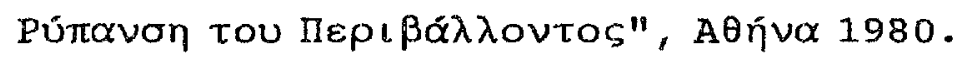

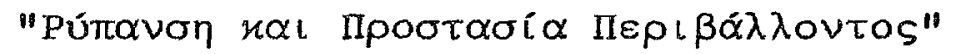

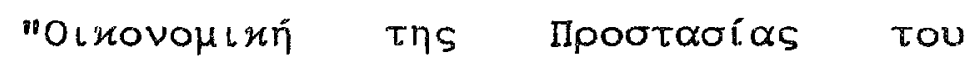

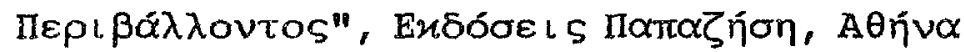
1975 
AEßE์นTท $\Gamma$.

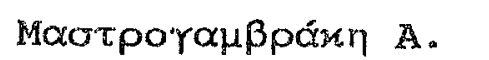

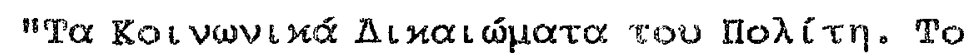

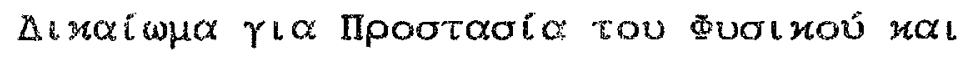

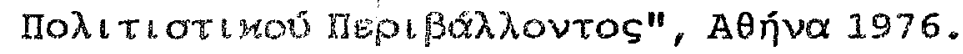

"Th

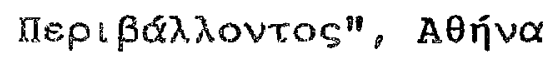

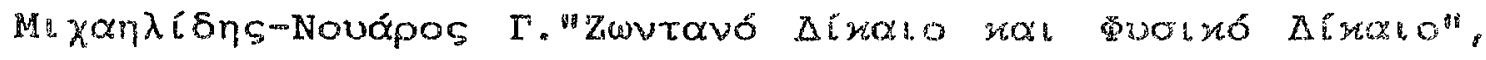
A 6 tivio 1982

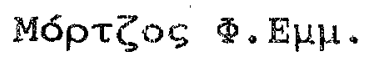

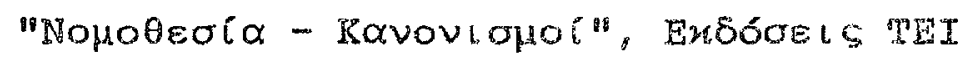
A67vos 1988 .

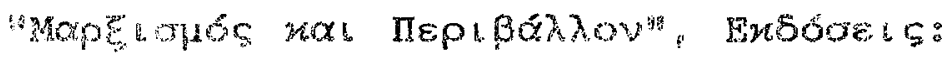

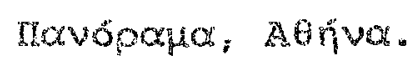

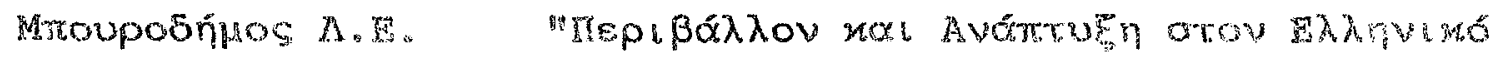

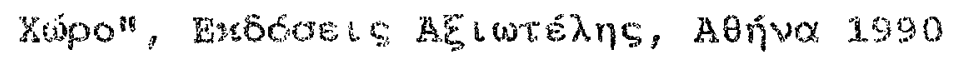

Mroútonい M. Póntepts A.

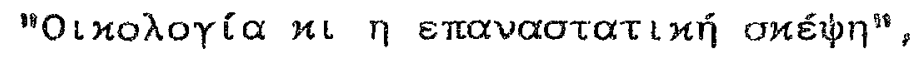

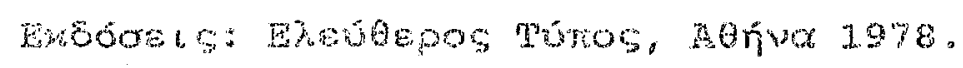


Nínas $\Delta$.

NTuนóv Pevé

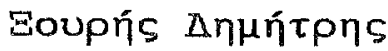

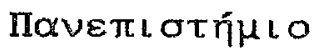

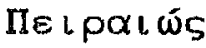

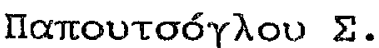

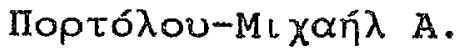

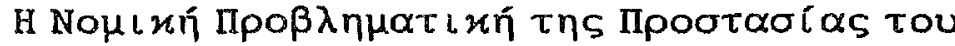

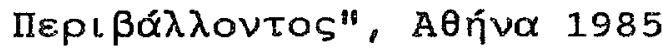

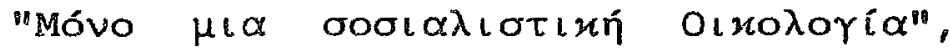

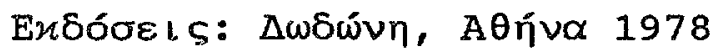

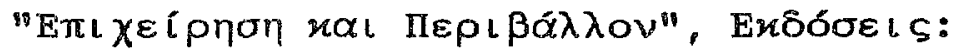

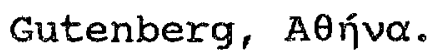

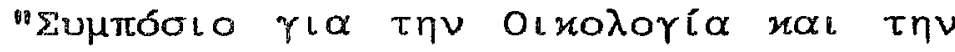

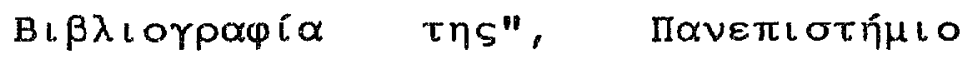

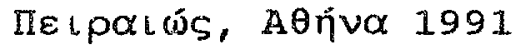

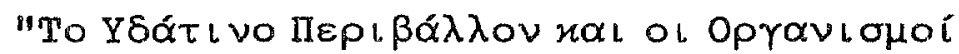

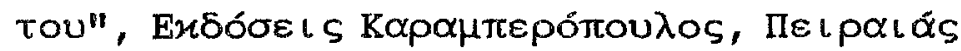

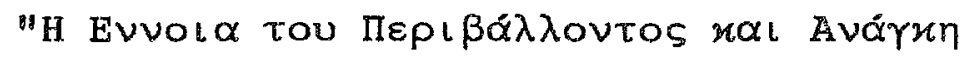

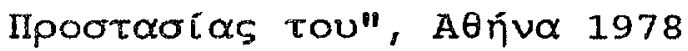

Pẃtกร B.

"Avoíruata tips Nohodorías rla tiv

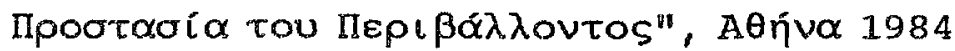




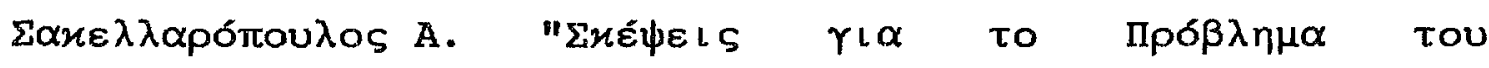

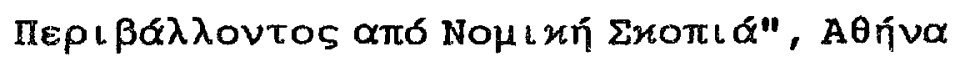
1982.

$\Sigma \alpha \mu \iota \omega ́ \tau \eta ⿻ ~ \triangle . \Gamma$.

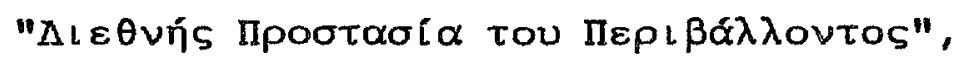

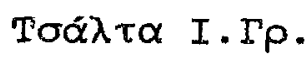

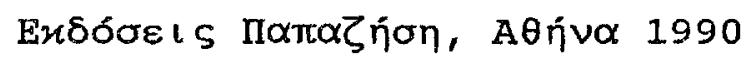

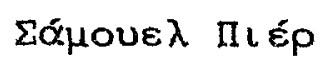

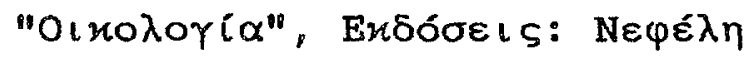

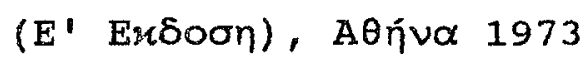

Táxos A.

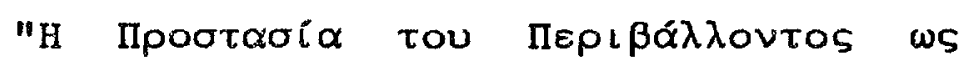

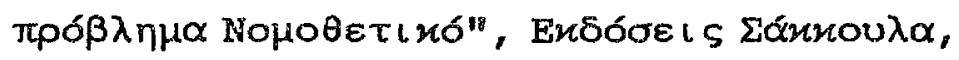
A iñua 1983.

Tóxos A.

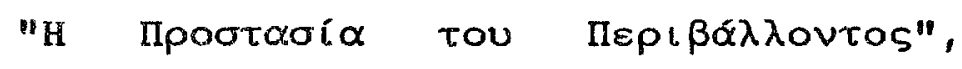

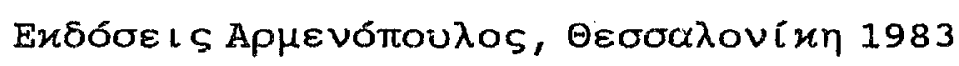

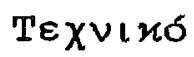

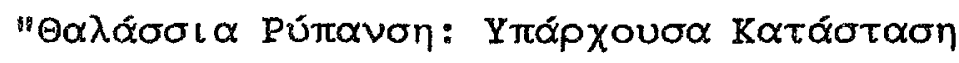

Eтเ $\mu \varepsilon \lambda \eta \tau \tilde{p}\llcorner о$

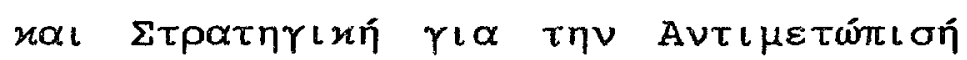

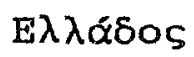

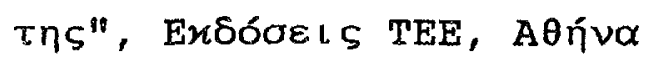

Tsakos Shipping

and Trading S.A. 
ФUTıóvos K.K.

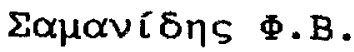

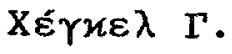

HELMEPA

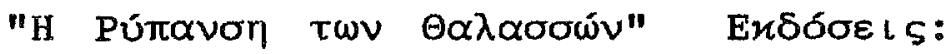

University studio Press, $\Theta \varepsilon \sigma \sigma \alpha \lambda o v i u \eta$

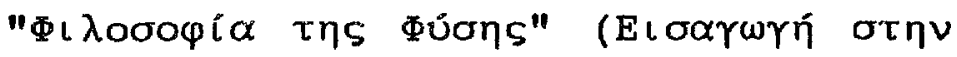

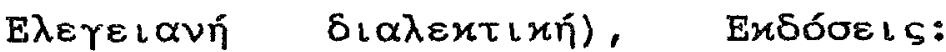

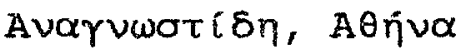

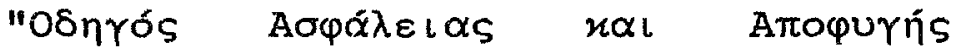

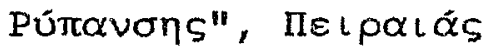


EANHNIKA IEPIOAIKA

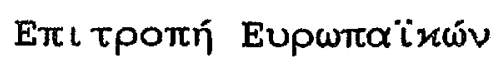
Kolvotńtwv

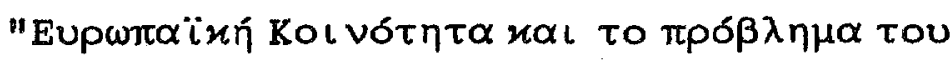

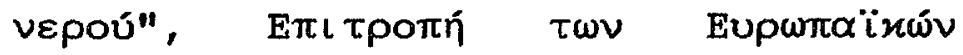
Kolvotítwv, 1987.

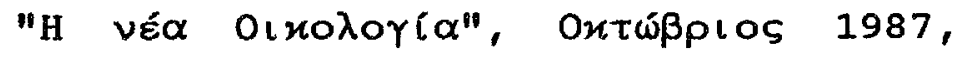
Teúxos 60 .

1987-1988

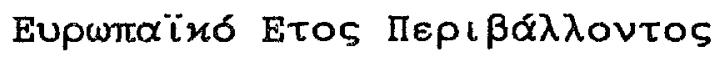

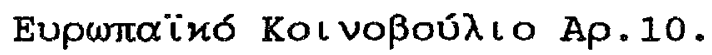




\title{
ВENH BIBAIOIPAФIA
}

\begin{abstract}
Abecassis W.David, "Oil Pollution from ships:
Jarashow L.Richard International, United Kingdom and United states Law and Practice". $2 \alpha$ ed. Stevens \& Sons, London 1985
\end{abstract}

Abecassis W.David IMO and Liability for Oil Pollution from ships: a retrospective. Lloyd's Maritime and Commercial Law Quarterly, Feb.1983, p. $45-49$.

\section{Bagwell David Asley}

"Liability under United States law for
spills of oil or chemicals from
vessels". Lloyd's Maritime and
Commercial Law Quarterly, Nov.1987,
p.488-522.




\begin{abstract}
Barnett C.Barry "Transnational pollution: agreement regarding marine pollution incidents. Agreement of Cooperation between the United States of America and the United Mexican states regarding pollution of the marine environment by discharge of hydrocarbons and other hazardous substances". Harvard International Law Journal, v.23, Spring 1982, p.177-185.

Bates John

"Oil pollution: "ships" and "liability". (The stolt Avance)". Lloyd's Maritime and Commercial Law Quarterly, Feb 1990, p. 14-16.
\end{abstract}

Baumol W.,

Becker L.Gordon
"The theory of environmental policy", Oates W. 1975 .

"Acronyms and Compensation for oil Pollution Damage from Tankers". Texas International Law Journal, v.10, summer 1983, p. 475-481. 
Bradley G.Paul

Brodecki $\mathrm{Z}$.

Brooks Jon Schuyler
"Marine oil spills: a Problem in Environmental Management". Natural Resources Journal, v.14, July 1974, p. $337-359$.

"New definition of pollution damage". Lloyd's Maritime and Commercial Law Quarterly, v.10, Aug 1985, p.382-391.

"Slick maneuvering: the Fifth circuit finds liability for oil pollution exists outside the Federal water Pollution Control Act". Maritime Lawyer, v.8, Spring 1983, p.171-186.

Brown

"Toward an economic theory of liability" Journal of Legal Studies, 1973, p. 323-349. 
Burrows, Rowley \& Owen

Burrows, Rowley \& Owen

Calabresi $\mathrm{G}$.

Caron D.David

Clausen Gerald
"Operational Dumping and the pollution of the sea by oil: An evaluation of preventive measures". Journal of Environmental Economy \& Management, 1974 p. 202-218.

"The Economics of Accidental Pollution by Tankers in Costal Waters". Journal of Public Economics, 1974, p.251-268.

"The costs of accidents: a legal and economic analysis", 1973.

"Liability for Transnational Pollution Arising from offshore Oil Development: A Methodological Approach" Ecology Law Quarterly, v.10, Spring 1983, p.641-683

"Liability for high seas oil pollution cleanup costs: domestic and international provisions". Hastings International and Comparative Law Review, v.3, Spring 1980, p.473-496. 
Cohen G.Lawrence

Davidson Neil

Davis Keith,

Blomstrom L.Robert

De Bievre F.M.Aline

Duncan M.William
"Revisions of TOVALOP and CRISTAL: Strong Ships for stormy Seas". Journal of Maritime Law and Commerce, v.18 No 4, October 1987, p.525-539.

"Marine pollution control: EEC and UK law". Journal of the Law Society of Scotland, v.27, Feb 1982, p.54(4).

"Business and Society: Environment and Responsibility". International student Edition. (3rd ed.).

"Liability and Compensation for Damage in connection with the carriage of Hazardous and Noxious Substances by Sea". Journal of Maritime Law and Commerce, v.17 No 1, January 1986, p. $61-88$.

"Liability of third parties for oil spill cleanup costs under the Federal Water Pollution Control Act and under General Maritime Law" . Maritime Lawyer, v.10, Spring 1985, p.25-45. 
Eaton R.Judith

Edelman S.Paul

Edelman S.Paul

Edelman S.Paul

Ehrlich R.Paul,
"Oil spill liability and compensationtime to clean up the law". George Washington Journal of International Law and Economics, v.19, Dec.1985, p. 787-827.

"Recent events in the Exxon Valdez disaster". New York Law Journal, v.203, May 1990, p.3 col.1.

"Pollution at sea-an update". New York Law Journal, v.192, Sept. 1984, p.1. $\operatorname{col} 1$.

"Since the Exxon Valdez hit the reef". New York Law Journal, v.202, August 1989, p.3 col. 1 .

"Population-Resources-Environment. Issues Ehrlich H. Anne on Human Ecology". W.H. Freeman and Company, San Francisco. 
Environmental

Law Reporter

Environmental

Policy \& Law

Fjermedal Glenn

Gallagher J.John
"Who pays for cleaning up the oil spill? Recent cases examine liability issues under the Water Act". Environmental Law Reporter, v.11, July 1981, p. 10140-10144.

"Information system for the control and reduction of pollution caused by the spillage of hydrocarbons and other harmful substances at sea". Environmental Policy \& Law, v.16 No 2, 1986, p. $87-88$.

"Federal oil spill fund legislation: a future standard". Albany Law Review, v.53, Fall 1988, p. 161-216.

Notes on the case of the S.S. Zoe Kolokotroni oil spill. Journal of Maritime Law and Commerce, v.12 No 2, November 1981, p.263-270. 
Gold Edgar

Goldie E.F.I.

Gunn T.Carter
International convention on $0 i l$ Pollution Preparedness Responce and Co-operation, 1990 Report". Journal of Maritime Law and Commerce, v.22 No 2 , April 1991, p.341-344.

"Liability for oil pollution disasters: international law and the delimitation of competences in a federal policy". Journal of Maritime Law and Commerce, v.6 No.3, April 1975, p.303-329.

"Limitation of liability: United States and convention jurisdictions". Maritime Lawyer, v.8, Spring 1983, p.29-68.

Guss Garland Bonnie "Interaction of the Federal water Pollution Control Act with the Limitation of Liability Act and the general maritime law" . Maritime Lawyer, v.6, Fall 1981, p.199-218. 
Hansen T.John,

w. Charles

Harrison F.Thomas

Hartje J.Volkmar

Hawks Neil

Hoffman T.Kevin
"Alaskan oil spill: legal fallout". Ray Trial, v.25, Oct 1989, p.26(7).

${ }^{10}$ Environmental Law: the growth and evolution of rights and liabilities". Chicago-Kent Law Review, v.56, winter 1980, p. 255-277.

"Oil Pollution Caused by Tanker Accidents: Liability Versus Regulation". Natural Resources Journal, v.24, Jan $1984, \mathrm{p} .41-60$.

"Oil pollution of the sea and the Concepts of Pollution and Environmental. Damage". Business Law Review, v.11, May 1980, p.139(3).

"The Campeche Bay blow-out: a strict liability approach to damages resulting from offshore drilling accidents". Fordham International Law Forum, v.3, Spring 1980, p.237-254. 
Jacobsen A.Douglas, Yellen D.James

Jacobsson Mans, Trotz Norbert

Johnston $R$.

Journal of

Maritime Law and

Commerce

Journal of

Maritime Law and

Commerce
"Oil Pollution: The 1984 London

Protocols and the Amoco Cadiz". Journal of Maritime Law and Commerce, v.15, No.4, October 1984, p.467-488.

"The Definition of Pollution Damage the 1984 Protocols to the 1969 Civil Liability Convention and the 1971 Fund Convention". Journal of Maritime Law and Commerce, v.17, Oct 1986, p.467-491.

"Oil pollution of the sea". Academic Press, 1976.

"International Convention on oil PollutionPreperedness, Responce and Co-operation, 1990. Documents". Journal of Maritime Law and Commerce, v.22 No 2. Apri1 1991, p.325-339.

"Protocol of 1984 to amend the International Convention on civil Liability for oil Pollution Damage, 1969". Journal of Maritime Law and Commerce, v.15, oct 1984, p.613-622. 
Journal of

Maritime Law and

Commerce

Juric Catherine

International

Legal Materials

International

Legal Materials
"Protocol of 1984 to amend the International Convention on the Establishment of an Inernational Fund for compensation for oil pollution Damage, 1971". Journal of Maritime Law and Commerce, v.15, Oct 1984, p.623-633.

"A Review of Liability Issues Arising from the Grounding of the oil Tanker, Amoco Cadiz". Trial Lawyer's Guide, v.31, Fall 1987, p.297-330.

"Draft protocol to the International Convention on civil Liability for oil Pollution Damage, 1969". International Legal Materials, v.23, Jan 1984, p. 177-195.

"Draft protocol to the International Convention on the Establishment of an International Fund for Compensation for oil Pollution Damage, 1971". International Legal Materials, v.23, Jan 1984, p.185-213. 
International

Petroleum Industry

Environmental

Conservation

Association

Kimball D.John

King John

Lummert

MacNeill J.
"The response of the oil industry to the problems of pollution of the sea from ships". Petroleum and Environmental Conservation, 1975, p. 199-213.

Pollution-Shipowner's Liability not limited by Limitation of Liability Act" . Journal of Maritime Law and Commerce, v.6 No 4, July 1975, p.661-664.

"Damages recoverable for environmental harm caused by an oil spill; Jurisdiction over foreign based insurers sued under a "direct action" statute". Journal of Maritime Law and Commerce, v.12, Jan 1981, p.263-273.

"Changes in Civil Liability concepts". Environmental Policy and Law, p.238. "Impacts of oil pollution". Environmental Policy and Law, v. 13 No $3-4,1984$, p. $87-95$. 
Maher E.Robert

McKaig Amy

Meese A. Sally

Milsten E.Donald "statutes of limitations in United states suits to recover oil spill cleanup costs under the Federal water Pollution Control Act". Fordham Law Review, v.53, March 1985, p.903-910.

"Liability for oil tanker spills". Southwestern Law Journal, v.44, September 1991, p.1599-1630.

"When Jurisdictional Interests collide: Internationa1, Domestic, and stateEfforts to Prevent Vessel source Oil Pollution". Ocean Development and International Law, V.12, winter-Spring1982, p.71-139.

"Enforcing International Law: USAgencies and the Regulation of 0 i 1 Pollution in American Waters". Journal of Maritime Law and Commerce, v. 6 No 2, January 1975, p.273-285. 
Nijkamp P.

Paulsen W.Gordon

Pearce D.

Pentis J.Carl

"Theory and applications of environmental economics", 1977.

"Why the United states should ratify the 1984 protocols of the International Conventions on civil Liability for oil Pollution Damages (1969) and the Establishment of an International Fund for Compensation for oil Pollution Damage(1971)". Forum, v.20, Fall 1984, p. 164-205.

"Environmental economics", 1976.

"A comparison between United States Domestic Pollution Acts and the International civil Liability and Fund Conventions". Northrop University Law Journal of Aerospace, Energy and the Environment, v.3, winter 1981, p. 155-179.

Posner R. "Economic analysis of Law" (2d ed.), 1977 . 
Preston D.Hilde

Raftopoulos G.Evan.
"Domestic and International Liability for the Bay of Campeche oil Spill". International Trade Law Journal, v.8, Fal1-winter 1980-81, p.55-76.

"The Mediterranean Action Plan in a functional perspective: a quest for law and policy". MAP Technical Reports SeriesNo.25, UNEP, Athens 1988.

Ramakrishna $K$. "Environmental Concerns and the New Law of the Sea". Journal of Maritime Law and Commerce, v.16 No 1 , January $1985, p \cdot 1-19$

Ramanlal Soni

"Control of Marine pollution in International Law". Inta \& Co. Lad, Cape Town 1985.

"New law puts strict liability on oil spilis; no question about it, corporations must pay for damages". The Los Angeles Daily Journal, v.99, Nov. 1988, p. 2 col. 2 。 
Ross Jaimie

Schenke ohare

schlesingex stwart,

Sanock. J. Edward

Schombaurif J. Thomas: "cleamp sost labitay for old sills: whener the mect (Federal Wor

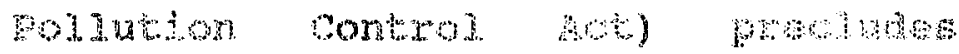
a termathe remed

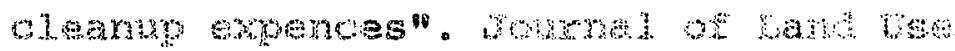

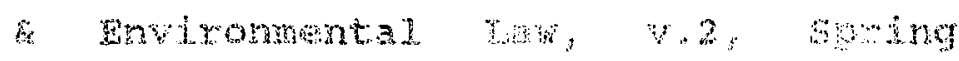
$1986, p, 1,63$

Wublity tox anates arising now an

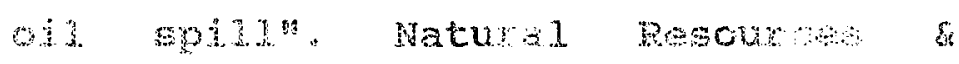

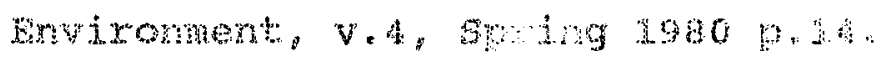

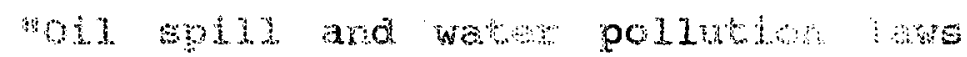
reviewed" New the Law "mman, v.201, May 2988, p. 3 1. 1 .

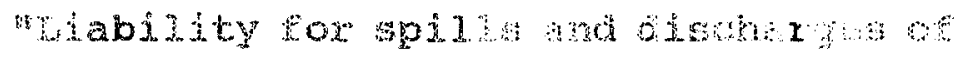
011 and harbous suberangs son vesseds". Forum, $1984, p \cdot 152-163$ 
Smets Henx

Straube Whohels

Sweeney C.Mary

Tiberg

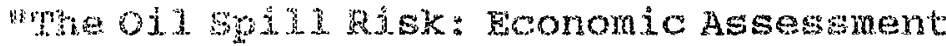
and conpenswhon Lint". Jourmal of Wawthe Lar and Commexce, $v .14$ No 1 , January 1983, $0.23-43$.

"ws ful compration possible for the damages resulting from the Exron valdezoil spind" Enviromental raw mepotsen, $10, \quad$ August 1989, p. $3.0338-20350$

Mabidit of shipower and other

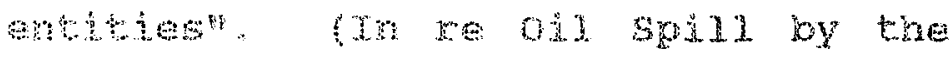
"Amoco Cadiz" off the coast of Franceon Waxch 16, agry). Trial Lawyex's Guide. 1. 20, sumer $1984,9.225 \times 243$.

Hugo "Oll poldution of the sea and the

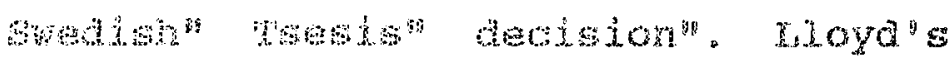

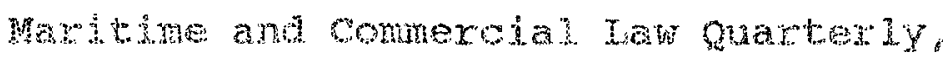
MaY $1984,218 \times 226$ 
United Nations

Environ. Prog. -

Intergovernmental

Dceanographic

Cormission

Van Hanswyk Beth

International

Wallace A.Sidney,

Ratcliffe L.Temple

Webb James
"Assessment of the state of pollution of the Mediterranean sea by petroleum hydrocarbons". MAP Technical Reports Series No. 19, UNEP, Athens 1988.

"The 1984 Protocols to the Convention on Civil Liability for oil Pollution Damages and the International Fund for Compensation for oil Pollution Damages: An Option for Needed Reform in United states Law". The International Lawyer, v.22, Summer 1988, p.31.9-343.

"Water pollution laws:can they be cleaned up? (Admiralty Law Institute: Symposium on American and International Maritime Law: Comparative Aspects of Current Importance)" . Tulane Law Review, V.57, June 1983, p.1343-1387.

"\$ 155 million Amoco Cadiz Damages". Chicago Dajly Law Bulletin, v.13, July 1990, p.1 col 2 . 
West M.James

Wood
"The Ixtoc I oil spill Litigation: Jurisdictional Disputes at the Threshold of Transnational Pollution Responsibility" . Texas International Law Journal, v.16, Summer 1981, p.475-532.

"Toward compatible International and Domestic Regimes of Civil Liability for oil Pollution in Navigable Waters". Environmental Report, issue 50, $1975, \mathrm{p} .116-145$. 